Institute of Inorganic Chemistry

Georg-August-Universität Göttingen

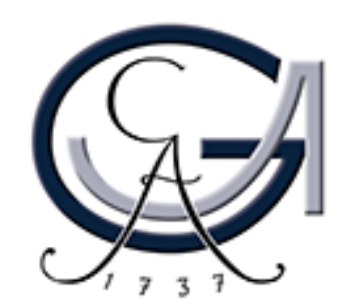

\title{
Metal-Mediated Coordination Cages: Endohedral Functionalization and Oligonucleotide Embedding
}

\author{
Dissertation \\ zur Erlangung des mathematisch-naturwissenschaftlichen \\ Doktorgrades \\ " Doctor rerum naturalium " \\ der Georg-August-Universität Göttingen \\ im Promotionsprogramm BioMetals \\ der Georg-August-University School of Science (GAUSS)
}

vorgelegt von

Marcel Krick

aus

Meschede

Göttingen, 2021 



\section{Betreuungsauschuss:}

Prof. Dr. Guido H. Clever

Institut für Anorganische Chemie, TU Dortmund

Prof. Dr. Franc Meyer

Institut für Anorganische Chemie, Georg-August Universität Göttingen

Prof. Dr. Claudia Höbartner

Institut für Organische Chemie, Julius-Maximilians-Unversität Würzburg

\section{Mitglieder der Prüfungskommission:}

Referent: Prof. Dr. Guido H. Clever

Institut für Anorganische Chemie, TU Dortmund

Korreferent: Prof. Dr. Franc Meyer

Institut für Anorganische Chemie, Georg-August Universität Göttingen

\section{Weitere Mitglieder der Prüfungskommission:}

Prof. Dr. Inke Siewert

Institut für Anorganische Chemie, Georg-August Universität Göttingen

Prof. Dr. Rikardo Mata

Institut für Physikalische Chemie, Georg-August Universität Göttingen

Dr. Holm Frauendorf

Institut für Organische und Biomolekulare Chemie, Georg-August Universität Göttingen

Dr. Michael John

Institut für Anorganische Chemie, Georg-August Universität Göttingen

Tag der mündlichen Prüfung: 26. März 2021 

Date of Creation: $\quad$ March 3, 2021

Supervisor: $\quad$ Prof. Dr. Guido Clever

Co-Supervisor: $\quad$ Prof. Dr. Franc Meyer 



\section{List of Publications and Conference Contributions}

\section{Publications}

- Carnarius, C.; Kreir, M.; Krick, M.; Methfessel, C.; Moehrle, V.; Valerius, O.; Brueggemann, A.; Steinem, C. \& Fertig, N., Green Fluorescent Protein Changes the Conductance of Connexin 43 (Cx43) Hemichannels Reconstituted in Planar Lipid Bilayers, The Journal of Biological Chemistry, 2011, 287, 2877-2886.

- Schulte, T. R.; Krick, M.; Asche, C. I.; Freye, S. \& Clever, G. H., Subtle backbone modifications control the interpenetration of dibenzosuberone-based coordination cages, RSC Advances, 2014, 4, 29724-29728.

- Frank, M.; Ahrens, J.; Bejenke, I.; Krick, M.; Schwarzer, D. \& Clever, G. H., LightInduced Charge Separation in Densely Packed Donor-Acceptor Coordination Cages Journal of the American Chemical Society, 2016, 138, 8279-8287.

- Krick, M.; Holstein, J.; Würtele, C. \& Clever, G. H., Endohedral dynamics of pushpull rotor-functionalized cages, Chemical Communications, 2016, 52, 10411-10414.

- Krick, M.; Holstein, J. J.; Wuttke, A.; Mata, R. A. \& Clever, G. H., TemperatureDependent Dynamics of Push-Pull Rotor Systems Based on Acridinylidene Cyanoacetic Esters, European Journal of Organic Chemistry, 2017, 2017, 5141-5146. 


\section{Awards}

- Poster prize for "Endohedral Dynamics of Push-Pull Rotor-Functionalized Cages" at the International Symposium for Macromolecular and Supramolecular Chemistry (ISMSC) 2017 in Cambridge.

\section{Conference Contributions}

- Talk, HeKKSaGon Summer School, 2014, Karlsruhe, Germany.

- Poster, Koordinationschemie Treffen, 2014, Kaiserslautern, Germany.

- Talk, IRTG 1422 Meeting, 2015, Katlenburg, Germany.

- Poster, International Symposium for Macromolecular and Supramolecular Chemistry, 2015, Straßbourg, France.

- Poster, IRTG 1422 Final Symposium, 2015, Göttingen, Germany.

- Poster, European Biologic Inorganic Chemistry Conference, 2016, Budapest, Hungary.

- Poster, Materials Chain, 2016, Bochum, Germany.

- Poster, SupraChem, 2017, Aachen, Germany.

- Talk, Tag der Chemie, 2017, Dortmund, Germany.

- Poster, International Symposium for Macromolecular and Supramolecular Chemistry, 2017, Cambridge, England. 


\section{Zusammenfassung}

Die kovalente Bindung zwischen zwei Atomen ist dafür verantwortlich, das diskrete Strukturen sich aus vielen Atomen bilden können und genau in dieser Anordnung bleiben und dem somit aufgebauten Molekül eine Stabilität und Lebensdauer verleiht. Lange Zeit war der Fokus der Chemiker auf das selektive und vor allem kontrollierte Brechen bzw. Ausbilden, solcher kovalenten Bindungen gesetzt. Jedoch wurde auch schnell klar, dass die Natur, wie wir sie kennen, ein hoch komplexes Konstrukt aus ineinander greifenden Prozessen ist, welche weder starr noch ausschließlich aus chemischen Reaktionen bestehen, indessen Bindungen gebrochen und wieder zusammengefügt werden. Abgeleitet aus dieser Erkenntnis, musste es weitere Bindungsarten geben, die solche Dynamiken innerhalb einer Struktur oder auch zwischen verschiedenen Strukturen ermöglichen. Genau mit solchen schwachen und reversiblen Bindungsarten beschäftigt sich der Bereich der supramolekularen Chemie, um die Welt hinter der kovalenten Bindung zu entdecken und zu verstehen. Mit Hilfe dieser Erkenntnisse können neue, größere und komplexere Konstrukte erschaffen werden, die völlig neue Eigenschaften und Möglichkeiten bieten, aber gleichzeitig auch helfen, die bio-chemischen Abläufe in der Natur zu verstehen und für die Menschheit zugänglich zu machen.
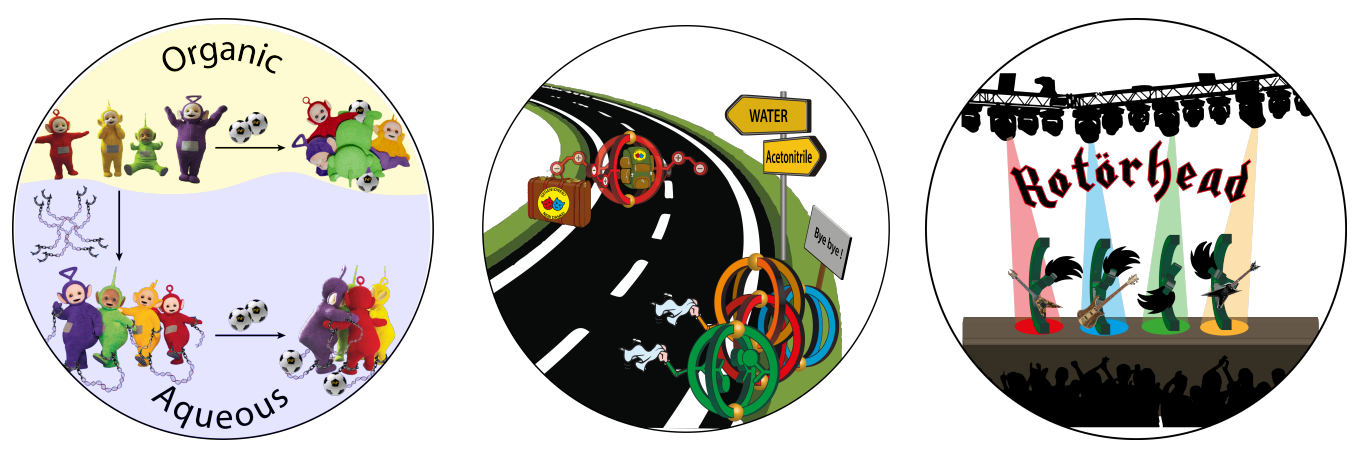

In dieser Dissertation werden drei Themen behandelt, alle stehen im Zusammenhang mit "Supramolekularen Koordinationskäfigen". Der erste Abschnitt beschäftigt sich mit der Integration von Ligandensystemen in artifiziell hergestellten Oligonukleotidstrukturen. Diese Ligandensysteme sind in der Lage in Anwesenheit von Metallkationen käfigartige Strukturen des Types $\left(\mathrm{L}_{4} \mathrm{M}_{2}\right)$ auszubilden, wodurch ein Konstrukt gebildet wird, der einen inneren Hohlraum beinhaltet. Dieser Hohlraum, kann abhängig von der Struktur, kleinere 
Moleküle aufnehmen und/oder Reaktionsabläufe begünstigen. Die Ausbildung dieser Käfigstruktur läuft zwar gerichtet, aber nicht kontrolliert ab. Das heißt, sobald mehrere unterschiedliche, aber strukturell ähnliche Liganden an der Assemblierung des Käfigs beteiligt sind, kommt es in den meisten Fällen zu einer unkontrollierten Zusammensetzung. Weder die Anzahl der einzelnen Liganden noch die Positionierung der Liganden im Käfig kann dabei gesteuert werden. Um ein kontrolliertes Zusammenfügen der Liganden im Käfig zu gewährleisten, wurden die Liganden in Oligonukleotidstränge eingebettet, welche sich selektiv und kontrolliert, abhängig ihrer Sequenzen zusammensetzen und ein stabiles, dennoch reversibles Gerüst aufbauen. So werden die Liganden im Zentrum dieser Gerüststruktur preorganisiert, um in einem abschließenden Schritt in Anwesenheit der Metallkationen einen Käfig auszubilden, welcher sowohl der Anzahl, als auch der Positionierung der Liganden im assemblierten Oligonukleotidgerüst entspricht. In diesem Projekt, sollte die allgemeine Machbarkeit eines solchen Systems gezeigt und gleichzeitig ein generelles Konzept geschaffen werden, um supramolekulare Käfige aus derivatisierten, aber strukturellverwandten Liganden selektiv und kontrolliert aufzubauen.

Im zweiten Abschnitt dieser Arbeit, wurde ein neuer Ansatz ausprobiert, basierend auf zwitterionischen Strukturen, um niedrig geladene supramolekulare Käfige aus dem organischen Lösungsmittel in reines Wasser zu überführen. Dazu wurde sich der Struktur der Sulfobetaine bedient, welche häufig auch in natürlich vorkommenden Verbindungen gefunden werden können und eine wichtige Rolle im Bereich der Tenside spielen. Der große Vorteil der hier eingesetzten Sulfobetaine ist die Selbststabilisierung der zwitterionischen Struktur, d.h. es werden keine zusätzlichen Gegenionen benötigt, wodurch dieses Motiv erstens relativ unabhängig von möglich koexistierenden Ionen und deren Konzentration ist und zweitens, in einem weiten $\mathrm{pH}$ Bereich existieren kann, ohne seine zwitterionische Form einzubüßen. Supramolekulare Käfige lassen sich mit unterschiedlichsten Ligandensystemen einfach und variabel zusammensetzen. Der hier beschriebene Käfig Typus $\left(\mathrm{L}_{4} \mathrm{M}_{2}\right.$, besitzt eine im inneren liegende Kavität, in der entweder kleinere Moleküle eingelagert oder sogar Reaktionen mit ungewöhnlicher Selektivität begünstigt werden, wodurch sie Interessant für ein breites Spektrum von Anwendungen im Bereich der medizinischen oder der katalytischen Chemie sind. Häufig jedoch sind diese Ligandensysteme sehr hydrophob, da ein rigides aromatisches Gerüst eingesetzt wird. Dadurch lassen sich die korrespondieren supramolekularen Strukturen nur in organischen Lösungsmitteln herstellen. Auch die gebildete Käfigstruktur, zeigt anschließend keine besonders vorteilhafte Affinität zu hydrophilen Umgebungen, wodurch sie sich nicht in vivo oder anderen wässrigen Umgebungen verwenden lassen. Ziel der Chemie, besonders im Bereich der "Grünen Chemie" ist es jedoch, den Verbrauch von organischem Lösungsmittel zu reduzieren und stattdessen vermehrt Wasser als Medium zu verwenden. Durch die Einführung des hier beschriebenen Ansatzes, könnten viele dieser bis dato hydrophoben supramolekularen Systeme in Wasser überführt werden, ohne die Käfigstruktur und deren Funktion massiv zu beeinflussen. 
Im dritten Abschnitt dieser Arbeit, wurde versucht ein System zu entwickeln, welches ermöglicht, dem System nach innen gerichtete funktionelle Gruppen hinzuzufügen. Ziel dieses Ansatzes ist es, die innen liegende Kavität der Käfigstruktur zu nutzen, um dort Moleküle aufzunehmen und anhand der zur Verfügung stehenden funktionellen Gruppen, eine selektive Bindung bestimmter Moleküle zu ermöglichen bzw. Reaktionen mit Hilfe der nach innen gerichteten funktionellen Gruppen zu erreichen. Dazu wurde das aus dem ersten Abschnitt bekannte acridon-basierende Ligandensystem modifiziert, sodass sich ein Käfig mit nach Innen gerichteten funktionellen Gruppen bilden konnte. Dabei wurde eine nicht erwartete Dynamik entdeckt, welche zunächst an einem Ligandenderivat mit reduzierter Komplexität intensiv untersucht und in einem zweiten Schritt, in eine Käfigstruktur transferiert wurde. Die untersuchte Dynamik wird durch ein intramolekulares elektronisches Push-Pull System verursacht, welches sich zwischen dem elektronenreichen Teil des Moleküls und dem elektronenarmen endohedralen Rotor einstellt und so zu einer temporären Ladungstrennung innerhalb der Struktur führt. Dadurch wird die Bindungsordnung der formalen Doppelbindung reduziert, sodass diese einen Einzelbindungscharakter erhält und eine temporäre Rotation um die verbrückende Bindung ermöglicht. Durch systematische Veränderung der äußeren Einflüsse und Derivatisierung des Moleküls, konnte gezeigt werden, dass die Rotationsrate stark abhängig von der Stabilisierung der zwitterionisches Form ist. Die Existenz konnte zusätzlich bewiesen werden, indem die negative Ladung in Anwesenheit von starken Säuren eine reversible Protonierung aufwies. Durch die Protonierung hat sich ein hoch-fluoreszierende positiv-geladene Struktur gebildet, welche durch seine markante Adsorptionsbanden, als Acridinium-Ion identifieziert werden konnte. Durch die Integration des Push-Pull Systems in die Ligandenstruktur, ging diese Eigenschaft verloren, jedoch blieb die Rotationsdynamik. Letztere konnte ebenfalls im gebildeten supramolekularen Käfig nachgewiesen werden, wo sich die nach innen-gerichteten Rotoren weiterhin drehten. Interessanterweise konnten trotz der nach innen gerichteten Gruppen, kleinere Moleküle reversible eingelagert werden. Die Einlagerung von Moleküle hatte einen signifikanten Einfluss auf die Rotationsraten, welcher durch geeignete VT-NMR Experimente nachgewiesen werden konnte. 



\section{Contents}

I Summary 1

II General Introduction 7

\begin{tabular}{lll}
\hline 1.1 & Supramolecular Chemistry \\
\hline
\end{tabular}

II.1.1 History . . . . . . . . . . . . . . . . . . . . . . . . . . . . . . . . . . . . . . . . 8

II.1.2 Concepts of Supramolecular Chemistry $\ldots \ldots \ldots \ldots$

II.1.3 Supramolecular Coordination Cages $\ldots \ldots \ldots \ldots$

\begin{tabular}{|lll}
11.2 & Desoxyribonucleic acid
\end{tabular}

II.2.1 Structural Motifs . . . . . . . . . . . . . . . . . . . . . . . . . . . 26

$\llbracket 1.2 .2$ Holliday Junction $\ldots \ldots \ldots \ldots$

III Ligands Embedded in Oligonucleotide Scaffolds 39

\begin{tabular}{lll}
\hline 11.1 & Introduction & 40
\end{tabular}

III.2 Results and Discussion 46

III.2.1 Idea and conception $\ldots \ldots \ldots \ldots$

III.2.2 Acridone-based ligand embedded in Holliday junction . . . . . . . . 48

III.2.3 Anisol-based ligand embedded in Holliday junction . . . . . . . . . . 66

\begin{tabular}{llll}
\hline 11.3 & Conclusion & 82
\end{tabular}

IV Water-soluble Cages 85

\begin{tabular}{lll}
\hline IV.1 & Introduction & 86
\end{tabular}

\begin{tabular}{lll}
\hline IV.2 & Results and discussion & 91
\end{tabular}

IV.2.1 Diethanolamin methoxy ligand . . . . . . . . . . . . . . . . . . . . 91

IV.2.2 Diethanolamin hydroxy ligand $\ldots \ldots \ldots \ldots \ldots$ 
IV.2.3 Sulfobetaine methoxy ligand . . . . . . . . . . . . . . . . . . . . . . 99

\begin{tabular}{|lll}
\hline IV.3 Conclusion & 104 \\
\hline
\end{tabular}

V Endohedrally Functionalized Cages 107

\begin{tabular}{lll}
\hline V.1 & Push-Pull Rotor Systems based on Acridinylidene Cyanoacetic Esters & 108
\end{tabular}

V.1.1 Introduction . . . . . . . . . . . . . . . . . . . . 109

V.1.2 Results and discussion . . . . . . . . . . . . . . . . . . . . . . . . 111

V.1.3 Protonation-Induced Fluorescence . . . . . . . . . . . . . . . . . . 136

V.1.4 Conclusion . . . . . . . . . . . . . . . . . . . . . . . . . . . . . . . . . . . . . . . . . . . 149

V.2 Endohedral dynamics of push-pull rotor-functionalized cages 151

V.2.1 Introduction . . . . . . . . . . . . . . . . . . . . . . . . . . 152

V.2.2 Results and discussion . . . . . . . . . . . . . . . . . . . 156

V.2.3 Conclusion . . . . . . . . . . . . . . . . . . . . . . . . . . . . . . 176

V.3 Azacrown-Cyano-Acridinylidne-Ethyl Acetate 177

V.3.1 Introduction . . . . . . . . . . . . . . . . . . . . . . 178

V.3.2 Results and discussion . . . . . . . . . . . . . . . . . . . . . . . . 179

V.3.3 Conclusion and Prospects . . . . . . . . . . . . . . . . . . . . . 190

VI Experimental Section 193

\begin{tabular}{|lll} 
VI.1 Syntheses of Oligonucleotide Incorporable Ligands & 194
\end{tabular}

VI.1.1 Synthesis of the Acridone-based Ligand . . . . . . . . . . . . . . . . . . 195

VI.1.2 Syntheses of the Methoxybenzylamine-based Ligand . . . . . . . . . . . . 214

\begin{tabular}{|lll}
\hline VI.2 & Syntheses of Water-Soluble Ligands \\
\hline
\end{tabular}

VI.2.1 Syntheses of Sulfobetaine Ligand . . . . . . . . . . . . . . . . . . . . . . 232

VI.2.2 Syntheses of Hydroxybenzylamine-based Ligand . . . . . . . . . . . . . . 239

\begin{tabular}{|lll}
\hline VI.3 Syntheses of Acridinylidene derivatives & 253 \\
\hline
\end{tabular}

VI.3.1 Syntheses of Backbone Derivatives . . . . . . . . . . . . . . . . . 254 25

VI.3.2 Syntheses of Ligand Derivatives . . . . . . . . . . . . . . . . . . . . . . 262

VI.3.3 Syntheses of Cage Derivatives . . . . . . . . . . . . . . . . . . 274

VI.3.4 Syntheses of Azacrown Ethyl Acridinylidine . . . . . . . . . . . . . . . . 281 
A Further Analytical Data 294

A.1 Oligonucleotides for Unmodified Holliday Junction MKDH01um. . . . . . 297

A.2 Oligonucleotide for Holliday Junction MKDH01 _ . . . . . . . . . . 306

A.3 Oligonucleotide for Holliday Junction MKDH03 … . . . . . . . . 315

A.4 Oligonucleotide for Holliday Junction MKDH04 … . . . . . . . . 320

A.5 Oligonucleotide for Holliday Junction MKDH05 . . . . . . . . . . 325

A.6 Oligonucleotide for Holliday Junction MKDH06 _ . . . . . . . . . . 330

A.7 Further Data . . . . . . . . . . . . . . . . . . . . . . 335

A.8 Crystal Data and Structure Refinement . . . . . . . . . . . . 345

B Methods 347

B.1 DNA Solid State Synthesis . . . . . . . . . . . . . . . . . . 349

B.2 Oligonucleotide Purification $\ldots \ldots \ldots \ldots \ldots \ldots$

B.3 Oligonuecleotide Characterization . . . . . . . . . . . . . 352

B.4 Spectroscopic Methods . . . . . . . . . . . . . . . . . . . 354

B.5 PAGE Gel Electrophoresis . . . . . . . . . . . . . . . . 357

B.6 $\quad$ NMR Line-Shape Analysis $\ldots \ldots \ldots \ldots \ldots$

\begin{tabular}{lll}
\hline & Overview & 360
\end{tabular}

C.1 List of Figures $\ldots \ldots \ldots \ldots \ldots \ldots$

C.2 List of Schemes . . . . . . . . . . . . . . . . . . . . 365

C.3 List of Tables . . . . . . . . . . . . . . . . . . . 367

C.4 Abbreviations . . . . . . . . . . . . . . . . . . 368

References 

Summary

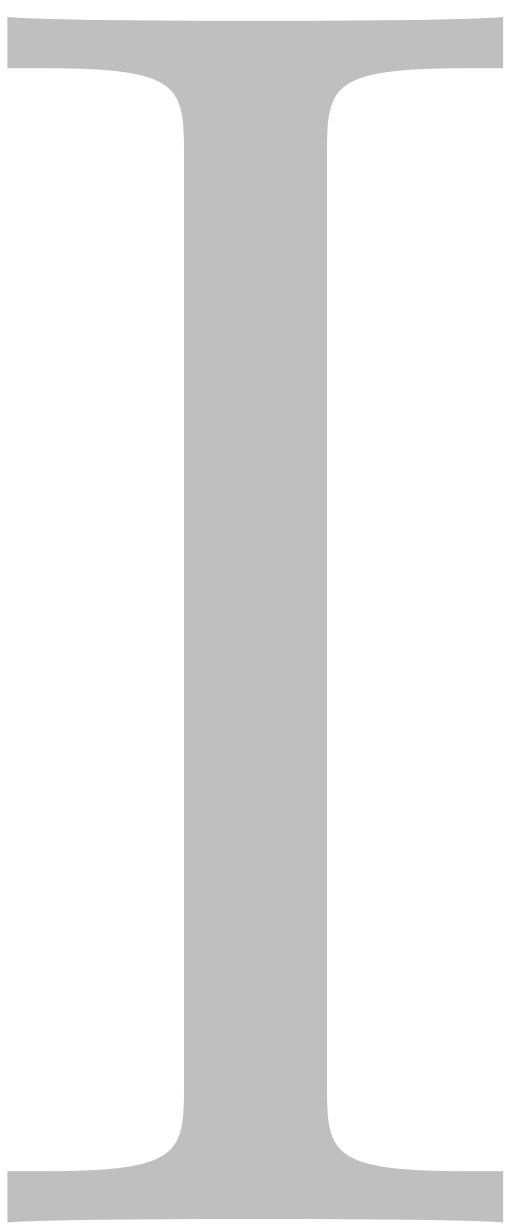


Enzymes are nature's workhorses, holding a vast variety of functional properties for essential processes in life, including catalyzing reactions, transporting molecules or being an auxiliary in protein/RNA folding. Especially, the catalytic properties of enzymes produced a lot of interest in all areas of science, reaching from biology, over physics and chemistry and is even found nowadays to be part of informatics. In the chemistry the catalytic functions of enzymes have long been in the focus of organic and bio-inorganic scientists, who accumulated their focus of interest rather on the very core mechanism and coordination between active center and reagents, but neglected the active sites shell. The contributions of the spatial arrangements and the involved inter- and intramolecular interactions are essential for the allover enzymes functionality. Although consisting of rather simple building units, connected by covalent bonds, the non-covalent inter- or intramolecular forces, make them become extremely versatile in shape and function. The field of supramolecular chemistry is investigating such interactions and scientist put all their effort in understanding this chemistry in all its facets, in order to be able to mimic and reach natures functionalities and beyond. Starting with pure organic compounds, soon after, the area of supramolecular chemistry was extended by using metal coordination motifs, what greatly expanded the design of possible structural topologies, especially with respect to three-dimensional arrangements. Besides, also the combination with natural occurring building blocks promoted the creation of new functional structures, giving new information and insights to understand why nature's workhorses have evolved in the way they are and how the enzymes' unique functions can be made accessible to mankind.

This work covers three projects dealing with the functionalization of supramolecular metal-mediated coordination cages. In the first two parts, two approaches, based on exohedrally functionalized ligands, are discussed. The first one aims for a controlled assembly of heteroleptic coordination cages. Those cages are interesting to create endohedrally functionlized cages, acting a molecular reaction vessel and mimicking the active site of an enzyme. In order to be able to control the arrangement of structurally related ligands into a cage structure, the assembly process is assisted by an externally attached DNA scaffold. In the second project, the anisole-based ligand design of the first project was reused to synthesize exohedrally modified charged and neutral ligands. These ligands are investigated for their ability to form low-charged water-soluble coordination cages in the presence of palladium(II) salts. In the last chapter the acridone-based ligand, taken from the first project, is endohedrally functionalized with various cyano acetate ester derivatives. The ligands, as well as their corresponding palladium-mediated coordination cages, are showing an unexpected but interesting intramolecular rotational motion. This motion is induced by a charge-separated state within its intramolecular push-pull system and can be significantly effected by various external influences, including guest encapsulation within the cage's cavity. 


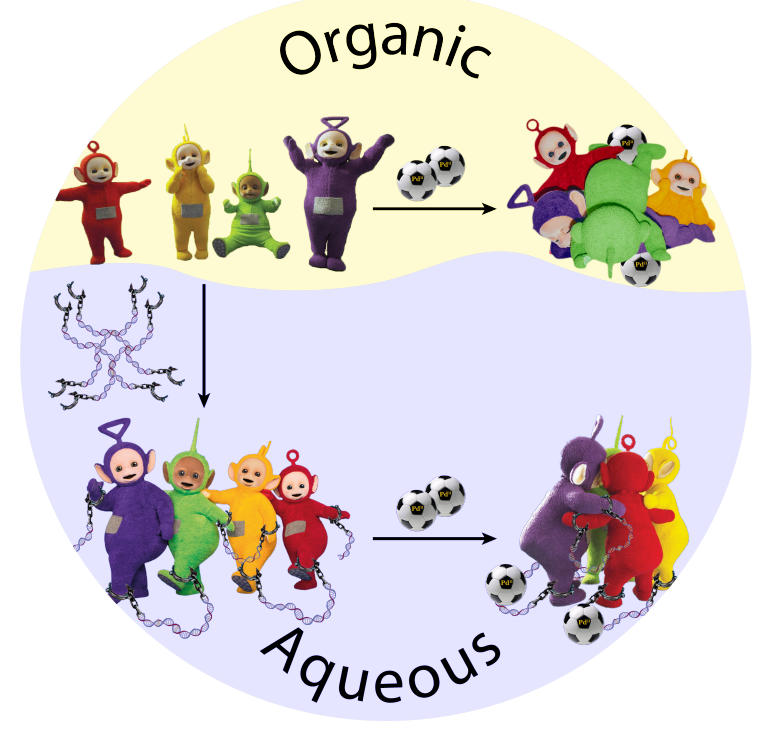

PART 1 The idea of a DNA scaffold supported assembly for a supramolecular architecture, is an approach to overcome the problem of statistical mixtures during the assembly of supramolecular heteroleptic metal-mediated coordination cages, from structurally related ligands. The system was designed to preorganize the involved ligands in a square-planar fashion by the preceded formation of an external DNA scaffold. For that purpose acridoneand anisole-based ligands were designed, synthesized and subsequently incorporated into DNA single strands, capable of forming a Holliday junction (HJ). The Holliday junction is a cruciform-shaped canonical DNA architecture, where the ligands shall be located at the central crossing section. In order to prove the approach on its feasibility and functionality, the following steps had to be accomplished.

In a first step the ligand was synthesized, including linkers and protecting groups, crucial for DNA solid phase synthesis, accompanied by a full characterization via NMR and ESI-MS and other methods. Followed by the incorporation of ligands into the respective DNA sequences with subsequent chromatographic clean-up and characterization by ESI-MS and common spectroscopic methods. As a third step, the assembly of the intended DNA structure was proved with help of thermal analysis, electrophoreses, fluorescence and circular dichroism spectroscopy. In sum, two ligand-containing Holliday junction systems were proved to be formed under the given conditions and their thermal stability were compared to an "unmodified" Holliday junction (containing no ligands), as a reference. In a last step the assembly of the intended metal-mediated coordination cage was investigated, in presence of the respective metal salts and the established conditions, aiming to achieve the proof of principle for the actual system.

None of the used transition metals gave a sufficient evidence for the formation of the intended embedded supramolecular coordination structure. However, the acridone-containing HJ exhibit an increase in denaturation temperature $\left(\mathrm{T}_{\mathrm{M}}\right.$ of $13{ }^{\circ} \mathrm{C}$ compared to the unmodified HJ. Moreover, an observable bathochromic shift in the UV-vis and fluorescence spectrum 
indicates the formation of a stable ligand involved J-aggregate. The aggregation of ligands does not only explain the increase of $\mathrm{T}_{\mathrm{M}}$, but also the inertness towards the metal cations. Moreover, a chiral transfer from the exterior DNA scaffold on the ligand aggregates within the Holliday junction is indicated by signals in circular dichroism spectra, vanishing upon complete disassembly. Although the formation of the embedded coordination structure could not be proved, to the best of our knowledge the here presented system is the first example of a fully assembled $\mathrm{HJ}$, with artificial organic compound incorporated at its central crossing section and moreover, also exhibit a stabilization due to these artificial modifications. Apart from that, the system bears a huge potential for the design of an easily accessible scaffold for the investigation of uncommon aggregation partners in solution.

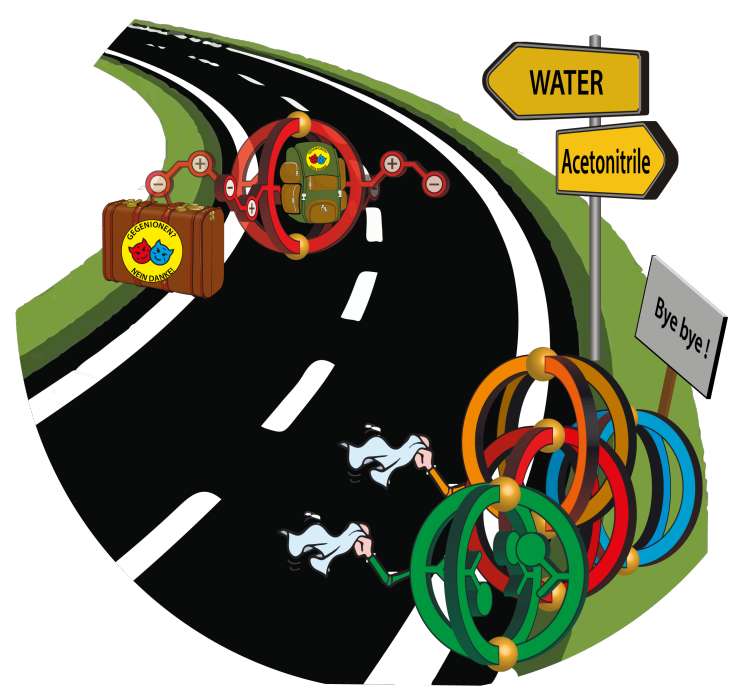

PART 2 The anisole-based ligand, that was also used for the DNA scaffold topic in part I, was exohedrally modified with either a diethanol amine group (neutral ligand) or a sulfobetaine motif (zwitterionc ligand). By introducing the polar groups, the allover polarity of both ligands is significantly increased. Thus, both can be solved in polar organic solvent or even aqueous medium. Furthermore, both ligands still allow the formation of supramolecular metal-mediated coordination cages, even in pure water. In addition, it was tried to introduce an endohedral functionality to the system, in form of phenolic hydroxyl group by simple deprotection of the anisole's inward pointing methoxy ether. The deprotection resulted in an unwanted cyclization reaction, giving an asymmetric ligand, that was not able to form any metal-mediated coordination structure at all. Although the approach of bringing coordination cages into aqueous medium by attaching polar residues, has been shown in literature before, the usage of self-stabilizing zwitter-ionic motifs, in order to create low-charged water-soluble coordination cages, has not been shown yet to the best of our knowledge. The synthesized ligands and cages were characterized by common analytical methods, as NMR, ESI-MS and further spectroscopic techniques. Unluckily, 
the here presented cages did not show any evidence of guest encapsulation, with a small selection of neutral guest molecules, probably due to the lack of space within the cavity. To overcome this problem, the ethynyl bridges could be exchanged i.e. with phenyl linkers, slightly increasing the size of the endohedral void. With this change, the unwanted described cyclization reaction would be probably obliterated as well. However, still the zwitter-ionic approach could be easily transferred on other supramolecular architectures, making it possible to investigate the host-guest chemistry or related functionalities in an aqueous environments, 1 providing for sure a quantity of new systems for the field of green chemistry.

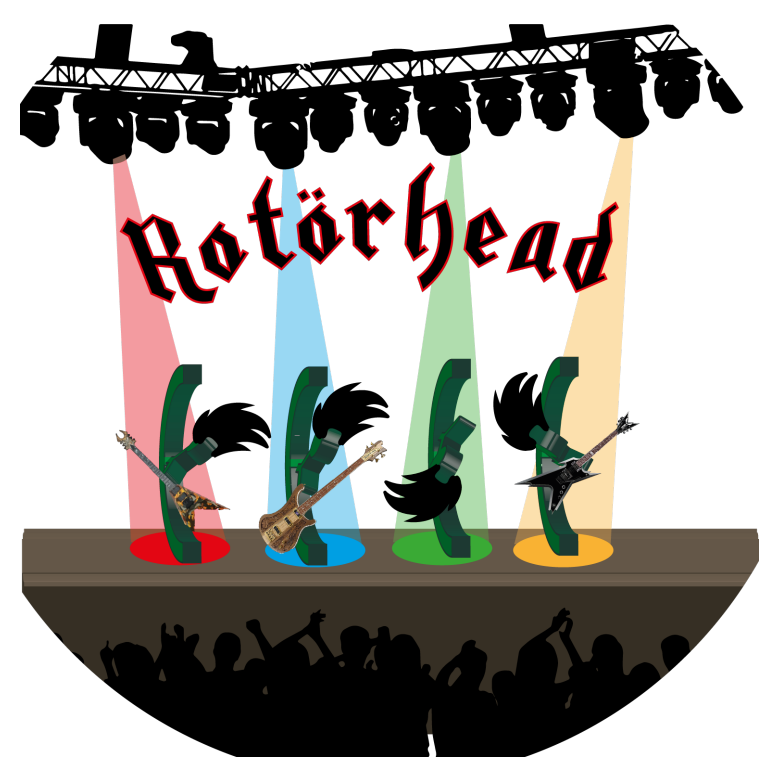

PART 3 In the last part an endohedral modification of the acridone-based ligand, related to the ligand used in part I, with various cyano acetate esters is discussed. All derivatives showed an unexpected intramolecular rotational motion, caused by a push-pull system with a certain degree of charge separation, between the acridone nitrogen and the opposed cyano acetate ester. This motion was thoroughly investigated on the modified acridone backbone, before it was transferred into a bis-dentate ligand-system and subsequently into a supramolecular palladium-mediated coordination cage. Interestingly, under certain conditions the charge-separated state within the acridone predominates, so that the negative charge could even function as a weak base. At this stage the system can be reversibly protonated in the presence of strong acids, forming a highly fluorescent cationic acridinium motif. The whole process of protonation was investigated in detail by NMR, UV-, fluorescence spectroscopy and mass spectrometry. Besides the interaction with strong acids, the influences of the various used ester derivatives, as well as other external influences on the intramolecular electronics, as for example temperature and solvent polarity, were studied as well. It became apparent that all these above mentioned influences have either more or less influence on the intermolecular electronics of the push-pull system, giving a tool for the 
adjustable control of the rotational motion. Based on these findings a system was designed in order to control the rotational motion by external stimuli. For that reason, the $\pi$-system was extended, ending in an azacrown ether motif, capable of interacting with metal cations. This interaction is intended to form an opposed electron-withdrawing motif, being in competition with the actual electron-withdrawing cyano acetate ester. The influence of the various metal cations on the intramolecular push-pull system was investigated by variable temperature NMR methods, as well as UV-vis spectroscopy. Nearly all investigated metal cations showed a clearly observable interaction with the azacrown ether, tracked by NMR and UV-vis spectroscopy. Yet, the effect on the intramolecular electronics was moderate and even not detectable for the rotational motion. As an exception, the protonation of the azacrown ether with strong acids is to be mentioned. Nevertheless, latter is not applicable for the usage with metal-mediated coordination cages, since strong acid can result in a disassembly of those structures. However, since some results indicating a small influence on the rotational motion, it is worth to continue the research on this topic, in particular since this approach could be used as a good starting point for several new systems in the field of supramolecular functional units, 233 molecular sensing 4 or organic light-emitting diodes (OLED). $[5$ 
General Introduction 


\section{chapter II. 1}

\section{Supramolecular Chemistry}

The main focus in the area of supramolecular chemistry is the design and creation of functional systems, assembled from smaller building units to a highly complex architecture with distinct functionalities. Compared to the classical synthetic chemistry, where it is the measure of all things to form a new covalent bond between two atoms, supramolecular chemistry utilize the non-covalent interactions between two molecules, to create uncommon spatial arrangements. As so often before nature is one of the most important paragons for the scientists and gave inspiration for structural motifs in the field of supramolecular chemistry. On the other hand, supramolecular structures are used to create biomimetics in order to understand the by far advantageous and well-developed functional units in biological systems, as for instance enzymes. Moreover, by combining artificial structural motifs from the supramolecular chemistry with natural occurring building block, the field of nanotechnology was pushed on a new level in the last years.

\section{II.1.1 History}

The field of supramolecular chemistry has its roots go back to the end of the 19th century, 6 but first became in 1987 officially an own field of research, after Jean-Marie Lehn, who later was awarded with the Nobel prize together with Cram and Pederson, introduces the term supramolecular chemistry in 1978. But also before some important findings for the field of supramolecular chemistry were made, which are still contemporary and frequently investigated in the modern supramolecular chemistry. In 1894, already Emil Fischer proposed the concept of lock and key, what is a commonly used model in biology and medicine as well as supramolecular host guest systems. 1953 the structure of DNA was solved by Rosalind Franklin as well as Crick and Watson, the latter both received the Nobel Prize for their work. Curtis, Busch and Jäger reported between 1961 and 1964 new 
macrocycles based on Schiffs bases, coordinating to various transition metals. Some years later, in 1967, Pederson published the first crown-ether, able to bind alkali and earth-alkali metals, making it possible to transfer them into organic solvents. 1969 the first cryptand was synthesized by Lehn, a three-dimensional analogon to the crown ether, but published it several years later. 8 Some years later, in 1973, Cram reported the synthesis of the first spherand, a more rigid version of a cryptand that provides a almost spherical preorganized coordination environment for enveloped cations.

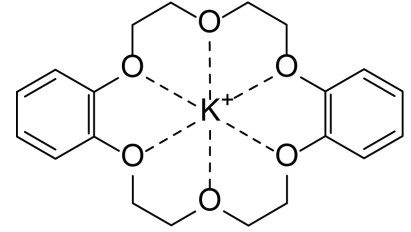

Pederson, 1967

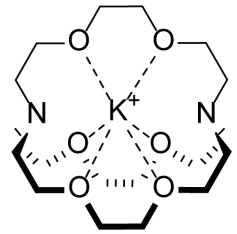

Lehn, 1978

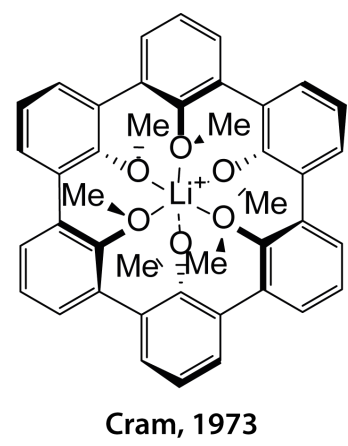

Cram, 1973

Figure II.1.1.1: Three supramolecular structrues, the noble prize winners Pederson, Cram and Lehn were honored for. From left to right: Crownether (Pederson, 1967), 7$]$ Cryptand (Lehn, 1978), 9] Spherand (Cram, 1973). 10]11]

These structures mark the birth of supramolecular chemistry. Since then, supramolecular chemistry did not only stick to the coordination of metal cations, but evolved to an area of research which bears the possibility to innovative architectures with high diversity and complexity for versatile applications. Starting from novel approaches for the directed formation of new topologically interesting complexes, modern supramolecular chemistry also provided the tools for potential systems as molecular devices and machines, catalytic molecular flasks and hosting units for molecular recognition, but also as containers for drug transport.

In 2016 another Noble prize for supramolecular chemistry was awarded to Sauvage, Stoddart and Feringa, the originators of the modern supramolecular chemistry. The foundation stone was laid by Sauvage et al. in 1983 by establishing a new strategy for the syntheses mechanically interlocked structures. 12 The so called "catenanes" are mechanically interlocked rings, $13 \mid 14$ which can only be separated by breaking a covalent bond withing one of these rings, thus, the structure can move freely but is highly stable at the same time. Structures like that have been reported before, but Sauvages' approach in synthesis was pioneering. By preorganizing the rings with an templating metal ion, before the rings were covalently interlocked, Sauvage could synthesize significant amounts of those catenane structures, which hitherto were synthesized under ultra high-dilution conditions with extremely low yields. Based on this strategy, further new intertwined topologies like links and knots could be designed.

Later, another supramolecular structure was established by Stoddart et al. The "rotaxanes" consists of an linear rod-like motif threaded through an molecular ring. Both ends of 
the rod are capped with bulky stopper motifs, giving the whole system a dumbbell shape. As for the catenanes, a templated preoganization of rod and ring by non-covalent interactions between both, before covalently adding the stoppers, is the key step for an high yielded synthesis. In 1991 Stoddart could prove, that a the ring could move along the rod-like thread. Furthermore, a controlled ring movement between to distinct states, triggered by external stimuli ( $\mathrm{pH}$, chemical trigger or light irradiation) led to the so called "shuttlebus rotaxane". Based on the same principle Stoddart designed even more complex systems, as the molecular muscle figure II.1.1.2a or the nano elevator .15 17

(a)

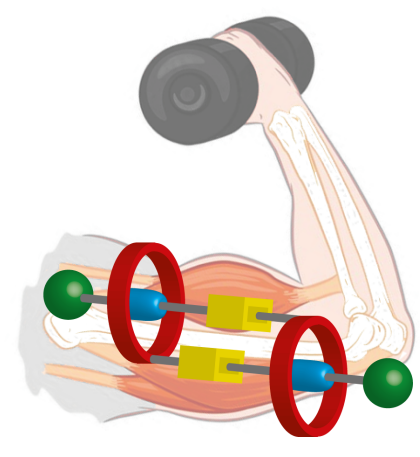

contracted

(b)

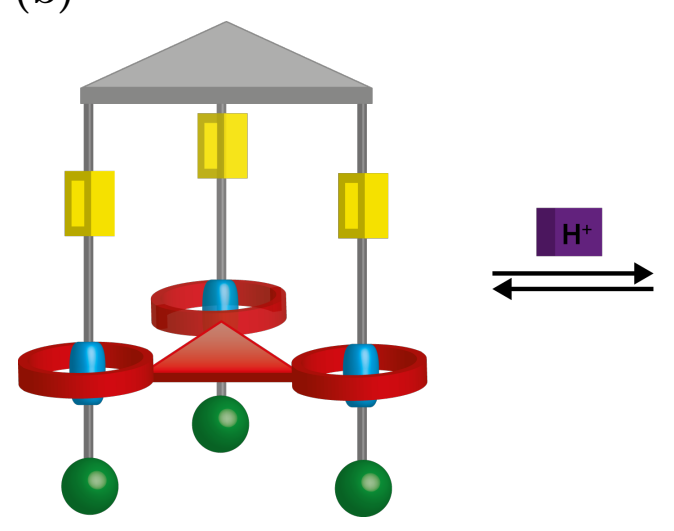

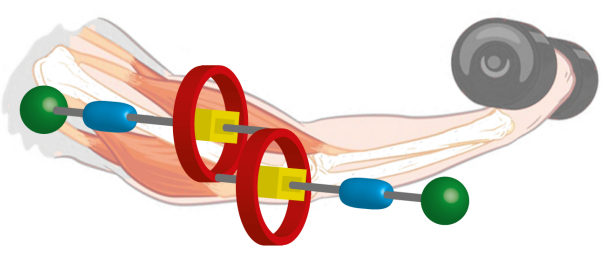

relaxed

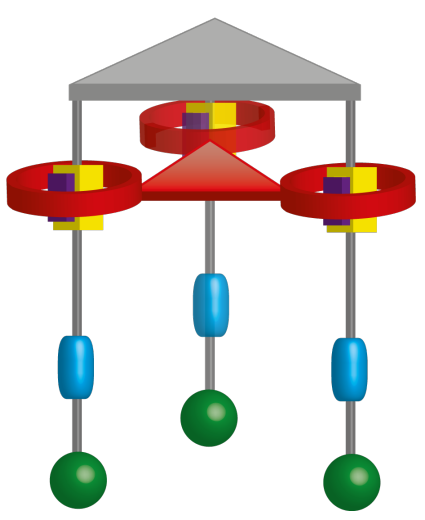

Figure II.1.1.2: The two famous supramolecular architectures of Stoddart et al., for which amongst others, he has received the noble prize in chemistry in 2016, together with Sauvage and Feringa. a) molecular muscle based on a "shuttlebus rotaxane" structure, where to topological states can be trigged by external stimuli. b) Based on the same principle, more complex structures, as the so called "molecular elevator", were designed and synthesized.

Already in 1999, Feringa et al. reported an unidirectional molecular rotor. Since it shows a controlled motion in one direction, it was one of the first artificial molecular motors. The principle of function is further discussed in chapter V.1. Although, this dynamic unit is not a supramolecular system, non-covalent interactions were crucial for the design of the world's smallest car. Feringa et al. created in 2011 a nanocar with four of their above 
mentioned molecular motors as wheels and with a total size of approx. $1 \mathrm{~nm}$. Due to the unidirectional motion of the four incorporated molecular motors, Feringa et al. could prove that the nano car structure was able to move forwards on a copper surface in a controlled unidirectional fashion, what was so far an unique breakthrough in the field of molecular machines.

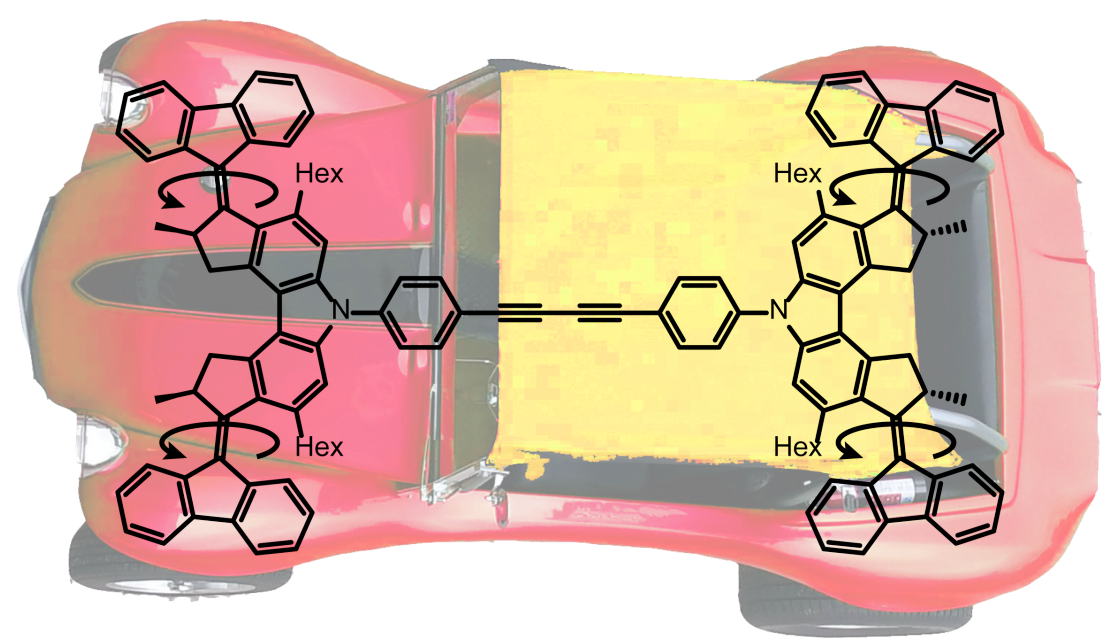

Figure II.1.1.3: Supramolecular nanocar by Feringa et al.. The whole structure consists of two identical building blocks merged together in the middle by briding alkyne units. It formed a consistently aromatic rigid system, with two molecular motor units at the front and two at the back. The location where the rotational motion takes place is marked with circular arrows.

\section{II.1.2 Concepts of Supramolecular Chemistry}

Supramolecular Chemistry investigates the "chemistry of the non-covalent bond", means every attractive inter- or intramolecular force, that do not lead to a sharing of electrons between two atoms. Hence, the term "non-covalent" includes interactions between ions, dipoles, induced dipoles or spontaneous transient dipoles. The diverse combinations can be subcategorized into ion-ion, ion-dipole, hydrogen bonds and so on (figure II.1.2.4). 
a)

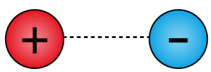

e)

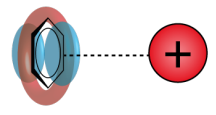

b)

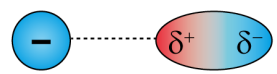

f)

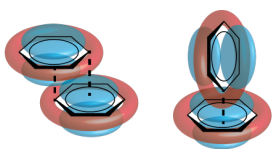

c)

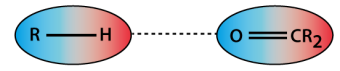

g)

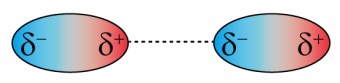

d)

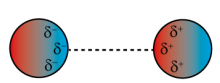

h)

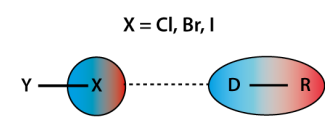

\begin{tabular}{lll}
\hline Interaction & Scheme & Energy $\left[\mathrm{kJ} \mathrm{mol}^{-1}\right]$ \\
\hline Ion-Ion & a) & $200-250$ \\
Ion-Dipole & b) & $50-200$ \\
Halogen Bond & h) & $50-180$ \\
Hydrogen Bond & c) & $4-100$ \\
Dipole-Dipole & g) & $5-50$ \\
Ion- $\pi$ & e) & $5-80$ \\
$\pi-\pi$ & f) & $1-50$ \\
Van-der-Waals & d) & $<5$ \\
\hline
\end{tabular}

Figure II.1.2.4: Non-covalent interactions in supramolecular chemistry. a)-h) show the non-coordinative interactions in a schematic fashion. In the table below, the above shown interactions are named and sorted by their strength, regarding the energy to break these attractive interactions, from strong to weak in a descending order.

Compared to covalent bonds with an bond energy of 300-400 $\mathrm{kJ} \mathrm{mol}^{-1}$, the non-covalent interactions a rather weak with energies between $<5-250 \mathrm{~kJ} \mathrm{~mol}^{-1}$, but the sheer amount of weak interactions in sum, that can occur within a systems, have a significant contribution to the allover system stabilization that should not to be underestimated. The strongest interactions with bond energies about $50-250 \mathrm{~kJ} \mathrm{~mol}^{-1}$ are the electrostatic interactions, including ion-ion, ion-dipole and dipole-dipole interactions with permanent dipoles. Ion-ion interactions a known from salts or acid-base pairs, ions or molecules with permanent charges of opposite polarity. Although the electrostatic forces are pretty strong between those pairs, yet the presence of water often makes these interactions break very easily. One of the most occurring and also extremely important dipole-dipole interactions are the hydrogen bonds. With an energy of maximum $100 \mathrm{~kJ} \mathrm{~mol}^{-1}$ hydrogen bonds are less than half as strong as ion-ion interactions. However, the formation of several hydrogen bonds within a system lead in sum to a significant stabilization, as it can be seen i.e. in DNA helix structures, but do also play an important role in the host-guest interaction of supramolecular systems. Back to the example of DNA, besides the above mentioned hydrogen bonds, the $\pi-\pi$ stacking between neighboring nucleobases have a substantial contribution in stabilization. These attractive forces can cause a gain in energy about $50 \mathrm{~kJ} \mathrm{~mol}^{-1}$ and play an important role in the guest encapsulation in supramolecular chemistry, especially with regard to highly aromatic guests, as for example fullerene. 
In the last years the rather weak but frequently occurring interactions moved into the focus of interest, like the Van-der-Walls interactions, which do also include London dispersion forces, belong to the weakest interactions with just $5 \mathrm{~kJ} \mathrm{~mol}^{-1}$ at maximum. They are caused by spontaneous formation of transient dipoles, by other ions, dipoles or induced dipoles and occur in all matter. The best known example for the immense forces that can be caused by Van-der-Walls interactions is the gecko reptile. Thanks to its special surface under its feet, it can form an enormous amount of Van-der-Walls interactions between itself and the surface it is walking on. All forces in total generate such a big attraction, that the gecko can walk smoothly headlong without becoming an victim of gravity.

Besides the here presented forces, which mainly contributes to the enthalpic part of energy, the hydrophobic effect has occupied an important role in the area of supramolecular chemistry, since more and more water-soluble systems appeared that offer the possibility of hydrophobic guest encapsulation. Although not fully understood yet, the hydrophobic effect seems to mainly influence the entropy with respect to the thermodynamics, at least at room-temperature and below. $\frac{18 \mid 19}{19}$ However, the contribution of the hydrophobic effect in combination with possible interactions between host and guest molecules, makes the encapsulation of guests within supramolecular cavities energetically so preferable, thus those systems become extremely attractive for the usage as containers or molecular reactions flask in aqueous medium.

With supramolecular structures the term "molecular self-assembly" is often connected. Self-assembly describes the process of congregation of building blocks, which arrange themselves to a supramolecular architecture of higher complexity. The composition of the formed construct depends on the involved non-covalent interactionsand lead to the most energetically favorable structure, since the system always tries to form the thermodynamically most stable product. Moreover, the reversibility of the non-covalent interactions plays an important role on the way during the process of self-assembly, because spontaneously occurring wrong connections can be broken again for the benefit of more stable non-covalent forces or coordination bonds. Nowadays industry is taking advantage of this reversible bond formation. Some materials are made of or coated with supramolecular polymers, where damages in the surface consolidate again, due to the reformation of the non-covalent bonds. These materials are called "self-healing". However, during the assembly of supramolecular structures non-covalent or dynamic-covalent bonds are broken and formed several times until the system reaches the final product. But even after the system is situated in an equilibrium, the building blocks are detached from and reattached to the supramolecuar structure. This situation is important to be considered in order to understand why the formation of heteroleptic supramolecular cages is not an easy task chapter III.1).

The process of self-assembly can be subcategorized into two types, $20[21]$ the strict self- 
assembly and the directed self-assembly. In the case of the strict self-assembly, the final product is formed entirely spontaneously when all components are mixed in the right ratio under certain conditions. In contrast, during the directed self-assembly process a templating compound is involved, i.e. ions, solvent molecule or organic compounds of a certain shape. Those auxiliaries can later be removed or are integrated parts of the end product. Moreover, the template can be essential for the formation of a discrete structure, which could not be achievable in a simple strict self-assembly, as for the synthesis of catenanes reported by Sauvage 22

The thermodynamics of supramolecular structure formation is given by the Legendre transformation equation (II.1.2), which is received from the Gibbs-Helmholtz equation equation (II.1.1):23.

$$
\begin{aligned}
& {\left[\frac{\delta G / T}{\delta T}\right]_{p}=-\frac{H}{T^{2}}} \\
& \Delta G=\Delta H-T \Delta S
\end{aligned}
$$

with $(\Delta G)$ as the free energy change in the system, $(\Delta H)$ as the enthalpy and $(\Delta S)$ as the change of entropy. The consideration of the contributions of $(\Delta H)$ and $(\Delta S)$ are crucial for a successful formation. The enthalpy includes the gain in energy due to the formation of non-covalent or dynamic covalent bonds within the supramolecular structure as well as from the enthalpy of solution. In contrary, the formation of an higher ordered architecture from several individual smaller molecules is less favorable with regard to the systems entropy. But since the assembly to an supramolecular structure mostly leads to a smaller overall solvation shell compared to those of the individual building blocks, the release of solvent molecules can push the entropic contribution in favor of the self-assembled structure. Nevertheless, due to the fact that both, enthalpy and entropy, have an effect on the thermodynamic favorability of the formation, it is not necessary that both values do favor the process, as long as one contribution has a predominant effect compared to the other. In general, the self-assembly of higher ordered structures from smaller building blocks can be distinguished in two assembly mechanism. The strict self-assembly (figure II.1.2.5a), where the building blocks spontaneously assemble to the desired supramolecular structure and the directed self-assembly, where an auxiliary templating molecule helps to direct the formation of the desired supramolecuar structure. The auxilary is interacting with the building units in a non-coordinative manner, in order to preogranize the building blocks, before the assembly takes place (figure II.1.2.5b). 
(a)

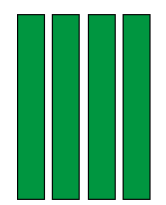

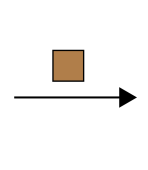

(b)

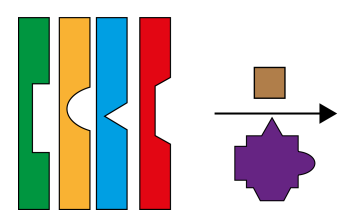

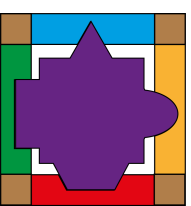

Figure II.1.2.5: Strict vs. directed self-assembly. a) Schematic representation of a strict self-assembly. The involved building units assemble spontaneously into a discrete structure without any outer auxiliary influences. b) In contrast, an auxiliary molecule is needed, i.e. functioning as an templating agent, in order to form the desired structure and preventing the formation of other possible assemblies.

Some of the supramolecular architectures including any inside cavity, where smaller molecules of the right size and shape can be taken up. This feature is of particular interest due to the ability to use those structures as transport containers or molecular reaction vessels chapters IV.1 and V.2. The investigation of such processes is called host-guest chemistry. The larger aggregate possessing the cavity to bind a molecule is referred as host $(\mathbf{H})$, while the encapsulated smaller compound is called guest (G), which can be a single ion or larger charged or neutral molecule. $20 \mid 24$ The resulting structure is called a host-guest complex $[\mathbf{G @ H}]$ (in literature also often shown as $[\mathbf{G} \subset \mathbf{H}]$, Figure II.1.2.6. 2125

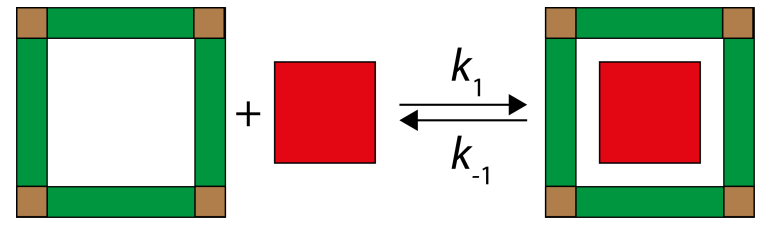

Figure II.1.2.6: Supramolecular assemblies containing an inner cavity, are capable in most cases to incorporate and smaller molecule. Those structures are mostly referred as HOSTs $[\mathbf{H}]$, whereas the uptaken molecules are called GUESTs (G). The fitting between the host cavity and guest molecule as well as the number and strength of existing interactions between host and guest, affect the equilibrium between host-guest complex and unoccupied guest. Also outer given conditions, like solvent polarity, $\mathrm{pH}$, etc. can have a tremendous effect on the host-guest equilibrium.

As for the assembly of the supramolecular structure, the formation of host-guest complex is in equilibrium, considering the uptake of the guest (G) into the host structure host $(\mathbf{H})$ and the release of guest from the cavity. The situation in equilibrium depends as before on the thermodynamics. Therefore, the quality of interactions between host and guest predominately predetermines how strong the guest is bound. The thermodynamic stability of the host-guest complex is determined in practice by determining the bindingor association constant $K_{\mathrm{a}}$ (equation (II.1.3)).

$$
K_{a}=\frac{k_{1}}{k_{-1}}=\frac{[G @ H]}{[G][H]}
$$


In some systems, the ions function as guest and template, as it was shown in the modified suberone cage of Freye et al., where the change of counterion in sterically hindered coordination cage, results in the formation of an interpenetrated double cage. chapter V.2.26 Furthermore, in some artificial supramolecular coordination structures, containing more than one binding position, positive allosteric effects or cooperative binding could be observed, $20 \mid 27$ as it is known from natural occurring systems, i.e. oxygen binding in hemoglobin. 28

\section{II.1.3 Supramolecular Coordination Cages}

The topologies of pure organic supramolecular nano-architectures are already highly diverse, 29 but can be even extended by combining them with the topologies of metal coordination environments. The introduction of these coordination geometries (figure II.1.3.7) expanded the possibly designable topologies in supramolecular assemblies by a multiple. Depending on the nature of the used metal ion, a various number of ligands can be connected in a certain angle over the metal ion. With this strategy the shape of the overall supramolecular structures is easier to design, since the outcoming spatial arrangement of the ligands is more predictable. For the connection of two ligands in a linear fashion, $\mathrm{Ag}^{+}$ions would be the first choice. Four ligands can be connected in a square planar fashion with palladium(II) or copper(II), for tetrahedral shapes $\mathrm{Cu}$ (I) or $\mathrm{Zn}$ (II) and octahedral coordination environment can be achieved with i.e. iron(II/III). Also, metal ions with higher coordination numbers, like lanthanides, have been used for the formation of supramolecular cages. $\frac{30}{30}$ The defined metal coordination environments give supramolecular chemists additionally an easy opportunity to go from two-dimensional to three-dimensional compounds, while keeping the overall structure rather small compared to pure organic structures. 31 In addition, rare topologies could be obtained, which would not be obtainable by conventional organic synthesis, as i.e. the already mentioned catennanes section II.1.1). 


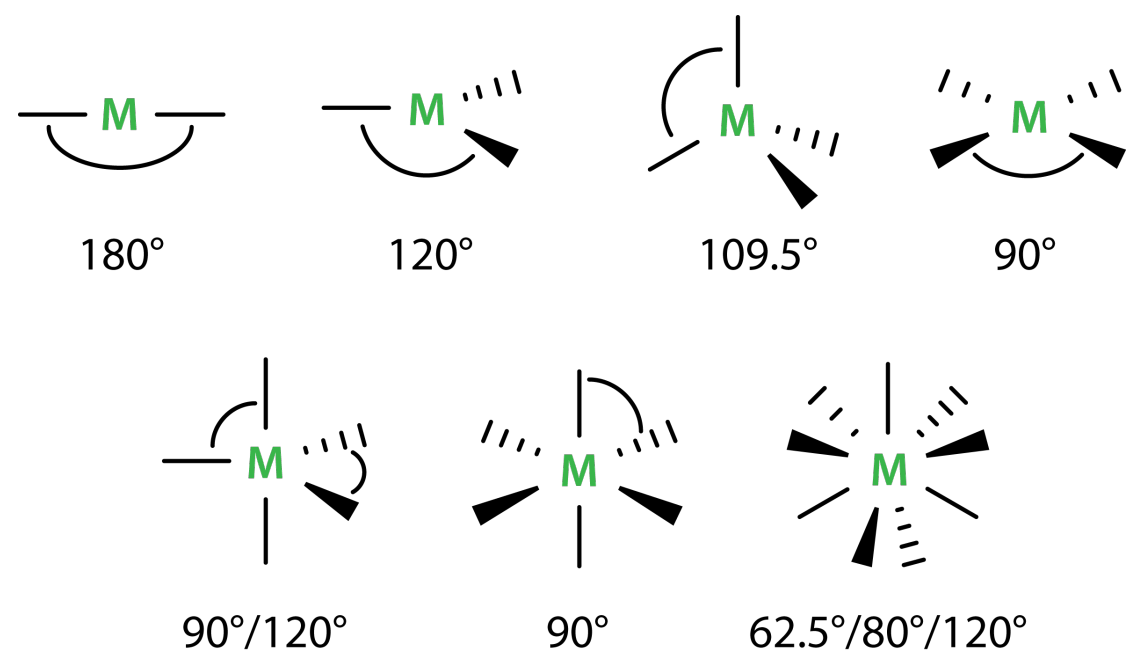

Figure II.1.3.7: The here shown geometric schemes represent various possible coordination geometries with different coordination numbers. The existing coordination geometry within a supramolecular structure, depends on the nature of the used metal, its state of oxidation, size and nature of the corresponding coordinating ligands and other factors.

Besides the precept of directionality by the metal coordination environment, the used organic ligands have a major impact on the spatial arrangement within the self-assembly, thus on the allover structure. The compound specific features of the ligands, like length, coordinating functional groups, angles, denticity, flexibility has to be considered as well as the metal preferable coordination environment. On top of this, the reaction conditions such as metal/ligand ratio, choice of counter anions, solvent, temperature, and reaction time will affect the cage formation. 32 Typical geometries obtained from homoleptic assemblies are in general highly symmetric, often resembling the rather simple Platonic solids, e.g. tetrahedr, 33 octahedra 34 or cubes 35 (figure II.1.3.8), but also more complex Archimedean solids are achievable. $36 \mid 37$ Moreover, other topologies of three-dimensional self-assembled coordination structures, like adamantoids, trigonal prism, helices, bowls, and many more were reported in the last years. 38 


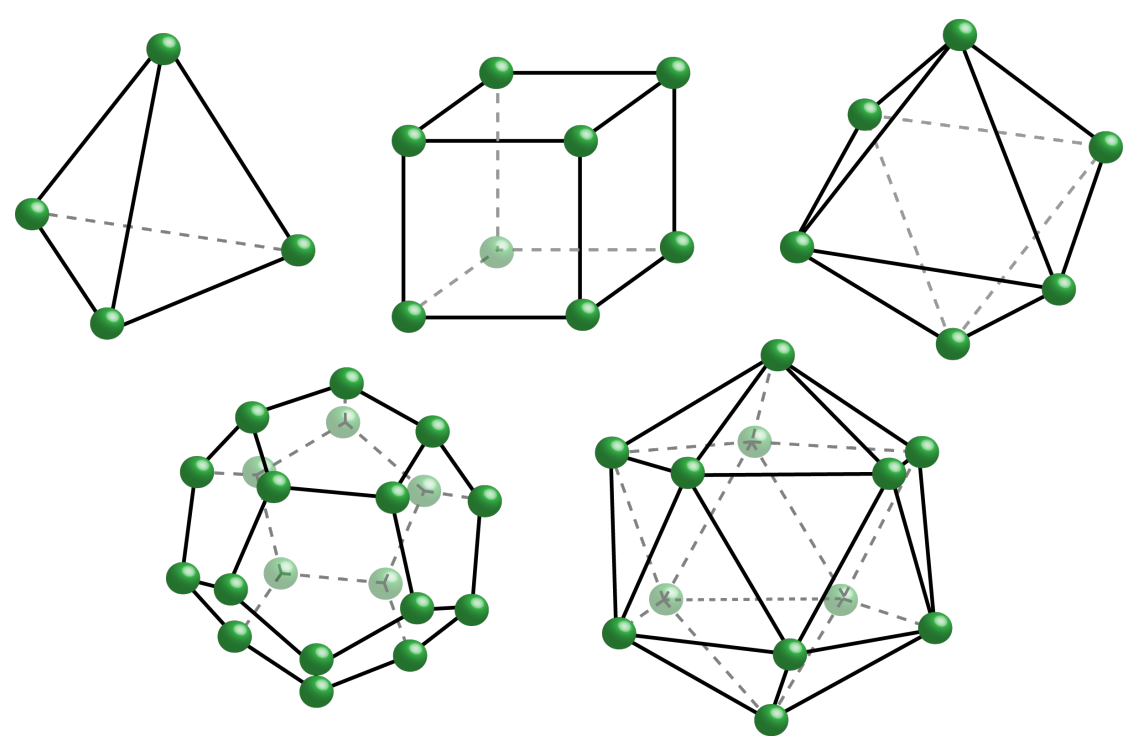

Figure II.1.3.8: The here presented topologies belong to the Platonic solids. These topoglies can be frequently found in supramolecular metal-mediated architectures and provide in most cases an endohedral cavity, which can function either as a container or reaction vessel in present of suitable guest molecules. It is worth to mention, that the above mentioned topologies are often formed by a combination of building blocks with a geometry deviating from the assembled structure.

Among all supramolecular coordination complexes, the three-dimensional structure of the supramolecular coordination cages have become a very promising architecture for a variety of applications, as containers or reaction environment on a nanoscalic level, since the assembled coordination cages have a discrete cavity, able to interact with smaller encapsulated guest molecules. Due to the fact, that a large number of coordination cages are positively charged, negatively charged molecules can be encapsulated inside their void, 39] but also the uptake of neutral guests are reported in some cases. $40[41$ Additionally, reactive species could be stabilized and prevented from further undesired reactions, by shielding them within the assemblies cavity. $22[43$ With regard to supramolecular structures as nanoscale reaction environment, examples of accelerated reactions were reported.44[45. In both cases, charged intermediates are stabilized while bound in the cavity of the highly charged supramolecular construct. A further example is unusual regioselectivity of a Diels-Alder cycloadditon within a highly charged coordination cage, due to the changed spatial situations wihtin the supramolecular cavity. 46 The highly charged supramolecular assemblies are furthermore soluble in water, compared to most other supramolecular structures, for this reason they gained interest in the field of green chemistry (chapter IV.1. Besides, further structures with unique functions, like catalytic reactivity, $\underline{39|47| 48}$ gas sequestration 49 or segregation of mixtures 50 could be realized in well-designed supramolecular metal-mediated coordination architectures.

Structures assembled from bis-mono dentate concave shaped ligands, have become one of the most prominent directions in the last years. $\frac{38 \mid 51}{3}$ Besides its mostly straight forward syntheses, the ligands can be combined with a variety of metal ions to form discrete coordination architectures with a stoichiometry of $\left[\mathrm{M}_{n} \mathrm{~L}_{2 n}\right]$. Examples in literature do 
include metal ions as copper, nickel, palladium, platinum, cobalt and different lanthanides (La, Ce, Sm, Eu, Tb). 51|52] For the latter, multi dentate ligands are used.53. Most reported ligands consisting of rigid, aromatic backbone, with the coordinating groups mainly connected via rigid alkyne or phenyl units, but also more flexible linker as piperazine, amides, ethers or simple alkane based linker have been reported.51. Compared to the coordination cages mentioned in previous paragraph, the total charge is rather low and located at the coordination sites, thus the cages are polar but not water-soluble and preferably form in aprotic polar solvents as acetonitrile or dimethylsulfoxide. Furthermore, due to their banana-shaped ligands, the cages contain an internal void, whose size depends on the ligands specific size, but also how the ligands are arranged around the coordination centers.

In figure II.1.3.9 a collection of possible cage structures derived from various banana-shaped ligand with the $\mathrm{d}^{8}$ metals $\mathrm{Pd}^{\mathrm{II}}$ or $\mathrm{Pt}^{\mathrm{II}}$, providing a square planer coordination environment for the ligands. All these structures were reported in the group of Prof. Clever, who has intensively researched $\left[\mathrm{M}_{\mathrm{n}} \mathrm{L}_{2 \mathrm{n}}\right]$ coordination cages the last years. But also Fujita, Shionoya, Crowley, Yoshizawa and many many others, continuously expanding the the pool of delightful palladium-mediated supramolecular architectures. 


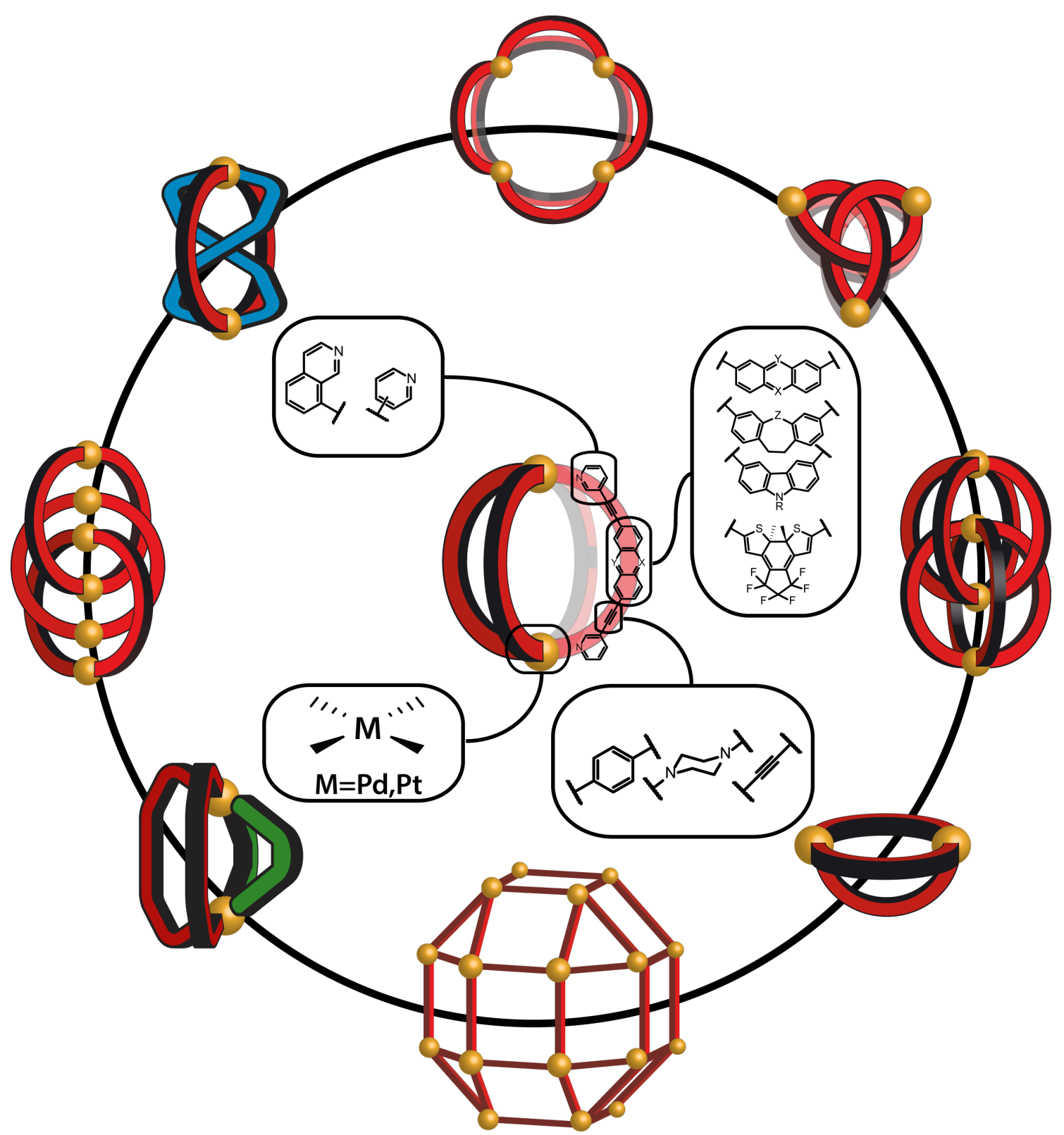

Figure II.1.3.9: Schematic representation of self-assembled architectures based on metal(II) cations (e.g. Pd(II) or $\mathrm{Pt}(\mathrm{II}))$ and banana-shaped bis-pyridyl ligands L. Resulting architectures in clockwise order $\left[\mathrm{M}_{3} \mathrm{~L}_{6}\right]$ ring, c) $\left[\mathrm{M}_{3} \mathrm{~L}_{6}\right]$ double-trefoil knot, d) $\left[\mathrm{M}_{4} \mathrm{~L}_{8}\right]$ ring, e) $\left[\mathrm{M}_{2} \mathrm{~L}_{3} \mathrm{X}_{2}\right]$ bowl $(\mathrm{X}=$ solvent $)$, f) $\left[\mathrm{M}_{2} \mathrm{~L}_{4}\right]$ coordination cage, g) $\left[\mathrm{M}_{4} \mathrm{~L}_{8}\right]$ interpenetrated coordination cage, $\left.\mathrm{h}\right)$ triple catenane trans- $\left[\left(\mathrm{MBr}_{2}\right)_{2} \mathrm{~L}_{2}\right] 3$ and i) rhombicuboctahedral sphere $\left[\mathrm{M}_{24} \mathrm{~L}_{48}\right]$.

The shown hollow cage assemblies are all capable to encapsulate anionic $54 \mid 55$ or, in specieal case, neutral guests. 5657. The choice of solvent and counter anions can have a dramatic influence on the formation of structure. Under specific conditions, monomeric cages do form in presence of a specific counter anion and the matching amount an interpenetrated dimeric $\left[\mathrm{M}_{4} \mathrm{~L}_{8}\right]$ cage, $\underline{58}$ while an additional change in solvent can prevent again the interpenetration process. Furthermore an excess of halide anion in general leads to the disassembly of the supramolecular structure, whereas in the structure of Zhu et al., the excess of halide anions result in the formation of triple catennane. 59. Substitution of the 
pyridine donor group by a quinoline group yielded a bowl shaped $\left[\mathrm{M}_{2} \mathrm{~L}_{3}(\mathrm{Solv})_{2}\right]$-structure, where the free coordination side of the missing ligand is occupied by two solvent molecules. Furthermore, a shorter version of the light switchable dithienylethene (DTE) based ligand, with an additional change in coordination angle forms in presence of $\mathrm{Pd}^{\mathrm{II}}$ a mixture of three- or four membered rings $\left(\left[\mathrm{Pd}_{3} \mathrm{~L}_{6}\right]\right.$ or $\left.\left[\mathrm{Pd}_{4} \mathrm{~L}_{8}\right]\right)$, when in open form. 36 The lightswitched closed isomer on the other hand forms a $\left[\mathrm{Pd}_{24} \mathrm{~L}_{48}\right]$ rhombicuboctahedral sphere. Although the architectures of self-assemblies are usually quite predictable, sometimes still surprising spatial arrangement occur, such as formation of an entangled double-trefoil knot 60. or the heteroleptic trans- $\left[\mathrm{M}_{2} \mathrm{~L}_{2}{ }_{2} \mathrm{~L}_{2}{ }_{2}\right]$ cage with a "doubly bridged figure eight" topology. 61

Since the interest in using the cavities of supramolecular architectures as catalytic units $62[63$. or highly selective containers for chemical segregation $[64$ or drug delivery 65 has grown drastically, the question for suitable systems arose, where several independent functionalities can be united. In the field of supramolecular coordination cages the realization of such a system turned out not to be an easy endeavor, since scientist facing the problem of statistical mixtures for multi-component cages. The lability of metal ligand coordination, that ensures the correct assembly of the desired end structure, is for the formation of heteroleptic supramolecular structures a fundamental issue (figure II.1.3.10). 66

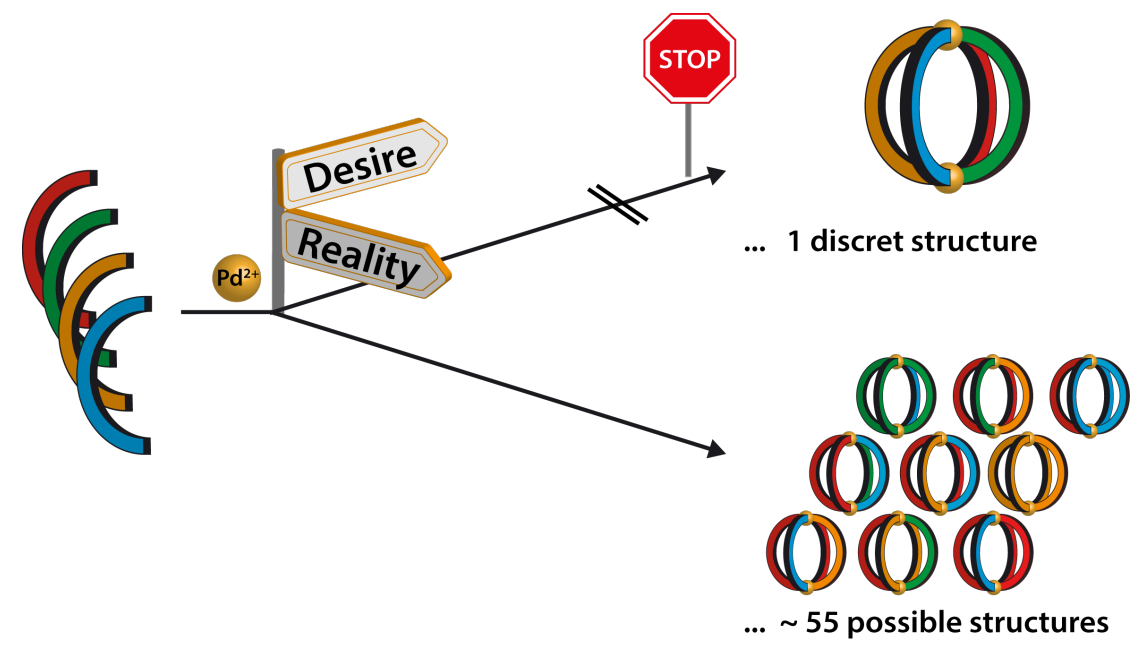

Figure II.1.3.10: Schematic representation of the assembly process for palladium-mediated heteroleptic cages. The assembly of heteroleptic highly symmetric architectures is still a challenging task in supramolecular chemistry, since a discrete assembly of those structures is hardly controllable without any geometric restriction or templating auxiliaries. Assuming an assembly of a supramolecular cage structure composed of two palladium ions and four different ligands $\left[\mathbf{P d}_{\mathbf{2}}\left(\mathbf{L}_{\mathbf{A}} \mathbf{L}_{\mathbf{B}} \mathbf{L}_{\mathbf{C}} \mathbf{L}_{\mathbf{D}}\right)\right]$, having all the same structure, only the backbones are slightly modified This would not lead to a single discrete non-racemic structure, but rather in a statistical mixture, where approx. 55 assembled cages can be found.

Most of the above shown examples figure II.1.3.9), are highly symmetric architectures that contain only one type of organic ligand and therefore only one functionality. Due to that fact, the chemistry of artificial supramolecular self-assemblies is still far apart from 
the level of structural and functional complexity encountered in biological macromolecular hosts. Especially in terms of those systems containing highly accurately arranged functionalities within its cavity, for a specific binding or chemical conversion of encapsulated molecules. In order to overcome this disparity, rational approaches have been emerged the last years to realize heteroleptic assembly in a regulating integrative self-sorting fashion between metal ions and multiple ligand components. Considering the $\left[\mathrm{M}_{2} \mathrm{~L}_{4}\right]$ coordination cage as an example, the possible resulting assemblies in a fully-dynamic two-ligand-system $\left(\mathrm{L}^{\mathrm{a}}\right.$ and $\left.\mathrm{L}^{\mathrm{b}}\right)$ can be categorized into three scenarios of self-sorting figure II.1.3.11a): 67 .

1. $\left[\mathrm{Pd}_{2} \mathrm{~L}^{\mathrm{A}}{ }_{n} \mathrm{~L}^{\mathrm{B}}(4-\mathrm{n})\right]$ - Statistical mixtures - forms cages with all imaginable ligand compositions, mostly encountered in the absence of complementary interactions between the two deployed ligands $\mathrm{L}^{\mathrm{a}}$ and $\mathrm{L}^{\mathrm{b}}$.

2. $\left[\mathrm{Pd}_{2} \mathrm{~L}_{4}^{\mathrm{a}}\right]+\left[\mathrm{Pd}_{2} \mathrm{~L}_{4}^{\mathrm{b}}\right]-$ Narcissistic self-sorting - generates several discrete homoleptic species, occurs due to a significant energetic penalty arising from the combination of ligands $\mathrm{L}^{\mathrm{a}}$ with $\mathrm{L}^{\mathrm{b}}$.

3. $\left[\mathrm{Pd}_{2} \mathrm{~L}_{2}^{\mathrm{a}} \mathrm{L}_{2}^{\mathrm{b}}\right]$ - Integrative self-sorting - giving a single preferred heteroleptic product, mostly caused by significant enthalpic factors originating from the combination of $\mathrm{L}_{\mathrm{a}}$ and $\mathrm{L}_{\mathrm{b}}$ in the assembly.

In the last years, several systems for the integrative self-sorting were reported, based on various interaction concepts. As an example for ligand interactions (figure II.1.3.11b) Hooley and co-workers examined in 2011 the formation of heteroleptic $\left[\mathrm{Pd}_{2} \mathrm{~L}_{2}{ }_{2} \mathrm{~L}_{2}\right]^{4+}$ cages by bis-monodentate ligands with identical geometries and donor types, but with endohedral modifications, owning different steric demands. Combination of the ligands containing an endohedral steric bulk with those that have a less steric demand, formed preferably $\left[\mathrm{M}_{2} \mathrm{~L}_{3}{ }_{3} \mathrm{~L}^{\mathrm{b}}\right]$ cages, containing only one sterically demanding ligand. 68

In 2015, the group of Yoshizawa reported the guest templated self-sorting of anthracenefunctionalized $\left[\mathrm{Pd}_{2} \mathrm{~L}_{2}{ }_{2} \mathrm{~L}_{2}{ }_{2}{ }^{4+}\right.$ cages using a fullerene as templating guest, $\underline{69}$ with short and long versions of a bis-monodentate ligand from a previous study. 70 In the absence of a guest template, an equimolar mixture of the two homoleptic cages was found to rearrange into a statistical mixture of cages, as in the reported system of Frank et al. 66 In contrary, the presence of $\mathrm{C}_{60}$ resulted in guest encapsulation and simultaneous reorganization to a single type of heteroleptic cage $\left[\mathrm{C}_{602} \mathrm{~L}_{2}^{\mathrm{a}} \mathrm{L}_{2}{ }_{2}\right]^{4+}$.

Bloch et al. reported in 2016 an heteroleptic $\left[\mathrm{Pd}_{2} \mathrm{~L}_{2}{ }_{2} \mathrm{~L}_{2}{ }_{2}\right]^{4+}$ assembly, based on the concept of shape complementarity. The combination of two ligands with complementary 
coordination angles $\left(\mathrm{L}^{\mathrm{a}}: 60^{\circ}\right.$ and $\mathrm{L}^{\mathrm{b}}$ : $120^{\circ}$ differing from the usual $180^{\circ}$, led to a tilt of the square planar coordination axis. Therefore, only the $c i s-\left[\mathrm{Pd}_{2} \mathrm{~L}_{2}{ }_{2} \mathrm{~L}^{\mathrm{b}}{ }_{2}\right]$ species shows the right ligand arrangement to occupy all coordination sites of the square planar the $\mathrm{Pd}^{\mathrm{II}}$ cation, which make this species enthalpically most favorable. 71

The utilization of steric control by introducing steric hindrance around the metal center has been widely investigated. $72 \mid 73$ However, this donor site engineering has been rarely used in the formation of assembling heteroleptic cages. Clever et al. reported in 2018 a system formed by acridone- and phenothiazine-based picolyl ligands with either in- or outward pointing methyl groups on the coordinating picolyl groups. The combination of acridone ligand with outward pointing picolyl and phenothiazine with inward pointing methyl group led to a clean formation of the cis- $\left[\mathrm{Pd}_{2} \mathrm{~L}_{2}{ }_{2} \mathrm{~L}_{2}{ }_{2}\right]$ cage. $24 \mid 75$. Apart from the steric control approach, secondary interactions can also initiate heteroleptic cage formation. Crowley and co-workers reported an ortho-amino-substituted pyridyl ligands capable to form an heteroleptic cis- $\left[\mathrm{Pd}_{2} \mathrm{~L}_{2}^{\mathrm{a}} \mathrm{L}^{\mathrm{b}}{ }_{2}\right]^{4+}$ coordination cages in combination with unmodified ligands through complementary hydrogen bonding. 76 

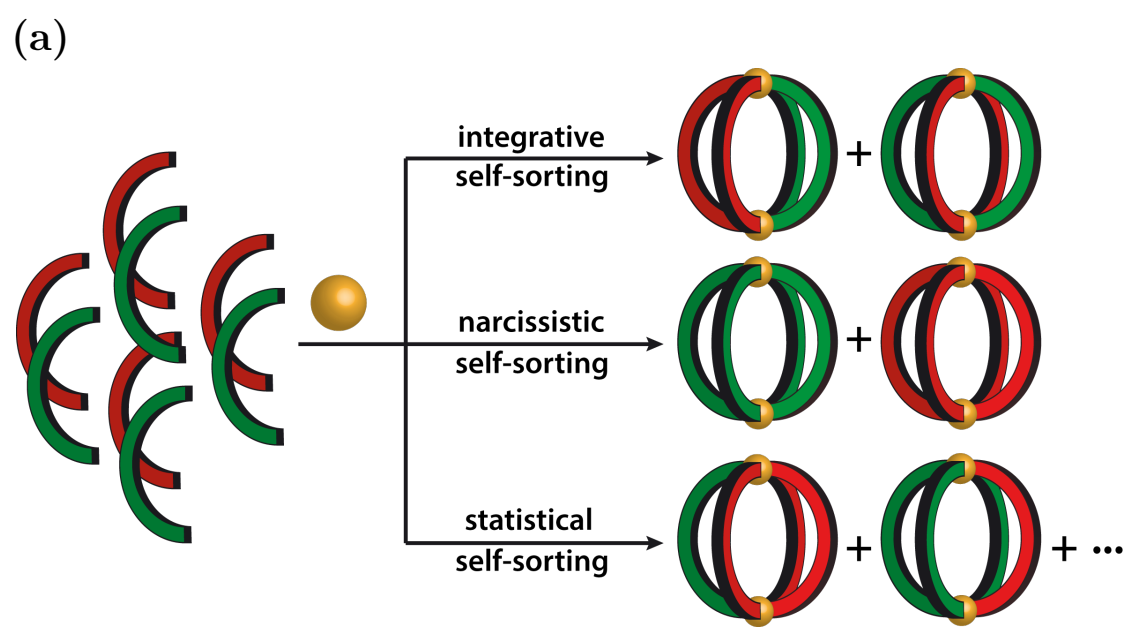

(b)

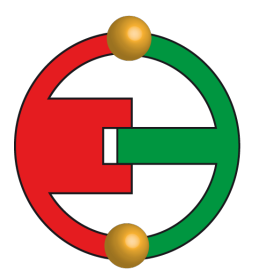

Ligand Interaction

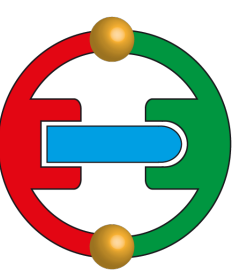

Guest Templation

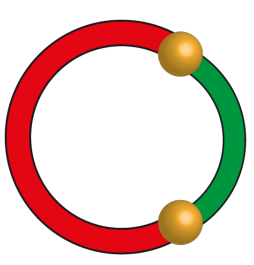

ShapeComplementary

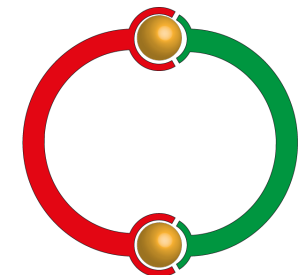

Donor-Site Engineering

Figure II.1.3.11: Concepts of self-sorting and designed sorting. 67 a) Three possible self-sorting outcomes for an $A B$-system (two different ligands). In the integrative self-sorting case gives a single heteroleptic product, but not necessarily enantiopure. The assembly is often driven by significant enthalpic factors that originate from interactions of vicinal components within the assembly and can be achieved by secondary interactions or geometric principles trough proper system design as shown in figure II.1.3.11b Narcisstic self-sorting lead to coexisting homoleptic cage species.- The outcome of statistical self sorting is described by $n^{k}$ species. In the here assumed AB-System, 6 species would be expected. b) Comparison of possible designs for a controlled assembly of heteroleptic structures: ligand interaction design is based on specific stabilizing interactions between opposing or neighbourd ligands. Guest templating design aims on the same, but is achieved by an auxiliary templating guest molecule. The next is an geometric approach, with designed shape-complementarity ligands, allowing only opposite ligands to assemble in the most energetically stable structure. Donor-site engineering concentrates on the design of the coordinating ligand parts, either by the approach of additional stabilizing interactions or steric hindrance. The first three design aims for the ligands backbone, while last approach focus on the donor site, more decoupled from ligands functionality. The disadvantage of the ligand interaction and guest templation approaches is the sacrifice of the cavity when using endohedral interactions.

Once, heteroleptic self-assembly strategies find a wider implementation in supramolecular design, the resultant interplay between tailored combinations of precisely positioned substituents promises enhanced functionality in nanoscale structures. However, the complexity of the assembled systems is even more increased by mixing more than two kinds of different ligands in presence of metal cations. Even now, there is no general approach for the formation of heteroleptic self-integrated coordination cages consisting two different ligands, not to mention assemblies with more than that. In part III a new possible approach for the formation of heteroleptic coordination cages is presented. The design is based on a highly programmable natural occurring polymer, also known as DNA, which could help to design a exterior scaffold for the preogranization of ligands within a self-assembly. 
Furthermore, this DNA scaffold could prevent the uncontrolled and undesired detaching or exchange of ligands. 


\section{Chapter}

\section{Desoxyribonucleic acid}

Since the discovery of its structure in 1953 by Franklin, Watson and Crick, the DNA was investigated intensively. Even after 65 years of research, still not all secrets of the DNA and its natural occurring structures could be revealed. Especially in medicine it becomes more and more important to understand the structure and behavior of DNA, in order to find the right therapies to treat diseases caused by defects or mismatches within the DNA. However, still there are unanswered question, that issue a challenge to scientist. In the last years DNA also appeared as a promising material in nanotechnology, due to its highly predictable nature. By observing the rules of the DNA assembly and well-designed sequences a portfolio of $2 \mathrm{D}-77$ and $3 \mathrm{D}-78 / 79$ functional nano-structures 80.83 could be obtained, where especially the research of Seeman and Rothemund played a key role. In combination with the toolbox of artificially synthesized organic, inorganic or polymeric building blocks, a new field of research, termed "supramolecuar DNA assembly" has been recently emerged. 84

\section{II.2.1 Structural Motifs}

Natural systems do provide both selectivity and functionality, but they also had a long time to evolve and develop effective solutions and to organize complex architectures that contain the desired sophisticated functions. Of the naturally evolved systems, DNA is a fascinating example of self-assembly and commonly known to be the carrier of our genetic code. Despite its rather simple building units and especially and with only four different nucleobases, it still can create a highly potent system which made it possible to stores the whole information of all existing living organisms. Having a closer look at the building units of DNA, the so called nucleotides, they can be subdivided into three parts figure II.2.1.1. 


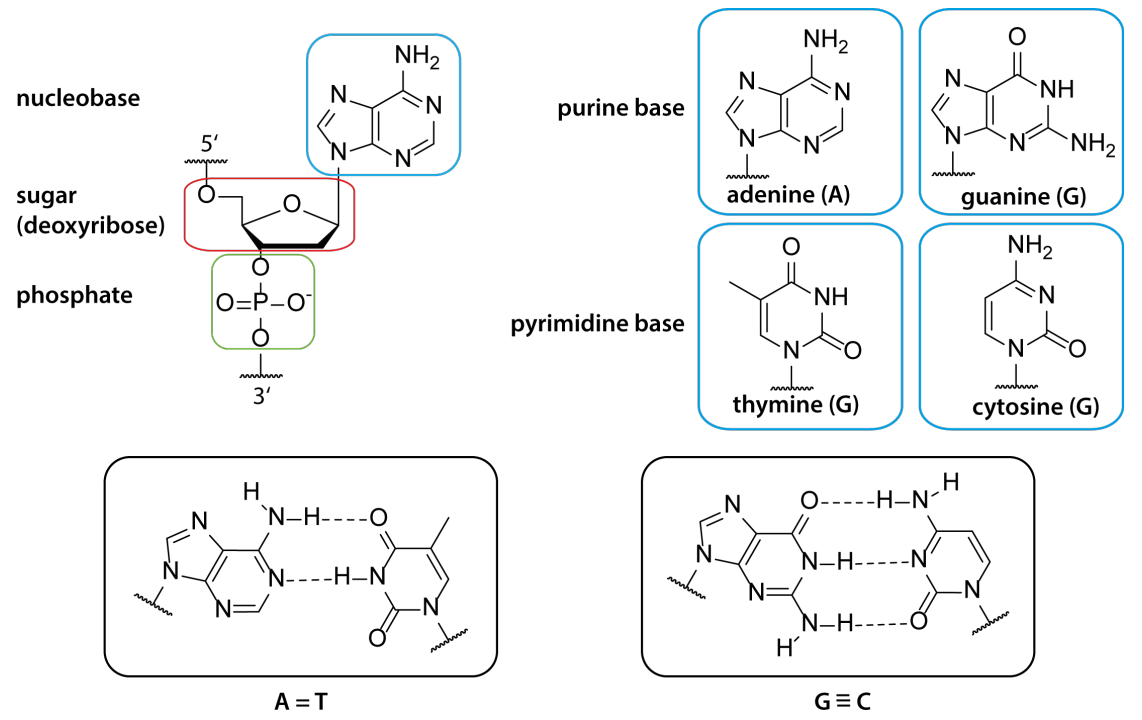

Figure II.2.1.1: General structure of nucleotides, DNA nucleobases and Watson-Crick base pairs. Single unit of DNA, the nucleotide, consists of two parts: the nucleoside, containing the nucleobase and the sugar backbone, for RNA this is the ribose, for DNA it is the deoxyribose and the phosphate. For the DNAs polymeric backbone, the deoxyribose and the phosphate are mainly responsible, by forming a covalent bond between the phosphate on the 3-C of the ribose, therefore formally called 3'-end and the alcohol group on the riboses' 5-C, formally called 5'-end. The naturally occurring nucleobases in DNA are subcategorized into purine bases, including the adenine (A) and guanine $(\mathbf{G})$ nucleobases and the pyrimidine base, where thymine $(\mathbf{T})$ and cytosine $(\mathbf{C})$ belong to. Base pair formations between adenine and thymine and between guanine and thymine are the most stable nucleobase pairs existing in a DNA. Those pairs are referred to as Watson-Crick base pairs and can be predominatly found in DNA double strands.

The repeating sugar moiety (deoxyribose) is connecting one of the four possible nucleobases (adenine $(\mathbf{A})$, cytosine $(\mathbf{C})$, guanine $(\mathbf{G})$ or thymine $(\mathbf{T})$ ) to the negatively charged phosphate group. The latter forms the highly negatively charged shell of DNA. This unfavorable accumulation of charges is primarily compensated by surrounding mono- or divalent cations, like $\mathrm{Na}^{+}, \mathrm{K}^{+}, \mathrm{Mg}^{+}$, or $\mathrm{Ca}^{+}$, but makes the DNA constructs watersoluble. 85 The inner core of the DNA structures is formed by the nucleobases. They are connected to the sugar and form the base pairs. Guanine and cytosine or adenine and thymine form the so called Watson-Crick base pairs, or canonical base pairs. They are the most occurring type of base pairing and can be found i.e. in mostly known DNA secondary structure, the B-DNA. The favored formation of three hydrogen bonds between $\mathbf{G}$ and $\mathbf{C}$ or two hydrogen bonds between $\mathbf{A}$ and $\mathbf{T}$, makes DNA so predictable and is next to the $\pi-\pi$ interaction between vicinal base-pairs, the predominant interactions responsible for the stabilization of the DNA secondary structures. Furthermore, nucleobases can show a syn- or anti-orientation in respect to the sugar, which plays an important role for the formation of secondary DNA structures. 86

Under physiological conditions, the most stable of the natural occurring canonical secondary structures is the B-DNA. Two complementary ssDNA stick together as right-handed double helix due to Watson-Crick base pairing and stabilized by the intramolecular hydrogen bonds and the above mentioned $\pi-\pi$ stacking between vicinal nucleobases. Besides this DNA construct, several additional structures have been found in the last decades, as 
i.e. the A-DNA, in which the B-DNA can be reversibly converted upon dehydration. At high concentrations of electrolytes, the left-handed Z-DNA can be formed, which has a completely different spatial arrangement to the other both structures and uses purine bases in syn configuration.

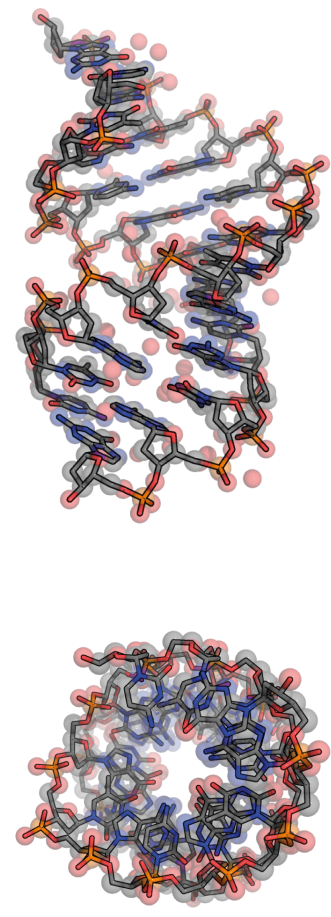

A-DNA
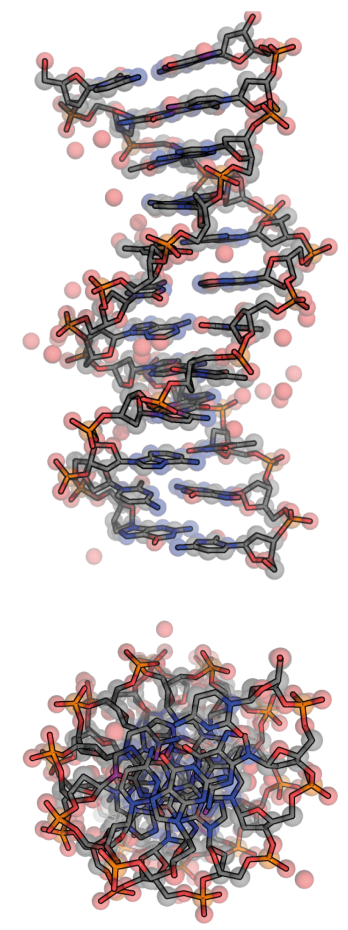

B-DNA
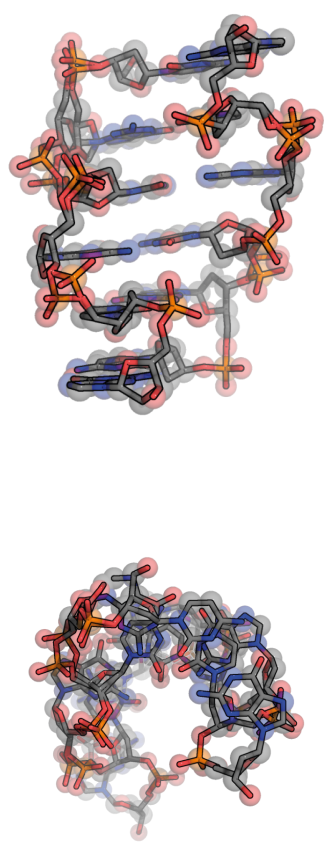

Z-DNA

Figure II.2.1.2: Crystal structure representation of the most common natural occurring DNA double strands. From left to right: A-DNA, 87 B-DNA, 88$]$ Z-DNA. 89$]$ The pale red dots represent counter cations detected during crystal analysis.

A further occurring structure is the so called DNA hairpin loop, which mainly formed in RNA, but can also be found in double stranded DNA structures. For this special phenomena the ssDNA has to contain palindromic or inverted repeating nucleobase sequences. 90 More commonly for DNA is the cruciform structure, which contain a complementary palindromic sequences in both strands, by what two opposing hairpins are formed.91. As the last canonical DNA structure, the Holliday Junction or four-way junction, is to be mentioned. Playing an important role during homologous recombination and the DNA repair mechanism, it is transiently formed by two crossing double stranded DNA fragments. This structure is further discussed in section II.2.2. 


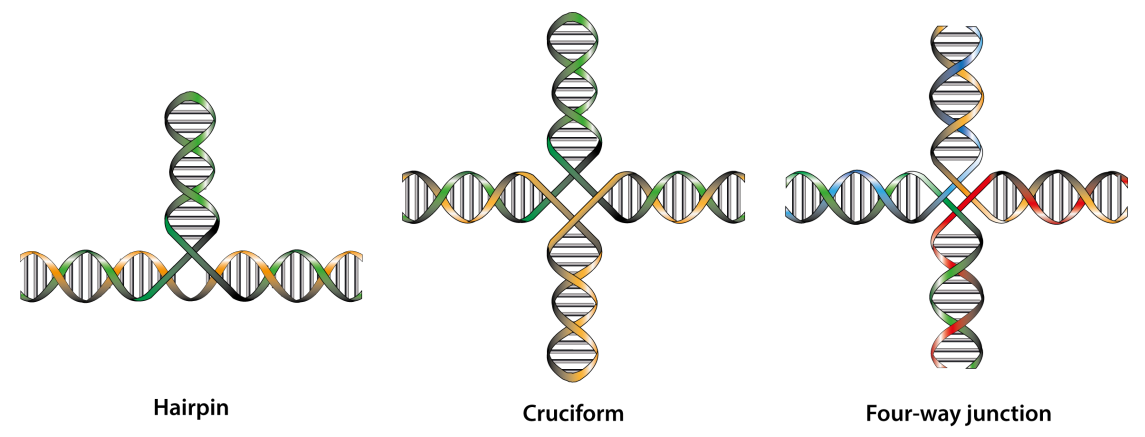

Figure II.2.1.3: Schematic representation of various canonical scondary non B-DNA structures. From left to right: Hairpin, cruciform, Holliday Junction. The hairpin structure exhibits an seperated palindorimic sequence wihtin one strand, which can form under certain conditions this intrastrand loop. The cruciform structure can be deduced from the hairpin, since it basically a double strand with two opposite hairpins, with one hairpin in each single strand, resulting in a cross-like topology. The Holliday Junction contains four single strands, where one partial sequence of each single is complementary to one of the other three strands, whilst the other partial sequence fits to another one of the remaining two strands. Applying this system to all of these strands result in two-dimensional four-stranded cruciform, which can transform under physiological conditions into other three-dimensional topologies figure II.2.2.7.

Besides the Watson-Crick base pairs an alternative hydrogen bond pattern between adjacent nucleobases can be found in secondary DNA structures. In the non-canonical secondary structures, 92 the so called Hoogsten base pairings take place, by what the formation of new DNA architectures are facilitated. In figure II.2.1.4 the most prominent representatives of the non-canonical secondary DNA structures are shown. 
(a)

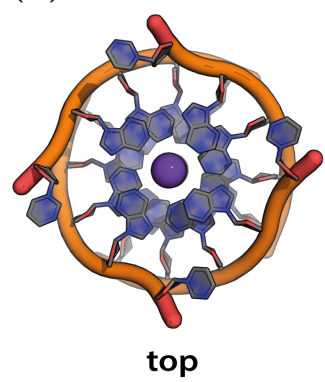

(b)

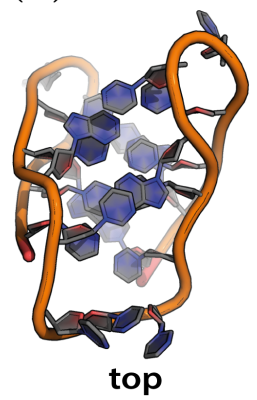

(c)

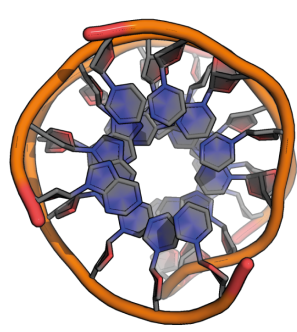

top

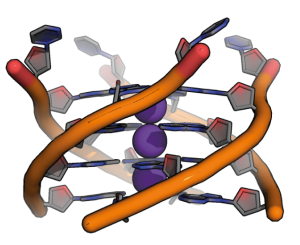

side

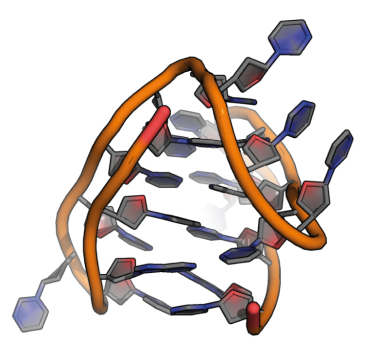

side
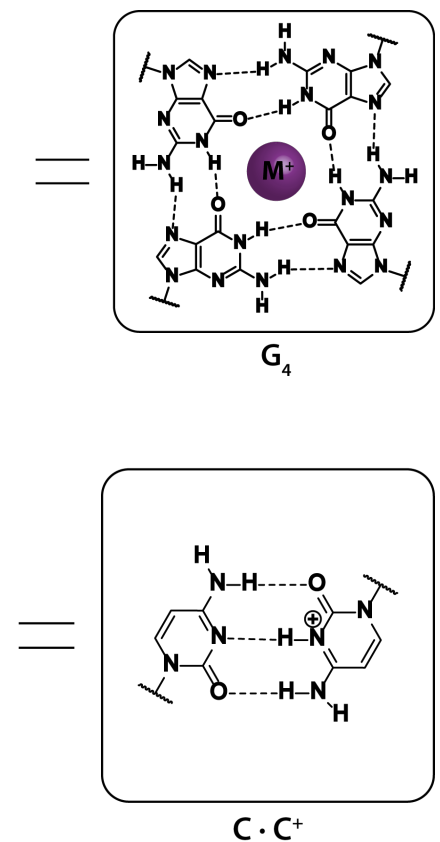

$\mathrm{C} \cdot \mathrm{C}^{+}$

Figure II.2.1.4: Crystal structures of non-canonical secondary DNA structures. The formation of those strucutres are promoted by forming the so-called Hoogsteen base pairs. a) The G-Quadruplex belongs to the most stable secondary DNA structures. It is formed mono- or poly-molecular guanine-rich sequences and develops square-planar layers, consisting of four guanines, one on each corner. Every gunanine forms two hydrogen bindings to each of its adjacent gunanines, four in total, by what an enormous stability is archived. Furthermore, the layers stick on each other and are further supported by ions, in general potassium cations, which are encapsulated between two layers, interacting with the inward directed guanine oxygens. b) The counterpart of the G-Quadruplexes are represented by the $i$-Motif, it can be formed by cytosin-rich sequences, but needs a lower pH, since the one of cytosine has to be protonated in order to form an $\mathrm{C} \cdot \mathrm{C}^{+}$base pair. c) The triplet structure is a normal DNA double strand structure, where a third single strand nestles into the double strand along the major groove. Besides the hydrogen bindings between adenine and thymine, known from Watson-Crick base pairing, adenine is forming further hydrogen bindings to another thymine of the third single strand, in a Hoogsteen-like fashion.

In figure II.2.1.4a the crystal structure of a tetramolecular G-quadruplex is illustrated. It is mainly formed in guanine-rich sequences, an consists of stacked planar G-quartets, held together by Hoogsten base pairing. In between those planar layers, small monovalent cations, like sodium or potassium, are enclosed, which contributes to the structures' stabilization. 93 The Hoogsten base pairing occur in G-quadruplexs between the $H 1$ and $C_{2}-\mathrm{NH}_{2}$ with the $C 6=\mathrm{O}$ and $N 7$ of the next guanine. Up next, the crystal structure of the i-motif is presented. The corresponding quadruple stranded secondary DNA structure to 
the G-quadruplex. In contrast to the G-quadruplex there are no four bases in one layer, but alternating layers of $\mathrm{C} \cdot \mathrm{C}^{+}$base pairs between opposite strand.94 Since an unusual hemiprotonated cytosin per base pair is crucial for its formation, the investigations the i-motif structure is mostly done under acidic conditions. Right below, the triplex structure is shown (figure II.2.1.4c). A helix consisting of three DNA single strand. The third ssDNA binds to the major groove of DNA double helix by Hoogsten base pairing to an existing Watson-Crick base pair. Besides the shown base triplet $\mathrm{T} \cdot \mathrm{A}=\mathrm{T}$, another possible triplet is $\mathrm{C}^{+} \cdot \mathrm{G} \equiv \mathrm{C}$, which also includes an hemiprotonated cytosine. 95 .

Apart from the natural occurring nucleotide building blocks, the exchange and modification of the individual nucleotide constituents, including phosphate backbone, sugar and nucleobase, was extensively investigated in order to create a toolbox, with which the features of DNA can be finely tuned. Especially during the design and creation of functional DNA architectures, as i.e. for drug delivery or cell biology applications, 83 the natural occurring negatively charged form of DNA is not ideal, since it makes the penetration of membranes almost impossible. As a consequence, a portfolio of artificial DNA hybrid structures have been designed and examined in the last years. In figure II.2.1.5 some nucleotide modifications are illustrated. 


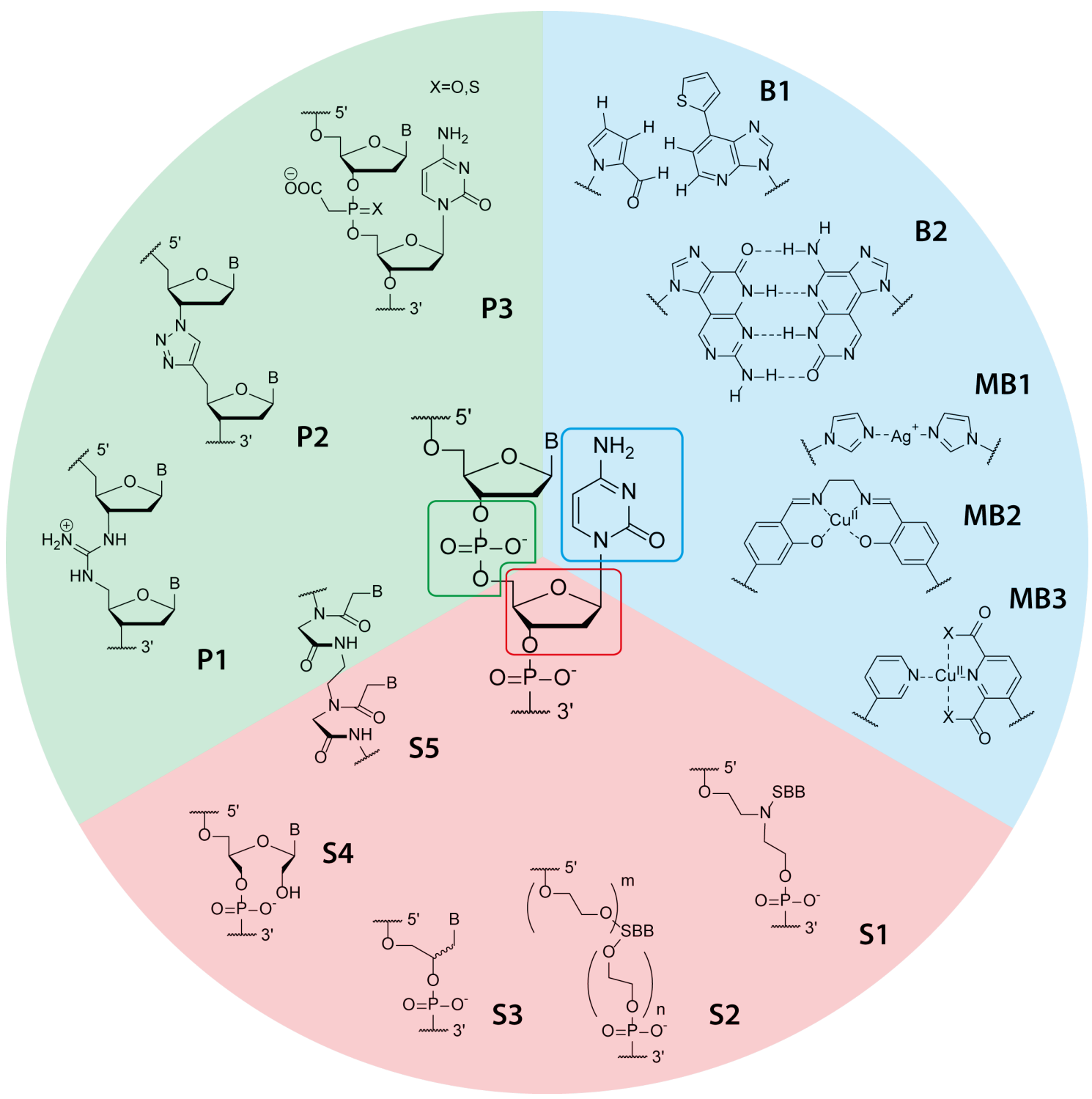

Figure II.2.1.5: Modifications of the individual nucleotide constituents. In the green third a selection of phosphodiester modifications are shown. The subtle alteration of the phophodiester $(\mathbf{P 1})$, uncharged triazole linker (P2) 96 and a positively charged guanidinium linkage (P3).97. In the red third a selection of sugar modifications and replacements are illustrated. An RNA based modification with open sugar for higher conformational freedom, named as unlocked nucleic acid UNA (S4) and it the counterpart of the locked nucleic acid. 98 S5 is an exception, since both the phosphodiester and the sugar are replaced by an uncharged peptide sequence. The modification is called peptide nucleic acid PNA). S1 and S2 are used linkers to incorporate supramolecular building block (SBB in DNA and will be used for the systems describedb in the next chapter. In the blue third a selection of artificial nucleobases is presented. MB1-3 show linkers for artificial nucleobases/ligands for metal base interaction. B2 shows a pair of artificial nucleobases that undergoes hydrogen bonding. As a contrast, another class of artificial nucleobases is made for hydrophobic interactions (B1).

Among the very first modifications made to oligonucleotides were alterations of the phosphodiester backbone to stabilize the oligonucleotides towards enzymatic degradation within cells. Therefore, a number of studies have focused on modifications of the native phosphodiester linkage, reaching from subtle modification as the replacement the free acidic oxygen by an organic acid (phosphonoacatate, P3), $\underline{96}$ over a complete exchange to an uncharged triazole linker (P2) to linkers with a positively charged guanidine linker, 
reversing the complete overall charge of the natural occurring DNA. Besides the phosphate backbone, also the sugar linker connecting the backbone with the nucleobase, has been frequently modified. In order to achieve a higher or lower degree of flexibility for the nucleobases various sugar analogs were investigated. As a result, nucleic acid variations as the RNA based unlocked nucleic acid (UNA), with an opened ribose ring (S4) or its counterpart the locked nucleic acid LNA, has been designed and examined. 98 Meggers et $a l$. have replaced the sugar completely with an glycol motif, connected with the phosphate backbone on 1- and 2-position. The nucleobase is attached to the 3-position (S3). Due to its containing stereocenter the effect of the incorporated enantiomers on stability and reactivity can be investigated. This motif has recently be used by our group to introduce pyridine or imidazole ligands in tetra- and telomeric G-quadruplex for various metal bindings. 199 . 101 An exceptional derivative is the peptide nucleic acid (PNA) (figure II.2.1.5. S5). The complete phosphordiester-sugar framework is replaced by a peptide sequence. In contrast to natural occurring DNA its backbone is not charged, similar to the backbone modification P2, unless additional charged groups are introduced. $102|103| \mathbf{S} 4$ is a frequently used linker for the incorporation of supramolecular building blocks in DNA, which gives a certain flexibility. Furthermore, the polyethylene glycol $(\mathrm{PEG}$ is easy to introduce to both the desired ligand and the oligonucleotide. $\frac{84}{} \mathbf{S 5}$ is based on diethanolamine. The ethanol chains are connected to the phosphate backbone, the nucleobase or $\mathrm{SBB}$ is attached on the amine nitrogen. This structural motif is further discussed in chapter III.2

The third possibility to modify the nucleotide is a variation of the nucleobases nature, in terms of shape or functionality. This area of research makes probably the largest part of nucleotide modifications in the last years. There are mainly four concepts of modifications. Firstly, the hydrogen bonds are retained, but the shape of nucleobases and possible formed hydrogen bonds are varied, as seen for B2. Secondly, the hydrogen bonds are discarded, instead the interactions of base pairs are based on hydrophobicity and shape complementary, as it is the case for B1. In the third place, the base pairing is neglected and the large mainly polycyclic aromatic functional compounds are introduced to one of the strands, while the other strand has only a small or even no nucleobase at the opposite position. Due to its planarity the compound can stack with the adjacent nucleobase pairs.

Lastly, the field of metal-base paring has to be mentioned. The fact that metal ions, can and do interact with nucleic acid is already known for some time past. Under physiological conditions, canonical as well as non-canonical architectures are stabilized by the biologically ubiquitary ions $\mathrm{Na}^{+}, \mathrm{K}^{+}, \mathrm{Mg}^{2+}, \mathrm{Ca}^{2+}$ mainly from the outside, since the highly negatively charged backbone is located there. The concentration and/or change of counter-cation has a direct influence on topology of secondary DNA structure, as i.e. can be observed for the Z-DNA, which is only formed at high electrolyte concentration or for the Holliday Junction, 
which switches from the open form to the stacked form in presence of small concentration of magnesium. 104 However these interactions are rather of unspecific nature, an example for more specific interactions with nucleic acid is the the G-quadruplex, whose structure is additionally stabilized by an cation ion sitting in between G-quartet layers and interact with the inward pointing guanine carbonyl groups, resembling to an ion sandwiched by two crownethers. 105 Most prominent specific interaction of metal ions to natural occurring nucleic acid is probably the DNA cross-links caused by the anti-cancer therapeutic cisplatin, which are preferably formed between adenine and guanine nucleobases. Besides, in the last years further metal base pair interactions in unmodified canonical DNA has been found. $106 \mid 107$ On the one hand there is the insertion of $\mathrm{Hg}^{2+}$ between and $\mathrm{T}-\mathrm{T}$ mismatch, on the other hand $\mathrm{Ag}^{+}$was found to bind in between a C-C mismatch; in both cases the involved nucleobases are deprotonated. Encouraged by this observation, a plethora of artificial nucleobases were developed, that can be introduced to canonical oligonucleotides, where one and more layers of base paring is replaced by an metal base pair. 108 Due to the confined space between the base pair layers, flat coordination environment (linear, square-planar) are preferred but under certain conditions or secondary DNA topologies other coordination environments are possible. In figure II.2.1.5 some examples are shown (MB1-3). Inspired by the above mentioned metal interactions between natural occurring nucleobases, Müller et al. replaced the cytosine by imidazole and could prove the existence of imidazole- $\mathrm{Ag}^{+}$-imidazole artificial metal base pair within an intact DNA double helix (MB1). 109. MB3 shows one of the first artificial metal base pairs reported by Schultz et al., a $\mathrm{Cu}^{2+}$ metal ion coordinated by pyridine and pyridine-2,6-dicarboxylate, or an nitrogen containing analogs, in a flat square planar fashion.110[11] As a last example, the DNA incorporated salen ligand reported by Clever et al. has to be mentioned. The combination of the metal base pair concept in combination with dynamic covalent chemist led to reversibly covalently bounded DNA double helix, showing an immense stabilization compared to the unmodified double strand.112|113

The above described system are coined as ligandosides 114 (figure II.2.1.6a), which includes all artificial ligands that are attached at the sugar backbone, where actually the normal nucleobases were located. 
(a)

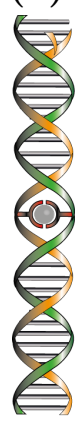

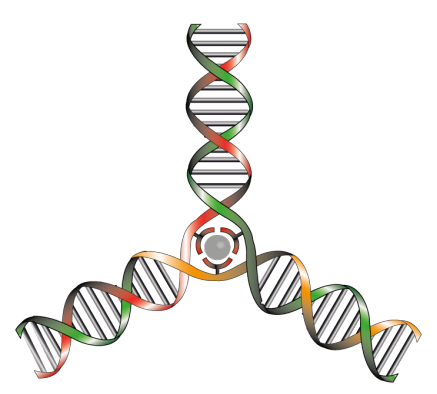

(b)

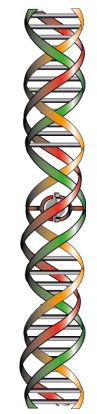

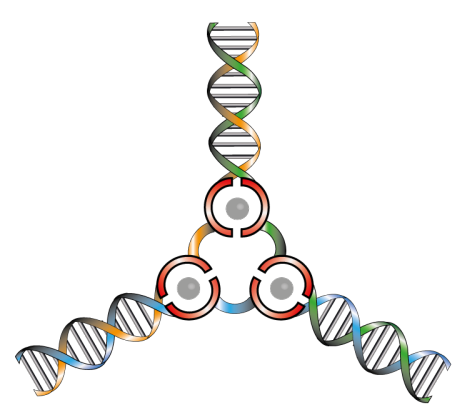

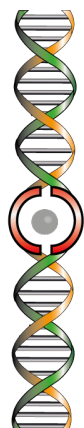

Figure II.2.1.6: Ligandoside vs. incorporated ligands. a) Schematic representation of published ligandoside containing secondary DNA structures. From left to right: double-stranded helix with opposite lying ligandosides coordinating to metal ion; 109 three-way iunction with ligandosides in the structures' central void capable of forming octahedral complexes with metal ions; 115 triplex stabilized by three ligandosides, one per strand, coordinating to metal ion. b) Similar structures as threeway junctions or normal double helix structures have been designed with incorporated artifical ligands, capable of metal coordination. 116. However, compared to the ligandoside approach, the sugar-phosphate backbone is suspended, what effects the stability of the secondary DNA architecture.

These ligandosides are rarely used in other secondary structures then the double helix, anyway, some examples have been reported by Shionoya et al. With the help of triple coordinated $\mathrm{Ag}^{+}$by pyridine ligandosides, the stabilization of a metal containing triplex structure could be obtained. 117. Later another ligandoside based on 2,2'-bipyrdine was used to stabilize a DNA three-way junction, with $\mathrm{Ni}^{2+}$ metal ion, coordinated in the middle of the DNA architecture by three ligandosides in an octahedral fashion.118 Some years later a fine tuned system was reported by Takezawa et al., where, depending on the presence of $\mathrm{Ni}^{2+}$ metal ions, the system can reversibly switched between duplex and three way junctions. 115 More commonly used in modified non-duplex DNA, especially in the field of "supramolecular DNA assemblies", are the here entitled "DNA incorporated ligands" figure II.2.1.6b). For this kind of modification the whole nucleoside is replaced by the respective ligand, which is directly bound to the phosphate backbone. Representatives of those systems can be found in hairpin, 119 duplex,"116 g-quadruplexes, 99[120 threeway junction structures 121 and other higher order assemblies. 84 These structures are further discussed in the next chapter (chapter III.1).

\section{II.2.2 Holliday Junction}

Already over 50 years ago Robin Holliday postulated a DNA construct consisting of two crosslinked DNA duplexes, which is formed during the process of intergenic homologous recombination, 122 responsible for the combinations of chromosomal DNA, representing genetic variation, which in turn enables populations to adapt during the course of evolution. 123 This cross-linked four-way junction was later named after R. Holliday, 
therefore this DNA architecture is nowadays mainly known as Holliday Junction (HJ). From that day on the motif Holliday junction was intensively investigated from both, chemists and biologists, in terms of topological and functional appearance under physiological conditions, 124 126 DNA-protein interactions, $127 \mid 128$ relevant mechanisms during the homologeous recombination 129 and of course structural elucidation. 130.132 In general the HJ consist of, as the alternative name four-way junction let assume, four individual ssDNA, which contain two sequence segments, each complementary to a sequence segment of two other ssDNA. Carrying on this assembly motif, four connected DNA helix structures are formed with a junction in the middle. For this structural arrangement four possible isomeric main topologies were postulated the HJ can switch between (figure II.2.2.7). The other possible intermediate topologies the $\mathrm{HJ}$ can assume while transforming from one stable topology to another are neglected here.133 In contrast to natural occurring HJs. the artificial designed $\overline{\mathrm{HJS}}$ are in most cases immobile, means that the complementary sequences are designed in a way that only one structure can be formed. In contrary, the huge genomic DNA contains several potential complementary sequence segments, meaning that the four individual arms may slide through the junction in a specific pattern till the largest possible complementary base pair overlap is obtained.

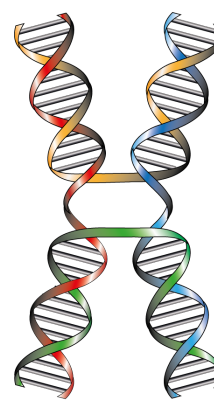

Stacked X (Anti-Parallel) Iso-I
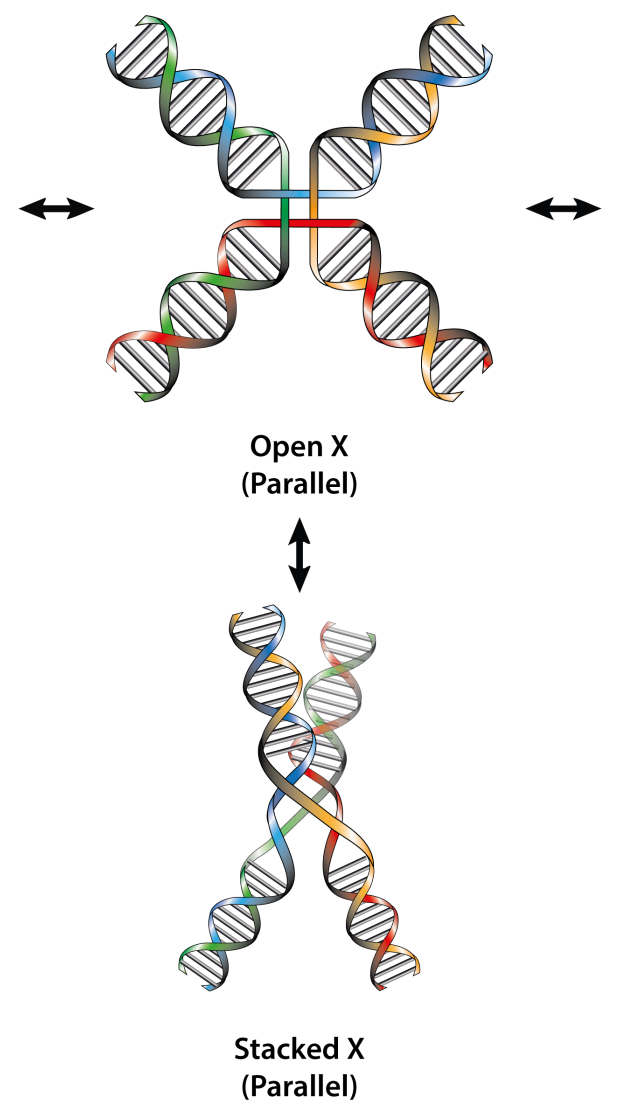

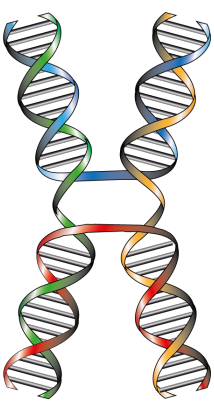

Stacked X (Anti-Parallel) Iso-II

Figure II.2.2.7: Schematic representation of the four known possible isomers of a natural occurring Holliday Junction. The isomers can be categorized into open and stacked forms, whereby the stacked form can be subcategorized into parallel stack, where two neighbored oligonucleotide strands have the same orientation $(3$ '- $>5$ ', 3'-> 5'), whereas in the antiparallel form, the strand orientation is alternating (3'-> 5', 5'-> 3'). Most common form is the antiparallel stacked form, which can isomerize from the ISO-I to the ISO-II form, by passing the open stacked form as an intermediate structure. 
The four major $\mathrm{HJ}$ species are the stacked $\mathrm{X}$ form, where a distinction is made between the parallel and anti-parallel assembly. In the parallel configuration two adjacent strands exhibit the same strand orientation, while the strand orientation in the anti-parallel isomer is alternating. Additionally, the anti-parallel orientated stacked $\mathrm{X}$ form of the HJ is further subcategorized into the Iso-I and Iso-II conformation, depending on its spatial arrangement. The open $\mathrm{X}$ form has a square planar topology (figure II.2.2.8a) and exists as the major species at low electrolyte concentrations, $104 \mid 134$ since in this form the repulsive forces between the negatively charged phosphate groups within the DNA backbone are minimized. It is worth to mention, that the open-X clearly shows a gap at the junction position, what should be kept in mind. From this topology the HJ can be directly converted into every other possible topology, which in turn mean that the open $\mathrm{X}$ is always an intermediate species, when the HJ transforms to any other topology. However, in silico investigation indicating that at higher salt concentrations the intermediate $\mathrm{HJ}$ conformer has already a rather tetrahedral character. 104 Under physiological conditions, with electrolyte concentration above $100 \mathrm{mM}$ or in presence of divalent cation $\left(>2 \mathrm{mM} \mathrm{Mg}^{2+}\right), 135$. the preferred conformer is the anti-parallel stacked X structure, which is more densely packed compared to the open form (figure II.2.2.8, since the negatively charged DNA backbone is better shielded by the present cations. Having a closer look at the crystal structure, it becomes quite obvious that the two respectively $\mathrm{HJ}$ arms are arranged in line, forming almost one consistent duplex. Both of these pseudo duplex structures are twisted against each other. This twist is characterized with the so called inter duplex angle (IDA) and is ranged between $40^{\circ}$ and $60^{\circ}$. Although often postulated, the parallel stacked-X isomer of the Holliday junction, was never really verified. Neither the results from the publication dealing with that topic have been clear enough to see the existence of this conformer as proofed, nor was this conformer ever crystallized.136|137 However, Lu et al. claim to create a parallel stacked-X $\mathrm{HJ}$, by connecting two involved ssDNA of opposite arms. 138 
(a)

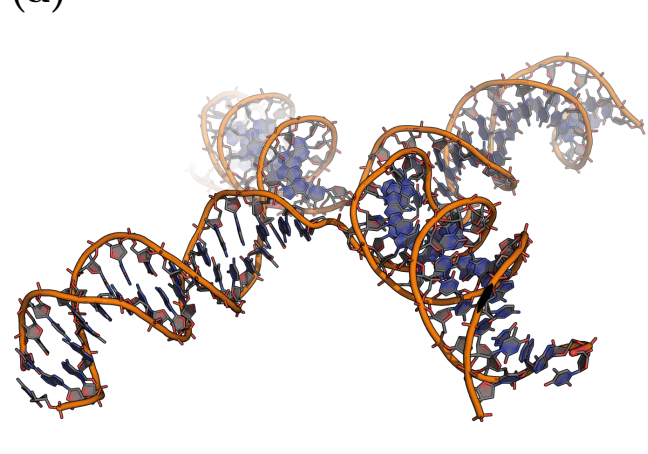

side

(b)

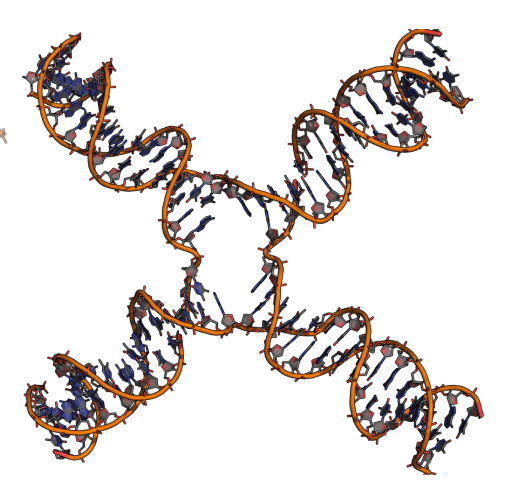

top

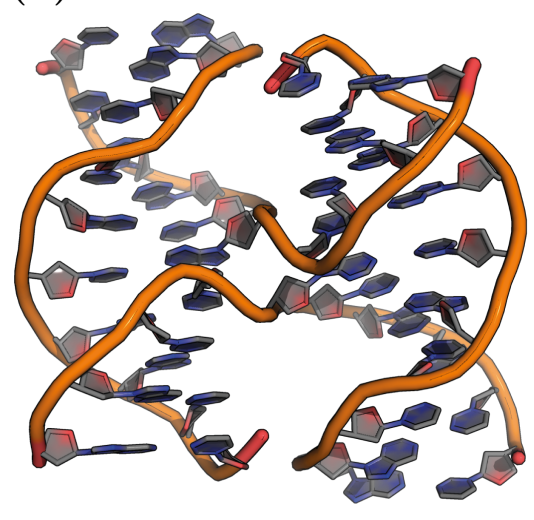

side

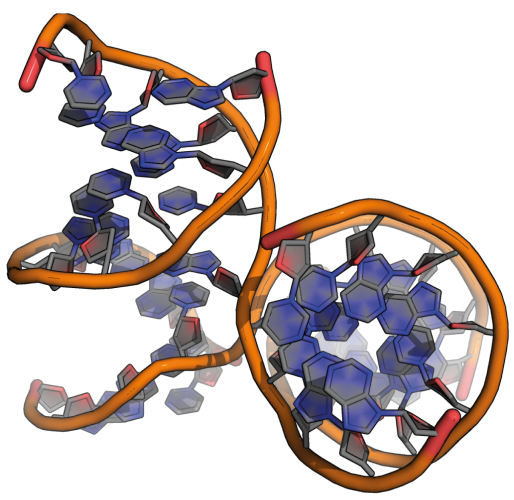

top

Figure II.2.2.8: Crystal strucutres of open- $X$ and stacked- $X$ Holliday junctions. The open-X139 form was extracted from protein-DNA co-crystallisation. The stacked-X form of the HJ was solved by Cardin et al.. [140]

In the recent past, Mergney et al. reported a system including a parallel stacked-X like four-way junction to preset the orientation of a G-quadruplex motif, formed within a junction environment. 141] This specific example will be further discussed in the next chapter (chapter III.1).

Concluding, the Holliday junction has been intensively investigated to the present day, however lots of structural and functional questions are still unanswered. That is probably one of the reasons, why the unmodified four-way junction motif is frequently used in quite well predictable DNA origami structures, $79[130$ but has never been used, to the best of our knowledge, in combination with metal base pairs or supramolecular ligand modifications, as it has been shown for almost all other known secondary DNA structures section II.2.1. 


\section{Ligands for Metal-Mediated \\ Coordination Cages Embedded in Oligonucleotide Scaffolds}

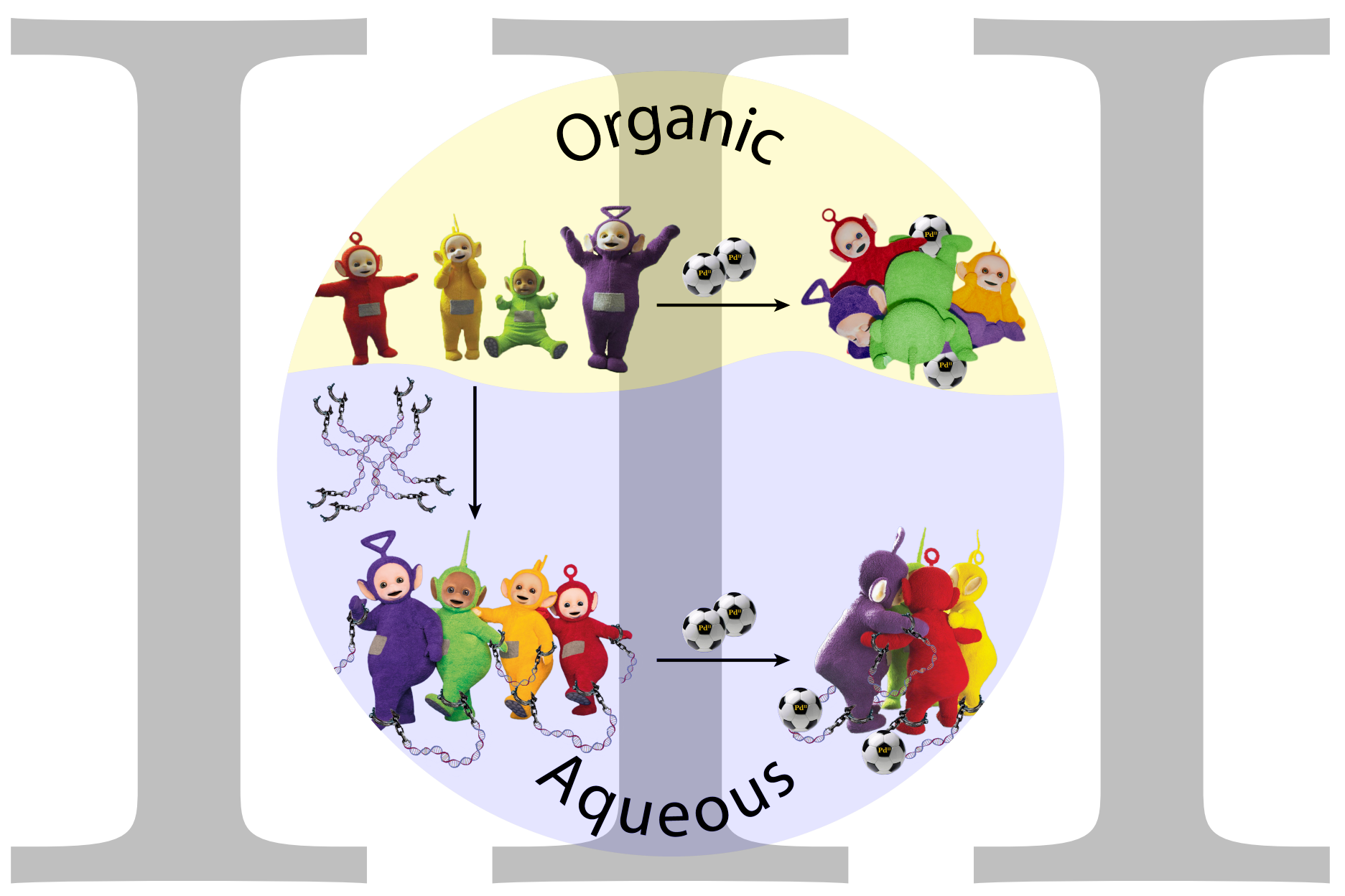

Related Publications:

T. R. Schulte, M. Krick, C. I. Asche, S. Freye, G. H. Clever, RSC Adv. 2014, 4, 29724-29728. 


\section{Chaperer III. 1}

\section{Introduction}

Historically, discrete assemblies derived from natural occurring building blocks (including DNA and proteins, but in this work only the DNA part is taken into account) were distinguished from those made of synthesized organic ligands, with or without the use of metal ions. Thus, although both, the field of supramolecular chemistry and the field of biotechnology, sharing certain approaches in the design, especially with regard to deployed symmetries, they evolved independently their own methods to create three-dimensional self-assembled structures. $142 \mid 143$ Even though most structural symmetries can be formed in both areas of research, they clearly differ in size. While the average cavity dimension for bio-derived systems ranges in the nanometer scale, the size of cavities for synthetic structures is rather in the region of several $\AA$. This difference in size is most commonly accompanied with a difference in the cavities' hydrophobicity, what is not surprising since the smaller cavities of the synthetic structures are delimited in most cases by poly-aromatic ligand backbones. In contrast, the larger cavities are more framed than delimited by the bio-derived building blocks, which rather led in combination with its commonly polar shells to a hydrophilic interior of cavity. Due to these differences the choice of the most suitable system is highly dependent on a respective issue to be investigated, therefore the actual intention of desired system must be known at a very early stage and will be discussed in the next chapter. However, for most of the bio-derived architectures the water-solubility is inherent, whereas the formation of three-dimensional supramolecular structures of artificial organic ligand in water was quite a challenge. A more detailed introduction to this topic can be found in Chapter IV.1.

On the other hand, the architectures formed from naturally occurring building blocks are often lacking in rigidity especially in terms of the formation of discrete angles and vertices. 144 The combination of both, bio-derived and synthetic building blocks gave completely new possibilities of designing three-dimensional structures, which showed even in large structures discrete shapes and a certain rigidity, compared to everything else that has been reported before in this area of research. In the following, some of those systems 
are presented.

In 2004 Sleiman et al. and Han et al. independently reported the incorporation of metal coordination environments into oligonucleotide strands. 145 146] In case of Han, a double strand triangle was designed, with iron(II) terpyridine complexes on both ends of the double strands forming the discrete vertices in the DNA structure. In a first step iron(II) is added to two non-complementary strands in order to from an octahedral iron complex between to single strands. Subsequently all strands are mixed together and the self-assembly of complementary olignucleotides lead to formation of the triangle figure III.1.0.1.

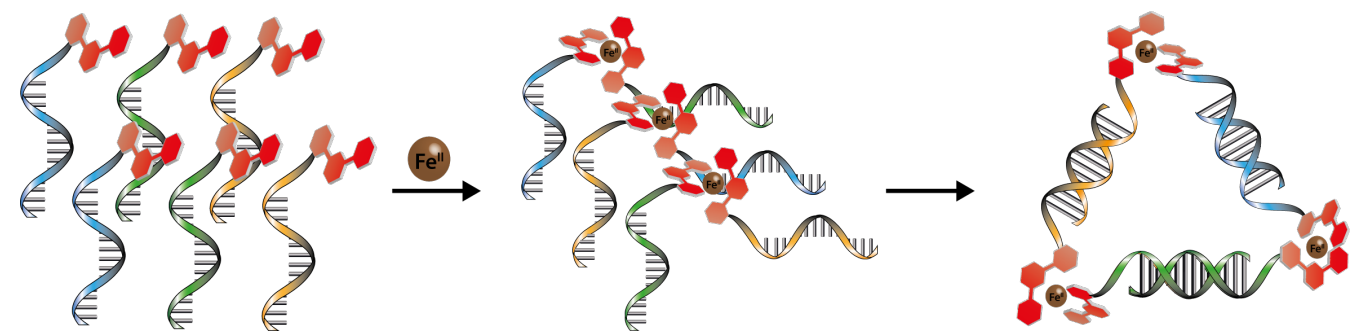

Figure III.1.0.1: Triangular DNA architercuture by Han et al.. The assembled structure consists of six ssDNA complementary to each other, having a terpyridine motif on one end. In presence of FeII cations, the single strand do form octahedral Fe(tpy-DNA) 2 complex, which assembles in a subsequent step to three bridging double strand helices, giving the final triangular DNA architecture. 145

One year later, Krämer et al. reported another system for the incorporation of metal coordination environments into DNA.147. Also based on terpyridine, the ligand was attached on both ends of an oligonucleotide single strand. In absence of $\mathrm{Fe}^{2+}$ or $\mathrm{Zn}^{2+}$ the modified strand hybridized with a complementary strand. Upon addition of one equivalent $\mathrm{Fe}(\mathrm{II})$ or $\mathrm{Zn}(\mathrm{II})$, the double strand preferentially disassembles to gain the flexibility and form an intramolecular octrahedral metal complex. In both cases this process is reversible, but interestingly, in contrast to $\mathrm{Fe}(\mathrm{II})$, a second equivalent of $\mathrm{Zn}$ (II) led to reformation of the double strand, where each terpyridne ligand is bound to one zinc cation figure III.1.0.2 This example nicely illustrates how strongly the formation of metal complexes can affect the overall oligonucleotide structure depending on the choice of the metal ligand system. 


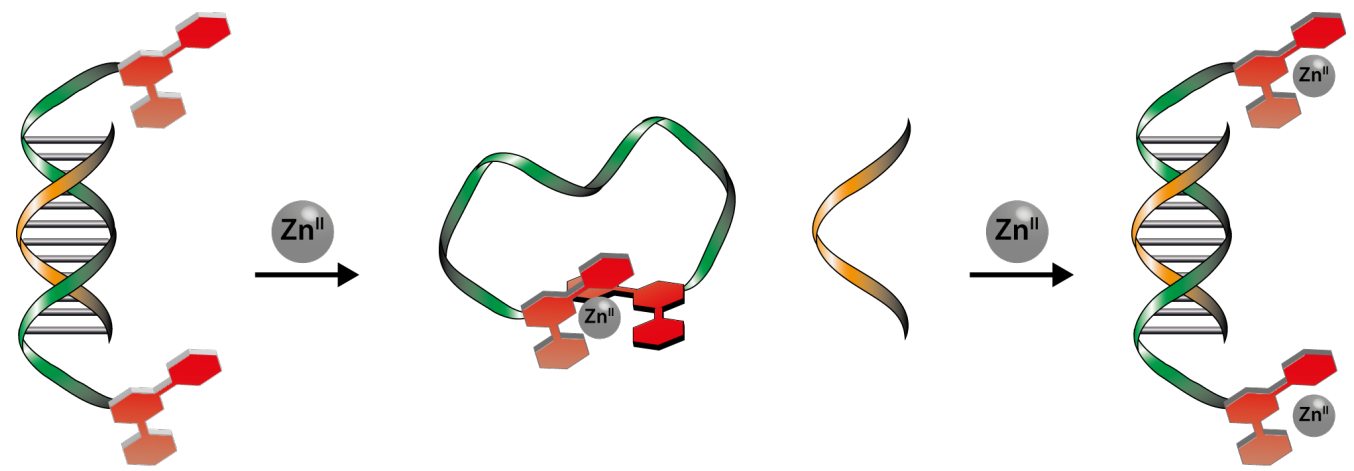

Figure III.1.0.2: Terpyridine-DNA structure by Krämer et al.. In contrast to Sleiman's system, Krämer's structure consists of a DNA single strand with terpyridine attached to both ends and a complementary strand without any artificial attachments. In absence of transition metals, the most stable form is described by a double helix structure. In the presence of one equivalent $\mathrm{Fe}(\mathrm{II})$ or $\mathrm{Zn}(\mathrm{II})$, the double helix disassembles and an intramolecular $\left[\mathrm{Zn}(\mathrm{II})(\text { terpy })_{2}\right]$ complex is formed. In contrast to $\mathrm{Fe}(\mathrm{II})$, a seconed equivalent of $\mathrm{Zn}(\mathrm{II})$ leads to reassemble of the starting double helix structure, but with both terpyridine motifs occupied by ion zinc cation. 147.

However, Sleiman et al. reported a system where the coordination environment of the metal is predetermined by the hybridization of two single strands. These strands contained either a terpyridine or a phenanthroline moiety. By mixing two complementary strand together, double strands with different coordination environments were created, which show depending on the ligand pairs, a preferred coordination behavior with respect to the tested metal cations (figure III.1.0.3), accompanied with a significant increase of denaturation temperature. Fe(II) showed as in the examples before the best match with two terpyridine ligands and the highest stabilization in denaturation temperature, while two phenantroline ligands form the most stable complex with $\mathrm{Cu}(\mathrm{I})$, that prefers an tetrahedral coordination environment. For the mixed ligand case $\mathrm{Cu}(\mathrm{II})$ binds strongest in a trigonal-bipyramidal fashion. Most interestingly, is the behavior of copper in the wrong coordination environment. Initially, $\mathrm{Cu}(\mathrm{I})$ binds weakly to the mixed ligand case, but after ca. $30 \mathrm{~min}$ it undergoes a spontaneous oxidation to $\mathrm{Cu}(\mathrm{II})$ to fit better into the trigonal-pyramidal coordination environment. The other way around with $\mathrm{Cu}(\mathrm{II})$ in the phenantroline environment is also observable, but in this case the spontaneous reduction to $\mathrm{Cu}(\mathrm{I})$ took over $12 \mathrm{~h} . \underline{116}$ 


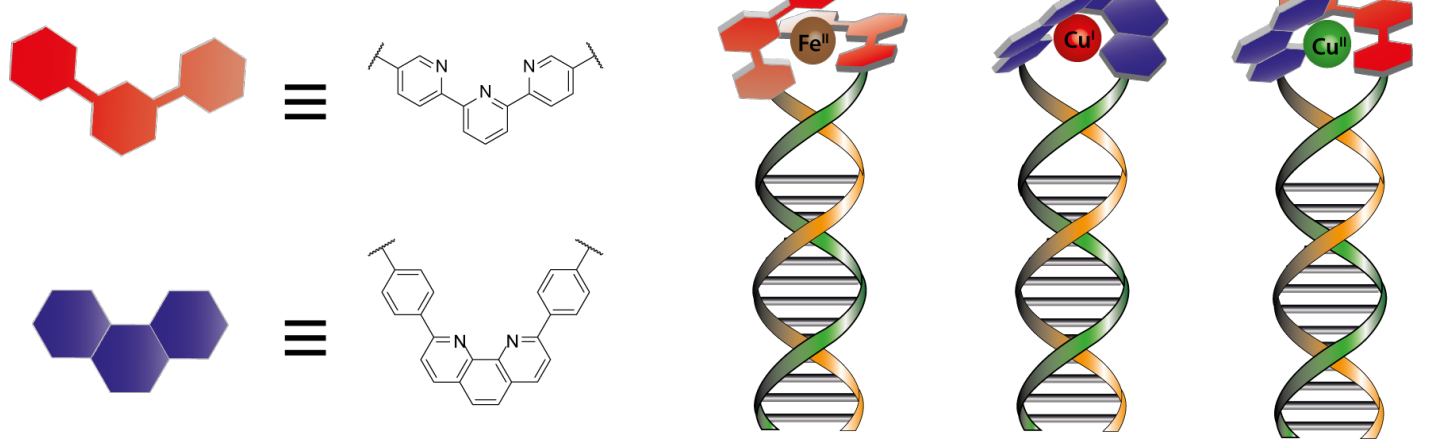

Figure III.1.0.3: Mixed coordination DNA structure by Sleiman et al. The system consists of two coordination motifs, phenantroline (phen) and terpyridine (terpy), attached to two complementary sequences. By assembling the complementary single strands to a stable double helix, three different coordination environments can be obtained. Each coordination environments, prefers a different metal cation to coordinate. From left to right: (terpy) 2 - $>$ $\mathrm{Fe}(\mathrm{II}),(\text { phen })_{2}->\mathrm{Cu}(\mathrm{I}),($ terpy)$($ phen $)->\mathrm{Cu}(\mathrm{II}) .116$

In another example, Sleiman et al. used the the phenantroline coordination environment within the oligonucleotidic strands. In the first one, a templated formation of a metal-DNA fourway single stranded junction with a chiral metal center is described. Two modified single strands with an internally integrated phenantroline ligand are brought together by an with help of an templating strand. Then the metal ion is added to from the chiral phenantroline metal complex. Subsequently the templating strand is removed and the chiral four-way single stranded junction with the metal complex in its branch remained figure III.1.0.4. 148 In a further example, Sleiman et al. designed a three-dimensional DNA prism with the phenantroline coordination environments located at each of its vertices, where matching metals can coordinate in order to give further stabilization and rigidity to the DNA construct. 149]

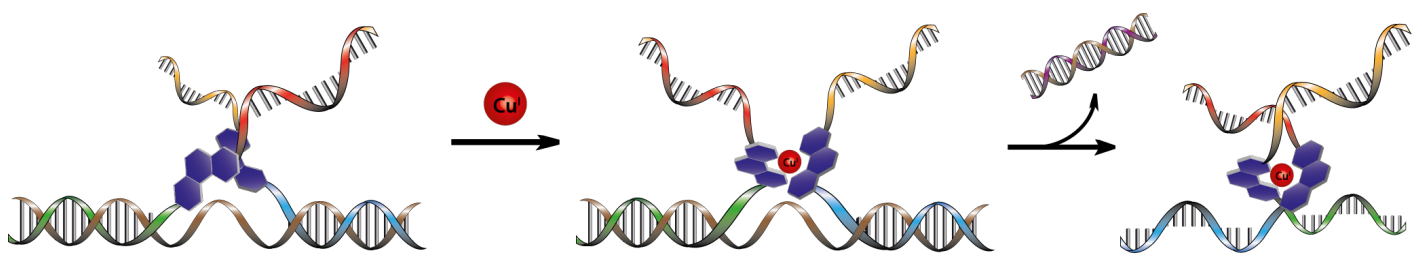

Figure III.1.0.4: Chiral single stranded Metal-DNA fourway junction by Sleiman et al.. The system consists of two DNA single strands with an central incorporated phenantroline ligand. A partial sequence of each single strand is complementary to a third single strand. The assembly of all DNA strands give a structure, where both phenantroline ligands are preorganized, before $\mathrm{Cu}(\mathrm{I})$ can coordinate in a subsequent step. In a last step, the nonmodified single strand is removed from the structure with help of complementary single strand, obtaining a single stranded Metal-DNA fourway junction with a chiral metal coordination center. 148.

Inspired by the Metal-DNA hybrid systems described above, we wanted to exploit the highly programmable folding patterns of DNA and the possibility to integrate metal coordinating ligand into oligonucleotides, 150 to design a new system which shall solve one of the still existing problems in the field of supramolecular coordination cages. The formation of multi-functional heteroleptic coordination cages. Especially, the controlled 
self-sorting between ligands with similar (or identical) coordinating groups is still a big challenge. Yet, it is highly desirable, as it would simplify the ligands synthesis and modular strategies to incorporate a combination of specific functionalities into the coordination cages. 151] Although there are some examples for narcissistic self-sorting $66[152$ and social selfsorting 153 for coordination cages, the systems lack a controlled formation, especially when more than two different ligands are present. Frank et al. showed this for some phenothiazine based $\left[\mathrm{Pd}_{4} \mathrm{~L}_{8}\right]$ interpenetrated coordination cages. As long as the ligand had a similar size and coordination profile, the self-assembly of ligands ended in a mixture of cages with various ligand combinations, represented by the sum formula $\left[\mathrm{Pd}_{4} \mathrm{~L}_{\mathrm{x}} \mathrm{L}_{8-\mathrm{x}}\right]$. An statistical mixture of cages even occurs when assembling the pure homoleptic interpenetrated coordination cages before mixing them. Due to the reversible palladium-nitrogen coordination bond, the ligands can exchange. On the one hand it is an important feature on the way to the thermodynamically most stable structure, but on the other hand it promotes the formation of the statistical cage mixtures, due to the similar energies of the different cages, making it easy to interconvert. (figure III.1.0.5).

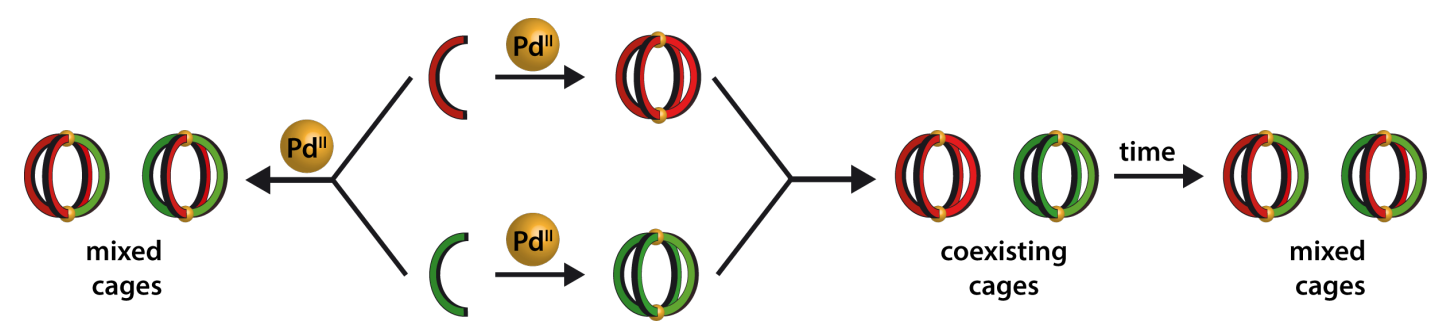

Figure III.1.0.5: Statistic mixtures of coordination cages occur during the assembly of $\left[\mathrm{Pd}_{2} \mathrm{~L}_{2}^{\mathrm{A}} \mathrm{L}^{\mathrm{B}}{ }_{2}\right]$ with structurally related ligands. By mixing all building blocks immediately together at once, a statistical mixture is obtianed. Assembly of the ligand pure coordination cages (green and red) in spatially separated environment was a possible approach,which allows the ligand pure cages to coexist after mixing them together. However, due to the reversible coordination bonds of the ligands to the palladium(II) cations, a thermodynamically more favorable statistical mixture of cages is obtained after a certain time. 66

In the last years several attempts were reported and a progress is clearly visible. Clever and Crowley reported palladium-mediated coordination cage systems that form quantitatively $\left[\mathrm{Pd}_{2} \mathrm{~L}_{2}{ }_{2} \mathrm{~L}_{2}{ }_{2}\right]$ cages in a integrative self-assembled manner, either by steric hindrance $75[76$ or shape-complementary 6171 However, a general approach for the quantitative formation of heteroleptic cages from two different ligands or more was not found yet.

In order to find a possible DNA scaffold for the controlled formation of $\left[\mathrm{Pd}_{2} \mathrm{~L}_{4}\right]$ coordination cages, Shionoyas' metal-stabilized three-way junction (figure II.2.1.6a, second structure) gave the inspiration to have a closer look at secondary canonical and non-canonical structures of DNA section II.2.1). Therefore, the DNA architecture had to fulfill two crucial requirements. First, it had to be possible to integrate four ligands into its structure, in a certain proximity. And second, the ligands had to be preogranized in a squareplaner manner, since the coordination of ligand to the metal occurs in the same geometry. Considering those two requirements, two structures were suitable for the desired purpose, the G-quadruplex and the HJ (4-way junction). But since the tetrameric G-quadruplex 
does not give any control over the self-sorting of ligands, the structure would also lead to a statistical mixture and was therefore excluded, by what the HJ became our first choice (figure III.1.0.6).
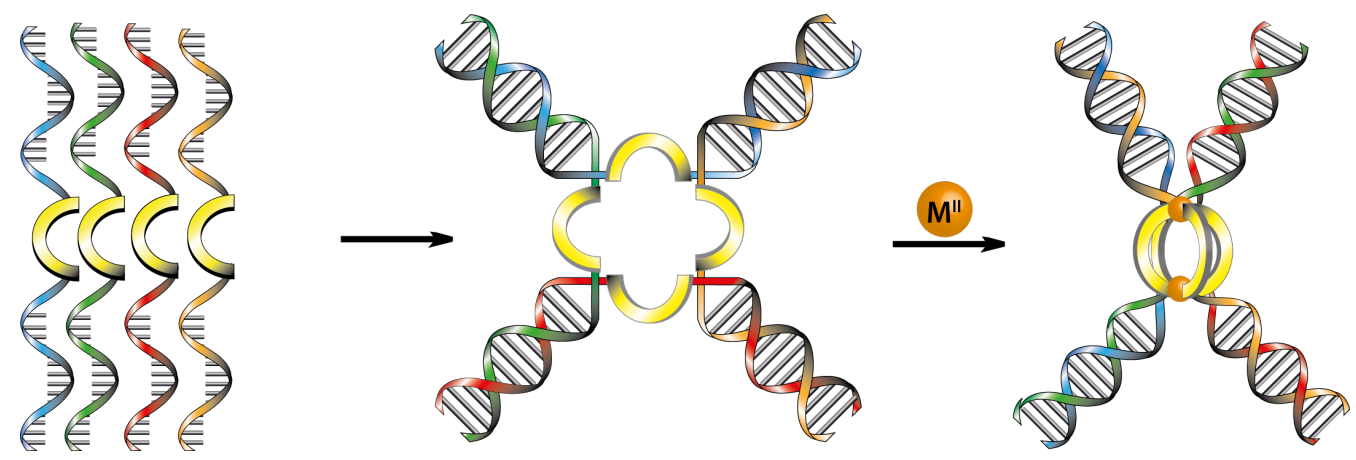

Figure III.1.0.6: Approach of a controlled assembly of a metal-mediated coordination cage, supported by a preassembled Holliday junction scaffold. The system shall consists of four DNA single strands, containing an incorporated bis-monodentate ligand. In a first step, the DNA single strands are assembled into a Holliday junction, forming the intended scaffold, preorganizing the ligands at the central position of the DNA architecture. Subsequently, the metal cations are added, in order to form the desired coordination cage. 


\section{Chapter}

\section{Results and Discussion}

\section{III.2.1 Idea and conception}

For a non-statistical formation of an $\left[\mathrm{M}_{2} \mathrm{~L}_{4}\right]$ cages derived from a ligand mixture with more than two different ligands, some basic requirements should to be fulfilled.

1. the formed cage structure must be significantly lower in energy compared to the other formed structures

2. the ligand exchange process must be inhibited

3. a preoganized positioning of ligands within the coordination structure.

As already mentioned in the introduction section II.1.3, several successful attempts were reported for the creation of heteroleptic $\left[\mathrm{Pd}_{2} \mathrm{~L}_{2}{ }_{2} \mathrm{~L}_{2}{ }_{2}\right]$ by Clever et al. based on bis-monodentate pyridine ligands with shape complementary or steric hindrance at the coordination site. Crowley et al. published an $\left[\mathrm{Pd}_{2} \mathrm{~L}_{2}{ }_{2} \mathrm{~L}_{2}{ }_{2}\right]$ cage with free amine groups at the coordination site for hydrogen bonds with neighbored exohedrally modified ligands, to from preferentially the cis-isomere of the $\left[\mathrm{Pd}_{2} \mathrm{~L}^{\mathrm{a}}{ }_{2} \mathrm{~L}^{\mathrm{b}}{ }_{2}\right]$ cage. Although all of these systems work for the specific ligands, they only fulfill the first requirement. Additional ligands would probably lead again to a statistical mixture, albeit with less possible species.

For the second requirement, the inhibition of the ligand exchange, no ideal concepts are known in the field of supramolecular coordination cages. Clever et al. have shown that the rate of ligand exchange is decreased in coordination structures, depending either on their complexity, means how strongly the ligands are interwoven with each other, $\underline{60}$ or on 
the metal-ligand interaction. When palladium(II) is replaced by platinum(II), the ligand exchange rate is drastically reduced, since the Pt-N coordination bond is kinetically more inert compared to the $\mathrm{Pd}-\mathrm{N}$ coordination bond.24] On the other hand the correction of malpositioned ligands during the assembly process is also inhibited, what leads to extremely decelerated formation rates of the desired coordination cage. A further possibility, that has been already shown for supramolecular architectures and coordination cages, where covalent connections of ligands was achieved in a post-synthetic approach.154 Leigh et al. created a 'Star of David' catenane, where the ligands were preorganized by templating metals and subsequently covalently connected in a metathesis reaction. 155 Thereafter, the ligands cannot exchange anymore. Transferred to the coordination cages, this method would require an initial formation of the heteroleptic cage that is stable for a certain time, until the post-synthetic reaction takes place. Furthermore the structure would be irreversibly connected, what's contrary the concept of supramolecular chemistry. However the connection of course could also be realized by dynamic covalent bonds.

The last requirement is the preorganized positioning of the ligands before forming the coordination cage, to avoid stereo isomers. For that reason it needs a system which assembles in a highly controllable and reproducible way. Since the integration of such function into the ligand system would probably be an unpredictable endeavor and nightmarish synthesis, outsourcing this function into an external scaffold would probably be the most auspicious approach.

The idea and concept of the here presented system actually was inspired by nature. Having a closer look to natural occurring enzymes, it becomes quite obvious that the actual active site, where the desired catalytic reaction takes place, is rather small, compared to the rest of the peptidic secondary structure. Although the residual structure does not necessarily contribute to the reactivity of the catalytic site, it is still the framework or scaffold, which places the functional groups within the active site at the right position, forming the cavity shape and shielding the active site from unwanted external influences. Additionally, it makes the active site soluble in water and contributes in many other ways. With other words, the peptidic scaffold around the active site is a crucial part of the enzyme structure, which offers additional features. However, creating such a peptidic scaffold of a specific shape is an highly complicated project. In nature enzymes are folded during the peptide synthesis in a certain way, often even with help of supporting enzymes, to achieve the desired functional topology. As soon as the structure is unfolded, e.g. due to high temperatures, the enzyme cannot simply be refolded into its native topology. This process, the so called 'denaturation', is accompanied with a loss of function. Therefore, it is still a complicated and unsolved problem, to predict the structure and function of secondary peptedic structures formed from an synthesized polypeptide. Hence, another natural occurring polymer was chosen, which folding patterns are highly predictable, making 
it suitable to create an external scaffold for the formation of heteroleptic coordination cages.

\section{III.2.2 Acridone-based ligand embedded in Holliday junction}

DNA is possibly the most predictable and programmable self-assembling material known, undergoing rapid, high fidelity association according to simple base-pairing rules. Though many self-assembling molecules are known, such predictable control over the resulting structure is scarce. DNA gives researchers a code to define which strands will pair, to from double helices that are structurally uniform and well-defined. For these reasons, DNA assembly has been exploited in the field of DNA nanotechnology to generate higher order functional architectures.

(a)

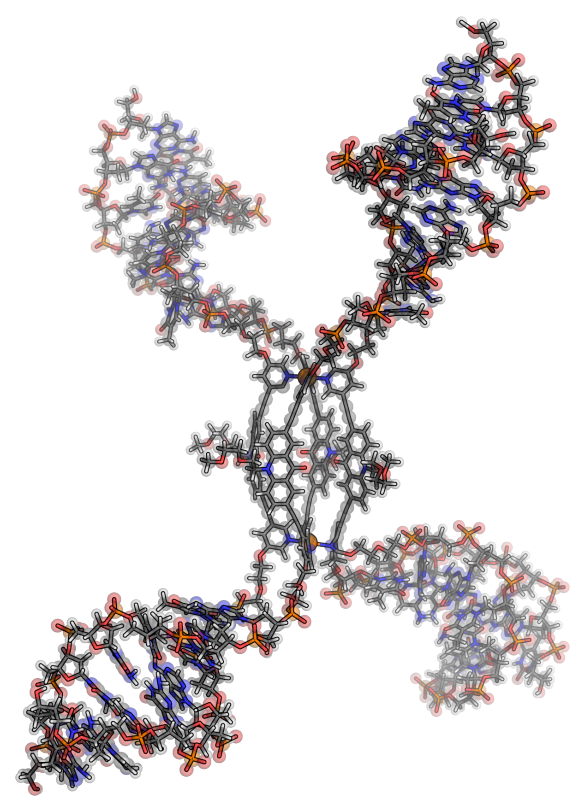

\section{III.2.2.1 Design and syntheses acridone-based ligand}

The first attempt to create a DNA incorporated ligand, suitable for an controlled cage formation, was based on the acridone backbone, which was previously used in various other supramolecular cages systems and is therefore already well characterized.20|41] For the incorporation in oligonucleotides, polyethylene glycol (PEG) linkers were chosen, which are known not to hinder the formation of self-assembled coordination cages from a previous publication. 32 The exohedral modifications on the ligands, for the increase of solubility, 
(b)

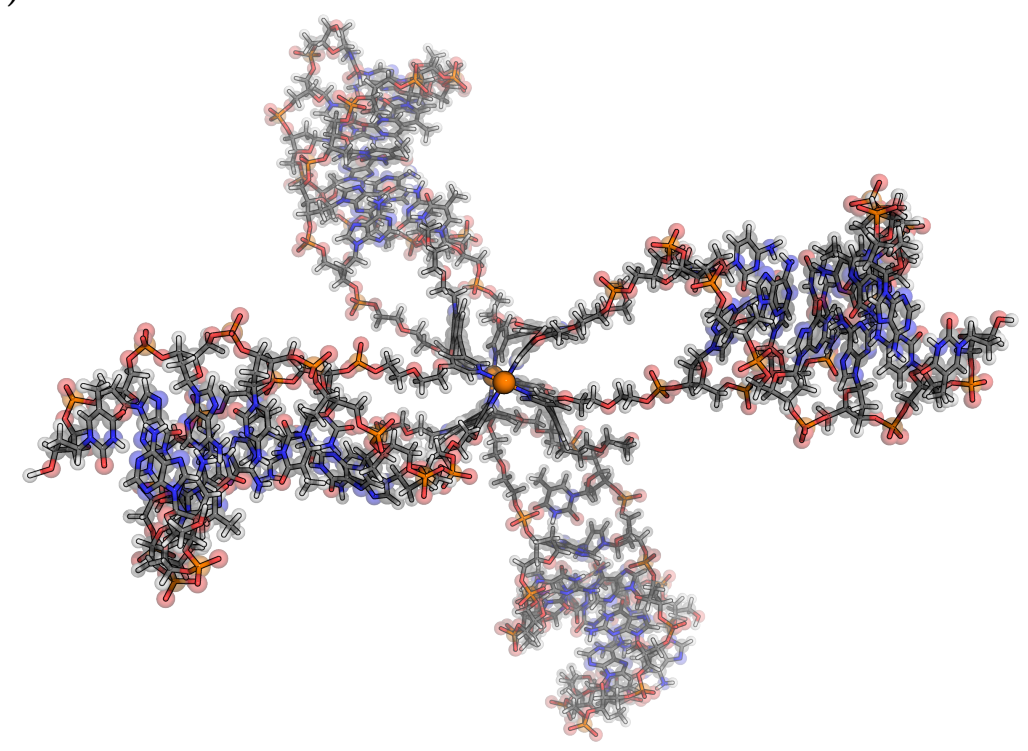

Figure III.2.2.1: MMFF model of first generation DNA-cage hybrid structure. Modelled in Sparten '10. 156 The ligands are coordinated to palladium. The ONS sequences contain and extra T-T mismatch next to the integrated ligand.

were tested on its ability to form supramolecular cage structures. It could be shown that the attachment of $\mathrm{PEG}$ groups at the coordinating pyridine motifs did not hinder the ligands in the formation of supramolecular cages or even interpenetrated double cages. On this basis, ligand 9 was designed with an acridone backbone and pyridine attached polyethylene glycol linkers, suitable for the integration in ONS during DNA solid-phase synthesis. The ligand was synthesized in an eight step synthesis, followed by two additional steps to introduce the 4,4'-Dimethoxytrityl (DMT) and $\beta$-cyanoethyl phophoramidite CEP groups, which are crucial for the subsequent DNA solid phase synthesis. 


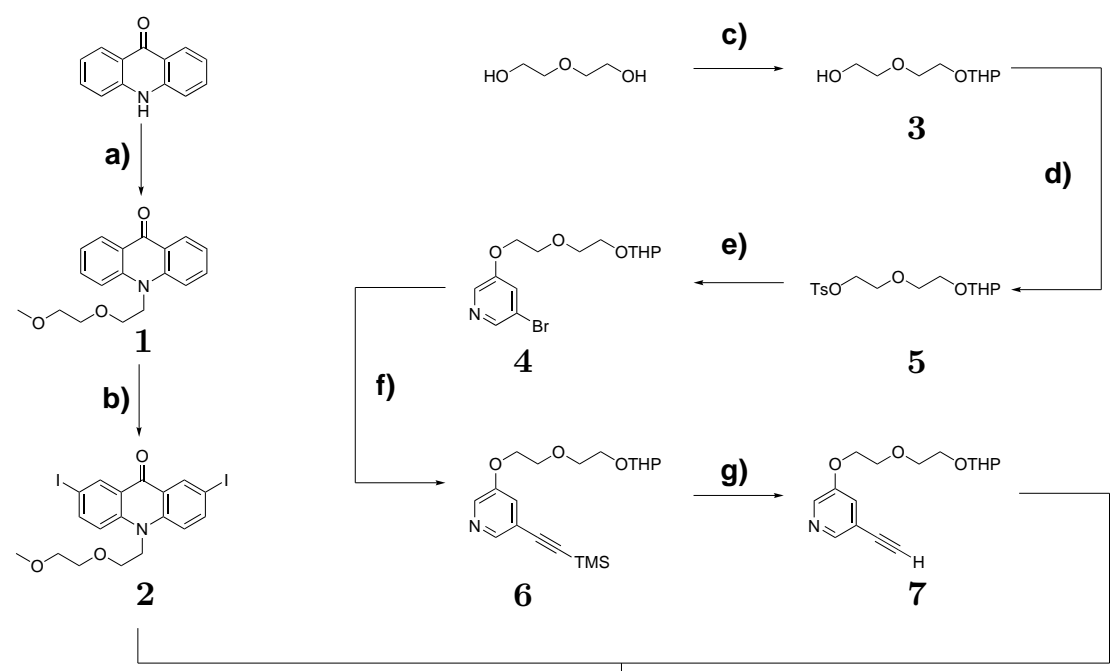

h)

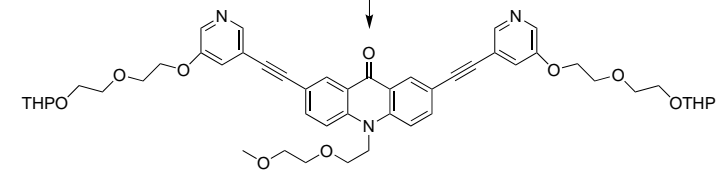

8
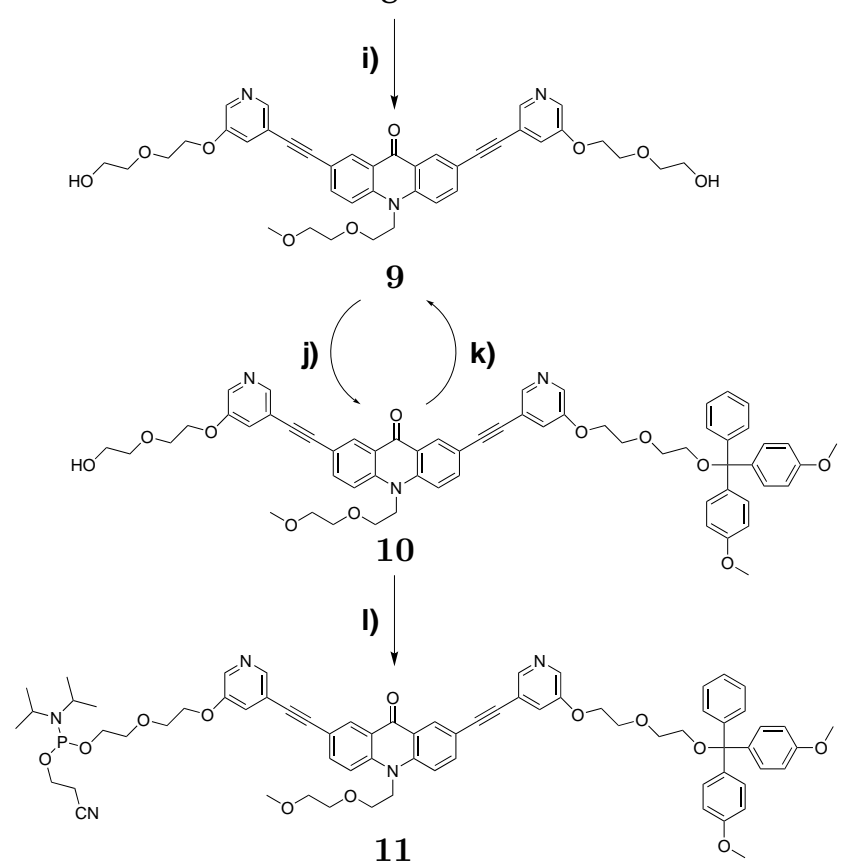

Scheme III.2.2.1: Synthetic path for ligand 11. a) $\mathrm{CH}_{3} \mathrm{O}\left(\mathrm{CH}_{2}\right)_{2} \mathrm{O}\left(\mathrm{CH}_{2}\right)_{2} \mathrm{Br}, \mathrm{K}_{2} \mathrm{CO}_{3}$, DMF; b) NIS, TFA, MeCN; c) DHP, TsOH, DCM; d) TsCl, NEt3, DCM; e) 3-Bromo-5-hydroxy pyrdine, $\mathrm{K}_{2} \mathrm{CO}_{3}$, NEt 3 ; f) Ethynyl-TMS, Pdcat., CuI, NEt ${ }_{3}$, THF; g) $\mathrm{K}_{2} \mathrm{CO}_{3}, \mathrm{MeOH}$; h) Pd-cat., CuI, NEt 3 , THF; i) TsOH, DCM; i DMT-Cl, 4-DMAP, NEt 3 , DCM; j) $\beta-\mathrm{PA}-\mathrm{Cl},(i \mathrm{Pr})_{2} \mathrm{EtN}, \mathrm{DCM}$.

The synthesis for $\mathbf{1 1}$ was achieved in two independent synthetic pathways, one for the acridone backbone and the other one for the pyridine arms with the attached protected PEG linkers. Subsequently both individual motifs ( $\mathbf{7}$ and $\mathbf{2}$ ) were merged in a Sonogashira cross-coupling reaction to obtain molecule 8 . In three further steps the DMT and CEP groups were asymmetrically introduced to the ligand. 
At first the THP protecting group was introduced to one side of the diethylene glycol chain. After, the other hydroxy group was converted into a better leaving group with tosylchloride. Subsequently, the tosylate leaving group was substituted with of 3-bromo-5-hydroxy pyrdine. In a palladium catalyzed cross coupling reaction ethynyl trimethyl silane was introduced to the 3-postion of the pyridine compound and the TMS group was removed under mild basic condition in the next step, to obtain $\mathbf{7}$. For the acridone, methoxy-ethoxy-ethyl bromide was attached at the acridone nitrogen in a first step, in order to increase the solubility of the later end product. Secondly, iodine was introduced regiospecificly to the 2- and 7-postion of the acridone in a TFA catalyzed electrophilic aromatic substitution reaction, by what the substituted acridone nitrogen exhibits a para-directing effect (+M effect), while the keto group contributes with an meta-directing effect (-M effect). The coupled product was deprotected under acidic condition with the organic acid p-toluenesulfonic acid ( $\mathrm{TsOH})$. In the subsequent step the DMT protecting group was introduced to one of the pyrdine attached $\mathrm{PEG}$ chains in a asymmetric substitution reaction. Since there was no regioselective control over the substitution reaction 10 was obtained with a max. yield of roughly $50 \%$ with $25 \%$ non-deprotected or double-protected compound, each, as side products. The non-protected ligand was immediately reused, while the the doubleprotectecd compound had to be deprotected in presence of $5 \%$ TFA in dichloromethane (DCM). As the last step the 2-cyanoethyl N,N-diisopropylchlorophosphoramidite was attached to the second unprotected $\mathrm{PEG}$ chain. The reaction was proceeded under inert conditions and also the product compound was stored in an inert atmosphere at low temperatures $\left(<-30^{\circ} \mathrm{C}\right)$, since the phosphorous(III) species is extremely sensitive towards oxygen or moisture. Therefore, preparation of $\mathbf{1 1}$ was performed on the same day, as it was used for the DNA solid phase synthesis section III.2.2.2.

\section{III.2.2.2 Synthesis of Ligand Embedded Oligonucleotides}

The synthesized ligand $\mathbf{1 1}$ was incorporated into artificial oligonucleotide sequences known from literature to form a four-way junction. Seeman et al.157|158 published a sequence, designed with respect to sequence symmetry minimization rules and without longer repeating $\mathrm{G}$ sequences, in order to avoid the formation of non-Watson-Crick pairs table III.2.2.1. Every single strand consists of two non-complementary sequences, whose complementary counterpart is located in the other single strands. In other words, the half of one single strand forms a double strand to another single strand, while the other half of the single strand is interacting with a third single strand. In this manner, four single strands can be wrapped up to the desired Holliday junction (HJ) structure (figure II.2.2.7. To give an easier overview, which sequences do match to each other, the complementary sequences, shown in table III.2.2.1, are highlighted in the same color. Besides, a second sequence was used from a recent paper by Mendoza et al.,141. also consisting of four single 
strands. Here the complementary parts do not just vary in the nucleotide composition, as in the case of Seeman, but also in the length of each matching sequence. Latter are only mentioned for the sake of completeness, however, since no formation of the desired structure could be observed for the sequences of Mendoza et al., neither by UV melting nor by PAGE electrophoresis experiments, the synthesized strands are not further discussed in the following course of this dissertation.

Table III.2.2.1: ONS sequences for MKDH01um and MKDH01. The sequences are known to form a Holliday Junction. In the case of the unmodified sequence, the position where the ligand is incorporated in MKDH01, is represented by an extra thymine, to create and T-T mismatches wihtin the center of the structure. Additional T-T mismatches next to the ligand are not necessary due to the long $\mathrm{PEG}$ linkers, which provides plenty of space in the central position, according to the MMFF model figure III.2.2.1).

\begin{tabular}{|c|c|c|}
\hline & MKDH01um Sequences 5' $\rightarrow 33^{\prime}$ & MKDH01 Sequences 5' $\rightarrow 3$ \\
\hline \hline A & GCC ATA GTT TGA GCA CG & GCC ATA GTL TGA GCA CG \\
\hline B & CGT GCT CAT GGA TTG CG & CGT GCT CAL GGA TTG CG \\
\hline C & CGC AAT CCT CCG AAT GC & CGC AAT CCL CCG AAT GC \\
\hline D & GCA TTC GGT ACT ATG GC & GCA TTC GGL ACT ATG GC \\
\hline
\end{tabular}

Besides the strands containing the incorporated artificial ligand, from now on referred as MKDH01, the same sequence was also synthesized without the ligand, but with a thymine as connecting link between the two non-complementary sequences within a single strand. The thymines shall form T-T mismatches in the middle of intended HJ structure, in order to imitate the void within the Holliday junction, caused by the sterical demanding ligands or cages. These strands, from now on referred as MKDH01um, or MKDH01-unmodified, shall function as an easy accessible reference system, to figure out, if the the intended Holliday junction, with the incorporated ligands, is able to be formed at all. And if so, what impact the ligands do have on the formation and stabilization of those ligand-containing modified Holliday junction, in comparison to the unmodified versions.

As already mentioned at the end of the previous section, the freshly synthesized phosphoamitide was instantly used for the oligonucleotide solid phase synthesis (SPS), since compound $\mathbf{1 1}$ was extremely labile and decomposed within hours, even under cooled inert conditions. Therefore the reaction mixture was filtered under inert conditions, to get rid of the formed solids and subsequently the solvent and residual Hünig base was evaporated in vacuo. Directly after, the weight of the residue was determined and a $50 \mathrm{mM}$ solution in anhydrous $\mathrm{MeCN}$ was prepared, on the supposition that the reaction yielded at least $50 \%$ of the desired product. The syntheses for the above mentioned oligonucleotides table III.2.2.1 were accomplished in DMT-ON mode, according to the procedure described in the appendix, section B.1 
Followed by a successful synthesis, the oligonucleotides were cleaved from the solid phase support over night in a 33\% aqueous ammonia solution, while all base labile protecting groups on the used oligonucleotides were removed as well. Subsequently the purification of the synthesized ONSs was performed via Reversed Phase HPLC as described in the appendix in section B.2.a, to separate the desired sequences from all other fail-sequences, formed during the oligonucleotide SPS. For that purpose, the ONSs were synthesized in DMT-ON mode, where the last DMT protecting group is not cleaved, but remains on the last coupled nucleotide. The DMT-group is a hydrophobic residue, which leads to an increased interaction with the solid phase during RP-HPLC cleanup. Therefore, it virtually functions as an anchor, so that the ONSs still containing DMT have a longer retention time on column, compared to the other $\mathrm{ONSs}$ and are easier to separate. HPLC traces recorded at $260 \mathrm{~nm}$ for the clean strands are shown in the appendix, chapter A. Since the incorporated ligands have an specific absorbance at $375 \mathrm{~nm}$, for MKDH01 strands a-d, HPLC traces at $375 \mathrm{~nm}$ were recorded as well. After HPLC clean up, the ONSs had to be desalted and the DMT protecting group was removed. For that reason each sample was transferred on solid phase support, as described in the appendix, section B.2.b. Under acid conditions the DMT moiety was cleaved from the ONSs and the ions were washed out, before the strands were released again from the solid phase support and dissolved in $1 \mathrm{~mL}$ of pure water, as a stock solution.

As a next step, the isolated ONSs were each characterized by ESI-MS analysis. The corresponding mass spectra for the specific ONS can be found as the HPLC traces before in chapter A of the appendix. As it can be clearly seen, there is no single peak for each charged state of the ONS, but rather accumulations of peaks. This phenomenon comes from statistical unspecific adducts of cations with the ONS During the whole process of synthesis and clean up various counter cations have been dragged along and were exchanged on the phosphate backbone of the ONS, so that several peaks do appear with similar molecular weight and various amounts of sodium, potassium or ammonium ions. Nevertheless, all peaks belong to the same ONS and therefore, prove the inegrity of the ONS As a last step the concentration of the $1 \mathrm{~mL} \mathrm{ONS}$ solutions for every single sequence was determined, as described in the appendix, section B.3.b. For that reason the extinction coefficient of the incorporated ligands at $260 \mathrm{~nm}$ was determined by dilution UV-vis experiments. Due to the bad solubility of ligand $\mathbf{9}$ in water, the extinction coefficient was determined in a 1:1 mixture of pure water and MeCN. Later a highly diluted solution of $\mathbf{9}$ in pure water was available, from which an extinction coefficient of $\varepsilon_{260 \mathrm{~nm}}=26618 \pm 470 \mathrm{~mol} \mathrm{~cm}^{-1}$ was determined (figure VI.2.3.1a). As a comparison, the same dilution series was also made for the 1:1 mixture of water and acetonitrile, by what an extinction coefficient of $\varepsilon_{260 \mathrm{~nm}}=26299 \pm 1605 \mathrm{~mol} \mathrm{~cm}^{-1}$ was obtained. Since both values are very similar, the MeCN seems not to have a significant influence on the absorption properties of the acridone ligand. Together with the revised extinction coefficients, 159 the concentration of each 
stock solution was calculated from the measured absorption at $260 \mathrm{~nm}$ of $1 \mu \mathrm{lONS}$ stock solution, filled up with pure water to a total volume of $400 \mu$ l. Knowing the concentration of each ONS solution, first samples could be prepared with all four necessary sequences, to form a Holliday junction structure.

\section{III.2.2.3 Holliday Junction with Embedded Acridone-Based Ligand Ligand}

\section{III.2.2.3.1 Holliday Junction MKDH01um}

First experiments were performed with the unmodified version of the desired Holliday Junction system. The samples were prepared with $2 \mu \mathrm{M}$ of each required single strand, in order to get a $2 \mu \mathrm{M}$ solution of the desired Holliday junction, $10 \mathrm{mM}$ of cacodylate buffer adjusted to a $\mathrm{pH}$ of 7.3, $100 \mathrm{mM}$ sodium perchloride and $5 \mathrm{mM}$ of magnesium perchloride with a total volume of $800 \mathrm{\mu l}$. The perchloride salts were used on purpose, since chloride ions can disturb the formation of coordination cages, especially when palladium salts are involved, while perchlorides belong to the class of non-coordinative anions, which only show an extremely weak interactions with metal cations. Under these conditions it is ensured, that excess amounts of anions within the sample solution does not compete with the coordination of ligand to the metal. The prepared sample was heated to $90{ }^{\circ} \mathrm{C}$ for a couple of minutes to disassemble any unspecific binding between the DNA single strands, ensued by the cooling down process, where the sample was cooled down with a rate of $0.5^{\circ} \mathrm{C} \mathrm{min}^{-1}$ to end temperature of $4{ }^{\circ} \mathrm{C}$. After, the sample solution was transferred into the cuvette, and topped with $200 \mu \mathrm{l}$ of silicon oil. The topped oil shall prevent evaporation, causing a higher concentration of the sample, making it difficult to compare the single melting and assembly rounds with each other. For every experiment two rounds of melting and assembly process were recorded. Additionally, for every UV-vis experiment the background was measured with the buffer and salt, but no DNA. The background was later subtracted from the actual experiments, getting only the effects caused by the DNA. Furthermore, for each melting experiment a second wavelength, where no absorption occurs (mostly $500 \mathrm{~nm}$ ), was recorded as well to exclude artefacts caused by the instrument. In an ideal experiment both passes should look identical. Since this is not the case, an average curve was calculated from all the data of one experiment, if applicable. From this curve the denaturation temperature $\left(\mathrm{T}_{\mathrm{M}}\right)$ could be determined in three different ways, as described in section B.4.b 160

1. Manually, by determining the midrule between the upper and lower baseline of the melting curve. The crossing point equals the approximate temperature where $50 \%$ of the the DNA structure is disassembled. 
2. Forming the first derivative of the melting curve and determine the point of inflection, which equals the approximate denaturation temperature

3. Calculating the fraction folded values and determine the $\mathrm{T}_{\mathrm{M}}$ at $\mathrm{y}=0.5$ (equals $\alpha$ $=0.5)$. In this work aprrox. $\mathrm{T}_{\mathrm{M}}$ value was determined with help of the DoseResp sigmoidal fitting, which is also used to determine the $\mathrm{LD}_{50}$ value.

In order to get a better and more detailed insight into the chosen HJ system, not only the denaturation temperature for the full assembled MDHO1um and MKDH01 HJs was determined, but also for every single pair of complementary oligonucleotide sequences contained in the HJ (shown in chapter A). Interestingly the single pairs, contributing to the overall stability of the full assembled HJ having in most cases a denaturation temperature very close to $\mathrm{T}_{\mathrm{M}}$ value of the corresponding Holliday junction table III.3.0.1). But it has to be taken into account, that partially complementary parts of the "non-complementary" sequence can also contribute to the determined denaturation temperature.

(a)

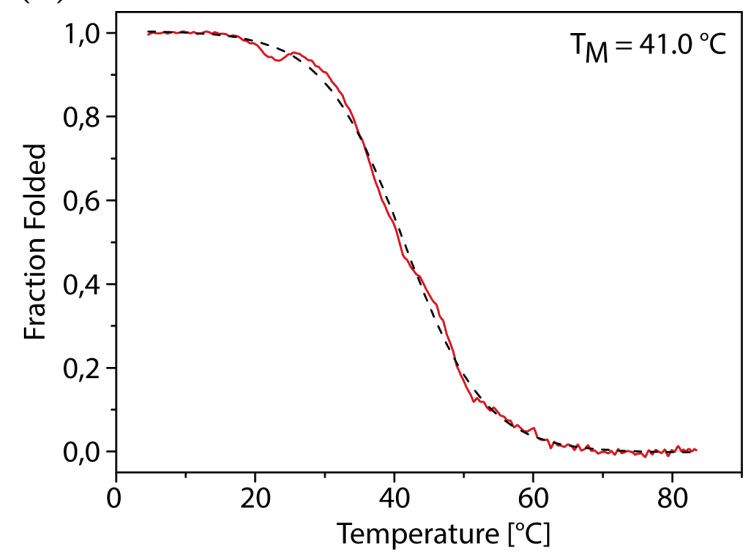

(b)

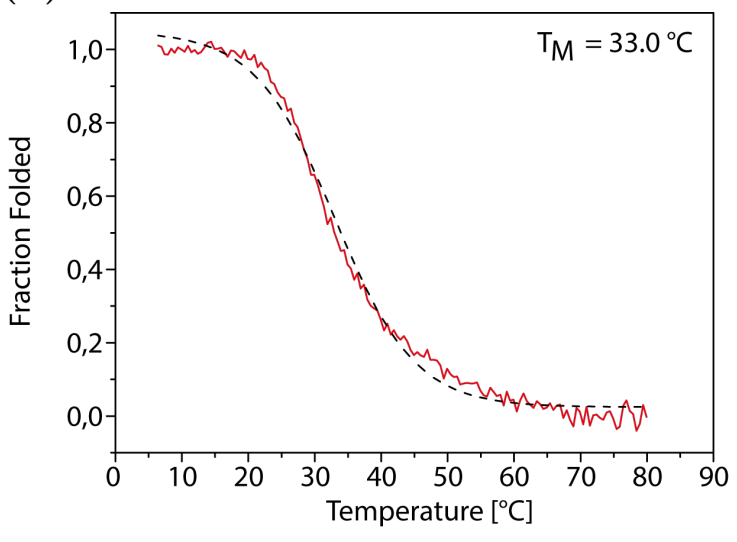

Figure III.2.2.2: Fraction folded plots for the unmodified HJ MKDH01um in presence (a) and absence (b) of magnesium cations. Both experiments were performed with $2 \mu \mathrm{M}$ of each required single strand, corresponding to $2 \mu \mathrm{M}$ Holliday junction in solution, $10 \mathrm{mM}$ of cacodylate buffer $\mathrm{pH}$ of $7.3,100 \mathrm{mM}$ sodium perchloride and $5 \mathrm{mM}$ of magnesium perchloride (a) or $0 \mathrm{mM}$ of magnesium perchloride with a total volume of $800 \mu \mathrm{l}$. From the measured melting curve the fraction folded values were calculated and plotted. $\mathrm{T}_{\mathrm{M}}$ was determined from an appropriate sigmoidal DoseResp fit.

Figure III.2.2.2a shows the fraction folded plot for the unmodified Holliday junction (red) and the corresponding sigmoidal fit (black dashed), from which a $\mathrm{T}_{\mathrm{M}}$ of $41.0^{\circ} \mathrm{C}$ was determined. The curve has a rather flat slope in the temperature range of $30{ }^{\circ} \mathrm{C}$ to $50{ }^{\circ} \mathrm{C}$, indicating a rather low temperature dependency on the affinity constant, as it was described in section B.4.b. ${ }^{160}$ In literature the presence of $\mathrm{Mg}^{2+}$ has been described as a crucial element in the stabilization of the $\mathrm{HJ}$, since the equilibrium of the predominantly existing isomer (open or stacked) seems to be sensitive to the magnesium ion concentration. This 
sensitivity is attributed to the phosphates located at the branch on the crossing over strands, which are assumed to differ in proximity, geometry or both. 138 However, the presence of magnesium does not only affect the choice of the prevailed isomer, but also has a significant effect on the stability of the Holliday junction In figure III.2.2.2b the $\mathrm{T}_{\mathrm{M}}$ was determined for the unmodified $\mathrm{HJ}$ under the same conditions as before, but without any $\mathrm{Mg}\left(\mathrm{ClO}_{4}\right)_{2}$. The drop of $\mathrm{T}_{\mathrm{M}}$ to $31.0{ }^{\circ} \mathrm{C}$ shows that the absence of magnesium has a significant impact on the stability of the Holliday junction. It it worth to mention, that no significant change could be observed for the slope during transition, compared to the experiment with $\mathrm{Mg}\left(\mathrm{ClO}_{4}\right)_{2}$. The slope of transition is nearly unchanged, appearing also in a temperature range of approx. $30{ }^{\circ} \mathrm{C}$. Suggesting, that there is no additional stability with respect to the temperature-dependency of the affinity constant.

(a)

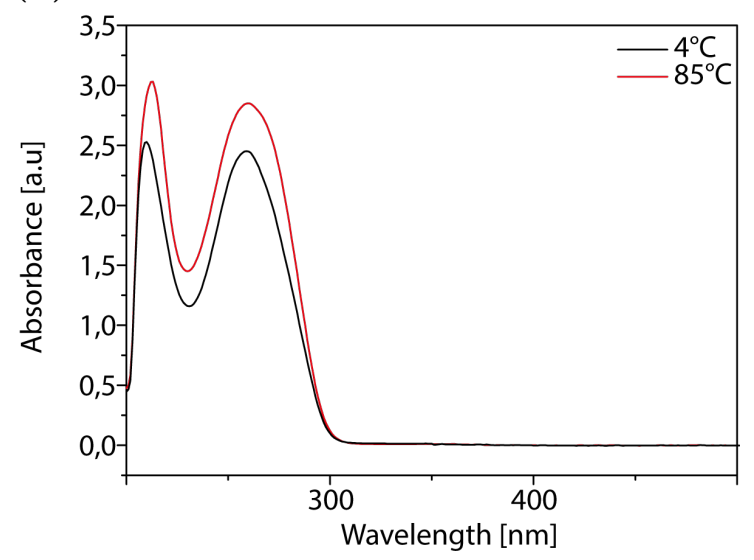

(b)

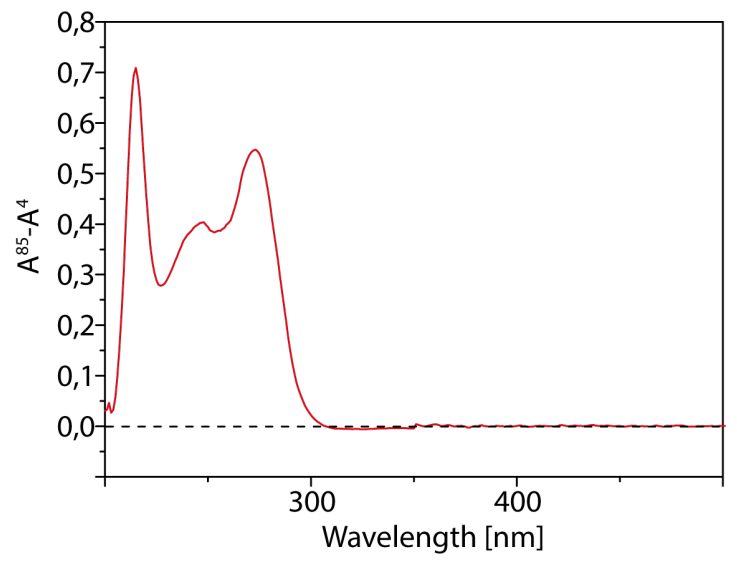

Figure III.2.2.3: UV-vis (a) and TDS(b) spectra for the unmodified Holliday junction, all recorded in presence $5 \mathrm{mM} \mathrm{Mg}\left(\mathrm{ClO}_{4}\right)_{2}$. In the UV-vis specturm (a) the absorbance patterns for the assembled (4 ${ }^{\circ} \mathrm{C}$, black) and the disassembled $\left(85^{\circ} \mathrm{C}\right.$, red) are shown. The assembled DNA structure has a lower absorbance, compared to the free oligonucelotide strand ONS. The TDS spectrum (b) shows the change in absorbance between the assembled and disassembled HJ For that reason the absorbance of the assembled HJ $\left(4^{\circ} \mathrm{C}\right)$ is subtracted from the disassembled HJ $\left(85^{\circ} \mathrm{C}\right)$.

Comparison of the UV-vis absorption pattern show a significant change in absorption at $260 \mathrm{~nm}$ between assembled (black) and disassembled (red) double stranded DNA dsDNA (figure III.2.2.3a), which is used in all melting experiments. This change is known and characteristic for the assembly or disassembly of DNA double helices. Due to the formation dsDNA, the extinction coefficient is lowered, since the ssDNAs are forming a highly defined structure, with lots of newly formed interactions in between the nucleobases, as e.g. hydrogen bonding, stacking or electrostatic effects. These interactions cause a change in the absorbance behavior. The predominant effect the formation of $\pi$ - $\pi$ - stacking, giving a lowering in absorbance up to approx. one third of the initial one. This decrease is used in general as indicator of an assembled DNA structure and makes it possible to determine the denaturation temperature via various temperature UV-vis experiments. $161 \mid 162$ In figure III.2.2.3b the difference in absorbance between the denatured 
DNA structure at high temperatures and the folded one at low temperature is plotted. These representation is called thermal differential spectrum (TDS), by what the change in absorbance between those two states can be shown in detail. Every TDS has a specific shape that is unique for each type of nucleic acid structure and can be seen as structural fingerprint. 163 .

Due to the fact that oligonucleotides have a chiral character, it is suitable for circular dichroism (CD). The nucleosides themselves rather have a small response in the CD. however, in a sequence of nucleic acids these small responses are summed up, giving a detectable signal in $\mathrm{CD}$ measurements. By assembling into the secondary or even tertiary architectures, the spacial arrangement of each structure produces a unique chiral profile that can be identified and investigated by circular dichroism measurement, from which structural information can be derived.164|165 Figure III.2.2.4a represents the recorded spectra for assembled (red) and the disassembled (green) unmodified HJ. The formation of the Holliday junction induces a significant change in the spectrum. The band at $250 \mathrm{~nm}$ decreases about $30 \mathrm{mdeg}$, while the band at $270 \mathrm{~nm}$ is not only increased by ca. $15 \mathrm{mdeg}$, but is also slightly shifted to approx. $280 \mathrm{~nm}$. These changes in CD between assembled and disassembled $\mathrm{HJ}$ are more clearly illustrated in the TDS of the $\mathrm{CD}$ measurements (figure III.2.2.4b). Furthermore, the change in profile indicates the formation of a higher ordered structure, which can not unambiguously assigned to the HJ structure, since the CD curve also resembles the profile of a B-DNA structure, what is not very surprising, since the flanks of the $\mathrm{HJ}$ virtually consists of B-DNA double helices. 166 However, most CD profiles for B-DNA do not show such an intense band at $250 \mathrm{~nm}$ and in fact, comparison with literature-given $\mathrm{CD}$ spectra of $\mathrm{HJs}$ confirms this intense band to be quite characteristic. 167. Unfortunately it seems not to be possible to determine the actual isomer HJ from the measured $\mathrm{CD}$, since the profile mainly depends on the sequences flanking the crossing section. 
(a)

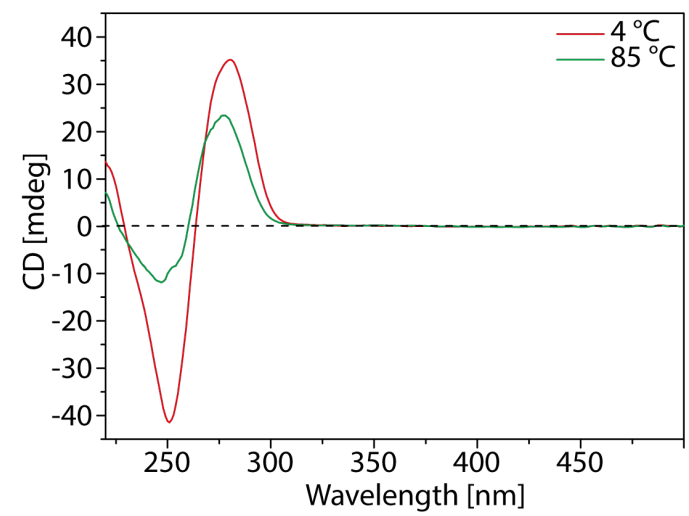

(b)

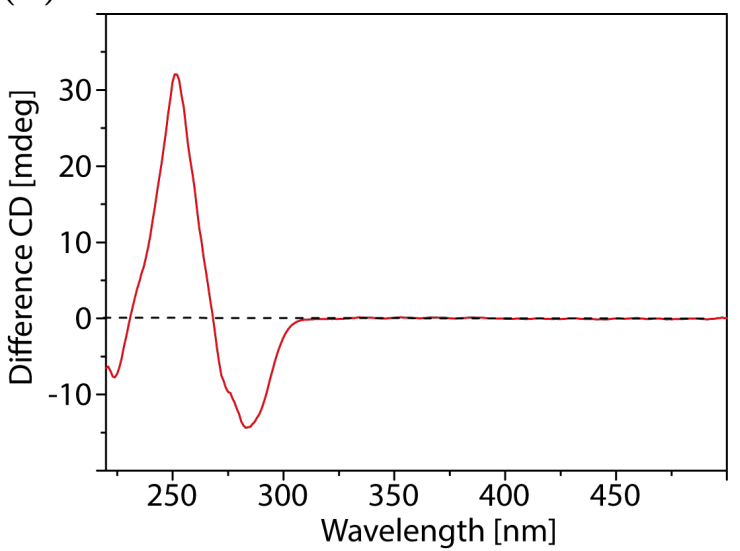

Figure III.2.2.4: $\mathrm{CD}$ spectra for the unmodified HJ MKDH01um. HJ MKDH01um sample contains $2 \mu \mathrm{M}$ of each ssDNA $100 \mathrm{mM} \mathrm{NaClO}_{4}, 5 \mathrm{mM} \mathrm{Mg}\left(\mathrm{ClO}_{4}\right)_{2}, 10 \mathrm{mM} \mathrm{NaCaco} \mathrm{pH}=7.2$. (a) shows the $\mathrm{CD}$ spectra of MKDH01um (red) in comparison to its disassembled (green) form. (b) The TDS illustrates the difference in signal amplitude between the spectrum at $85^{\circ} \mathrm{C}$ and the one at $4{ }^{\circ} \mathrm{C}$, giving a unique shape for the respective DNA structure.

Although, the CD experiment indicates that a formation of the desired Holliday junction happens, the system was investigated with help of an additional method, in order to proof whether the intended $\mathrm{HJ}$ is formed from the four ssDNA. The polyacrylamide gel electrophoresis PAGE experiment is a very common method, 168 either to identify DNA strands or to separate them. For that reason small amounts of DNA is run through a polyacrylamide gel in a saline buffer solution, while applying an electric field, which attracts the negatively charged DNA and forces it to traverse the polyacrylamide network. The method is comparable to the size exclusion chromatography (SEC). Since the secondary or tertiary DNA structure shall be kept intact during the experiment, the gel was run under non-denaturing conditions as described in section B.5. It is worth to mention, that all salts and buffers were already added to the unpolymerized gel solution, thus, the gel already contained the same salt concentration, as the running buffer. That shall avoid upcoming salt barriers during the sample run, which can have a tremendous effect on the running behavior of the samples, resulting in sophisticating observations. Furthermore, the experiments were performed under constant temperature control, in order to avoid unwanted disassembly of the samples or cracking of the used glass apparatuses, due to thermal stress. Additionally, in order to get the best possible resolution, provisions were made to obtain as sharp bands as possible for each single sample in the final gel. First of all, the gels were prepared with a stacking gel, by what the sample shall enter the separating gel as concentrated as possible and secondly, the density of each sample was increased before injecting them to the sample pocket. The function of the stacking gel is easily explained and a common method. By topping the more dense separation gel by the less dense stacking gel, the sample can run much faster at the beginning through the polyacrylamide network, thus, the sample is concentrated at the interface of the two gels, giving much sharper band during the PAGE experiment. To support the effect additionally, the samples 
were mixed with a $40 \%$ sucrose solution, increasing the density of the sample, by what the sample solution sinks to the very bottom of the pocket, avoiding a quick diffusion of the sample into pockets and without interfering the mobility of the DNA in the electric field. Figure III.2.2.5 shows the gel for the unmodified HJ. Besides each single strand (a-d) and of course the Holliday junction (hj) itself, all pairwise combinations of the ssDNAs were run as well (ab,ac,ad,bc,bd,cd). All samples were run side by side in one gel, making the differences in travel distance, depending on the size of the investigated structures, easier to compare. In addition, a DNA ladder, containing defined double stranded helices ranging from 10 base pairs bp up to $150 \mathrm{bp}$ in $10 \mathrm{bp}$ steps, was also run on the same gel as a reference $(\mathbf{M})$. The lower gel illustrates the same experiment as above and only serves for a better overview. The combinations, where an higher ordered structure would be expected, are highlighted in green, while for the red marked lines only ssDNA should be observed.

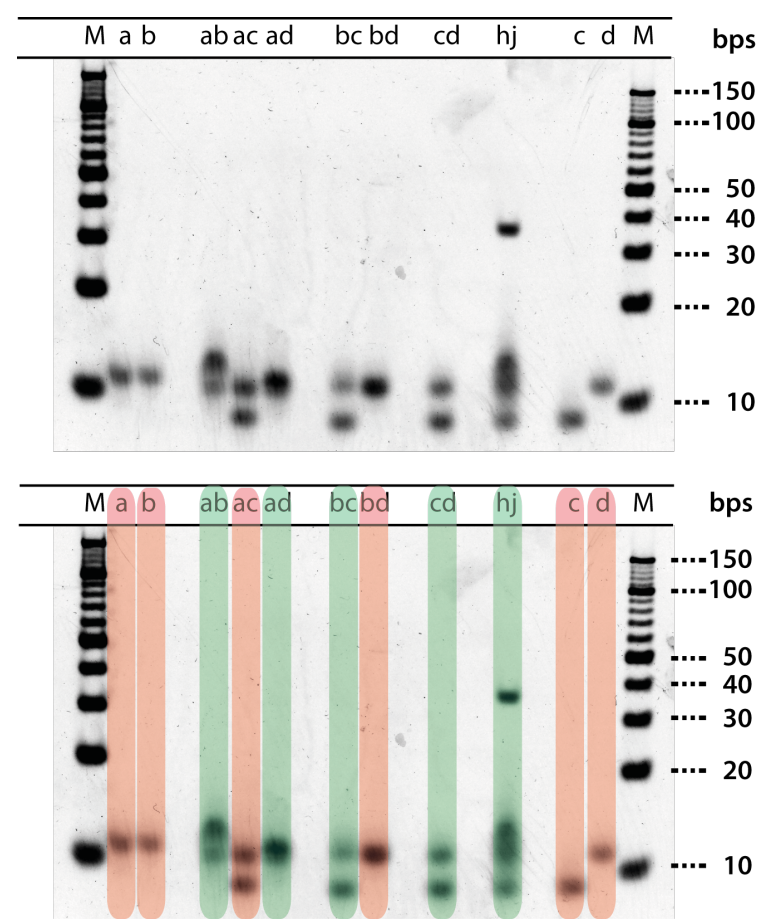

Figure III.2.2.5: PAGE gel for the unmodified Holliday junction MKDH01um. The samples (125 ng per strand) were run in a $8 \%$ polyacrylamide gel with TBM running buffer, containing $2 \mathrm{mM} \mathrm{Mg}\left(\mathrm{ClO}_{4}\right)_{2}$ at $200 \mathrm{~V}$ for about $3 \mathrm{~h}$. Besides the samples, a DNA ladder (M) containing double helix DNA 10-150 glsbp in 10 bp steps, was run as well as a reference for travel distance and size orientation. The higher the band the bigger the size of the respective DNA structure. The single band in the hj indicates the existence of the supposed HJ]

The band for the supposed HJ has an obviously smaller travel distance compared to all other samples, indicating a clear difference in size. Although it is not one-to-one comparable with the here used DNA ladder, from the height of the observed band it can be deduced that the present structure consists of 30 to 40 base pairs, what fits to the HJ with its $32 \mathrm{bp}$. Taking into account that the more bulkier structure of the HJ, compared to double stranded helix, cause a concomitant shape-originated lower mobility within the polyacrylamide structure, the travel distance of the $\mathrm{HJ}$ is in full accordance to the 
expected traverse and also fits to literature-given examples. 169 Surprisingly, only for the combination $\mathbf{a b}$ a band for a higher ordered structure was observable, indicating a lack of robustness under the given conditions. With regard to the determined denaturation temperature $\left(\mathrm{T}_{\mathrm{M}}\right)$, shown in chapter A actually more band for the pairwise combinations were expected. Nevertheless, since the unmodified Holliday junction sequences seem to be capable of forming the intended DNA structure, in a next step the ligand-containing modified sequences were investigated in the same way.

\section{III.2.2.3.2 Holliday Junction MKDH01}

To our surprise, the denaturation temperature, received from the melting curve of the modified HJ, is $13{ }^{\circ} \mathrm{C}$ higher figure III.2.2.2a with respect to the unmodified one figure III.2.2.2a under the same conditions. In absence of $\mathrm{Mg}\left(\mathrm{ClO}_{4}\right)_{2} \mathrm{~T}_{\mathrm{M}}$ is decreased to $47.5{ }^{\circ} \mathrm{C}$, giving a difference of $6.5{ }^{\circ} \mathrm{C}$ or $14.5{ }^{\circ} \mathrm{C}$ with respect to the unmodified HJ under the same conditions. As already observed before, the range of transition is approx. 30 celsius, thus the presence of magnesium cations do not effect the range of transition, merely its positioning is affected.

(a)

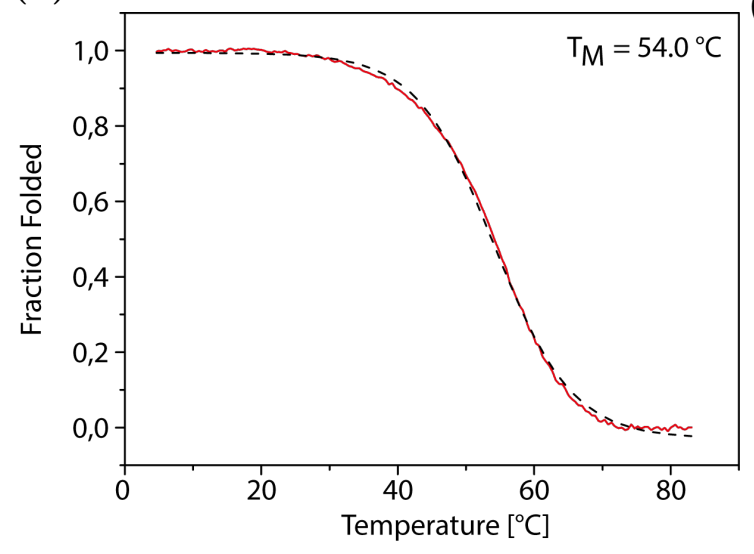

(b)

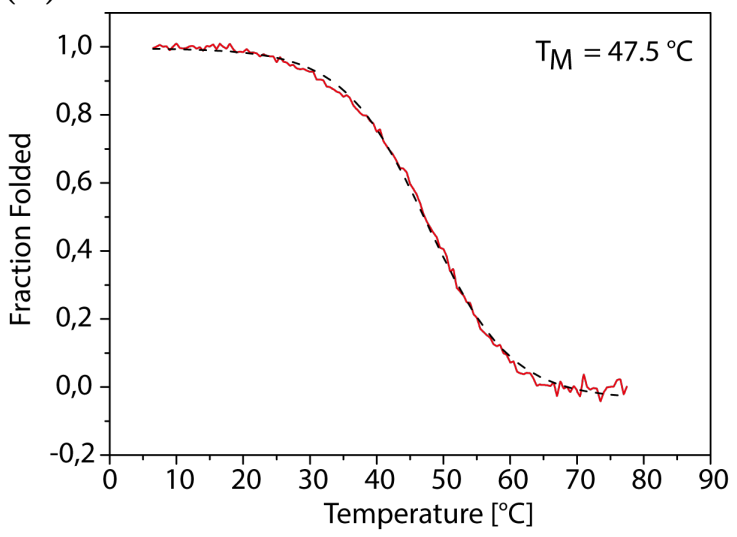

Figure III.2.2.6: Fraction folded plots for the modified HJMKDH01 in presence (a) and absence (b) of magnesium cations. Both experiments were performed with $2 \mu \mathrm{M}$ of each required single strand, corresponding to $2 \mu \mathrm{M}$ Holliday junction in solution, $10 \mathrm{mM}$ of cacodylate buffer $\mathrm{pH}$ of $7.3,100 \mathrm{mM}$ sodium perchloride and $5 \mathrm{mM}$ of magnesium perchloride (a) or $0 \mathrm{mM}$ of magnesium perchloride (b) with a total volume of $800 \mu \mathrm{l}$. From the measured melting curve the fraction folded values were calculated and plotted. $\mathrm{T}_{\mathrm{M}}$ was determined from an appropriate sigmoidal DoseResp fit.

Having a look at the UV-vis spectra of the modified HJ, at high (black) and low temperatures, reveals the expected decrease in absorbance for the region between $220 \mathrm{~nm}$ and $300 \mathrm{~nm}$, caused by the assembling process of the ssDNA. Next to this region, the characteristic absorption bands of the acridone ligand at $325 \mathrm{~nm}$ and $360 \mathrm{~nm}$ can be 
found. ${ }^{20}$ In this context, it is noteworthy to mention, that those experience a bathochromic shift upon the assembly of HJ, suggesting an interaction of the ligands, either with the surrounding nucleic acids or among themselves. These interactions will be discussed in more detail later during this chapter. In the thermal differential spectrum figure III.2.2.7b the lucid red-shifting about 10-15 $\mathrm{nm}$ of the acridone absorption is more clear. In the region above $300 \mathrm{~nm}$, the postive bands belong to the ligand of the disassembled DNA structure, while the subzero band represent the shifted acridone bands in the assembled HJ.

(a)

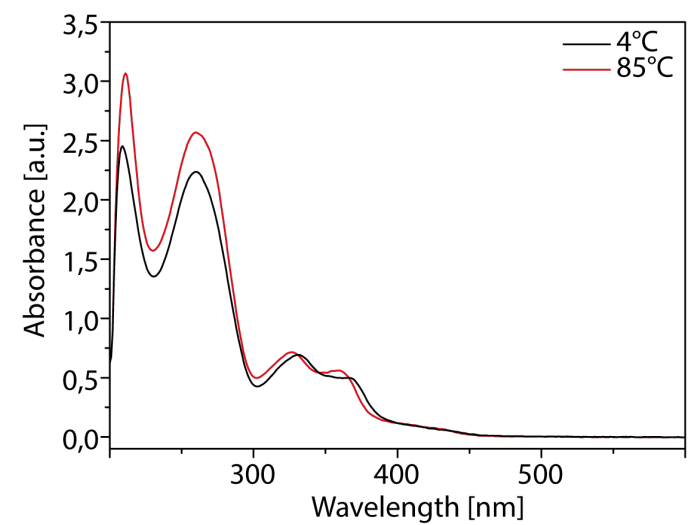

(b)

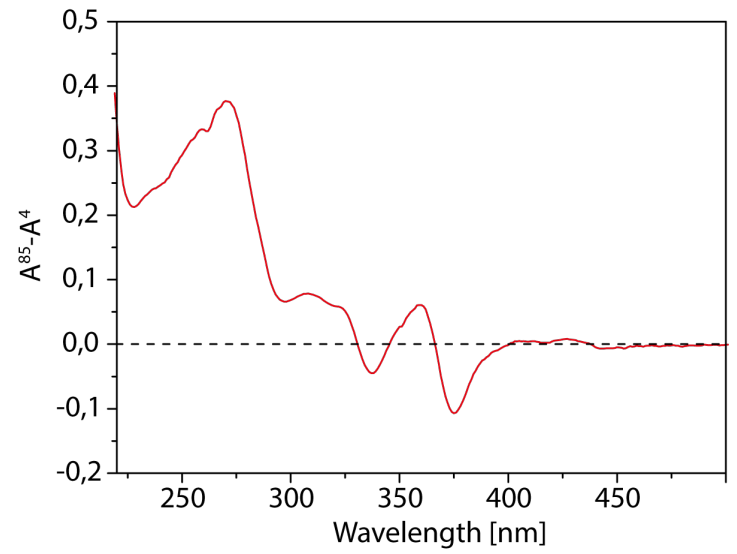

Figure III.2.2.7: UV-vis (a) and TDS (b) spectra for the modified Holliday junction MKDH01, all recorded in presence of $5 \mathrm{mM} \mathrm{Mg}\left(\mathrm{ClO}_{4}\right)_{2}$. In the UV-vis specturm (a) the absorbance patterns for the assembled $\left(4^{\circ} \mathrm{C}\right.$, black) and the disassembled $\left(85^{\circ} \mathrm{C}\right.$, red) are shown. The assembled DNA structure has a lower absorbance, compared to the free oligonucelotide strand ONS. Furthermore the ligand region above $300 \mathrm{~nm}$ experience a bathochromic shift upon assembly of the HJ (b) A detailed illustration of the differences in absorption is displayed in the thermal differential spectrum (TDS).

Since the polyacrylamide gel electrophoresis (PAGE) gave so far the best visual evidences for an existing fully assembled Holliday junction, polyacrylamide gels were prepared for the ligand-containing modified ssDNA, as described before. In figure III.2.2.8 the PAGE is illustrated, again, the lines with an expected higher order structure are highlighted in green, while the others, where solely ssDNA should be observed, are marked in red. In this gel four separately prepared $\mathrm{HJ}$ samples we inserted into adjacent line, all of them showing sharp and intense short traveled bands, suggesting a successful and reproducible assembly of the desired $\mathrm{HJ}$ architecture. The HJ bands are located this time between $40 \mathrm{bp}$ and $50 \mathrm{bp}$, thus even a little higher as the unmodified HJ (figure III.2.2.5). But taking in consideration that the there are big aromatic ligands incorporated at the central position, this result is not very surprising. Although it is apparent that more bands for the dimeric combination ( $\mathbf{a b}, \mathbf{a d}, \mathbf{b c}$ and $\mathbf{c d}$ ) are visible, besides, also the pale shadow band do attract attention, especially those which were not expected to exist. As example, in the line bd no higher ordered dimeric structure would be expect, however, there is one. In line ad a very light but still visible band appears on the same height as the HJs. The 
fact that all bands show a slight fluorescence when illuminated at $312 \mathrm{~nm}$, portends that all bands contain the ligand-containing ssDNAs. This finding supports the theory, that the ligand either intercalates into the formed double helices or stack on each other, since these bands are not observable for the unmodified HJ (figure III.2.2.5). Yet, one would probably expect a more intense band for the intercalaton of the ligands to the DNA, since acridone derivatives are known to intercalate quite strongly with a high affinity. 170 Therefore the shaded bands rather speak for the less stable but still powerful ligand stacking.
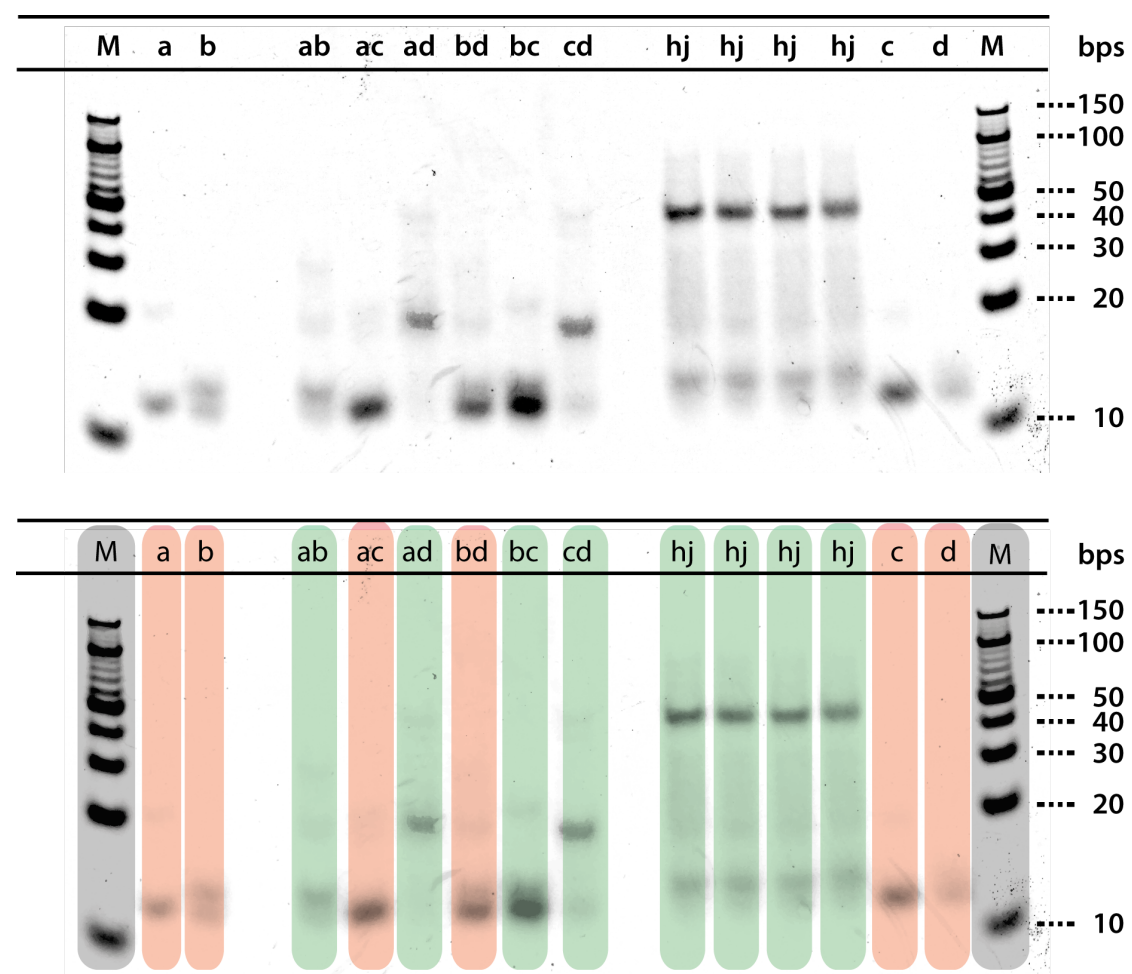

Figure III.2.2.8: PAGE gel for the modified Holliday junction MKDH01. The samples (125 ng per strand) were run in a $8 \%$ polyacrylamide gel with TBM running buffer, containing $2 \mathrm{mM} \mathrm{Mg}\left(\mathrm{ClO}_{4}\right)_{2}$ at $200 \mathrm{~V}$ for about $3 \mathrm{~h}$. Besides the samples, a DNA ladder (M) containing double helix DNA 10-150 glsbp in 10 bp steps, was run as well as a reference for travel distance and size orientation. The higher the band the bigger the size of the respective DNA structure. The single band in the $\mathbf{h j}$ indicates the existence of the supposed HJ

Since the ligands are embedded into a chiral environment, it was of interest to examine if the ssDNAs transfer its chiral character on the ligands and if this chirality can also be found in the assembled $\mathrm{HJ}$ structure, respectively. For that purpose, circular dichroism (CD) spectroscopic measurements were performed for the modified HJ system at high and low temperatures, as described in section B.4.c. In consense with the unmodified HJ, the disassembled structure (green) shows a postive band at $275 \mathrm{~nm}$ and a slightly weaker negative band at $250 \mathrm{~nm}$, according to the postive Cotton effect already seen for the unmodified Holliday junction. In the region of ligand above $300 \mathrm{~nm}$, there could be a small 
broad band, but that cannot be confirmed with certainty, since the the spectra seems to be a little offset, although the spectrum was background corrected. As for the unmodified HJ, the assembly of the modified HJ led to an increased band at $280 \mathrm{~nm}$ and a strongly decreased band at $250 \mathrm{~nm}$, supporting the evidences for a fully assembled HJ. Interestingly, in the ligand region above $300 \mathrm{~nm}$, two new bands appeared at approx. $330 \mathrm{~nm}$ and $375 \mathrm{~nm}$, in total accordance to the bands appeared in the UV-vis spectra (figure III.2.2.7b). These bands can be seen more clearly in the thermal differential spectrum (TDS), figure III.2.2.9b and have the same sequence, negative band $->$ postive band, as the pattern caused by the Holliday junction, indicating a chirality transfer from the DNA scaffold to the incorporated ligands within the assembled structure.

(a)

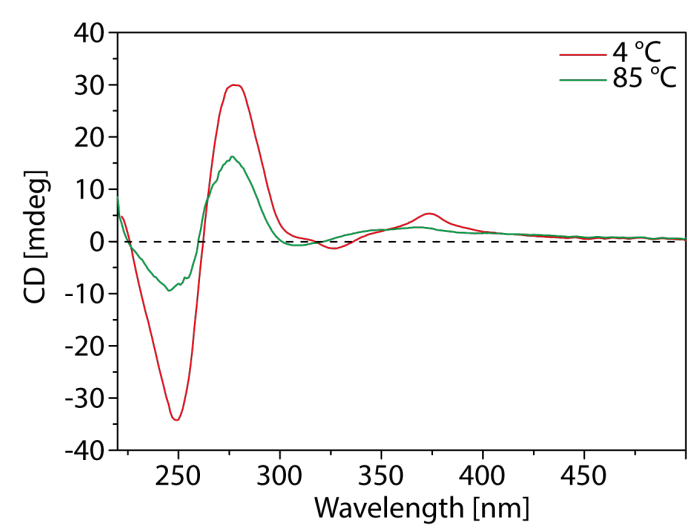

(b)

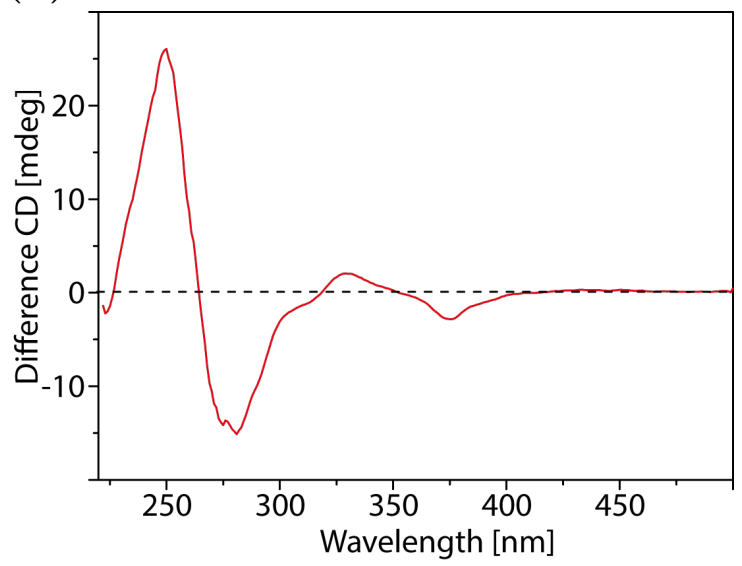

Figure III.2.2.9: $\mathrm{CD}$ spectra for the modified HJ MKDH01. HJ MKDH01 sample contains $2 \mu \mathrm{M}$ of each ssDNA $100 \mathrm{mM} \mathrm{NaClO}_{4}, 5 \mathrm{mM} \mathrm{Mg}\left(\mathrm{ClO}_{4}\right)_{2}, 10 \mathrm{mM} \mathrm{NaCaco} \mathrm{pH}=7.2$. (a) shows the CD spectra of MKDH01 (red) in comparison to its disassembled (green) form. (b) The TDS illustrates the difference in signal amplitude between the spectrum at $85{ }^{\circ} \mathrm{C}$ and the one at $4{ }^{\circ} \mathrm{C}$, giving a unique shape for the respective DNA structure.

Furthermore, as mentioned before, due to the incorporated ligands, the ssDNAs are fluorescent in comparison to the unmodified strands. Therefore the differences in fluorescence for the assembled and disassembled $\mathrm{HJ}$ were also investigated by various temperature fluorescence spectroscopy (figure III.2.2.10). The disassembled structure at $85{ }^{\circ} \mathrm{C}$ (red) has maximum emission a $445 \mathrm{~nm}$ and smaller side band at $525 \mathrm{~nm}$. In comparison, the maximum emission is shifted to $470 \mathrm{~nm}$ for the assembled HJ, while the side band is not only red-shifted about $10 \mathrm{~nm}$ to $535 \mathrm{~nm}$, but also the intensity is more than doubled. The bathochromic shift observable in fluorescence spectroscopy between disassembled and assembled HJ is probably caused by aggregation of the ligands, what is in good accordance to the results of the previous experiments. What kind of aggregation takes place is really hard to say from the here presented results, but will be discussed in detail at the end of this chapter. 


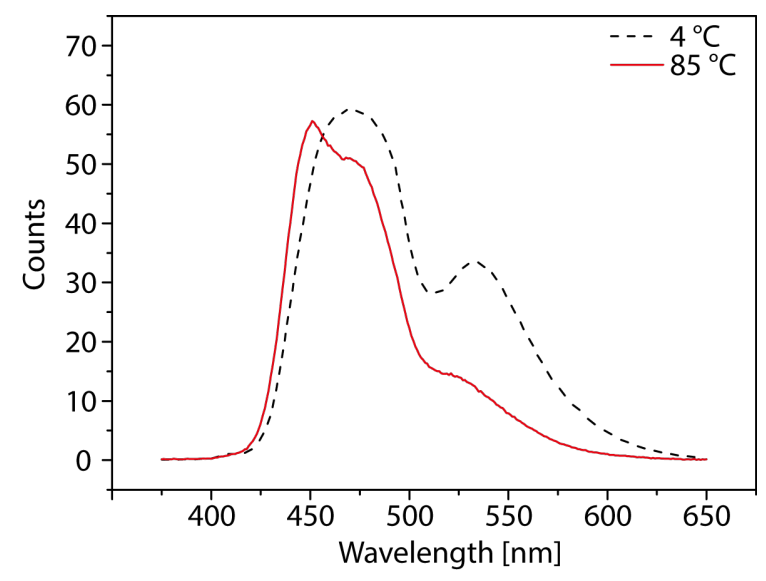

Figure III.2.2.10: Fluorescence emission spectra for the modified HJ MKDH01 at $85{ }^{\circ} \mathrm{C}$ (red) and $4{ }^{\circ} \mathrm{C}$ (black). The HJ system shows an emission at $450 \mathrm{~nm}$, when irradiated at $360 \mathrm{~nm}$. Upon assembly of the HJ the emission band experience a bathochromic shift from $445 \mathrm{~nm}$ to $470 \mathrm{~nm}$. Furthermore, the small band at $525 \mathrm{~nm}$ is shift about $10 \mathrm{~nm}$ and the intensity of emission is tripled.

The logical next step was the attempt of forming the intended embedded coordination cage. For that purpose the addition of the transition metal salts $\mathrm{Pd}\left(\mathrm{NO}_{3}\right)_{2}, \mathrm{Cu}\left(\mathrm{ClO}_{4}\right)_{2}$, $\mathrm{Ni}\left(\mathrm{ClO}_{4}\right)_{2}$ to the $\mathrm{HJ}$ and their influence on the melting behavior was investigated. In figure III.2.2.11a the fraction folded representation for the melting experiment of MKDH01 in abscence and presence palladium(II) cations is shown. The green curve was recorded after the addition of 2 equivalence $\mathrm{Pd}\left(\mathrm{NO}_{3}\right)_{2}$, since two palladium(II) cations are needed for the formation of the coordination cage. It is obvious that there is no significant difference in the shape of the melting curve and also the denaturation temperature remained unchanged, what does not necessarily speak for an unsuccessful formation of a coordination cage within the $\mathrm{HJ}$, but it is very unlikely that $\mathrm{T}_{\mathrm{M}}$ does not change after the formation of a metal coodination complex, especially when compared to other DNA system where metal-coordination occurs. 84[118|120] Likewise, there was also no significant difference in the UV-vis spectra between both experiments. However, the fact that the sample still showed fluorescence, what can be seen by the naked eye when irradiated at $365 \mathrm{~nm}$ with a UV lamp, rather speaks for no formation of a coordination cage. Particularly, because it is known that the fluorescence of acridone is quenched upon coordination to palladium(II) cations. Encouraged by the systems of Engelhard 120 and Yoshizawa, $171 \mid 172$ the melting behavior in presence of nickel(II) or copper(II) cations was investigated as well (figure III.2.2.11b). In order to avoid a miscarriage of the experiment due a lack of metal cations, by i.e. unspecific interactions with the DNA scaffold, the experiments were performed with an excess in metal cations (10 equiv.), in praticular since it is very unlikely that a slight excess of metal cations would prevent the formation of the embedded cage, as one might expect it for a supramolecular cage, consisting of isolated ligands. Under the same conditions the experiment was also performed with 10 equiv. of $\operatorname{Pd}\left(\mathrm{NO}_{3}\right)_{2}$, but unfortunately no usable data were obtained. It is assumed that this happened due to a technical issue, but cannot be said with certainty. However, since all measured samples 
used in this experiment were derived from the same batch, it is assumed that it was not a problem of the sample composition, especially because all other samples showed an intact $\mathrm{HJ}$ system. However, there was no time to repeat, therefore the melting curve for palladium is missing. For the HJ, in absence of any transition metal, a denaturation temperature ( $\left(\mathrm{T}_{\mathrm{M}}\right)$ of $56{ }^{\circ} \mathrm{C}$ was determined. In contrast, the same sample showed in presence of 10 equiv. $\mathrm{Cu}\left(\mathrm{ClO}_{4}\right)_{2}$ a lower $\mathrm{T}_{\mathrm{M}}$ by about $2{ }^{\circ} \mathrm{C}$, whereas the sample with 10 equiv. $\mathrm{Ni}\left(\mathrm{ClO}_{4}\right)_{2}$ has a $1.5^{\circ} \mathrm{C}$ higher $\mathrm{T}_{\mathrm{M}}$. Although a change in melting temperature can be observed, the results rather speak for unspecific interactions of the metal cations with the DNA scaffold. The contrary effects on the denaturation temperature are maybe associated with the different preferred interaction of the here used metal cations with nucleic acids. While nickel preferably interact with the negatively charged phosphate backbone, leading to a stabilization of the double stranded helices, copper(II) exhibit a high affinity to the nucleobases, preventing an interaction with opposite nucleic acids, destabilizing the double helix. 173 Since the metals are deployed in extremely low amounts, accordingly, the resulting effects on the $\mathrm{HJ}$ is weak as well. Moreover, as in the previous experiment the fluorescence is still observed after the experiment. If this can be used as an evidence for a not-existing coordination of ligand, cannot be said, since no suitable system could be found as a reference.

(a)

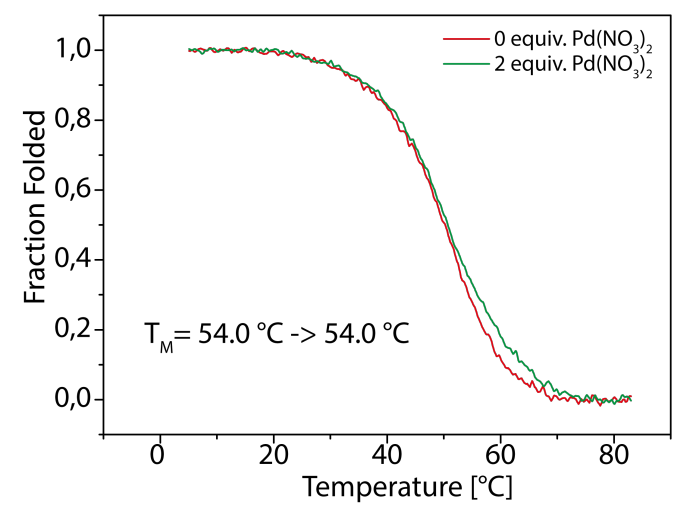

(b)

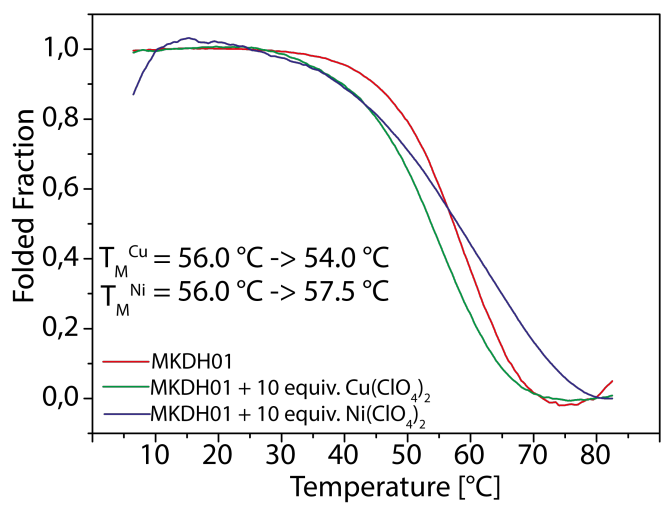

Figure III.2.2.11: UV-vis melting experiments for MKDH05 in presence of (a) $\left.\mathrm{Pd}_{(} \mathrm{NO}_{3}\right)_{2}$ and $\left.(\mathbf{b}) \mathrm{Ni}_{(\mathrm{ClO}}\right)_{2}$ or $\mathrm{Cu}\left(\mathrm{ClO}_{4}\right)_{2}$. The samples contain $2 \mu \mathrm{M}$ of each ssDNA $100 \mathrm{mM} \mathrm{NaClO}_{4}, 5 \mathrm{mM} \mathrm{Mg}\left(\mathrm{ClO}_{4}\right)_{2}, 10 \mathrm{mM} \mathrm{NaCaco} \mathrm{pH}$ $=7.3$. a) $\mathrm{MKDH} 01$ in presence of $\left.4 \mu \mathrm{M} \mathrm{Pd}\left(\mathrm{NO}_{3}\right)_{2} \cdot \mathbf{b}\right) \mathrm{MKDH} 1$ in presence of excess $\left.\mathrm{Ni}_{(}\left(\mathrm{ClO}_{4}\right)_{2} \mathrm{Or} \mathrm{Cu}_{(\mathrm{ClO}}\right)_{2}$. From the same experiment with $\mathrm{Pd}\left(\mathrm{NO}_{3}\right)_{2}$ no usable data were received.

In the here presented chapter, it was proven, that an organic banana-shaped bismonodentate ligand, suitable for the formation of $\mathrm{Pd}_{2} \mathrm{~L}_{4}$ and $\mathrm{Pd}_{4} \mathrm{~L}_{8}$ coordination cages, could be incorporated into ssDNA. These modified oligonucleotides were assembled into the intended Holliday junction (HJ), which was verified by UV-vis, CD and PAGE experiments, as well as by comparison to an appropriate reference system. However, the last step, the formation of a coordination cage within the $\mathrm{HJ}$ architecture could not be proven, neither 
in presence of palladium(II), nor copper(II) or nickel(II) cations. The interaction between metal cations and ligands is probably prevented due to strong stacking between the highly aromatic ligands. The strength of the stacking effect is reflected by an stabilization of the HJ by $13{ }^{\circ} \mathrm{C}$ compared to the unmodified system. This stabilization could be caused either by aggregation of the ligands or by intercalation of the ligands into the close by double helices. Latter is mostly accompanied with quenching of fluorescence. 174 Since this is not the case, a stacking between the ligands is more likely. Furthermore, a bathochromic shifting in the ligands' absorption and emission profile could be observed, indicating the formation of excimers or j-aggregates, what is confirmed by literature.175|176 Moreover, it is worth to mention that for the assembled Holliday junction circular dichroism signals are appearing for the ligands' region of absorption. Since the signals show the same positive Cotton effect as the HJ itself, it is most likely that the DNA structure is transferring its chiral character on the ligands, resulting in chiral J-aggregates embedded in a HJ scaffold.

\section{III.2.3 Anisol-based ligand embedded in Holliday junction}

Based on the results presented in the previous chapter, the ligand system was completely redesigned in order to eliminate the problems arising from the the first generation ligand. The main focus in the ligand design was set to the reduction of the aromatic backbone to avoid the effect of stacking interactions between the ligand or the nucleobases. Furthermore the site for the linker attachment to the oligonucleotides was shifted from the pyridine arms to the ligand backbone in order to provide a higher degree of freedom for the ligand within the secondary DNA scaffold and preventing a disfavored arrangement of the ligand within the Holliday Junction that hinders the formation of the supramolecular cage. As before, a MMFF model of the desired embedded cage system was prepared, estimating the amount of oligonucleotide mismatches and the PEG linker length, needed to provide enough space for the supramolecular cage in the center of the DNA scaffold. 
(a)

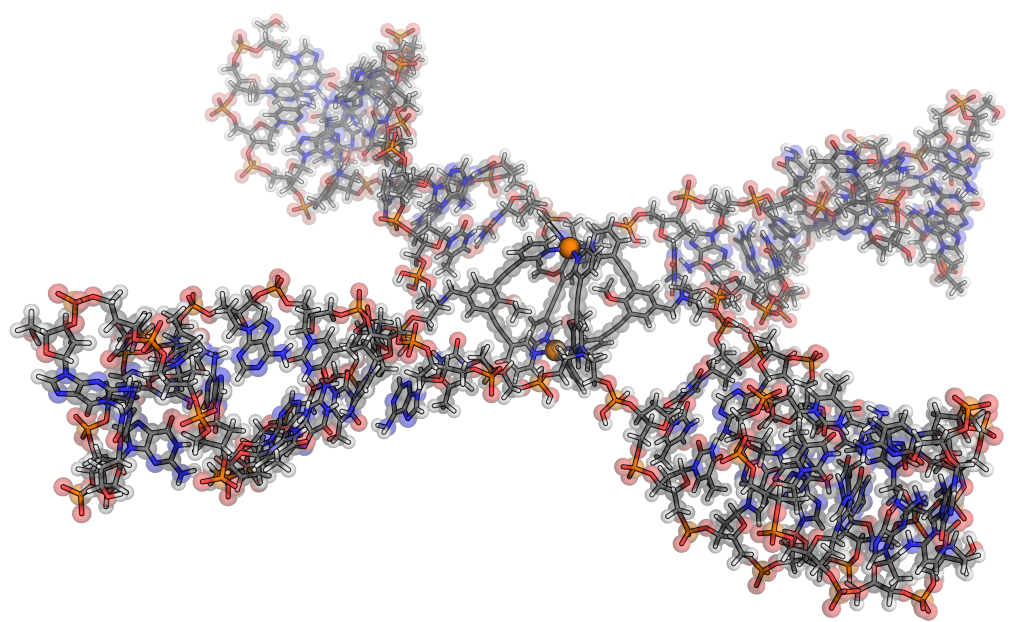

(b)

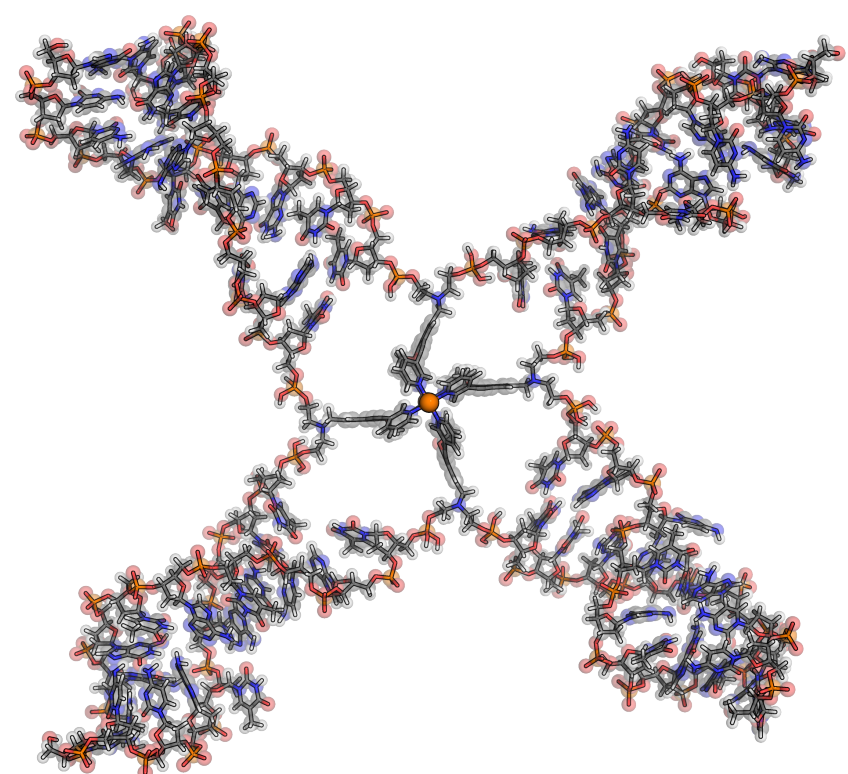

Figure III.2.3.12: MMFF model of 2nd generation DNA-cage hybrid structure. Modelled in Sparten '10.156. The ligands are coordinated to palladium. The ONS sequences contain and extra T-T mismatch next to the integrated ligand.

\section{III.2.3.1 Design and syntheses anisol-based ligand}

As mentioned above, the backbone and ONS coupling positions were redesigned for the new embedded ligand system. The aromatic tricyclic acridone backbone, was replaced by an simple aromatic benzene motif, which still provides the flat and rigid character of the acridone, but provides a clearly reduced aromatic plane for stacking effects. Furthermore, this motif was already used several times before in literature for the design of endo- or exohedral modified metal-mediated coordination cages. $56|74| 177$ The pyridine arms must 


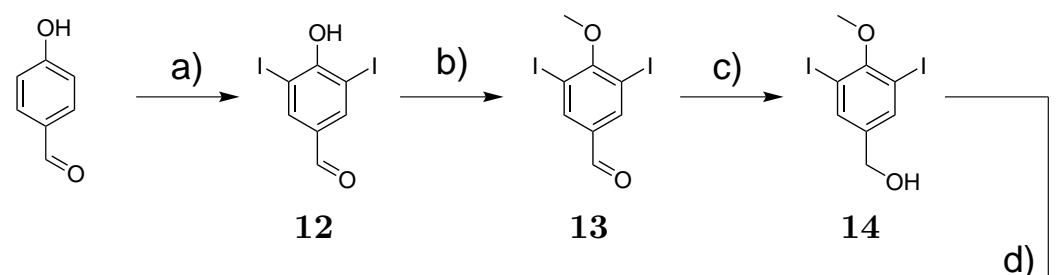

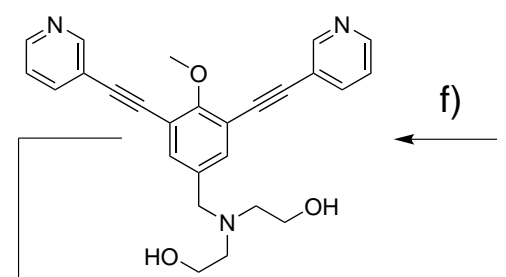

g)
17

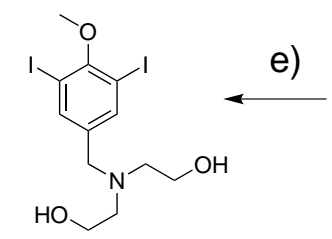

16 d)

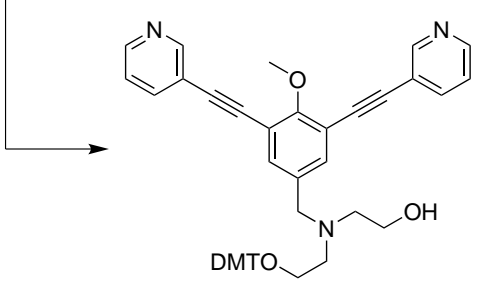

18

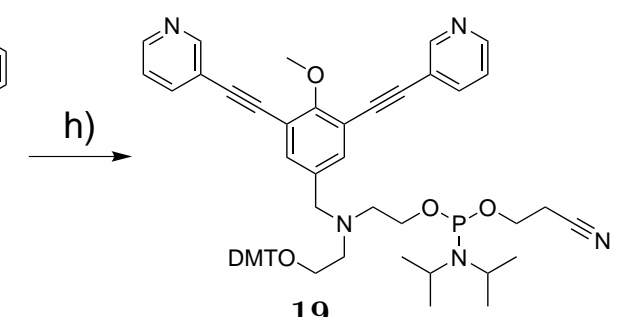

19

Scheme III.2.3.2: Synthetic path for ligand 17. a) $\mathrm{KIO}_{3}, \mathrm{KI}, \mathrm{KCl}, \mathrm{AcOH}, \mathrm{H}_{2} \mathrm{O}$; b) $\mathrm{MeI},\left(\mathrm{CH}_{3}\right)_{2} \mathrm{CO}$; c) $\mathrm{NaBH}_{4}$,

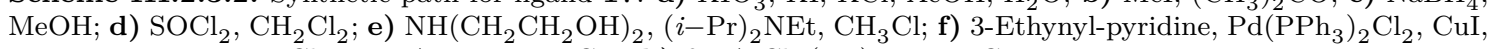
$\mathrm{NEt}_{3}, \mathrm{DMF} ; \mathbf{g}$ DMT-Cl, 4 -DMAP, $\left.\mathrm{NEt}_{3}, \mathrm{DCM} ; \mathbf{h}\right) \beta-\mathrm{PA}-\mathrm{Cl},(i \mathrm{Pr})_{2} \mathrm{EtN}, \mathrm{DCM}$.

be attached in 2- and 6-position to generate the typical banana-shape for the ligands. To achieve this objective, the phenol motif was used as starting compound, since its hydroxy group acts as a directing group during the synthesis. As the ligand shall be integrated into the ONS in way that it has more freedom in movment, the linkage had to be moved to a central position of the backbone, therefore it must be inevitably positioned in para position to the phenol hydroxy group. Furthermore the linkage motif has to be branched, ending in two terminal hydroxy groups, where the nucleotides can be attached during solid phase synthesis. Diethanolamine as an commercially available compound fulfilled all the requirement and seemed to be suitable for this purpose. However, first attempts to attach the diethanolamine directly to the aromatic backbone failed, what is why the phenolic backbone was replaced by 4-hydroxybenzaldehyde. The newly designed ligand $\mathbf{1 7}$ could be synthesized in an eight step synthesis, followed by two additional steps to introduce the DMT and CEP groups scheme III.2.3.2).

Outgoing from the simple 4-hydroxybenzylaldehyde, the compound was regioselective iodinated at the 3 - and 5-position (2- and 6- position, considering the hydroxy group on position 1), by generating elementary iodine in situ. In a next step the hydroxy group was protected by etherification with $\mathrm{MeOH}$. Subsequently the benzylic position was prepared for the introduction of the diethanolamine. First the benzyl aldehyde was reduced to the benzyl alcohol in presence of $\mathrm{NaBH}_{4}$. In a second step, the newly formed benzyl alcohol was 
immediately converted to benzyl chloride in the presence of thionylchloride. Subsequently the chloride was substituted with diethanolamine, followed by the introduction of the pyridine arms in palladium catalyzed Sonogashira cross-coupling to obtain the ligand 17, whose chemistry is discussed in section IV.2.1. As in acridone-based ligand case scheme III.2.2.1, again two equivalent hydroxy groups exist, by what the DMT group has to be introduced in a desymmetrization reaction, with a moderate yield. The double substituted side-product can be simply treated with acid to recover the starting material. In the last step the 2-cyanoethyl N,N-diisopropylchlorophosphoramidite (CEP was attached to the second unprotected free hydroxy group under inert conditions and used for the DNA solid phase synthesis on the same day, due to the same reasons mentioned before at the end of section III.2.2.1.

\section{III.2.3.2 Synthesis of Ligand Embedded Oligonucleotides}

For the next approach the sequence of the HJ system was kept, also the position of the ligand stayed the same. Synthesis of the oligonucelotide strand ONS via solid phase synthesis (SPS) worked straight forward, although it was not known whether the new link would couple at all during SPS. Due to the fact that the polyethylene glycol linkers are only half the size of those used before, the ligands were incorporated in a second sequence containing additional thymines on each side of the ligand, which should form T-T mismatches at the HJ crossing section, generating a little more space for the ligands at the central gap, as it is shown in the MMFF model (figure III.2.3.12). Also strands were synthesized with two T-T mismatches next to the ligands, but no usable melting curves could be obtained from this system, therefore they are neglected in this work.

Table III.2.3.2: ONS sequence table for MKDH05 and MKDH06. The sequences of MKDH05 are equal to those used for the first approach with the incorporated ligand $\mathbf{5 4}$ shown in the previous chapter (section III.2.2.1, but contains the more flexible second generation ligand 17. In the sequences of MKDH06 extra T-T mismatches on both sides of the incorporated ligand are given, in order give more space for the assembly of the intended supramolecular cage structure at the central position of the Holliday junction.

\begin{tabular}{|c|c|c|}
\hline & MKDH05 Sequences 5' $\rightarrow 3$ & MKDH06 Sequences $5{ }^{\prime} \rightarrow 3$ \\
\hline \hline A & GCC ATA GTL TGA GCA CG & GCC ATA GTTL TTGA GCA CG \\
\hline B & CGT GCT CAL GGA TTG CG & CGT GCT CATL TGGA TTG CG \\
\hline C & CGC AAT CCL CCG AAT GC & CGC AAT CCTL TCCG AAT GC \\
\hline D & GCA TTC GGL ACT ATG GC & GCA TTC GGTL TACT ATG GC \\
\hline
\end{tabular}

Moreover into the same sequences shown in table III.2.3.2 a simple imidazol ligand was incorporated instead of the bis-dentate ligands, in order to simplify the coordination 
environment and check whether a coordination within the Holliday junction is possible anyway. Those sequences were completely characterized chapter A, MKDH03 and MKDH04) and the the analytic give evidence for a successful formation of a HJ. It is worth to mention, that the sequence without additional T-T mismatches, shows a slightly smaller $\mathrm{T}_{\mathrm{M}}\left(39{ }^{\circ} \mathrm{C}\right)$ compared to the unmodified Holliday junction $\left(41{ }^{\circ} \mathrm{C}\right)$, indicating destabilization of the DNA structure, due to the artificial ligand. This effect becomes more apparent as additional T-T mismatches next to imidazol ligand were added. In that system the $\mathrm{T}_{\mathrm{M}}$ was even decreased to $\left(34^{\circ} \mathrm{C}\right)$, giving a clear evidence for disturbance of HJ structure caused by the T-T mismatches, by what a destabilization occurs and actually was also expected for the other used ligands. However, since the interaction with transition metal cations was not investigated yet, the imidazol containing $\mathrm{HJ}$ systems are not further discussed in this thesis.

\section{III.2.3.3 Assembly and characterization of Holliday junction with embedded anisol-based ligand}

\section{III.2.3.3.1 Holliday junction MKDH05}

The preparation of the sample for MKDH05 was performed under the same condition as for the the previously discussed system MKDH01. In section III.2.3.3.1 the fraction folded plot, generated from the UV-vis melting experiments, is illustrated, from which a denaturation temperature $\left(\mathrm{T}_{\mathrm{M}}\right)$ of $44^{\circ} \mathrm{C}$ could be obtained. This $\mathrm{T}_{\mathrm{M}}$ is only slightly higher compared to the unmodified HJ $\left(41{ }^{\circ} \mathrm{C}\right)$ and significantly smaller compared to MKDH01 $\left(54{ }^{\circ} \mathrm{C}\right)$, speaking for weaker interaction between the ligands or with the surrounding

oligonucletotidic scaffold, as it was intended. Since it is known from literature, 104|135 that under the used condition, high salt concentration and especially in presence of magnesium, the $\mathrm{X}$-stacked $\mathrm{HJ}$ isomers preferably forms, the melting experiments were also performed in presence of small salt concentration $(<10 \mathrm{mM})$ and in absence of magnesium, in order to generate conditions where the formation of the open HJ isomer is prevailed. But no usable data was obtained from those melting experiments, giving rise to the assumption that the $\mathrm{HJ}$ is not formed at all under the given conditions, forcing us to stay with the high salt concentrations. But since the determined $\mathrm{T}_{\mathrm{M}}$ speaks for a rather weak interaction of the ligands, further experiments were continued with the high salt concentrations. 


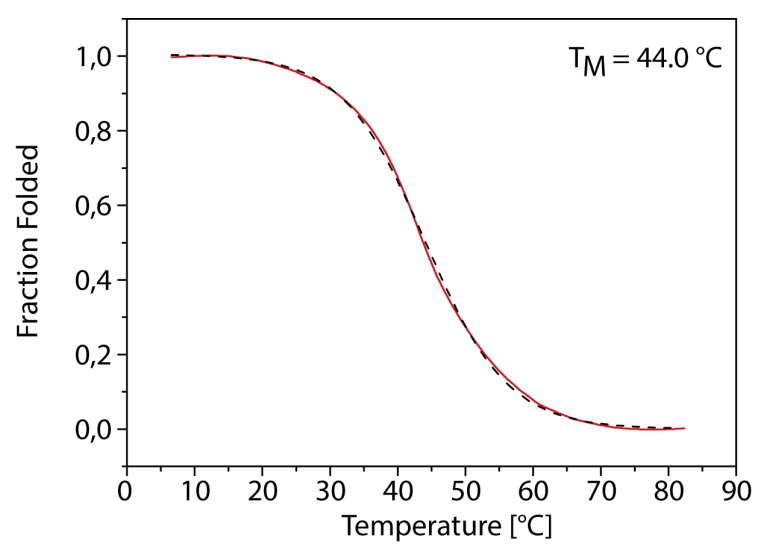

Figure III.2.3.13: Fraction folded plots for the modified HJ MKDH05, performed with $2 \mu \mathrm{M}$ of each required single strand, in order to get a $2 \mathrm{\mu M}$ solution of the desired Holliday junction, $10 \mathrm{mM}$ of cacodylate buffer adjusted at a $\mathrm{pH}$ of $7.3,100 \mathrm{mM}$ sodium perchloride and $5 \mathrm{mM}$ of magnesium perchloride with a total volume of $800 \mu \mathrm{l}$ From the measured melting curve the fraction folded values were calculated and plotted. $\mathrm{T}_{\mathrm{M}}$ was determined from an appropriate sigmoidal DoseResp fit.

The first hints for the aggregation of ligands within the $\mathrm{HJ}$ structure in the first generation system (MKDH01) were received from the UV-vis spectroscopy and its TDS. For the assembled structure as significant bathochromic shift in the ligand region was observed, known from the formation of J-aggregates. For the here discussed ligand that approach is a little more difficult, due to fact that the ligands' main absorption band (figure IV.2.1.2a) is overlapping with the absorption region of the oligonucleotides. Nonetheless, by comparing the UV-vis absorption of the unmodified HJ, it becomes apparent that the small band above $300 \mathrm{~nm}$ must belong to the ligand. On the first glance it looks like the ligand is experiencing a tiny bathochromic shift, as it can also be seen in the TDS figure III.2.3.14b at approx. $310 \mathrm{~nm}$. What also can be seen, there is an jump at $350 \mathrm{~nm}$, infrequently caused the UV-vis machine, as it changed the light sources and due to the sporadic occurring this jump was not obliterated by background corrections. Therefore, the observed bathochromic shift cannot be proofed with certainty, but since the shift is minimal compared to that one observed for the first generation ligand, it can be assumed that only weak interactions occur, if at all. 
(a)

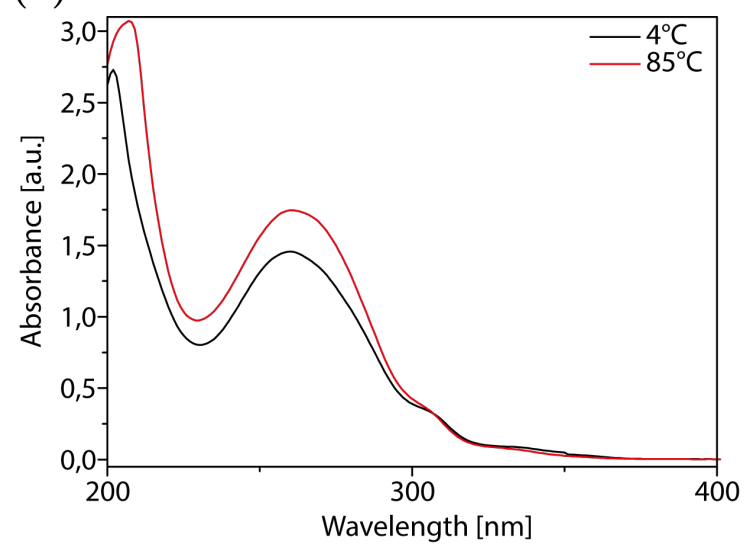

(b)

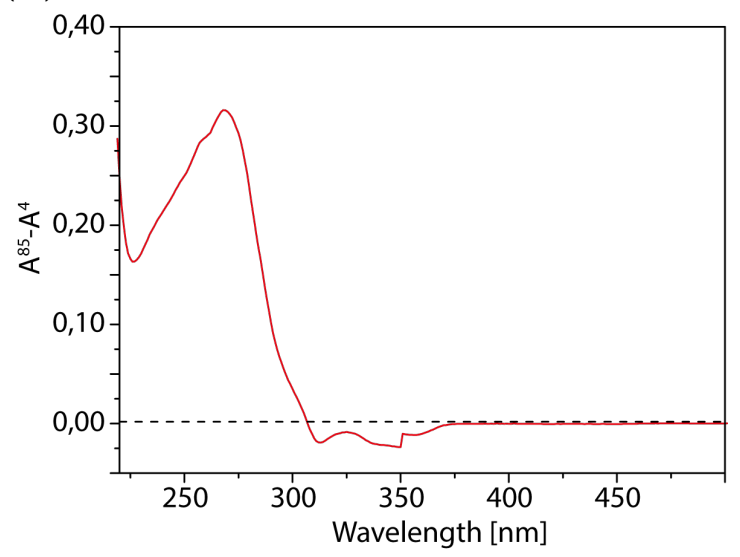

Figure III.2.3.14: UV-vis (a) and TDS (b) spectra for the modified Holliday junction MKDH05, all recorded in presence of $5 \mathrm{mM} \mathrm{Mg}\left(\mathrm{ClO}_{4}\right)_{2}$. In the UV-vis spectrum (a) the absorbance patterns for the assembled (4 ${ }^{\circ} \mathrm{C}$, black) and the disassembled $\left(85^{\circ} \mathrm{C}\right.$, red) are shown. The assembled DNA structure has a lower absorbance, compared to the free oligonucelotide strand ONS, indicating a successful assembly of the intended HJ

$\mathrm{CD}$ measurements do support the previous findings. As for the previous investigated HJs, the same positive Cotton effect with the bands at $250 \mathrm{~nm}$ and $275 \mathrm{~nm}$ could be observed and they do exactly behave the same way upon the assembly process. Means, both bands become more intense and show nearly the same value for the negative as well as for positive amplitude. Likewise, the thermal differential spectrum (TDS) does not show any divergent results, not to speak of any evidence for a chirality transfer to the ligand, as it was seen for the MKDH01 system. Considering that only the linker moiety is incorporated into the DNA scaffold, but not the ligand itself, it would have been surprising if chirality had been transferred on the ligand. Especially, since link and ligand are connected over a flexible bridge, where rotation can occur, so that the ligand can adjust its positioning within the assembled structure and is not forced to follow the arrangement of the DNA scaffold. Therefore, it appears to be logic, that no signal was observed for the ligand region in the $\mathrm{CD}$ spectrum. 
(a)

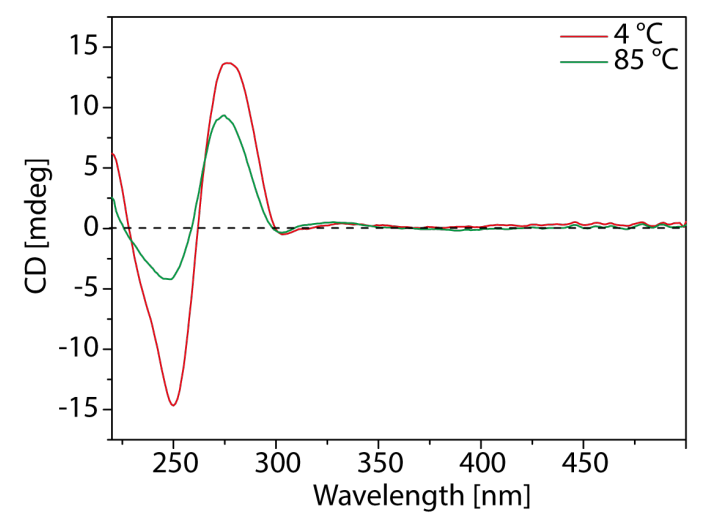

(b)

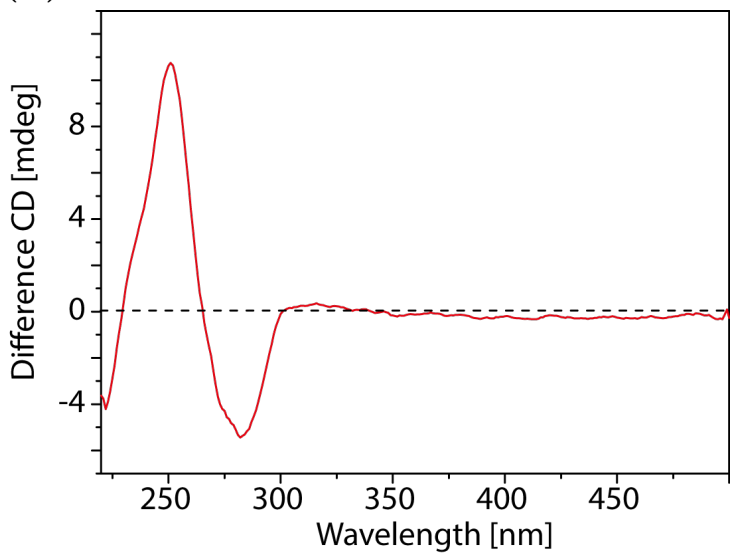

Figure III.2.3.15: $\mathrm{CD}$ spectra for the unmodified HJ MKDH05. HJ MKDH05 sample contains $2 \mu \mathrm{M}$ of each ssDNA $100 \mathrm{mM} \mathrm{NaClO} 4,5 \mathrm{mM} \mathrm{Mg}\left(\mathrm{ClO}_{4}\right)_{2}, 10 \mathrm{mM} \mathrm{NaCaco} \mathrm{pH}=7.2$. (a) shows the CD spectra of MKDH05 (red) in comparison to its disassembled (green) form. (b) The TDS illustrates the difference in signal amplitude between the spectrum at $85{ }^{\circ} \mathrm{C}$ and the one at $4{ }^{\circ} \mathrm{C}$, giving a unique shape for the respective DNA structure.

As it can be seen in figure IV.2.1.2b the ligand is fluorescent and shows emission at $375 \mathrm{~nm}$, when irradiated with $280 \mathrm{~nm}$ light. Even though it is not as intense as the acridone-based ligand, it can still be detected in the assembled HJ. Nevertheless, another mechanism must take place for the here presented system compared to the aggregation in the acridonebased system, since the intensity of fluorescence is almost doubled, while for MKDH01 figure III.2.2.10 the emission is red-shifted and even a new band arose. It is really hard to say by what effect this increase in emission is initiated. Stacking effects can have multiple influences on the fluorescence of molecules, depending on the way of how the molecules are stacked.176|178 Hence, the current data is not sufficient to make a qualified statement about the reason. However, the fact that there is detectable fluorescence in the assembled structure, giving a good starting point for later investigation with respect to the formation of the intended coordination cage, as the fluorescence is totally quenched upon coordination to palladium(II) salts, as it is described in section IV.2.1. 


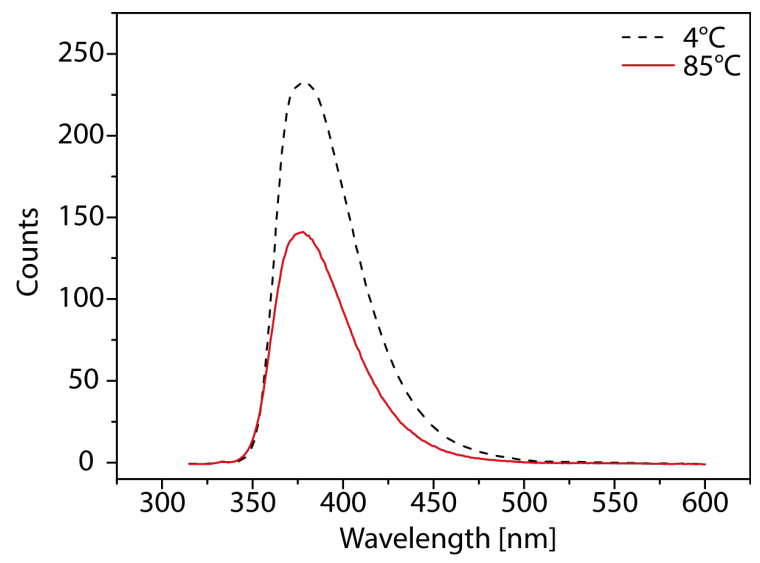

Figure III.2.3.16: Fluorescence emission spectra for the modified HJ MKDH05 at $85^{\circ} \mathrm{C}$ (red) and $4{ }^{\circ} \mathrm{C}$ (black). The HJ system shows an emission at $375 \mathrm{~nm}$, when irradiated at $280 \mathrm{~nm}$. Upon assembly of the HJ the amplitude of emission is increased about $50 \%$ of the starting intensity.

Although the formation of the fully assembled $\mathrm{HJ}$ is proved with a reasonable certainty from the results of the previous experiments, yet, the system was still examined in PAGE experiments. And in fact, again it was possible to observe a band, representing the supposed HJ (figure III.2.3.17, line $\mathbf{h j}$ ) Not only the $\mathrm{T}_{\mathrm{M}}$ is very similar to the unmodified Holliday junction, but also the appearance of the polyacrylamide gel. There are no visible bands for higher ordered structures, which would be expected for the pairwise combination of the strands $\mathbf{a b}, \mathbf{a d}, \mathbf{b c}$ and $\mathbf{c d}$. But obviously the structures stability was not sufficient to survive in the electric field under the given conditions. This finding is showing again, that the thermal stability does not represent the allover stability of a system and can be totally different, depending on the forces acting on it. Further, it supports the assumption of a reduced or weaker stacking between the ligands in the actual system, otherwise one would expect the appearance of weak bands in the gel, similarly as it was observed for acridone-containing HJ (figure III.2.2.8). 


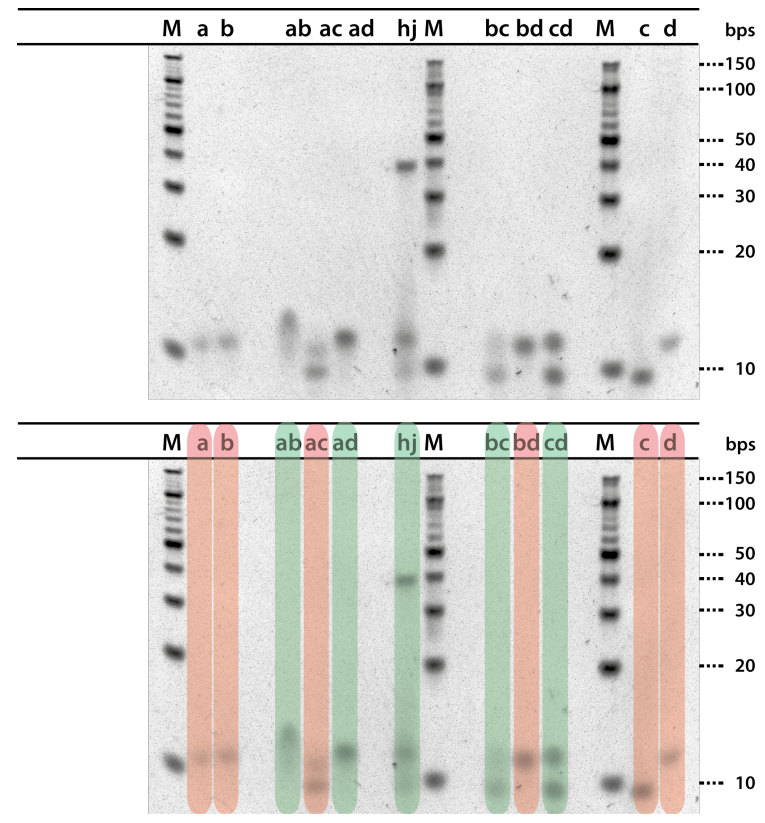

Figure III.2.3.17: PAGE gel for the modified Holliday junction MKDH05. The samples (125 ng per strand) were run in a $8 \%$ polyacrylamide gel with TBM running buffer, containing $2 \mathrm{mM} \mathrm{Mg}\left(\mathrm{ClO}_{4}\right)_{2}$ at $200 \mathrm{~V}$ for about $3 \mathrm{~h}$. Besides the samples, a DNA ladder (M) containing double helix DNA 10-150 glsbp in 10 bp steps, was run as well as a reference for travel distance and size orientation. The higher the band the bigger the size of the respective DNA structure. The single band in the hj indicates the existence of the supposed HJ]

\section{III.2.3.3.2 Holliday junction MKDH06}

The obtained results for the HJ system MKDH06, which only differs from MKDH05 in two additional thymine, enclosing the incorporated ligands, show a pretty similar melting behavior. The $\mathrm{T}_{\mathrm{M}}$ is $42{ }^{\circ} \mathrm{C}$, a decrease of $2{ }^{\circ} \mathrm{C}$ compared to the previously presented HJ. what is consistent with the denaturation temperature of the unmodified Holliday junction MKDH01um. The additional thymines are forming T-T mismatches, leading on the one hand to a higher disorder in the central position of the HJ, accompanied with a decrease in stability. On the other hand the gap at the cross section in increased, giving the ligands more space to move and the formation of the intended coordination cage. Very similar to MKDH05, melting experiments were also performed at low salt concentrations $(<10 \mathrm{mM})$ in order to shift the equilibrium to the open $\mathrm{HJ}$ isomer. However, the experiments resulted as before in unusable melting curve, giving no evidence for a formation of a higher ordered structure. Therefore, all subsequent analytics were also performed under the common conditions described in this thesis. 


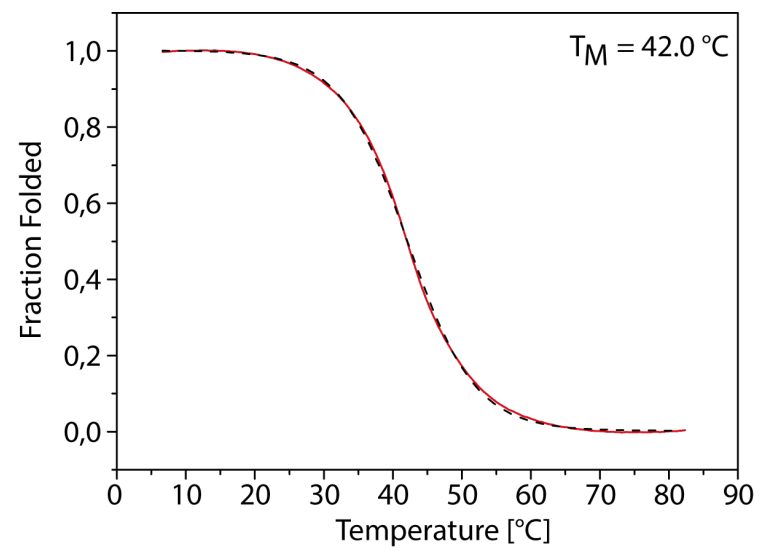

Figure III.2.3.18: Fraction folded plots for the modified HJ MKDH06, performed with $2 \mu \mathrm{M}$ of each required single strand, in order to get a $2 \mu \mathrm{M}$ solution of the desired Holliday junction, $10 \mathrm{mM}$ of cacodylate buffer adjusted at a $\mathrm{pH}$ of $7.3,100 \mathrm{mM}$ sodium perchloride and $5 \mathrm{mM}$ of magnesium perchloride with a total volume of $800 \mathrm{\mu l}$. From the measured melting curve the fraction folded values were calculated and plotted. $T_{M}$ was determined from an appropriate sigmoidal DoseResp fit.

Analog to the UV-vis spectrum recorded for MKDH05, the spectrum of MKDH06 shows a small band above $300 \mathrm{~nm}$ which can be assigned to the ligand. Likewise, the TDS is indicating a slight bathochromic shift of the ligand band, suggesting weak interactions of the ligand with the surrounding DNA scaffold or with vicinal ligands. The jump at $350 \mathrm{~nm}$ is caused by the UV-vis instrument during the change of the light source, as explained before. Since this artefact was happening infrequently, it could not be compensated by background corrections.

\section{(a)}

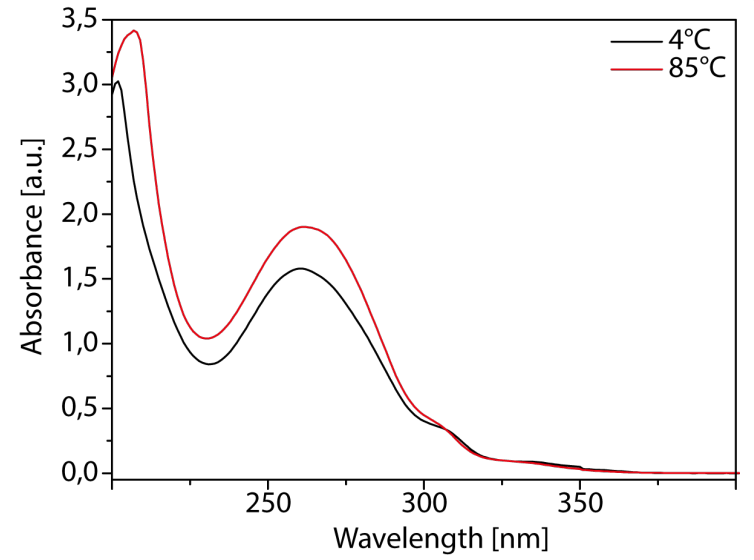

(b)

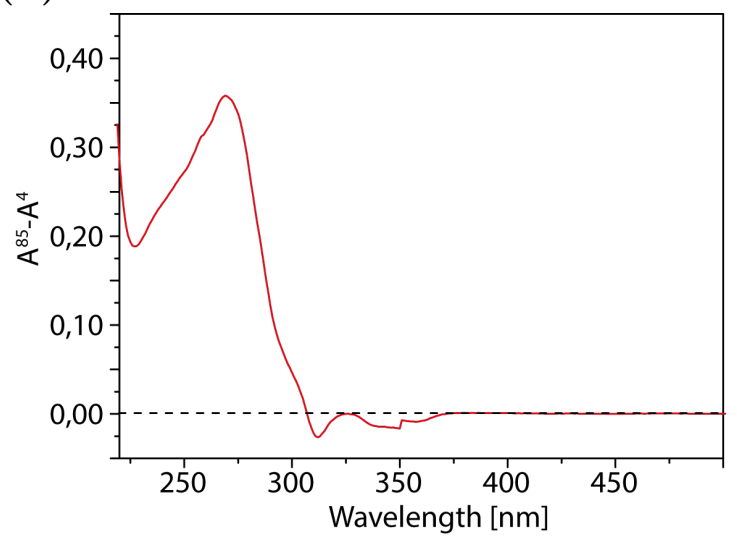

Figure III.2.3.19: UV-vis (a) and TDS (b) spectra for the modified Holliday junction MKDH06, all recorded in presence of $5 \mathrm{mM} \mathrm{Mg}\left(\mathrm{ClO}_{4}\right)_{2}$. In the UV-vis spectrum (a) the absorbance patterns for the assembled $\left(4^{\circ} \mathrm{C}\right.$, black) and the disassembled $\left(85^{\circ} \mathrm{C}\right.$, red) are shown. The assembled DNA structure has a lower absorbance, compared to the free oligonucelotide strand ONS, indicating a successful assembly of the intended HJ.

The CD spectrum shows the typical bands at $250 \mathrm{~nm}$ and $275 \mathrm{~nm}$ with a positive Cotton effect, as it was observed before. Likewise, the bands are increased upon assembly of the 
HJ, so that the negative band at $250 \mathrm{~nm}$ and the positive band at $275 \mathrm{~nm}$ exhibit nearly the same height in amplitude, indicating a successful assembly of the supposed HJ. In contrast to the shorter MKDH05 system, a significant negative CD band could be observed, which can be better seen in the TDS. Interestingly, at $310 \mathrm{~nm}$ only one negative peak can be observed, therefore it is assumed that a second positive peak lies under the $275 \mathrm{~nm}$ peak, which result in a negative Cotton effect. If this is the case, the chriality is probably not transferred from the DNA scaffold to the ligand, since for that case the same orientation of the $\mathrm{CD}$ signals would be expected. 6 However, although both do not share the same orientation, the formation of the HJ must have somehow induced an inverse chirality at the ligands.

(a)

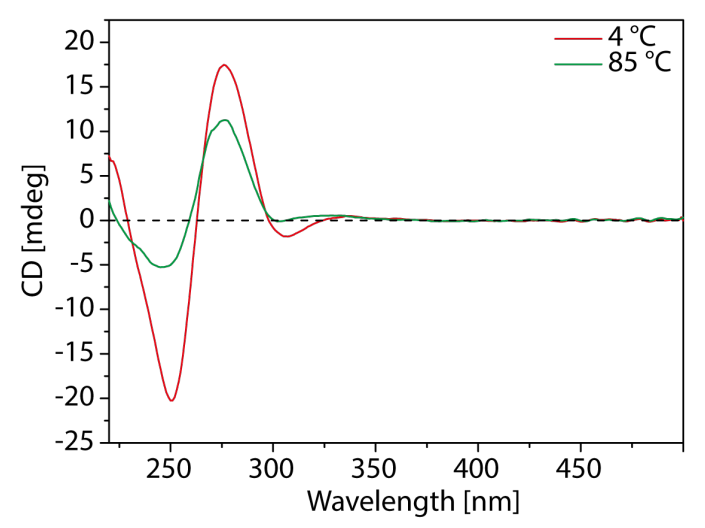

(b)

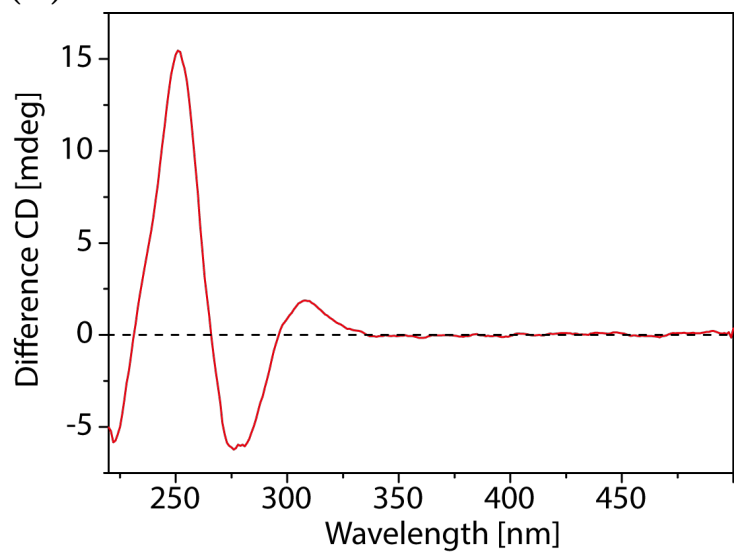

Figure III.2.3.20: $\mathrm{CD}$ spectra for the unmodified HJ MKDH06. HJ MKDH06 sample contains $2 \mu \mathrm{M}$ of each ssDNA $100 \mathrm{mM} \mathrm{NaClO} 4,5 \mathrm{mM} \mathrm{Mg}\left(\mathrm{ClO}_{4}\right)_{2}, 10 \mathrm{mM} \mathrm{NaCaco} \mathrm{pH}=7.3$. (a) shows the CD spectra of MKDH06 (red) in comparison to its disassembled (green) form. (b) The TDS illustrates the difference in signal amplitude between the spectrum at $85{ }^{\circ} \mathrm{C}$ and the one at $4{ }^{\circ} \mathrm{C}$, giving a unique shape for the respective DNA structure.

The fluorescence spectrum for the HJ system shows as before an emission at $375 \mathrm{~nm}$, when it is irradiated with $280 \mathrm{~nm}$ light. The fluorescence is caused by the incorporated ligand as it was shown in figure IV.2.1.2b. Upon assembly of the HJ the florescence is significantly increased, even more than in the previous case for MKDH05. Assuming, this increase in fluorescence is caused by ligand involved stacking effects, the higher intensity of emission in MKDH06 correlates with the increased space at the HJ crossing section. This extra space seems to give the ligand the possibility of a better overlap with the vicinal ligands, causing an enhanced fluorescence not by means of higher or lower energy gaps, but a higher probability to get electrons into an excited state, thus, an increased yield. 


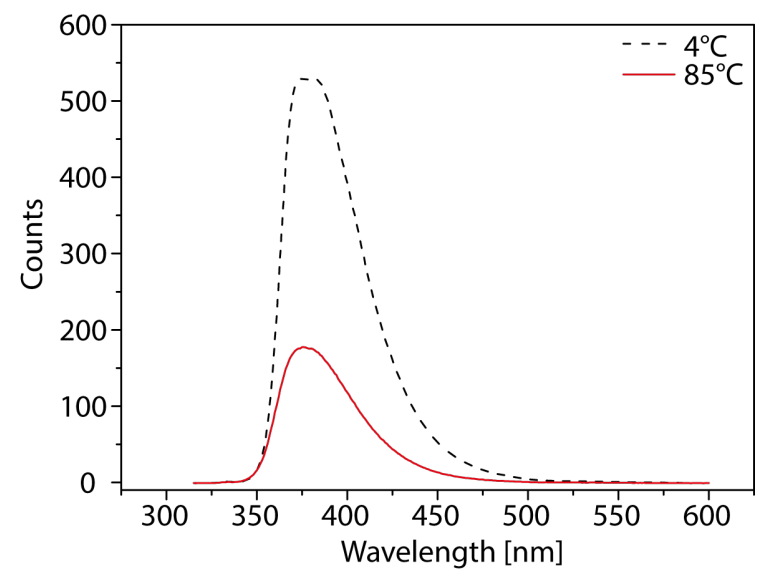

Figure III.2.3.21: Fluorescence emission spectra for the modified HJ MKDH06 at $85{ }^{\circ} \mathrm{C}$ (red) and $4{ }^{\circ} \mathrm{C}$ (black). The HJ system shows an emission at $375 \mathrm{~nm}$, when irradiated at $280 \mathrm{~nm}$. Upon assembly of the HJ the amplitude of emission is three times higher, compared to the disassembled case.

The PAGE experiment for MKDH06 (figure III.2.3.22 does not show any surprising results and lines up with the prior shown polyacrylamide gels. In line $\mathbf{h} \mathbf{j}$, containing all four ssDNAs, a band for the supposed HJ is found, giving an additional evidence for an successful assembly of the intended Holliday junction. As mentioned before, it was expected to find also higher ordered structures for the lines $\mathbf{a b}, \mathbf{a d}, \mathbf{b} \mathbf{c}$ and $\mathbf{c d}$, since those contained two semi-complementary ssDNAs, capable to form a double stranded structure. However, under the given conditions, those seem not to be stable, thus, they could not be observed in the actual gel.

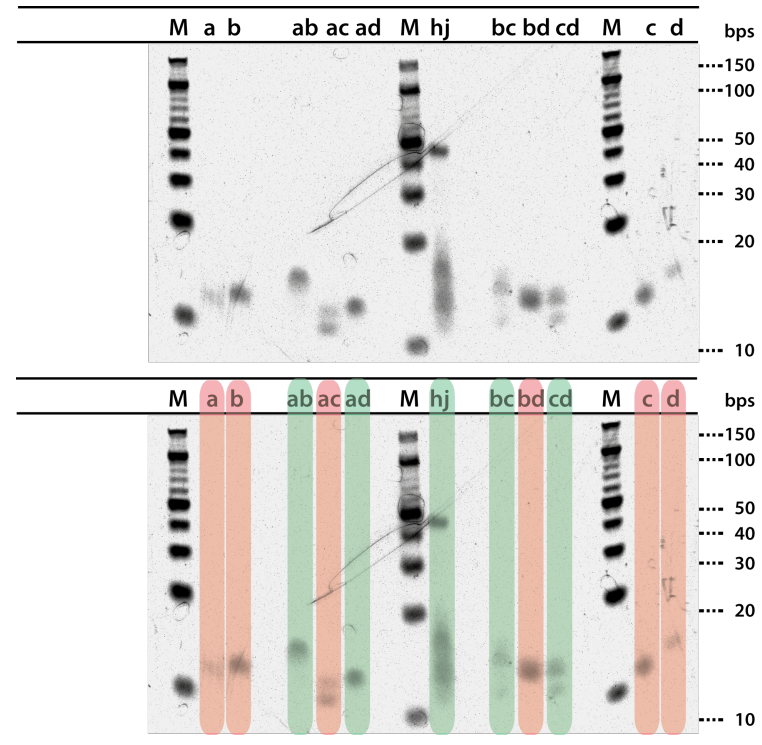

Figure III.2.3.22: PAGE gel for the unmodified Holliday junction MKDH06. The samples (125 ng per strand) were run in a $8 \%$ polyacrylamide gel with TBM running buffer, containing $2 \mathrm{mM} \mathrm{Mg}\left(\mathrm{ClO}_{4}\right)_{2}$ at $200 \mathrm{~V}$ for about $3 \mathrm{~h}$. Besides the samples, a DNA ladder (M) containing double helix DNA 10-150 glsbp in 10 bp steps, was run as well as a reference for travel distance and size orientation. The higher the band the bigger the size of the respective DNA structure. The single band in the $\mathbf{h j}$ indicates the existence of the supposed HJ 


\section{III.2.3.4 Melting behavior in presence of transition metals}

As it was already shown for the MKDH01 system, the melting behavior was also investigated in presence of the transition metals palladium(II), copper(II) and nickel(II), in order to find any evidence for the formation of an coordination structure. For that reason, three succeeding experiments were accomplished for the HJ systems MKDH05 and MKDH06. After each run a certain amount of the respective metal salt was added. The first run was performed in absence of any metal, followed by the second run, where two equivalents of the respective metal salt were added, the minimum amount to form the intended $\mathrm{M}_{2} \mathrm{~L}_{4}$ structure. In the last run an excess of the metal salt was added, ending up with a total concentration of $20 \mu \mathrm{M}$ (5 equiv.) of the corresponding metal. Compared to MKDH01 with copper (figure III.2.3.23a) or nickel figure III.2.3.23b), MKDH05 did not show any significant differences in $\mathrm{T}_{\mathrm{M}}$ even in presence of 10 equiv. of the respective metal salt. One would have at least expected a similar behavior as seen before for MKDH01 figure III.2.2.11, even though the metals hardly changed the denaturation temperature In contrast, the addition of $\mathrm{Pd}\left(\mathrm{NO}_{3}\right)_{2}$ to the $\mathrm{HJ}$ gave a change in the melting profile and an increase in $\mathrm{T}_{\mathrm{M}}$ about $3{ }^{\circ} \mathrm{C}$ compared to the starting situation. Nevertheless, it is believed that this increase in thermal stabilization is rather caused by an unspecific interaction of the palladium cations with the DNA scaffold, since after the experiment there was still fluorescence visible when irradiated with light of the wavelength $254 \mathrm{~nm}$, what does not match with the quenched fluorescence observed for the ligand. In figure IV.2.1.2b it was shown that the fluorescence is totally quenched due to the assembly of the coordination cages with palladium(II). 
(a)

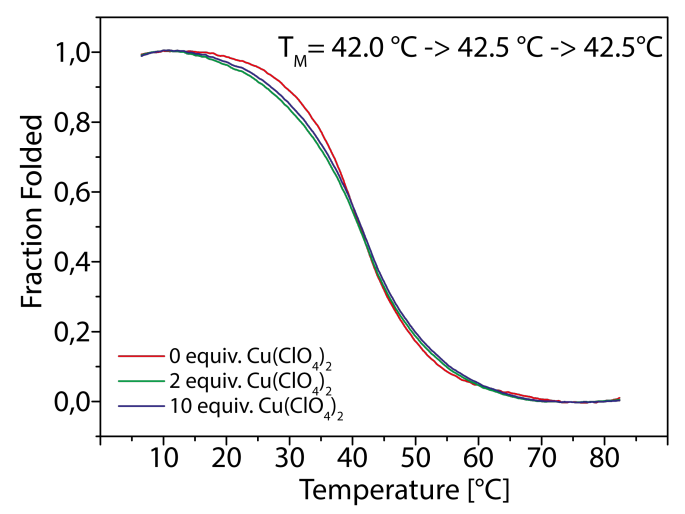

(b)

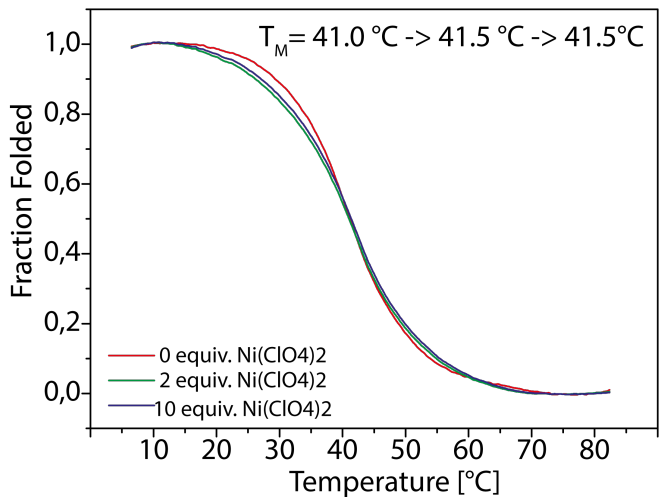

(c)

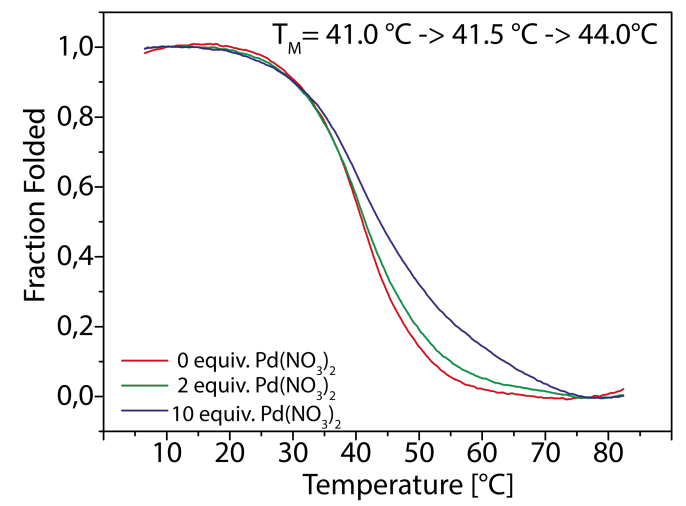

Figure III.2.3.23: UV-vis melting experiments for MKDH05 in presence of (a) $\left.\mathrm{Cu}\left(\mathrm{ClO}_{4}\right)_{2},(\mathbf{b}) \mathrm{Ni}_{(\mathrm{ClO}}\right)_{2}$ and (c) $\mathrm{Pd}\left(\mathrm{NO}_{3}\right)_{2}$. The samples contain $2 \mu \mathrm{M}$ of each ssDNA $100 \mathrm{mM} \mathrm{NaClO} 4,5 \mathrm{mM} \mathrm{Mg}\left(\mathrm{ClO}_{4}\right)_{2}, 10 \mathrm{mM} \mathrm{NaCaco}$ $\mathrm{pH}=7.3$. For each metal three succeeding melting curves were recorded. 1 . in absence of metal, 2 . in presence of $4 \mu \mathrm{M}$ metal salt, 3 . with an excess $(20 \mu \mathrm{M}$ of the respective metal cation.

For MKDH06 the melting profiles look pretty divergent, although all samples were taken from the same batch. The sample with copper is only shown for the sake of completeness, however, since the melting profile looks already strange and the sample with 10 equiv. did not show any usable data, it is omitted in this discussion. In figure III.2.3.24b the addition of nickel gives a minimal stabilization about $2{ }^{\circ} \mathrm{C}$, what correlates to the observation made for MKDH01 in presence of nickel(II) cations. Although the the starting $\mathrm{T}_{\mathrm{M}}$ for the last experiment figure III.2.3.24c is too low for some reasons, it looked so far most promising, since in the presence of an excess palladium(II) salt, the melting profile seems to contain two denaturation processes. By fitting the curve with a BiDoseResp function two temperatures could be determined. The denaturation temperature for the first process is $\mathrm{T}_{\mathrm{M}}=43.5^{\circ} \mathrm{C}$ and $\mathrm{T}_{\mathrm{M}}=64.0^{\circ} \mathrm{C}$ for the second melting process. Unfortunately, comparison of the UV-vis spectra did not give any hint on second species or the formation of a cage. But having figure IV.2.1.2a in mind, one would not expect any big changes in the UV-vis 
spectrum. Checking the sample with $254 \mathrm{~nm}$ light by hand, revealed again fluorescence which could be seen with the naked eye.

(a)

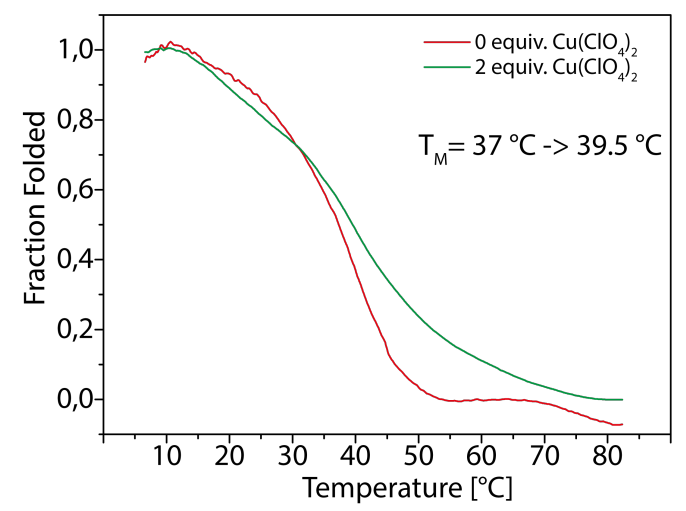

(b)

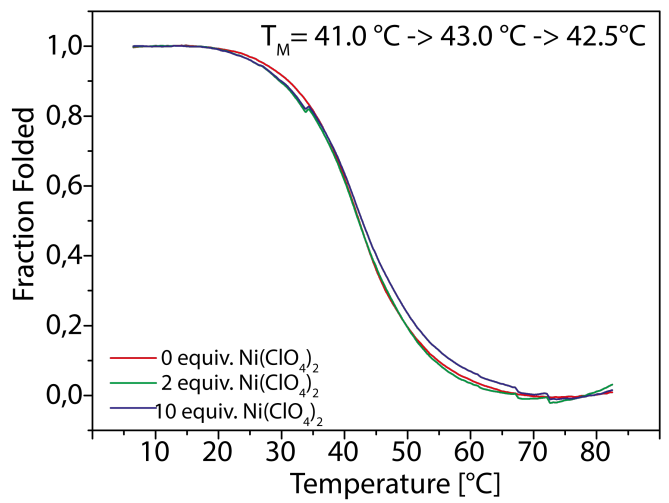

(c)

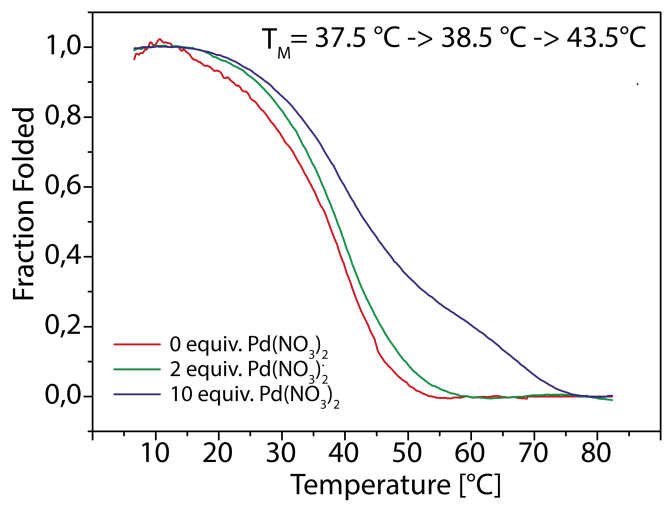

Figure III.2.3.24: UV-vis melting experiments for MKDH06 in presence of (a) $\mathrm{Cu}\left(\mathrm{ClO}_{4}\right)_{2},(\mathbf{b}) \mathrm{Ni}\left(\mathrm{ClO}_{4}\right)_{2}$ and (c) $\mathrm{Pd}\left(\mathrm{NO}_{3}\right)_{2}$. The samples contain $2 \mu \mathrm{M}$ of each ssDNA $100 \mathrm{mM} \mathrm{NaClO}_{4}, 5 \mathrm{mM} \mathrm{Mg}\left(\mathrm{ClO}_{4}\right)_{2}, 10 \mathrm{mM} \mathrm{NaCaco}$ $\mathrm{pH}=7.3$. For each metal three succeeding melting curves were recorded. 1 . in absence of metal, 2 . in presence of $4 \mu \mathrm{M}$ metal salt, 3 . with an excess $(20 \mu \mathrm{M}$ of the respective metal cation.

Anyway, in this case the fluorescence does not exclude the existence of coordination cage. On one hand, based on the melting profile two species seem to exist, therefore fluorescene can still be detected, if one of those is still fluorescence active. On the other hand it is not possible to say from a single measurement if this second species really exist or if it is just an artefact, therefore, the experiment has to be repeated in order to give a convincing answer to this question. Up to that point, it is a "Schrödinger" metal-mediated coordination cage embedded in a oligonucleotide scaffold. 


\section{Chapter}

\section{Conclusion}

In this chapter a new approach was investigated to form heteroleptic metal-mediated coordination cages in a controlled non-statistical fashion, by preorganizing the ligands in an exterior oligonucleotide scaffold. For that reason, four banana-shaped bis-monodentate ligands were incorporated into four different semi-complementary oligonucleotide sequences, capable to from a Holliday junction ( $(\mathrm{HJ})$. In this cross-shaped secondary DNA structure, the ligands are gathered in the central position in order to form a cage in presence of suitable transition metal cations. The first acridone-based ligand was successfully synthesized and subsequently incorporated into the respective single stranded DNA ssDNA. After cleanup and identification of the desired strands, the system was tested on its ability to form the intended Holliday junction. As a reference a second sequence was synthesized, where the ligand was replaced by a single thymine nucleobase, which should form T-T mismatches at the central position in the HJ, simulating a disorder within the structure, as it is caused by the incorporated ligands.

Table III.3.0.1: Comparison of denaturation temperature $\mathrm{T}_{\mathrm{M}}$ for the investigated Holliday junction (HJ) systems. The $\mathrm{T}_{\mathrm{M}}$ was determined for $2 \mu \mathrm{M}$ of the respective $\mathrm{HJ}$ with $100 \mathrm{mM} \mathrm{NaClO}, 5 \mathrm{mM} \mathrm{Mg}\left(\mathrm{ClO}_{4}\right)_{2}$ and $10 \mathrm{mM} \mathrm{NaCaco}$ at pH 7.3 in demineralized ultra-pure water. 11] Preliminary value, does not necessarily reflect the reality.

\begin{tabular}{ccccccc}
\hline System & $\mathrm{T}_{\mathrm{M}}\left[{ }^{\circ} \mathrm{C}\right]$ & $\mathrm{HJ}$ & $\left.\Delta \mathrm{T}_{\mathrm{M}}\right]^{\mathrm{Cu}}\left[{ }^{\circ} \mathrm{C}\right]$ & $\left.\Delta \mathrm{T}_{\mathrm{M}}\right]^{\mathrm{ii}}\left[{ }^{\circ} \mathrm{C}\right]$ & $\Delta \mathrm{T}_{\mathrm{M}}{ }^{\mathrm{Pd}}\left[{ }^{\circ} \mathrm{C}\right]$ & Cage \\
\hline \hline MKDH01um & 41.0 & $\boldsymbol{V}$ & - & - & - & - \\
MKDH01 & 54.0 & $\boldsymbol{V}$ & -2.0 & 2.0 & - & $\mathbf{x}$ \\
MKDH05 & 44.0 & $\boldsymbol{V}$ & 0.5 & 0.5 & 3.0 & $\mathbf{x}$ \\
MKDH06 & 42.0 & $\boldsymbol{V}$ & - & 1.0 & $6.0 \& 26.5^{[1]}$ & $\mathbf{x}$ \\
\hline
\end{tabular}

Both system were investigated with help of UV-vis melting experiments, various temperature fluorescence spectroscopy, various temperature CD spectroscopy and polyacrylamide gel electrophoresis (PAGE) experiments. The existence of both HJs could be proven by melting curves and especially, by a distinct band in the PAGE gel. Interestingly, the modified HJ has 
a $13{ }^{\circ} \mathrm{C}$ higher denaturation temperature compared to the unmodified HJ table III.3.0.1). This behavior can be explained by a stacking of the polyaromatic ligands within the HJ structure, comparable to the effect described in a three-way junction by Laing et al. 179 Either the ligands intercalate into the nearby double helices or more likely, they do interact with each other, forming an aggregate. This aggregation is indicated by a significant bathochromic shift, observable for the ligand-specific UV-vis band, caused by the assembly of $\mathrm{HJ}$ and is known from molecules forming so called J-aggregates or excimers. What kind of aggregate is formed could not be elucidated with the here presented experiments. However, what is for sure, since all experiments are performed in a polar environment, the stacking process additionally benefits from the hydrophobic effect, making the aggregate so stable that no coordination cage could be formed in presence of any investigated transition metal salt.

Therefore the ligand system as well as how it was incorporated into the oligonucleotide was complete redesigned. The aromatic backbone was minimized to avoid the stacking efficiency and the linker motif was replaced by a diethanol amine motif and relocated to the ligands backbone, giving the whole system more flexibility. This new ligand motif was incorporated into the same sequence as it was used in the first approach. In addition, a second sequence with thymines, enclosing the ligand, were synthesized, in order to form T-T mismatches at the crossing section of the HJ. That approach gave more space for the formation of the intended coordination structure, since the shortened DNA linkers could provide too few space for this purpose. From the accomplished experiments the proof of an successful assembly of Holliday junction for both sequences was obtained, still, the results indicating an interaction of the ligands with each other or the surrounding oligonucleotide scaffold, even though in a mitigated form. Nevertheless, again no trustworthy evidences could be found for a formation of the intended coordination cage, neither in presence of palladium, nor nickel or copper.
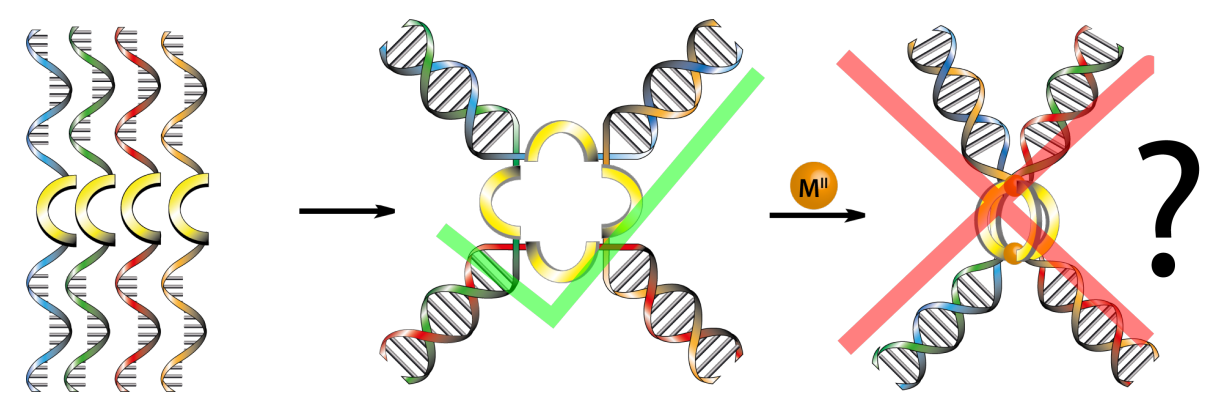



\section{Water-soluble Metal-Mediated \\ Coordination Cages}

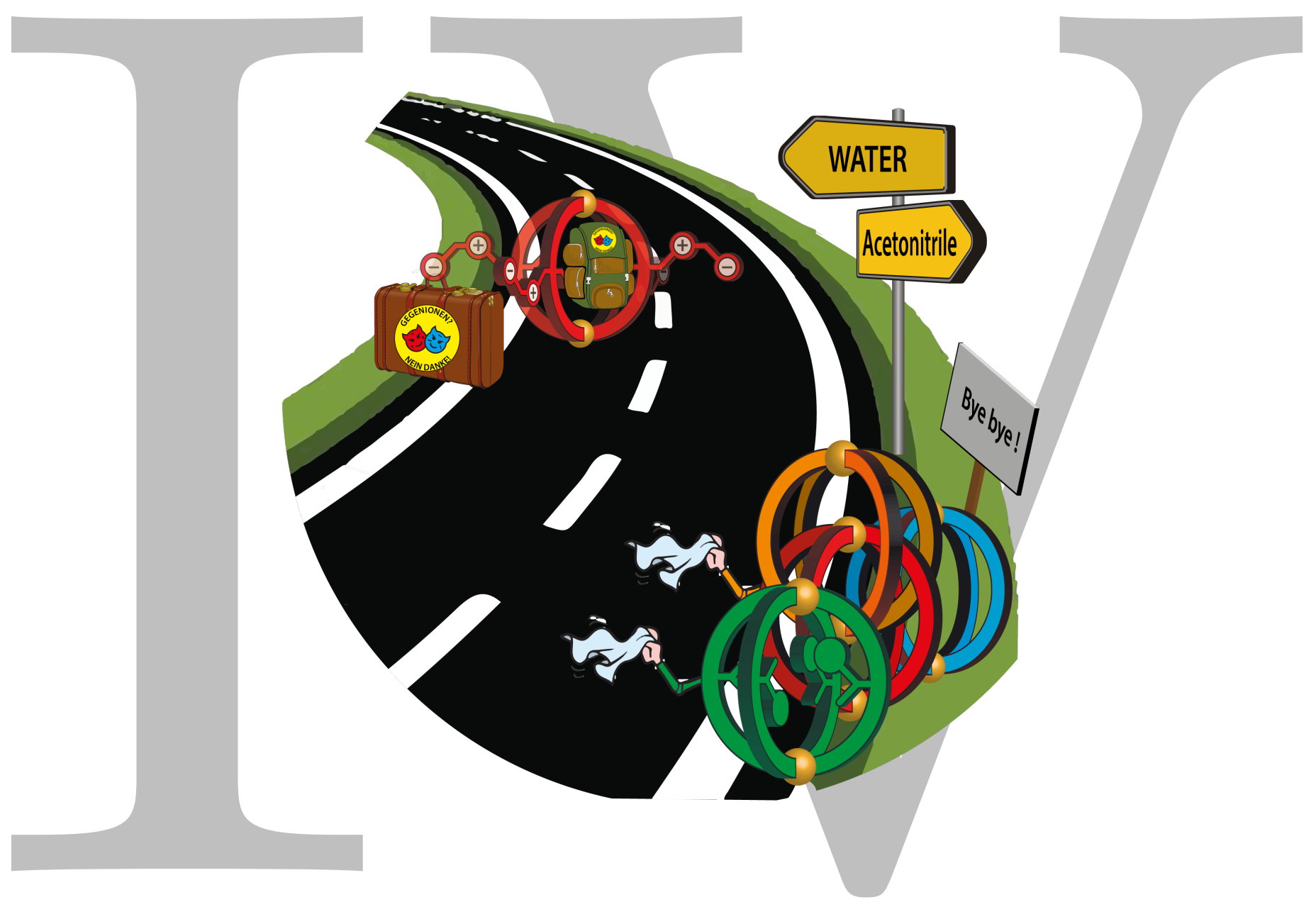




\section{Chaperer IV.1}

\section{Introduction}

In the context of the world wide chemical production, solvents are widely recognized to be one of greatest hazards for the environment. Apart from the high effort and energy consumption that is needed for production and processing of suitable amounts of organic solvents for industrial applications, they are highly toxic when exposed to organisms. 180 Moreover, their inflammability or even explosion hazards led in the last years to several incidents in both academics and industry, some of them were even lethal. 181. Hence, the reduction of the global organic solvent usage is one of the primary and probably most important aim in the new field of research "green chemistry". 182 That presupposes of course the availability of alternative methods that are equal to existing solvent based procedures. Moreover, especially with regard to industrial applications, the economic sustainability of those methods is an important factor to be considered during the development, to make the changeover attractive to the companies. 183

In the last 20 years, since green chemistry became an own area of research, new solvent systems like ionic liquids, 184 organic-aqueous tuneable system (OATS), 185 switchable solvents 180 or supercritial fluids, 186 to name some, have been investigated and proofed to be adequate alternatives to many volatile organic compounds. However, they often suffer from a lack of flexible applicability and their costs. 187 Concerning the economic factor and availability, water became more and more the focus of attention. Although at the first glance water seems to be furtherest from the classical organic solvents, what is the truth, new innovations combined with the physical and chemical knowledge, how organic compounds solvate in water, helped to transfer a range of classical organic syntheses into aqueoues medium.188 Moreover, the usage of water as solvent can potentially improve reactivities and selectivities or simplify the workup procedures. In addition, in the context of organic chemistry in water, in some reaction uncommon reactivities and reverse selectivities could be observed compared to the classic reaction in organic solvents. 189 .

In biological systems almost all reaction proceed in water, where superior control of reactivity and selectivity can be obtained, even under highly complex reaction mixtures, 
due to nature's well-developed functional units. To emulate this level of complexity, the interaction between solvent, reagent and functional units has to be understood, what also includes the physical properties of water and its uniqueness towards other solvents. For that reason, as so often before, scientist created model compounds based on artifical bio-derived biopolymers, oligonucleotides and -pepteides, $190 \mid 191]$ or metal-organic frameworks, 192 that can mimic the functionalities in natural occurring structures. Especially, the field of supramolecular chemistry provided in the last years a versatile playground for the creation of complex three-dimensional architectures, from which several beautiful examples of water-soluble functional constructs derived. 144

An early example is reported by Raymond et al. consisting in a supramolecular polyanionic compound, where six bis-catechol based ligands self-assemble with four Ga(III) cations to form a tetrahedral water-soluble cage (Figure IV.1.0.1).
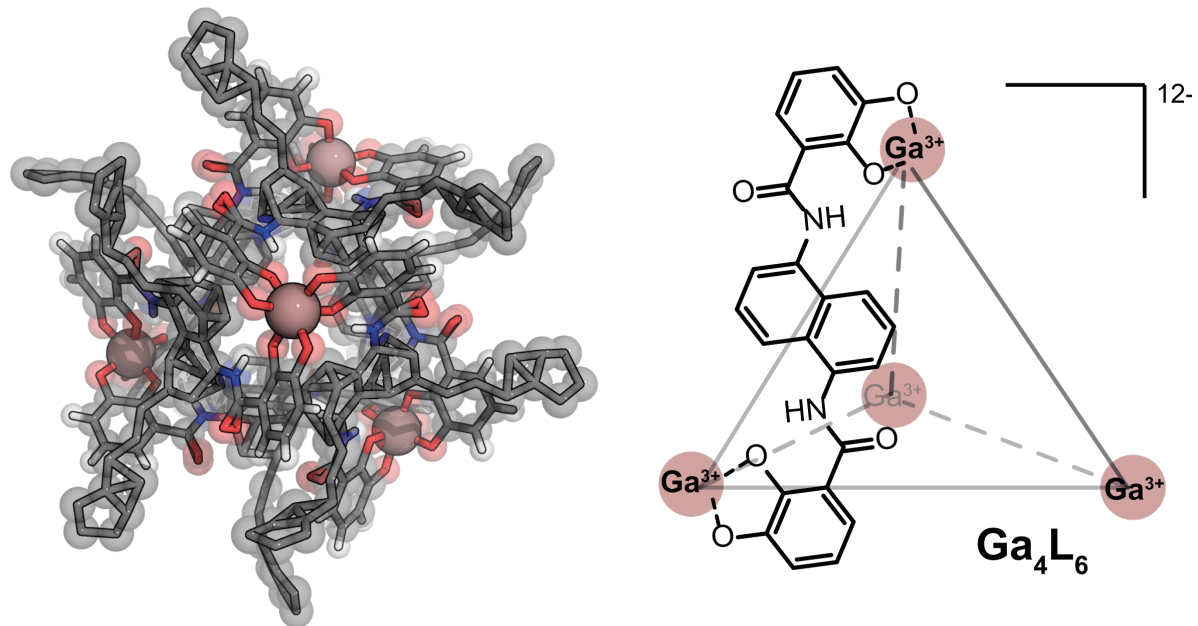

Figure IV.1.0.1: Supramolecular gallium-mediated coordination cage of Raymond et al..193. The cage has a tetraedic topology, with gallium(III) cations sitting on the corners, connected by six ligands. Each gallium is coordinated by 3 ligands in octahedral fashion. The ligand is symmetric with catechol motif for coordination on both ends. Each ligand is bridging two vicinal gallium cations, giving a total charge of -12 . Due this highly negative charge, this compact supramolecular architecture is soluble in aqueous medium.

Besides the fact, that Raymonds' cage is water-soluble, it additionally promotes an alternative reaction route of citronellal in water, when added to the reaction in catalytic amounts. While in abscence of the gallium cage a dimerization of the substrate occurs, an intramolecular cyclization is preferred when the substrate is encapsulated in the polyanionic structure. This change in reaction selectivity is caused by several factors. First of all, due the hydrophic effect the substrate goes preferentially inside the cages' cavity, further due to the highly charged environment the concentration of oxonium ions is spatially increased, as well as cationic transtion states or intermediates are better stabilized.44|193] An inversed charged principle can be found in the cobalt cube of Ward et al.194 or the ocathedral cage of Fujita et al. Figure IV.1.0.2. 4 .45 
(a)
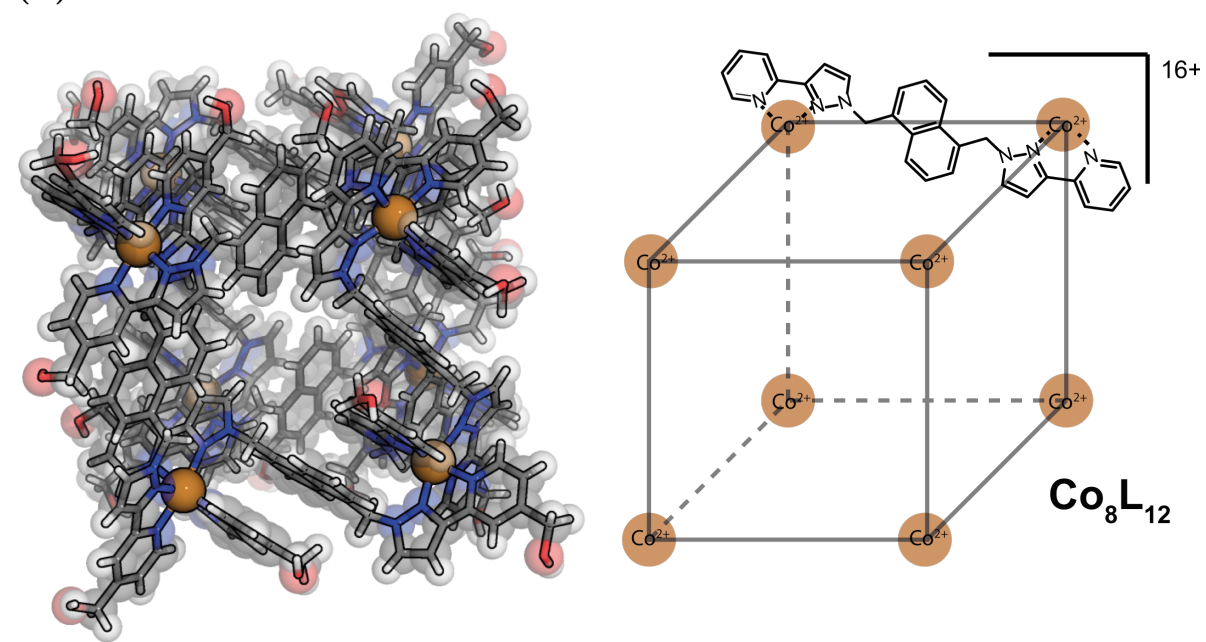

(b)
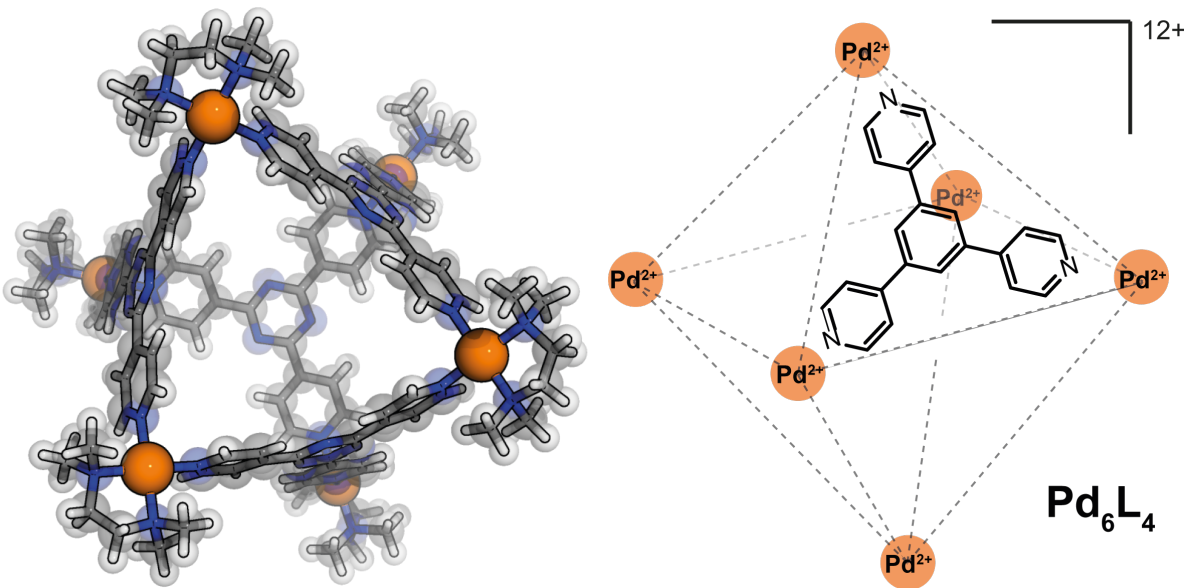

$P d^{2+}$

Figure IV.1.0.2: Highly charged cages by Ward and Fujita, both soluble in aqueous medium. a) The cage of Ward et al. shows a cubic topology with cobalt(II) ions sitting on each corner. Each cobalt is coordinated by three ligands in an octahedral fashion, therefore the cage consists of 8 cobalt(II) ions and 12 neutral ligands, lying on the cubes' edges. The supramolecular structure has an allover charge of $16+$. b) Fujita's palladium coordination cage has a octahedral topology, with palladium(II) cations on each corner. The whole cage contains only four tridentate neutral ligands, which coordinate to three neighbored palladium ions, spanning a triangluar surface. Since the palladium is coordinated in a square planar fashion and only two coordinating positions are occupied by the ligands, the residual coordination sites are capped by an ethylendiamine motif on each palladium cation. Each pyramidal substructure consists of two opposite lying ligands, turned 90 in respect to the second substructure. The allover charge is $12^{+}$.

Further water-soluble cages of other geometries and charges were also reported by Fujita with an pyramidic structrure, 46 Nitschke with a negatively charged tetrahedral complex or Yoshizawa with a positively charged $\left[\mathrm{Pd}_{2} \mathrm{~L}_{4}\right]$ cage, containing neutral bis-pyridyl ligands with polyethylene glycol (PEG) chains. Figure IV.1.0.3). 195 Comparing all these water-soluble structures, regardless whether they have catalytic function or serve only as container, all of them are highly charged $(\geq 8+)$, what seems to be one crucial requirement to get the accumulation of aromatic ligands into solution. As a second requirement, the charges have to be well distributed over the whole struture, as for example, Clever et al. showed several examples of highly charged $\left[\mathrm{Pd}_{4} \mathrm{~L}_{8}\right]^{8+}$ interpenetrated cages that 
are not soluble in water at all.58 However, both Yoshizawa and Crowley reported supramolecular structures based on bis-pyridyl ligands forming $\left[\mathrm{Pd}_{2} \mathrm{~L}_{4}\right]^{4+}$ cages that are soluble in aqueous medium. Both achieved this extra solubility in the organic solvent water mixture by adding polyglycolic chains or free hydroxy groups to the ligands backbones. $42 \mid 76$

(a)

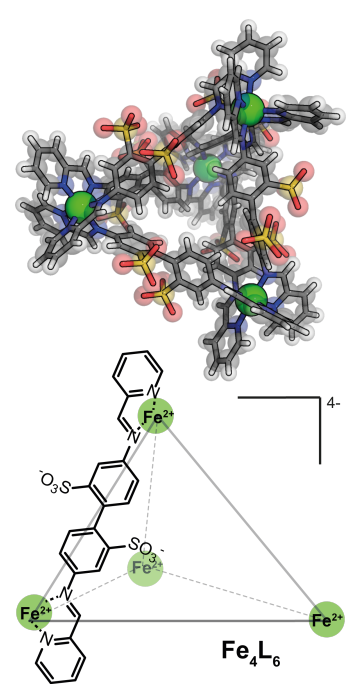

(b)
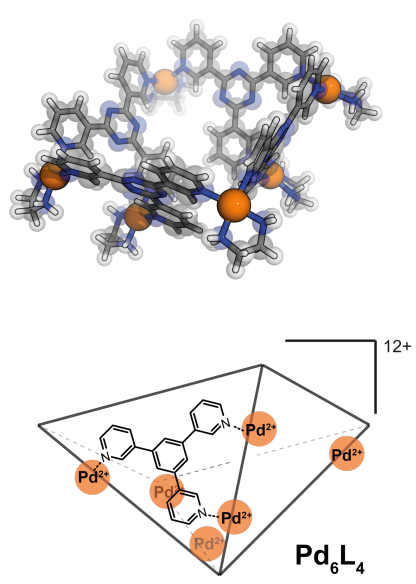

(c)
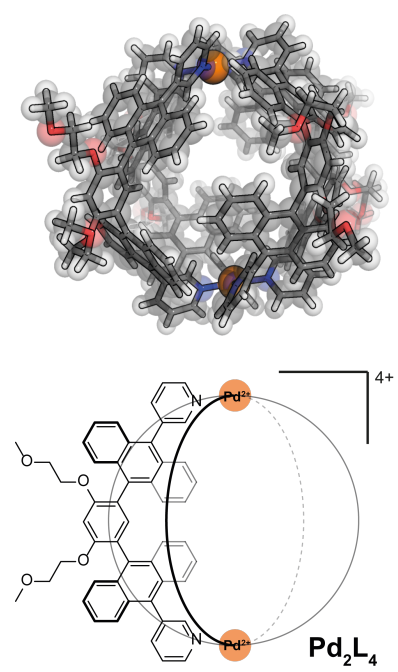

Figure IV.1.0.3: Charged cages by Nitschke, Fujita and Yoshizawa. a) Tetrahedral cage by Nitschke et al., consisting of iron(II) cations, sitting on each corner, bridged by six negatively charged ligand. In comparison to Raymond's gallium-mediated cage, the metal ions are coordinated by neutral nitrogen ligands, with negatively charged sulfonate groups located at the ligands backbone, which do not interact with the iron cations. Therefore, each charged motif within the supramolecular structure, needs its own counter ion, giving 16 counter ions in total and a total charge of 4- for the coordination cage itself. b) By changing the substitution pattern of the pyridines from para to meta, Fujita et al. synthesized a derivative of the previously described ligand (Figure IV.1.0.2b), which rather forms a bowl structure than an octahedral coordination cage, in presence of palladium(II). c) Yoshizawa et al. attached $\mathrm{PEG}$ chains to the backbone of the banana-shaped bi-dentate ligand, making its corresponding cage suitable to be solved in aqueous medium. A little later, introduction of a third PEG chain to the ligands backbone, led to a completely water-soluble capsule. 196

There are also some concepts of adding charged residues, like trimethylammonium or sulfonate groups, to the ligand backbones, which led also to an increase in solubility, but generates as well a highly charged species with lots of counter-anions, which can have a tremendous influence on the formation 31 or solubilty 197 of the intended supramolecular architecture. In the previous chapter, it has already been shown that the addition of either glycol chains or hydroxy groups to the ligand backbone, does not necessarily drive the system to be water soluble. (Chapter III.2).

In this chapter, the anisole-based ligand (55), known from the previously discussed topic Section III.2.3 is examined for its ability to form a $\left[\mathrm{Pd}_{2} \mathrm{~L}_{4}\right]^{4+}$ cage in pure water. Furthermore, instead of the diethanolamine motif, a new concept of self-stabilizing zwitterionic moiety was attached to the ligands backbone, causing a significant increase in water-solubility for the ligand itself and its resulting supramolecular architecture without changing the overall charge. The reported strategy to introduce zwitter-ionic moieties, 
successfully achieved to avoid organic solvents, and it shows an elegant general strategy that can be used to tune the cage solubility, without changing the net charge, resembling a similar approach found in natural compounds. 


\section{Chapter}

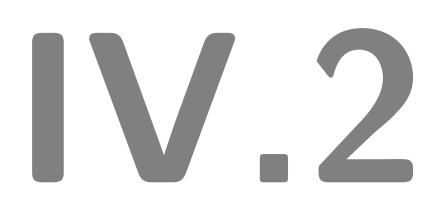

\section{Results and discussion}

\section{IV.2.1 Diethanolamin methoxy ligand}

In chapter III.2 the formation of cage with ligand $\mathbf{L}^{\mathbf{A c r}}(\mathbf{9})$ could not be verified due to the insufficient solubility of ligand and cage in water (Section III.2.2), otherwise the preferred stacking of the acridone ligands maybe could have been noticed earlier. However, also this time the ligand $\mathbf{1 7}$ was checked for the formation of $\left[\mathrm{Pd}_{2} \mathrm{~L}_{4}\right]$ cage in water, to be sure that ligand can do form a supramolecular cage in water in the presence of palladium(II) cations, before it was build into the oligonucleotides. For that reason a $2.8 \mathrm{mM}$ solution of ligand $\mathbf{L}^{\text {DEA }}(\mathbf{1 7})$ in $\mathrm{D}_{2} \mathrm{O}$ was prepared. Since the ligand itself showed a rather low solubility in water at room temperature, the solution was heated to $60{ }^{\circ} \mathrm{C}$, until a clear transparent homogeneous solution was obtained. After, $500 \mathrm{\mu l}$ of the ligand solution were mixed together with an $50 \mathrm{\mu l}$ solution of $\mathrm{Pd}\left[(\mathrm{MeCN})_{4}\right]\left(\mathrm{BF}_{4}\right)_{2}$ or $\mathrm{Pd}\left(\mathrm{NO}_{3}\right)_{2}\left(15 \mathrm{mM}\right.$ in $\left.\mathrm{D}_{2} \mathrm{O}\right)$ and heated for $1 \mathrm{~h}$ at $70{ }^{\circ} \mathrm{C}$. The resulting mixture examined via ${ }^{1} \mathrm{H}$-NMR and ESI-MS Figure IV.2.1.1. At the beginning the samples were prepared with the palladium salts solved in MeCN or DMSO, since the solvation in pure $\mathrm{D}_{2} \mathrm{O}$ quickly led to formation of the reduced palladium $(0)$ species, precipitating as a black solid. The formation of palladium(0) species already occurs at room temperature and was even accelerated when solvent was heated in order to bring the palladium(II) salts into solution. To obtain a suitable palladium(II) solution, a preceded cooling of the deuterated water was necessary. Subsequently the palladium salt was added and solved by only applying ultra-sonification until a homogeneous solution emerged. The freshly prepared palladium(II) solution was immediately added to the ligand, leading to the formation of the cage species. As soon the metal ion is coordination by the pyridine motifs, the palladium(II) seems to be stable, since no further black precipitation was observable. In ${ }^{1} \mathrm{H}-\mathrm{NMR}$ the typical significant down-field shift of the pyridine related proton signals (especially $\mathbf{a}$ and $\mathbf{b}$ ) was observed for both samples, the one with $\left[\mathrm{Pd}(\mathrm{MeCN})_{4}\right]\left(\mathrm{BF}_{4}\right)_{2}$ and the one with $\operatorname{Pd}\left(\mathrm{NO}_{3}\right)_{2}$. Additionally, the methoxy signal is also significantly down-field 
shifted, indicating a formation of the cage, since the methoxy group pointing inwards the cage, having the positively charged palladium(II) cations in direct vicinity. Although it was not in the focus of interest, it is worth to mention that the formation of cage was also observed by NMR in polar organic solvents, like MeCN and DMSO in the presence of the $\operatorname{Pd}\left[(\mathrm{MeCN})_{4}\right]\left(\mathrm{BF}_{4}\right)_{2}$ salt.

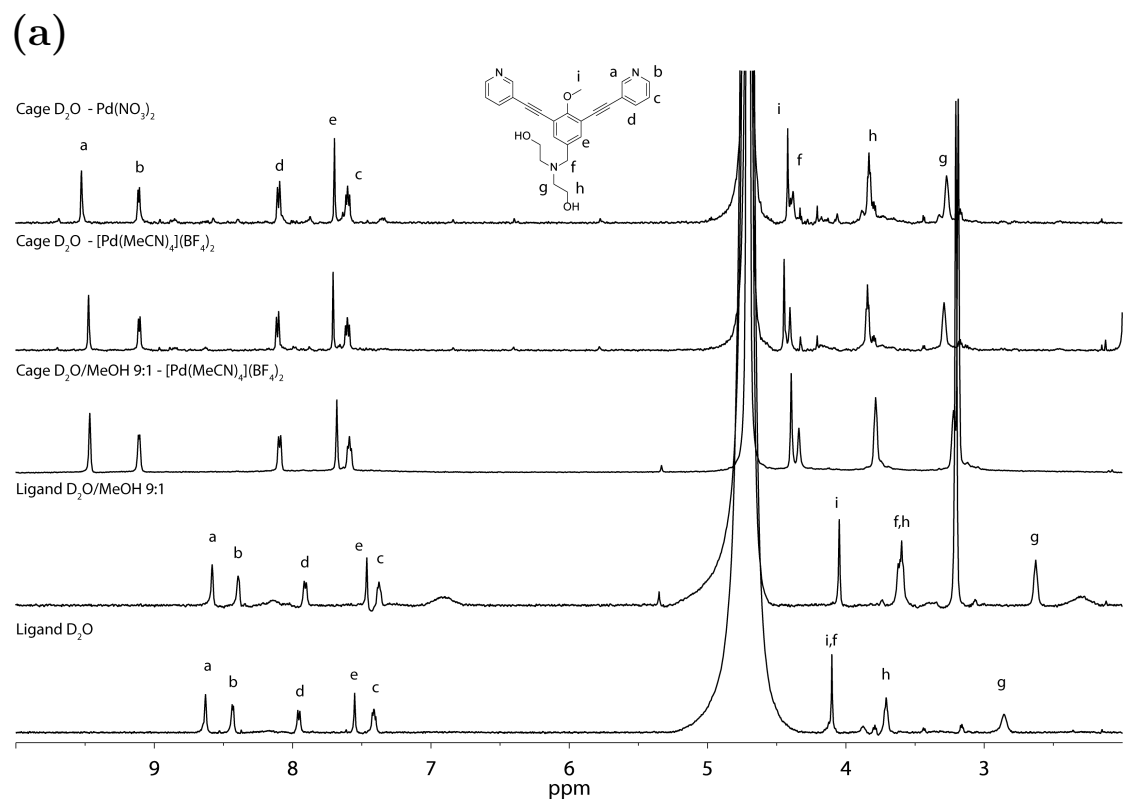

(b)

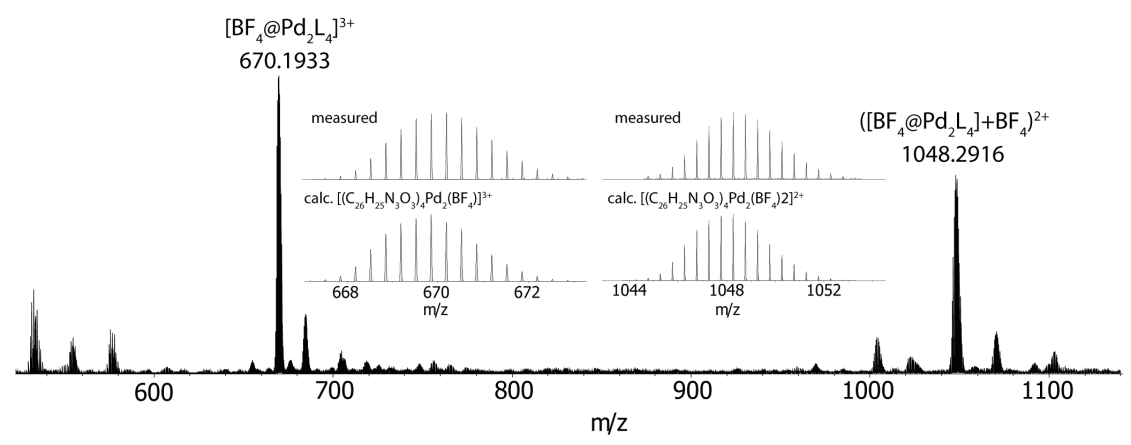

Figure IV.2.1.1: (a) ${ }^{1} \mathrm{H}$ NMR $(500 \mathrm{MHz}) .\left[\mathrm{Pd}_{2} \mathrm{~L}_{4}\right]$ cage can be formed in pure deuterated water with both $\left[\mathrm{Pd}(\mathrm{MeCN})_{4}\right]\left(\mathrm{BF}_{4}\right)_{2}$ as well as $\mathrm{Pd}\left(\mathrm{NO}_{3}\right)_{2}$. (b) ESI-MS of the cage formed from ligand $\mathbf{1 7}$ with $\left[\mathrm{Pd}(\mathrm{MeCN})_{4}\right]\left(\mathrm{BF}_{4}\right)_{2}$ in $\mathrm{D}_{2} \mathrm{O}$. Since ligand $\mathbf{1 7}$ is pourly soluble in water at room temperature, additional spectra were recorded with $10 \%$ of deuterated methanol to increase the amount of solved ligand in the NMR sample. The cage was formed upon addition of 0.5 equiv. $\left[\mathrm{Pd}(\mathrm{MeCN})_{4}\right]\left(\mathrm{BF}_{4}\right)_{2}$ in deuterated methanol. The sample was taken from NMR sample in pure $\mathrm{D}_{2} \mathrm{O}$. For the cage formed in presence of $\mathrm{Pd}\left(\mathrm{NO}_{3}\right)_{2}$ no cage peak was detectable in ESI-MS, but the formation was verified by NMR and fluorescence spectroscopy.

Due to the fact that the ligand showed a rather poor solubility in pure water and partially precipitated when the solution cooled down to room temperature, an additional NMR experiment was accomplished in an aqueous solvent mixture containing $10 \%$ deuterated methanol. The solubility of the ligand was significantly increased, thus a clear transparent solution was obtained at room temperature. The ligand compound showed the same chemical shifts for the aromatic region in NMR, merely the diethanol amine signals are 
slightly upfield shifted in the aqueous solvent mixture. Nevertheless, upon addition of $\left[\mathrm{Pd}(\mathrm{MeCN})_{4}\right]\left(\mathrm{BF}_{4}\right)_{2}$, also solved in $\mathrm{d}^{4}-\mathrm{MeOH}$, almost exactly the same NMR spectrum could be obtained, compared to the cages measured in pure water.

Samples for ESI-MS measurements were directly taken from the pure water NMR samples and measured. The mass spectra clearly showed two main peaks, which could be assigned to the $3+$ and $2+$ species of $\left[\mathrm{Pd}_{2} \mathrm{~L}^{\mathrm{DEA}}{ }_{4}\right]$ cage with tetrafluoroborate as counter anions. It is worth to mention, that the $4+$ species was not found in the ESI-MS spectrum, thereof it may be inferred that one $\mathrm{BF}_{4}{ }^{-}$anion is encapsulated within the cage cavity, which in turn explains why only the $3+$ and $2+$ species can be seen. On the other hand it just could be a matter of the used condition during the ESI-MS experiment. For the cage formed with $\mathrm{Pd}\left(\mathrm{NO}_{3}\right)_{2}$ no satisfying ESI-MS spectra could be obtained, however, in order to verify its formation, further experimental measurements with help of UV-vis and fluorescence spectroscopy were performed (figure IV.2.1.2).

(a)

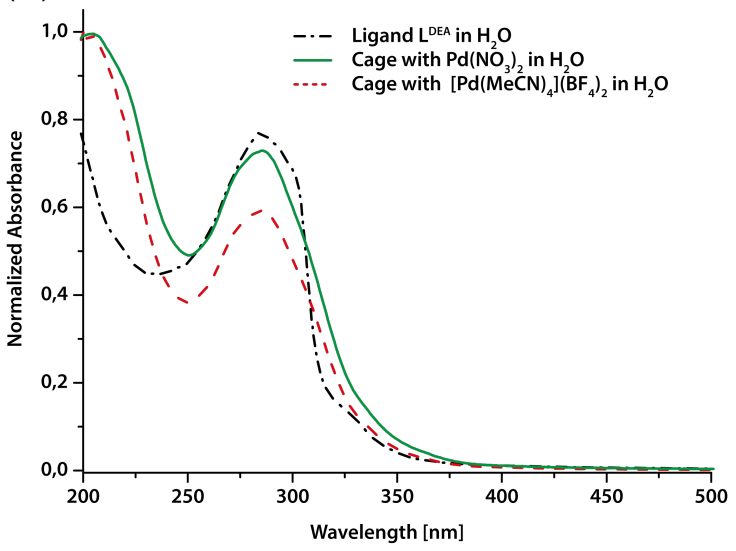

(b)

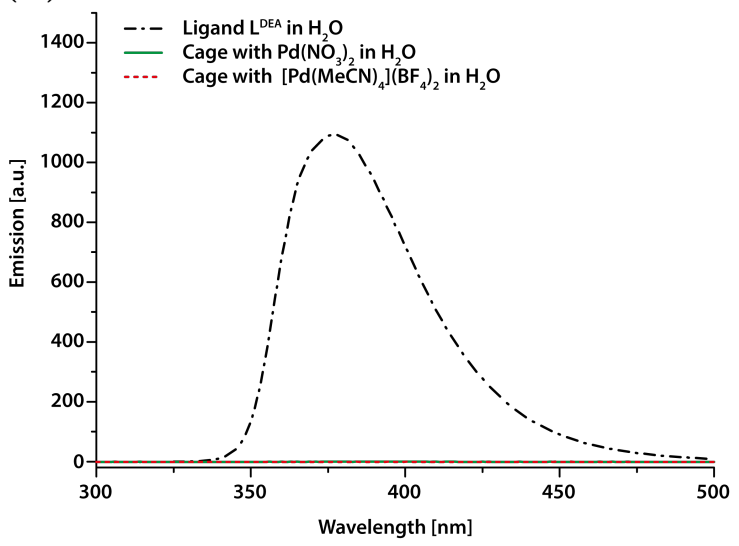

Figure IV.2.1.2: UV-vis and fluorescence spectra for $\mathbf{L}^{\mathbf{D E A}}$ and $\left[\mathrm{Pd}_{2}\left(\mathbf{L}^{\mathbf{D E A}}\right)_{4}\right] \mathrm{X}_{2}\left(\mathrm{X}=\mathrm{NO}_{3}\right.$ or $\left.\mathrm{BF}{ }_{4}\right)$. a) $\mathrm{All}$ UV-vis spectra were recorded in $\mathrm{H}_{2} \mathrm{O}$ with a total ligand concentration of $0.035 \mathrm{mM}$. b) All fluoresence spectra were recorded in $\mathrm{H}_{2} \mathrm{O}$ with a total ligand concentration of $0.005 \mathrm{mM}$, irradiated with a wavelength of $280 \mathrm{~nm}$. The ligand is fluorescent and emits light in the range of $350 \mathrm{~nm}$ and $500 \mathrm{~nm}$, with a maximum of approx. $377 \mathrm{~nm}$. In contrast, the assembled cage does not show any fluorescence.

In the UV-vis measurements a difference between the spectra of the ligand $\mathbf{L}^{\mathrm{DEA}}$ and those of the cage solutions is visible. In addition the shape of absorption bands for both cage measurements are pretty similar, even though they differ in absorbance. Anyway, a clear proof for the cage formation with $\mathrm{Pd}\left(\mathrm{NO}_{3}\right)_{2}$ as metal salt could not be obtained from this method. As a consequence, the fluorescence of the same samples were measured, since it is known from previous works, that if the ligand shows any fluorescence, it is usually quenched upon coordination to the palladium cation. And in fact, for both cage sample no fluorescence was detectable. In contrast, the free non-coordinated ligand shows a significant emission band at $377 \mathrm{~nm}$ when irradiated with $280 \mathrm{~nm}$. 
(a)

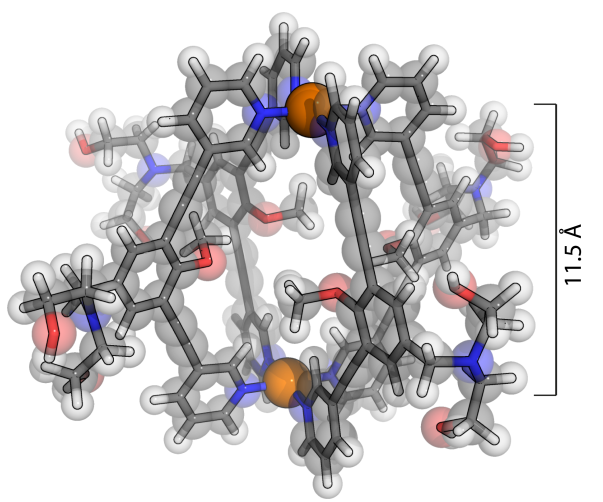

(b)

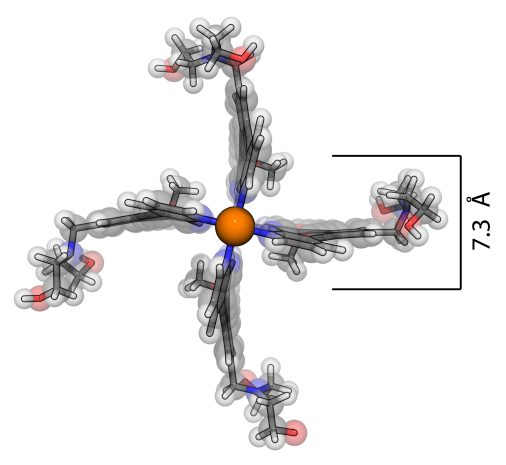

Figure IV.2.1.3: PM6 model of cage $\left[\mathrm{Pd}_{2}\left(\mathbf{L}^{\mathbf{D E A}}\right)_{4}\right]$. The cage has a Pd-Pd distance of $11.5 \AA$ and an average distance of $7.3 \AA$ between two opposite methoxy groups. The values do match with those of the sulfobetaine cage figure IV.2.3.7a, what is not really surprising, since both consists of the same backbone and only differ in the exohedrally attached motif.

Although plenty of crystallization attempts under various condition were tried, it was not was possibly to obtain a crystal suitable for single crystal X-ray diffraction experiments. Due to that reason, a computer assisted model of the cage structure was created in silico figure IV.2.1.3).

\section{IV.2.2 Diethanolamin hydroxy ligand}

Due to the fact that the cage based on diethanol amine ligand $\mathbf{L}^{\mathbf{D E A}}$ with the inward pointing methoxy group did not show any evidence for an encapsulation of small unpolar guest, we decided to synthesize an analog structure with a less steric endohedral group. Since the hydroxy group is needed in the first step of synthesis, it had to remain, therefore only the methyl group could be omitted. Anyway, from PM6 model the average distance to the opposite ligand was increased in average by $1 \AA$, which approximates the cavity size to the double cage inner cavity, described in figure IV.2.3.7. Concurrently, the cavity would of course become less hydrophobic, lacking the methyl motif, which makes is less favorable for unpolar guests. However, the new motif also can give the opportunity for a new range of possible applications, since the hydroxy groups are actually phenolic groups, means they are weak organic acids. Phenols are often used in the context of supramolecular structures or macrocycles for anion recognitions, but also small molecules with hydrogen bond acceptors. 198 Moreover, a post-synthetic deprotonation could make the cavity suitable for metal cations or small positively charged molecules. 
The synthesis of ligand $\mathbf{3 1}$ was accomplished analogously to the previous synthesis. The only change, was the introduction of a protection group for the phenol hydroxyl group. As several reactions has to be performed under basic conditions, the phenol would probably deprotonated, leading to side reaction or product. From pre-experiments with various protecting groups, including methoxymethyl acetal (MOM) and methyl ether, tertbutyldimethylsily (TBDMS) turns out to be the best choice. After introducing TBDMS in the second step, all further product could be obtained with high yields. As a last step the TBDMS had to be removed from compound 30, but during the purification process, it was spontaneously converted to compound 32 scheme IV.2.2.3.

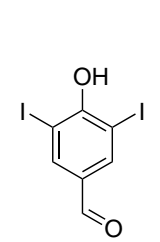

12

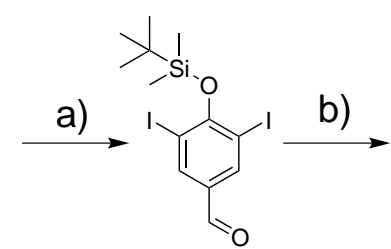

26<smiles>CC(C)(C)Oc1c(I)cc(CO)cc1I</smiles>

27

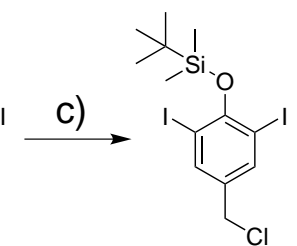

28
31

32

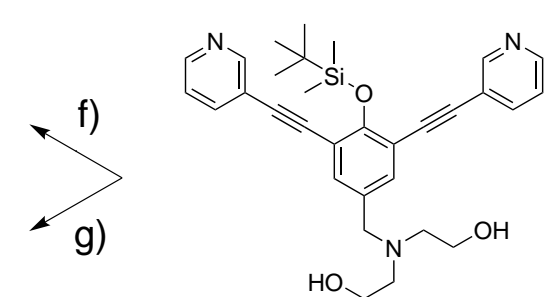

30

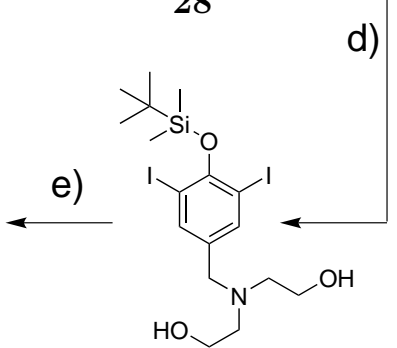

29

Scheme IV.2.2.3: Synthesis of compound 30. a) TBDMS-Cl, $\mathrm{NEt}_{3}, \mathrm{EtOAc}$; b) $\mathrm{NaBH}_{4}, \mathrm{MeOH}$ c) $\mathrm{SOCl}_{2}$, $\mathrm{NEt}_{3}, \mathrm{DCM}$; d) DEA, (i-Pr) ${ }_{2} \mathrm{NEt}, \mathrm{CHCl}_{3}$; e) 3-ethynyl-pyridine, $\mathrm{Pd}\left(\mathrm{PPh}_{3}\right)_{2} \mathrm{Cl}_{2}, \mathrm{CuI}_{\text {, NEt }}$, DMF. In step f) a deproctection was planed with tetrabutylammonium fluoride to obtain $\mathbf{3 1}$, but in presence of $\mathbf{g}) \Delta \mathrm{T}$ and moisture, $\mathbf{3 0}$ spontaneously reacted to $\mathbf{3 2}$ scheme IV.2.2.4.

After column purification of the compound 30, there were still visible signals from DMF, which was used during synthesis. Therefore, the residue was dried for several hours in high vacuum at $60{ }^{\circ} \mathrm{C}$. Suddenly the compound showed a lot of new signals in the aromatic region, from which several looked doubled. Interestingly, the signals from the side chain were unchanged as well as signals of the TBDMS protecting groups were also still there, but a little up-field shifted. Mass analysis with ESI-MS showed peaks, fitting to the desired deprotected ligand $\mathbf{3 1}$ (shown in Figure IV.2.2.4), but there were no peaks for the substrate 30, as an evidence for the thermally induced deprotection of the tert-butyldimethylsilyl group. However, ESI-MS analysis did not allow to identify the origin of the new NMR signals in the aromatic region. As a consequence, the mixture was purified via GPC] and the isolated compounds were analyst. The resulting ${ }^{1} \mathrm{H}-\mathrm{NMR}$ spectrum still showed the doubled aromatic signals, but the TBDMS signal were vanished. By further 2D-NMR experiments the resulted compound could be identified as the asymmetric compound 32. The compound has undergone a intramolecular cyclization reaction between the 
deprotected phenol hydroxyl group and one of the ortho-substituted 3-alkynyl pyridine arms, by what the whole system becomes asymmetric, thus the aromatic signals get doubled. Additionally, the formed hetero-aromatic five-membered ring contains a new proton, showing a typical chemical shift in the aromatic region, which explains the same mass as the desired unprotected compound 31. This proton could be assigned to signal j. Since the additional proton was not provided anyway, it must come either from air moisture or the wet solvent.

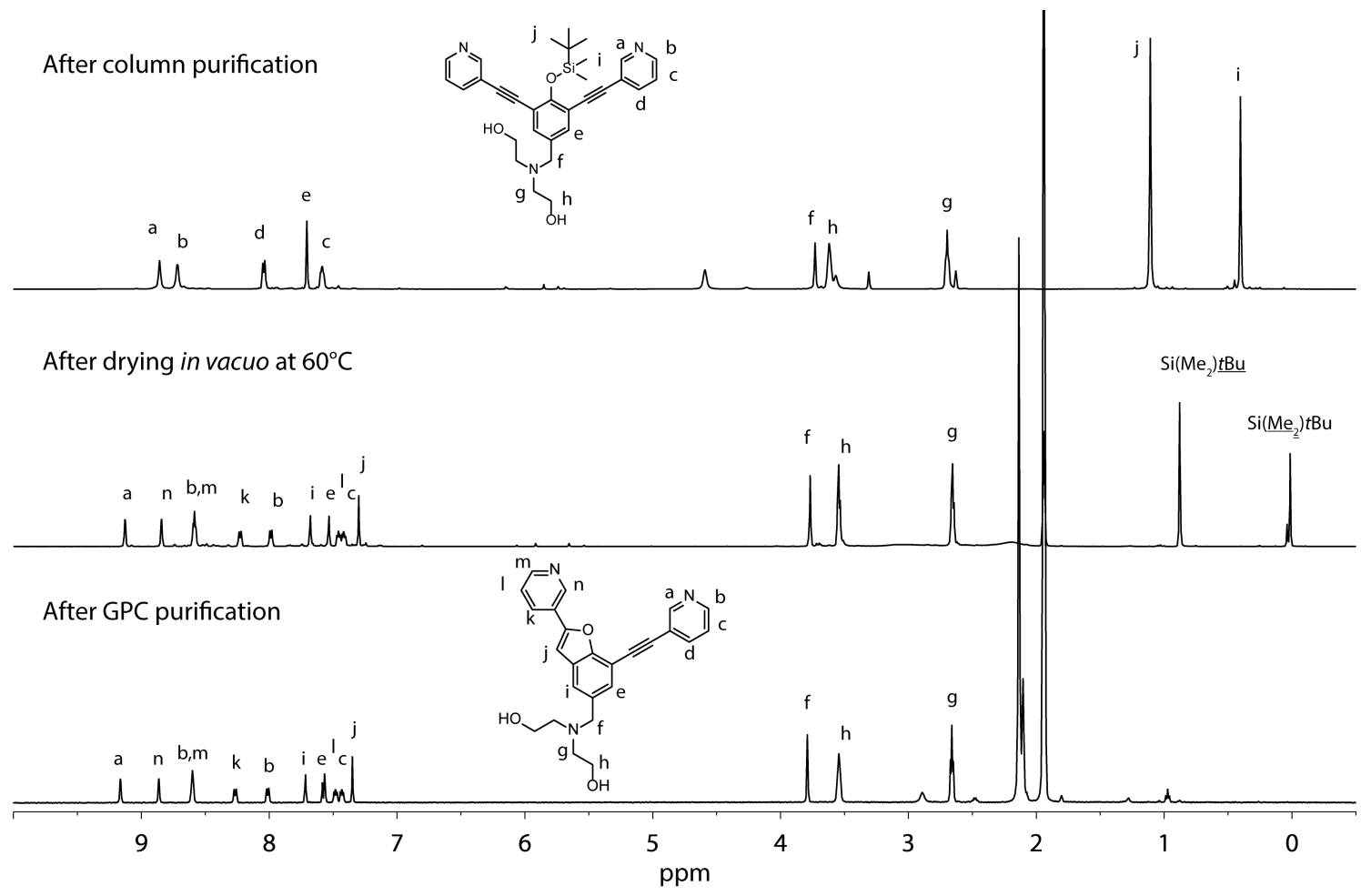

Figure IV.2.2.4: ${ }^{1} \mathrm{H}$ NMR $(500 \mathrm{MHz})$. After synthesis of ligand 30 the compound was dried in vacuo to get rid of the residual solvent at $60{ }^{\circ} \mathrm{C}$. The dried compound showed a doubling of the aromatic signals. Additionally, the signals of the tert-butyl-dimethyl silyl protecting group are slightly highfield shifting. After repurification the signals of the tert-butyl-dimethyl silyl protecting group vanished.

Since the complete ligand $\mathbf{3 0}$ was converted to the cyclized ligand $\mathbf{3 2}$, the still available methoxy ligand $\mathbf{1 7}$ got again in the focus of interest, since the methoxy group is also protecting group for phenols. To avoid the undesired cyclization reaction, the deprotection reaction was accomplished with $\mathrm{BBr}_{3}$ and dry solvent at low temperatures scheme IV.2.2.4. 


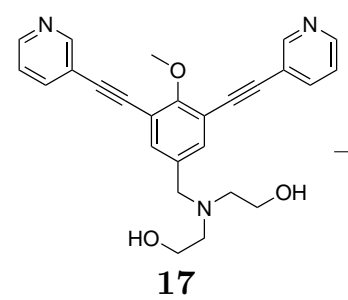

a)

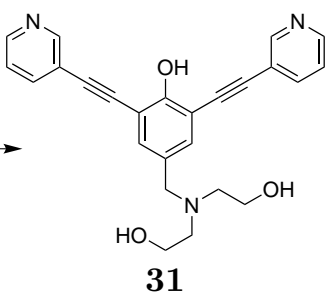

b)

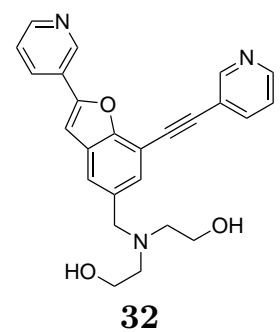

Scheme IV.2.2.4: Deprotection of $\mathbf{1 7}$ and subsequent intramolecualr cyclisation. a) $\left.\mathrm{BBr}_{3}, \mathrm{DCM} ; \mathbf{b}\right) \Delta \mathrm{T}, \mathrm{moisture}$

Indeed, the deprotected ligand could be obtained. In ${ }^{\mathbf{1}} \mathbf{H}-\mathbf{N M R}$ all relevant signals could be observed, including the phenol hydroxy signal (i) (figure IV.2.2.5), since it was measured in DMSO. Attempts to measure the compound 31 in $\mathrm{D}_{2} \mathrm{O}$ failed, the initially clear ligand solution turns within minutes into a suspension. Probably the cyclization reaction immediately occurs, when the unprotected ligand is exposed to water. Also wet organic solvents and time led to the intramolecular cyclization. The NMR sample measured in DMSO, was remeasured after two weeks, stored at room temperature. The resulting NMR spectrum, shows a almost complete conversion to the cyclized ligand $\mathbf{3 2}$, this clearly indicates the instability of ligand $\mathbf{5 6}$ and the tendency to convert to ligand 32 .

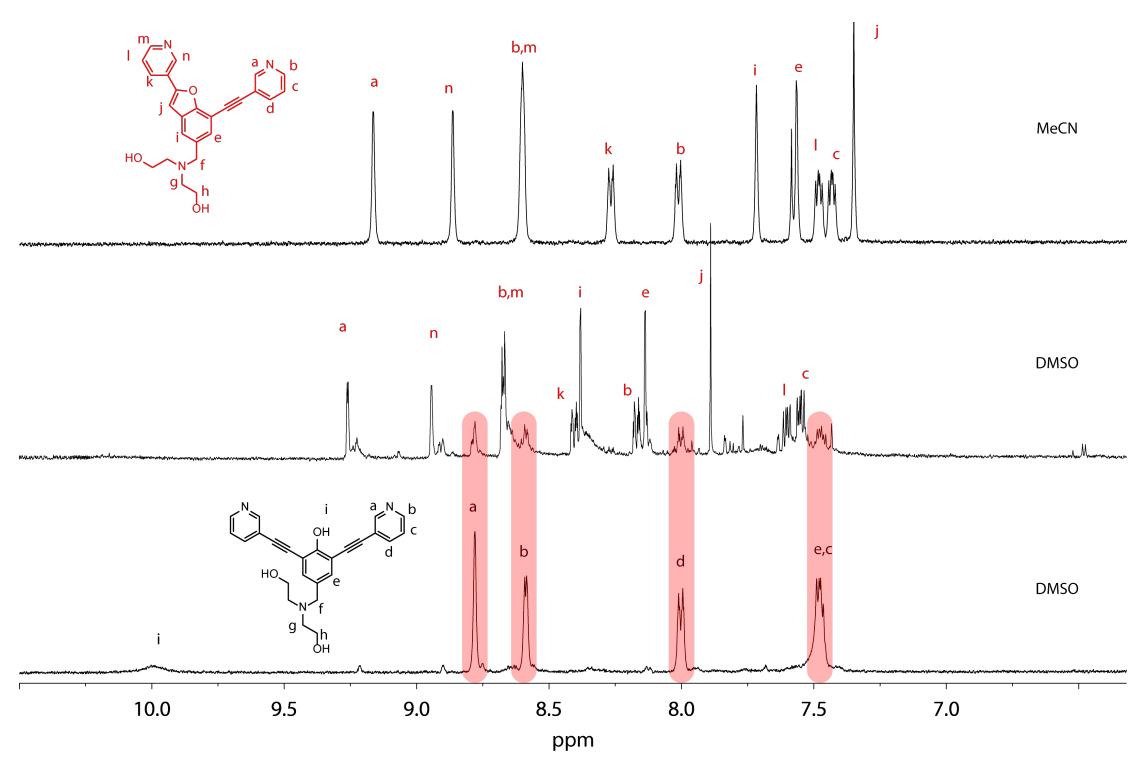

Figure IV.2.2.5: ${ }^{1} \mathrm{H}$ NMR $(500 \mathrm{MHz})$. Solving ligand 31 in organic solvent like DMSO does not lead to a direct intramolecular cyclization. Therefore, the existence of $\mathbf{3 1}$ is be able to verify via NMR But, remeasuring the same sample after two weeks (stored at room temperature) revealed that the intermolcecular cyclisation still happens. All signals of compund $\mathbf{3 2}$ can be found, although signals (i,e and $\mathbf{j}$ ) show a quite big downfield shift in the solvent DMSO compared to the solvent MeCN. Some non-converted compound $\mathbf{3 1}$ can also be found in the spectra, indicated with the red bars.

The attempt to preserve the deprotected ligand $\mathbf{3 1}$ by assembling it into a supramolecular coordination cage also failed. From $\mathrm{NMR}$ measurements only an nonassignable mess of 
signals could be obtained. The standand self-assembly conditions used in this work requires heating of the cage solution at $60 \check{\mathrm{r}} \mathrm{C}$ for at least 1 hour to obtain a completely assembled cage in solution for NMR. Since it was assumed, that heat expedites the cyclization reaction, another sample was prepared. The sample was stored at room temperature and measured every day for five days, giving the same result as described before, a intramolecular cyclisation of the product.

Additonally, the cyclized ligand was investigated for its ability to assemble in a supramolecular construct in the presence of $\left[\mathrm{Pd}(\mathrm{MeCN})_{4}\right]\left(\mathrm{BF}_{4}\right)_{2}$. The experiements were performed in acetonitile, because ligand $\mathbf{3 2}$ was not soluble in water at all anymore. The resulting spectra showed absolutely no shifting of the pyrdine signals, but suprinsingly the signals for the diethanol amine DEA motif, plus the adjacent aromatic signals, exhibit a significant downfield shift, suggesting a favorable interaction between palladium and the the DEA motif. In literature a few systems are reported, where triethanol amine or DEAffunctionalized polymers acting as ligand for palladium(0) species in phosphine-free catalyst for Suzuki or Heck cross-coupling reactions.. 199201 In contrast, the methoxy ligand $\mathbf{1 7}$ did not show any evidence of an interaction between palladium(II) and the DEA motif, thus the geometry of ligand 32 probably became so unfavorable for the coordination to the metal center, due to the intramolecular cyclization preventing the formation of a supramolecular metal-mediated coordination cage. In turn, the coordination to the diethanol amine came probably into favor, since clear shifts of the signals assigned to DEA could be observed, in presence of palladium(II). But to really proof this assumption, further experimental evidence is needed. 


\section{IV.2.3 Sulfobetaine methoxy ligand}

In the last part of this chapter, the introduction of an new motif to supramolecular coordination cages is described, which make them water-soluble but does not change the total charge of the supramolecular architecture. As already mentioned in the introduction of this chapter all water-soluble supramolecular cages have a high total charge, which is additionally well distributed over the whole structure. The lower charged coordination cages contain methyl capped PEG groups or free hydroxyl groups, as shown for the ligand 17.

Another coordination cage with an low total charge is reported by Nitschke et al.. The tetrahedral structure (figure IV.1.0.3a) consists of four iron(II) metal ions, each coordinated by three bis-bidentante nitrogen-based ligands. Each ligand contains two sulfonic acid moieties at the backbone, which are deportonated in solution. Taken together, the total charge is consequently -4. However, since the architecture is very labil towards acidic conditions, 12eq. tetramethylammonium hydroxide had to be added, to neutralize the protons released by the sulfonic acid motifs and further providing non-coordinative countercations for the sulfonates. Additionally, each metal corner has a sulfonate coounter-anion, makes in total 16 counter-ions for the whole structure. As a result a water-soluble supramolecular structure is obtained, with postive charges at metal vertices and negative charges at the edges. 202

Herein we introduce a new general approach for water-soluble coordination cages with zwitter-ionic sulfobetaine moities, in order to create low-charged coordination cages, which have a lower dependency on the needed counter-ions and are more attractive for "Green Chemistry" with respect to bio-compatibility and atom-economics. The sulfobetaines moiety belongs to the amphoteric tensides and is an organic zwitter-ionic group, containing a positively charged tertiary ammonium motif and a negatively charged sulfonate group, hence the formal charge is neutral. They are often used in cosmetics, but also find applications as hydrotropes in chemical industry. Especially the self-stabilizing forms $\mathrm{R}-\mathrm{NMe}_{2}{ }^{+}-\left(\mathrm{CH}_{2}\right) n-\mathrm{SO}_{3}{ }^{-}(\mathrm{n}=3,4)$ retain their zwitter-ionic character over a wide range of $\mathrm{pH}$, which makes them suitable to use under acidic and basic conditions. 203|204 Additionally, they are highly bio-compatible and their water-solubility is largely independent from the surrounding ions. 205|206

Due to above listed points, the sulfobetaine motif could be very interesting and promising for water-soluble supramolecular coordination architectures, especially with regard to drug delivery containers and nanoscalic reaction vessels.

Synthesis of the ligand scheme IV.2.3.5 is based on the already presented ligand $\mathbf{L}^{\text {DEA }}$ (17). The first four steps of synthesis are identical. In contrast to the ligand $\mathbf{L}^{\mathbf{D E A}}$, dimethyl amine was introduced to the benzylic position. Subsequently, the sulfobetaine 
motif was formed as precipitate in a ring opening reaction of the highly carcinogenic 1,3-propane sultone. The collected solid was further converted to the desired ligand by attaching the 3-ethynyl-pyridine arms with help of palladium-catalyzed Sonogashira crosscoupling reaction. Due to the fact that the ligand had to be purified by elaborate reversed phase liquid chromatography methods, it was also tried to couple the pyridine arms firstly and forming the sulfobetaine motif subsequently, but the the 1,3-propane sultone reacted with the pyridine as well, resulting in an even more complex product mixture, with lower yield and more side-products that had to be separated, hence, the first synthetic pathway is preferred.<smiles>O=Cc1cc(I)c(O)c(I)c1</smiles>

12

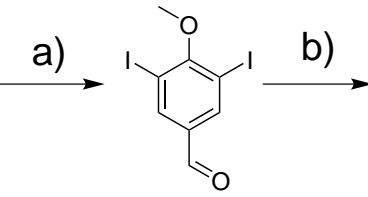

13<smiles>COc1c(I)cc(CO)cc1I</smiles>

14

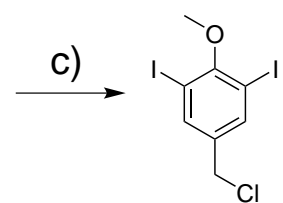

15

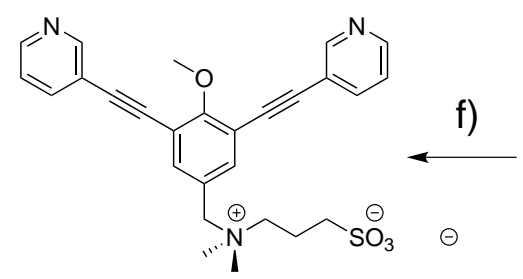

24

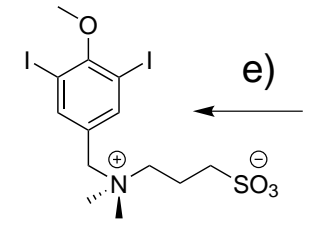

23

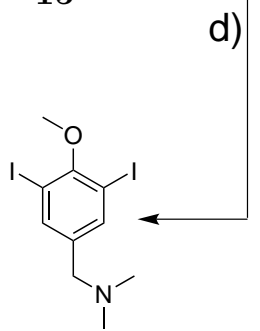

22

Scheme IV.2.3.5: Synthetic path for ligand 24. a) MeI, $\left(\mathrm{CH}_{3}\right)_{2} \mathrm{CO}$; b) $\left.\mathrm{NaBH}_{4}, \mathrm{MeOH} ; \mathbf{c}\right) \mathrm{SOCl}_{2}, \mathrm{DCM}$; d) $\mathrm{HNMe}_{2}, \mathrm{THF} / \mathrm{H}_{2} \mathrm{O}$; e) propane-1,3-sultone, 1,4-dioxane; 3-ethynylpyridine, $\mathrm{Pd}\left(\mathrm{PPh}_{3}\right)_{2} \mathrm{Cl}_{2}, \mathrm{CuI} \mathrm{NEt}_{3}, \mathrm{DMF}$.

After the full characterization of ligand, the system was investigated for its ability to form palladium-mediated coordination cages in water, as it was supposed to do. In fact, in the presence of $\mathrm{Pd}\left(\mathrm{NO}_{3}\right)_{2}$ the NMR spectra show a significant down-field shift of the pyridine related proton signals, indicating a coordination to the palladium(II) cations figure IV.2.3.6. Additionally, as it was already described for the $\left[\mathrm{Pd}_{2} \mathrm{~L}^{\mathrm{DEA}}{ }_{4}\right]$, a down-field shift for the inwards pointing methoxy group was observed as well. In contrary, the sulfobetaine signals $(\mathbf{f}-\mathbf{j})$ stayed virtually unchanged, indicating no interaction between the sulfobetaine and the palladium cations. Due to the fast reduction of $\mathrm{Pd}^{\mathrm{II}}$ to the black $\mathrm{Pd}^{0}$ species in the used aqueous $\mathrm{Pd}\left(\mathrm{NO}_{3}\right)_{2}$ solution, first cage formation experiments were accomplished with a $\mathrm{Pd}\left(\mathrm{NO}_{3}\right)_{2}$ solution in deuterated DMSO (figure IV.2.3.6a), lower cage spectrum), as it was already mentioned in section IV.2.1. However, it was expected, that the here described ligand $(\mathbf{2 4})$ can also form a coordination cage in pure water without any additional organic solvent, since it was also possible for the less water-soluble ligand $\mathbf{L}^{\text {DEA }}$. Especially, considering that the ligand on its own shows already a quite good solubility in water. Indeed, as it can be seen in figure IV.2.3.6a (upper cage spectrum) the cage is formed in presence of $\operatorname{Pd}\left(\mathrm{NO}_{3}\right)_{2}$ and deuterated water as solvent. Moreover, the 
cage formation was further verified by ESI-MS experiments (figure IV.2.3.6b). Peaks for the $+\mathbf{3}$ species with $\mathrm{NO}_{3}{ }^{-}$and $\mathrm{Cl}^{-}$could be found as well as traces of the $+\mathbf{4}$ species. It is worth to mention that also a $\left[\mathrm{PdL}_{2}\right]_{2}{ }^{+}$species was found, superimposed by the +4 species of the cage. The fact that the main peaks contain free ligand, suggesting that the chosen ESI-MS settings for the experiment are not ideal and have to improved, on the other hand, regarding the usage of the standard condition for ESI-MS measurements of cages, indicating a lower relative stability of the cage in comparison to other cages, as e.g. $\left[\mathrm{Pd}_{2}\left(\mathbf{L}^{\mathrm{DEA}}\right)_{4}\right]$ in figure IV.2.1.1b. To make a certain statement about this assumption, more evidences have to be supplied by further experiments.

(a)

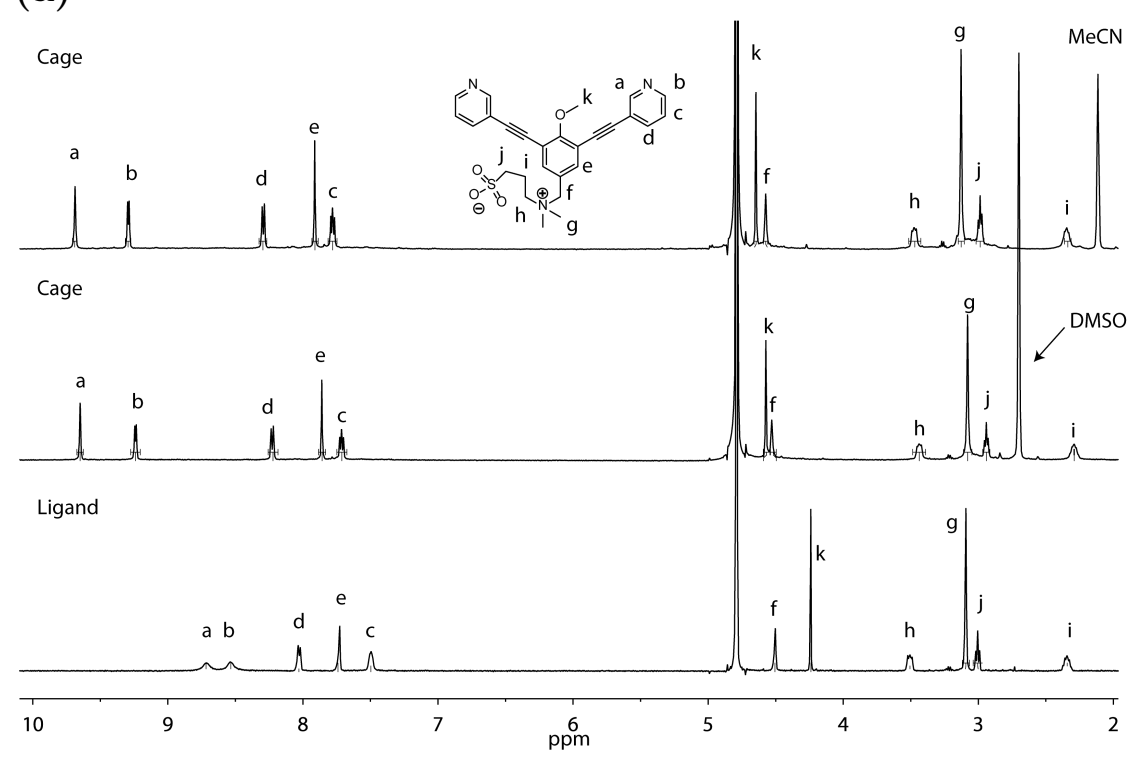

(b)

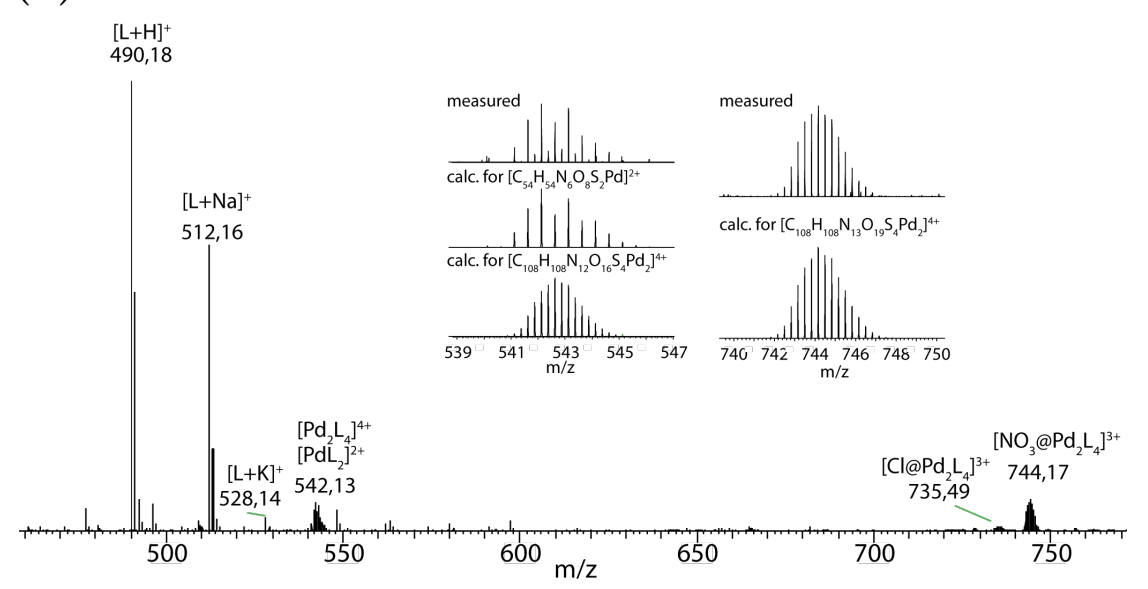

Figure IV.2.3.6: (a) ${ }^{1} \mathrm{H}$ NMR $(500 \mathrm{MHz})$. The ligand 24 is soluble in pure deuterared water $(500 \mu \mathrm{l}, 2.8 \mathrm{mM})$. Addition of $\mathrm{Pd}\left(\mathrm{NO}_{3}\right)_{2} 50 \mu \mathrm{l}$, in deuterated $\mathrm{H}_{2} \mathrm{O}$ (upper cage spectrum) or DMSO (lower cage spectrum) lead to formation of the $\mathrm{Pd}_{2} \mathrm{~L}_{4}$ cage. The typical downfield shift of the signals $\mathbf{a}$ and $\mathbf{b}$ is observable due to coordination of the pyrdine motif to the $\mathrm{Pd}_{2}{ }^{+}$cation. Furthermore the methoxy signal is also shifting downfield, which is a hint that the group is pointing inwards to the cages cavity. (b) The measured ESI-MS shows besides the peaks for the ligand 24, the peak of the $\left[\mathrm{Pd}_{2} \mathrm{~L}_{4}\right]^{4+}$ as well as $\left[\mathrm{Pd}_{2} \mathrm{~L}_{4}\right]^{3+}$ with either chloride or nitrate as counteranion. 
Crystals could be obtained from slow evaporation of THF into an aqueous cage solution. The crystals were picked under low temperature conditions in cryo stream, however, unfortunately they were not suitable for X-ray diffraction analysis. Therefore the here presented structures (figure IV.2.3.7a) were obtained in silico from semi-emperical PM6 geometry optimization calculations. From the model a Pd(II)-Pd(II) distance of $12.06 \AA$ was extracted, as well as an average distance of 7.5-8.6 A between the methoxy group, what is in agreement with the values obtained from the PM6 calculation of structural related $\left[\mathrm{Pd}_{2} \mathrm{~L}^{\mathrm{DEA}}{ }_{4}\right]$ cage (figure IV.2.1.3a).

Having a low-charged palladium-mediated coordination cage in water, it was of interest to investigate the encapsulation of small apolar guest molecules. Especially with regard to take advantage of the hydrophobic effect. For that reason exemplary ${ }^{\mathbf{1}} \mathbf{H}-\mathbf{N M R}$ titration experiments figure VI.1.7.1) were made with cyclohexane, naphtalene and 1,4benzoquinone, which was used by Lusby et al. in a structural related cage of similar size. 207 Unfortunately none of these guest molecules showed any evidence for encapsulation. In all cases, the signals of guest molecules could be seen in NMR, but there was no observable change in chemical shift of the inward pointing hydrogen signals, neither from the aromatic hydrogens nor from those of the methyl groups. This behavior rather suggest that no encapsulation occurs in presence of the guest molecules.

Two possible reasons, why the cage did not take up a guest molecules, are taken from topological deliberations. Either the cavity is to small, or the cavity is more polar than expected and rather prefers charged guest/anions over the uncharged ones. As a reference the crystal structure of the previously reported double cage is used, reported by Löffler et al., since it was able to bind neutral guest molecules. 20 From the PM6 model of the cage, the obtained distance between two opposite ligands is in average $0.5 \AA$ smaller (ca. 6.8-7.7 $\AA$ ) compared to the inner cavity of the double cage (ca. 7.7-8.6 $\AA$ ), where the binding of the neutral guest molecules occurs. Since the methyl groups are rather directed to the sides of the cage, the oxygen of the inward pointing methoxy groups, consumes most of the cavities' space, so that small molecules, as the ones used for titration, should have enough space. Also the distance between the palladium cations ( $\mathrm{Pd}-\mathrm{Pd}->$ $11.5 \AA$ ) is comparable to the distance found in the interpenetrated double cage (Pd-Pd -> $10.5-11.0 \AA$ ). In contrary, the charge of palladium cations is less shielded in the small cage, compared to the interpenetrated double cage, where the chloride anions show a close interaction with the palladium metal centers. Therefore the encapsulation of the counter-anion could be preferred towards the neutral guest molecule. Another difference to be mentioned are the open sites of the cage, which allows an easy uptake and release of the guest molecules, compared to the densely packed inner cavity of the interpenetrated double cage. However, to prove this theory, more titration experiments have to be made, especially with small anionic guest, in order to figure out whether the cage is just not 
able to encapsulate neutral guests or whether the cage is not able to encapsulate guest molecules at all.

(a)
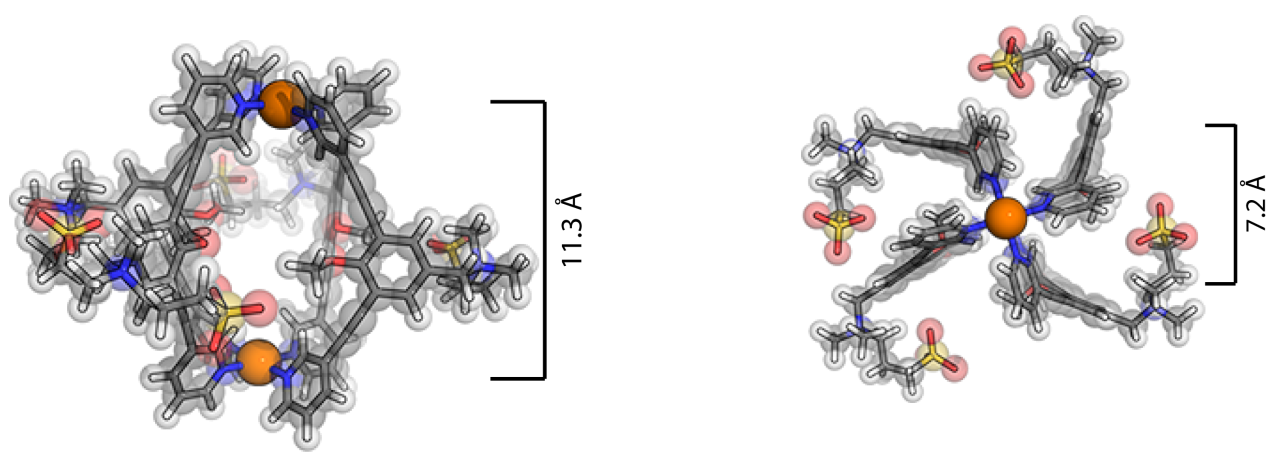

(b)
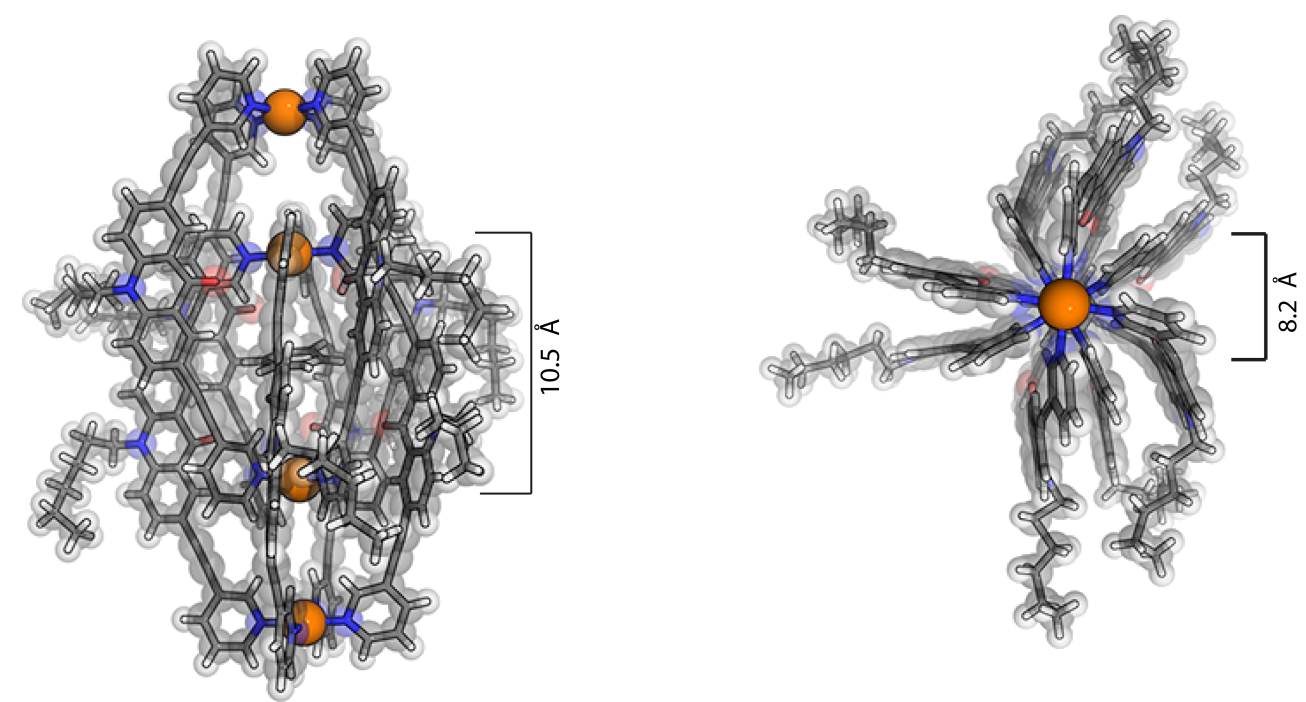

Figure IV.2.3.7: Comparison PM6 model of sulfobetaine cage with acridone interpenetrad double cage crystal structure $\left[\mathrm{Cl}_{2}+\mathrm{C}_{6} \mathrm{H}_{6} @ \mathrm{Pd}_{2} \mathrm{~L}_{4}\right]_{8}{ }^{+} .20$ All conterions and guest molecules were omitted in order to have a better view on the cavities. The chloride containing acridone interpenetrated double cage was chosen on purpose, since it was the first cage in the Clever group capable of encapsulating neutral guest molecules. Compared to the sulfobetaine cage, its central cavity is slightly lower with $10.5 \AA$ (11.3 $\AA$ for the sulfobetaine cage), but also a little broader with $8.2 \AA$ ( $7.2 \AA$ for the sulfobetaine cage). Furthermore, the sulfobetaine cage has rather open sites, which are occupied by the ligands in the acridone double cage. This situation makes it harder for the guest to enter the cavity, but also the leaving and there are more possibilites for interactions between host and guest, giving a higher sum of potential stabilizing forces. 


\section{chaperer IV.3}

\section{Conclusion}

In this chapter two new motifs were presented to make low charged supramolecular $\left[\mathrm{Pd}_{2} \mathrm{~L}_{4}\right]$ cages soluble in pure water. Although the diethanol amine motif was actually chosen as linker for the incorporation of ligand $\mathbf{L}^{\text {DEA }}(\mathbf{1 7})$ into oligonucleotides, it gives the small aromatic backbone the extra hydrophilicity to be solved in pure water at elevated temperatures. In presence of palladium(II) salts the ligand $\mathbf{L}^{\mathbf{D E A}}$ can form a supramolecular coordination cage, which is soluble in water as well as in polar organic solvents, such as MeCN and DMSO. This result was important to continue the oligonucleotide project (section III.2.3.3), since it was not clear if the first generation olignucleotide ligand section III.2.2 can not form at all in aqueous medium or just in the oligonucleotide scaffold. Furthermore, the here presented results is the subsequent development of Yoshizawa's PEG chain approach and Crowley's hydroxyl group approach to make supramolecular $\left[\mathrm{Pd}_{2} \mathrm{~L}_{4}\right]$ water-soluble and can be seen as a proof of principle, that a branched, dendron-like side chains ending in free hydroxyl groups is a viable motif for this purpose. At the same time, the cage has not lost its ability to be solved in polar organic solvents, resulting in interesting supramolecular containers for applications in phase transfer reactions, as it has been shown by Nitschke et al.208|209]

The synthesis of an analogous cage with an endohedral hydroxyl group instead of an inward pointing methoxy group failed, due to formation of an unexpected side product. In literature similar systems have been synthesized succesfully without mentioning any synthetic problems. $210 \mid 211$ However, the side product could be identified as intramolecular cyclization product, resulting in the asymmetric benzofuran containing ligand (32). With an alternative synthesis route, the desired ligand $\mathbf{3 1}$ could be obtained, but it converted in presence of water directly to the intramolecular cyclized ligand, which made this system impractical for the intended application. It is worth to mention, that the assembly of the asymmetric ligands to a palladium-mediated coordination cage failed, probably due to the reduction of the angle between the coordination sites. Thus, the coordination to the metal center became less favored. In turn, the palladium cations seem to be coordinated by the 
backbone-attached diethanol amine (DEA moiety instead.

For the last system, the DEA motif was replaced by an zwitter-ionic sulfobetaine. The zwitter-ionic structure has a formal charge of zero, therefore it does not change the total charge in a supramolecular coordination structure, when introduced, compared to supramolecular cage consisting of neutral ligands. Furthermore it is self-stabilizing, means it does not need any additional counter ions, which is not only an advantage in terms of atom-economy, but also ensures that its water-solubility is not dependent on the provided counter ions. In addition, the here used sulfobetaine motif is known to keep its zwitter-ionic character over a large $\mathrm{pH}$ range, 203|204 making the system suitable for reactions under basic or acidic conditions. Additionally, the sulfonbetaine structure has shown an excellent biocompatibility, hence it was used for drug carrier systems in recent publications. 212[213] The here presented approach bears a huge potential, not only giving low-charged water-soluble coordination cages, but also gives a chance to bring neutrally charged supramolecular architectures in to aqueous medium, concerning its suitability for applications in Green Chemistry. 



\section{Endohedrally Functionalized \\ Metal-Mediated Coordination Cages}

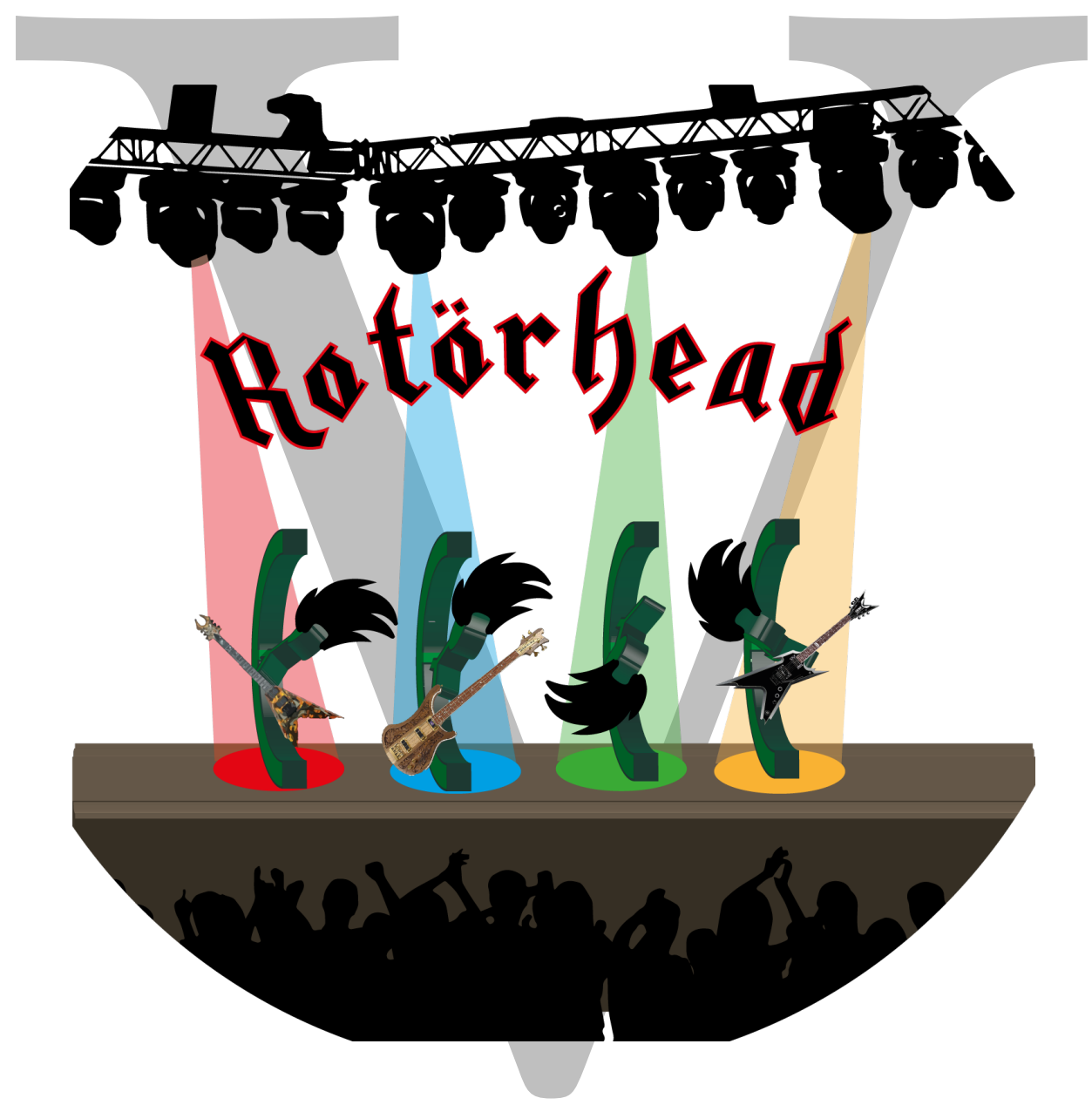

Related Publications:

M. Krick, J. Holstein, C. Würtele, G. H. Clever, Chem. Commun. 2016, 52, 10411-10414.

M. Krick, J. J. Holstein, A. Wuttke, R. A. Mata, G. H. Clever, Eur. J. Org. Chem. 2017, 2017,

$5141-5146$. 


\section{Chapter}

\section{Push-Pull Rotor Systems based on Acridinylidene Cyanoacetic Esters}

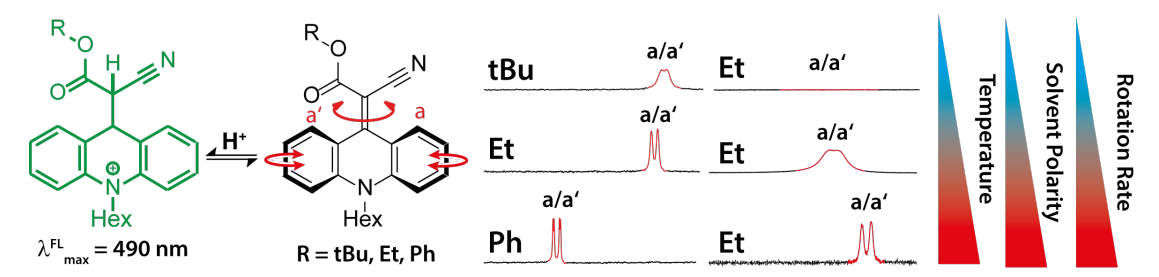

\section{Contents}

V.1.1 Introduction . . . . . . . . . . . . . . . . . . . . . . . 109

V.1.2 Results and discussion . . . . . . . . . . . . . . . . . . 111

V.1.2.1 Synthesis . . . . . . . . . . . . . . . . . . . . . . . 111

V.1.2.2 Influence of Temperature on Rotation $\ldots \ldots$. . . . . . 114

V.1.2.3 Influence of Solvent Polarity on Rotation . . . . . . . . . . 118

V.1.2.4 Determination of activation enthalpy and entropy. . . . . . . 121

V.1.2.5 Cyclic voltammetry . . . . . . . . . . . . . . . . . . . 127

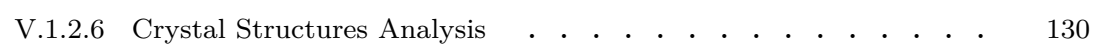

V.1.2.7 Chemoinformatic Studies ． . . . . . . . . . . . . . 133

V.1.3 Protonation-Induced Fluorescence ～. . . . . . . . . . 136

V.1.3.1 Position of Protonation . . . . . . . . . . . . . . . . . 137

V.1.3.2 ${ }^{1} \mathrm{H}$ NMR titration experiments with|TFA $\ldots \ldots . . . . \quad 140$

V.1.3.3 Determination of associations constants from|UV-vis $\mid$ titration experiments with TFA . . . . . . . . . . . . . . . . . . . . 143

V.1.3.4 Fluorescence titration experiments with TFA $\ldots . . . .145$

$\mathbf{V . 1 . 4}$ Conclusion $\ldots \ldots \ldots \ldots$ 


\section{V.1.1 Introduction}

The study of dynamics in all size scales of molecular systems is an important area of research, especially in respect of understanding and controlling the dynamics in such important natural occuring systems, like enzymes. In order to delve into the operating principle and function of those highly specialized and complex systems, smaller systems, ranging from simple alkanes, 214|215] over more complex organic 216|217] or organometallic compounds, 218|219] catalysts, 220|221] to biopolymers such as DNA, 222] frequently delved. The integration of such dynamic systems in higher order supramolecular scaffolds can help to create biomimetics systems, giving hints about nature's chemistry or even develop artificial analogues. A prominent example for the latter one is the rotaxane based supramolecular peptide synthesis machine reported by Leigh et al.223|224

In the context of dynamic systems in artificial supramolecular constructs, 225 functionalities such as gears, 226 wheels, 227 and rotaxanes 228,230 have been used as components in molecular-scale machines. 231 However, most of these dynamic systems are lacking of directional control. 232 Feringa et al. hurdled this problem by designing a system, consisting of photo-interconvertible helical isomers, which are thermally stable. 233|234 In 2010, Feringa et al. reported an ultra-fast rotating motor, operating in the manner as his previous systems, but based on a N-Boc protected acridinylidene stator, with an asymmetric substituted bulky fivemembered ring as rotor. 235.

Interestingly, Löffler et al. were also able to synthesize similar acrdinylidene system with a sterically demanding adamantyl group instead of the five-membered ring. 24 Moreover, since the the system was based on the the acridone ligand from previous works, 20 four of those molecules were able to form an endohedrally functionalized supramolecular coordination cage. Compared to Feringa's acridinyldine compound, the adamantyl system didn't show any rotation but a dynamic flipping motion, by what the ligand is mirror-inverted. This motion could be observed for the free ligand, for the assembled cage and even with an encapsulated guest. It is noteworthy to mention, that the flipping rate is decreasing from free ligand to cage to the cage with encapsulated guest.

Motivated by this new way to modify the rather inert keton postion in our banana-shaped ligands, we were interested in introducing functional groups to the inward pointing position, in order to create functional units for specific molecular recognition 236|237 or catalyzed reactions within the cage cavities. 238

For the acridone-based ligand we were able to introduce malonitrile and 2-cyano acetate ethyl ester at the keto-position while maintaining the double bond. Altough being aware of having a potential flipping motion, the found predominant rotational motion within the ligand was not expected, which is only observable in the asymmetric substituted derivatives. First evidence of an intramolecular dynamic was seen in the comparison of the NMR signals from ligands $\mathbf{L}^{\mathrm{CN}} 4 \mathbf{4 0}$ and $\mathbf{L}^{\mathbf{E t}} \mathbf{4 2}$ in figure V.1.1.1. 


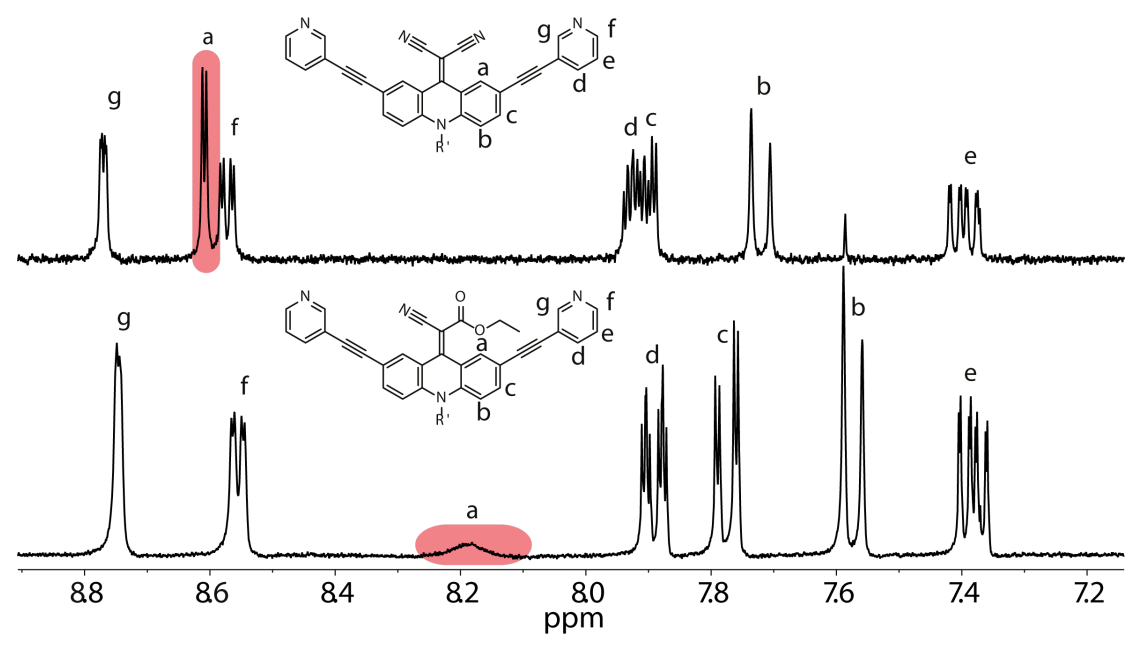

Figure V.1.1.1: Comparison of ${ }^{\mathbf{1}} \mathbf{H}$ NMR spectra $\left(400 \mathrm{MHz}, 298 \mathrm{~K}, \mathrm{~d}^{3}-\mathrm{MeCN}\right)$ for ligands $\mathbf{L}^{\mathbf{C N}}$ and $\mathbf{L}^{\mathbf{E t}}$. The signals of a and/or a', highlighted in red, belong to the protons with the closest proximity to the malononitrile or 2-cyano-acetate ester substitutent. The 2-cyano-acetate ester derivative shows a less downfield shifted broad signal in comparison to the the symmetric ligand.

Contrary to the expectations the asymmetric ligand $\mathbf{L}^{\mathrm{Et}}$ showed only one set of signals (figure V.1.1.1), what was at the first glance not a problem, but the absence of an proton signal was. The signal for the protons a could not be found. However, changing the solvent from chloroform to acetonitrile revealed the potion of a very broadened peak, we assumed to be a. It is noteworthy that similar derivatives of substituted acridinylidenes were already synthesized in the mid 1950s by condensation of malononitrile or 2-cyanoacete esters with acridone, by Dimroth et al.,239] but this report is lacking of any NMR spectroscopic data. But interestingly, they already proposed the existence of an intramolecular push-pull system and a zwitterionic structure, what gave a first hint towards the cause of the rotation. More recent publications examined only symmetrically substituted dicyano derivatives, which do not reveal this dynamic motion in NMR spectroscopic investigations. 240|241]

During our studies, it became apparent that we could not investigate the rotational motion by dynamic NMR spectroscopy (DNMR) in the ligand structure, due to spin convolution in the ${ }^{\mathbf{1}} \mathbf{H} \mathbf{N M R}$ experiments between the a signals and some signals belonging to the pyridine arms. Therefore, we decided to investigate the dynamic motion in a simplified structure without the attached the pyridyl arms. 


\section{V.1.2 Results and discussion}

\section{V.1.2.1 Synthesis}

In the synthesis of the endohedral substituted admantyl ligand,24 acridone and adamanton were merged with help of the McMurry reaction, a reductive coupling reaction. 242 This reaction is taking place in presence of $\mathrm{TiCl}_{3}$ and a reducing agent. $\mathrm{TiCl}_{3}$ plays the key figure in this reaction, since, due to its oxophilic character, it firstly activates the rather unreactive keto group, secondly brings the reactants close together by forming a titanium(III)-carbonyl complex, before the reduction occurs and lastly removes the oxygen from the system by forming $\mathrm{TiO}_{2}$. As a result the double bond between the two reactants is formed. A similar reaction has been used for a controllable molecular motor of Feringa et al.243. Starting from the commercially available acrid-9(10H)-one, all derivatives $(\mathbf{3 4}, \mathbf{3 5}$ and $\mathbf{3 7})$ were synthesized in a two step synthesis. To increase solubility, a hexyl chain was introduced to the acridone nitrogen via $\mathrm{S}_{\mathrm{N}} 2$ substitution reaction using 1-bromhexane and $\mathrm{K}_{2} \mathrm{CO}_{3}$. Subsequent, the cyano acetate esters were coupled to the $N$-hexyl acrid-9(10H)-one with help of a variation of the Knoevenagel condensation reaction. 244 The Lehnert modification 245|246. uses besides the base, titanium(IV) species to couple methylene compounds with aromatic ketone, where the titanium species is also responsible for the activation, followed by the elimination of the carbonyl oxygen.<smiles>O=c1c2ccccc2[nH]c2ccccc12</smiles>
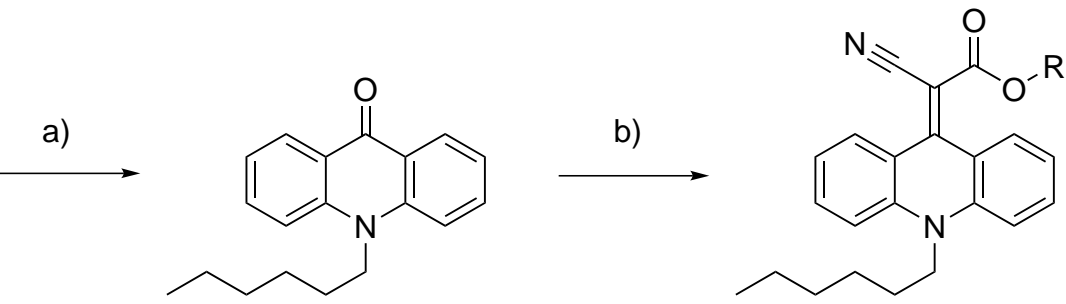

34,35 and 37

Scheme V.1.2.6: Syntheses of aridiynlidne cyanoacetic esters $\mathbf{B}^{t \mathbf{B u}} \sqrt[34]{\mathbf{3 4}}, \mathbf{B}^{\mathbf{E t}} \mathbf{3 5}$ and $\mathbf{B}^{\mathbf{P h}} \mathbf{3 7}$ a) $\mathrm{K}_{2} \mathrm{CO}_{3}$, Hexylbromide, DMF. b) Cyano acetate derivatives ( $\mathrm{R}=t \mathrm{Bu}, \mathrm{Et}, \mathrm{Ph}), \mathrm{NEt}_{3}, \mathrm{TiCl}_{4}, \mathrm{CH}_{2} \mathrm{Cl}_{2}$

After full characterization of all derivatives at room temperature (section VI.3.1), there was still no evidence of a second set of proton signals, although the asymmetric substitution should cause this. Since, we still expected a dynamic motion within the molecule, a deep temperature ${ }^{\mathbf{1}} \mathbf{H}$ NMR at $-80{ }^{\circ} \mathrm{C}$ of $\mathbf{B}^{\mathbf{E t}}(\mathbf{3 5})$ in $d^{8}$-THF was measured. And indeed a second set of signals appeared and become distinguishable. Signals a/a' as well as $\mathbf{b} / \mathbf{b}$ ' show a quite big difference in chemical shift, while $\mathbf{c} / \mathbf{c}^{\prime}$ and $\mathbf{d} / \mathbf{d}$ ' show just a slight difference ffigure V.1.2.6). Which is not surprising, concerning the different proximities of the protons toward the cyano acetate substituent. Anyway, from ${ }^{\mathbf{1}} \mathbf{H} \mathbf{~ N M R}$ it was not 
possible to assign the signal a', neither to the proton closest to the cyano nor to the ester substructure. And same for the signal a. Regarding the proximity of the substituent to the tricyclic backbone, we expected to find through-space contacts between the ethyl ester group and the proton signal a', but not to proton a. First of all an ${ }^{1} \mathrm{H}-{ }^{1} \mathrm{H}-\mathrm{COSY}$ experiment was measured at $-80{ }^{\circ} \mathrm{C}$ (creffig:bet-cosy), where all aromatic signals could be categorized to the left $(\mathbf{a}-\mathbf{d})$ or the right $\left(\mathbf{a}^{\prime}-\mathbf{d}\right.$ ') substructure.

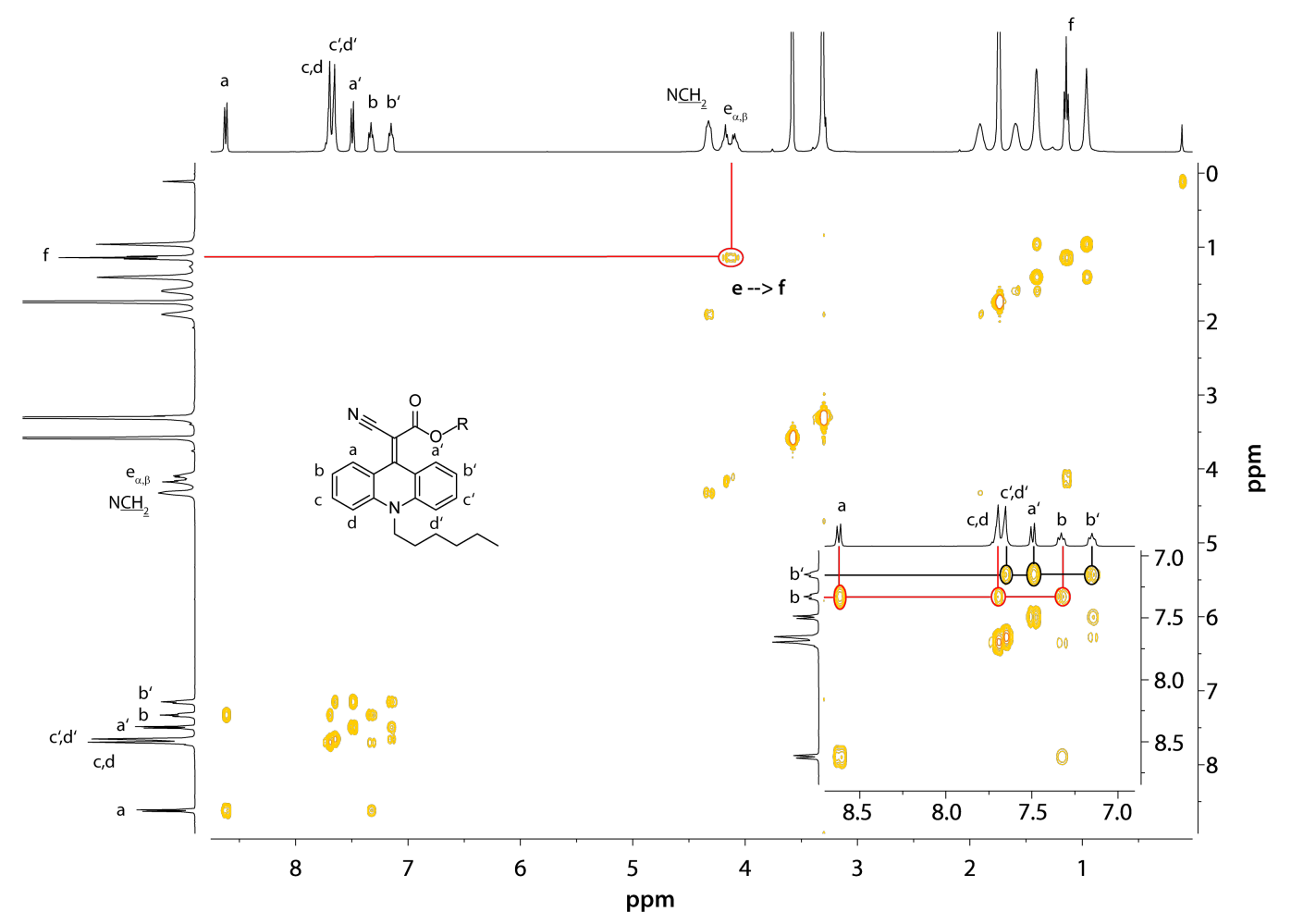

Figure V.1.2.2: ${ }^{1} \mathrm{H}-{ }^{1} \mathrm{H}-\mathrm{COSY}$ of 35. $2.8 \mathrm{mM}$ in $\mathrm{d}^{8}-\mathrm{THF}$ at $-80{ }^{\circ} \mathrm{C}$. The enlarged NMR segment shows the coupling between the aromatic proton signals a-d and a'-d'.

The ${ }^{1} \mathrm{H}-{ }^{1} \mathrm{H}-\mathrm{NOESY}$ experiment at $-80{ }^{\circ} \mathrm{C}$ allowed us to assign the signals a and $\mathbf{a}$ ' to the right proton position in $\mathbf{B}^{\mathbf{E t}}(\mathbf{3 5})$, as we found through-space contacts between the ethyl ester group (e and $\mathbf{f}$ ) and the proton signal $\mathbf{a}$ ', but not to proton a figure V.1.2.3. Furthermore, smaller cross peaks were observable between ethyl ester signal $\mathbf{e}$ and $\mathbf{b}$ ' , as well as between $\mathbf{f}^{\prime}$ and b' and even c'. Therefore, it quite clear that the less shifted signals (a'-d') belonging to the site of the aromatic compound closest to the ester group, while (a-d) is on site of the cyano substituent. 


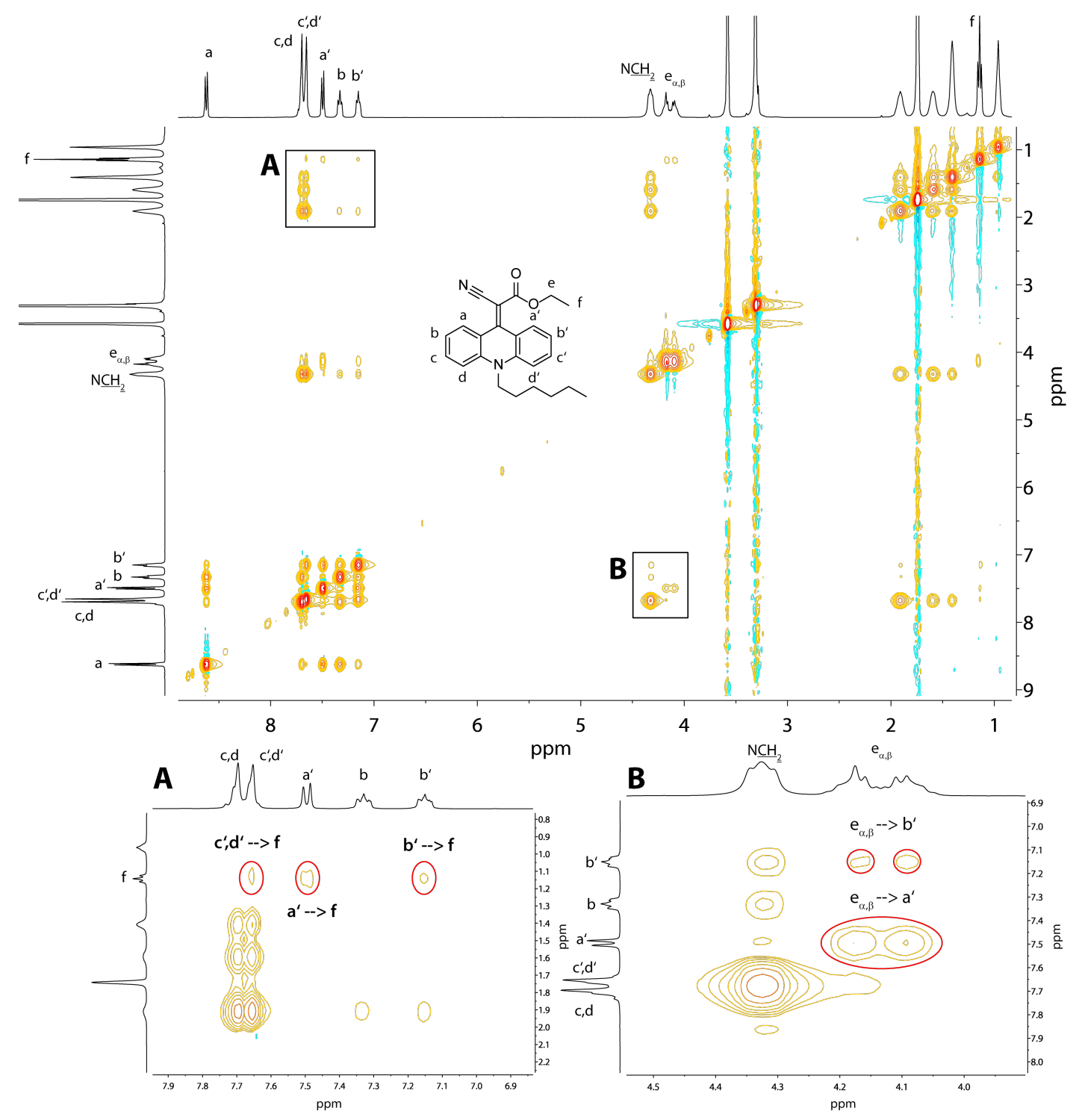

Figure V.1.2.3: ${ }^{1} \mathrm{H}-{ }^{1} \mathrm{H}-\mathrm{NOESY}$ of 35. $2.8 \mathrm{mM}$ in $d^{8}$-THF at $-80{ }^{\circ} \mathrm{C}$. In the enlarged section $\mathrm{A}$ the contacts between the $\mathrm{CH}_{3}$ group of the ethyl ester group (signal f) and the aromatic signals $\mathbf{a}$ ', b' and c' are shown. The enlarged section B shows the contact of the aromatic proton signals a' and 'b' with the methylene group (signal e) of the ethyl substituent.

Although it was clear at this point, that the dynamic within the molecule seems to depend on the temperature and that the motion can drastically decelerated by cooling down the whole system, the question still remained what kind of dyanmic motion is responsible for this effect. In similar systems $24 \mid 247$ a flipping motion of the aromatic scaffold was reported .In our case a flipping would not explain the observed chemical exchange between a' and $\mathbf{a}$ in the NMR Regarding the reported twist of the dicyano substituent in a dianionic form of an acirdinylidene derivate by Sutherland et al.248 and the proposed zwitterionic structure of Dimroth, 239 a rotation of the cyano acetate ester is therefore the most likely explanation. The zwitter-ionic state occurs due to the intramolecular push-pull system between the rather electron-rich nitrogen and the electron-withdrawing substituent. 
While having the charges separated, the former double bond that connect the stator and rotor, getting temporarily a single bond character, by what the substituent can rotate freely.

\section{V.1.2.2 Influence of Temperature on Rotation}

To get a better idea what parameters can have influence on the rotation motions, two further derivatives were synthesized. The tert-butyl derivative (34), should have similar influence on the system as the ethyl derivative, but is more bulky. In contrast, the phenyl derivative should show a different effect on the electronics compared to the aliphatic esters residues, while having a similar demand as the tert-butyl group. In figure V.1.2.4a all the aromatic region in ${ }^{\mathbf{1}} \mathbf{H} \mathbf{~ N M R}$ at room temperature for all three derivatives are shown.

(a)

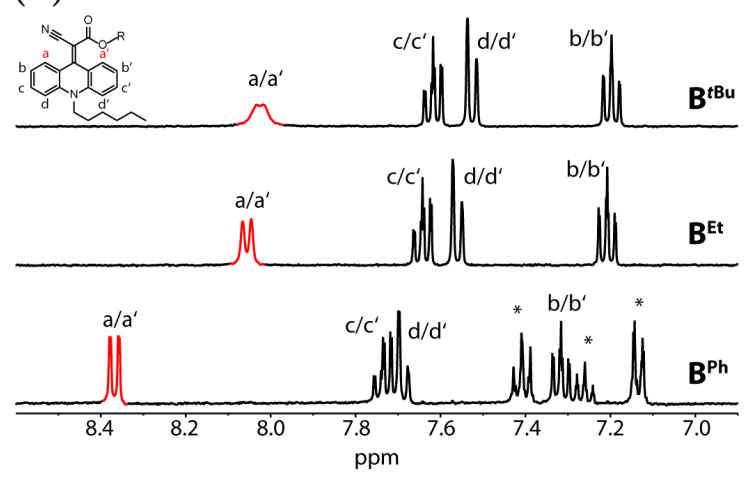

(b)

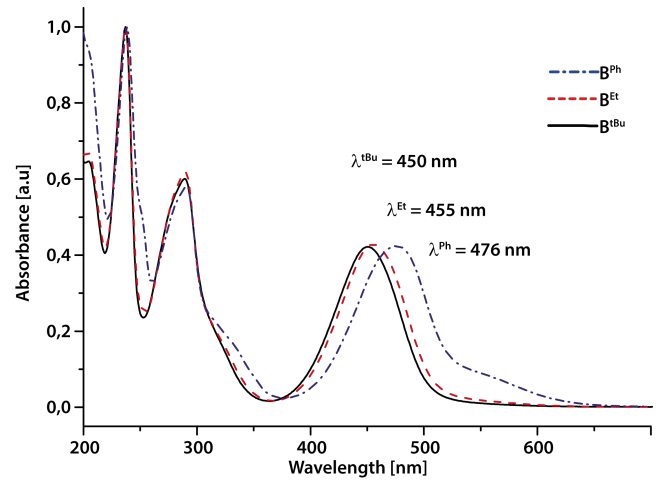

Figure V.1.2.4: Comparison of 34 35 and 37 All ${ }^{\mathbf{1}} \mathbf{H}-\mathbf{N M R}$ spectra were recorded in $\mathrm{d}^{3}-\mathrm{MeCN}$ at $298 \mathrm{~K}$. Aromatic signals of phenyl group are asterisked. Reprinted with permission from reference. 249] Copyright $\odot 2017$ Wiley-VCH

Considering again the spectra in figure V.1.1.1, it becomes quite obvious that the slower rotation in $\mathbf{L}^{\mathbf{E t}}$ is indicated by the broadening of signal $\mathbf{a} / \mathbf{a}$ ', whereas the the ester's less electron-withdrawing characteristic causes a fewer chemical shift of the signal a/a' Keeping this in mind, the least shifted but most broadened signal $\mathbf{a} / \mathbf{a}$ ' in the spectrum of $\mathbf{B}^{t \mathbf{B u}}(\mathbf{3 4})$, shows qualitatively as decrease in rotation, as well as a slight more electronpushing effect of the tert-butyl group, as compared to $\mathbf{B}^{\mathbf{E t}}(\mathbf{3 5})$. In contrary, $\mathbf{B}^{\mathbf{P h}}(\mathbf{3 7})$ has a higher chemical shift and sharper signal a/a' under the same measuring conditions, thus, it exhibits probably a higher spinning rate and has a higher electron-withdrawing character, as compared to the ethyl ester derivative. Therefore, it is obvious, that the size seems to have rather a minor influence on the rotation. The influence of the ester substituent on the intramolecular electronics seems to be the predominant effect, that changes the rate of rotation. Next, the influence on temperature on the three derivatives was investigated. For 
that reason VT NMR measurement were made from $-90^{\circ} \mathrm{C}$ to $60{ }^{\circ} \mathrm{C}$ in $10{ }^{\circ} \mathrm{C}$ steps. All VT NMR measurements were made in $d^{8}$-THF, since it showed the best solubility, while giving the best range of measurable temperatures for the system. Figure V.1.2.5 shows the VT NMR measurement of $\mathbf{B}^{t \mathbf{B u}}(\mathbf{3 4})$. First thing to be noticed is that a splitting into the signals a and a' already occurs a room temperature. The broadened signal at with chemical shift of $8.55 \mathrm{ppm}$ belongs to the proton a and $7.55 \mathrm{ppm}$ to the proton $\mathbf{a}$ '. In comparison, the same compound showed in $d^{3}-\mathrm{MeCN}$ a single broadened signal at ca. $8.0 \mathrm{ppm}$. Hence, the solvent polarity seems to be another factor to influence the rotation in the system, which is described in section V.1.2.3.

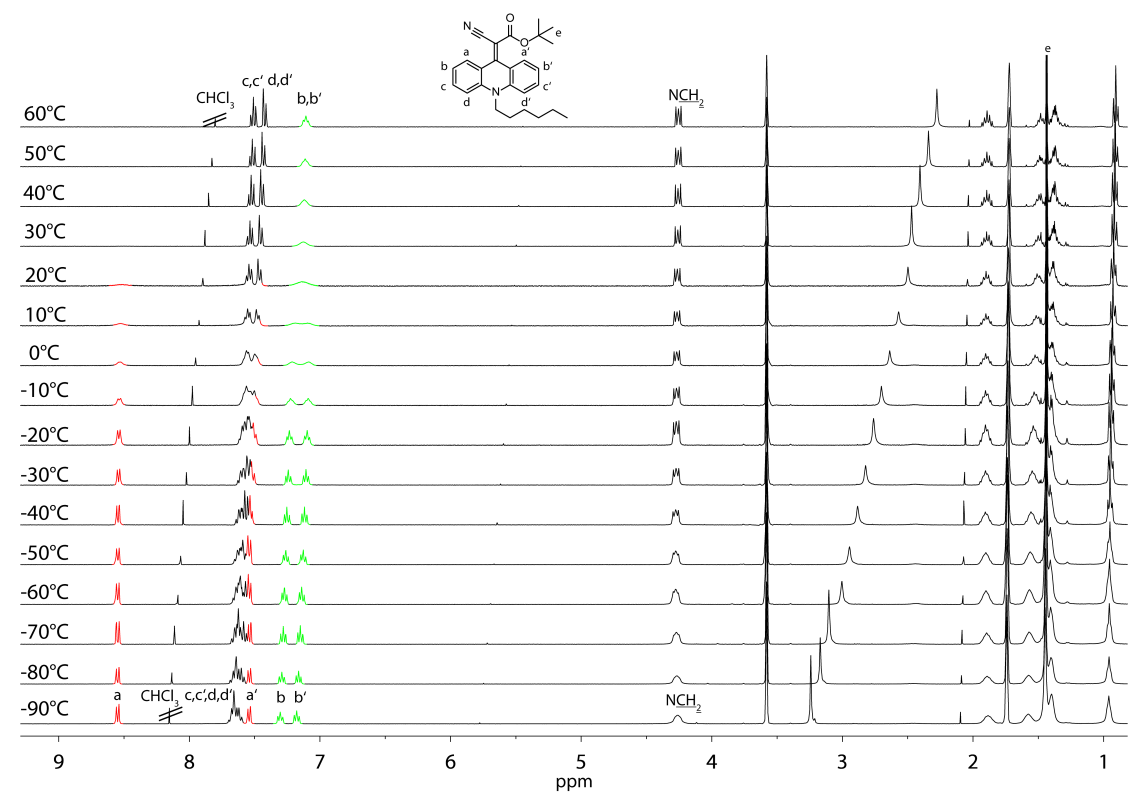

Figure V.1.2.5: VT- ${ }^{1} \mathbf{H}$ NMR (400 MHz) form $-90{ }^{\circ} \mathrm{C}$ to $60{ }^{\circ} \mathrm{C}$ of $\mathbf{B}^{t \mathbf{B u}} 2.8 \mathrm{mM}$ in $\mathrm{d}^{8}$-THF. Signals of protons $\mathbf{a} / \mathbf{a}^{\prime}$ are highlighted in red. Signals of protons $\mathbf{b} / \mathbf{b}$ ' are highlighted in green. The coalescence cannot be clearly determined, but lies approximatly between $50^{\circ} \mathrm{C}$ and $60^{\circ} \mathrm{C}$

As expected signals (a/a') are separated at very low temperatures. The difference in chemical shift is ca. $1.00 \mathrm{ppm}(400 \mathrm{~Hz})$. Likewise, the nearest neighbored protons $(\mathbf{b} / \mathbf{b}$ ') show as well a significant difference in chemical shift, ca. $0.13 \mathrm{ppm}(50 \mathrm{~Hz})$. Since we could not observe the merged signal above coalescence, the $\mathrm{T}_{\mathrm{C}}$ is guessed and estimated to be between $50{ }^{\circ} \mathrm{C}$ and $60{ }^{\circ} \mathrm{C}$. Compared to $\mathbf{B}^{t \mathbf{B u}}(\mathbf{3 4}), \mathbf{B}^{\mathrm{Et}}(\mathbf{3 5})$ has a bigger difference in chemical shift between the signals a and a' (ca. $1.13 \mathrm{ppm}$ or $450 \mathrm{~Hz}$ ), as well as for the signals of $\mathbf{b}$ and $\mathbf{b}^{\prime}(0.18 \mathrm{ppm}$ or $73 \mathrm{~Hz})$. For this case the coalescence temperature is approx. $20{ }^{\circ} \mathrm{C}$. Furthermore, it is worth to mention that we observed a splitting of the ester's methylene signal $\mathbf{e}$ in $\mathbf{B}^{\mathbf{E t}}(\mathbf{3 5})$ at very low temperatures figure V.1.2.6. highlightet in dark green), which gave a first hint to a further dynamic process within the system, since the acutal rotation is already frozen-out. The signal splitting probably occurs due to inequivalence of the now diastereotopic protons $\mathbf{e}_{\boldsymbol{\alpha}}$ and $\mathbf{e}_{\beta}$, caused by the molecule's deviation from a flat structure and emergence of axial chirality in the frozen-out 
conformation (figure V.1.2.20, Rapid flipping between both degenerate conformations occurs at ambient temperatures but slows down at the lowest temperatures reached in our NMR investigation. Estimation of the flipping frequency $\left(\mathrm{k}_{\mathrm{flip}}=\frac{\pi \Delta \nu_{0}}{\sqrt{2}}\right) \underline{250}$ gave a value of about $100 \mathrm{~Hz}$ at a $\mathrm{T}_{\mathrm{C}}$ of $-75^{\circ} \mathrm{C}$ with an maximal peak separation $\left(\Delta v_{0}\right)$ approximated to $45 \mathrm{~Hz}$.

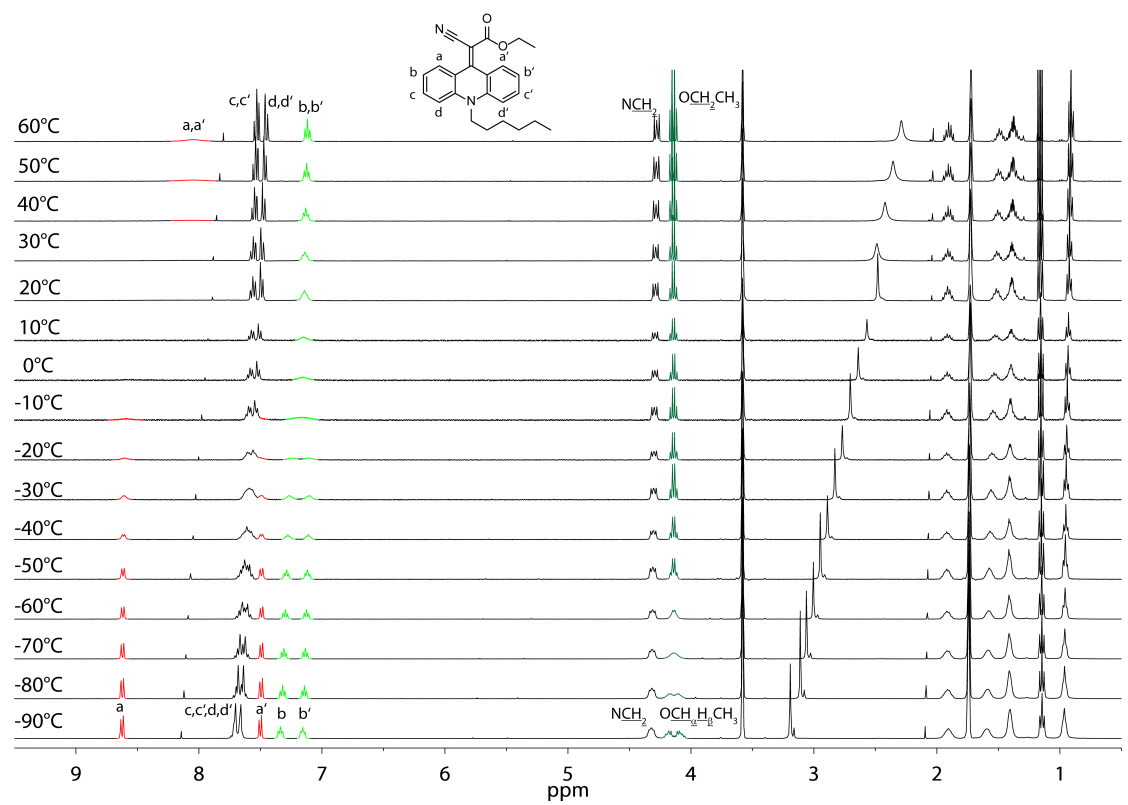

Figure V.1.2.6: VT-1 ${ }^{1} \mathbf{H}$ NMR (400 MHz) form $-90{ }^{\circ} \mathrm{C}$ to $60{ }^{\circ} \mathrm{C}$ of $\mathbf{B}^{\mathbf{E t}} 2.8 \mathrm{mM}$ in d $\mathrm{d}^{8}$ THF. Signals of protons $\mathbf{a} / \mathbf{a}^{\prime}$ are highlighted in red. Signals of protons $\mathbf{b} / \mathbf{b}$ ' are highlighted in green. Furthemore, the signal of the methylene group of the ethyl ester residue $\left(\mathrm{CH}_{2} \mathrm{CH}_{3}\right)$ is additionally highlighted in dark green. Coalescence temperature is approx. $20{ }^{\circ} \mathrm{C}$.

This flipping motion was already described for a similar systems that contains a bent acridinylidene motif, 24 mentioned in chapter V.1, carrying sterically demanding substituents at this position. As it already become apparent in figure V.1.2.4a $\mathbf{B}^{\mathbf{P h}}(\mathbf{3 7})$ has a much faster rotation rate compared to the other both derivatives at the same temperature. In this case it was not possible to frozen-out the rotation, since even at $-90{ }^{\circ} \mathrm{C}$ the signals $\mathbf{a} / \mathbf{a}^{\prime}$ are already separated but still quite broad. And for the signals $\mathbf{b} / \mathbf{b}^{\prime}$ a splitting was not even observable. 


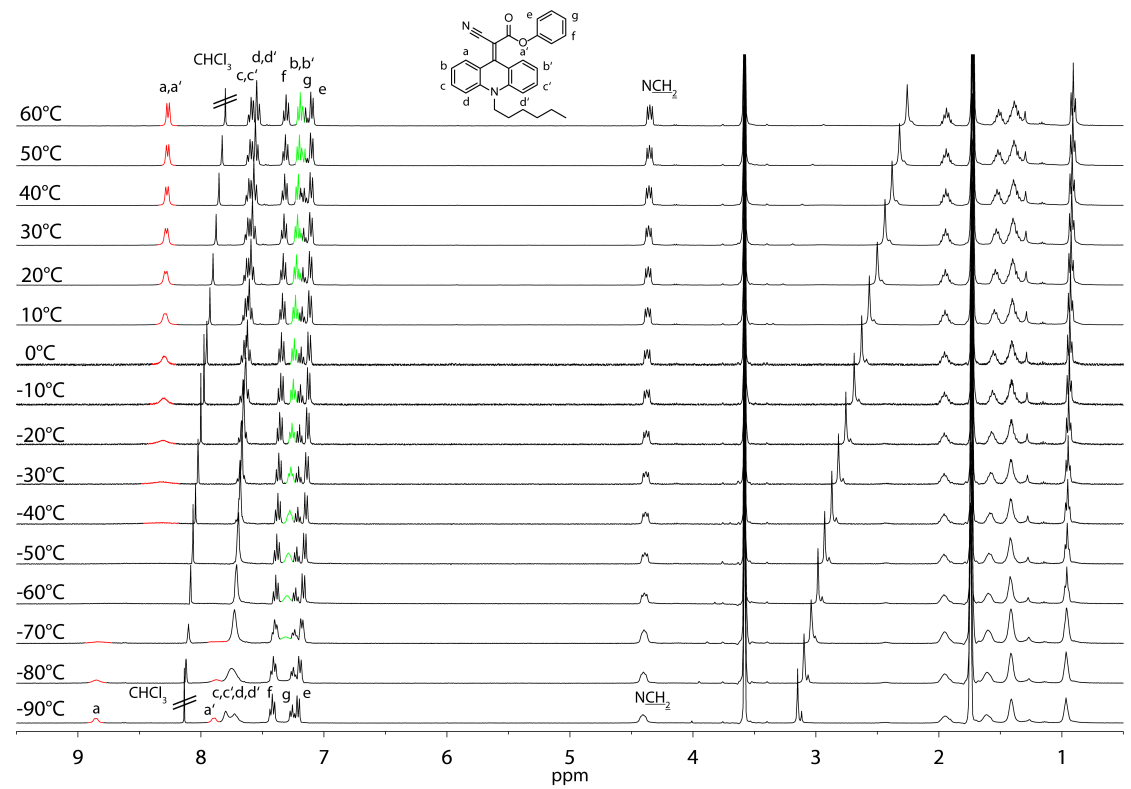

Figure V.1.2.7: VT- ${ }^{1}$ NMR $(400 \mathrm{MHz})$ form $-90{ }^{\circ} \mathrm{C}$ to $60{ }^{\circ} \mathrm{C}$ of $\mathbf{B}^{\mathrm{Ph}} 2.8 \mathrm{mM}$ in $d^{8}$-THF. Signals of protons $\mathbf{a} / \mathbf{a}^{\prime}$ are highlighted in red. Signals of protons $\mathbf{b} / \mathbf{b}$ ' are highlighted in green. The coalscence temperature is approx. $-55{ }^{\circ} \mathrm{C}$

The small change of the ester substituent causes already a difference in $\mathrm{T}_{\mathrm{C}}$ of roughly $40{ }^{\circ} \mathrm{C}$ between $\mathbf{B}^{t \mathbf{B u}}$ and $\mathbf{B}^{\mathbf{E t}}$. Going to the phenyl derivative the difference to the next highest coalescence temperature becomes even $75{ }^{\circ} \mathrm{C}$. Therefore the rotation motion predominantly dependent on the intramolecular push-pull system. Furthermore it is obvious that all dynamic motions in the here investigated derivatives are also dependent on temperature. By chance, a further flipping motion is revealed in VT NMR experiment due to the splitting of diasterotopic proton signals $\mathbf{e}_{\boldsymbol{\alpha}}$ and $\mathbf{e}_{\beta}$ in $\mathbf{B}^{\mathbf{E t}}$. In the next section the above mentioned dependency on the solvent polarity is further investigated. 


\section{V.1.2.3 Influence of Solvent Polarity on Rotation}

As already implied in the previous section, there seems to be not only dependencies from temperature or electronic situation of the ester substituents on the rotation, but from the polarity of the used solvent as well. To get a better understanding of what effect the solvent polarity has on the speed of rotation, NMR and UV-vis experiments in different solvents were performed and compared. The system $\mathbf{B}^{t \mathbf{B u}}$ is in $d^{3}-\mathrm{MeCN}$ above coalescence temperature, indicated by a broadened but discrete signal for $\mathbf{a} / \mathbf{a}$ '. Lowering the solvent polarity by using chloroform, pushes the coalescence temperature to higher temperatures, therefore the system is closer to coalescence temperature at $25^{\circ} \mathrm{C}$ and the signal $\mathbf{a} / \mathbf{a}$ ' is more broadened. THF has the lowest solvent polarity, hence the system has the highest coalescence temperature $\mathrm{T}_{\mathrm{C}}$ with $50-60{ }^{\circ} \mathrm{C}$ (figure V.1.2.5). Thus, the system must be definitively below $\mathrm{T}_{\mathrm{C}}$ at $25{ }^{\circ} \mathrm{C}$ and indeed $\mathbf{a}$ and $\mathbf{a}^{\prime}$ are split but still quite broadened. The signal a' is lying under the signals $\mathbf{c} / \mathbf{c}^{\prime}$ and $\mathbf{d} / \mathbf{d}$ '.

(a)

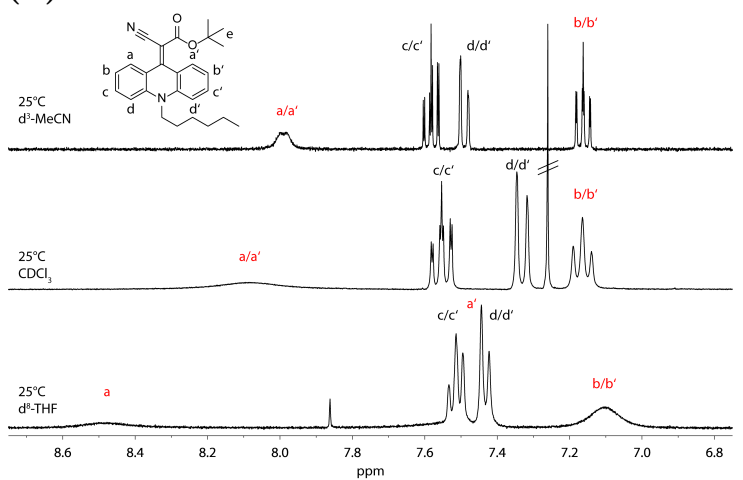

(b)

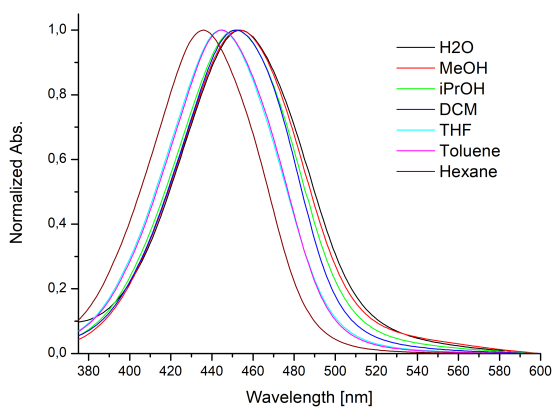

(c)

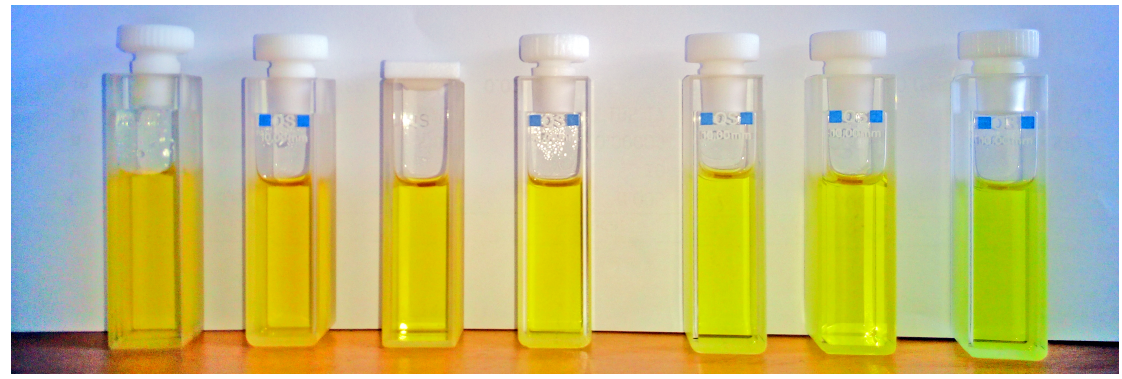

Figure V.1.2.8: a) ${ }^{\mathbf{1}} \mathbf{H}-\mathbf{N M R}(400 \mathrm{MHz})$ of $\mathbf{B}^{\boldsymbol{t} \mathbf{B u}}$ in various solvents of different polarity at $298 \mathrm{~K}$. The polarity decreases from upper the spectrum to the bottom one. The proton signals $\mathbf{a} / \mathbf{a}$ ' and $\mathbf{b} / \mathbf{b}$ ' are highlighted in red. The sharpness of signals decrease with solvent polarity, qualitatively correlating with a decrease of the rotational frequency. b) UV-vis absorbance of $\mathbf{B}^{t \mathbf{B u}} 0.005 \mathrm{mM}$ in various solvents of different polarities. The system shows the highest bathochromic shift in water $\lambda_{\max }\left(\mathrm{H}_{2} \mathrm{O}\right)=454 \mathrm{~nm}$ and highest hypsochromic shift in hexane $\lambda_{\max }($ hexane) $=436 \mathrm{~nm}$. c) $\mathbf{B}^{t \mathrm{Bu}} 0.005 \mathrm{mM}$ in various solvents of different polarities, see V.1.2.8b Solvent polarity decreases from left $\left(\mathrm{H}_{2} \mathrm{O}\right)$ to right (hexane).

In the $\mathrm{UV}$-vis experiments various spectra of $\mathbf{B}^{t \mathbf{B u}}$ in different solvent were recorded, reaching from very low solvent polarity (hexane) to a very high solvent polarity $\left(\mathrm{H}_{2} \mathrm{O}\right)$. 
Considering the comparison of all three systems in acetonitrile figure V.1.2.4b, $\mathbf{B}^{\mathrm{tBu}}(\mathbf{3 4})$ is expected to absorb at the lowest wavelength. From hexane $\left(\Delta_{\max }=436 \mathrm{~nm}\right.$ to water $\left(\Delta_{\max }=454 \mathrm{~nm}\right.$ the system undergo a bathochromic shift of $18 \mathrm{~nm}$ (figure V.1.2.8b). The color of the solution go from slightly greenish to yellow as seen in figure V.1.2.8c. $\mathbf{B}^{\mathbf{E t}}(\mathbf{3 5})$ is settled in a similar range, it shows an absorbance of $\Delta_{\max }=439 \mathrm{~nm}$ in hexane and $\Delta_{\max }=$ $461 \mathrm{~nm}$ in water, thus it has an bathochromic shift of $22 \mathrm{~nm}$, what is only a slight change of the absorption behavior compared to $\mathbf{B}^{t \mathbf{B u}}$ figure V.1.2.9b).

(a)

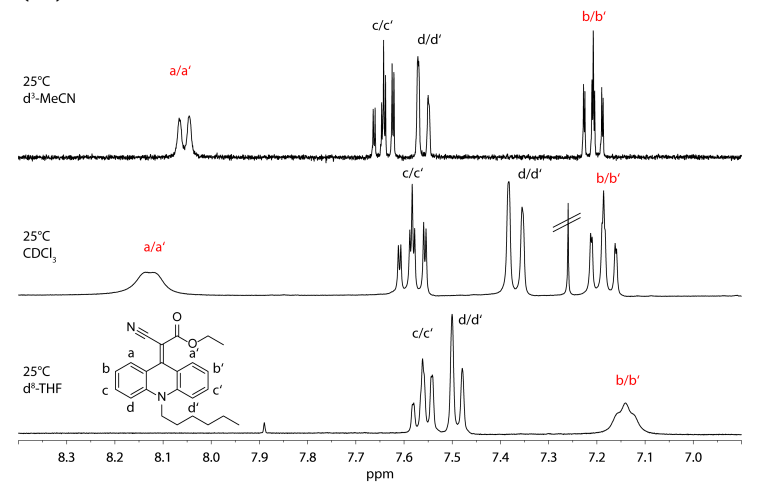

(b)

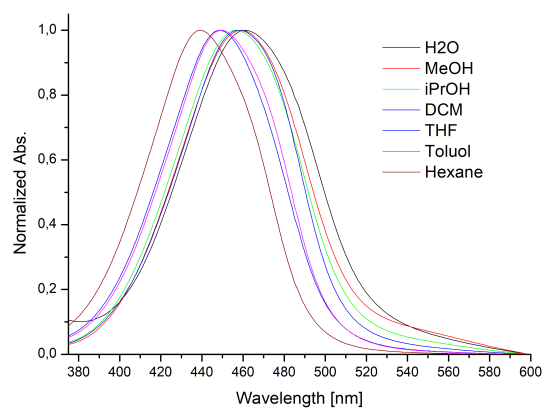

Figure V.1.2.9: a) ${ }^{\mathbf{1}} \mathbf{H}-\mathbf{N M R}(400 \mathrm{MHz})$ of $\mathbf{B}^{\mathbf{E t}}$ in various solvents of different polarity at $298 \mathrm{~K}$. The polarity decreases from the upper spectrum to the bottom one. The proton signals $\mathbf{a} / \mathbf{a}^{\prime}$ and $\mathbf{b} / \mathbf{b}$ ' are highlighted in red. The sharpness of signals decrease with solvent polarity, qualitatively correlating with a decrease of the rotational frequency. In $d^{8}$-THF the a/a' signal is too broad to be distinguished from noise. b) UV-vis absorbance of $\mathbf{B}^{\mathbf{E t}}$ $0.005 \mathrm{mM}$ in various solvents of different polarities. The system shows the highest bathochromic shift in water $\lambda_{\max }\left(\mathrm{H}_{2} \mathrm{O}\right)=461 \mathrm{~nm}$ and highest hypsochromic shift in hexane $\lambda_{\max }($ Hexane $)=439 \mathrm{~nm}$.

Likewise, the $\mathrm{NMR}$ shows, similar to the UV-vis, rather tiny changes (figure V.1.2.9a). In THF the coalescence temperature $\mathrm{T}_{\mathrm{C}}$ is around $20^{\circ} \mathrm{C}$, therefore the signal $\mathbf{a} / \mathbf{a}$ ' is extremely broadened and not observable. The signals $\mathbf{a} / \mathbf{a}^{\prime}$ are merely sharpened in the other two NMR spectra. As expected, all signals of the a/a' protons are above coalescence in the recorded NMR spectra (figure V.1.2.10a) and the UV-vis spectra show a bathochromic shift, but double as high as these of $\mathbf{B}^{t \mathbf{B u}}$ and $\mathbf{B}^{\mathbf{E t}}$ with $42 \mathrm{~nm}$ (figure V.1.2.10b). Since the absorption in more polar solvent occurs at wavelength up to $493 \mathrm{~nm})$, the colors of the used solution go from yellow, for low solvent polarity, to orange (figure V.1.2.10c). 


\section{(b)}

(a)
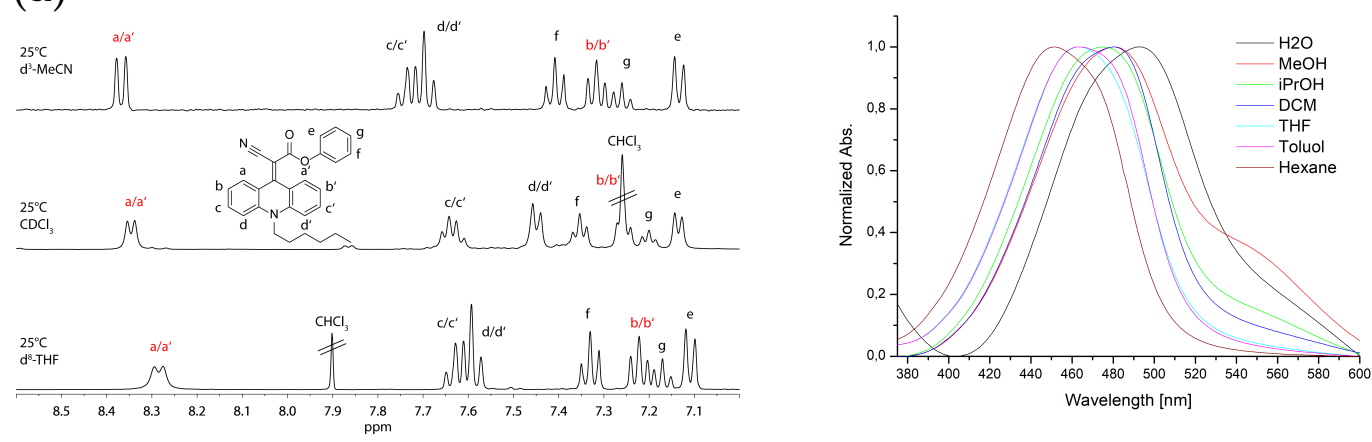

(c)

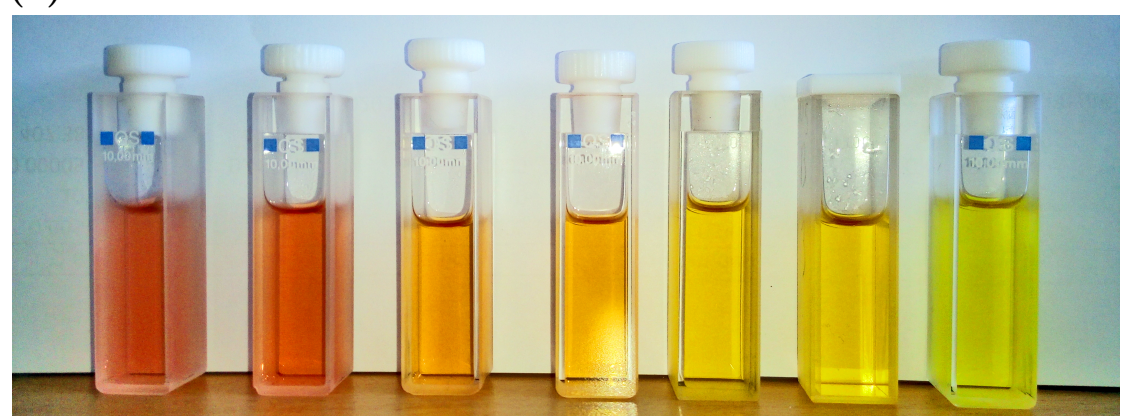

Figure V.1.2.10: a) ${ }^{\mathbf{1}} \mathbf{H}-\mathbf{N M R}(400 \mathrm{MHz})$ of $\mathbf{B}^{\mathbf{P h}}$ in various solvents of different polarity at $298 \mathrm{~K}$. The polarity decreases from the upper spectrum to the bottom one. The proton signals $\mathbf{a} / \mathbf{a}^{\prime}$ and $\mathbf{b} / \mathbf{b}$ ' are highlighted in red. The sharpness of signals decrease with solvent polarity, qualitatively correlating with a decrease of the rotational frequency. b) UV-vis absorbance of $\mathbf{B}^{\mathbf{P h}} 0.005 \mathrm{mM}$ in various solvents of different polarities. The system shows the highest bathochromic shift in water $\lambda_{\max }\left(\mathrm{H}_{2} \mathrm{O}\right)=493 \mathrm{~nm}$ and highest hypsochromic shift in hexane $\lambda_{\max }(\mathrm{Hexane})$ $=451 \mathrm{~nm}$. c) $\mathbf{B}^{\mathbf{P h}} 0.005 \mathrm{mM}$ in various solvents of different polarities, see V.1.2.10b Solvent polarity decreases from left $\left(\mathrm{H}_{2} \mathrm{O}\right)$ to right (hexane).

From the here presented results, it can be concluded so far that the dynamic rotation really takes place due to the proposed charge-separated transition state induced by the intramolecular push-pull system. All three systems show positive solvatochromism upon increase of solvent polarity from hexane to $\mathrm{H}_{2} \mathrm{O} . \mathbf{B}^{\mathbf{P h}}$ shows the largest bathochromic shift, $\mathbf{B}^{t \mathbf{B u}}$ the smallest $\left(\mathbf{B}^{t \mathbf{B u}}<\mathbf{B}^{\mathbf{E t}}<\mathbf{B}^{\mathbf{P h}}\right)$. The effect of solvent polarity can also be seen in ${ }^{\mathbf{1}} \mathbf{H}$ NMR measurements, since in unpolar solvents a clear broadening of the signals $\mathbf{a} / \mathbf{a}^{\prime}$ and $\mathbf{b} / \mathbf{b}$ ', closest to the rotor, is observed. The low solvent polarity disfavors the charge separation state, hence the single bond character, accompanied by a decrease of rotation rate. In the case of $\mathbf{B}^{t \mathbf{B u}}$ the rotation is decelerated to a spinning rate that is slow enough to observe a splitting of the signal a/a' into two distinguishable signals in ${ }^{\mathbf{1}} \mathbf{H}$ NMR. 


\section{V.1.2.4 Determination of activation enthalpy and entropy}

In order to obtain quantitative information about the thermodynamics of the intramolecular rotational motion, the principle of DNMR was used to obtain rate constants of rotation $\mathbf{k}_{\text {rot }}$ at various temperature from VT NMR experiemnts. With the help of the Full Line Shape Analysis, 250 the rotation rate can be calculated from the peak widths of the exchanging proton signals. Since the rotation changes dependent on the measured temperature, the peak widths of every single peak of the observed exchanging signals $\mathbf{a} / \mathbf{a}^{\prime}$ and $\mathbf{b} / \mathbf{b}^{\prime}$ were extracted from the different temperatures in the NMR spectra (figures V.1.2.5 to V.1.2.7). With the equations equations (V.1.1) to (V.1.3) the rate constants could be calculated. For slow exchange, means signals below coalescence temperature $\mathrm{T}_{\mathrm{C}}$ the equation (V.1.1) was used. For fast exchange or signals above $\mathrm{T}_{\mathrm{C}}$ equation (V.1.3) is applied. It should be noted that signals with small differences in chemical shift need different equations, both above and below $\mathrm{T}_{\mathrm{C}}$. But since the gap between the exchanging signals is quite big, the signals around $\mathrm{T}_{\mathrm{C}}$ become very broadened and hardly distinguishable from noise, thus it is hard to determine the peak width. To avoid thereof resulting mistakes, these signals were not considered (figure V.1.2.11c).

$$
k=\frac{\pi}{\sqrt{2}}\left(\Delta \nu_{0}^{2}-\Delta \nu_{e}^{2}\right)^{\frac{1}{2}}
$$

$\Delta \nu_{0}=$ Distance between signals of exchange protons when no rotation occurs $\Delta \nu_{e}=$ Distance between observed signals of exchange protons

$$
\begin{gathered}
k=\frac{\pi \Delta \nu_{0}}{\sqrt{2}} \\
k=\frac{\pi \Delta_{0}^{2}}{2}\left(\left(\Delta \nu_{e}\right)_{1 / 2}-\left(\Delta \nu_{0}\right)_{1 / 2}\right)^{-1} \\
\left(\Delta \nu_{0}\right)_{1 / 2}=\text { FWHM peak no rotation } \\
\left(\Delta \nu_{e}\right)_{1 / 2}=\text { FWHM observed peak }
\end{gathered}
$$


(a)

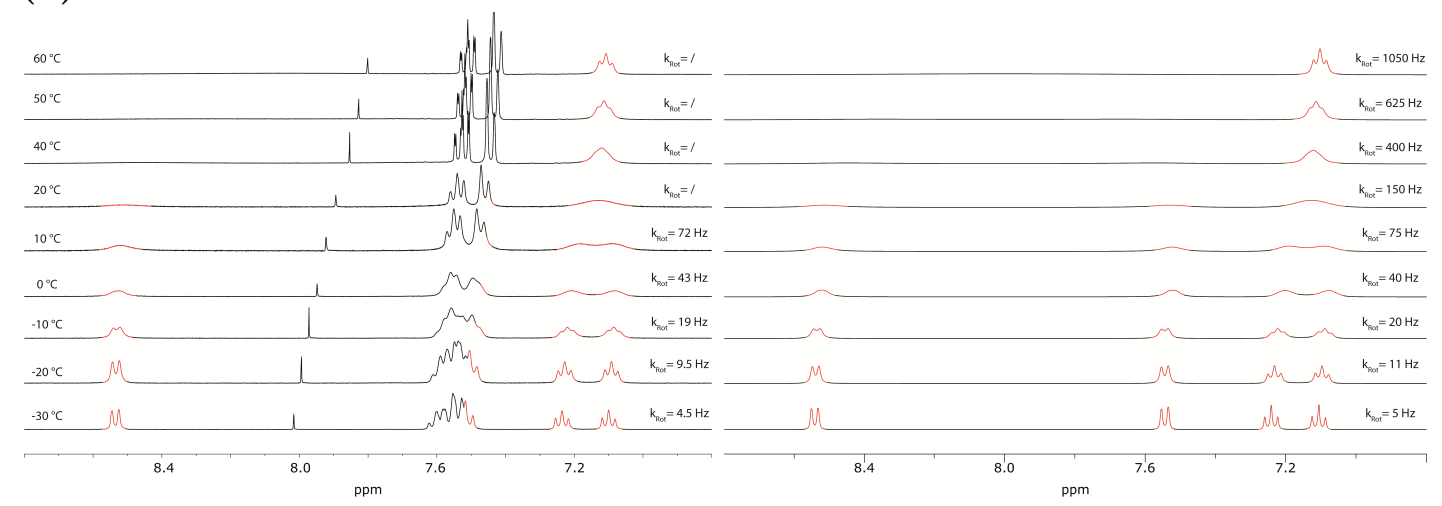

(b)

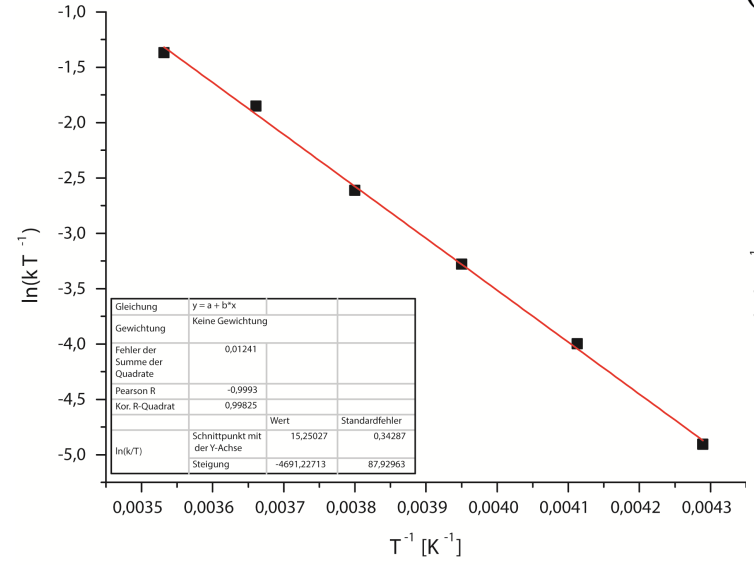

(c)

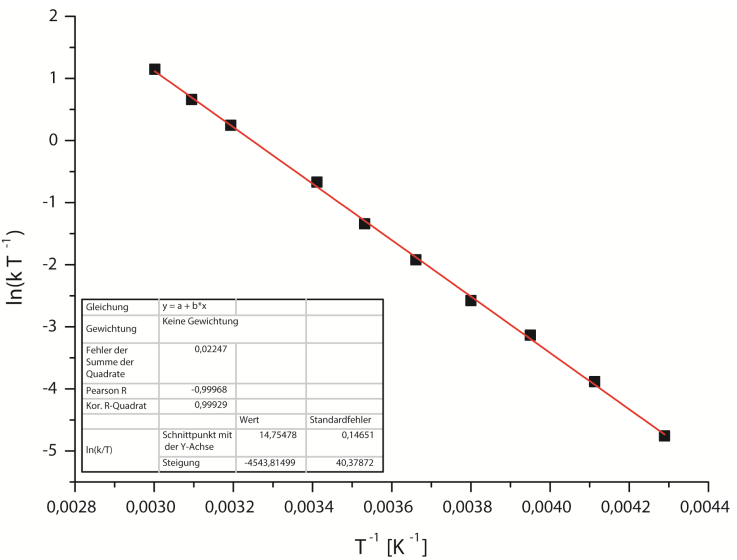

Figure V.1.2.11: a) Determination of rotational constants from NMR data of $\mathbf{B}^{t \mathrm{Bu}}$. The spinning rates were determined with full line shape analysis from experimental data at different temperatures. b) Additionally, rate constants were also obtained from DNMR simulations of the proton signals $\mathbf{a} / \mathbf{a}^{\prime}$ and $\mathbf{b} / \mathbf{b}$ ', which are closest to the rotor group and show the biggest signal splitting.

Additionally, all measured spectra were simulated with SpinWorks 251 and the rotation rates were also extracted from the simulations to compare them with the calculated values. Due to the restriction in the number of simulated exchanging nuclei to a total amount of five, the protons signals of $\mathbf{a} / \mathbf{a}^{\prime}$ and (b/b') were only simulated, since the show the biggest dynamic effect in the NMR spectra. To obtain the simulations as accurate as possible, chemical shift, coupling constants and peak-width of the simulated signals were fitted to signals of measured spectra at $-90{ }^{\circ} \mathrm{C}$, assuming that no rotation occurs anymore. For $\mathbf{B}^{\mathbf{P h}}(\mathbf{3 7})$ this was not possible, for this reason the chemical shifts for the non rotating situation were roughly estimated.

With help of the Eyring-Polanyi equation, 252 equation (V.1.4) it is possible to obtain from kinetic value $\mathrm{k}_{\text {rot }}$ and the temperature the thermodynamic values: enthalpy of activation $\Delta^{\ddagger} H^{\ominus}$ and the entropy of activation $\Delta^{\ddagger} S^{\ominus}$ equation (V.1.6). 


$$
\begin{gathered}
k=\frac{\kappa k_{B} T}{h} \exp \left(-\frac{\Delta^{\ddagger} G^{\ominus}}{R T}\right) \\
\Delta^{\ddagger} G^{\ominus}=\Delta^{\ddagger} H^{\ominus}-T \Delta^{\ddagger} S^{\ominus} \\
k=\frac{\kappa k_{B} T}{h} \exp \left(\frac{-\Delta^{\ddagger} H^{\ominus}}{R T}+\frac{\Delta^{\ddagger} S^{\ominus}}{R}\right)
\end{gathered}
$$

converted to equation (V.1.7), plotting $\ln \left(\mathrm{kT}^{-1}\right)$ versus $\mathrm{T}^{-1}$ gives a linear correlation, where $\Delta^{\ddagger} H^{\ominus}$ can be extracted from the slope of the plot and $\Delta^{\ddagger} S^{\ominus}$ from the yintercept.

$$
\ln \frac{k}{T^{-1}}=\frac{-\Delta^{\ddagger} H^{\ominus}}{R} \cdot \frac{1}{T}+\ln \frac{\kappa k_{B}}{h}+\frac{\Delta^{\ddagger} H^{\ominus}}{R}
$$


(a)
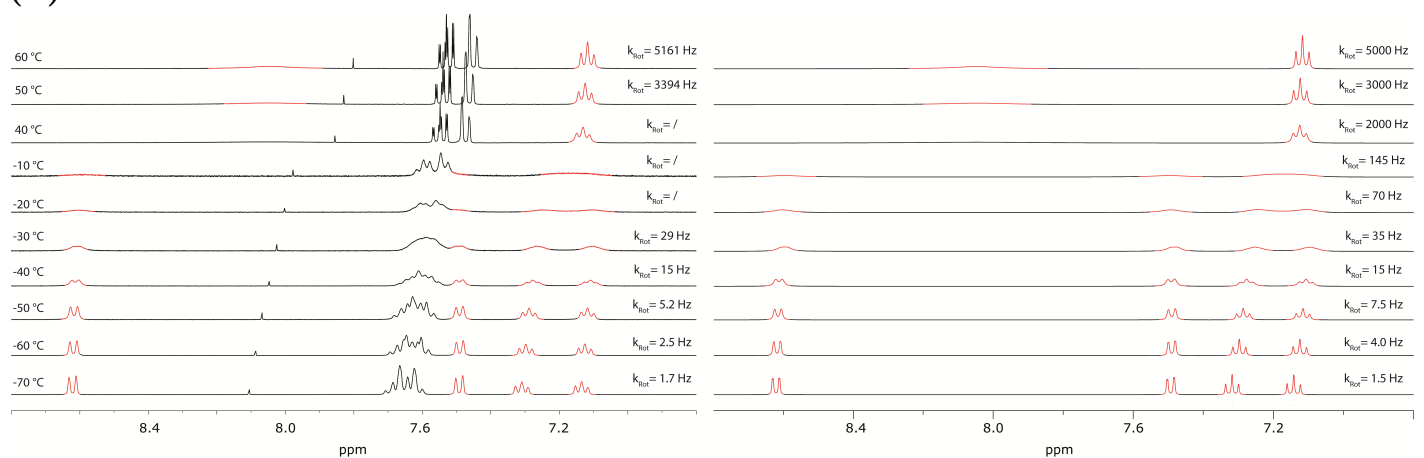

(b)

(c)
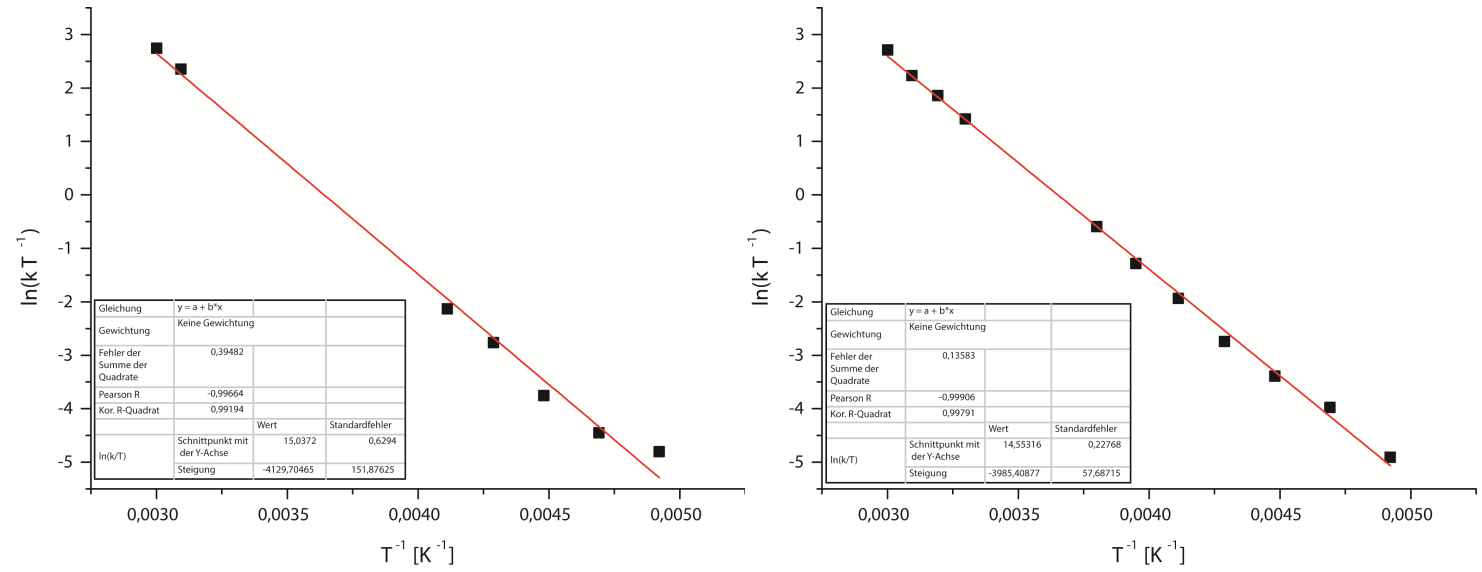

Figure V.1.2.12: Determination of rotational constants from NMR data of $\mathbf{B}^{\mathbf{E t}}$. The spinning rates were determined with full line shape analysis from experimental data at different temperatures. b) Additionally, rate constants were also obtained from DNMR simulations of the proton signals $\mathbf{a} / \mathbf{a}$ ' and $\mathbf{b} / \mathbf{b}$ ', which are closest to the rotor group and show the biggest signal splitting.

Experimentally determined rotation constants $\mathrm{k}_{\text {rotexp }}$, as well as from simulation $\mathrm{k}_{\text {rotsim }}$ were plotted (figure V.1.2.11b- V.1.2.13c) and the thermodynamic values $\Delta^{\ddagger} H^{\ominus}$ and $\Delta^{\ddagger} S^{\ominus}$ were calculated from linear fit of plotted data points. The activation entropies and enthalpies received from experimental determination and simulation table V.1.2.1 are in good agreement to each other. As expect, the enthalpy for the rotation decreases from $\Delta H^{\ddagger}=39.0 \mathrm{~kJ} \mathrm{~mol}^{-1}$ for the tert-butyl derivative to $\Delta H^{\ddagger}=34.3 \mathrm{~kJ} \mathrm{~mol}^{-1}$ to the fastest rotor with an activation enthalpy of $\Delta H^{\ddagger}=26.3 \mathrm{~kJ} \mathrm{~mol}^{-1}$. Since the rotation rate $\mathrm{k}_{\text {rot }}$ increasing at higher temperature and decreases at lower temperatures it is obviously an endothermic process, therefore the positive value of $\Delta^{\ddagger} H^{\ominus}$ is in full accordance with the empirical evidences. 
(a)

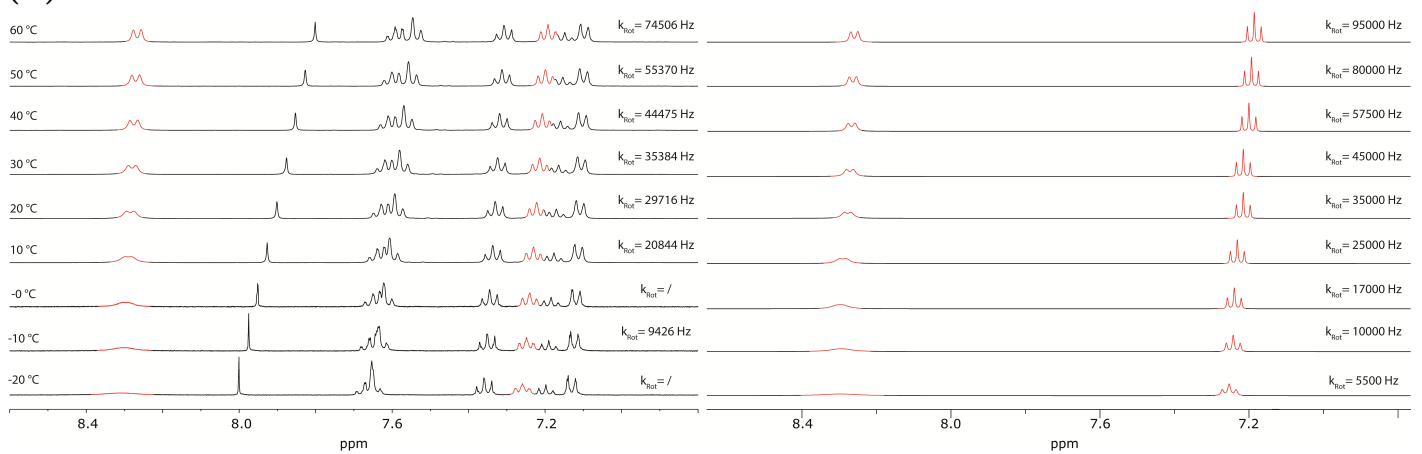

(b)

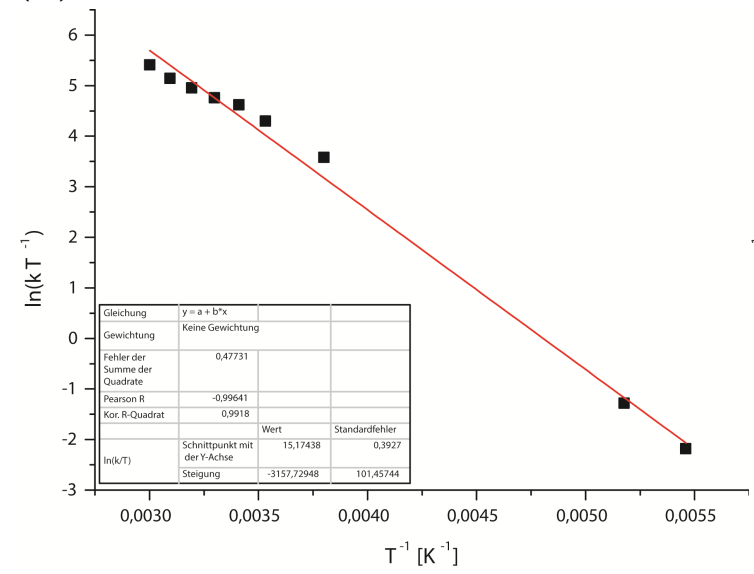

(c)

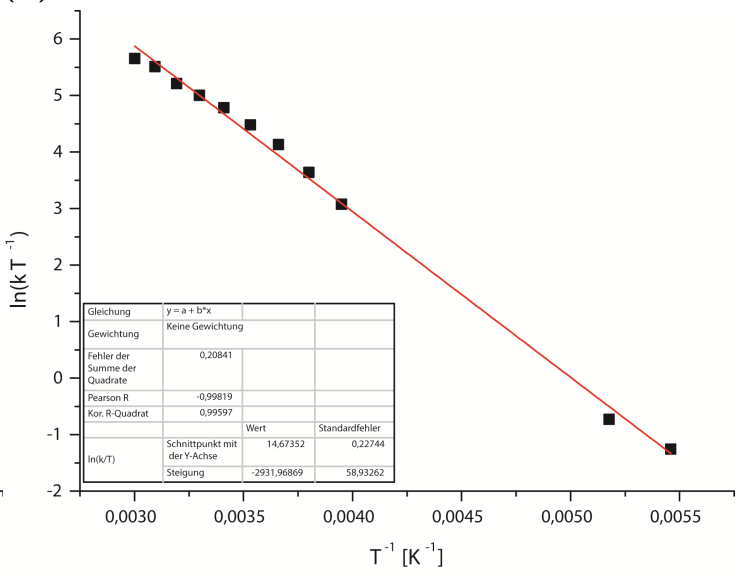

Figure V.1.2.13: Determination of rotational constants from NMR data of $\mathbf{B}^{\mathbf{P h}}$. The spinning rates were determined with full line shape analysis from experimental data at different temperatures. b) Additionally, rate constants were also obtained from DNMR simulations of the proton signals $\mathbf{a} / \mathbf{a}^{\prime}$ and $\mathbf{b} / \mathbf{b}$ ', which are closest to the rotor group and show the biggest signal splitting.

Interestingly, all derivatives show all nearly the same negative value for the entropy of activation $\left(-72 \mathrm{~kJ} \mathrm{~mol}^{-1} \mathrm{~K}^{-1}\right)$, which indicates that the pathway for the rotation has a similar nature in all derivatives. The negative value could be explained by the rearranging of the surrounding solvent molecules to a higher ordered structure, due to the formation of the assumed charged-seperated transistion state. 253 Comparing the entropies of activation for all derivatives the order is as expected as in all previous experiments $\left(\mathbf{B}^{\mathbf{P h}}>\mathbf{B}^{\mathbf{E t}}>\right.$ $\left.\mathrm{B}^{\mathrm{tBu}}\right)$. 
Table V.1.2.1: Activation enthalpies, entropies and Gibbs' free energies of $\mathbf{B}^{t \mathbf{B u}}, \mathbf{B}^{\mathbf{E t}}$ and $\mathbf{B}^{\mathbf{P h}}$ determined from experimental and simulated rate constants.

\begin{tabular}{|c|c|c|c|}
\hline & $\mathrm{B}^{t \mathrm{Bu}}(34)$ & $\mathrm{B}^{\mathrm{Et}}(35)$ & $\mathrm{B}^{\mathrm{Ph}}(37)$ \\
\hline$\Delta H^{\ddagger} \exp \left[\mathrm{k} J \mathrm{~mol}^{-1}\right]$ & $39.0 \pm 0.7$ & $34.3 \pm 1.3$ & $26.3 \pm 0.8$ \\
\hline$\Delta S_{\exp }^{\ddagger}\left[\mathrm{k} J \mathrm{~mol}^{-1} \mathrm{~K}^{-1}\right]$ & $-70.8 \pm 2.9$ & $-72.5 \pm 5.2$ & $-71.4 \pm 3.3$ \\
\hline$\Delta G^{\ddagger}{ }_{\exp }^{298 \mathrm{~K}}\left[\mathrm{k} J \mathrm{~mol}^{-1}\right]$ & 60.1 & 56.0 & 47.5 \\
\hline$\Delta H_{\operatorname{sim}}^{\ddagger}\left[\mathrm{k} J \mathrm{~mol}^{-1}\right]$ & $37.8 \pm 0.3$ & $33.1 \pm 0.5$ & $24.4 \pm 0.5$ \\
\hline$\Delta S^{\ddagger} \operatorname{sim}\left[\mathrm{k} J \mathrm{~mol}^{-1} \mathrm{~K}^{-1}\right]$ & $-74.9 \pm 1.2$ & $-76.6 \pm 1.9$ & $-75.5 \pm 1.9$ \\
\hline$\Delta G^{\ddagger}{ }_{\operatorname{sim}}^{298 K}\left[\mathrm{k} J \mathrm{~mol}^{-1}\right]$ & 60.1 & 56.0 & 46.9 \\
\hline
\end{tabular}




\section{V.1.2.5 Cyclic voltammetry}

Since the dynamic motion is caused by an induced charge-separation within the intramolecular push-pull system, it was interesting to investigate how the electrochemical properties would change between the three derivatives. Cyclic voltammetry measurements were performed for that reason in dichloromethane with 1 molar tetra- $n$-butyl ammonium tetrafluoroborate. The cyclic voltagramms of $\mathbf{B}^{t \mathbf{B u}}, \mathbf{B}^{\mathrm{Et}}$ and $\mathbf{B}^{\mathrm{Ph}}$ figures V.1.2.14b to V.1.2.14d) show all together a fully reversible reduction between $-1.3 \mathrm{~V}$ and $-1.6 \mathrm{~V}$ (vs. $\mathrm{Ag} / \mathrm{AgNO}_{3}$ ) with an increasing anodic shift from the tert-butyl to the phenyl derivate. The cyclic voltagramms (figures V.1.2.14b to V.1.2.14d of all derivatives show a fully reversible reduction between $-1.3 \mathrm{~V}$ and $-1.6 \mathrm{~V}$.

(a)

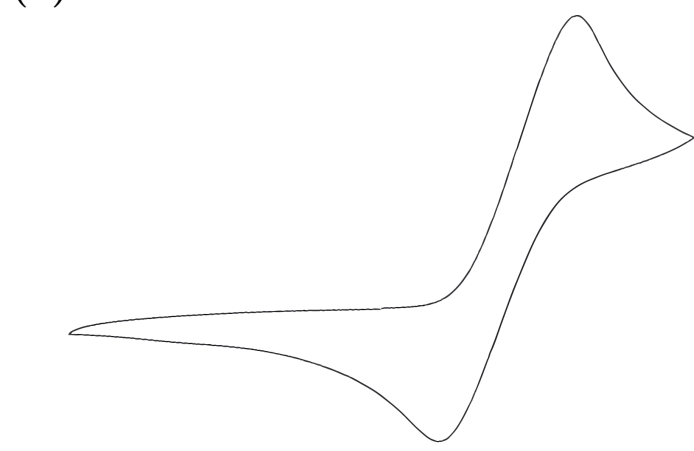

(b)

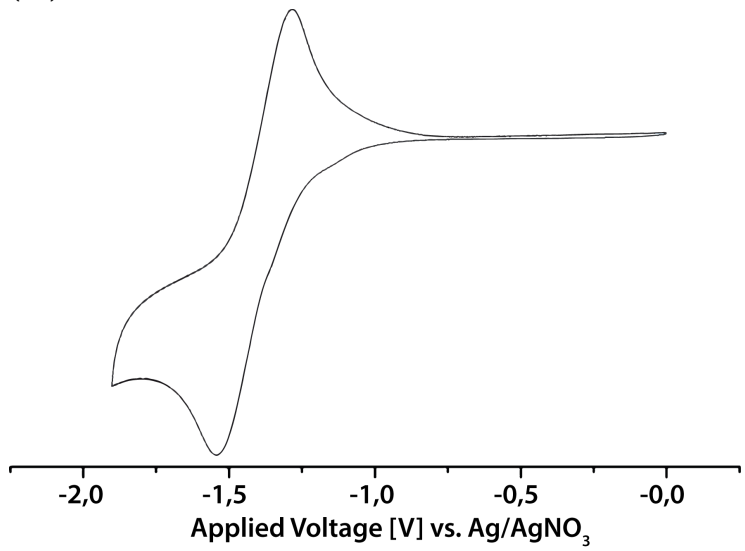

(d)

(c)
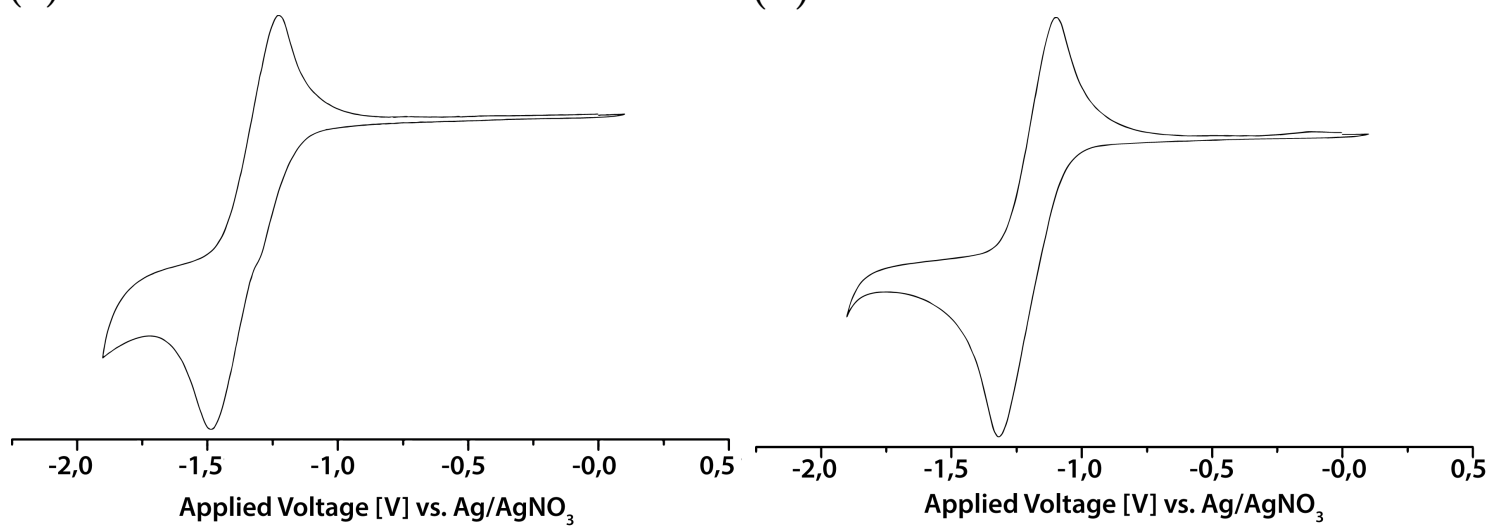

Figure V.1.2.14: a) Cyclic voltammogram of Ferrocene, $2.8 \mathrm{mM}$ in DCM, $0.1 \mathrm{M} \mathrm{nBu}_{4} \mathrm{BF}_{4}$, recorded with a scan rate of $100 \mathrm{mV} \mathrm{s}^{-1}$. b) Cyclic voltammogram of $\mathbf{B}^{t \mathrm{Bu}}, 2.8 \mathrm{mM}$ in DCM, $0.1 \mathrm{M} \mathrm{nBu}_{4} \mathrm{BF}_{4}$, recorded with a scan rate of $100 \mathrm{mV} \mathrm{s}^{-1}$. c) Cyclic voltammogram of $\mathbf{B}^{\mathbf{E t}}, 2.8 \mathrm{mM}$ in DCM, $0.1 \mathrm{M} \mathrm{nBu}_{4} \mathrm{BF}_{4}$, recorded with a scan rate of $100 \mathrm{mV} \mathrm{s}^{-1}$. d) Cyclic voltammogram of $\mathbf{B}^{\mathbf{P h}}, 2.8 \mathrm{mM}$ in DCM, $0.1 \mathrm{M} \mathrm{nBu}{ }_{4} \mathrm{BF}_{4}$, recorded with a scan rate of $100 \mathrm{mV} \mathrm{s}^{-1}$

The measured voltages for reduction and oxidation were referenced versus $\mathrm{Fc} / \mathrm{Fc}^{+}$, as internal standard to make them better comparable for the literature table V.1.2.2 254 
Comparison of the cyclic voltammetric measurements is shown in figure V.1.2.15. It is obvious that an increasing anodic shift happen from $-1.66 \mathrm{~V}$ for $\mathbf{B}^{t \mathbf{B u}}$, over $-1.60 \mathrm{~V}$ for $\mathbf{B}^{\mathbf{E t}}$ to $-1.45 \mathrm{~V}$ for $\mathbf{B}^{\mathrm{Ph}}$ (all vs $\mathrm{Fc} / \mathrm{Fc}^{+}$). This observation is in full agreement with the absorption spectroscopic investigations (figure V.1.2.4b).

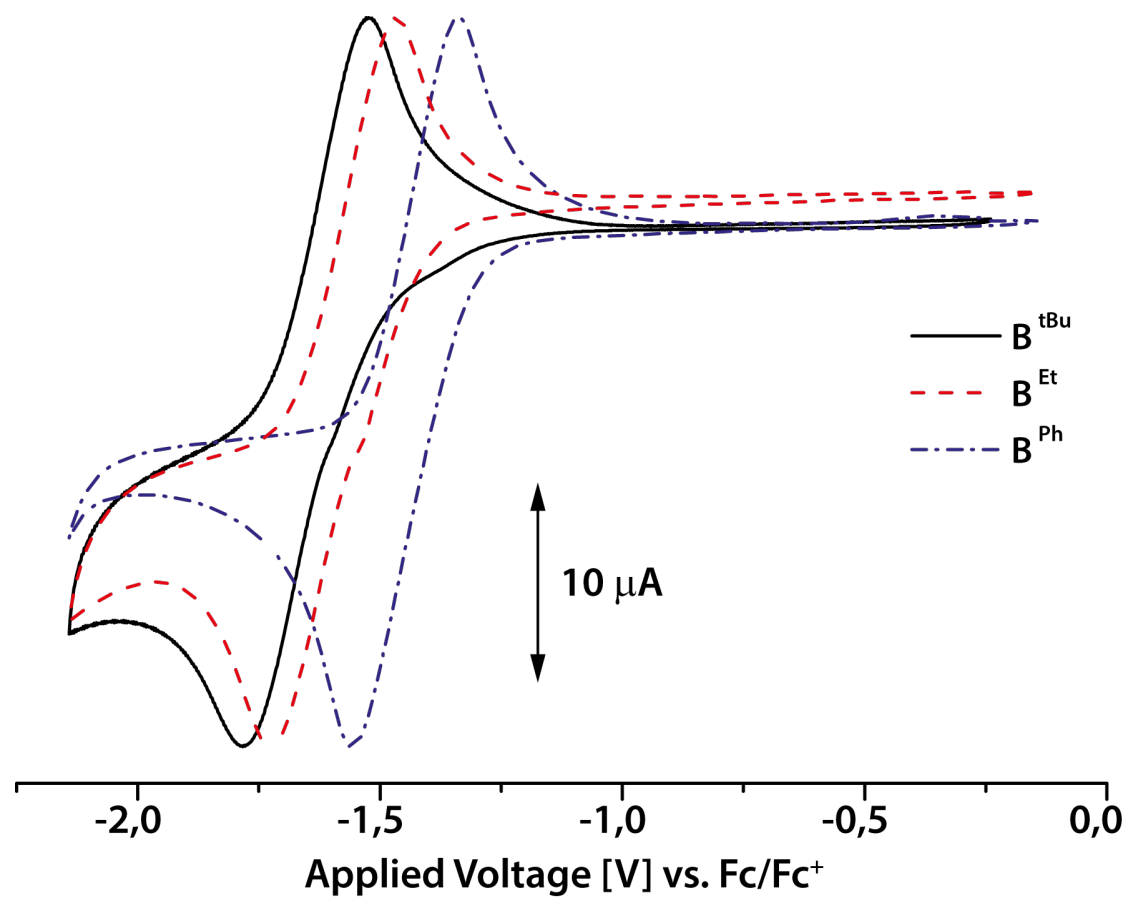

Figure V.1.2.15: Comparison of cyclic voltammetry measurements of all derivatives. Potentials corrected to $\mathrm{Fc} / \mathrm{Fc}+$. An anodic shift about $210 \mathrm{mV}$ is observed from $\mathbf{B}^{t \mathbf{B u}}$ to $\mathbf{B}^{\mathbf{P h}}$.

Table V.1.2.2: Reduction and oxidation voltages for $\mathbf{B}^{t \mathrm{Bu}}, \mathbf{B}^{\mathrm{Et}}, \mathbf{B}^{\mathrm{Ph}}$ extracted from cyclic voltammetry experiments. E1/2 values were referenced to $\mathbf{F c} / \mathbf{F c}^{+}$. Since the symmetrically substituted $\mathrm{B}^{\mathrm{CN}}$ was not available for $\mathrm{CV}$ the values were taken from literture. $241 / 255$

\begin{tabular}{lcccc}
\hline & $\mathbf{E}_{\text {red }}[\mathbf{V}]$ & $\mathbf{E}_{\text {red }}[\mathbf{V}]$ & $\mathbf{E}_{\mathbf{1} / \mathbf{2}}[\mathbf{V}]$ & $\begin{array}{c}\mathbf{E}_{\mathbf{1} / \mathbf{2}} \mathbf{v s} \\
\mathbf{F c}_{\mathbf{F}} \mathbf{F c}^{+}[\mathbf{V}]\end{array}$ \\
\hline \hline $\mathbf{F c} / \mathbf{F c}^{+}$ & 0.11 & 0.38 & 0.24 & \\
$\mathbf{B}^{\mathbf{C N}}$ & & & $-1.07[241]$ & $-1.4125]$ \\
$\mathbf{B}^{\mathbf{P h}}$ & -1.32 & -1.10 & -1.21 & -1.45 \\
$\mathbf{B}^{\mathbf{E t}}$ & -1.49 & -1.23 & -1.36 & -1.60 \\
$\mathbf{B}^{t \mathbf{B u}}$ & -1.54 & -1.28 & -1.42 & -1.66 \\
\hline
\end{tabular}

To compare the experimental obtained energy values for the HOMO/LUMO gaps $\left(\mathbf{E}_{\mathbf{g}}\right)$ from spectroscopic measurement section V.1.2.2 and cyclic voltammetry with those obtained from DFT calculations section V.1.2.7), additional CV measurements were made from $-2.0 \mathrm{~V}$ to $+2.0 \mathrm{~V}$. From the CV measurements (figure V.1.2.16) the oxidation $E_{\text {onset }}^{o x}$ and reduction $E_{\text {onset }}^{\text {red }}$ onset for every derivative was graphically determined by taking the 
potentials from the the intersection of the tangents between the baseline and the signal current (red dashed lines). 256

(a)

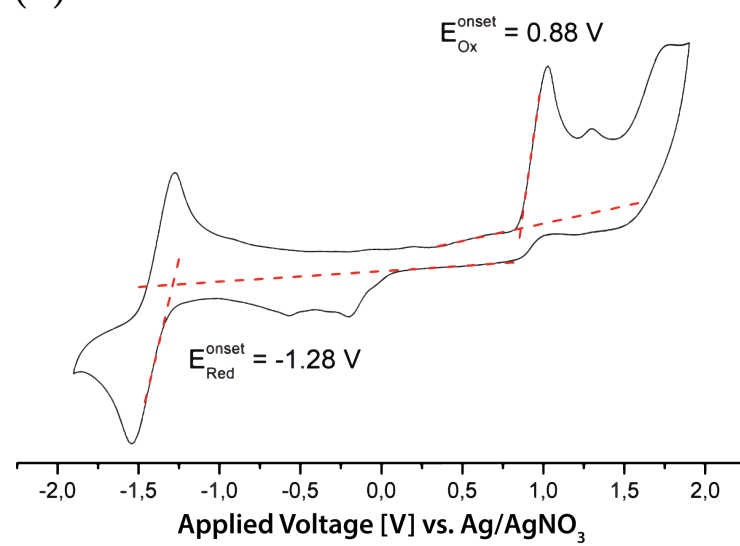

(c)

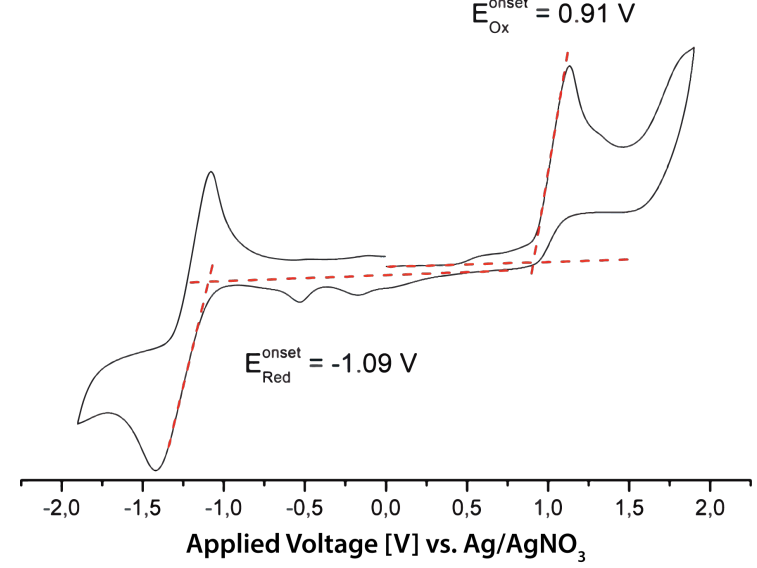

(b)

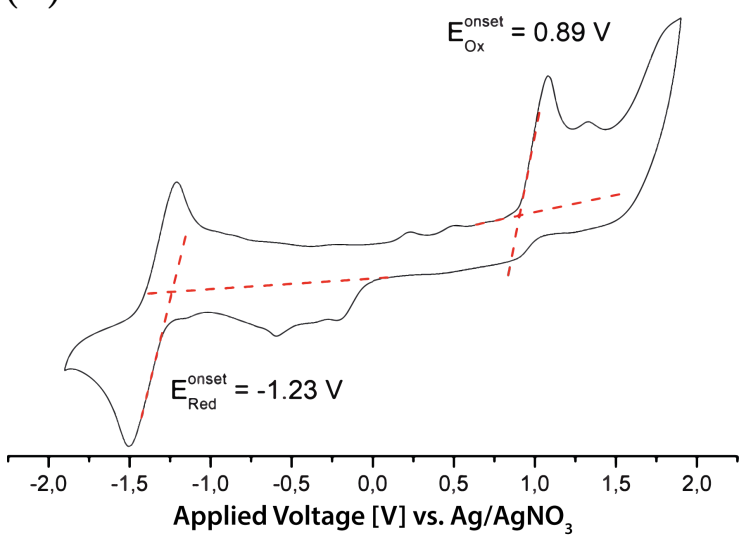

$E_{0 x}^{\text {onset }}=0.91 \mathrm{~V}$ 


\section{V.1.2.6 Crystal Structures Analysis}

To get an better idea of the spatial arrangment of the here investigated derivates, crystalization attemps were made, to obtain crystals suitable for x-ray diffracton measurements. For that reason soltuions of $\mathbf{B}^{t \mathbf{B u}}, \mathbf{B}^{\mathbf{E t}}$ and $\mathbf{B}^{\mathbf{P h}}$ in various solvents (THF, $\mathrm{CH}_{2} \mathrm{Cl}_{2}, \mathrm{CHCl}_{3}$, $\mathrm{MeCN}$ ) were prepeared for either slow evaporation or liquid-liquid diffusion. Crystals of $\mathbf{B}^{t \mathbf{B u}}$ and $\mathbf{B}^{\mathbf{E t}}$ were obtained by liquid-liquid diffusion of $\mathrm{MeOH}$ into a solution of the respective compound in $\mathrm{MeCN}$. For $\mathbf{B}^{t \mathbf{B u}}$ only an derivative with iodine on the 2- and 7-postion $\left(\mathbf{I}_{2}-\mathbf{B}^{t \mathbf{B u}}\right)$ was suitable for x-ray crystal analysis. Both structures are shown in figure V.1.2.17. Addtionally the already existing crystal structure of the dicyano derivate $\left(\mathbf{B}^{\mathbf{C N}}\right)$ is included,241|247 which resembles the hexyl chain derivative, although it merely has a butyl chain.
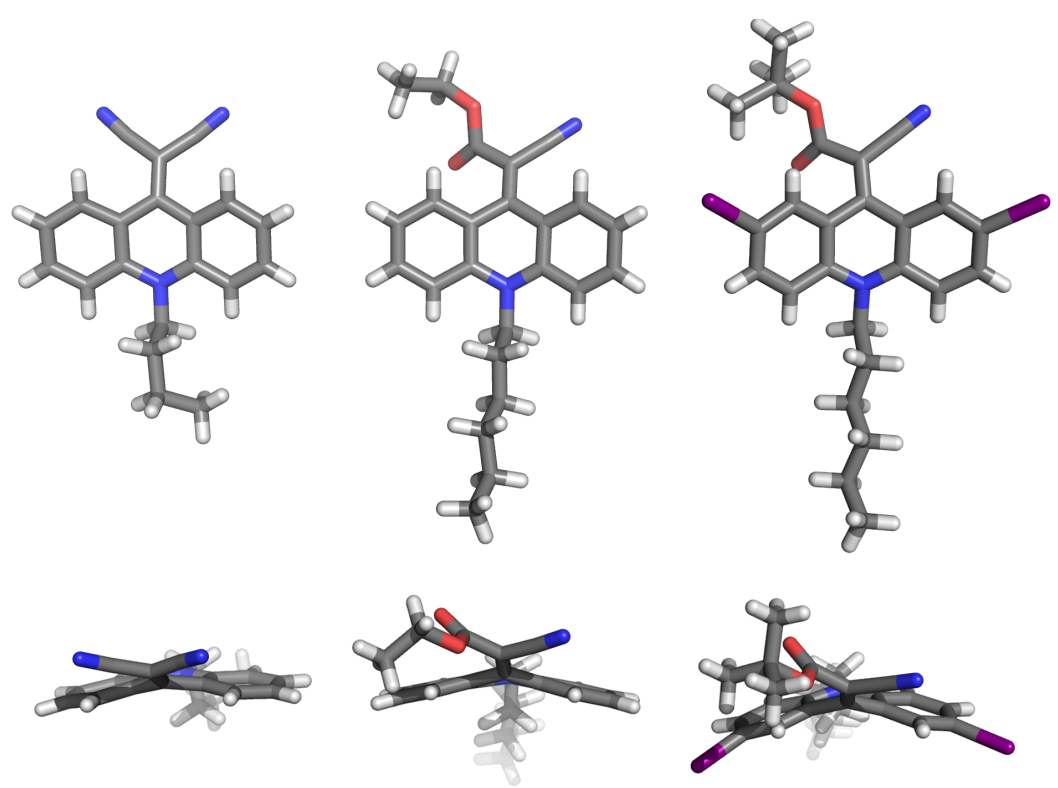

Figure V.1.2.17: Comparison crystal structures of acridinylidene derivatives, from left to right: $b t_{y l-\mathbf{B}^{\mathbf{C N}}}, \mathbf{B}^{\mathbf{E t}}$, $\mathbf{I}_{2}-\mathbf{B}^{t \mathbf{B u}}$. The dicyano derivative crystal structure is already known and is shown here for a better comparison of the rotation group influence on the whole structure. The tert-butyl residue, a crystal with iodine on the 2- and 7-postion of the acridone was only suitable for X-ray crystal structure analysis. The dicyano acridinylidene derivative shows the fewest bending of tricyclic backbone, whereas the derivative with the tert-butyl ester shows the biggest bending of the backbone. Furthermore, compared to the symetric dicyano derivative, the others show a slight tilt of the rotating group in respect to the backbone.

It is easy to see that the molecules have a slightly bent butterfly structure, where the bending of the aromatic stator is increasing from the dicyano derivative to the tert-butyl one. $\mathbf{B}^{t \mathrm{Bu}}$ shows the most bent structure with an angle of $\Theta=149^{\circ}$ section V.1.2.6. whereas $\mathbf{B}^{\mathbf{E t}}$ has an angle of $\Theta=154^{\circ}$ and $\mathbf{B}^{\mathbf{C N}}$ only of $\Theta=164^{\circ}$. It is apparent that the derivatives show again the same order as in previous experiment. Regarding this order one would expect the structure of $\mathbf{B}^{\mathbf{P h}}$ to be in between $\mathbf{B}^{\mathbf{C N}}$ and $\mathbf{B}^{\mathbf{E t}}$. Furthermore, all structures show a twisted rotor with regard to the stator, also increasing from $\mathbf{B}^{\mathbf{C N}}$ to 
$\mathbf{B}^{t \mathbf{B u}}$. The dihedral angle for $\mathbf{B}^{t \mathbf{B u}}$ is $\varphi=19^{\circ}$, decrease for $\mathbf{B}^{\mathbf{E t}}$ to $\varphi=11^{\circ}$ and $\mathbf{B}^{\mathbf{C N}}$ almost does not show any twist with $\varphi=2^{\circ}$. The twist probably occurs as a reaction of the system to minimize the repulsion between the rotating substituent and the aromatic protons next to the bridging double bond.

$\mathrm{I}_{2}-\mathrm{B}^{\mathrm{BB}}$

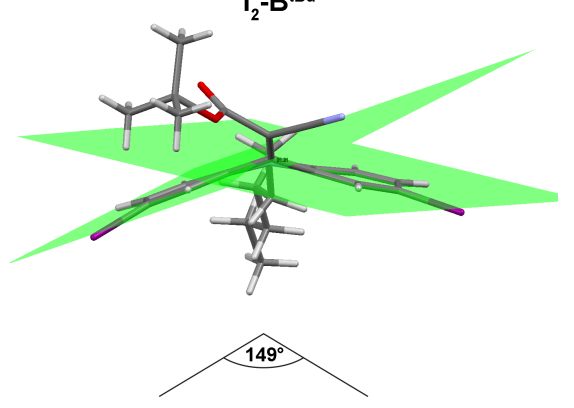

$\mathbf{B}^{\mathrm{Et}}$

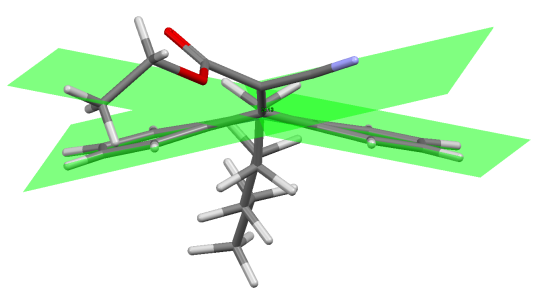

$\mathrm{B}^{\mathrm{CN}}$
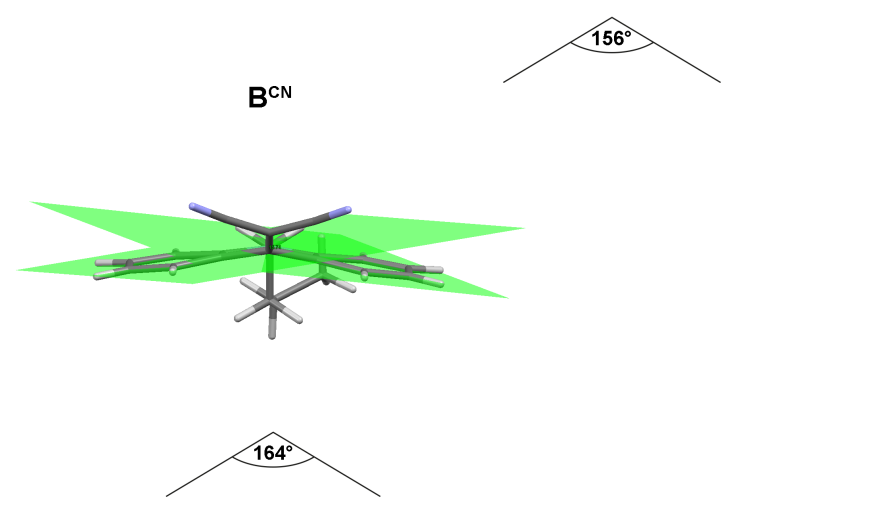

Figure V.1.2.18: Angles of bended backbones extracted from the obtained crystal structures. The $\mathbf{I}_{2}-\mathbf{B}^{t \mathbf{B u}}$ shows the biggest bending with a angle of $149^{\circ}$. The other derivatives are as expected less bended with angles of $156^{\circ}$ for $\mathbf{B}^{\mathbf{E t}}$ and $164^{\circ}$ for $\mathbf{B}^{\mathbf{C N}}$. $\mathbf{B}^{\mathbf{P h}}$ is expected to be between $\mathbf{B}^{\mathbf{E t}}$ and $\mathbf{B}^{\mathbf{C N}}$.

Due to the asymmetric rotor group and the bent tricyclic aromatic stator, the structure should owe an axial chirality what give rise to the existence of enantiomeric conformations of all investigated derivatives. In the case of $\mathbf{B}^{\mathbf{E t}}$ the asymmetric unit contained four molecules, and luckily two molecules of both enantiomers were found (figure V.1.2.19). In the crystal structure the enantiomers can exist side by side long enough to be distinguishable, since the dynamics are frozen-out. In solution, however, both enantiomers are quickly interconverting by either the rotation or the flipping motion. 


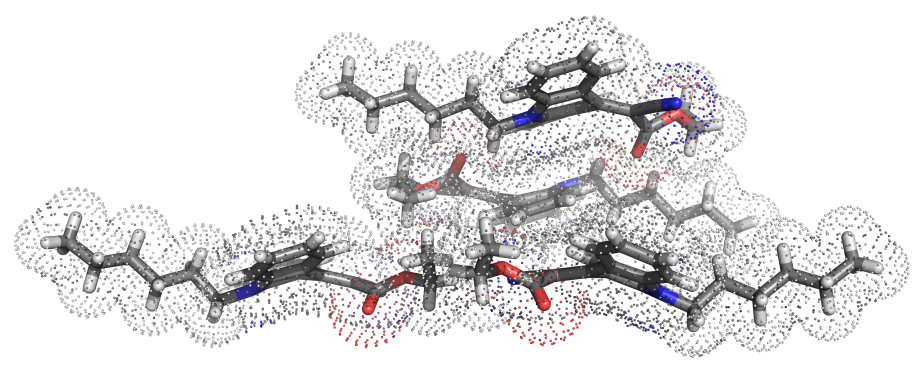

Figure V.1.2.19: The asymetric unit of the measured crystal of $\mathbf{B}^{\mathbf{E t}}$ contains all in all four molecules, two of each enantiomer. The single enantiomers a shown in figure V.1.2.20.

Moreover, the conformation shown in the crystal structure of $\mathbf{B}^{\mathbf{E t}}$ (figure V.1.2.20) supports the assumption of an additional flipping motion in the molecules, since the methylene protons of the ester substituent also show a distinguishable chemical environment $\left(\mathrm{e}_{\alpha}\right.$ and $e_{\beta}$ ), exactly those protons which show a splitting in the VT NMR experiments at very low temperatures, when rotation was already frozen-out. Additional analysis of the bond lengths in the $\mathrm{x}$-ray structure indicates a quinoidal character for $\mathbf{B}^{\mathbf{E t}}$, what indicates that the bridging connection between rotor and stator exist with a prevalent double bond character in solid state.

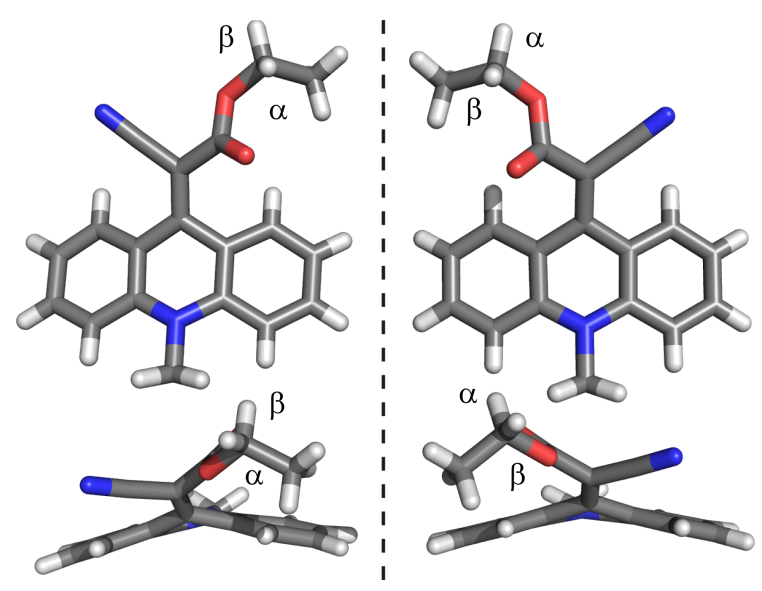

Figure V.1.2.20: The two existing enantiomers of $\mathbf{B}^{\mathbf{E t}}$, extracted from the asymetric unit of the measured crystal. The diasterotopic protons, which are probably responsible for the splitting of the esters' methylene group in the VT-NMR of $\mathbf{B}^{\mathrm{Et}}$ (figure V.1.2.6) are labelled with $\alpha$ and $\beta$. The hexyl chain is omitted for a better view. 


\section{V.1.2.7 Chemoinformatic Studies}

Comparison of the UV-vis spectra of $\mathbf{B}^{t \mathbf{B u}}, \mathbf{B}^{\mathbf{E t}}$ and $\mathbf{B}^{\mathbf{P h}}$ support this picture, since increasing the charge stabilization within the push-pull system goes along with a larger bathochromic shift $\left(26 \mathrm{~nm}\right.$ in $\mathrm{MeCN}$ from $\mathbf{B}^{t \mathbf{B u}}$ to $\left.\mathbf{B}^{\mathbf{P h}}\right)$ of the longest-wavelength maximum, corresponding to a decreasing magnitude of the HOMO and LUMO transition figure V.1.2.4b).

To get a better understanding of the electronic situation and substituent effects in the system we performed density-function theory (DFT) geometry optimization for all derivatives (B3LYP/6-311+g(d,p) in PCM-MeCN) based on the crystal structure of $\mathbf{B}^{\text {Et }}$. Furthermore, DFT transition state optimization (B3LYP/6-311+g(d,p)) were performed for all derivatives. Therefore, the structure of the transition state was assumed to be a flat tricyclic aromatic system with the rotor twisted $90^{\circ}$ in respect to the stator, bridged by a single bond. Calculations gave for all three derivatives only a single imaginary frequency, which give a hint that the provided structure is similar to the real transition state, but do not necessarily represent reality.

Furthemore TD-DFT (CAMB3LYP-6311 $+\mathrm{g}(\mathrm{d})$ in PCM-MeCN) calculations were employed to simulate UV-vis spectra figure V.1.2.21) and assign the experimentally observed absorption bands to MO transitions. $258 \mid 259]$ By default gaussian estimates a deviation of $0.4 \mathrm{eV}$ from the calculated value. 260 Therefore, the simulated spectra show pretty accurate predicted uv-vis band, although the the wavelength in $10 \mathrm{~nm}$ to short on average. Merely the phenyl derivative show higher deviation from the empirically observed wavelength, as well as the difference to $\mathbf{B}^{\mathbf{E t}}$ is to small with only $7 \mathrm{~nm}$ instead of $22 \mathrm{~nm}$. But this is probably attributed to the rather simple used level of TD-DFT calculation for here investigated system. However, it is worth to mention, that there are more accurate and complex methods that can better handle an intramolecular charge transfer. 
(a)

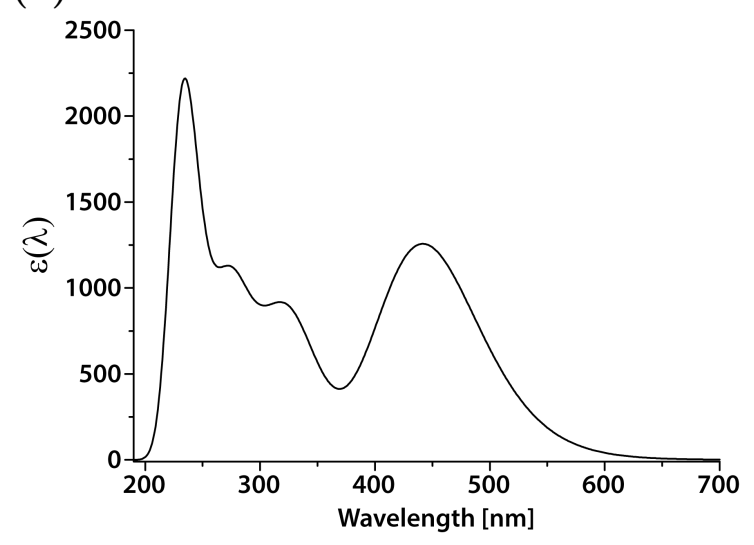

(c)

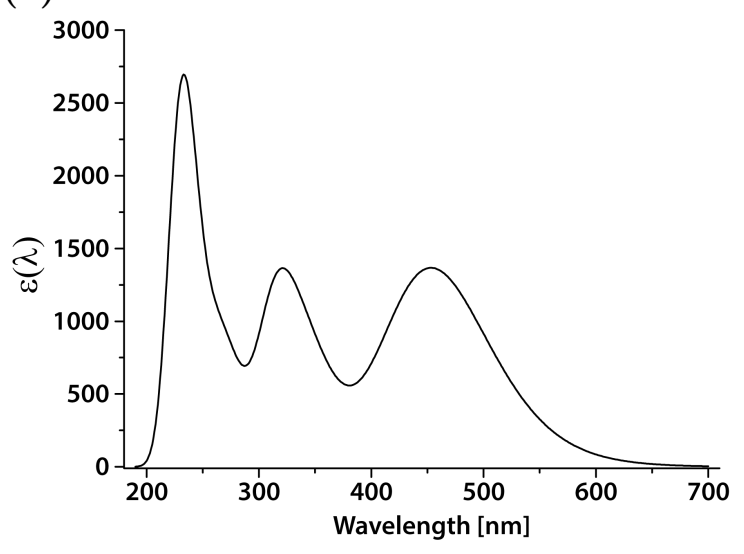

(b)

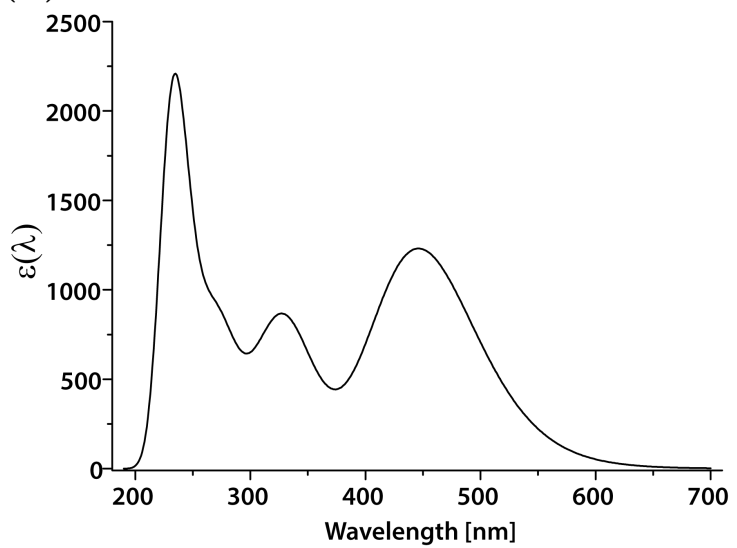

(d)

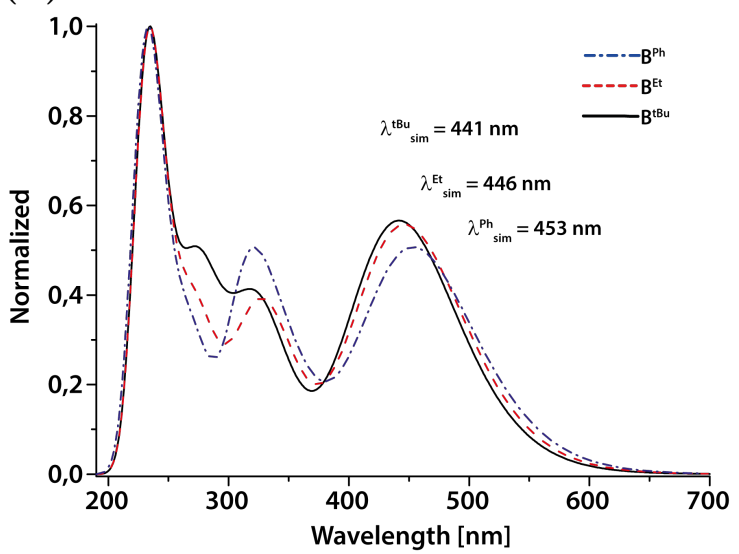

Figure V.1.2.21: Simulated UV-vis spectra from TD-DFT calculations (CAM B3LYP-6311+g(d) in PCM-MeCN) for $\left.\mathbf{a}) \mathbf{B}^{t \mathbf{B u}}, \mathbf{b}\right) \mathbf{B}^{\mathbf{E t}}$ and $\left.\mathbf{c}\right) \mathbf{B}^{\mathbf{P h}}$ made with Gaussien '09.261. d) Comparison of the simulated UV-vis spectra.

Table V.1.2.3: Comparison of HOMO-/ LUMO-energy levels for all three derivatives. All values are given in eV. [*] HOMO and LUMO energies were calculated from CV measurments (figure V.1.2.16). [**] Optical HOMO/LUMO gap were calculated from $\lambda_{\max }$ values observed in the UV-vis measurements.

\begin{tabular}{lccccccc}
\hline & $\begin{array}{c}\mathrm{E}_{\mathrm{HOMO}} \\
(\mathrm{DFT})\end{array}$ & $\begin{array}{c}\mathrm{E}_{\mathrm{LUMO}} \\
(\mathrm{DFT})\end{array}$ & $\begin{array}{c}\mathrm{E}_{\mathrm{g}} \\
(\mathrm{DFT})\end{array}$ & $\begin{array}{c}\mathrm{E}_{\mathrm{HOMO}} \\
(\mathrm{CV})^{[*]}\end{array}$ & $\begin{array}{c}\mathrm{E}_{\mathrm{LUMO}} \\
(\mathrm{CV})^{[*]}\end{array}$ & $\begin{array}{c}\mathrm{E}_{\mathrm{g}} \\
(\mathrm{CV})\end{array}$ & $\begin{array}{c}\mathrm{E}_{\mathrm{g}} \\
(\mathrm{opt})^{[* *}\end{array}$ \\
\hline \hline $\mathbf{B}^{\mathbf{t B u}}$ & -5.82 & -2.58 & 3.24 & -5.43 & -3.28 & 2.15 & 2.76 \\
$\mathbf{B}^{\mathbf{E t}}$ & -5.87 & -2.66 & 3.21 & -5.45 & -3.33 & 2.12 & 2.73 \\
$\mathbf{B}^{\mathbf{P h}}$ & -5.94 & -2.78 & 3.16 & -5.47 & -3.47 & 2.00 & 2.60 \\
\hline
\end{tabular}

Anyway, as expected, modifications of the acceptor moiety mainly affects the LUMO energies with $\mathrm{E}_{\mathrm{LUMO}}$ increasing from $-2.78 \mathrm{eV}$ for $\mathbf{B}^{\mathrm{Ph}}$ over $-2.66 \mathrm{eV}$ for $\mathbf{B}^{\mathrm{Et}}$ to -2.58 $\mathrm{eV}$ for $\mathbf{B}^{t \mathrm{Bu}}$. All values, empirically or in silico determined, are shown in table V.1.2.3 Although the values from $\mathrm{CV}$ measurements are underestimated, while the calculated ones are overestimated in respect to the energies received from UV-vis experiments, all together have the trend. Energy of HOMO-LUMO gap is decreased from $\mathbf{B}^{t \mathbf{B u}}$ over $\mathbf{B}^{\mathbf{E t}}$ to $\mathbf{B}^{\mathrm{Ph}}$. 


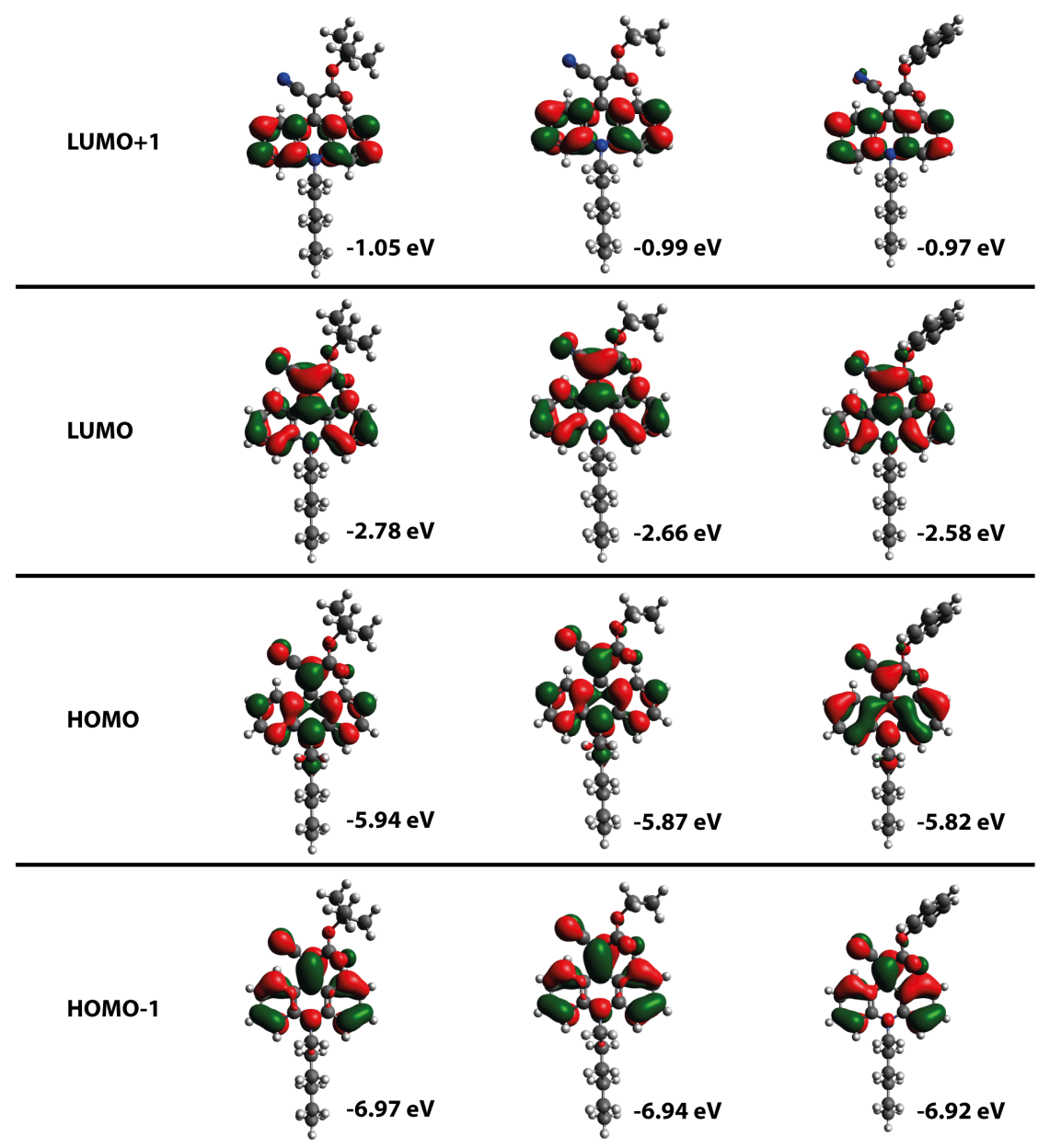

Figure V.1.2.22: Calculated frontier orbitals (B3LYP/6-311+g(d) in PCM-MeCN) of all examined derivatives in comparison. The energy level of the respective molecular orbitals is displayed next to the MO visualisation. 


\section{V.1.3 Protonation-Induced Fluorescence}

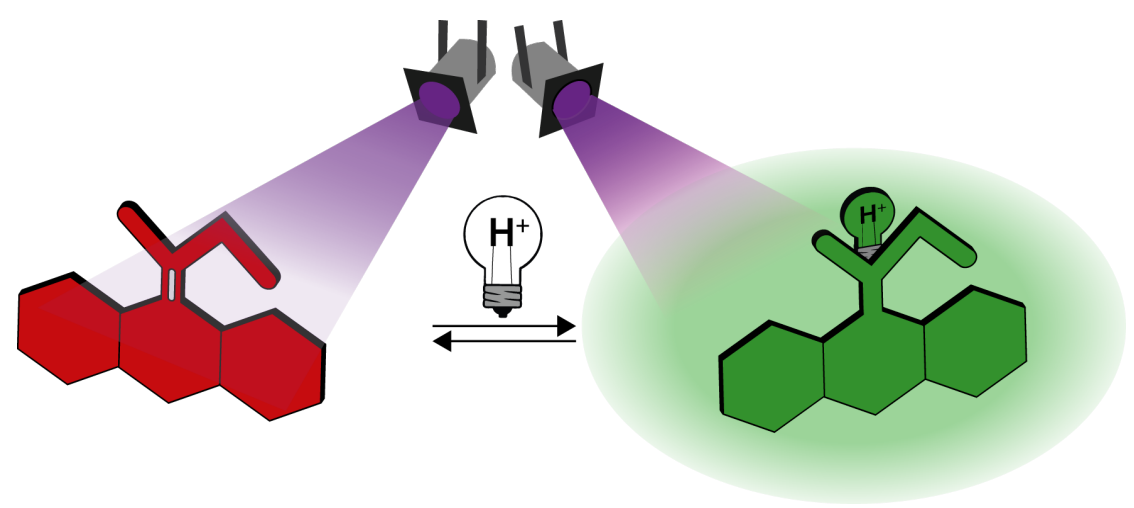

Due to the idea of the charge-separated zwitterionic transistion state, the question appeared if the negativ charge, located at the rotating group, can be used to protonate the system. And if this is possible, what influence the different ester substituent have on the grade of basicity. Dimorth et al. described a possible protonation of a similar system with weak acids, associated with a change in color.239. First tries with weak acids like ammonium chloride and acetic acid didn't lead to the desired result. A slight change in color of the solution was observable, which is rather attributed to the change in the allover polarity of the solution due to the addition polar acids molecules, which leads to a better stabilization of the charge-separated state, as described in section V.1.2.3. Anyway, using hydrochloric acid, a change in color from dark red to dark green was observed. Unfortunatly NMR delivered no usable information, since the spectrum showed a lot new signals which couldn't be assigned to any species. The usage of even stronger acids with non-coordinating conjugated bases, like trifluor acetic acid, showed a tremendous change in color. The addition of TFA led to change in color from dark red to slight greenish clear solution. Furthermore, upon irridation with ultraviolet light at $365 \mathrm{~nm}$ the solution showed a strong fluorescence (figure V.1.3.23a). Intrestingly, all derivatives (34, 35 and 37) showed the same reaction upon the addition of an excess of TFA. Comparing the acidified solution in UV-vis, all three derivates showed exactly the same spectra, with a absorbtion maxima at $\lambda_{\max }=260 \mathrm{~nm}$ and a second one at $\lambda_{\max }=370 \mathrm{~nm}$ (figure V.1.3.23c Additional invegstigation of the solutions via NMR showed a very similar chemical shift of all aromatic protons, as well as for the methylene group attached to the aromatic nitrogen. These results leaves only the conclusion that in all three cases the similar structural motif must be formed. Comparing the measured UV-vis spectra with similar compounds from literature revealed that the observed absorption bands are highly characteristic for the acridinium motif, where a positive charge is located on the nitrogen of the tricyclic backbone. $262 \mid 263$ 
(a)

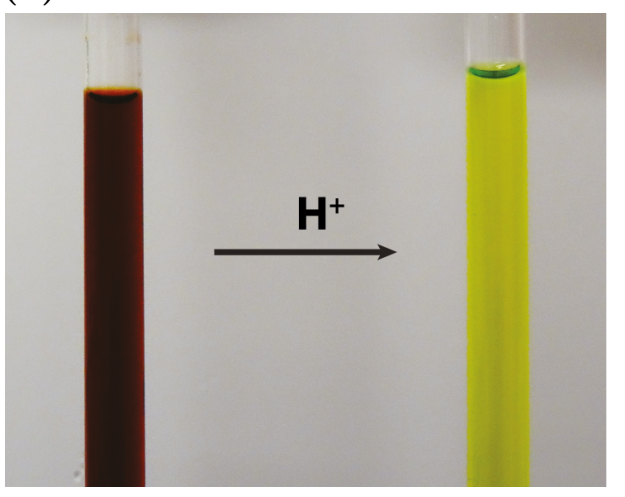

(b)

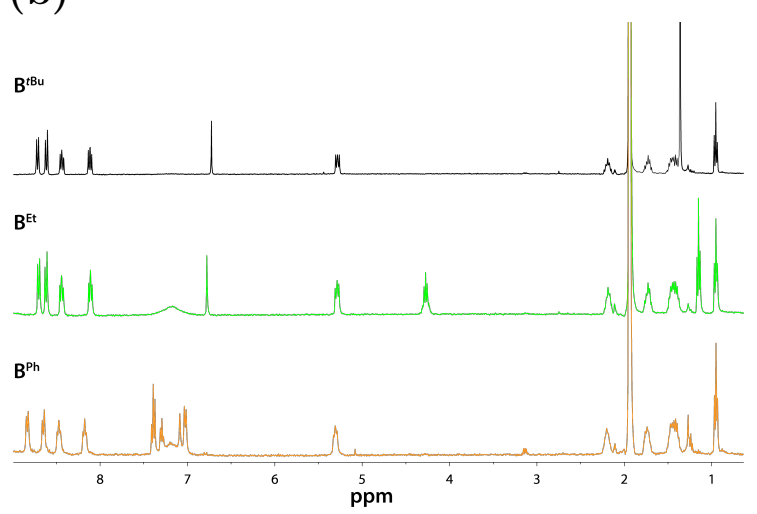

$365 \mathrm{~nm}$

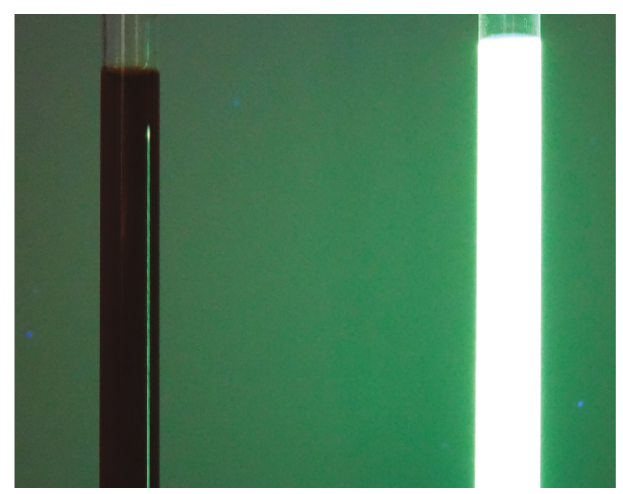

(c)

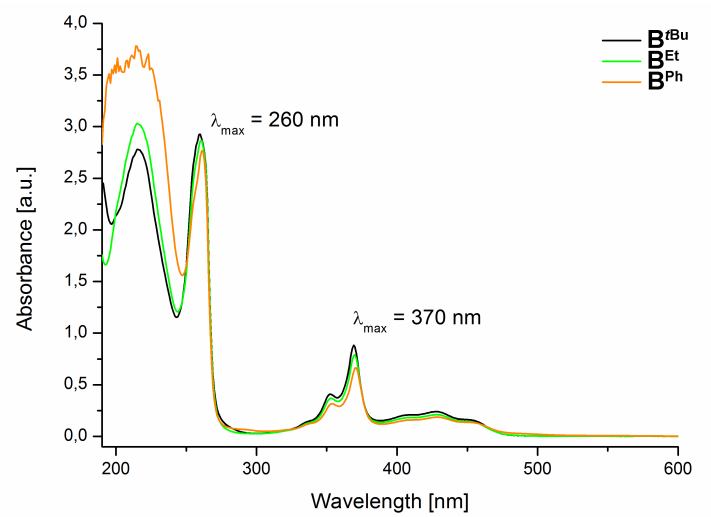

Figure V.1.3.23: a) Protonation of $\mathbf{B}^{\mathrm{Et}}$ with TFA leads to change in color (left). Upon irradiation with UV light $(1=365 \mathrm{~nm})$, the protonated species shows a strong fluorescence (right). b) Comparison ${ }^{\mathbf{1}} \mathbf{H}-\mathbf{N M R}$ spectra of protonated derivatives. textbfB ${ }^{t \mathrm{Bu}}$ (black), $\mathbf{B}^{\mathbf{E t}}$ (green), $\mathbf{B}^{\mathbf{P h}}$ (orange). $2.8 \mathrm{mM}$ in $d^{3}$-MeCN with $0.2 \mathrm{M}$ TFA c) Comparison UV-vis spectra of protonated derivatives. $\mathbf{B}^{t \mathbf{B u}}$ (black), $\mathbf{B}^{\mathbf{E t}}$ (green), $\mathbf{B}^{\mathbf{P h}}$ (orange). $0.05 \mathrm{~m} \overline{\mathrm{M}}$ in $\mathrm{MeCN}$ with $0.2 \mathrm{M} \mid \mathrm{TFA}$

\section{V.1.3.1 Position of Protonation}

In order to figure out where the protonation within the system occurs, the compounds 34, 35 and $\mathbf{3 7}$ were investigated by NMR in acidified solutions. In scheme V.1.3.7 the two possible positions of protonation are proposed. In theory both positions are feasible, since in both cases the negative charge, generated in the charge-separated state, would be stabilized. Furthermore, in both cases the bridging double bond is reduced to single bond, by what the system is loosing its chirality and the cyano ester group can rotate freely. However, compound $\mathbf{5 8}$ is a very unlikley system, since no comparable motif could be found in literature and was just here presented for the sake of completeness. 


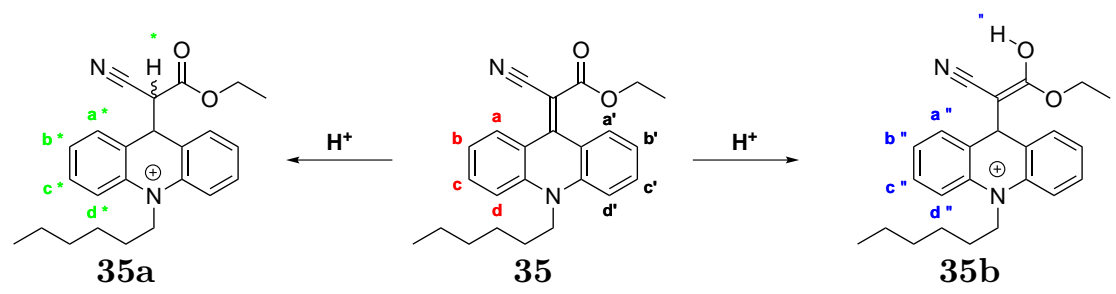

Scheme V.1.3.7: Protonation of 35. The two possible species protonated species $\mathbf{3 5 a}$ or $\mathbf{3 5} \mathbf{b}$, where the double bond is suspended.

Comparison of the $\mathrm{NMR}$ spectra of the non-protonated $\mathbf{B}^{\mathbf{E t}}(\mathbf{3 5})$ and the protonated species, show a general downfield shift of all aromatic protons upon addition of acid. It is noticeable that signal for the hydrogen atoms of $\mathbf{d}$ and the methylene group $\mathbf{N C H}_{\mathbf{2}}$, both located closest to the aromatic nitrogen, experience the highest chemical downfield shift (figure V.1.3.24). What is in consensus with the proposed formation of an postively charged nitrogen within the acridinium motif. Additionally a new signal appeared at ca. $6.75 \mathrm{ppm}(*)$. The new signal $(*)$ corresponded to the protonated potions.

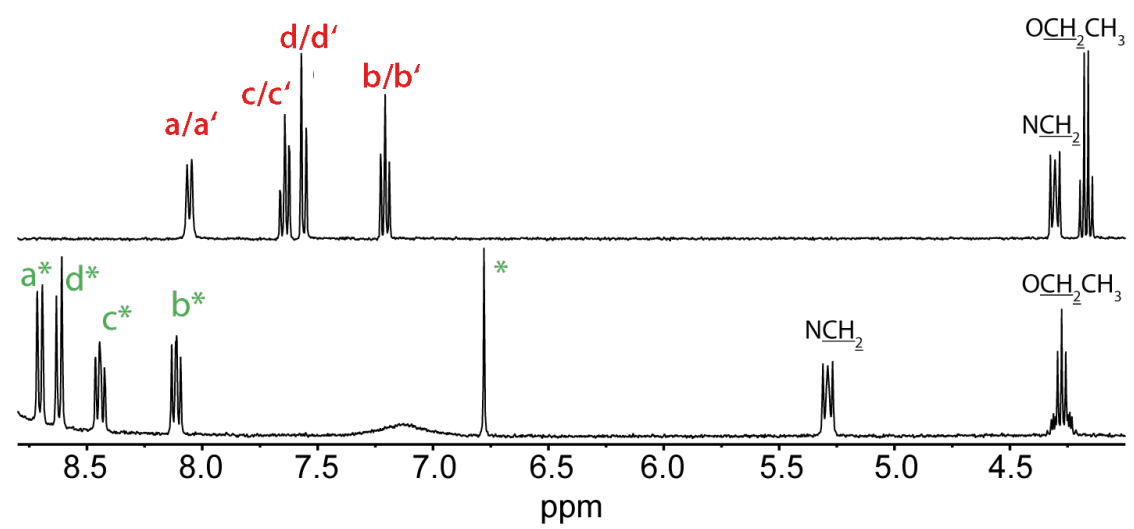

Figure V.1.3.24: Comparison of $\mathbf{3 5}$ and its protonated species $\mathbf{3 5 a}$ or $\mathbf{3 5 b}$. $2.8 \mathrm{mM}$ in $d^{3}-\mathrm{MeCN}$ (top) and with addtional $0.5 \mathrm{M}$ TFA (bottom).

To find out the exact position, where the rotating groups are protonated, various ${ }^{1} \mathrm{H}$ and ${ }^{13} \mathrm{C}$ NMR experiments were performed ffigure V.1.3.25). After assigning all ${ }^{1} \mathrm{H}$ and ${ }^{13} \mathrm{C}$ signals figures V.1.3.25a and V.1.3.25b, the 2D NMR experiments led to the conclusion that 35a was formed, as expected. Following findings speak for the formation of 35a. First of all in the ${ }^{1} \mathrm{H}-{ }^{1} \mathrm{H}-\mathrm{COSY}$ experiment figure V.1.3.25c only the diagonal peak is observable for signal $*$, what indicates that proton is isolated, since no coupling to other protons occurs. In the ${ }^{1} \mathrm{H}-{ }^{13} \mathrm{C}-\mathrm{HSQC}$ experiment, the signal $\left(^{*}\right)$ couples to signal 8 , what is assumed to be the non-aromatic bridging carbon of the substituent figure V.1.3.25d, therefore a protonation at the ester carbonoxy group can be excluded. The ${ }^{1} \mathrm{H}_{-}{ }^{13} \mathrm{C}-\mathrm{HMBC}$ confirmed this assumption, since signal ${ }^{*}$ ) couples to the carbon signals 6, 7, 9 and 10 what is only possible from assumed position. 
(a)

(b)
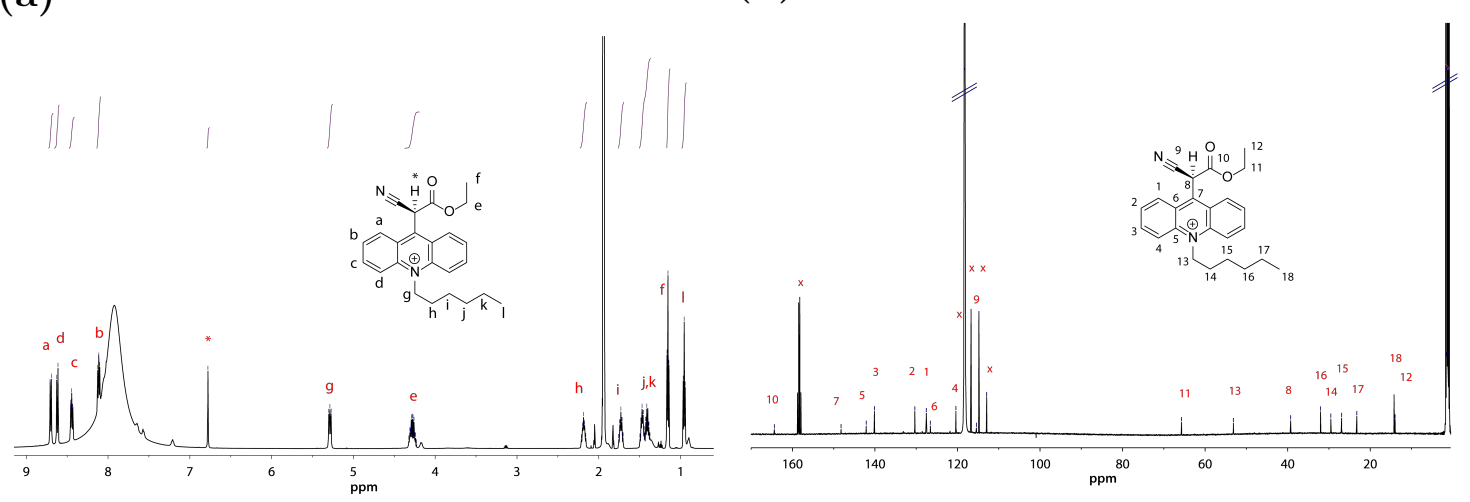

(c)

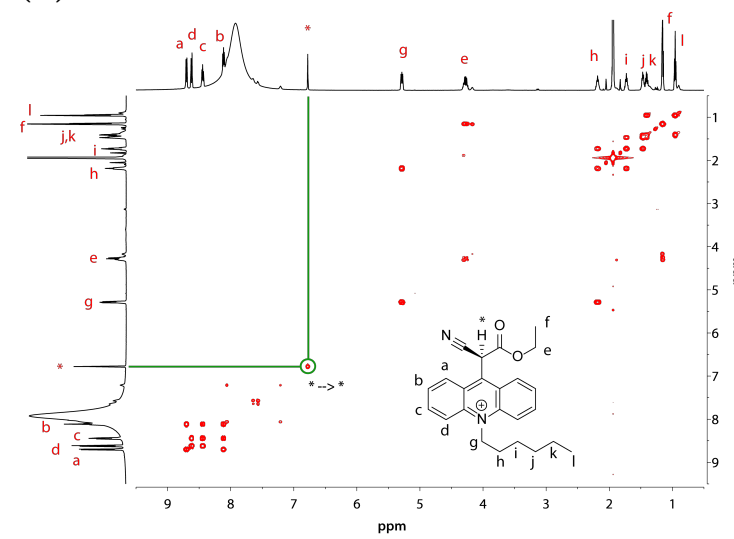

(d)

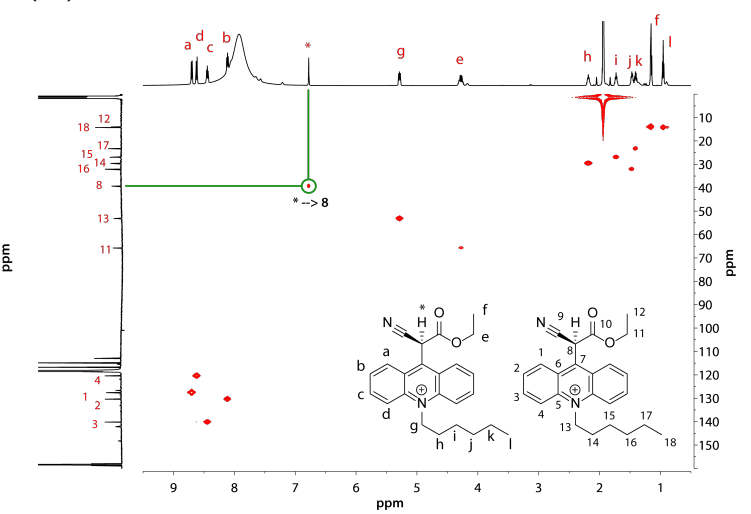

Figure V.1.3.25: a) ${ }^{\mathbf{1}} \mathbf{H}$ NMR $(400 \mathrm{MHz})$ of protonated $\mathbf{B}^{\mathbf{E t}}(\mathbf{3 5 a}) 2.8 \mathrm{mM}$ in $d^{3}$-MeCN with $140 \mathrm{mM}$ trifluoroacetic acid (TFA) b) ${ }^{13} \mathrm{C}-\mathrm{NMR}(176 \mathrm{MHz})$ of protonated $\mathbf{B}^{\mathrm{Et}}$ (35a) $2.8 \mathrm{mM}$ in $\mathrm{d}^{3}$-MeCN with $140 \mathrm{mM}$ TFA The signals marked with $\mathrm{x}$ belong to the TFA molecules. Splitting of these signals occurs due to ${ }^{13} \mathrm{C}-{ }^{19} \mathrm{~F}$ coupling. c) ${ }^{1} \mathrm{H}-{ }^{1} \mathrm{H}-\mathrm{COSY}$ of protonated $\mathbf{B}^{\mathbf{E t}}$ (35a) $2.8 \mathrm{mM}$ in $d^{3}$-MeCN with $140 \mathrm{mM}$ TFA The *-proton shows no coupling to other protons, what supports its isolated positioning at the rotor headgroup. d) ${ }^{1} \mathrm{H}_{-}{ }^{13} \mathrm{C}-\mathrm{HSQC}$ of protonated $\mathbf{B}^{\mathrm{Et}}$ (35a) $2.8 \mathrm{mM}$ in $d^{3}-\mathrm{MeCN}$ with $140 \mathrm{mM}$ TFA The *-proton couples to a C-atom, which is assumed to be at the postion 8 . 


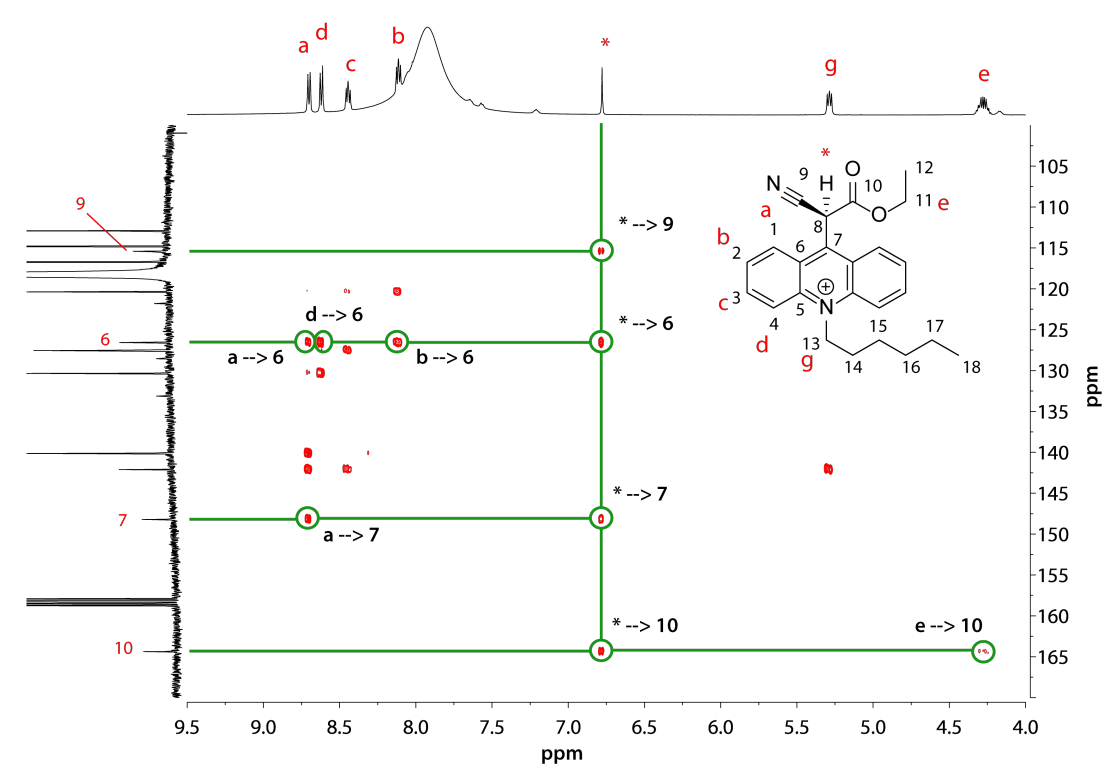

Figure V.1.3.26: ${ }^{1} \mathrm{H}^{13} \mathrm{C}-\mathrm{HMBC}$ of protonated $\mathbf{B}^{\mathbf{E t}}$ (35a) $2.8 \mathrm{mM}$ in $d^{3}$-MeCN with $140 \mathrm{mM}$ TFA Since there are no protons that can couple to the C-atom at position 8 , the coupling of the proton signal * to neighbored C-atoms were investigated. All ${ }^{2} J$ and ${ }^{3} J$ couplings of signal $*$ are marked with green lines. Additionally, some other proton carbon couplings are highlighted to confirm the right assignment of the ${ }^{13} \mathrm{C}$ signals.

\section{V.1.3.2 ${ }^{1} \mathrm{H}$ NMR titration experiments with TFA}

In order to get more information about the process of protonation and especially the differences among the three derivatives $\mathbf{B}^{t \mathbf{B u}}, \mathbf{B}^{\mathbf{E t}}$ and $\mathbf{B}^{\mathbf{P h}}$, NMR titration experiments were performed (figures V.1.3.27, V.1.3.28 and V.1.3.30). In contrast to the VT NMR experiments from section V.1.2.2, all described experiments were in MeCN, due to solubility issues of the charged species in THF. Following the process of protonation it is noticeable, that $\mathbf{B}^{\mathrm{Et}}$ seems to form the protonated species without any intermediates or byproduct, what is confirmed by UV-vis section V.1.3.3). Furthermore, the reaction equilibrium is slowly shifted towards the protonated species with increasing TFA concentrations. At a concentration of aprrox. $13 \mathrm{mM}$ first peaks of the protonated species appear for $\mathbf{B}^{t \mathbf{B u}}$ figure V.1.3.27 and $\mathbf{B}^{\mathbf{E t}}$ figure V.1.3.28). In contrast $\mathbf{B}^{\mathbf{P h}}$ first signal of the protonated species appear at a concentration of approx. $54 \mathrm{mM}$ TFA figure V.1.3.30, giving rise to the first evidence that the better stabilized negative charge is harder to be protonated. In the case $\mathbf{B}^{t \mathbf{B u}}$ and $\mathbf{B}^{\mathbf{E t}}$ the non-protonated species is not detectable anymore at a concentration above $130 \mathrm{mM}$, unluckily the steps in concentration are too far apart to make out a further difference between both derivatives. In contrary, $\mathbf{B}^{\mathbf{P h}}$ the signals of the non-protonated species disappearing first at higher concentration above $200 \mathrm{mM}$, as expected. 


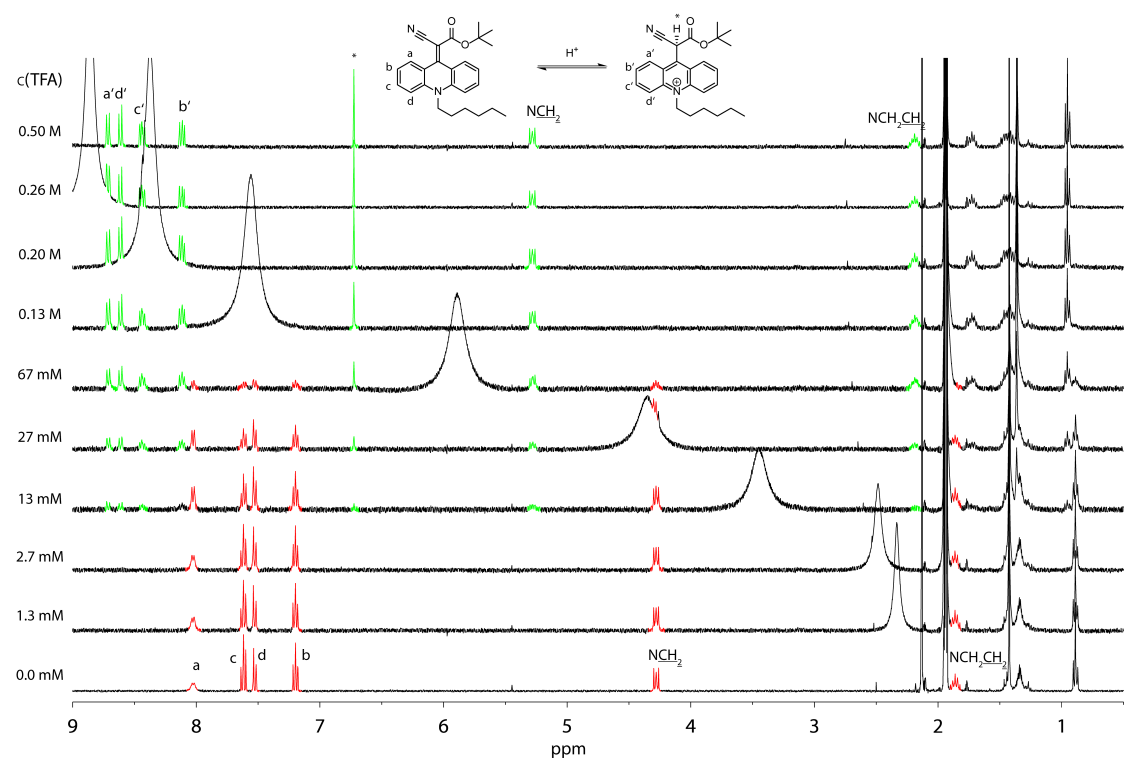

Figure V.1.3.27: ${ }^{1} \mathrm{H}$ NMR titration experiment of $2.8 \mathrm{mM} 34$ with TFA in deuterated MeCN. The titration was proceeded to an final TFA concentration of $0.5 \mathrm{M}$. It is clearly visible, that 34 (red) is converted into a new protonated species (green) by increasing the TFA concentration. All aromatic protons experience significant upflied shift, where signals for the protons $\mathbf{b}, \mathbf{d}$ and $\mathbf{N C H}_{\mathbf{2}}$ show the highest upfield shift.

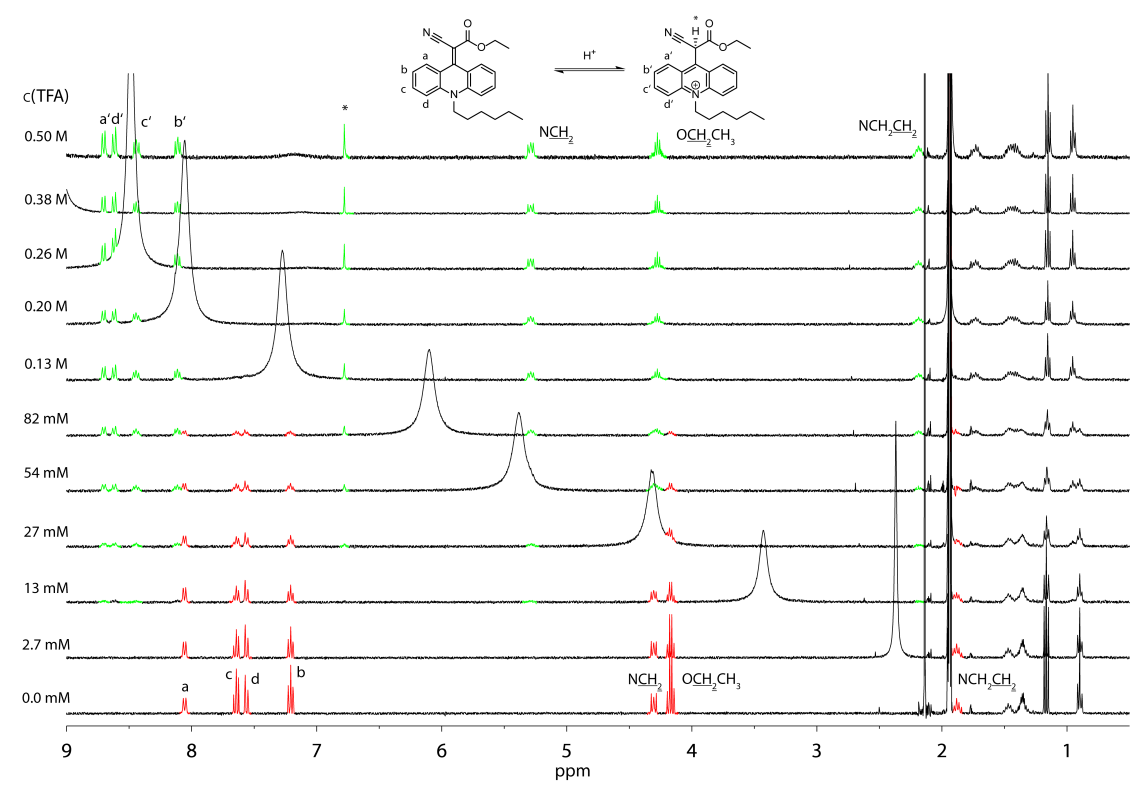

Figure V.1.3.28: ${ }^{1} \mathrm{H}$ NMR titration experiment of $2.8 \mathrm{mM} 35$ with TFA in deuterated MeCN. The titration was proceeded to an final TFA concentration of $0.5 \mathrm{M}$. It is clearly visible, that $\mathbf{3 5}$ (red) is converted into a new protonated species (green) by increasing the TFA concentration. All aromatic protons experience significant upfield shift, where signals for the protons $\mathbf{b}, \mathbf{d}$ and $\mathbf{N C H}_{\mathbf{2}}$ show the highest upfield shift.

Additonally to the titration experiments, the reversibility of the protonation reaction of the system was also investigated. For this reason a further titration experiment was performed on $\mathbf{B}^{\mathbf{E t}}$, exemplaric for all derivatives figure V.1.3.29). The compound solution was acidified with 125 equivalents of TFA till the derivative was completely protonated, subsequently 125 equivalents of the base triethylamine was added in several steps to 
neutralize the excess of TFA and deprotonate the protonated species of $\mathbf{B}^{\mathrm{Et}}$. In fact, already after first addition of the base (25 equiv. $\mathrm{NEt}_{3}$ ) the signals of $\mathbf{B}^{\mathrm{Et}}$ reappeared. After the second addition (50 equiv. $\mathrm{NEt}_{3}$ ) the deprotonated species was already completely formed back.

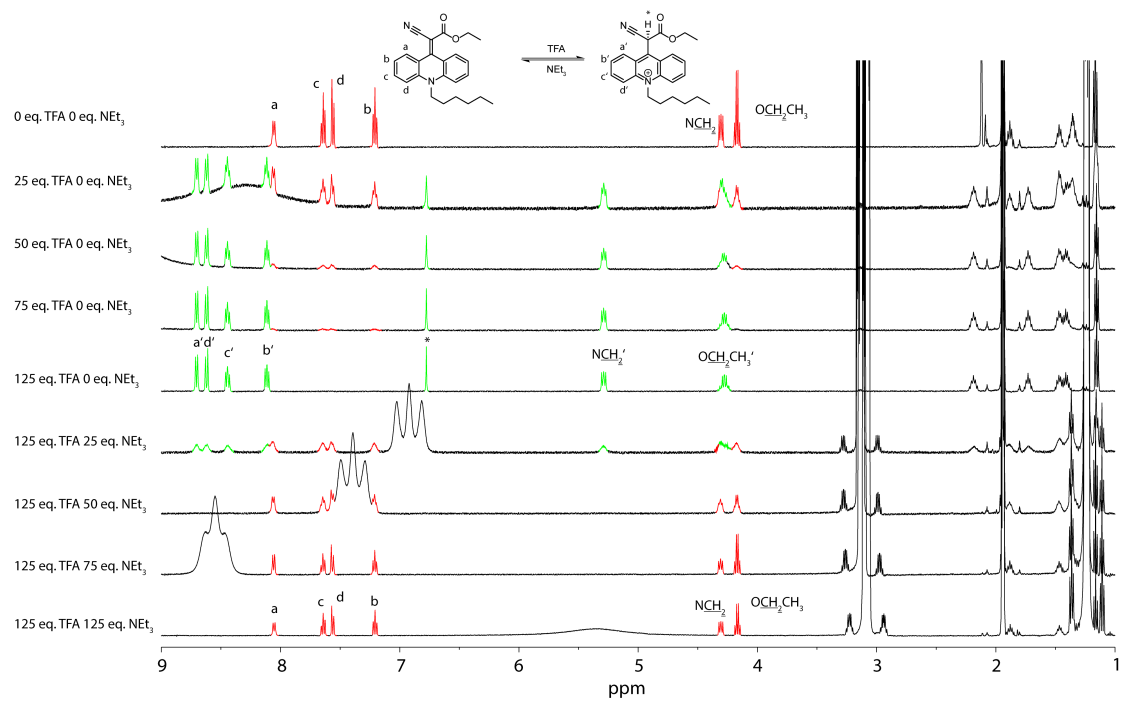

Figure V.1.3.29: ${ }^{1} \mathrm{H}$ NMR titration experiment of $2.8 \mathrm{mM} 35$ with TFA in deuterated MeCN. TFA was added until no unprotonated species (red) was detected in NMR anymore. After, NEt 3 was added until all TFA was completly neutralized and the unprotonated species was regained. This experiment proves the easy reversibility of the protonation process and the forth and back conversion between acridinylidene and acrdinium species.

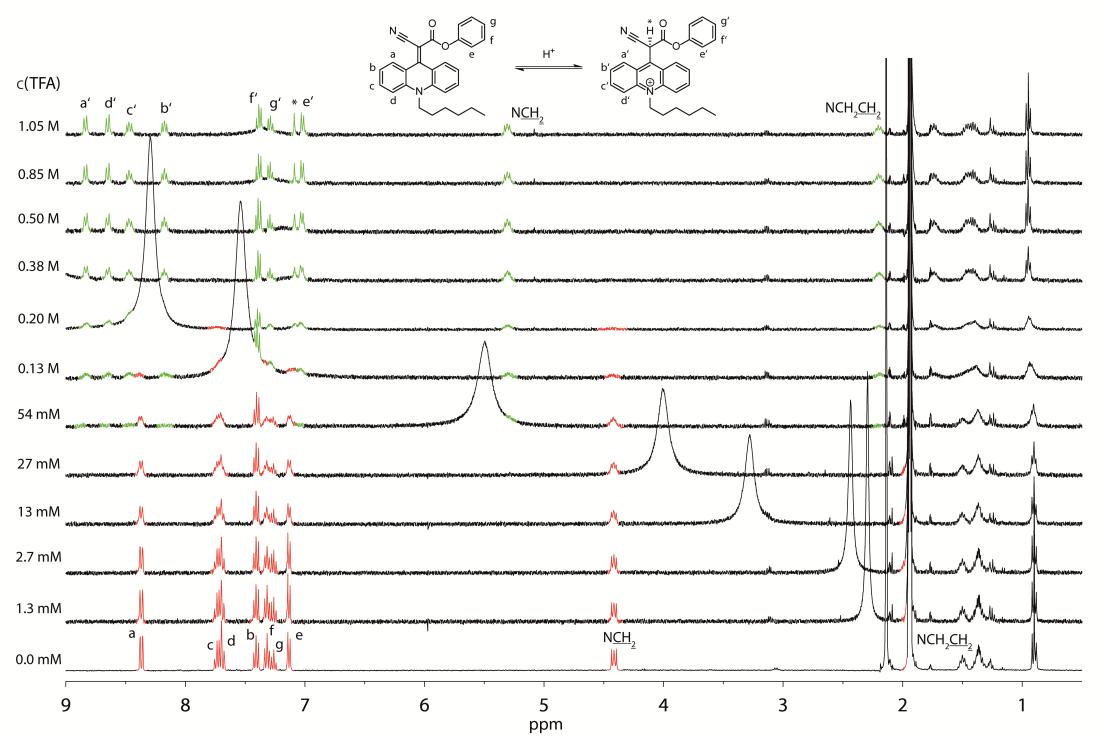

Figure V.1.3.30: ${ }^{1} \mathrm{H}$ NMR titration experiment of $2.8 \mathrm{mM} 37$ with TFA in deuterated MeCN. The titration was proceeded to an final TFA concentration of $1.05 \mathrm{M}$. It is clearly visible, that $\mathbf{3 7}$ (red) is converted into a new protonated species (green) by increasing the TFA concentration. All aromatic protons experience significant upfield shift, where signals for the protons $\mathbf{b}, \mathbf{d}$ and $\mathbf{N C H}_{\mathbf{2}}$ show the highest upfield shift. 


\section{V.1.3.3 Determination of associations constants from UV-vis titration experiments with TFA}

Additionally to the NMR experiments described in the previous section, the process of protonation for the derivatives $\left(\mathbf{B}^{t \mathbf{B u}}, \mathbf{B}^{\mathrm{Et}}, \mathbf{B}^{\mathrm{Ph}}\right)$ was examined in $\mathrm{UV}$-vis experiments. In all three cases TFA was added stepwise to a solution of $\left(\mathbf{B}^{\text {tBu }}, \mathbf{B}^{\mathrm{Et}}\right.$ or $\mathbf{B}^{\mathrm{Ph}}$ with an concentration of $0.035 \mathrm{mM}$, until no change in absorption was detectable. The absorption band at approx. $\lambda=450-470 \mathrm{~nm}$ show in all experiments a hypochromic shift as well as the absorption band $\lambda=290 \mathrm{~nm}$, whereas the acridinium specific absorption bands at $\lambda=370$ and $\lambda=260 \mathrm{~nm}$ arise. Moreover, isosbestic points can be found at the wavelength $\lambda_{\text {isosb }}=405 \mathrm{~nm}, \lambda_{\text {isosb }}=335 \mathrm{~nm}$ and $\lambda_{\text {isosb }}=270 \mathrm{~nm}$. This finding indicates a direct formation of the protonated species outgoing from starting material, in accordance with the ${ }^{1} \mathrm{H}$ NMR titration experiments described above section V.1.3.2.

(a)

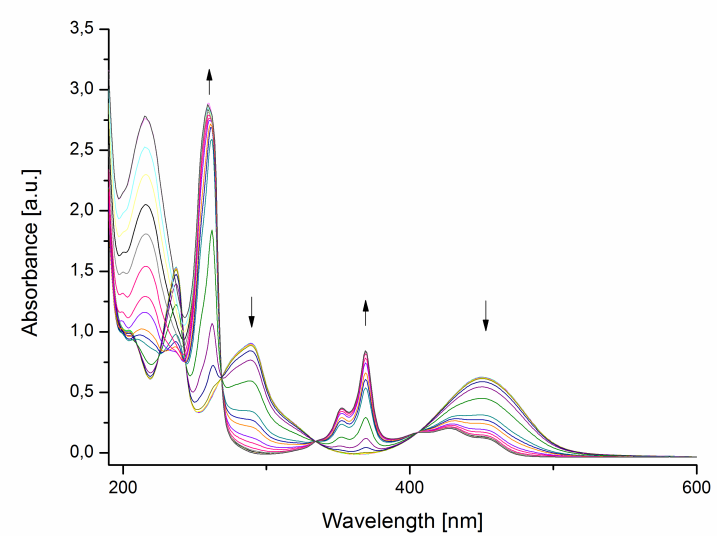

(b)

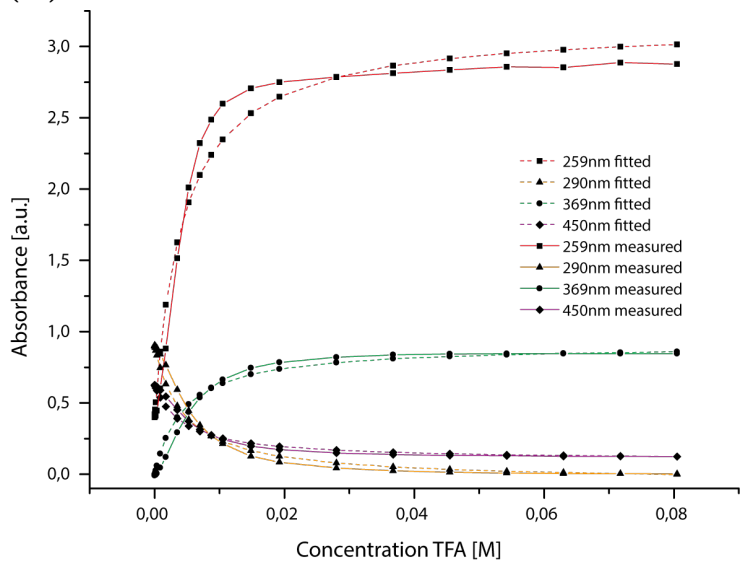

Figure V.1.3.31: a) UV-vis TFA titration of $\mathbf{B}^{t \mathbf{B u}}$ to protonated $\mathbf{B}^{t \mathbf{B u}} \mathbf{-} \mathbf{H}^{+}(\mathbf{3 4 a}), 0.035 \mathrm{mM}$ in $\mathrm{MeCN}$. Arrows indicate the hyper or hypochromic shift from low TFA - to high TFA concentration. b) Determination of association constant from UV-vis TFA titration experiment of $\mathbf{B}^{\text {tBu }}$. Data was globally fitted with BindFit v0.5. 264 265. Measured and fitted data points are shown for the wavelengths $259 \mathrm{~nm}, 290 \mathrm{~nm}, 369 \mathrm{~nm}$, 450nm. The determined constants are shown in table table V.1.3.4 
(a)

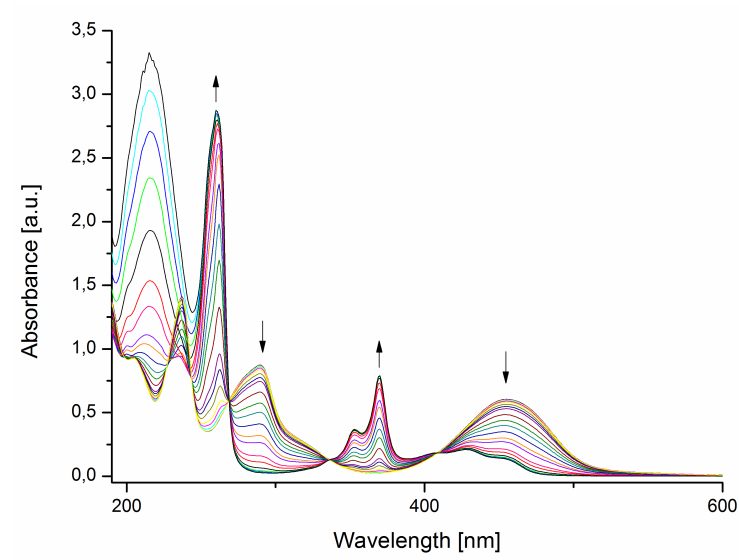

(b)

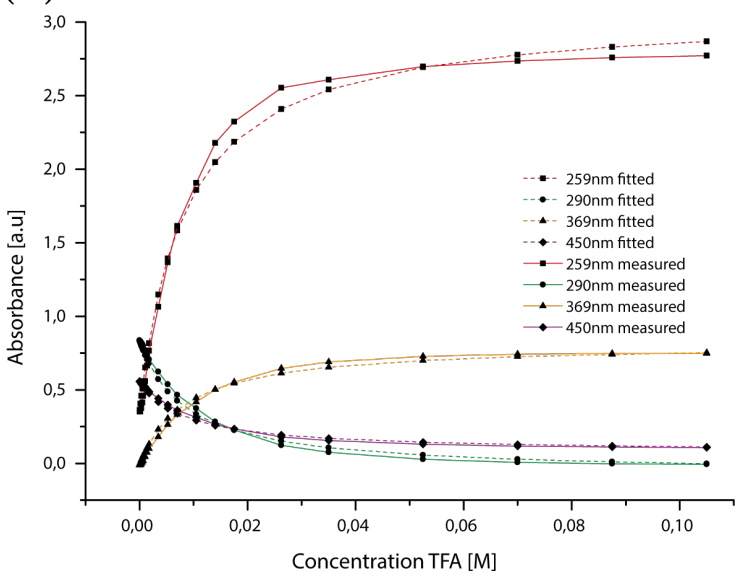

Figure V.1.3.32: a) UV-vis TFA titration of $\mathbf{B}^{\mathbf{E t}}$ to protonated $\mathbf{B}^{\mathbf{E t}} \mathbf{-} \mathbf{H}^{+}(\mathbf{3 5 a})$, $0.035 \mathrm{mM}$ in $\mathbf{M e C N}$. Arrows indicate the hyper - or hypochromic shift from low TFA - to high TFA concentration. b) Determination of association constant from UV-vis TFA titration experiment of $\mathbf{B}^{\mathbf{E t}}$. Data was globally fitted with BindFit v0.5. 264 265. Measured and fitted data points are shown for the wavelengths $259 \mathrm{~nm}, 290 \mathrm{~nm}, 369 \mathrm{~nm}, 450 \mathrm{~nm}$. The determined constants are shown in table V.1.3.4

In accordance to the NMR titration experiments, increasing amounts of acid are needed to fully convert the different derivatives into their corresponding protonated species. While the protonated $\mathbf{B}^{t \mathbf{B u}}$ was completely formed after 2300 equiv. of TFA (ca. $80 \mathrm{mM}$ ) figure V.1.3.31). $\mathbf{B}^{t \mathrm{Bu}}$ already needed approx. 700 more equivalents (ca. $105 \mathbf{B}^{t \mathrm{Bu}}$ ) to reach complete conversion (figure V.1.3.32. The highest concentration by far was used for $\mathbf{B}^{\mathrm{Ph}}$, no change in absorption was reached after approx. 5000 equiv. TFA (ca. $190 \mathrm{mM}$ ) figure V.1.3.33.

(a)

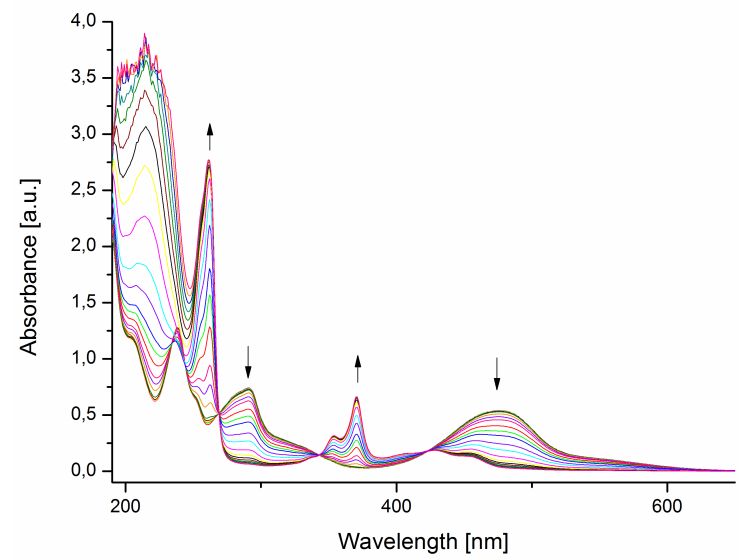

(b)

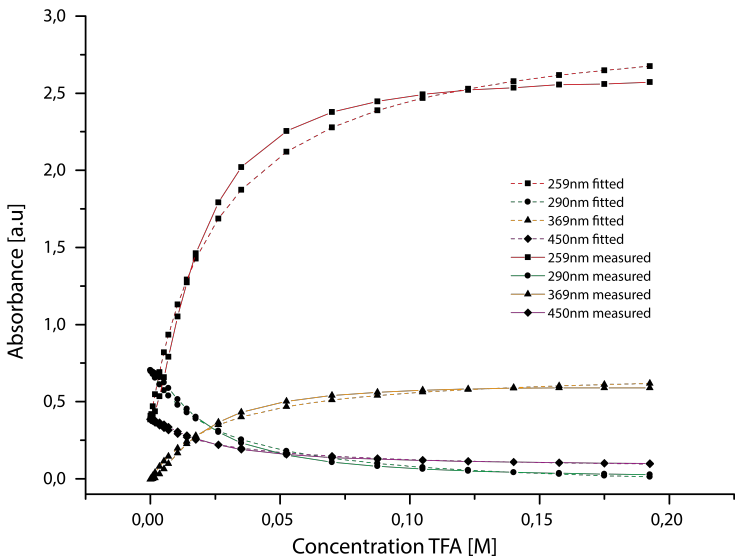

Figure V.1.3.33: a) UV-vis TFA titration of $\mathbf{B}^{\mathbf{P h}}$ to protonated $\mathbf{B}^{\mathbf{P h}} \mathbf{- \mathbf { H } ^ { + }}(\mathbf{3 7 a})$, $0.035 \mathrm{mM}$ in $\mathrm{MeCN}$. Arrows indicate the hyper or hypochromic shift from low TFA - to high TFA concentration. b) Determination of association constant from UV-vis TFA titration experiment of $\mathbf{B}^{\mathbf{P h}}$. Data was globally fitted with BindFit v0.5. 264 265 Measured and fitted data points are shown for the wavelengths $259 \mathrm{~nm}, 290 \mathrm{~nm}, 369 \mathrm{~nm}$, 450nm. The determined constants are shown in table V.1.3.4 
In order to determine the association constant of the protonation reaction for each derivative, the recoreded data from UV-vis titration experiments was fitted with the help of BindFit.264[265] On that account the concentration was plotted versus the corresponding absorption for the wavelengths 259nm, 290nm, 369nm, 450nm. In figures V.1.3.31b V.1.3.32b and V.1.3.33b the measured data is plotted together with the fitted data for corresponding derivate. All fits show a systematic divergence from the measured data, especially for the wavelength $\lambda=259 \mathrm{~nm}$. However, for the remaining wavelength the fit seems to match quite good. The association constants obtained from the fitted data are presented in table V.1.3.4 As expected the order association constant is $\mathbf{B}^{t \mathbf{B u}}>\mathbf{B}^{\mathrm{Et}}$ $>\mathbf{B}^{\mathbf{P h}}$. The easier the protonation can occur the higher the association constant. By implication, it is harder to protonate the better the negative charge is stabilized by the surrounding electron-withdrawing groups. Therefore, for the rotation, which is dependent on the stabilization of charge-separated structure, the order is reversed $\left(\mathbf{B}^{\mathbf{P h}}>\mathbf{B}^{\mathrm{Et}}>\right.$ $\left.\mathrm{B}^{t \mathrm{Bu}}\right)$.

Table V.1.3.4: Associations constants for protonation with TFA determined from UV-vis TFA titrations experiments for textbfB ${ }^{t \mathrm{Bu}}, \mathbf{B}^{\mathbf{E t}}$ and $\mathbf{B}^{\mathbf{P h}}$.

\begin{tabular}{cccc}
\hline & $\mathbf{B}^{t \mathbf{B u}}$ & $\mathbf{B}^{\mathrm{Et}}$ & $\mathbf{B}^{\mathbf{P h}}$ \\
\hline \hline $\mathrm{K}_{\mathrm{A}}{ }^{\text {TFA }}\left[\mathrm{mol}^{-1}\right]$ & $230.6 \pm 19.6$ & $118.7 \pm 5.3$ & $38.2 \pm 1.7$ \\
\hline
\end{tabular}

\section{V.1.3.4 Fluorescence titration experiments with TFA}

Besides the change in color, the here investigated compounds became fluorophores upon protonation, with a strong green fluorescene emission with maximum at $\lambda^{\mathrm{em}}=490 \mathrm{~nm}$, caused by the formation of the acidinium motif. 266. The dependency of the fluorescence properties on the concentration of acid was examined similar to the UV-vis experiments. To obtain the best excitation wavelength to get the maximal fluorescence, 3D fluorescence measurement were performed with $1 \mu \mathrm{M}$ in presence of $72.5 \mathrm{mM}$ TFA In these measurement fluorescence spectra from $400 \mathrm{~nm}$ to $700 \mathrm{~nm}$ were recorded at various excitation wavelength ranging from $220 \mathrm{~nm}$ to $400 \mathrm{~nm}$ in $5 \mathrm{~nm}$ increments. The results of these experiments show for all derivatives a maximum emission wavelength of $\lambda^{\mathrm{em}}=490 \mathrm{~nm}$ for the excitation wavelengths $\lambda^{\mathrm{ex}}=260 \mathrm{~nm}$ and $\lambda^{\mathrm{ex}}=370 \mathrm{~nm}$ (figures V.1.3.34a, V.1.3.35a and V.1.3.36a). It is noticeable that for both excitation wavelength the system is emitting at the same wavelengths $\left(\lambda^{\mathrm{em}}=490 \mathrm{~nm}\right)$. Therefore it is pretty likely, that a non-radiative internal conversion takes place for the both excited states to the same lower energy level, before the radiative process occurs. 
(a)

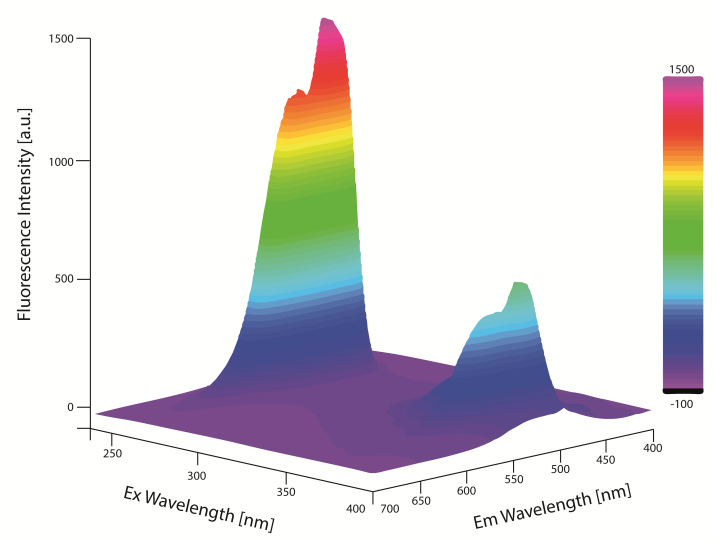

(c)

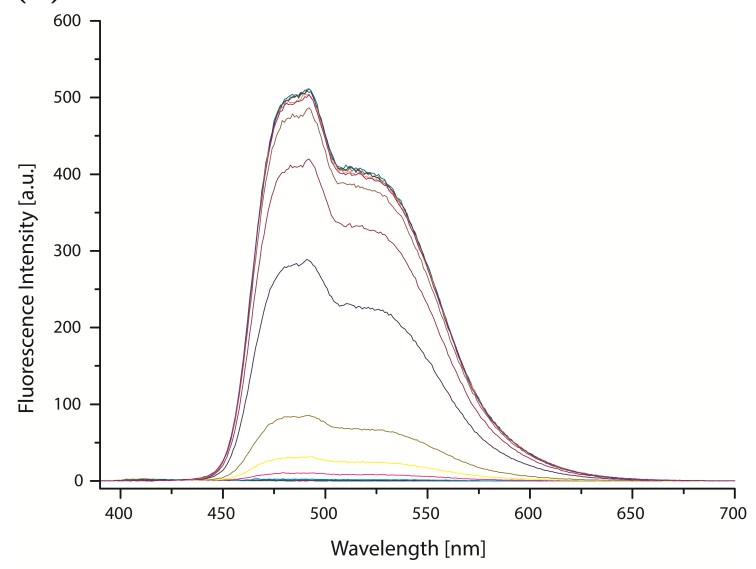

(b)

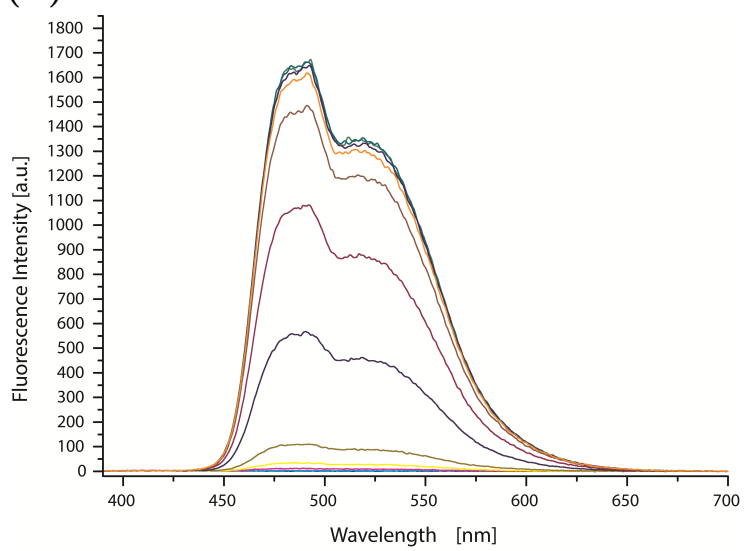

(d)

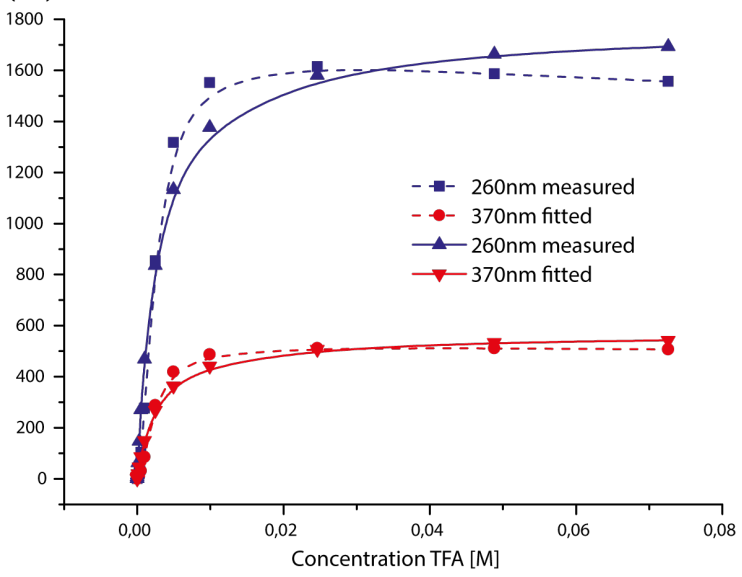

Figure V.1.3.34: a) 3D fluorescence measurement of $0.005 \mathrm{mM} \mathbf{B}^{t \mathrm{Bu}}$ in $\mathrm{MeCN}$ with $72.5 \mathrm{mM}$ TFA b) TFA fluorescence titration of $0.005 \mathrm{mM} \mathbf{B}^{t \mathbf{B u}}$ in MeCN, $\lambda^{\mathrm{ex}}: 260 \mathrm{~nm}$. c) TFA fluorescence titration $0.005 \mathrm{mM} \mathbf{B}^{t \mathrm{Bu}}$ in $\mathrm{MeCN}, \lambda^{\mathrm{ex}}: 370 \mathrm{~nm}$. d) Plot fluorescence intensity vs. TFA concentration of $\mathbf{B}^{t \mathbf{B u}}$ for the excitation wavelengths $\lambda^{\mathrm{ex}}=260 \mathrm{~nm}$ and $\lambda^{\mathrm{ex}}=370 \mathrm{~nm}$. Data was globally fitted with BindFit v0.5.264|265. Measured and fitted data points are shown for the emssion measured with excitation wavelengths $260 \mathrm{~nm}$ and $370 \mathrm{~nm}$. 
(a)

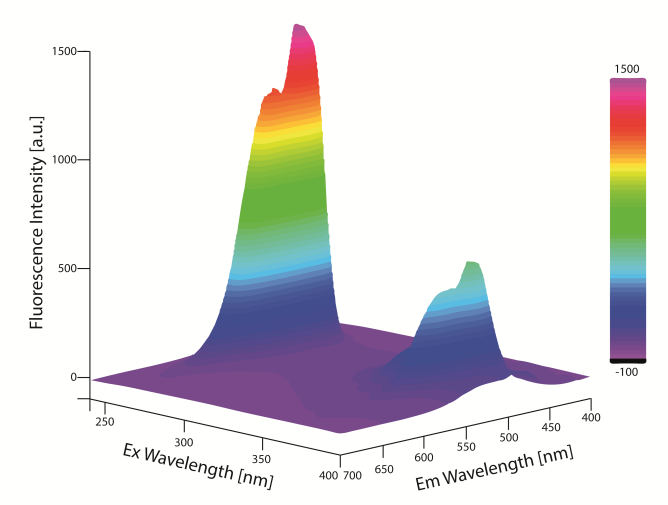

(c)

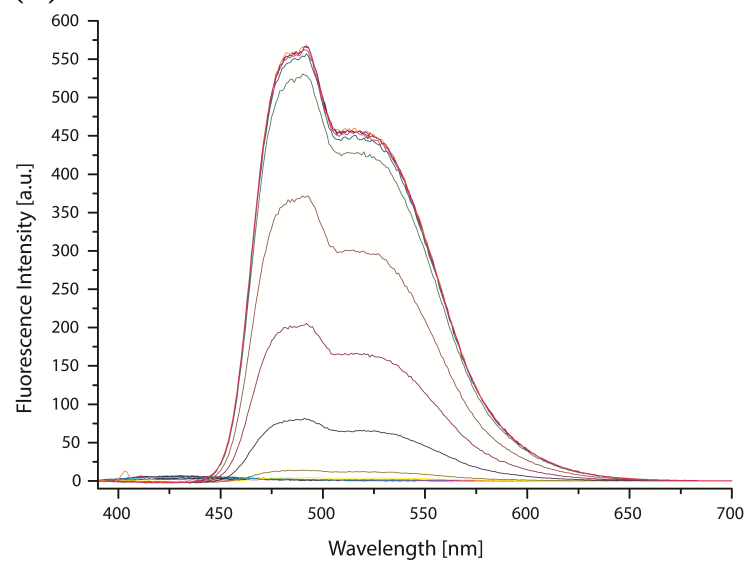

(b)

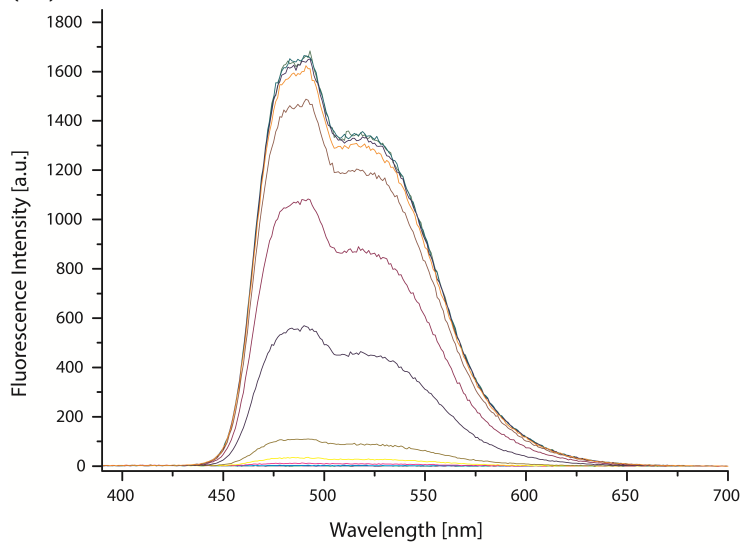

(d)

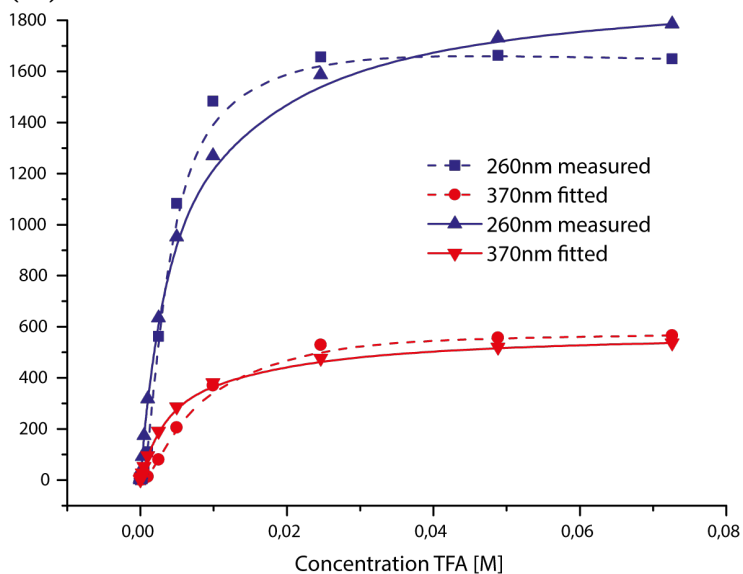

Figure V.1.3.35: a) 3D fluorescence measurement of $0.005 \mathrm{mM} \mathbf{B}^{\mathbf{E t}}$ in $\mathrm{MeCN}$ with $72.5 \mathrm{mM}$ TFA b) TFA fluorescence titration of $0.005 \mathrm{mM} \mathbf{B}^{\mathrm{Et}}$ in $\mathrm{MeCN}, \lambda^{\mathrm{ex}}: 260 \mathrm{~nm}$. c) TFA fluorescence titration $0.005 \mathrm{mM} \mathbf{B}^{\mathrm{Et}}$ in $\mathrm{MeCN}, \lambda^{\mathrm{ex}}: 370 \mathrm{~nm}$. d) Plot fluorescence intensity vs. TFA concentration of $\mathbf{B}^{\mathbf{E t}}$ for the excitation wavelengths $\lambda^{\mathrm{ex}}=260 \mathrm{~nm}$ and $\lambda^{\mathrm{ex}}=370 \mathrm{~nm}$. Data was globally fitted with BindFit v0.5.264|265] Measured and fitted data points are shown for the emssion measured with excitation wavelengths $260 \mathrm{~nm}$ and $370 \mathrm{~nm}$. 
(a)

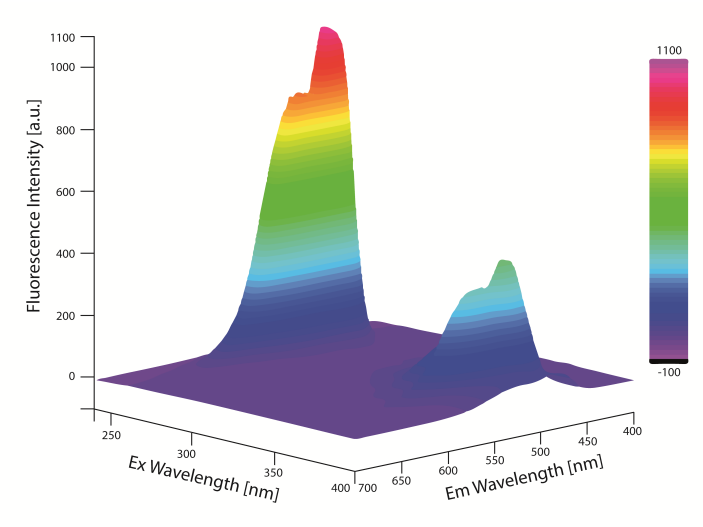

(c)

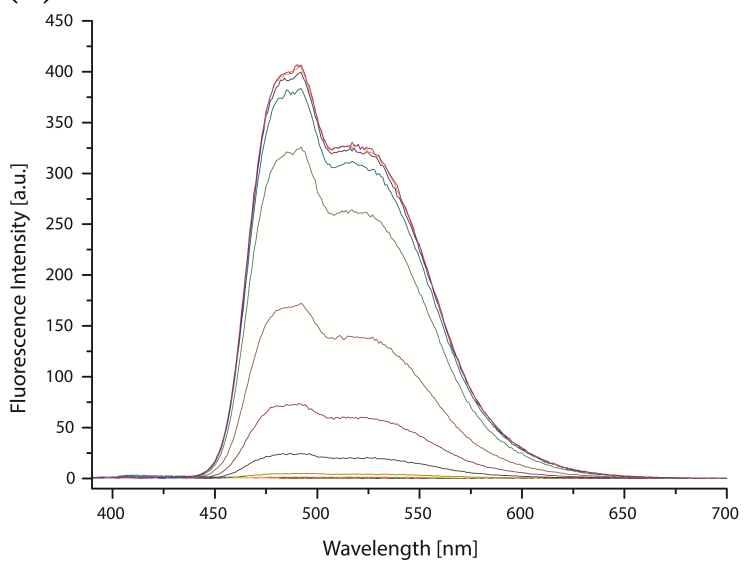

(b)

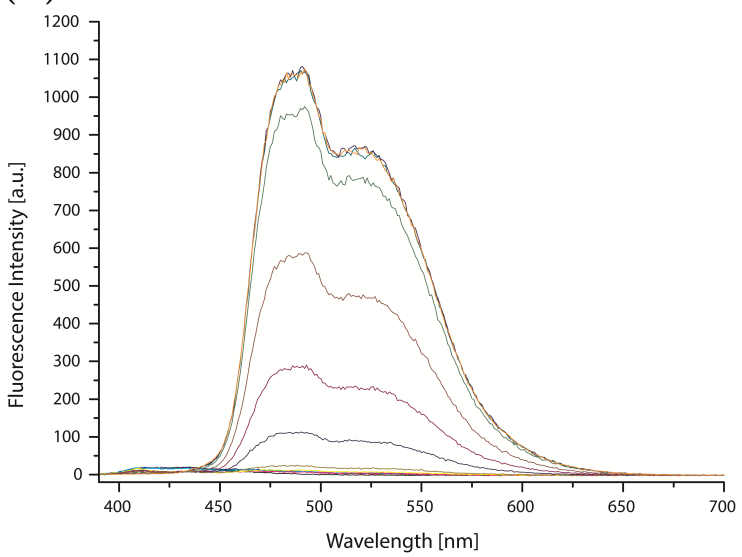

(d)

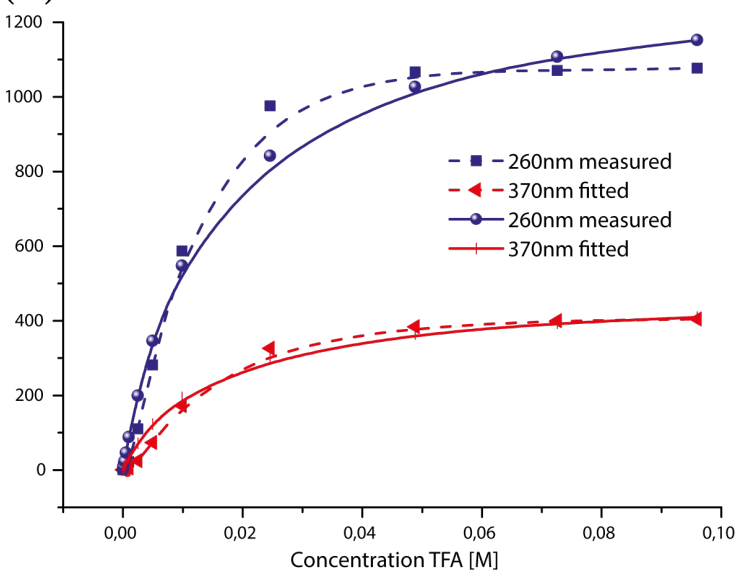

Figure V.1.3.36: a) 3D fluorescence measurement of $0.005 \mathrm{mM} \mathbf{B}^{\mathbf{P h}}$ in $\mathrm{MeCN}$ with $72.5 \mathrm{mM}$ TFA b) TFA fluorescence titration of $0.005 \mathrm{mM} \mathbf{B}^{\mathbf{P h}}$ in $\mathrm{MeCN}, \lambda^{\mathrm{ex}}: 260 \mathrm{~nm}$. c) TFA fluorescence titration $0.005 \mathrm{mM} \mathbf{B}^{\text {Ph }}$ in MeCN, $\lambda^{\mathrm{ex}}: 370 \mathrm{~nm}$. d) Plot fluorescence intensity vs. TFA concentration of $\mathbf{B}^{\mathbf{P h}}$ for the excitation wavelengths $\lambda^{\mathrm{ex}}=260 \mathrm{~nm}$ and $\lambda^{\mathrm{ex}}=370 \mathrm{~nm}$. Data was globally fitted with BindFit v0.5.264|265] Measured and fitted data points are shown for the emssion measured with excitation wavelengths $260 \mathrm{~nm}$ and $370 \mathrm{~nm}$.

Table V.1.3.5: Associations constants for protonation with TFA determined from fluorescence TFA titrations experiments for $\mathbf{B}^{t \mathrm{Bu}}, \mathbf{B}^{\mathrm{Et}}$ and $\mathbf{B}^{\mathrm{Ph}}$.

\begin{tabular}{cccc}
\hline & $\mathbf{B}^{\text {tBu }}$ & $\mathbf{B}^{\mathbf{E t}}$ & $\mathbf{B}^{\mathbf{P h}}$ \\
\hline \hline $\mathrm{K}_{\mathrm{A}}{ }^{\text {TFA }}\left[\mathrm{mol}^{-1}\right]$ & $365.6 \pm 77.8$ & $200.6 \pm 44.1$ & $0.07 \pm 0.01$ \\
\hline
\end{tabular}

Based on the results from the 3D measurements, fluoresence titration experiments were performed at the above mentioned excitation wavelength. (figures V.1.3.34b, V.1.3.34c, V.1.3.35b V.1.3.35c V.1.3.36b and V.1.3.36c Likewise to the UV-vis titration experiments the concentration of TFA was increased until no change in fluorescene intensity was observerd, whereby, as before the amount of acid needed to reach this steady state increased from $\mathbf{B}^{t \mathbf{B u}}$ to $\mathbf{B}^{\mathbf{P h}}$. The measured data plotted as fluourescene intensity vs. acid concentration and the curves are fitted with BindFit.264|265] Since BindFit is actually 
not conceptualized for fluorescence data, the obtained association constants are not really expressive table V.1.3.5, but explains the rather big deviation in comparison with the association constants determined by UV-vis. However, the values show the same trend as in the UV-vis experiments figure V.1.3.37).

(a)

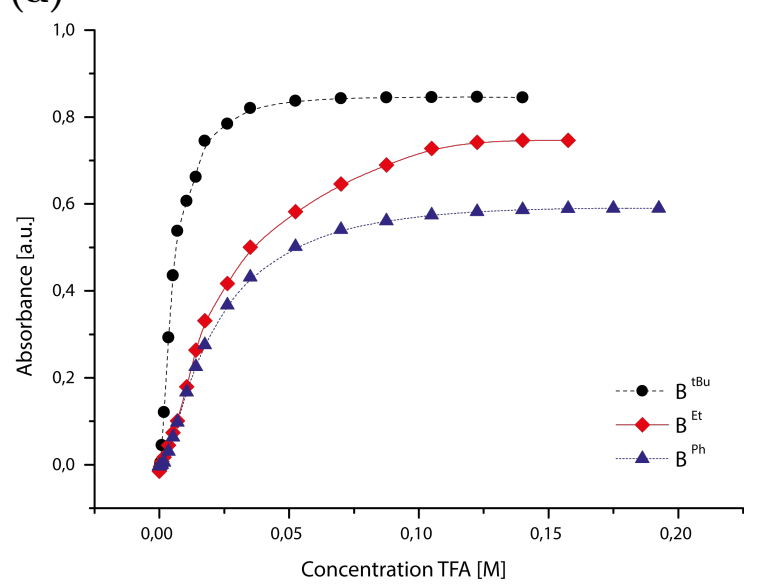

(b)

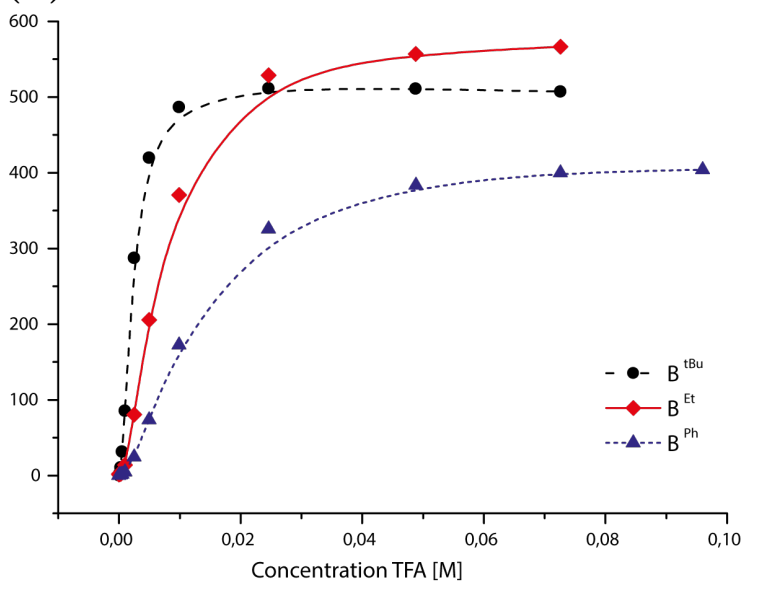

Figure V.1.3.37: a) Comparison of measured UV-vis data during TFA titration of all three drivatives $\mathbf{3 4}, \mathbf{3 5}$ and $\mathbf{3 7}$ for the absorption wavelength $369 \mathrm{~nm}$. b) Comparison of measured fluorescence data during TFA titration of all three drivatives $\mathbf{3 4}, \mathbf{3 5}$ and $\mathbf{3 7}$ for the exitation wavelength $370 \mathrm{~nm}$.

\section{V.1.4 Conclusion}

This chapter dealt with the syntheses of three asymmetrically substituted acridinylidene derivatives based on acridone, where the keton potion was substituted in a Knoevenagel-like reaction with various cyano acetate esters, without effecting the double bond situation. By introducing these electron-withdrawing substituents, a intramolcular push-pull system is formed, which induces a charge-separated state, by what the formal $\mathrm{C}=\mathrm{C}$ double bond is significantly weakened and a spinning motion of the cyano acetate ester can occur. The rate of rotation is highly dependent on the electronic character of the ester substituend, temperature (section V.1.2.2) and solvent polarity (section V.1.2.3), what have been examined in various NMR and UV-vis experiments. Moreover, the choice of ester substituents revealed that the esters contribution to the electronic situation within the molecule has a predominant effect, towards its steric demand, on the rate of rotation. The rotation motion was observable by dynamic NMR spectroscopy, since a chemical exchange of the protons, closest to the asymmetric rotor occurs in terms of NMR time scale. As a result the NMR shows only one set of signals above coalescence temperature (high spinning rates) which is split into two signals below coalescence temperature (low spinning rate). Furthermore, kinetic data was extracted from VT NMR experiments with help of Full Line Shape Analysis, by what thermodynamic parameters $\Delta^{\ddagger} H^{\ominus}$ and $\Delta^{\ddagger} S^{\ominus}$ for each derivatives 
could be determined. Thus, following order of derivatives can be made with regard to the rates of rotation: $\mathbf{B}^{t \mathbf{B u}}<\mathbf{B}^{\mathbf{E t}}<\mathbf{B}^{\mathbf{P h}}$. Results from UV-vis section V.1.2.3 and $\mathrm{CV}$ section V.1.2.5 experiments as well as data from crystal structure analysis section V.1.2.6 and DFT computational section V.1.2.7) were found to be in full agreement with the NMR observations, showing a decrease of activation energy from electron donating to rather electron withdrawing ester substituents. Furthermore, treatment with trifluoro acetic acid induces strong fluorescence in all derivatives, due to reversible protonation section V.1.3.2 of the bridging double bond between stator and rotor section V.1.3.1), linked to the formation of an acridinium ion. Determination of the associations constants by UV-vis and fluorescence titration experiments section V.1.3.3 revealed a reversed order $\left(\mathbf{B}^{t \mathbf{B u}}\right.$ $>\mathbf{B}^{\mathbf{E t}}>\mathbf{B}^{\mathbf{P h}}$ ) for the ability to be protonated. What is in consensus with the previous results, since the better stabilized negative charge at carbon leads to a lower basicity. In the next chapter, the here described derivatives were integrated into banana-shaped ligands suitable for metal-mediated supramolecular cage formation. The supramolcular cages are investigated for their host-guest chemistry and what is the effect of guest uptake on the rotation motion. 


\section{Chapter}

\section{Endohedral dynamics of push-pull rotor-functionalized cages}

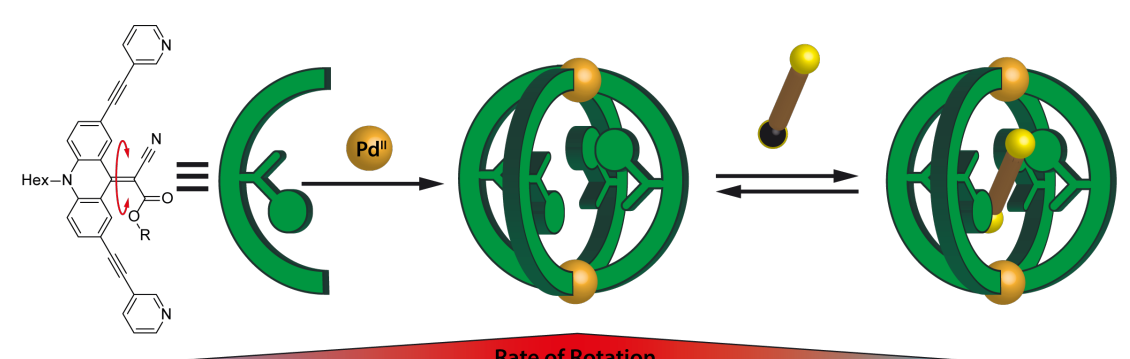

Contents

$\mathbf{V . 2 . 1}$ Introduction . . . . . . . . . . . . . . . . . . . . . . 152

$\mathbf{V . 2 . 2}$ Results and discussion . . . . . . . . . . . . . . . . 156

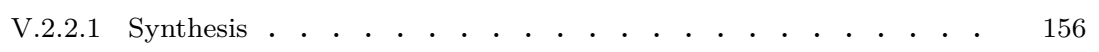

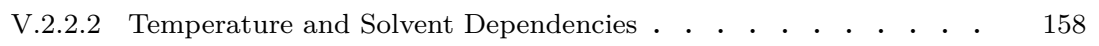

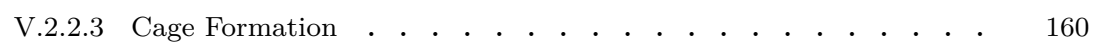

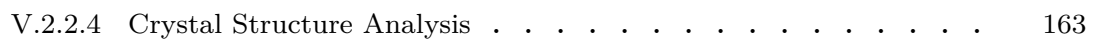

\begin{tabular}{l}
\hline .2 .2 .5 \\
Chemoinformatic Studies $\ldots \ldots$
\end{tabular}

\begin{tabular}{|l|l}
\hline .2 .2 .6 & Protonation $\ldots \ldots 6$
\end{tabular}

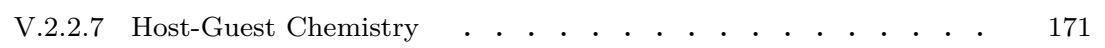

$\mathbf{V . 2 . 3}$ Conclusion $\ldots \ldots \ldots \ldots \ldots \ldots$ 


\section{V.2.1 Introduction}

Dynamic processes can be observed in most functional structures in nature. Whole domains in proteins show dynamics which are triggered upon binding of substrates and can lead to a change in the overall structure and its reactivity. 267 But also confined molecular environment can be found, which contain a combination of rigid and flexible elements, where the dynamics are often involved in the regulation of reactivity, 268 adaption of structure for an specific binding 269|270] or the shielding of catalytic sites. 271] On account of these features, nature still can selectively perform reactions in highly complex biological system. Therefore, the investigation and understanding of those dynamics is crucial to develop and create new highly specialized systems for medicinal or industrial production purposes.

However, strategies for the development of adequate artifical environments are needed first, since the investigation within natural occuring systems is usually as complex as the acquisition. Especially the field of supramolecular chemistry transpired to be a potential supplier, as it provides a vast portfolio of organic structures with interesting noncovalently connected topologies, which can even be extended, by including metal-mediated self-assemblies. The latter turned out to be a particularly successful strategy for the generation of a large degree of structural and functional architectures, with helicates, 272 entangled architectures 273 and coordination cages, 51 to be named as representatives. Yet, amongst those structures, the introduction of endohedral modifications remains a challenge and only few examples have been reported including inward-pointing hydrogen bond donors 274 and acceptors, 56 reactive residues inside helicates, cages and spheres, $275 \mid 276$ organocatalysts, 277 and metal-coordination sites. $278 \mid 279$

The term "endohedral" orignally derives from the fullerene chemistry. 280 Atoms, 281 molecules, 282 ions 283 or clusters $284[285$ are encapsulated, rarely reversible, into fullerene, by what the inner volume is occupied, thus an structural modification of the fullerene is only possible on the outer shell. In covalent organic 286 or non-covalent supramolecular constructs the situtation is different. On account of the feasibility to create larger structures with more voluminous cavities, exohedral $287 \mid 288$ and endrohedral 289 291 modifications are possible without loosing the abilitly of guest encapsulation. 24|292|293 In the last decades, countless self-assemblies with inlying cavities have been syntheszied. These constructs were vigorously examined for their properties in selective recognition, 294 host guest exchange, 295|296 or catalytic conversions. 297 Moreover, in recent years, the focus of research moved to the development of strategies for the incorporation of functionalities such as redox units, 298. photo switches 299|300 or specific binding sites. 236 In addition complex supramolecular systems were equipped with advances features, like stimuli-responsiveness, tuneable reactivity or allosteric regulation, functions mostly known from enzymes.

Clever et al. specialized in the structural motif of metal-mediated self-assembled coordination cages in form of $\left[\mathrm{Pd}_{n} \mathrm{~L}_{2 n}\right]$, which turns out to be a suitable for versatile structural and 
functional application. Ranging from allosteric binding 20 over light-responsive structural conversion 300 and light induced charge separation 301 to the formation of heteroleptic 61302 and also endohedral functionalized cages $24|236| 292|293|$

First attemps to create endohedral functionalized $\operatorname{Pd}_{2 n} \mathrm{~L}_{4 n}$ with banana-shaped ligands were shown by Freye et al.,26 Bulky aryl substituens were introduced at the keto position in the suberone-based ligands via Grignard addition. Although the endohedrally modified ligand does contain a hydroxy group, the mere presence of steric bulk led to the new behaviour towards its formation. Due to the change of available space, the ligand was not able to form a interpenetrated double cage in the presence of the inital $\mathrm{BF}_{4}{ }^{-}$anions. First the addition of one equivalent chloride anions was shifting the equilibrium towards a thermodynamically more stable double cage structure, where a single chloride anion occupied the inner cavity, due to the change in available space. In comparison to the unmodified suberone and acridone cage, $20 \mid 27$ the modified version showed a increased volume in the outer pockets and a smaller one for the inner pocket, where the chloride is located. The examination of the cages' spacial arrangement in crystal structure revealed that the endohedral substituents point rather to the sides of the cages than into central cavity. What is not surprising, since the carbonyl carbon exist in a $s p^{3}$ hybridized fashion after Grignard reaction took place (Figure V.2.1.1a). Therefore a new strategy to make the endohedral substituend pointing more inwards the cavity was needed.

(a)

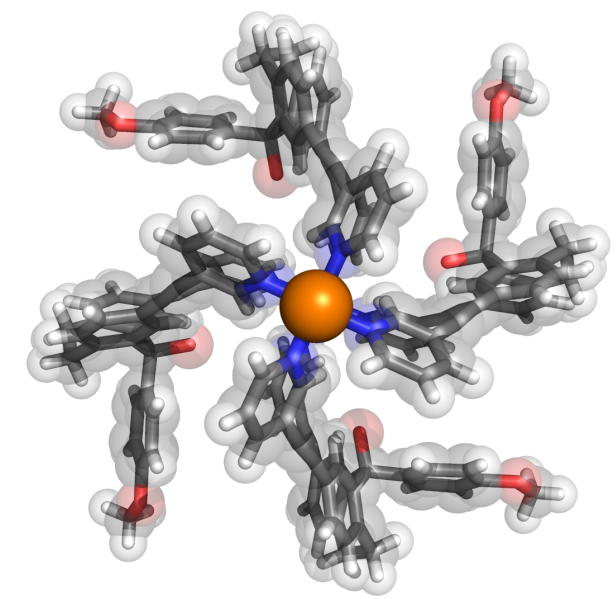

(b)

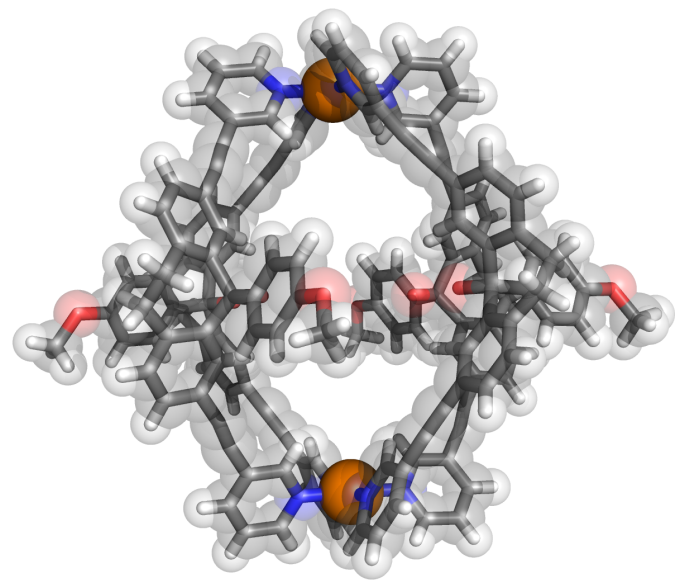

Figure V.2.1.1: Model of the published structure of the endohedrally modified suberone based cage by Freye et al.26. a) Top view on the cage. The single hydrogen atom is almost perfectly pointing into the cages central cavity, whilst the aromatic motif, that was introduced in a Gringard reaction is turned to the outsides, occupying the cages open sites, what can be better seen in the cages' site view b). Therefore the inner cavity remains free, allowing still the formation of an interpenetrated double-cage.

Also the change from the rigid aromatic ligand scaffold to a slightly more flexible ligand on the base of polynorbonene, similar to the system reported by Clever et al., 55 resulted in centrally functionalized ligands, that do self-assemble into $\left[\mathrm{Pd}_{2} \mathrm{~L}_{4}\right]$ coordination cages 
featuring endohedrally oriented strong H-bond donors. Due to the inwards pointing donor functionalities the cage was capable to selectively bind guest molecules with square-planar or octahedral orientated H-bond acceptors. 236

(a)

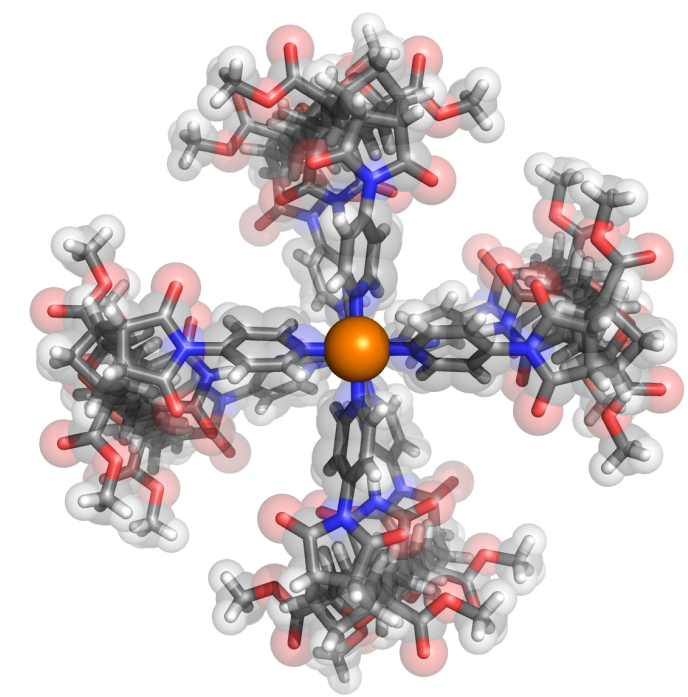

(b)

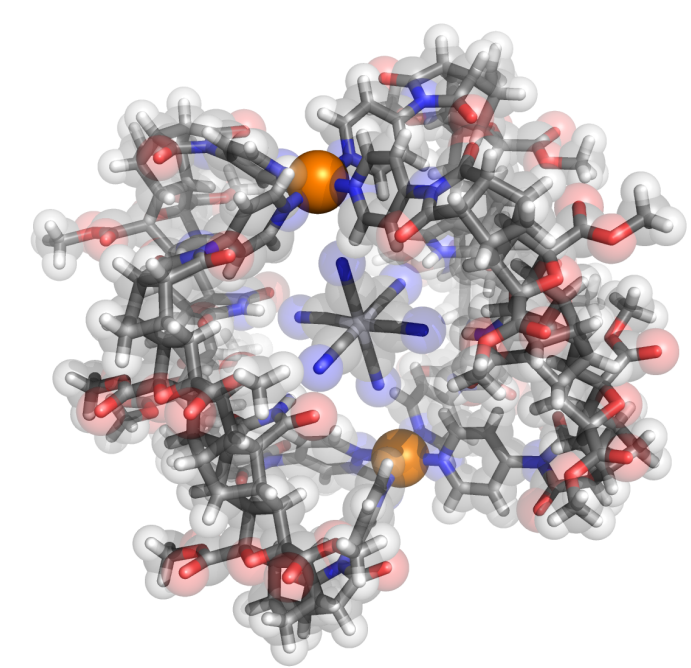

Figure V.2.1.2: Polynorbonene-Cage by Johnstone et al.236] a) Top view on the highly symmetric polynorbone cage, with the four banana-shaped ligands coordinated to palladium(II) ions. b) Side view, with encapsulated $\left[\mathrm{Fe}(\mathrm{CN})_{6}\right]$ complex as guest. The guest complex is perfectly aligned with the ligands inward pointing hydrogen bond acceptors.

In 2016 Löffler et al., published an endohedral functionalized cages, with four bulky admantyl subsituents. The functionalized cage units are based on the previously published acridone ligand, but with an adamantyl substituent at the former keton introduced by an McMurry reaction. It is worth to mention, that the double bond between the hetero-aromatic tricycle and the adamantyl is maintained, what was actually the crucial requirements of the ligands design, since this should make the substituent pointing inwards the cages cavity. But, crystal structure analysis of cage revealed, a bending of the acridone unit, by what the bulky substituent protruding sidewards from its concave face. This spacial arrangement causes the adamantyl substituents occupying the entrances of the cage and only partially the inner cavity. Therefore, there was still free space within the cage available, thus an encapsulation of an guest molecules could still occur. However, compared to the modified suberone cage, the adamantyl ligand was only able to form a single cage, no matter what counter anion was used, due to the bulky subsituents. Interestingly, the ligand showed a rapid flipping motion, by what the adamantyl ligand inverted itself. Interestingly, this motion even still happened for all ligands in the assembled cage, but due to the lack of space much slower and could even more decelerated upon the encapsulation of guest molecules. 
(a)

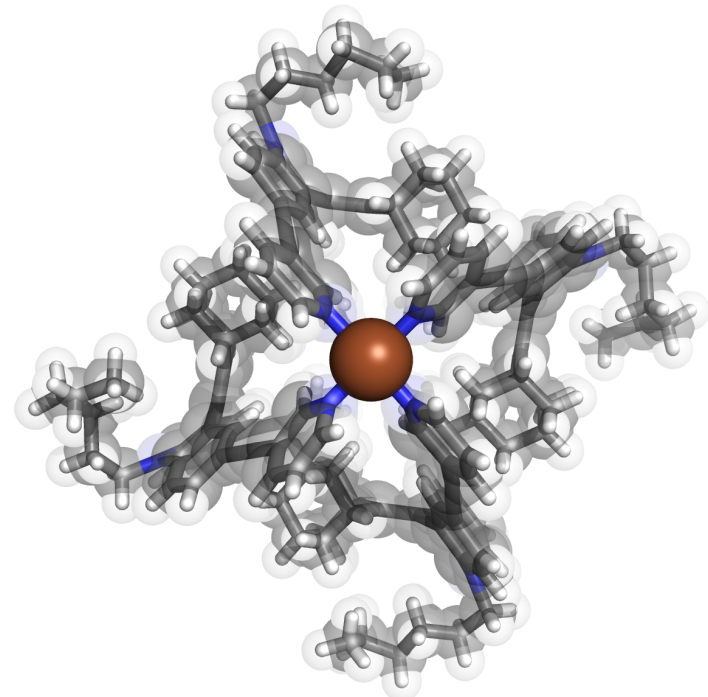

(b)

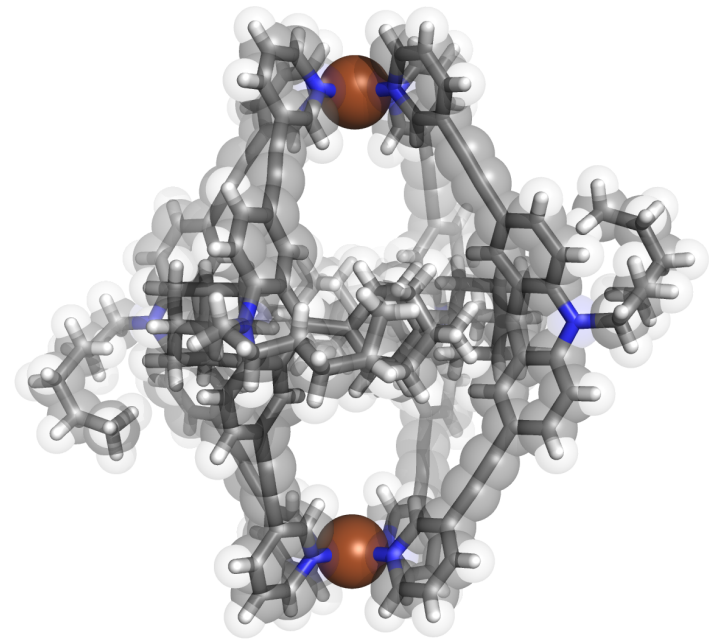

Figure V.2.1.3: Crystal structure adamantyl cage by Löffler et al. 24] a) Top view, the bulky endohedral adamantyl substituents are bended to the open sites of the cage, thus, rather pointing to a vicinal ligand than into the cages cavity. Due to that fact, the inner void provides enough space for guest encapsulation. However, in contrast to Freye's cage Figure V.2.1.1 the adamantyl cage is not able to form an interpenetrated double-cage. b) Side view, adamantyl substituents occupying cage's open sites.

Encouraged by the previous results, we designed a next generation endohedrally functionalized ligand based on the acridone backbone with a inward pointing group of smaller size to create less repulsion between substituent and aromatic backbone and additionally introducing functional groups.

The previous chapter dealt with a detailed characterization of a rotational motion around a double bond within an acridone based compound, enabled by an charge separated state. This charge separation was induced by an intramolecular push-pull system, which has reduced the bond order between stator and rotor, allowing a rotation. This chapter deals with the integration of the dynamic motion into a ligand system, capable of forming a metal-mediated supramolecular cage structure. The ligand properties are compared to its model compound with regard to the rotation. Furthermore, the formation of the cage structure, the host-guest chemistry and especially the influence of guest uptake in the rotational motion are investigated. 


\section{V.2.2 Results and discussion}

\section{V.2.2.1 Synthesis}

Similary to the cyano acetate ester derivatives described in the previous chapter, the synthetic pathway started with the introduction of an hexyl chain to improve the solubilty. Outgoing from the commercially available acrid-9/10H-one, hexylbromide was attached to the acridone via simple substitution reaction, with $\mathrm{K}_{2} \mathrm{CO}_{3}$ as base in DMF.<smiles>O=c1c2ccccc2[nH]c2ccccc12</smiles><smiles>[Li][13CH]</smiles><smiles>CCCCCCn1c2ccccc2c(=O)c2ccccc21</smiles>

33<smiles>CCCCCCn1c2ccc(I)cc2c(=O)c2cc(I)ccc21</smiles>

38<smiles>[R]OC(=O)C(C#N)=C1c2cc(C#Cc3cccnc3)ccc2N(CCCCCC)c2ccc(C#Cc3cccnc3)cc21</smiles>

41,42 and 43 d)

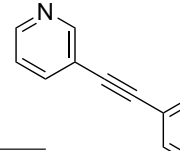<smiles>CCCCCCn1c2ccccc2c(=O)c2cc(C#Cc3cccnc3)ccc21</smiles>

39

Scheme V.2.2.8: Synthesis ligand derivatives 59, 42 and 43. a) hexylbromide, $\mathrm{K}_{2} \mathrm{CO}_{3}$, DMF; b) NIS, TFA, DMF; c) 3-ethynyl-pyridine, $\mathrm{NEt}_{3}, \mathrm{Pd}\left(\mathrm{PPh}_{3}\right)_{2} \mathrm{Cl}_{2}, \mathrm{CuI}$, THF; d) $(\mathrm{CN}) \mathrm{CH}_{2} \mathrm{COOR}$, TiCl4, $\mathrm{NEt}_{3}$, DCM.

In contrast to the published synthesis of the acridone ligand 24 iodine was introduced as leaving group for the later performed palladium cross-coupling reaction. The reaction was realized under catalytic amounts of TFA, that forms in presence of $N$-iodosuccimide in situ an activated $\mathbf{I}^{+}$species, suitable for the nucleophilic aromatic substitution. Moreover, + I-effect of hetero-aromatic nitrogen and -I-effect of the carbonyl within acridone led to a selective substitution in 2- and 7-postilion. After, 3-ethynyl-pyridine arms were attached at 2- and 7-potion with help of Sonogashira cross coupling reaction. At last malononitrile or cyano acetate esters were coupled to tricyclic aromatic backbone with the same procedure as described in section V.1.2.1 to obtain the symmetric ligand $\mathbf{L}^{\mathrm{CN}}(\mathbf{4 0})$ and the three asymmetric ligands $\mathbf{L}^{t \mathrm{Bu}}(\mathbf{4 1}), \mathbf{L}^{\mathrm{Et}}(\mathbf{4 2})$ and $\mathbf{L}^{\mathrm{Ph}}(\mathbf{4 3})$. 
(a)

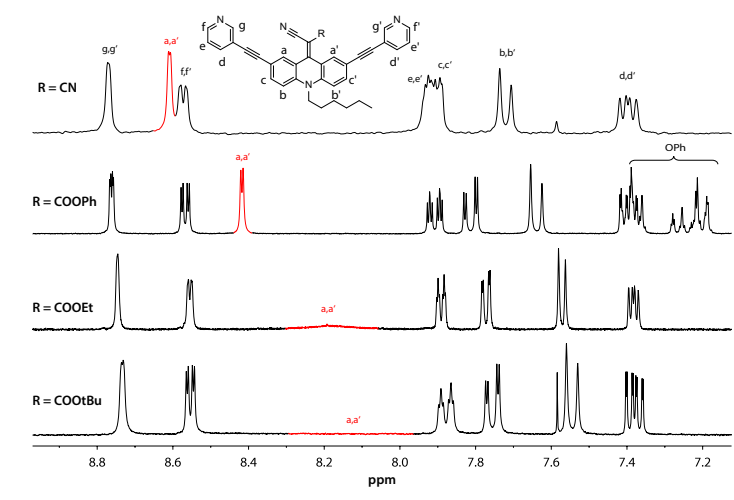

(b)

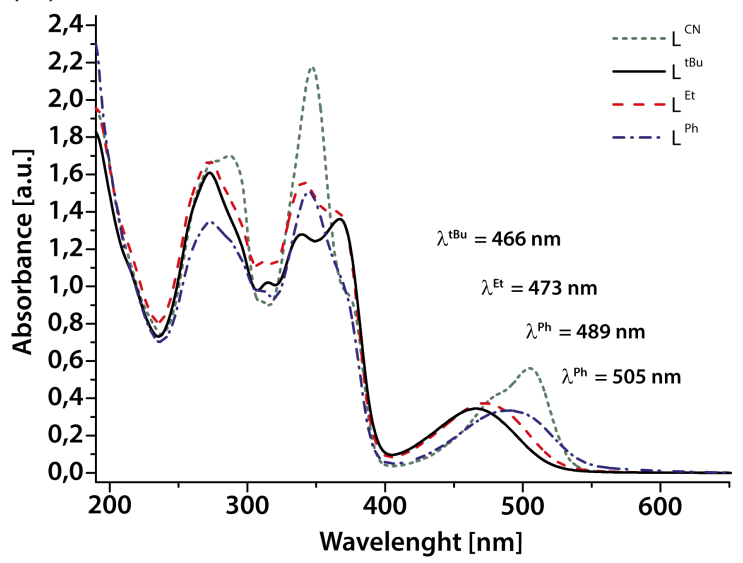

Figure V.2.2.4: a) Comparison NMR of the ligands $\mathbf{L}^{t \mathbf{B u}}, \mathbf{L}^{\mathbf{E t}}, \mathbf{L}^{\mathbf{P h}}$ and $\mathbf{L}^{\mathbf{C N}}$ in $\mathrm{MeCN}$ at $298 \mathrm{~K}$. The signal a/a' is highlighted in red. b) UV-vis spectra of all ligands. The bathochromic shift from $466 \mathrm{~nm}$ for the ligand $\mathbf{L}^{\mathbf{t} \boldsymbol{B} \boldsymbol{u}}$ (red) to $489 \mathrm{~nm}$ for the $\mathbf{L}^{\mathbf{P h}}$ (blue) correlates with the observations made in the NMR experiments. The more pronounced the electron withdrawing character of the headgroup, the stronger the bathocromic shift and the higher the single bond character of the exocyclic $\mathrm{C}=\mathrm{C}$ bond, leading to faster rotation. This is effect is in accordance with literature described systems.

As for the non-ligand compound $\left(\mathbf{B}^{t \mathrm{Bu}}(\mathbf{3 4}), \mathbf{B}^{\mathrm{Et}}(\mathbf{3 5}), \mathbf{B}^{\mathrm{Ph}}(\mathbf{3 7})\right.$ the ligands show all together only one set of signals for the aromatic protons, although two sets, one for each molecules site, are expected, since the introduction of the cyano acetate ester substituent decreased the compounds symmetry. Therefore it is obvious that the same intramolecular dynamic seems to take place.

Dynamic NMR spectroscopy revealed the same behavior in ${ }^{\mathbf{1}} \mathbf{H} \mathbf{~ N M R}$ measurements as the previously investigated systems (figure V.2.2.4a). While signal (a/a') is extremely broadened for $\mathbf{L}^{t B u}$,so that it is not distinguishable from noise, $\mathbf{L}^{\mathbf{E t}}$ show already a small bump at ca. $8.2 \mathrm{ppm}$. In contrast $\mathbf{L}^{\mathbf{P h}}$ has a quite sharper signal at $8.4 \mathrm{ppm}$. It is noteworthy that again there is not only the broadening of signal, indicating qualitatively the difference in speed of rotation, but the average signal a/a' is downfield shifted the higher the electron-withdrawing character of the observed ligand derivative. Therefore, the symmetric dicyano ligand $\mathbf{L}^{\mathbf{C N}}$ has the highest downfield shift in NMR and the sharpest peak, but latter is contributed to the unsuitability to observe the rotation by $\mathrm{nmr}$ in this symmetric system. However, the comparison of UV-vis measurements for all four ligands (figure V.2.2.4b), show again the distinctive bathochromic shift going along with an increased charge stabilization within the intramolecular push-pull system $\left(\mathbf{L}^{\mathbf{t} \mathbf{B u}}>\mathbf{L}^{\mathbf{E t}}\right.$ $>\mathbf{L}^{\mathrm{Ph}}>\mathbf{L}^{\mathrm{CN}}$. 


\section{V.2.2.2 Dependence on temperature and solvent polarity}

To get a better overview of how similar the ligands behave to their smaller model compounds from the previous chapter, their dependencies on temperature and solvent polarity, was also investigated. For that reason, for all derivatives VT ${ }^{\mathbf{1}} \mathbf{H}$ NMR experiments were performed in THF as solvent, since it showed the best ratio in solubility to temperature range. In figure V.2.2.5 the VT NMR experiment for $\mathbf{L}^{\mathbf{E t}}$ is shown in comparison with the $\mathbf{B}^{\mathbf{E t}}$. Qualitatively, both compounds seems to behave virtually identical in terms of change in rotation rate towards variation of temperature. Furthermore, although the signals a and a' have a slightly different chemical shift in both compounds $(\mathbf{a}=7.50 \mathrm{ppm}, \mathbf{a}=8.62 \mathrm{ppm}$ for $\mathbf{B}^{\mathbf{E t}}$ and $\delta_{\mathbf{a}}=7.70$ and $\left.\delta_{\mathbf{a}},=8.75\right)$ the gap in between both signals is nearly the same (420-450 Hz), another indication for similar rate of rotation. 


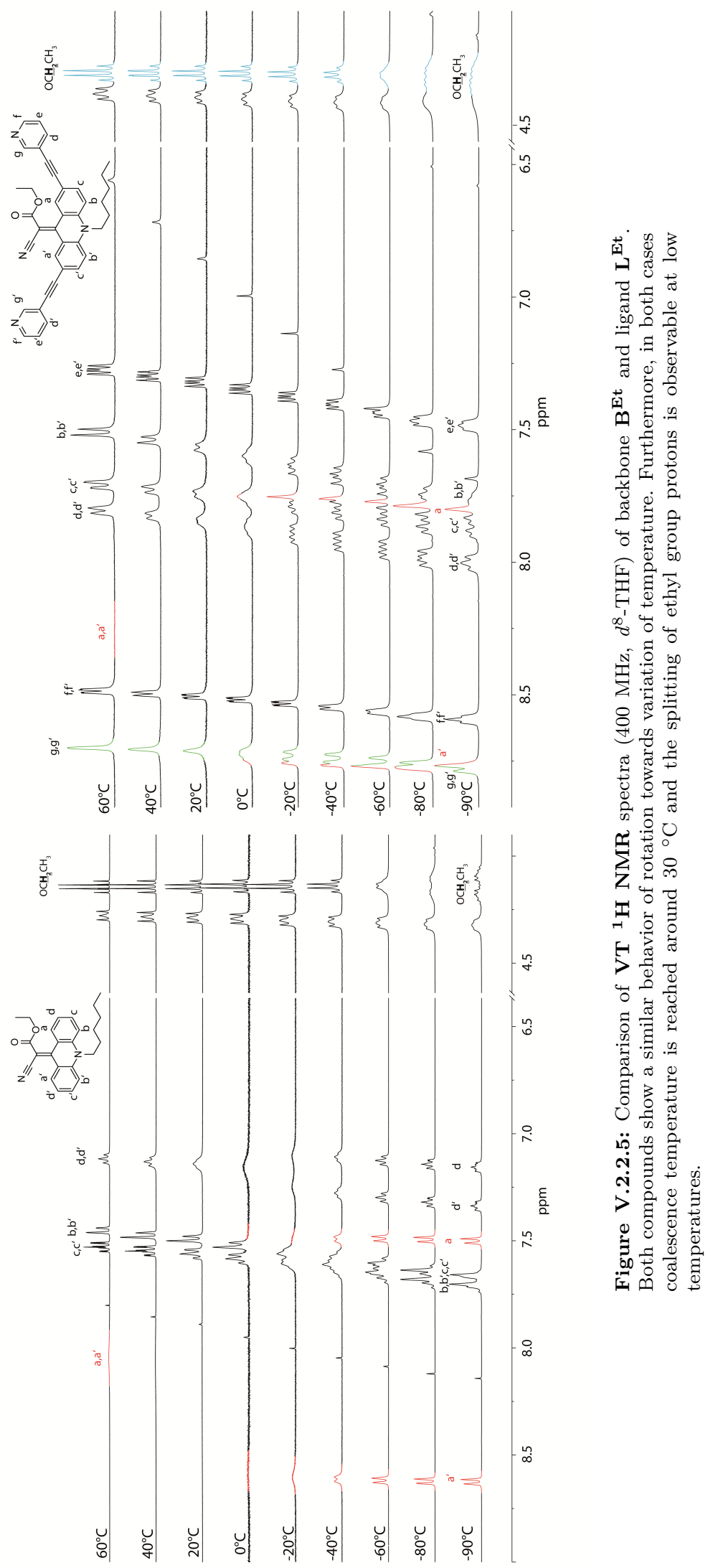


To examine the dependency on the solvent polarity further NMR experiments were recorded (Figure V.2.2.6). For that reason, exemplary NMR measurement were performed for the derivative $\mathbf{L}^{\mathrm{Et}}$ (42) and its corresponding cage $\mathbf{C}^{\mathbf{E t}}$ (46) in various solvent. Since the charged cages $\left[\mathrm{Pd}_{2} \mathrm{~L}_{4}\right]^{4+}$ are only soluble in polar solvent, following solvents $\mathrm{MeCN}, \mathrm{MeNO}_{2}$, DMSO, MeOH were used. As expected, the peak width of the signal $\mathbf{a} / \mathbf{a}$ ' is decreased (increased rotation rate) with the increase of solvent polarity. However, the system has a higher rotation rate in DMSO compared to $\mathrm{MeCN}$ and $\mathrm{MeNO}_{2}$, although DMSO has actually a lower relative polarity. 303 A possible explanation for that is the amount of water within the solvents, since non-dry deuterated solvents were used.

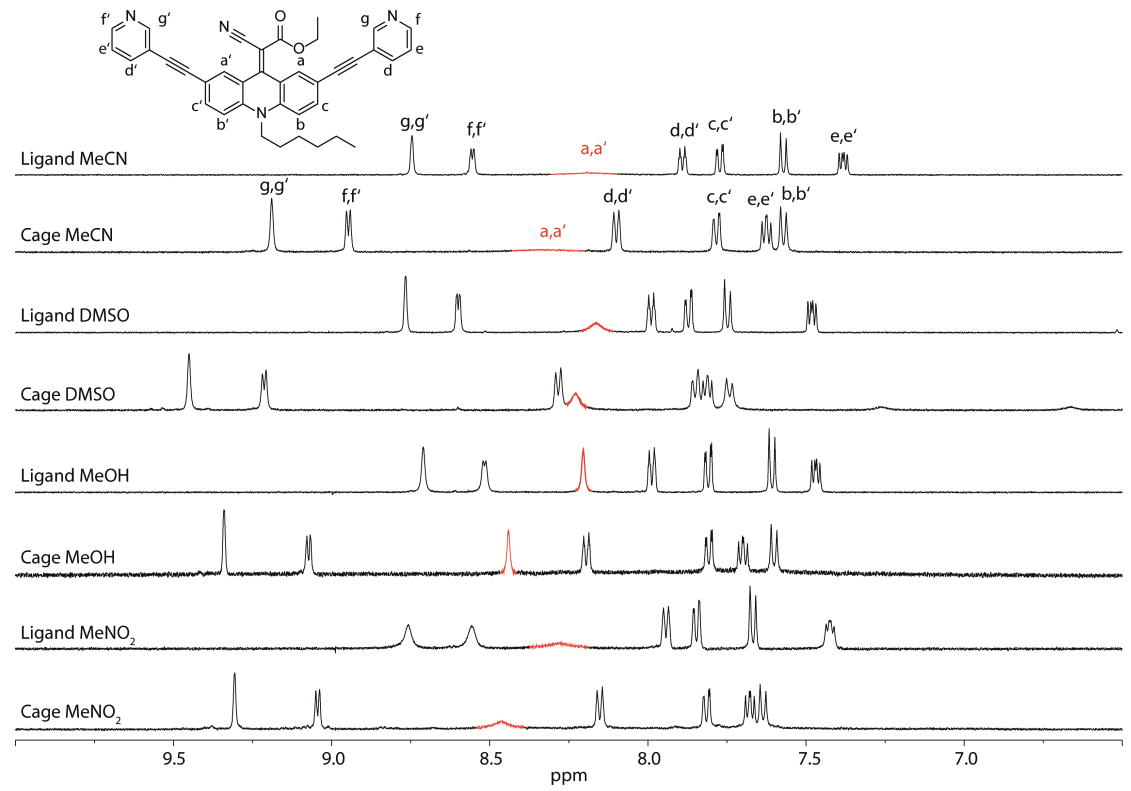

Figure V.2.2.6: ${ }^{\mathbf{1}} \mathbf{H} \mathbf{N M R}$ spectra $(400 \mathrm{MHz}, 298 \mathrm{~K})$ of ligand $\mathbf{L}^{\mathbf{E t}}$ and cage $\mathbf{C}^{\mathbf{E t}}\left[\mathrm{Pd}_{2} \mathbf{L}^{\mathbf{E t}}{ }_{4}\right]$ in different solvents. The spinning rate of the rotor groups and the associated sharpness of the signal a/a' (red) depends on the polarity of the solvent with the rotational rate rising in the order $\mathrm{MeCN}>\mathrm{MeNO}_{2}>\mathrm{DMSO}>\mathrm{MeOH}$. In all cases, the sharpness of the a/a' signal increases upon cage formation.

\section{V.2.2.3 Cage Formation}

After the succeful synthesis and characterization of the ligands $\mathbf{L}^{t \mathbf{B u}}, \mathbf{L}^{\mathrm{Et}}, \mathbf{L}^{\mathbf{P h}}$ and $\mathbf{L}^{\mathbf{C N}}$, the ability of the ligand derivatives to form supramolecular cages in the presence of the palladium cations was tested. Hence, a $2.8 \mathrm{mM}$ solution of the ligands in deuterated acetonitrile were prepared and 0.5 equiv of $\left[\mathrm{Pd}(\mathrm{MeCN})_{4}\right]\left(\mathrm{BF}_{4}\right)_{2}$ were added. The mixture was heated to $70{ }^{\circ} \mathrm{C}$ for $1 \mathrm{~h}$. Subsequently the formation was examined by NMR Comparison of the NMR spectra of ligands and cage, shows that in both cases the amount of signal is the same, indicating the formation of a simple supramolecular cage in form of $\left[\mathrm{Pd}_{2} \mathrm{~L}_{4}\right]$ likewise to the adamantyl cage. 24 Furthermore, the characteristic general downfield shift of the pyridine signals, but especially for $\mathbf{g} / \mathbf{g}$ ' and $\mathbf{f} / \mathbf{f}^{\prime}$ ' sitting closest to the nitrogen, are observable. The shifting for those signals occurs due to the coordination of the ligand to 
the palladium(II) cation and the consequent change in the electronic situation. Signal $\mathbf{a} / \mathbf{a}$ ' shows in systems a slight downfield shift as well, except of in the for $\mathbf{L}^{\mathbf{P h}}$, there the chemical shift for $\mathbf{a} / \mathbf{a}$ ' stays almost constant.

(a)

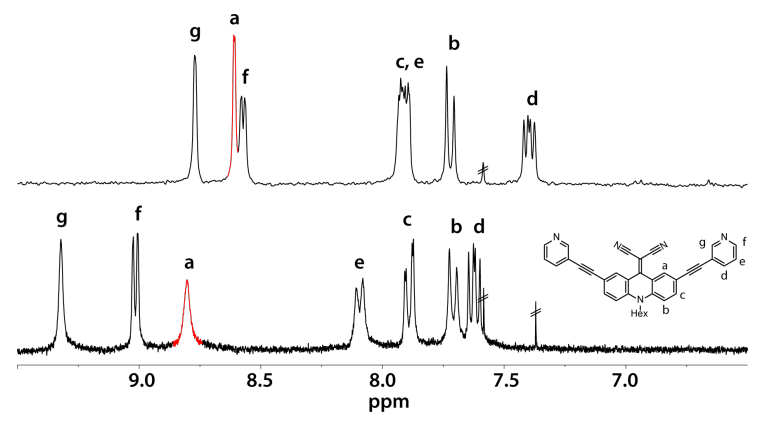

(c)
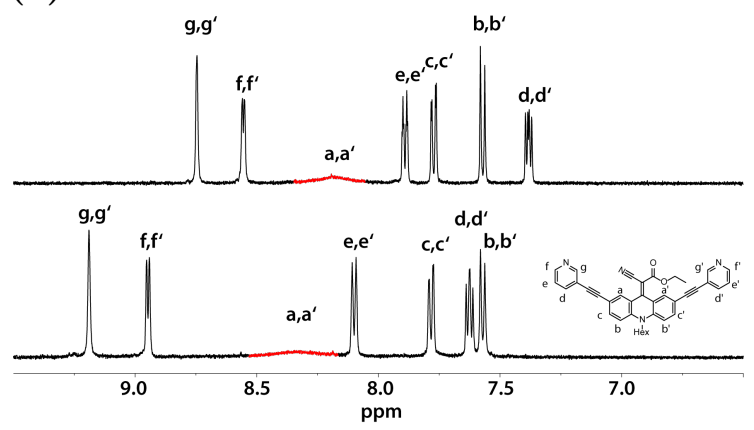

(b)

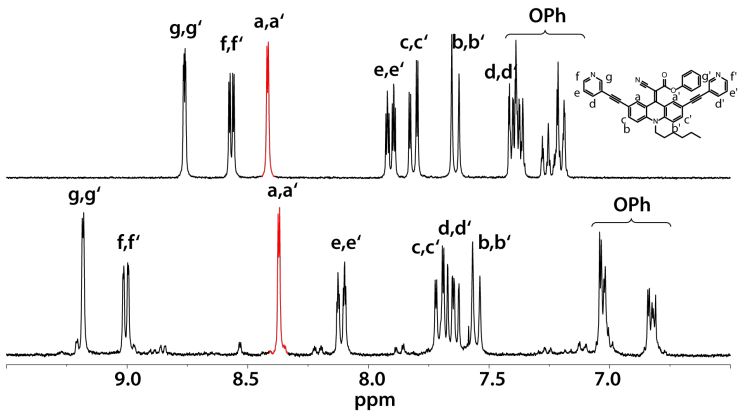

(d)

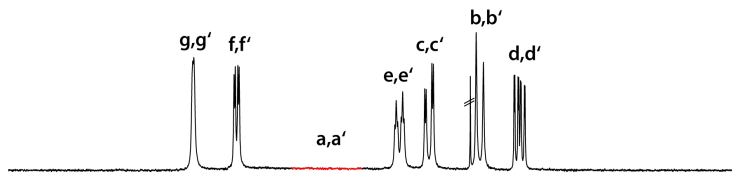

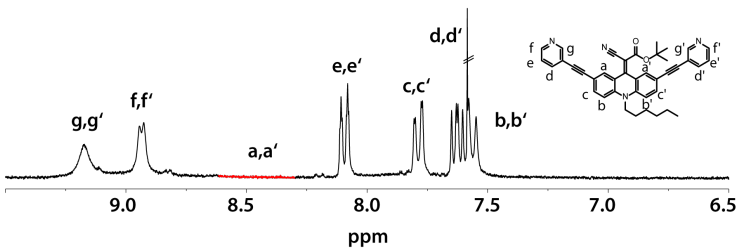

Figure V.2.2.7: Comparison ${ }^{\mathbf{1}} \mathbf{H}$ NMR ligand and assembled cage for 40, 42, 43 and 59 . Top spectra belongs to the free ligand $(2.8 \mathrm{mM})$, buttom one is the assembled cage $\left((0.7 \mathrm{mM})\right.$ with $\left[\mathrm{Pd}(\mathrm{MeCN})_{4}\right]\left(\mathrm{BF}_{4}\right)$. Everything was measured in deuterated MeCN. a) 40 and 44; b) 60 and 45; c) 42 and 46; d) 43 and 47 . The signal of the protons (a/a'), closest to the rotating group, are highlighted in red.

It is worth to mention, that the rate of rotation is increased upon cage formation (Figure V.2.2.6). Besides the slight downfield shift of the signal a/a' compared to the ligand spectra, the signal seems also to be sharpened in the cage spectra. This finding can be easily explained, since the electron withdrawing part of the ligand and therefore the negative part of the charge separated state, is pointing inwards the cage's cavity, which is known from other examples in literature to host either the non-coordinationg counteranions or negatively charged guest molecules. $24|51| 304$ Thus, environment within the cage structure probably contributes to the stabilization of the charge-separated state, therefore the rotation rate is increased compared to the free ligand.

Moreover, the formation of cages $\mathbf{C}^{t \mathbf{B u}}, \mathbf{C}^{\mathbf{E t}}$ and $\mathbf{C}^{\mathbf{P h}}$ was verified by ESI-MS. The samples for the mass were directly taken from the solution used in the NMR measurements. In all cases the $\left[\mathrm{Pd}_{2} \mathrm{~L}_{4}\right]^{4+}$ peak was found to be the main species, besides, a species with five ligands was also observable. Although, the NMR spectra figures V.2.2.7b to $\mathrm{V.2.2.7 \textrm {d }}$ showed a clean formation of the ligands, the ESI-MS measurement showed $\mathrm{s}$ 
significant a amount of the free ligand with various additives, what could be a indication for a rather low stability, at least under the experimental conditions for the ESI-MS measurements.

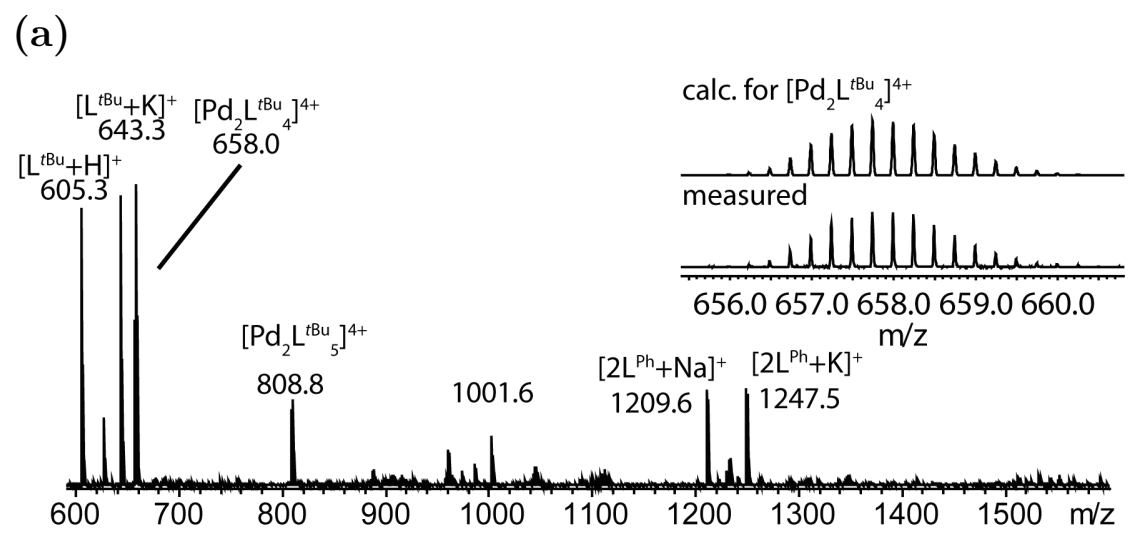

(b)

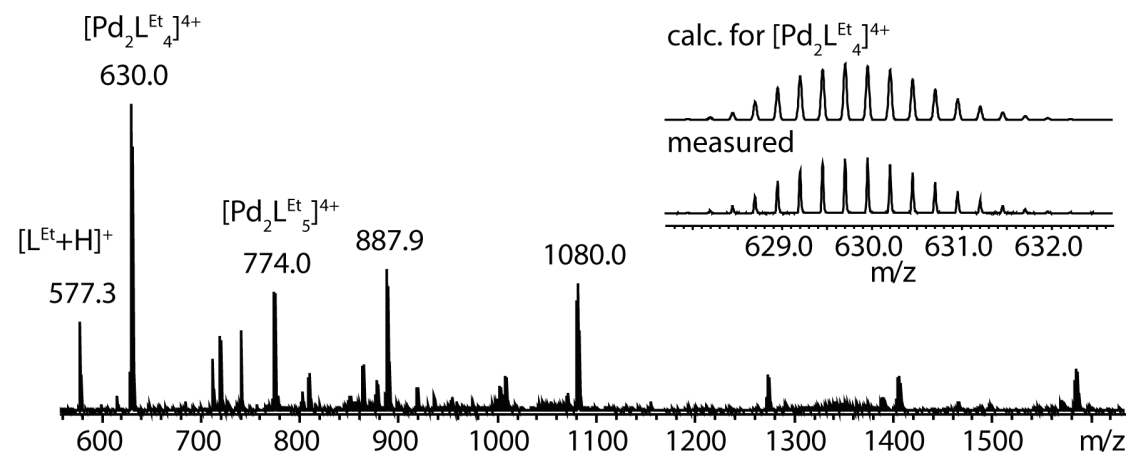

(c)

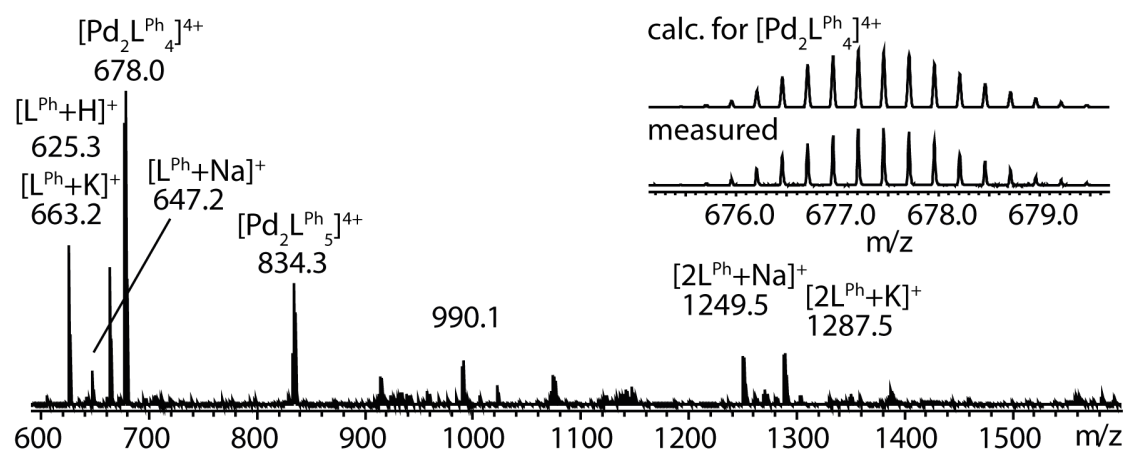

Figure V.2.2.8: ESI-MS of cages (a) $\left[\mathbf{P d}_{2} \mathbf{L}^{\mathbf{E t}}{ }_{4}\right]$ (b) $\left[\mathbf{P d}_{2} \mathbf{L}^{t \mathbf{B u}}{ }_{4}\right]$ and (c) $\left[\mathbf{P d}_{2} \mathbf{L}^{\mathbf{P h}}{ }_{4}\right]$. Samples were directly taken from NMR samples shown above.

In figure V.2.2.9 the comparison of UV-vis spectra for $\mathbf{L}^{\mathbf{E t}}$ and $\mathbf{C}^{\mathbf{E t}}$ are depicted. There is a significant difference in the absorption between $200 \mathrm{~nm}$ and $400 \mathrm{~nm}$, however, the absorption band distinctive for the stability push-pull system (or HOMO-LUMO gap) Sections V.1.2.1 and V.1.2.7 stays almost unchanged, indicating that the intramolecular push-pull system stays intact, although a slight bathochromic shift from $\lambda_{\max }{ }^{\mathrm{Et}}=472 \mathrm{~nm}$ to $\lambda_{\max }{ }^{\mathrm{Et}}=$ $476 \mathrm{~nm}$, what is not a huge difference, but correlates to the increased rotation rate upon 
the cage formation, observed in the NMR experiments (Figure V.2.2.7) and the proposed stabilization of charge-separated state within the cage cavity.

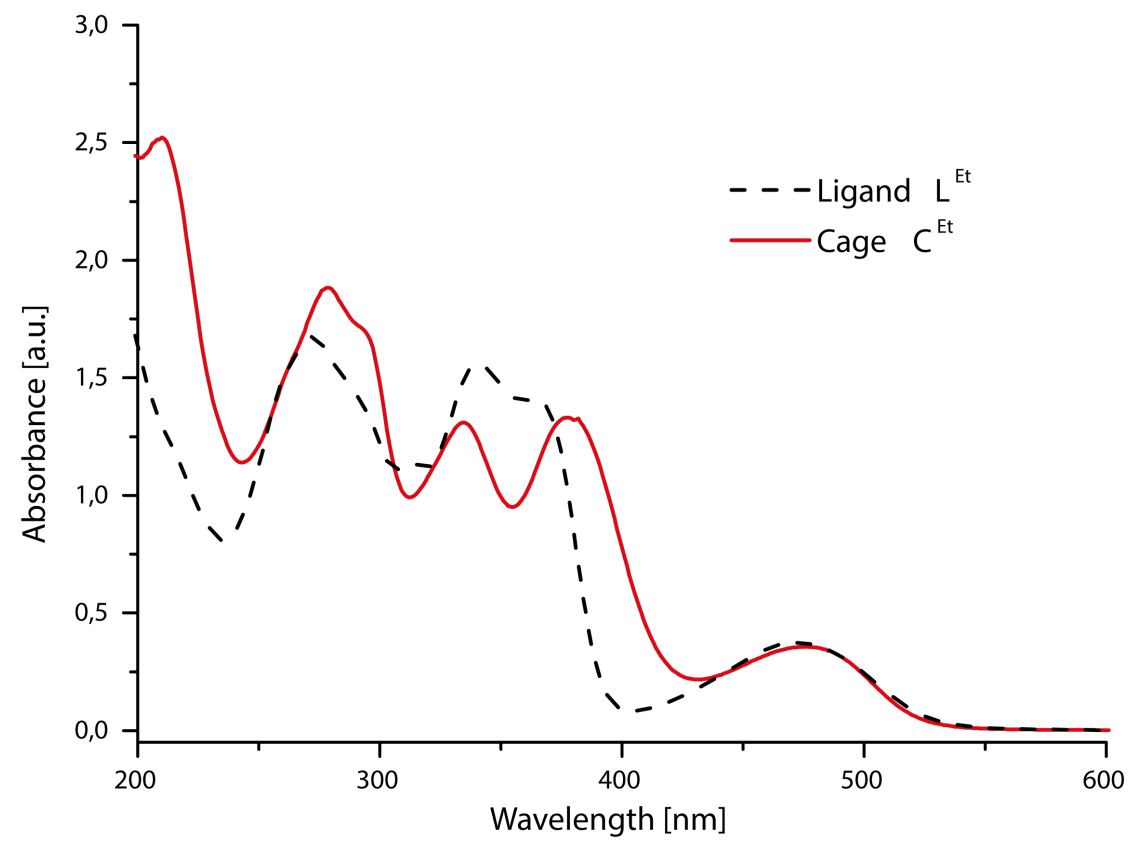

Figure V.2.2.9: Comparison UV-vis spectra of $\mathrm{L}^{\mathrm{Et}}(\mathbf{3 5})$ and $\mathrm{C}^{\mathrm{Et}}$ (46. Both spectra were recorded in $\mathrm{MeCN}$ with the same ligand concentration $(0.035 \mathrm{mM})$. The maximum at $472 \mathrm{~nm}$ for the ligand stayed intact, but is slightly shifted to $476 \mathrm{~nm}$ for the cage.

\section{V.2.2.4 Crystal Structure Analysis}

Crystals of $\mathbf{L}^{\mathbf{C N}}, \mathbf{L}^{\mathbf{P h}}$ and $\mathbf{L}^{\mathbf{E t}}$ were suitable for X-ray diffraction measurements and from all of them a crystal structure could be obtained. Unfortunately, for $\mathbf{L}^{\mathbf{B u}}$ no crystal could be grown, what is the reason why the structure of $\mathbf{L}^{t \mathbf{B u}}$ was optimized in DFT calculation (B3LYP/6-311+g(d,p) in PCM-MeCN) outgoing from the crystal structure of $\mathbf{L}^{\mathbf{E t}}$. It is not surprising that as in the model compounds $\left(\mathbf{B}^{\mathrm{Et}}\right.$ etc.) the tricyclic aromatic backbones are bent and the rotor group in slightly twisted depending on the substituent. Comparison of the angles shows immediately that the order from the most bent structure to the least bent structure is the same as for the model compounds $\left(\mathbf{L}^{t \mathbf{B u}}>\mathbf{L}^{\mathbf{E t}}>\mathbf{L}^{\mathbf{P h}}>\mathbf{L}^{\mathbf{C N}}\right)$, thus the angles are also very similar to their smaller analogs. $\mathbf{L}^{\mathbf{C N}}$ has a $2^{\circ}$ smaller bend compared to $\mathbf{B}^{\mathbf{C N}}\left(164^{\circ}\right.$ Figure V.1.2.18), whereas $\mathbf{L}^{\mathbf{E t}}$ is more bent about $6^{\circ}$ in comparison to $\mathbf{B}^{\mathrm{Et}}$. The calculated structure $\mathbf{L}^{t \mathbf{B u}}$ has exact the same angle as the crystal structure of $\mathbf{I}_{2}-\mathbf{B}^{t \mathbf{B u}}$, but since the calculation does not consider any packing effect and the model is calculated in solution, the angle in crystal structure could differ from the here present one. Furthermore, this time no coexisting enantiomers could be found in the asymmetric cell, anyway, from the previous VT NMR experiments Figures VI.1.7.2a to VI.1.7.2c it is obvious that the rotation motion takes place in all derivatives, hence, 
for the three asymmetric derivatives both enantiomers should exist, in solid as well as in solution.
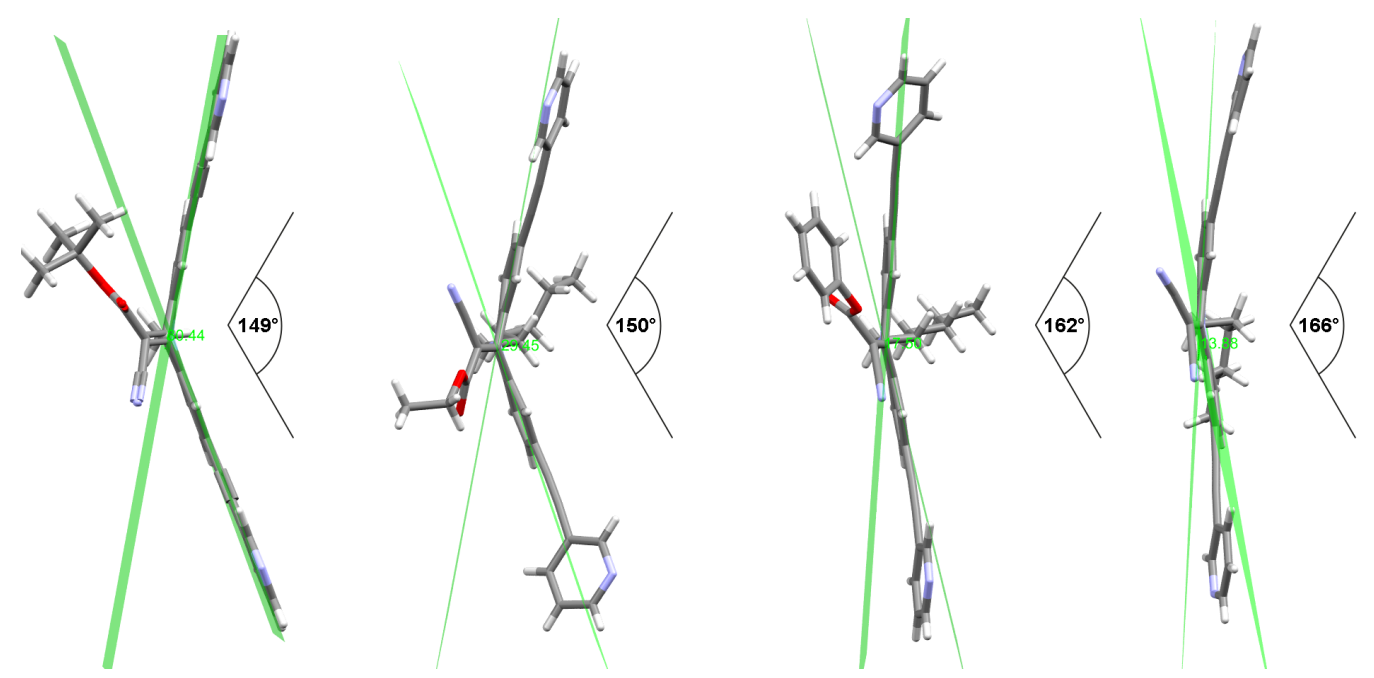

Figure V.2.2.10: Comparison crystal structures of ligand derivatives $\mathbf{L}^{\mathbf{C N}}, \mathbf{L}^{\mathbf{E t}}$ and $\mathbf{L}^{\mathbf{P h}}$. The here used structure of $\mathbf{L}^{t \mathbf{B u}}$ was created in silico (B3LYP-6-311+g(d,p) in PCM-MeCN), since no crystal was suitable for X-ray diffraction analysis. The crystal structures represents the bending angles within the respectives ligands, what is in total accordance to the general trend shown by all other experiments made so far $\left(\mathbf{L}^{t \mathbf{B u}}(149 \check{\mathrm{r}})<\mathbf{L}^{\mathbf{E t}}(150 \check{\mathrm{r}})\right.$ $<\mathbf{L}^{\mathbf{P h}}(162 \check{\mathrm{r}})<\mathbf{L}^{\mathbf{C N}}(166 \check{\mathrm{r}}) . \mathbf{L}^{t \mathrm{Bu}}$ is calculated presence of solvent, thus, the here shown angle may differ form the real one.

\section{V.2.2.5 Chemoinformatic Studies}

Since no crystals suitable for X-ray diffraction analysis could be obtained for any of the cages $\mathbf{C}^{t \mathbf{B u}}, \mathbf{C}^{\mathbf{E t}}$ or $\mathbf{C}^{\mathbf{P h}}$, models for $\mathbf{C}^{\mathbf{E t}}$ with the possbile arrangement of the inward pointing rotors (Table V.2.2.1) were calculated and the thereof obtained relative energies were compared. Unexpectedly, the cage with two neighbored rotors of the same orientation (cis-2up/2down) has the lowest energy and not as expected the confomer, where the rotors of opposite ligands show the same orientation of the cyano acetates. (trans-2up/2down). However, finding is in accordance with the recent publication of Zhu et al.,75] where sterical demanding picolyl ligands, designed for the formation of heteroleptic coordination cages, rather form cis coordinated $\left[\mathrm{Pd}_{2} \mathrm{~L}_{2}^{\text {in }} \mathrm{L}_{2}{ }_{2}\right.$ out cages than trans isomer, with the supposed less crowded palladium coordination sphere. Nevertheless, since the difference in energy is only $2.6 \mathrm{~kJ} \mathrm{~mol}^{-1}$, it is very likely that both isomers exist in solution, presumed that the energy barrier of interconversion between the cis and trans isomer is not too high. Furthermore, as expected, the most crowded isomer ("all on one side") has a the highest realtive energy, about 10 times higher with respect to the trans isomer $26.3 \mathrm{~kJ} \mathrm{~mol}^{-1}$, indicating a lower probabilty to be formed as compared to the cis and trans isomers. 
Table V.2.2.1: Comparison of relative energies of various cage stereoisomers, with respect to the positioning of vinical rotation group within the cage cavity. Values were determined in silico from DTF calculations

(B3LYP-6-311+g(d,p) in PCM-MeCN). Lowest enegertic level was found for the cis isomer, with two opposite equi-directed adjoined cyano actete ester groups.

Symmetry

Rel. E [kJ mol $\left.{ }^{-1}\right]$

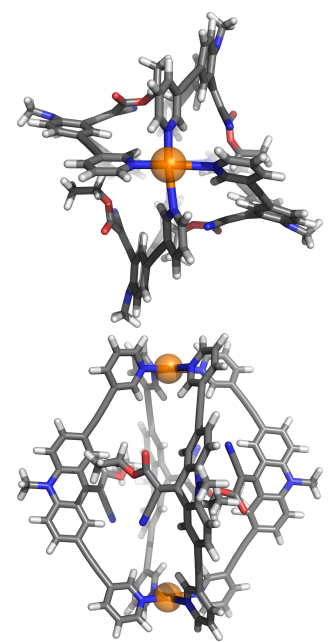

cis-2up/2down

$\mathrm{C}_{\mathrm{i}}$

0

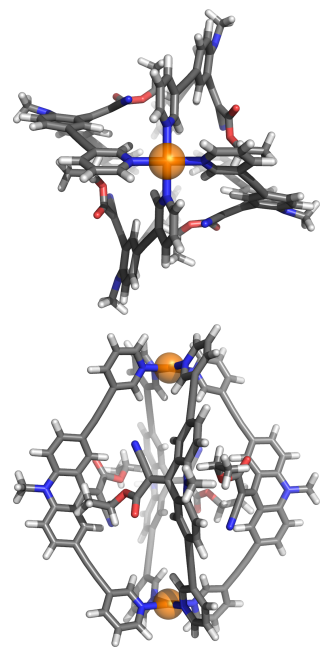

trans-2up/2down

$\mathrm{S}_{4}$

$+2.6$
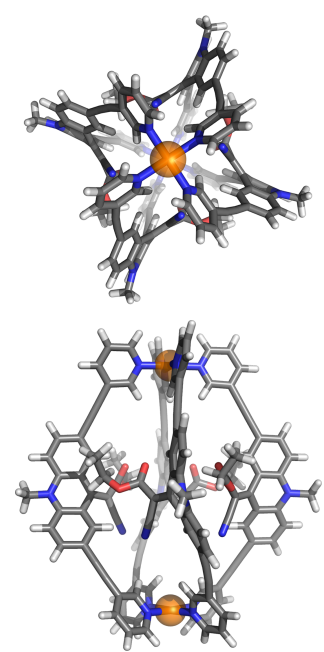

all on one side

$\mathrm{C}_{4}$

$+26.3$ 


\section{V.2.2.6 Protonation with TFA}

Before the system was investigated for its host-guest chemistry, we were interested whether the ligand can form the same fluorescence active acridinium species as described for the model compounds (Section V.1.3), or if the pyridine motives interfere the protonation of the rotor group. Addition of an excess of TFA to a solution of $\mathbf{L}^{\mathbf{E t}}$ in $\mathrm{MeCN}$ gave immediately the answer to that question. The solution showed only a slight change in color, as well as no fluorescence was observable upon irradiation with a $365 \mathrm{~nm}$ light. Neither further addition of TFA nor the direct solving of the ligands in pure TFA led to the desired formation of the fluorophore. To take a closer look what is happening to ligand in presence of the acid TFA, UV-vis titration experiments were performed. Interestingly, all of them showed at least a very similar behavior. While there is a hyperchromic shift for the absorption bands at approx. $300 \mathrm{~nm}$ and $395 \mathrm{~nm}$, a hypochromic shift of the absorption band at approx. $280 \mathrm{~nm}$ and $350 \mathrm{~nm}$ are clear observable. However, the absorption band at $460-490 \mathrm{~nm}$, which was used as indicator for the quality of the intramolecular push-pull system, stays almost unchanged, but experience a slight hypsochromic shift. At the first glance this behaviour is counter-intuitively, since one would rather expect a stabilization of the push-pull system due to the increased polarity, as it was observed before for the backbone system. Additon of TFA to the backbone derivatives led to a initial stabilization, thus a higher rotation rate due to the increase in solvent polarity, before concentration was high enough to protonate the rotor group (Figure V.1.3.27, 0-13 mM TFA). But obviously the protonation of the pyridine arms has a negative impact on the intramolecular electronic situation, which has a predominant effect on the push-pull system in comparison to change of solvent polarity. For $\mathbf{L}^{\mathbf{E t}}$, as an example, the titration experiment with TFA was also monitored in ${ }^{\mathbf{1}} \mathbf{H}$ NMR measurement Figure V.2.2.12. 
(a)

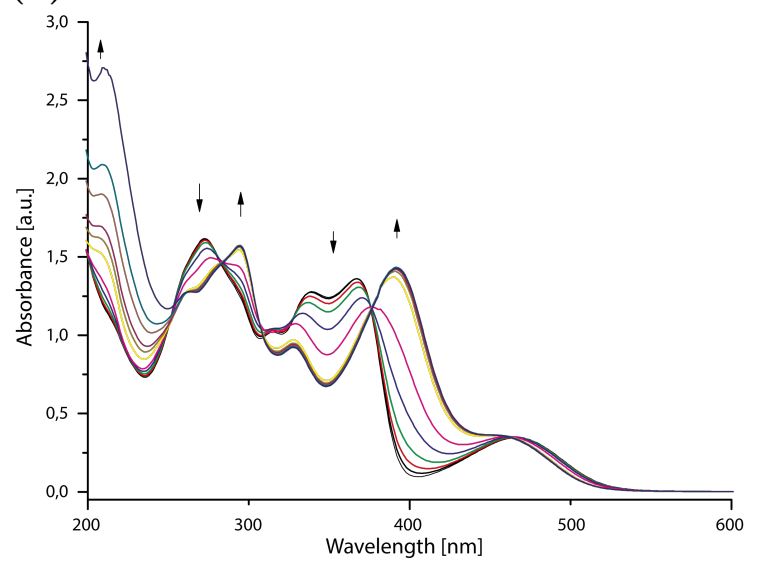

(c) (b)

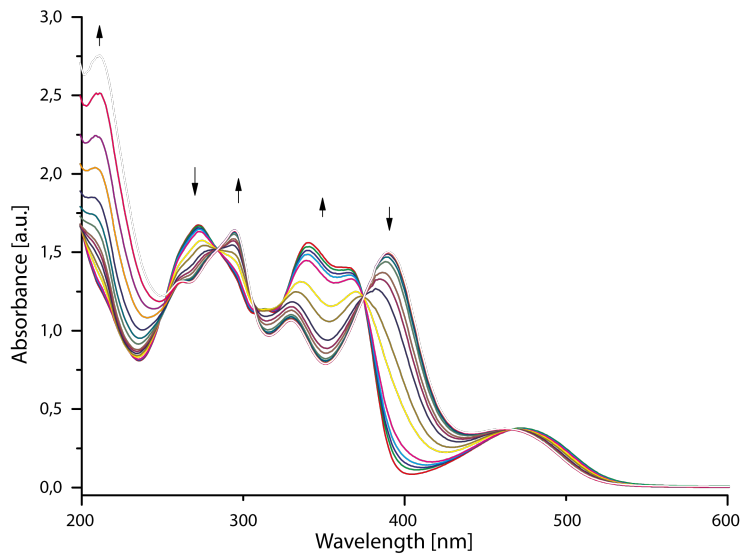

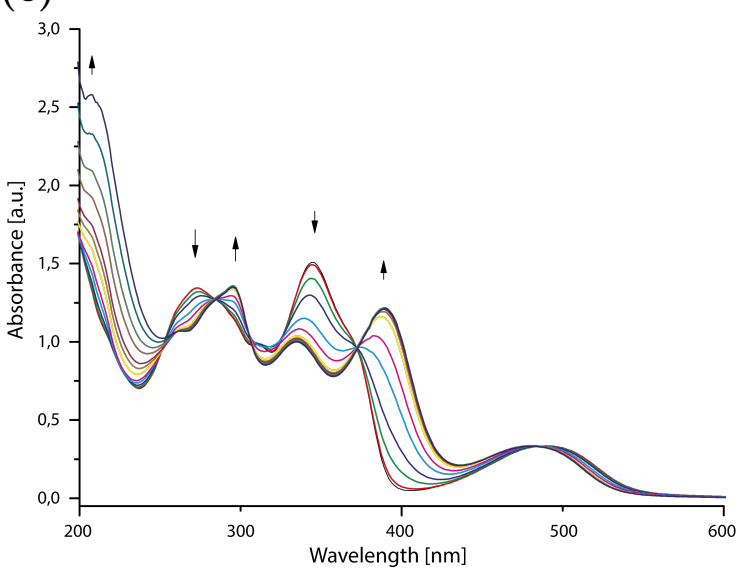

Figure V.2.2.11: UV-vis titration experiemts of all cyano acetate ligand derivatives a) $\left.\left.\mathbf{L}^{\mathbf{t B u}}, \mathbf{b}\right) \mathbf{L}^{\mathrm{Et}}, \mathbf{c}\right) \mathbf{L}^{\mathbf{P h}}$ $(0.035 \mathrm{mM})$ with TFA in MeCN. The TFA was added in 13 steps to an allover concentration of $0.13 \mathrm{M}$. Hyperand hypochromic shifts are indicated by arrows. In all spectra isosbestic points can be found, speaking for a concerted conversion into the protonated ligand species. Furthermore, a slight hypsochromic shift of $460 \mathrm{~nm}$ band, chararteristic for the quality of the push-pull system, can be observed for all derivatives, indicating a less favored conditions for a charge separated state.

As expected, upon protonation of the pyridines the proton signals $\mathbf{g} / \mathbf{g}^{\prime}$ and $\mathbf{f} / \mathbf{f}^{\prime}$ are downfield shifted, but only half as much $(0.15 \mathrm{ppm})$ with respect to the shift occurring upon coordination to palladium(II) cation during the cage formation (approx. $0.4 \mathrm{ppm}$ ). Whereas the signals $\mathbf{e} / \mathbf{e}^{\prime}$ and $\mathbf{d} / \mathbf{d}$ ' experience a rather large downfield shift $(0.8 \mathrm{ppm}$ and $0.65 \mathrm{ppm}$ ), while the shift in the cage is merely the half (Figure V.2.2.7c). However, the signals of the backbone are hardly effected by the protonation, although, against expectations the $\mathbf{a} / \mathbf{a}$ ' signals goes from a very broadened peak to a sharper and more defined peak with increasing TFA concentration, what indicates a increased rotation rate. That observation contradicts the hypsochromic shift in the UV-vis spectra, but shows definitely that rotating motion is maintained despite the presence of an excess TFA 


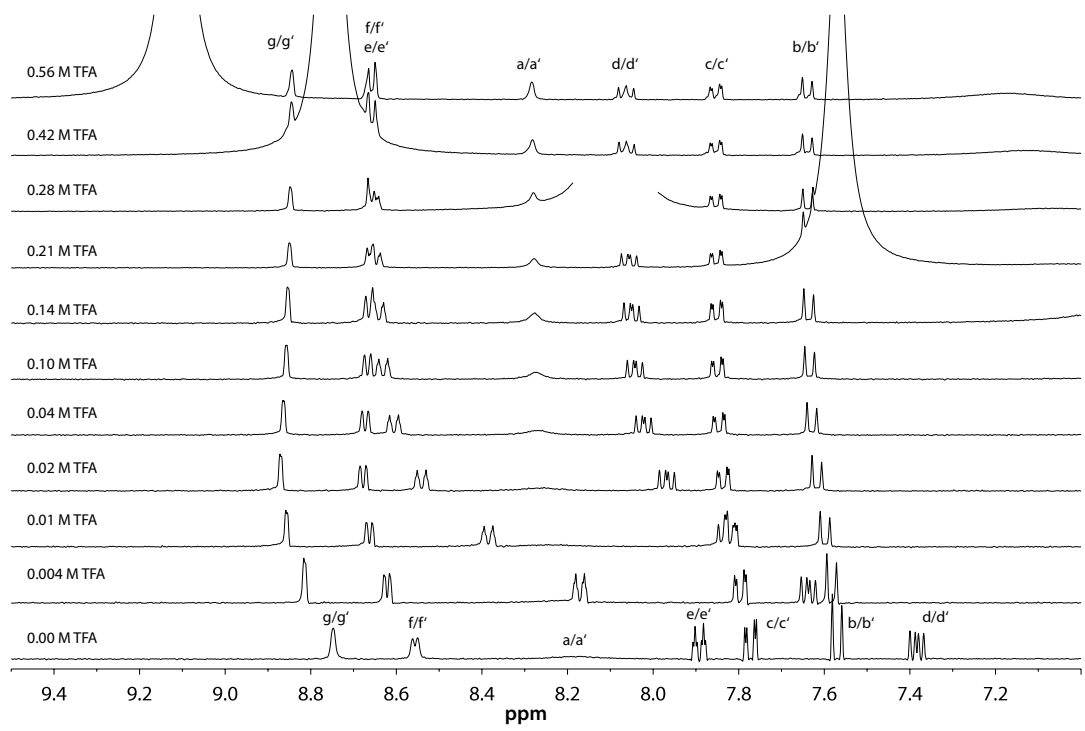

Figure V.2.2.12: ${ }^{1} \mathbf{H}$ NMR titration experiment of $\mathbf{L}^{\mathrm{Et}}(\mathbf{4 2})$ with TFA. All pyridine signals $(\mathrm{g} / \mathrm{g}$ ', f/f', e/e', d/d') are clearly downfield shifted, while the signals on the backbone expierence only a slight downfield shift. In presence of high concentration of TFA the a/a' signal gets sharpen due to the increased allover solvent polarity.

Neither the formation of cage does effect the rotation motion significantly, nor does the coordination of the pyridines to the palladium. In contrast, in presence of TFA the pyridines are protonated first and seem to prevent the protonation of the rotor group. Therefore, the titration of cage with TFA was investigated, since in metal-mediated supramolecular structure, the pyridines are blocked due the coordination to the the palladium(II) cations. Thus, it could be possible to protonate the rotor group again and generating the acridinium species, before the cage disassembles due to the high TFA concentration, resulting in a highly charged cage $(8+)$ with a strong fluorescence. 
(a)

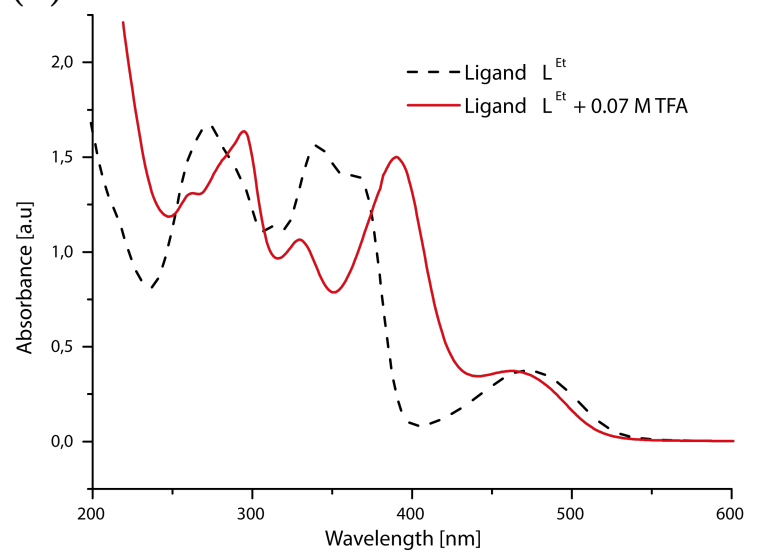

(c)

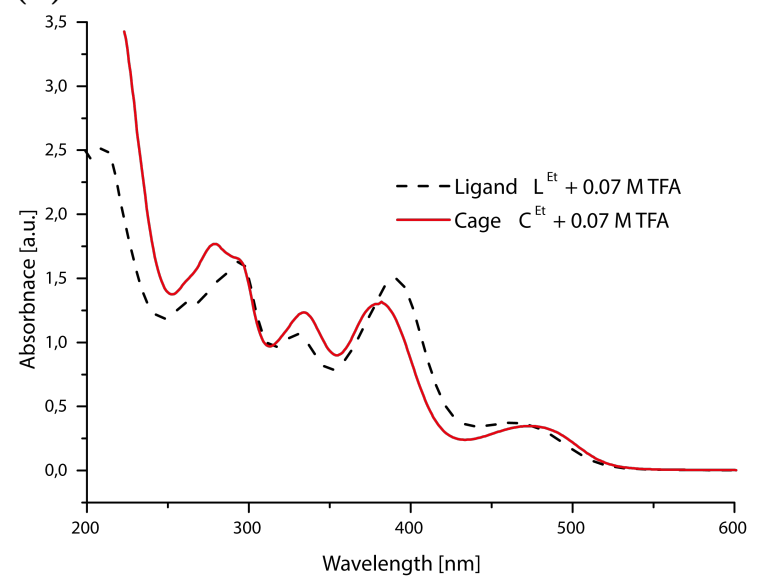

(b)

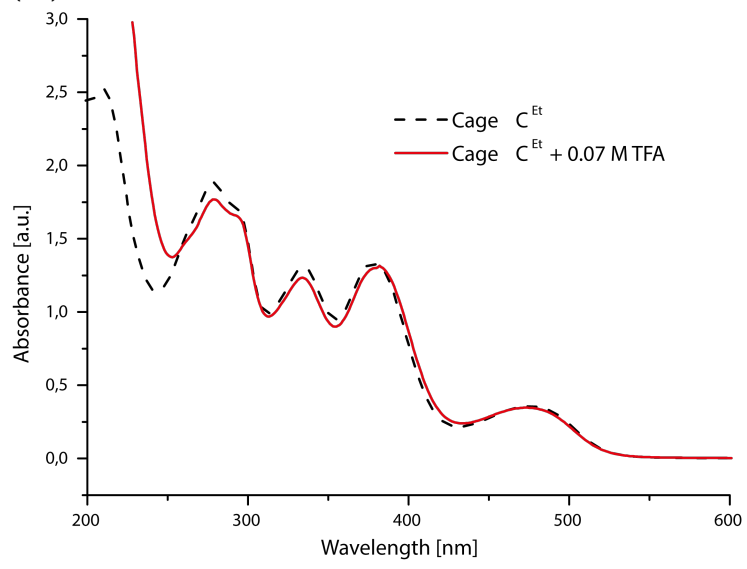

(d)

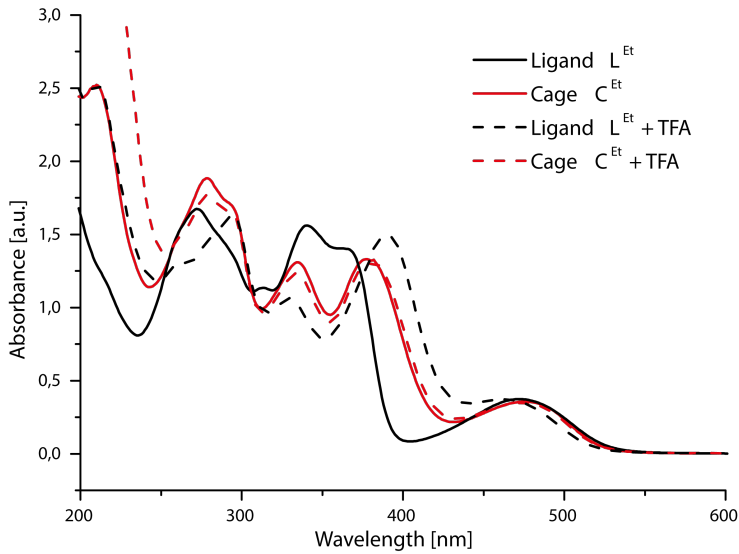

Figure V.2.2.13: UV-vis comparison of $\mathbf{L}^{\mathbf{E t}}$ and $\mathbf{C}^{\mathbf{E t}}$ in presence and abscence of TFA. All measuements were performed in MeCN with a total ligand concentration of $0.035 \mathrm{mM}$. a) Comparison between $\mathbf{L}^{\mathbf{E t}}$ its corresponding protonated species. b) Comparison between the cage $\mathbf{C}^{\mathbf{E t}}$ in presence and abscence of TFA. c) Comparison of ligand and cage in presence of TFA. d) Comparison of ligand and cage in presence or abscence of TFA.

The titration experiment (Figure V.2.2.13b) shows no real change in its absorption pattern upon addition of excess of acid, not even after addition of 4000 equiv. TFA (aprrox. $70 \mathrm{mM}$ ). The slight general hypochromic shift is caused by dilution. Comparison of figures V.2.2.13a and $\mathrm{V} .2 .2 .13 \mathrm{~b}$ elucidates the huge difference in absorption changes for the ligand in the presence of acid, while there is virtually no change for the cages' case. Furthermore, figure V.2.2.9 compares the spectra for the cage in presence of acid and the protonated ligand. Although the spectra exhibit a certain resemblance, the difference was still sufficient to clearly distinguish both species. On the one hand, this finding suggest that the cage can withstand the presence of acid, but on the other hand, it shows that the backbone is neither protonated in the cage nor in the free ligand. Nevertheless, this apparent resistance of the cage towards acid was suspicious, hence, a second titration experiment was made, but monitored by ${ }^{\mathbf{1}} \mathbf{H}$ NMR spectroscopy (Figure V.2.2.14). 


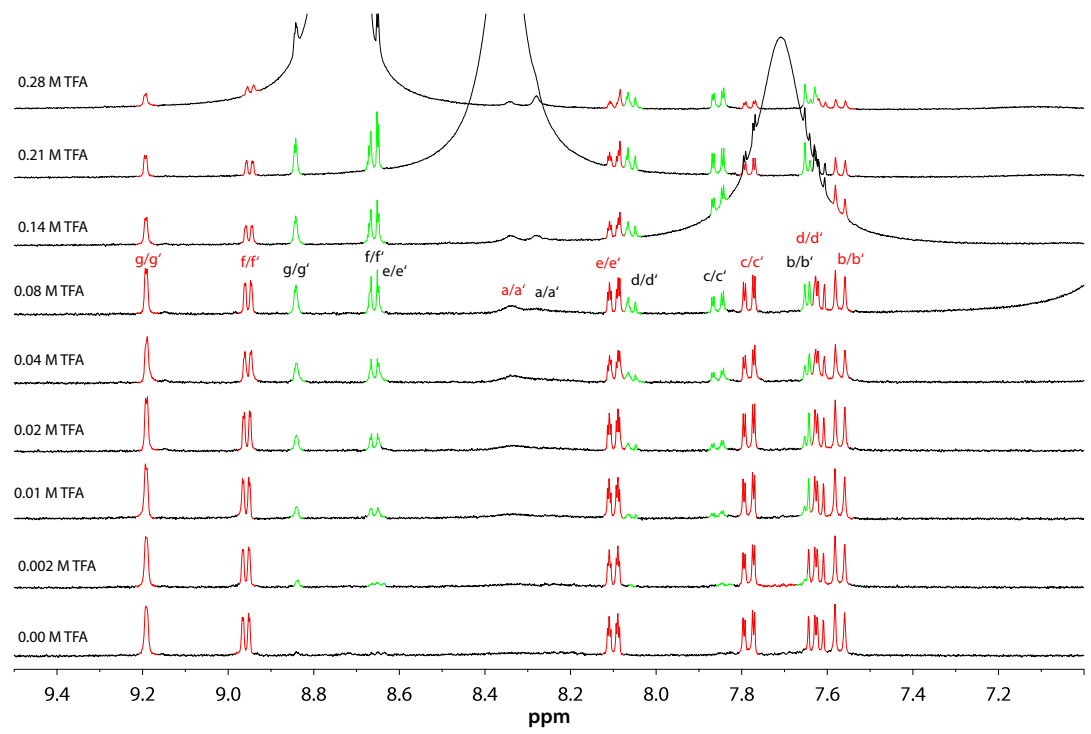

Figure V.2.2.14: ${ }^{\mathbf{1}} \mathbf{H}$ NMR $\left(400 \mathrm{MHz}, \mathrm{d}^{3}-\mathrm{MeCN}\right)$ titration experiment of $\mathbf{C}^{\mathbf{E t}}(2.8 \mathrm{mM})$ with TFA. Upon addition of TFA a new species is rising up, becoming more predominent with increased TFA concentrations (highlighted in green), while the signals of the starting material are slowly vanishing (highlighted in red).

And in fact, already at a concentration of $2 \mathrm{mM}$ TFA, first small signals appear (green), which grow with increasing TFA concentration, while the cage signals slowly disappear. In figure V.2.2.15 the spectrum of the $\mathbf{C}^{\mathbf{E t}}$ in presence of $80 \mathrm{mM}$ TFA is compared with the the spectra of the protonated ligand $\mathbf{L}^{\mathbf{E t}}$ (green) and of the cage $\mathbf{C}^{\mathbf{E t}}$ in absence of acid (red). And indeed, the appearing new signals belong the protonated ligand. In summary, the cage disassembles in presence of acid, but it takes a abundant excess of acid to shift the equilibrium to the ligands side, what was probably the reason why the disassembling could not be seen in the UV-vis titration experiments. 


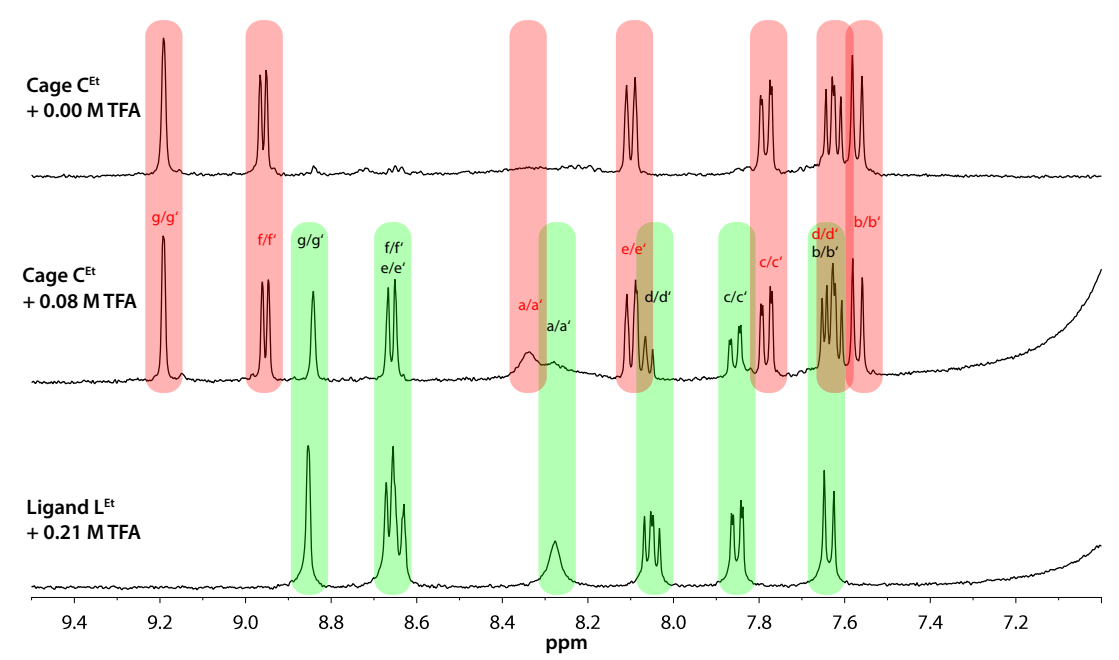

Figure V.2.2.15: Comparison of ${ }^{1} \mathrm{H}$ NMR spectra of cage 46 and ligand 42 in presence of TFA. All spectra were recorded in $\mathrm{d}^{3}-\mathrm{MeCN}$. It can be clearly seen, that the generated species is the protonated ligand (highlighted in green) and coexists with the assembled cage (highlighted in red).

\section{V.2.2.7 Guest encapsulation and its influence on the rotation}

In previous $\left[\mathrm{Pd}_{2} \mathrm{~L}_{4}\right]$ cage systems $\left.51|305| 306\right]$ it became apparent that small anionic molecules are particularly suitable for the investigation of the host guest chemistry. In the structural related adamantyl system, 24 Löffler et al. mainly used small aromatic bis-sulfonates. To get a better comparison of these two system, a selection of these bis-sulfonate guest molecules were used for the host-guest titration experiments (Scheme V.2.2.9). However, the focus of these studies was to find a combination, that represents the influence of the guest uptake on endohedral dynamic motion.

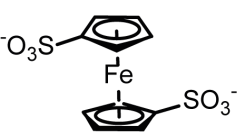

$\mathbf{G}^{1}$<smiles>O=S(=O)([O-])c1ccc(S(=O)(=O)O)cc1</smiles>

$\mathbf{G}^{2}$

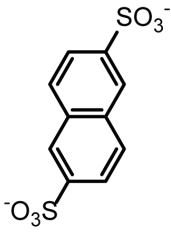

$G^{3}$<smiles>O=[SH]c1ccc(-c2ccc(O)cc2)cc1</smiles>

$\mathbf{G}^{4}$<smiles>O=S(=O)(O)c1ccc2ccc(S(=O)(=O)O)cc2c1</smiles>

$\mathbf{G}^{5}$

Scheme V.2.2.9: Guest molecules $G^{1}-G^{5}$ for ${ }^{1} \mathbf{H}-N M R$ host-guest titration experiments. All guests come with two tetra-n-butylammonium cations $\left.(\mathrm{nBu})_{4} \mathrm{~N}^{+}\right)$as counter-ions.

Löffler et al. described three cases for the encapsulation of guests in the host-guest titration experiments: 
1. Slow exchange: The guest is encapsulated in the cage. Due to slow exchange new signals appear belonging to the $\left[\mathrm{G}_{\mathrm{OPd}} \mathrm{L}_{4}\right]$ species that a growing with every addition of guest, while the signals of the free host disappear. Furthermore, the inward pointing protons experience a significant change in chemical shift, due to the changed chemical environment and/or interaction with the encapsulated guest molecule. Moreover the signals of the encapsulated guest can be observed as well. An excess of guest lead to a precipitation of the the cage.

2. Fast exchange: The guest is encapsulated in the cage. Due to fast exchange the signals of the chemical shift of the inward pointing protons is changing gradually with every addition of guest. An excess of guest leads to the precipitation of cage.

3. No encapsulation: The signals of the free cage do not change at all. The signals of the free guest appear. An excess of guest leads to the precipitation of cage.

In the beginning, the same conditions for the titration experiments were used as Löfler et al. have described for their adamantyl system. A solution of the respected guest was added to the preassembled adamantyl cage in acetonitrile. The procedure was repeated for the cage system $\mathbf{C}^{\mathbf{E t}}$ with the guests $\mathbf{G}^{\mathbf{1}} \mathbf{G}^{\mathbf{5}}$ Figures VI.1.7.3 to VI.1.7.7). Similar to the adamantyl cage $\mathbf{G}^{2}$ and $\mathbf{G}^{4}$ did show a slight shift for the the pyridine signals, indicating an encapsulation of guest but in fast exchange mode. The same behavior is seen for ferrocene guest $\left(\mathbf{G}^{\mathbf{1}}\right)$ which showed an encapsulation with slow exchange in the adamantyl system. However, since we expected to have a better chance to observe the influence of the guest uptake on the rotation motion in a system where the encapsulation occurs in a slow exchange fashion, the titration experiments were performed with the more steric demanding guests $\mathbf{G}^{\mathbf{3}}$ and $\mathbf{G}^{\mathbf{5}}$. Both experiments showed upon the first addition an extreme broadening of all signal in the NMR spectrum, though a broad significantly downfield shifted signal appeared, assumed to be inward pointing proton $\mathrm{g} / \mathrm{g}$ '. This spectrum differs from the one, where fast exchange of the guest is assumed to occur. Probably in this case the encapsulation process is proceeded with a slow-exchange mechanism, what would explain the significant downfield shift of $\mathbf{g} / \mathbf{g}^{\prime}$, however, the reason for the broadening of all signals upon guest encapsulation could be found.

Since the spectra showing broadening of the signals were not suitable for investigation of guest uptake influence on the dynamic motion, we concentrated on finding a good combination which shows fast exchange in the NMR titration experiments. Considering the previous findings Section V.2.2.2, the titration experiments were repeated with the already used host guest combinations, but with various solvents and at different temperatures Figures VI.1.7.8 to VI.1.7.10. The here shown examples are all from the 
titration experiments with guest $\mathbf{G}^{\mathbf{5}}$, as it emerged to be the most promising combination. Interestingly, in nitromethane the before described broadening occurs again, likewise to the experiments in acetonitrile. By performing the experiments at $60{ }^{\circ} \mathrm{C}$ the signals became again slightly sharper but were still to broad to gain any usable information (Figure VI.1.7.10). Under the same conditions but with methanol as solvent, the investigated system showed suddenly an encapsulation with a fast exchange rate. However, under both conditions precipitation of the cage was observable after adding more than 0.4 equivalents of guest to the cage solution. The encapsulation of the bis-sulfonate guest decreases the overall charge of the cage construct and therefore the solubility in methanol (Figure VI.1.7.9). In DMSO the combination $\mathbf{C}^{\mathbf{E t}}$ and $\mathbf{G}^{\mathbf{5}}$ showed already at room temperature very promising results. Signal $\mathbf{g} / \mathbf{g}$ ' is slightly downfield shifted, therefore the encapsulation seems to take place with a fast exchange rate. However, for the first time, a significant broadening of signal $\mathbf{a} / \mathbf{a}^{\prime}$ is visible with increasing concentration of guest (Figure VI.1.7.8), but since the spectrum suffered from ample signal broadening, the experiment was repeated at $60{ }^{\circ} \mathrm{C}$ (Figure V.2.2.17).

(a)

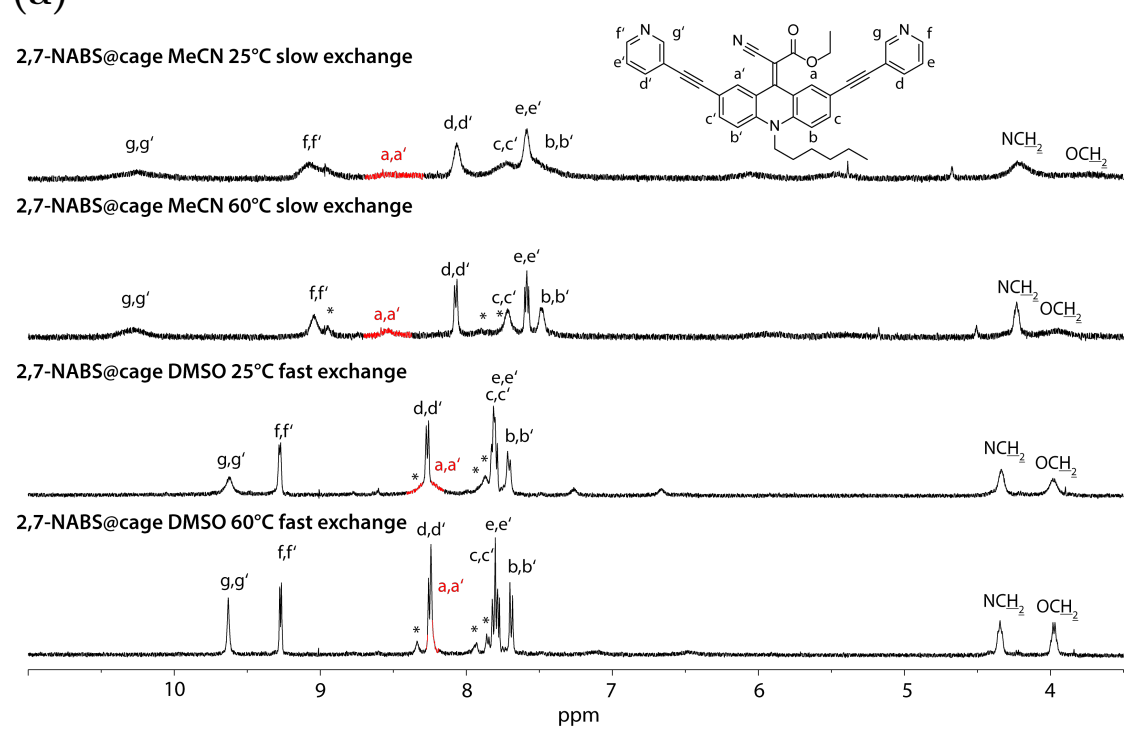



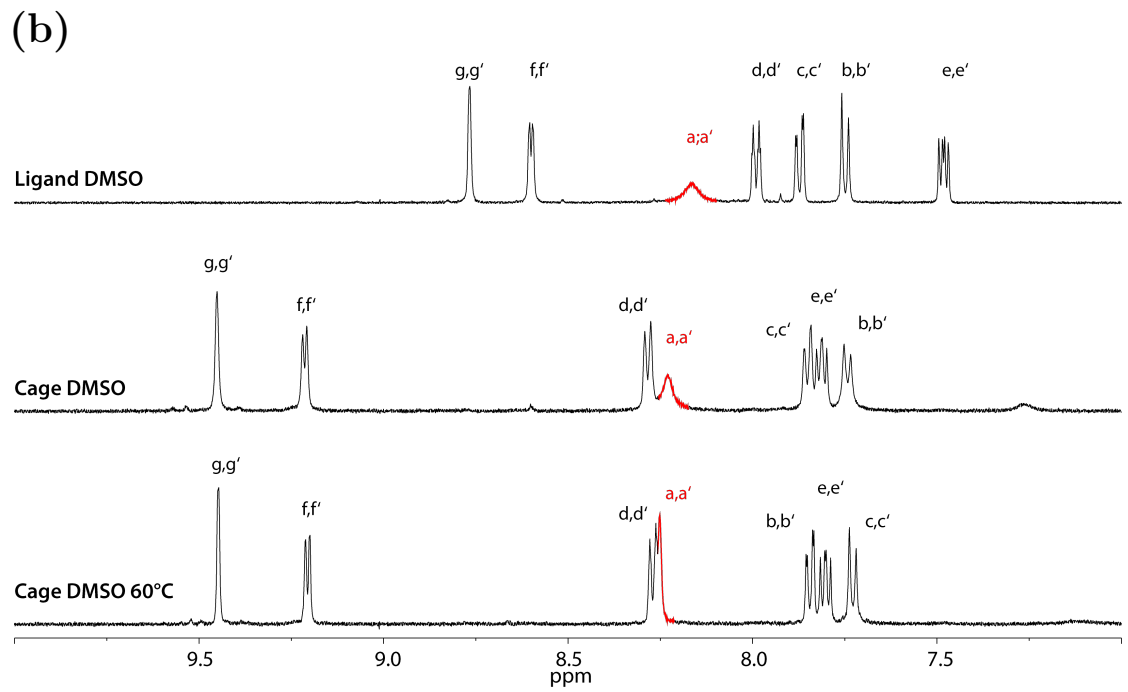

Figure V.2.2.16: Screening of proper conditions for host-guest experiemtens with $\mathbf{C}^{\mathbf{E t}}$ and $\mathbf{G}^{\mathbf{5}}$. a) NMR experiments with $\mathbf{C}^{\mathbf{E t}}$ and guest $\mathbf{G}^{\mathbf{5}}$ proceed in MeCN and DMSO at $298 \mathrm{~K}$ or $333 \mathrm{~K}$. In MeCN host and guest undergo a slow exchange process, since a new singal appear, while the actual cage signal do disapper. Nevertheless, the whole spectra extremly boradend at $298 \mathrm{~K}$ and $333 \mathrm{~K}$, making it difficult to assign the signals or determine any linewidth. In comparison, in DMSO host and guest undergo a fast exchange. The signals are much sharper especially the a/a' signal, making it easier so observe any changes in linewidth upon guest addition. The signals of the respective guest molecules are marked by an asterisk. b) Comparison of signal a/a' for ligand $\mathbf{L}^{\mathbf{E t}}(\mathbf{4 2})$ and cage $\mathbf{C}^{\text {Et }}(\mathbf{4 6})$ in DMSO at $298 \mathrm{~K}$ and $333 \mathrm{~K}$.

To clarify again what influence solvent polarity and temperature do exert on the system, in figure V.2.2.16a the NMR spectra of same cage guest combination $\left(0.7 \mathrm{mM} \mathrm{\mathbf {C } ^ { \mathrm { Et } }}\right.$ with 1.0 equiv. $\mathbf{G}^{\mathbf{5}}$ ) are illustrated, in acetonitrile at $25^{\circ} \mathrm{C}$ and $60{ }^{\circ} \mathrm{C}$ and in DMSO at the same temperatures. First observation to be mentioned, the exchange rate of guest is changing from slow exchange to fast exchange in terms of the NMR timescale, when going from acetonitrile to DMSO. Furthermore, an increase in temperature accelerates the dynamics in the whole system, what makes the NMR signals which are liable to a dynamic sharper in terms of their relative peak width. Exactly this case is observable in figure V.2.2.16a for both experiments. By going from $25{ }^{\circ} \mathrm{C}$ to $60{ }^{\circ} \mathrm{C}$ all signals become sharper, what is in total accordance to VT NMR experiments Section V.2.2.2), because all signals are liable to the intramolecular dynamic motion. The same effect occurs of course also in absence of the guest (Figure V.2.2.16b). A disadvantage of using DMSO as solvent is the overlapping of signals $\mathbf{d} / \mathbf{d}^{\prime}$ and $\mathbf{a} / \mathbf{a}^{\prime}$. It is noteworthy, that the measurement of the system in acetonitrile at $60{ }^{\circ} \mathrm{C}$ let most signals spawn from noise, what support our assumption that the extremely broadened spectra are caused by encapsulation on a slow exchange rate.

Despite the overlapping of signals, a careful analysis and line fitting including the resonance of the $\mathbf{a} / \mathbf{a}^{\prime}$ protons revealed that the encapsulation of the bis-sulfonate guest $\mathbf{G}^{\mathbf{5}}$ slows down the spinning of the endohedral rotors. The line fitting of the signals $\mathbf{a} / \mathbf{a}$ ' and $\mathbf{d} / \mathbf{d}$ ' is shown on the right of figure V.2.2.17. The increase in peak width is accompanied by 
the typical downfield shifts for the cage protons in contact with the guest (signals $\mathbf{g} / \mathbf{g}$, $\mathbf{a} / \mathbf{a}^{\prime}, \mathrm{OCH}_{2} \mathrm{CH}_{3}$ ). In figure V.2.2.18 the change of peak width for signals $\mathbf{a} / \mathbf{a}$ ' and $\mathbf{d} / \mathbf{d}$ ' are plotted vs. the concentration of guest.

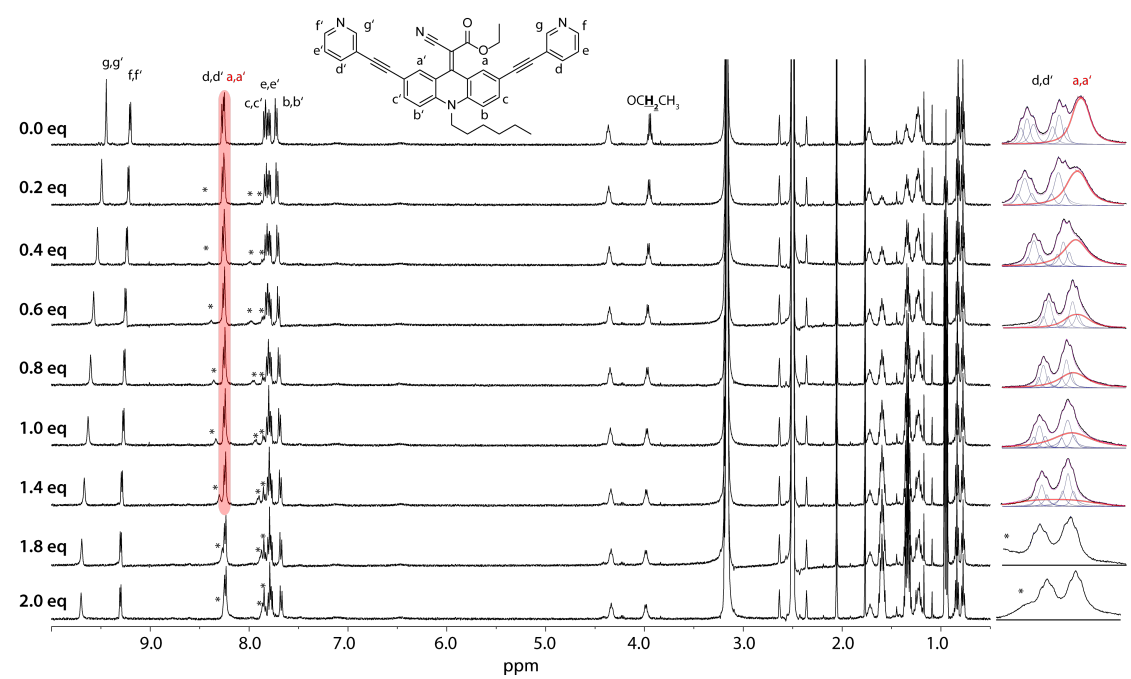

Figure V.2.2.17: $1 \mathrm{H}$ NMR titration $\left(400 \mathrm{MHz}, 333 \mathrm{~K}, d^{6}\right.$-DMSO) of $\left[\mathrm{Pd}_{2} \mathrm{~L}^{\mathrm{Et}}{ }_{4}\right]$ with $\left(\mathrm{NBu}_{4}\right)_{2} \mathrm{G}^{5}$. The chemical shifts of theinward pointing protons change gradually due to a fast exchange of the guest on the NMR time scale. Guest signals are marked with *. On the right line fittings analyses of the deconvoluted signals d/d' and a/a' are shown. Whereas the $\mathbf{d} / \mathbf{d}$ ' signals do not broaden upon guest addition, the signal assigned to a/a' clearly broadens with increasing amounts of the guest which indicates that the encapsulated guest slows down the spinning of the rotors inside the cavity.

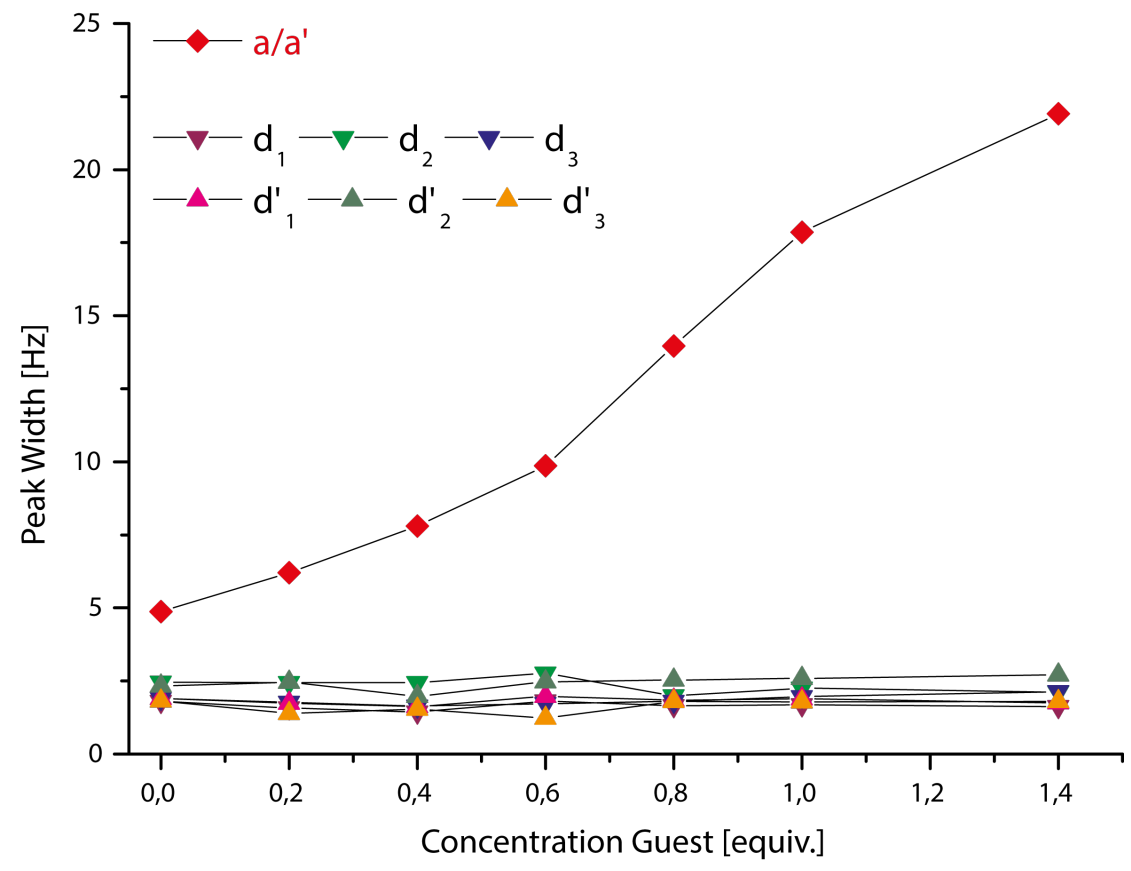

Figure V.2.2.18: Correlation of peak width with the concentration of guest $\mathbf{G}^{\mathbf{5}}$ from line fitting analysis of the NMR titration data shown in figure V.2.2.17. The significant change in peak width of the a/a' signal indicates a decrease of rotation rate upon guest uptake, while the peak width of other signals stays nearly constant. 


\section{V.2.3 Conclusion}

This chapter described the synthesis and characterization of four new endohedrally functionalized bis-monodentate banana-shaped ligands $\left(\mathbf{L}^{t \mathbf{B u}}, \mathbf{L}^{\mathbf{E t}}, \mathbf{L}^{\mathbf{P h}}\right.$ and $\left.\mathbf{L}^{\mathbf{C N}}\right)$ based on the acridone backbone. These ligands possess electron-withdrawing substituents, either a symmetric malononitril group or an asymmetric cyano acetate ester group (tert-butyl, ethyl or phenyl) protruding sideways from its concave face. Although connected by a double bond, the substiuents exhibit an rotational motion in respect to the polycyclic backbone, caused by an intermolecular backbone. This rotation motion is qualitatively observable in the asymmetric substituted derivatives by dynamic NMR spectroscopy. In contrast to the small model compound described in the previous chapter, the ligand does not form an acridinium moiety in presence of strong acid and therefore does not show any strong fluorescence. Instead, the pyridine arms are protonoated, which does not seem to influence the intramolecular push-pull system considerably. The presence of palladium(II) cations lead to a clean formation of supramolecular homoleptic coordination cages, what could be verified by NMR and ESI-MS measurements. Moreover, the rotation is maintained within the cage and even slightly accelerated compared the free ligand. In presence of TFA the cage $\mathbf{C}^{\mathbf{E t}}$ could not withstand, but relatively high concentrations of strong acid were needed for a complete disassembly. Furthermore, several host guest titration experiment were carried out, to find a system that clearly shows an effect on the endohedral rotational motion caused by the guest uptake. 2,7-naphtalene bis-sulfonate slowed the rotation significantly down, when encapsulated in the cage $\mathbf{C}^{\mathbf{E t}}$. This guest-induced "breaking effect" complements the previous findings concerning the flipping motion in a related adamantyl cage. 24 Based on the here present results, we were curious if the cages' guest uptake affinity can be changed by an stimuli responsive tuning of the endohedral rotation. 


\section{Chapter}

\section{Azacrown-Cyano-Acridinylidne-Ethyl Acetate}

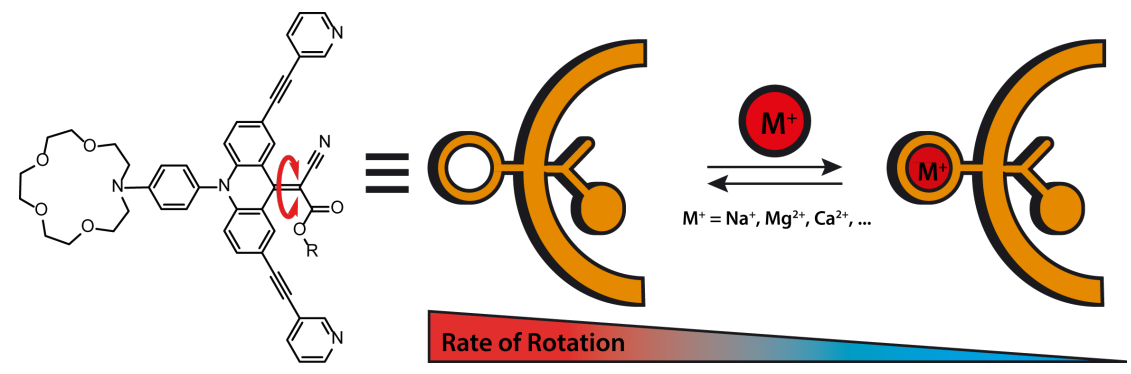

Contents

V.3.1 Introduction . . . . . . . . . . . . . . 178

$\mathbf{V . 3 . 2}$ Results and discussion . . . . . . . . . . . . . . . . . 179

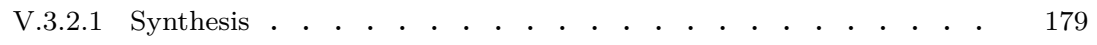

V.3.2.2 Comparison of $\mathbf{B}_{\mathbf{A z a c r o w n}}{ }^{\mathrm{Et}}$ to $\mathbf{B}^{\mathrm{Et}}$ in terms of rotational motion 181

V.3.2.3 Effect of Metal uptake in Azacrown on Acridinyline System . . . 184

\begin{tabular}{|l}
\hline V.3.2.4 Influence of alkali metal binding on the rotation rate in $\mid$ VT NMR $\mid$ \\
experiments . . . . . . . . . . . . . . . . . .
\end{tabular}

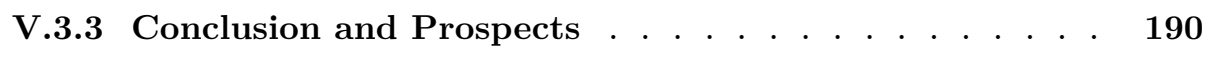




\section{V.3.1 Introduction}

In the previous chapters we could show that the rotation of the electron-withdrawing substituent was induced by a reduced bond order, caused by the intramolecular push-pull system induced charge separation. The quality of the push-pull system could be significantly tuned by small variations in the electronic contribution of the ester substituent what led to a better stabilization of the charge separated state and therefore to a faster or slower rotation. Furthermore we were able to integrate this dynamic system in supramolecular coordination cage while maintaining the rotational motion in the inner cavity. Moreover, the uptake of an bis-anionic guest into the supramolecular construct resulted in a distinct deceleration of the rotation rate. Since the the guest uptake seems to influence the dynamic in the cage, the question was if the guest uptake/release behavior can be influenced by the speed of rotation.

Especially with regard to idea of using the cages' cavity as supramolecular container, 307 reaction vessel $45[308$ or as drug delivery system,, 309 a stimuli responsive control over the host-guest chemistry could be of advantage. Concerning the reaction vessel, these system sometimes suffer from product inhibition, $\underline{310}$ meaning, the formed product stays inside the cavity, since it has a higher affinity compared to the substrate. A stimuli-responsive change in binding affinity could resolve such problems. Far more interesting is this approach for the latter topic. In several publication already dozens of attempts for stimuli responsive drug delivery systems were presented and discussed, 311 going from stimuli by $\mathrm{pH}, 312$ redox, 313 heat, 314 light 315 to magnetic 316 or even ultrasound. 317

In a previous work Han et al. could already show that the guest uptake and release behavior can be controlled by a light-triggered conversion from a flexible into rigid form of supramolecular coordination cage and vise versa. 300 Motivated by these results we wanted to design a system based molecular rotor system where the electronics of the intramolcular push-pull system can be influenced on the electron-donating side. Hence it had to be a functionality that can be attached to the aromatic nitrogen instead of the hexyl chain.

Since, our cages are normally not soluble in water and the coordination cages do not tolerate higher concentration of acid, we decided for a crown ether motif to bind alkali or earth alkali metals, which seem not to have a significant effect on the metal-ligand coordination as long as their counter-anions are of non-coordinative nature. 31 In literature some examples can be found, where azacrown ethers are integrated to the $\pi$ system of an chromophore, whose intramolecular electronic situation is manipulated by binding of cations to the azacrown ether, accompanied by a change of color. This process is generally called as ionochroism. 318 In the case of azacrown ether containing push-pull systems, the azacrown ether can be part of the electron-withdrawing motif, engendering a positive ionochroism 319 within the system (indicated by a bathochromic shift in the UV-vis absorption), or on the other hand, becomes part of the electron-donating motif, causing a weakening of electron-pushing effect by binding a cation (indicated by a hypsochromic 
shift in UV-vis absorption). 320.322 Therefore, a system was designed fitting the latter approach, in order to get a weakening of the intramolecular push-pull system, causing a deceleration in rotation, intended to give a change in the host-guest affinity.

\section{V.3.2 Results and discussion}

\section{V.3.2.1 Synthesis}

As the synthesis turned out to be quite challenging, we did not immediately plan to synthesis the whole ligand, but had a recourse to our model compound $\mathbf{B}^{\mathbf{E t}}$, so that the design of the system could be tested first before it is transferred into a ligand or coordination cage.

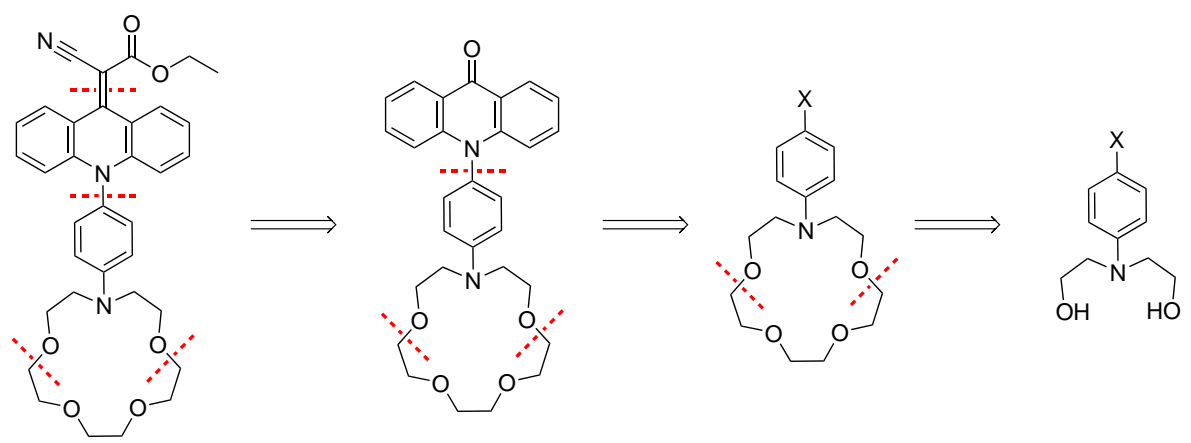

Scheme V.3.2.10: Retrosynthetic analysis of the intended azacrown ether system. The final product is cut up in four parts. The cyano acetate ester, the acridone backone and the opposing azacrown ether, splitted into the phenyl linker and the macrocycle itself.

From a retrosynthetic perspective, the azacrown ether was synthesized separately, before it was merged to the acridone backbone. In a last step the electronwithdrawing malonic acid derivative was introduced to complete the synthesis Scheme V.3.2.10 


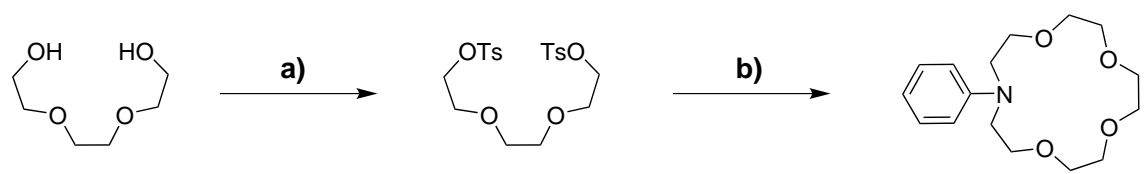

48

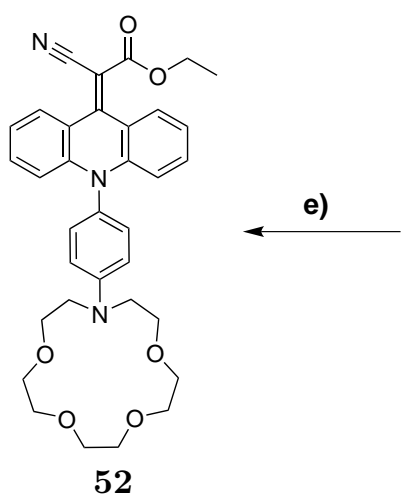<smiles></smiles><smiles>Nc1ccc(N2CCOCCOCCOCC2)cc1</smiles>

51
49

c)

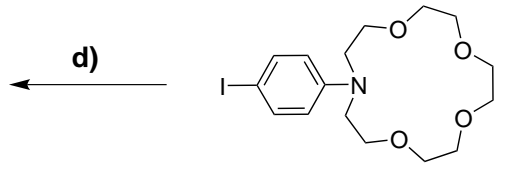

50

Scheme V.3.2.11: Synthesis path for compound $\mathbf{B}_{\text {Azacrown }}{ }^{\mathbf{E t}} \mathbf{5 2}$. a) TsCl,NEt ${ }_{3}, \mathrm{DCM}$; b) N-phenyl diethanol amine,NaH,THF; c) $\mathrm{KI}_{2} \mathrm{H}_{5} \mathrm{IO}_{6}, \mathrm{DCM} / \mathrm{H}_{2} \mathrm{O}$; d) acridone,dipivaloylmethane, $\mathrm{N}(\mathrm{nBu})_{4} \mathrm{OH}, \mathrm{CuI}, \mathrm{DMF} ;$ e) cyano ethyl acetate, $\mathrm{TiCl}_{4}, \mathrm{NEt}_{3}, \mathrm{DCM}$.

Based on the retrosynthetic analysis the proposed synthetic steps Scheme V.3.2.11) were processed by Halil Calik, in order to obtain $\mathbf{B}_{\text {Azacrown }}{ }^{\mathbf{E t}}$ (52). In a first step, tosylat was introduced to both hydroxy groups of the triethylene glycol to have a better leaving group for the next step in synthesis, where the azacrown ether is formed. The syntheses of the $N$-phenyl-aza-15-crown-5 ether was performed under high dilution conditions, as described in literature, 323 to avoid the formation of byproducts. In a further reaction iodine was introduced to the phenyl ring in para position to the azacrown ether, what was crucial for the C-N coupling reaction in the next step. Since the reaction was only known for simple iod-phenyl compound but not for its anilin derivatives, a palladium catalyzed BuchwaldHartwig amination reaction was performed, but without success. Despite variation of catalytic compounds, base and solvent the desired product could not be obtained. Also the change to a copper catalyzed Ullmann reaction initially did not lead to the desired product. Only after using bases with sterically demanding, non-coordinating conjugated acids, like tetrabutylammonium hydroxide, the coupled product could be isolated from reaction mixture. In the subsequent Lehnert modified Knoevenagel reaction the end product could be obtained as a red solid. 


\section{V.3.2.2 Comparison of $\mathrm{B}_{\mathrm{Azacrown}}{ }^{\mathrm{Et}}$ to $\mathrm{B}^{\mathrm{Et}}$ in terms of rotational motion}

At first both systems $\mathbf{B}^{\mathbf{E t}}$ and $\mathbf{B}_{\text {Azacrown }}{ }^{\mathbf{E t}}$ were investigated on their spinning behavior. Therefore VT NMR experiments were performed for $\mathbf{B}_{\text {Azacrown }}{ }^{\mathbf{E t}}$ and the spectra were compared to those of $\mathbf{B}^{\mathrm{Et}}$ (Figure V.3.2.1). Qualitatively both spectra show a similar behavior on the variation of temperature, although the $\mathbf{B}_{\text {Azacrown }}{ }^{\mathbf{E t}}$ derivative seems to be slightly faster, what can be noticed by comparing the peak shape of the signal a at $-40{ }^{\circ} \mathrm{C}$. Furthermore, the signal e admittedly shows a $-80{ }^{\circ} \mathrm{C}$ a broadening of the signal, but is not yet split into $\mathbf{e}_{\boldsymbol{\alpha}}$ and $\mathbf{e}_{\beta}$ as it can be seen in the ${ }^{\mathbf{1}} \mathbf{H}$ NMR spectrum of $\mathbf{B}^{\mathbf{E t}}$. These findings indicating a slightly enhanced interaction of the electron-withdrawing and -donating function within $\mathbf{B}_{\text {Azacrown }}{ }^{\mathbf{E t}}$, what is not uprising, though, the azacrown nitrogen acts as an additional electron-donating part. This become quite apparent on the chemical shift of the signal $\mathbf{d} / \mathbf{d}$. While all signals of the acridone exhibit only tiny almost negligible changes, d/d' experience an significant upfield shift (approx. 0.5 ppm), indicative for an increased electronic density. 


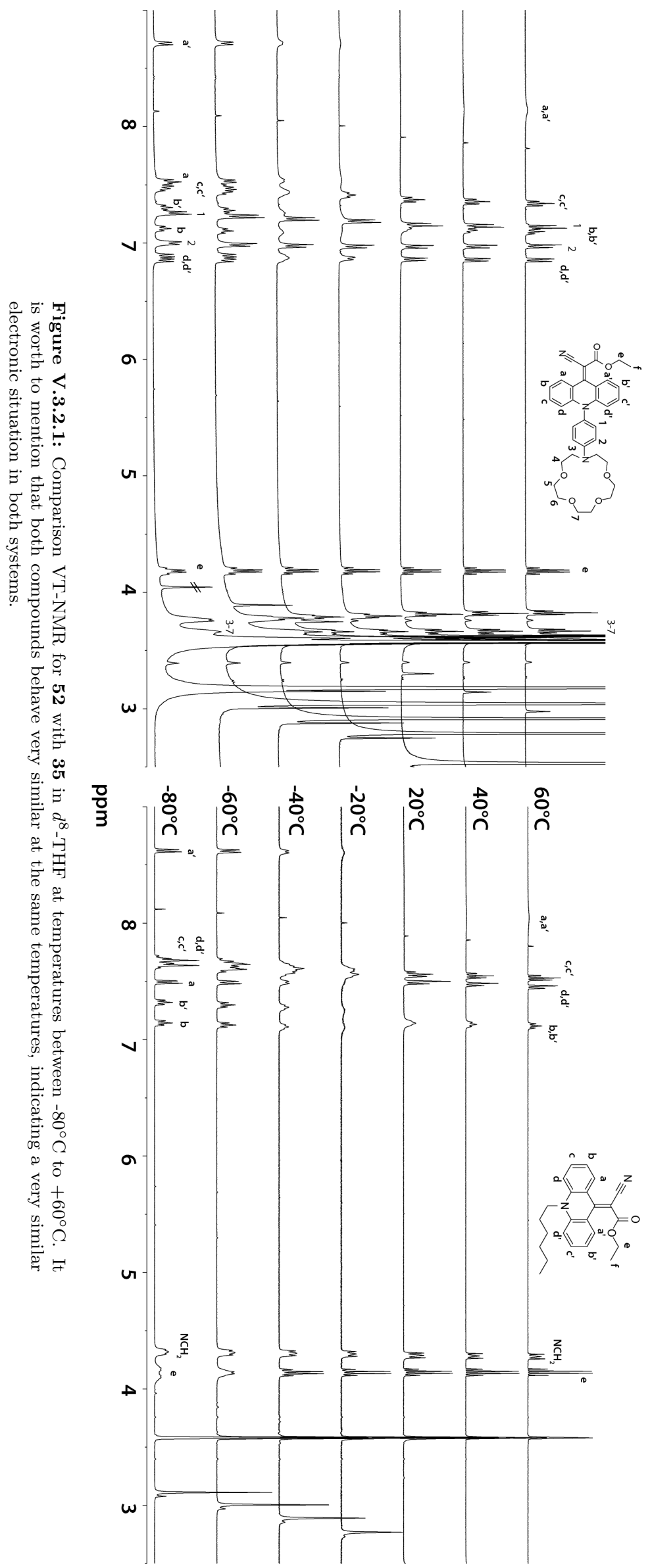


To confirm the above mentioned differences, a qualitative analysis of the obtained data was accomplished. Therefore, the thermodynamic parameters $\Delta H^{\ddagger}$ and $\Delta S^{\ddagger}$ were determined from experimental and simulated data, as it was already done before for $\mathbf{B}^{\mathrm{Et}}$ Section V.1.2.4. The rate constants extracted from experimental and simulated VT NMR spectra are plotted in figure V.3.2.2. From the corresponding Eyring plots the enthalpy and entropy of activation were calculated, which are presented in table V.3.2.1 in comparison to those determined for hexyl chain analogon $\mathbf{B}^{\mathbf{E t}}$.

(a)

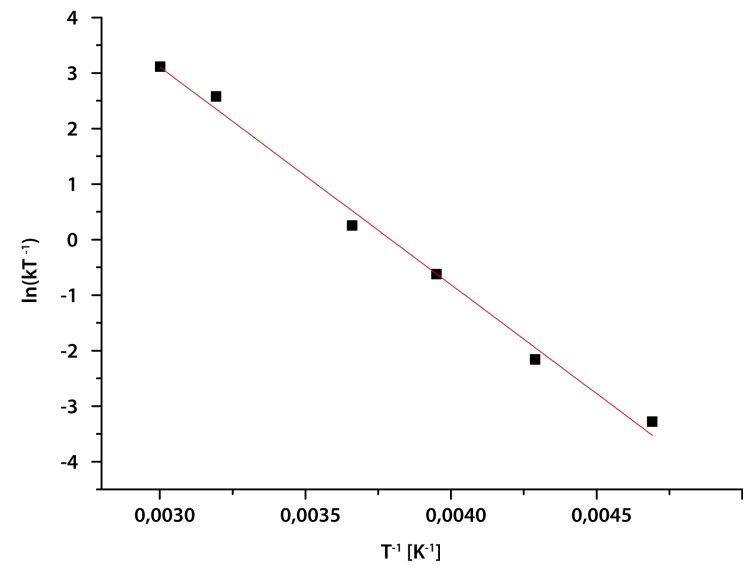

(b)

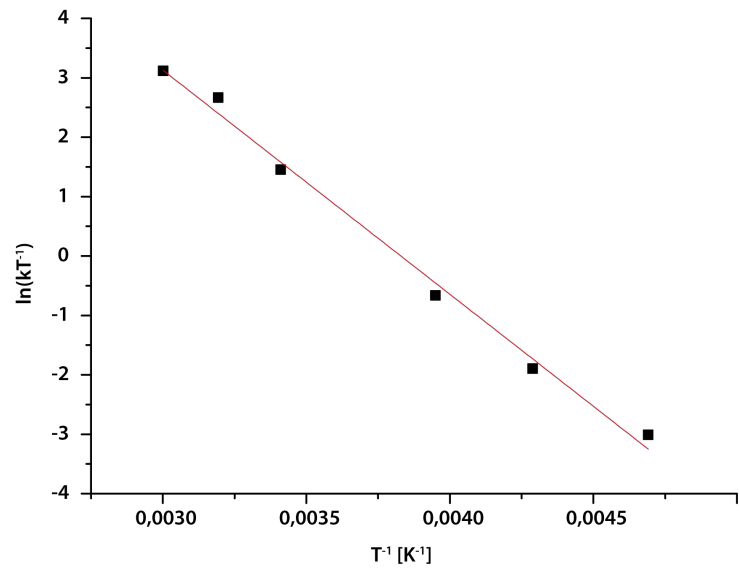

Figure V.3.2.2: Eyring plots of experimental determined (a) and simulated (b) rotation rates. The determined thermodynamic values from these plots are presented in table V.3.2.1

Table V.3.2.1: Activation enthalpies, entropies and Gibbs' free energies of $\mathbf{B}_{\text {Azacrown }}{ }^{\mathbf{E t}}$ in comparison to $\mathbf{B}^{\mathbf{E t}}$ calculated from experimentally determined rate constants and from simulations.

\begin{tabular}{lcc}
\hline & B $_{\text {Azacrown }}{ }^{\text {Et }}(\mathbf{5 2})$ & $\mathbf{B}^{\mathbf{E t}}(\mathbf{3 5})$ \\
\hline \hline$\Delta H^{\ddagger}{ }_{\exp }\left[\mathrm{k} J \mathrm{~mol}^{-1}\right]$ & $32.6 \pm 1.3$ & $34.3 \pm 1.3$ \\
$\Delta S^{\ddagger}{ }_{\exp }\left[\mathrm{k} J \mathrm{~mol}^{-1} \mathrm{~K}^{-1}\right]$ & $-73.9 \pm 5.3$ & $-72.5 \pm 5.2$ \\
$\Delta G^{\ddagger}$ exp $298 \mathrm{~K}\left[\mathrm{k} \mathrm{mol}^{-1}\right]$ & 54.6 & 56.0 \\
$\Delta H^{\ddagger} \operatorname{sim}\left[\mathrm{k} J \mathrm{~mol}^{-1}\right]$ & $31.4 \pm 1.3$ & $33.1 \pm 0.5$ \\
$\Delta S^{\ddagger}{ }_{\operatorname{sim}}\left[\mathrm{k} J \mathrm{~mol}^{-1} \mathrm{~K}^{-1}\right]$ & $-77.4 \pm 5.0$ & $-76.6 \pm 1.9$ \\
$\Delta G^{\ddagger}{ }_{\operatorname{sim}}^{298 \mathrm{~K}}\left[\mathrm{~kJ} \mathrm{~mol}^{-1}\right]$ & 54.5 & 56.0 \\
\hline
\end{tabular}

Undoubtedly, there is a difference in the enthaply of activation for $\mathbf{B}_{\mathbf{A z a c r o w n}}{ }^{\mathbf{E t}}$, which is in total accordance to the qualitatively made assertion. It is noteworthy, for the value for the entropy of activation there is nearly no deviation, what suggest that the mechanism for the rotational motion is unchanged compared to the model compounds $\mathbf{B}^{t \mathbf{B u}}, \mathbf{B}^{\mathbf{E t}}$ and $\mathbf{B}^{\mathbf{P h}}$. Interestingly, in the UV-vis absorption spectrum there is virtually no difference, the maximum of absorbance is merely blue-shifted about $1 \mathrm{~nm}$ compared to $\mathbf{B}^{\mathrm{Et}}$ (Figure V.3.2.3). 


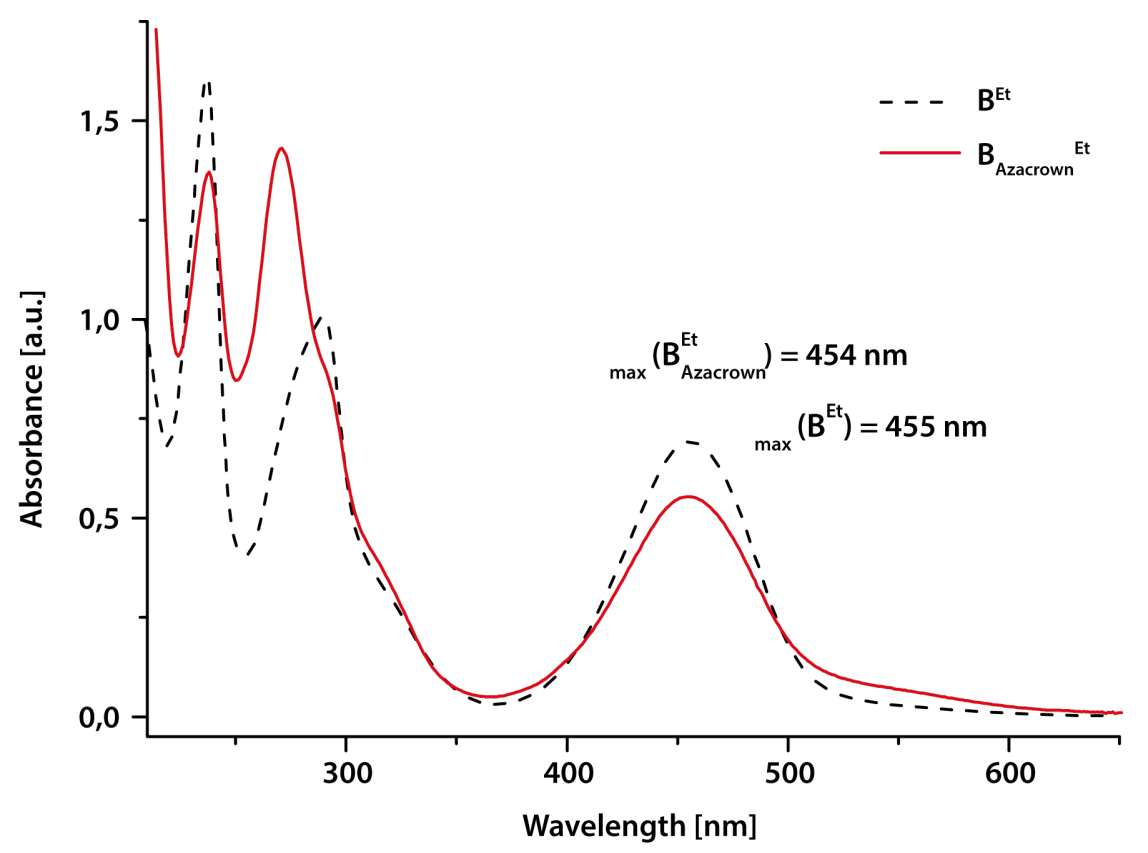

Figure V.3.2.3: Comparison UV-vis of $\mathbf{B}_{\text {Azacrown }}{ }^{\mathbf{E t}}$ and $\mathbf{B}^{\mathbf{E t}}$. Both spectra were recorded with $2.8 \mathrm{mM}$ of the respective compound in $\mathrm{MeCN}$

\section{V.3.2.3 Effect of Metal uptake in Azacrown on Acridinyline System}

Based on literature $\stackrel{321}{=}$ sodium, magnesium, calcium, and barium perchloride were chosen to investigate their impact on the push-pull system and the rotational motion when coordinating to the aza-15-crown-5 ether. For that reason UV-vis and NMR titration experiment were carried out with the respective metal and compound $\mathbf{B}_{\text {Azacrown }}{ }^{\mathbf{E t}}$. Furthermore, control titration experiments were accomplished with $\mathbf{B}^{\mathbf{E t}}$ to exclude unspecific metal interaction with the remaining system.

The UV-vis titration experiment with sodium perchlorate show a marginal hypsochromic shift in the region of interest Figure VI.1.7.11b. Whereas in ${ }^{1} \mathrm{H}$ NMR a significant downfield shift is observed for protons signals $\mathbf{1}$ and $\mathbf{2}$ of the bridging phenyl motif Figure VI.1.7.11a, red and green line indicate the approximate shifting routes), by what the the phenyl signal 2 even passes signal 1. In contrary, the proton signal $\mathbf{3}$ experience a slight upfield shift, while the residual crown ether signal stay nearly unchanged. All that speaks for an weak interaction between sodium cation and crown ether, therefore it is not surprising that no observable change in rotation rate happened. Next, the higher charged earth alkali metal magnesium in form of $\mathrm{Mg}\left(\mathrm{ClO}_{4}\right)_{2}$ was investigated. In NMR a clearly more intense downfield shift of both phenyl signals occurs. Additionally, all protons in the crown ether next to an oxygen show a clear downfield shift as well, while signals 3 experience again a slight upfield shift. The more surprising it was to see absolutely no change in UV-vis (the general hypochromic shift is due to dilution) Figure V.3.2.4a and section V.3.2.3). However, on the first glance also in this experiment did not show 
any changes. Through the control experiment with $\mathrm{Mg}\left(\mathrm{ClO}_{4}\right)_{2}$ titrated to a solution of $\mathbf{B}^{\mathbf{E t}}(\mathbf{3 5})$ an unspecific interaction could be excluded, since neither in NMR nor UV-vis a change could be noted.

(a)
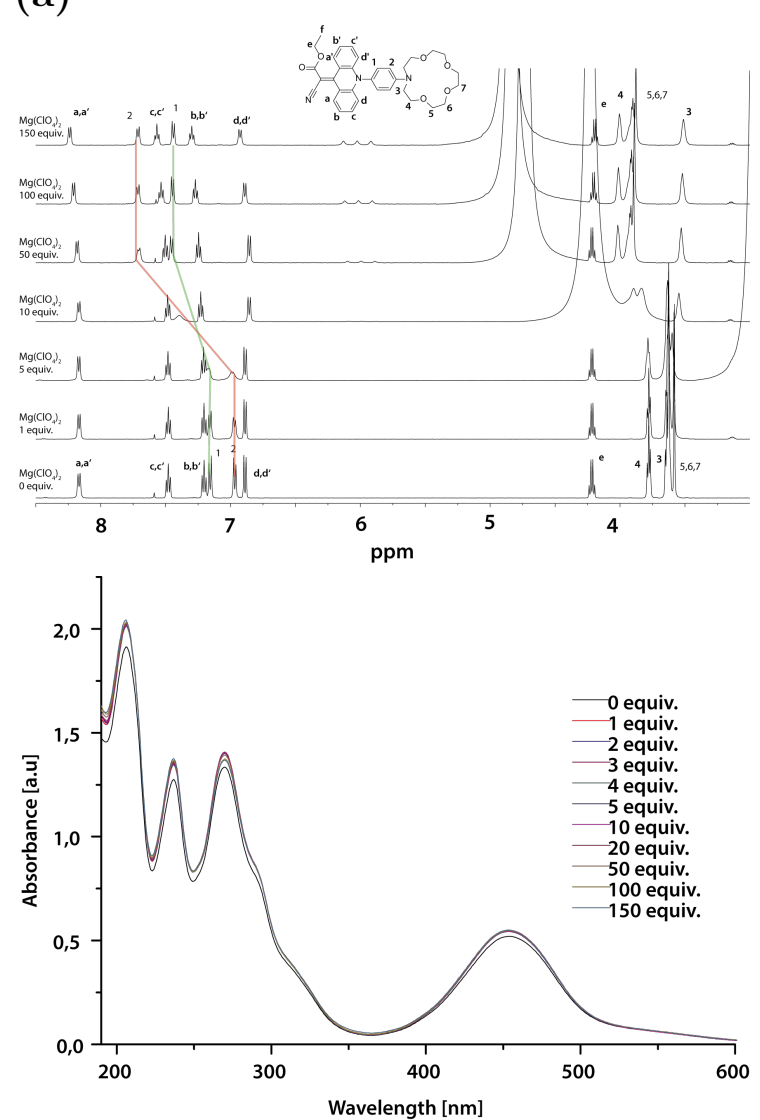

(b)
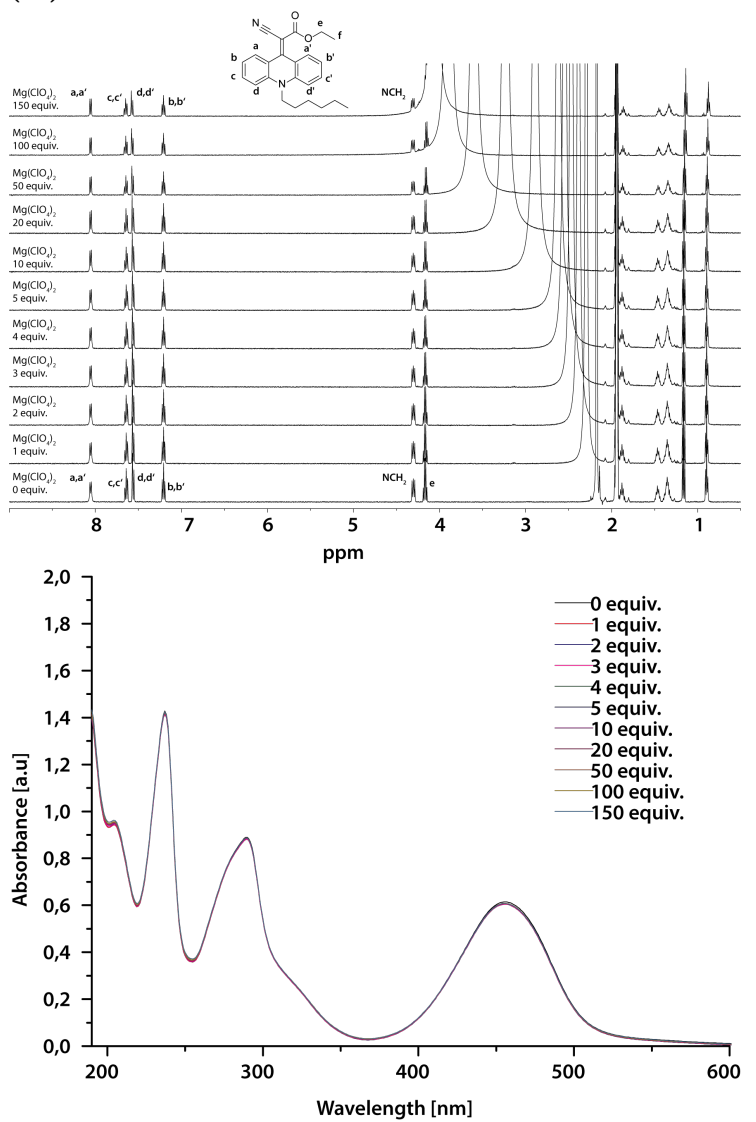

Figure V.3.2.4: a) ${ }^{\mathbf{1}} \mathbf{H}$ NMR (400 MHz, $d^{3}$-MeCN) titration of $\mathbf{5 2}(2.8 \mathrm{mM})$ with $\mathrm{Mg}\left(\mathrm{ClO}_{4}\right)_{2}$. The approximate shifting routes for the signals $\mathbf{1}$ and $\mathbf{2}$ are indicated by a green or red line. Below, UV-vis titration experiment of $52(0.035 \mathrm{mM})$ in MeCN. Upon addtion of the magnesium salt, no significant hypsochromic shift could be observed. Only a slight hypochromic shift, what could also be caused by dilution. b) NMR and UV-vis control experiments under the same conditions with $\mathbf{B}^{\mathbf{E t}}(\mathbf{5 2})$. Compound $\mathbf{3 5}$ does not show any evidence for an interaction with $\mathrm{Mg}\left(\mathrm{ClO}_{4}\right)_{2}$, neither in NMR nor in UV-vis experiments.

In literature it has been shown that calcium rather fits in the aza-15-crown-5 ether than potassium. The latter is more suitable for next bigger azacrown ether. Thus, the effect of calcium perchlorate was used in the next binding studies (Figure V.3.2.5). Similar to $\mathrm{Mg}\left(\mathrm{ClO}_{4}\right)_{2}$ the proton signals of the phenyl motif are downfield shifted, signal $\mathbf{1}$ about $0.2 \mathrm{ppm}$ and signal 2 about $0.6 \mathrm{ppm}$. At a approximate concentration $8 \mathrm{mM}$ of $\mathrm{Ca}\left(\mathrm{ClO}_{4}\right)_{2}$ signal 2 passes signal 1. In addition crown ether signals 5-7 experience a stronger downfield shift than 4 . In contrast the methylene groups bound to the azacrown ether nitrogen are shifted upfield. UV-vis shows first time a significant blue-shift about $9 \mathrm{~nm}$ and a more intense hypochromia for the $270 \mathrm{~nm}$ absorption band compared to sodium. As in the examples before, the control experiment do not indicate any unwanted interaction between 
the push-pull rotor compound a the used metal salt.

As a next salt, barium perchlorate was used for titration. Barium is compared to the previous used metals softer, thus it should better prefer the interaction with the nitrogen. On the other hand, it has a lower binding affinity towards the azacrown ether since the cation is too large to fit intro the crown ethers cavity. Still, it is literature-known that the barium cation forms a 2:1 complex instead with the azacrown ethers. $324 \mid 325$ Nevertheless, titration experiments have shown a weaker interaction compared to calcium perchlorate (Figure VI.1.7.12). Although an interaction between azacrown and metal can be regarded as certain, the studies suffer from a lack of evidence for a clear decrease in the rotation rate upon metal binding. Hence, as a next step we tried to protonate the azacrown nitrogen, what should be the strongest reversible reaction for this system to gain a electron-withdrawing counterpart in the opposite direction in respect to the rotating cyano acetate ester.

(a)
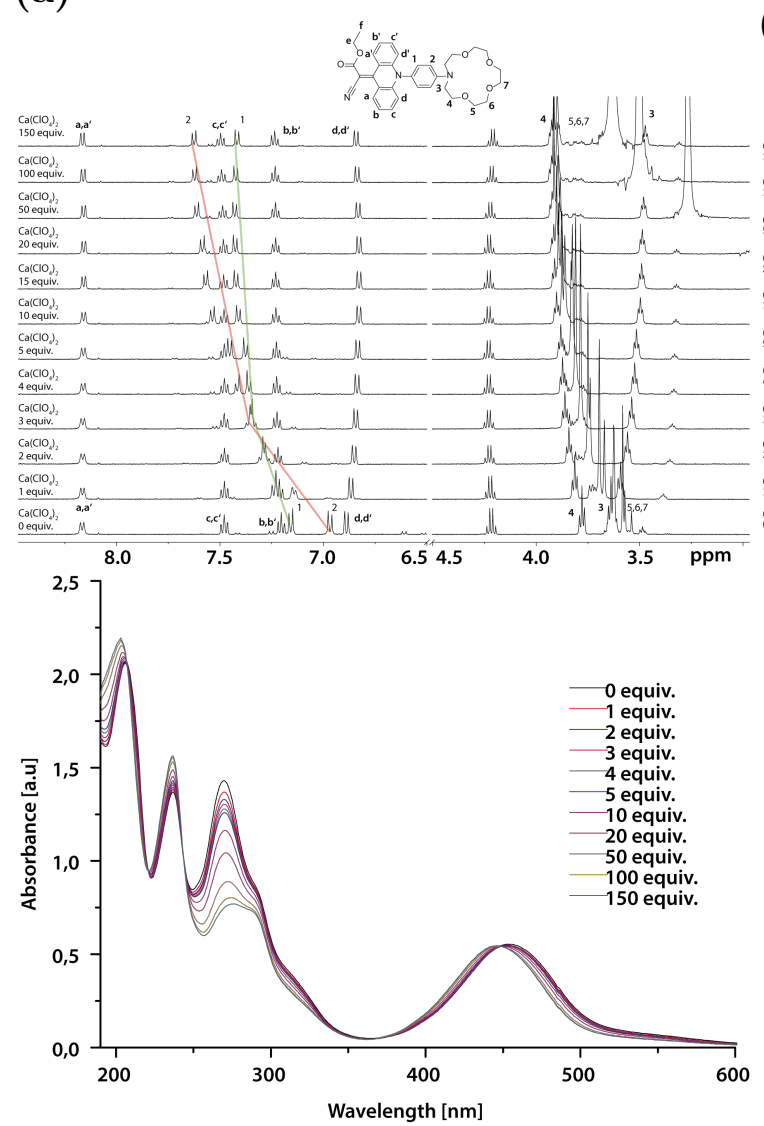

(b)
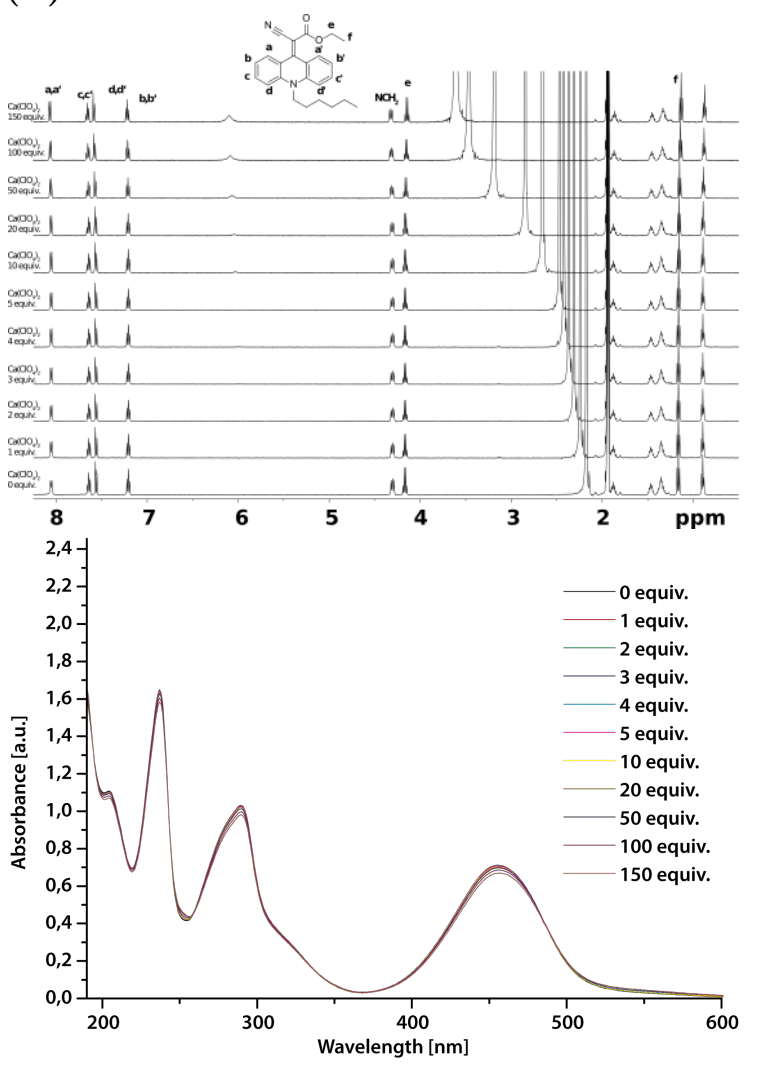

Figure V.3.2.5: a) ${ }^{\mathbf{1}} \mathbf{H}$ NMR (400 MHz, $\left.d^{3}-\mathrm{MeCN}\right)$ titration of $\mathbf{5 2}(2.8 \mathrm{mM})$ with $\mathrm{Ca}\left(\mathrm{ClO}_{4}\right)_{2}$. The approximate shifting routes for the signals $\mathbf{1}$ and $\mathbf{2}$ are indicated by a green or red line. Below, UV-vis titration experiment of $\mathbf{5 2}$ $(0.035 \mathrm{mM})$ in MeCN. Upon addtion of the calcium salt, a maximum blue-shift of $9 \mathrm{~nm}$ was observed. b) NMR and $\mathrm{UV}$-vis control experiments under the same conditions with $\mathbf{B}^{\mathbf{E t}}(\mathbf{5 2})$. In comparison to $\mathbf{5 2}$, compound $\mathbf{3 5}$ does not any chemical shift in NMR upon addition of $\mathrm{Ca}\left(\mathrm{ClO}_{4}\right)_{2}$, neither does it show any evidence of interaction with the calcium cation in UV-vis titration experiments. 
Indeed, the protonation with TFA (Figure V.3.2.6) showed the strongest effect on comparison to the other experiments for both NMR and UV-vis. In UV-vis the addition of 100 equiv. TFA resulted in a hypsochromic shift of $16 \mathrm{~nm}$. What equals almost the hypsochromic shift $\mathbf{B}^{\mathbf{E t}}$ has experienced by changing the solvent from water to hexane Figure V.1.2.9. Moreover, the phenyl signal $\mathbf{2}$ in NMR makes an tremendous downfield jump ending with a highest chemical shift in the recorded spectra. Besides, for the first time, signal $\mathbf{3}$ downfield shifted as well, while the reaming crown ether signals are slight shifted to a lower chemical shift. Both observation are a clear indication for the successful protonation of the azacrown nitrogen and for a significant change in the compounds intramolecular electronic situation. But the most interesting is the fact that the signal $\mathbf{a} / \mathbf{a}$ ' is getting considerably broader up to a concentration of 20 equiv. (approx. $60 \mathrm{mM}$ ), while the the remaining aromatic signals on the acidinylidene motif stay unchanged. This proofs that a electron-withdrawing motif in the reverse direction lead to a distinct deceleration of an dynamic motion. Anyhow, still the deceleration is only achieved by protonation, which is not desired in terms of making the system suitable for supramolecular coordination cages, hence the cages can disassemble under acidic conditions, as shown in figure V.2.2.14. Furthermore, the presence of an excess in ionic compounds or polar compounds induces a better stabilization of the charge separated state, responsible for the rotation, thus it accelerates the rotational motion again. This effect can be nicely seen in figure V.3.2.6a. The peak width of signal $\mathbf{a} / \mathbf{a}$ ' is decreased again with every addition of TFA above 20 equivalents.

(a)

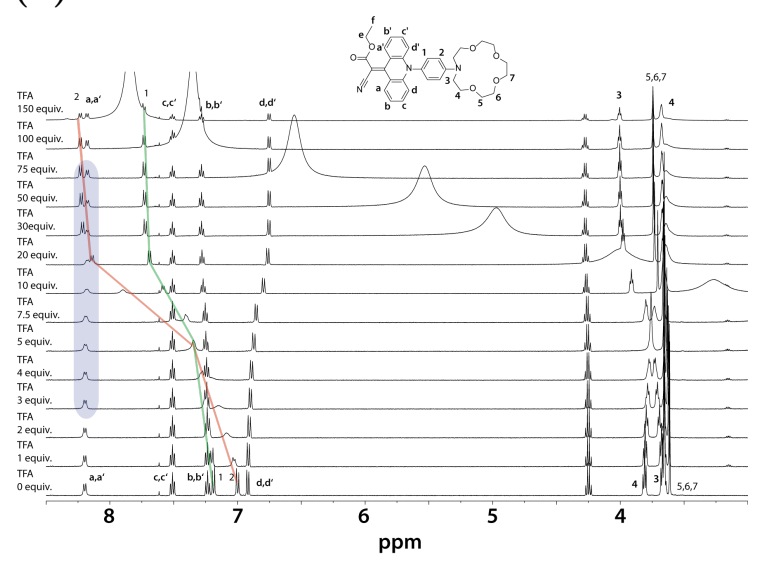

(b)

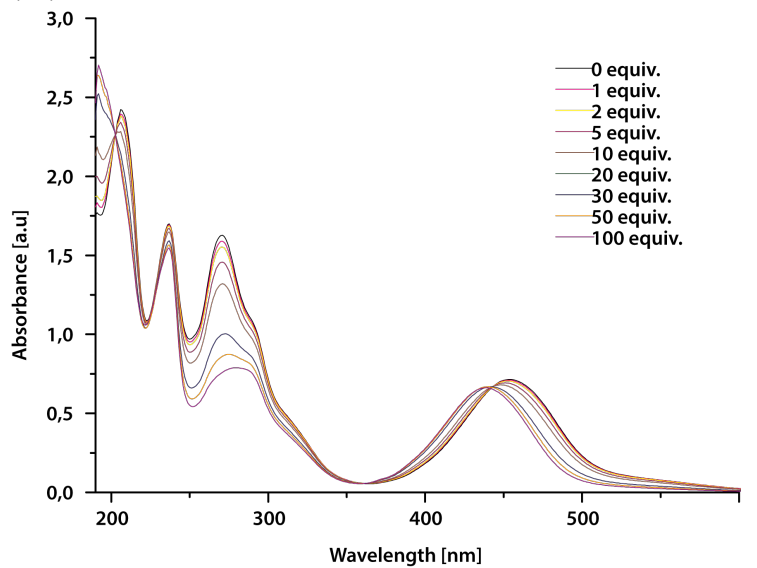

Figure V.3.2.6: NMR and UV-vis titration experiments of compound $\mathbf{5 2}$ and TFA. a) ${ }^{\mathbf{1}} \mathbf{H} \mathbf{N M R}(400 \mathrm{MHz}$, $\left.d^{3}-\mathrm{MeCN}\right)$ titration with TFA. The approximate shifting routes for the signals $\mathbf{1}$ and $\mathbf{2}$ are indicated by a green or red line. The region where a change in the width of the a/a' signal can be observed in highlighted in blue. Until the addition of 10 equiv, a slight broadending of signal a/a' can be observed. Further addition leads to a sharpen of the signal again. It seems like two contrary effects are taking place. On the one hand the protonation of the azacrown ether nitrogen, accompanied by weakening the push-pull system and on the other hand the stabiliziation of the charge seperated state by increasing the overall polarity due to addition of higher amounts of TFA. Furthermore, compared to the other titration experiments, signal $\mathbf{3}$ exhibit a significant downfield-shift. b) The UV-vis titration experiments of $\mathbf{5 2}(0.035 \mathrm{mM}, \mathrm{MeCN})$ with TFA. The maximum hypsochromic shift of $16 \mathrm{~nm}$ is achieved upon a total addition of 100 equiv. of TFA. Also a significant hypochromic shift for the band at $270 \mathrm{~nm}$ could be observed. 
In order to overcome the minor effects caused by weak interaction of alkali or earth alki metals with the azacrown nitrogen, the focus was set on transition metals. The coordination of nickel, copper, zinc and cadmium to the azacrown ether were investigated by UV-vis. Additionally manganese was used, since it is the closest transition metal analogon to magnesium in biological systems. 326 The experiments showed for manganese perchlorate and zinc tetrafluoroborate a blue-shift of $9 \mathrm{~nm}$, thus comparable to calcium perchlorate Figures VI.1.7.13a and VI.1.7.13b). Cadmium perchlorate led to a maximal hypsochromic shift of $11 \mathrm{~nm}$, what is not an outstanding improvement, apart from that, cadmium belongs rather to the higher toxic transistion metal and was therefore discarded for further experiments Figure VI.1.7.13c.

Nickel and copper showed a completely new behavior. While nickel looked very promising in the beginning (Figure V.3.2.7a), since addition of 10 equiv. $\mathrm{Ni}\left(\mathrm{ClO}_{4}\right)_{2}$ already gave a blue-shift of $12 \mathrm{~nm}$ ( $6 \mathrm{~nm}$ for 10 equiv. TFA), anyway, further addition ended in the generation of a well-known absorption pattern (Figure V.1.3.23c). The control experiment with $\mathbf{B}^{\mathbf{E t}}$ led to the same result (Figure V.3.2.7b). A further NMR titration experiments with $\mathbf{B}^{\mathbf{E t}}$ proved the suspicion about the protonation of the rotating group (Figure V.3.2.7c). Irradiation with UV light led to the characteristic fluorescence. Therefore we suspect the used $\mathrm{Ni}\left(\mathrm{ClO}_{4}\right)_{2} \times 6 \mathrm{H}_{2} \mathrm{O}$ to function as strong acid and providing the $\mathrm{H}^{+}$for the formation of the protonated acrdinium species. In the case of copper perchlorate, addition led to the same result for $\mathbf{B}^{\mathbf{E t}}$ Figure VI.1.7.14b), whereas for $\mathbf{B}_{\mathbf{A z a c r o w n}}{ }^{\mathbf{E t}}$ the addition of the first equivalent brought off an broad absorption band going from $400 \mathrm{~nm}$ to $800 \mathrm{~nm}$, whats typical for an radical species. Second equivalent led to another unknown absorption pattern, all further additions gave the already known acridinium specific absorption pattern (Figure VI.1.7.14a).

(a)

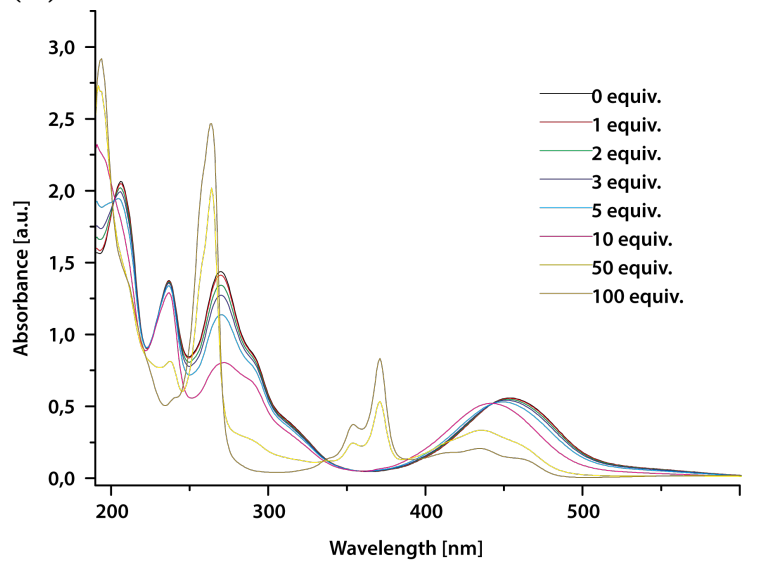

(b)

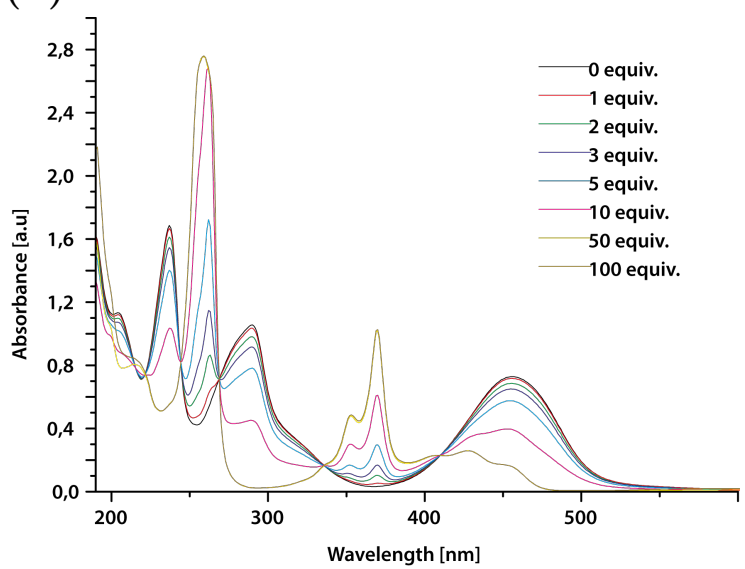




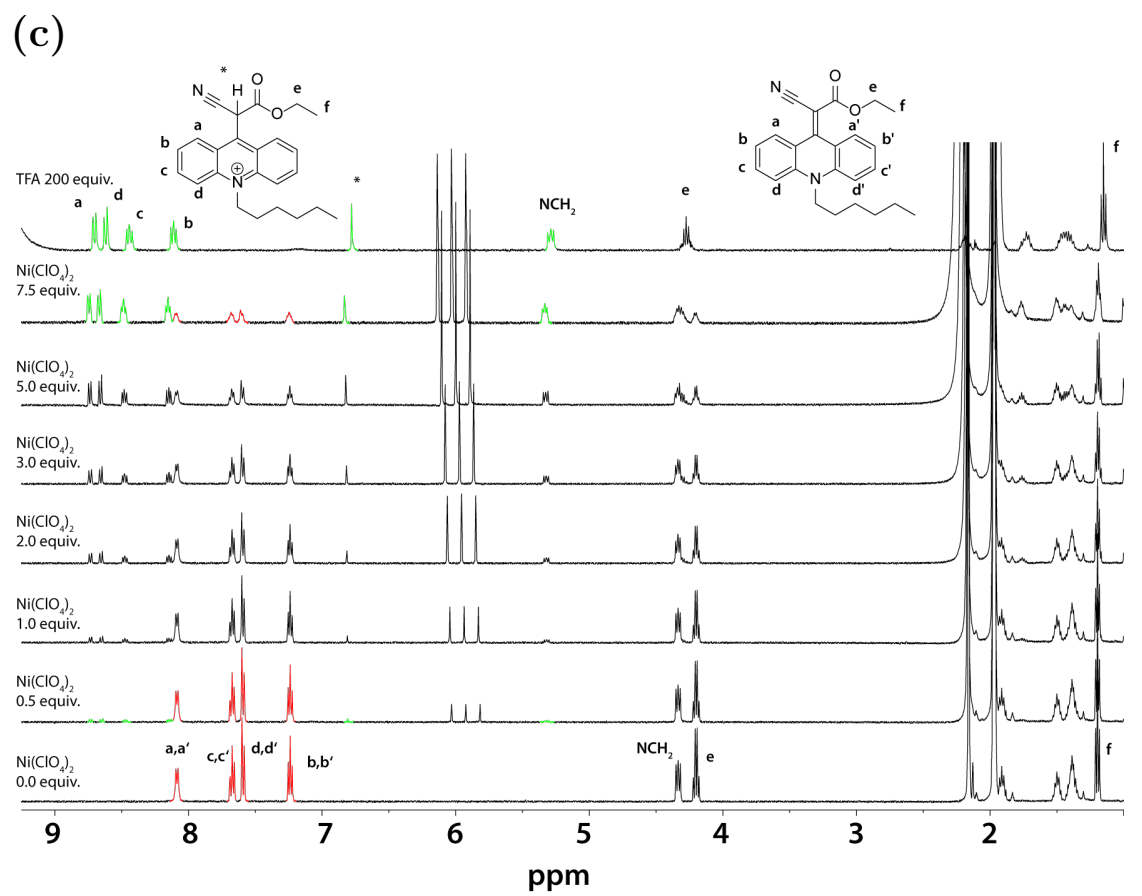

Figure V.3.2.7: UV and NMR titration experiments with $\mathbf{B}_{\mathbf{A z a c r o w n}}{ }^{\mathbf{E t}} / \mathbf{B}^{\mathbf{E t}}$ and $\mathrm{Ni}\left(\mathrm{ClO}_{4}\right)_{2}$. a) $\mathrm{UV}$-vis titration experiment with $\mathrm{B}_{\text {Azawcrown }}{ }^{\text {Et }}(\mathbf{5 2}, 0.035 \mathrm{mM}, \mathrm{MeCN})$ with TFA. Upon addition of 10 equiv. nickel salt a hypsochromic shift of $12 \mathrm{~nm}$ was observable. Further addition led to a conversion of $\mathbf{5 2}$ to the corresponding protonated acridinium species, indicated by the charateristic absorption pattern (Figure V.1.3.23c). b) Control experiment with $\mathbf{B}^{\mathbf{E t}}$ and the corresponding nickel salt. A formation of the protonated acridinium species is observable from the the very first addition and is totally converted at total addition of 50 equiv. $\mathrm{Ni}\left(\mathrm{ClO}_{4}\right)_{2}$. c) ${ }^{\mathbf{1}} \mathbf{H}$ NMR $\left(400 \mathrm{MHz}, d^{3}-\mathrm{MeCN}\right)$ titration experiments with $\mathbf{B}^{\mathbf{E t}}(\mathbf{3 5})$ and $\mathrm{Ni}\left(\mathrm{ClO}_{4}\right)_{2}$. The starting material is highlighted in red, the protonated acridinum species in green. For comparison purposes, a spectrum of the acridinium species, protonated with TFA, is shown on the very top.

\section{V.3.2.4 Influence of alkali metal binding on the rotation rate in VT NMR experiments}

Since the desired effect on the rotation rate was not clearly visible under standard conditions, we have compared the influence of temperature on the rotation rate in presence and absence of coordinating metals. Therefore VT NMR measurements in acetonitrile from -40 celsius to $70{ }^{\circ} \mathrm{C}$ were accomplished, hence the metal salts had only a poor solubility in the preferable solvent THF. In figure V.3.2.8 the outcome for both measurements are compared. It is obvious, that a broadening of signals already occurs at $20^{\circ} \mathrm{C}$, while in the other case no visible change in the peak widths occurred. Aside from that, at $-20{ }^{\circ} \mathrm{C}$ and below it looks like a splitting of the the signals already occurs, while in the spectra without $\mathrm{Mg}\left(\mathrm{ClO}_{4}\right)_{2}$, the system is still above coalescence temperature, means still one set of signals. Unfortunately, we could not further follow the process, due to the fact, that acetonitrile freezes at $-45{ }^{\circ} \mathrm{C}$, thus as decrease in temperature was not possible. Furthermore, in virtue of the general broadening of the signals in the spectra, it was not possible to assign the newly upcoming peaks to any proton, therefore we can only assume that the system 
remained intact. But, it is definitive indication that the presence of the earth alkali metal effects the system.

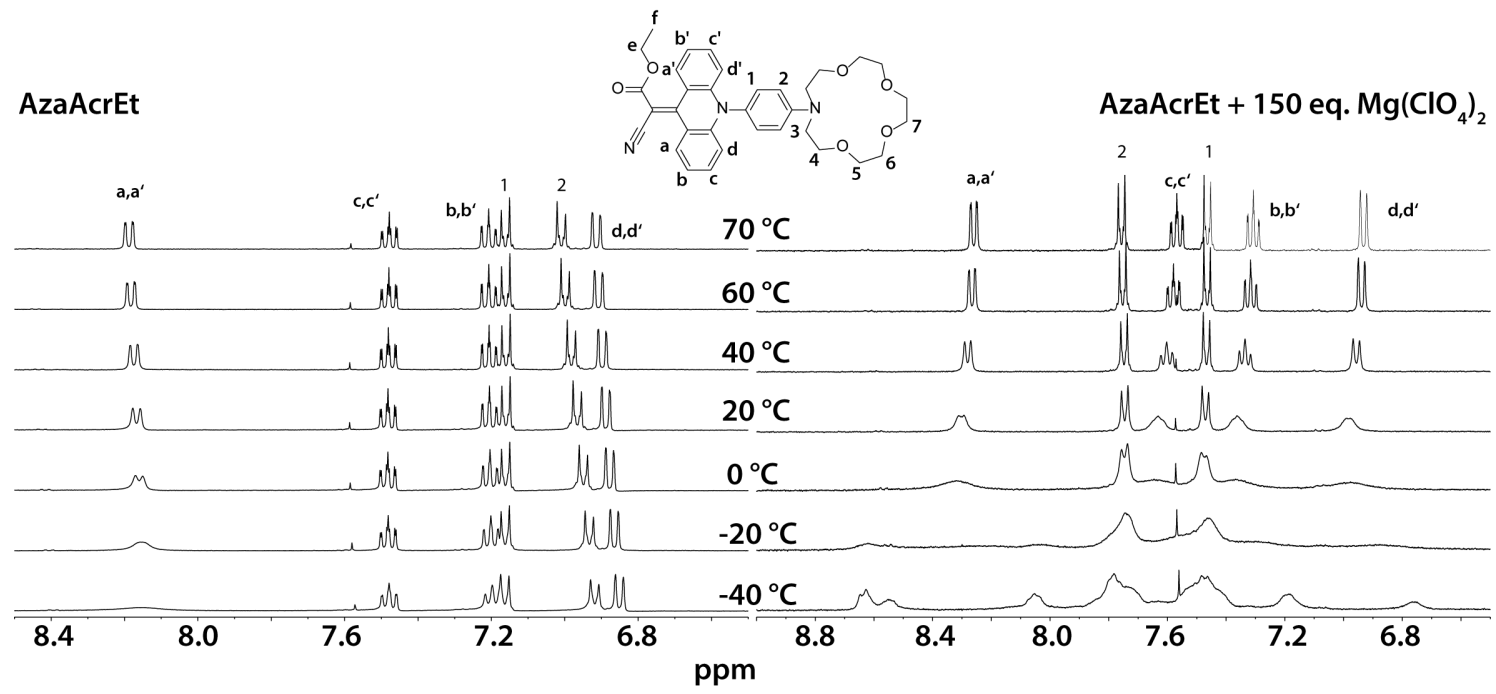

Figure V.3.2.8: Comparison of VT-NMR experiments with a) $\mathbf{B}_{\text {Azacrown }} \mathbf{E t}(2.8 \mathrm{mM})$ in $\mathrm{MeCN}$ and b) $\mathbf{B}_{\text {Azacrown }}{ }^{\mathbf{E t}}(2.8 \mathrm{mM})+150$ eq. $\mathrm{Mg}\left(\mathrm{ClO}_{4}\right)_{2}$ in $\mathrm{MeCN}$. For both the spectra between $-40{ }^{\circ} \mathrm{C}$ and $+70{ }^{\circ} \mathrm{C}$ are shown. Due to the broadening and convolution of the signal it was not possible to assign the signals to the corresponding hydrogens in a reliable way.

\section{V.3.3 Conclusion and Prospects}

In conclusion, the here presented system was designed to decrease the rate of rotation in the already described push-pull rotor system. Since the rate of rotation and the rotational motion itself strongly depends on the quality of the intramolecular push-pull induced charge separation, in terms of stabilization, we tried to initiate a reverse directed electron-withdrawing motif that lowers the electron-donating ability of the heteroaromatic nitrogen towards the electron-withdrawing cyano acetate ester. Moreover, the system should satisfy the requirements of reversible formation of this electron-withdrawing moiety and a suitability for a later incorporation into supramolecular coordination cages. For that purpose the motif of the azacrown ether was chosen. Coupled to the $\pi$ system, on the first glance it rather contributes as an electron donator, but can form in presence of suitable metals an reversible complex that exerts an electron-withdrawing effect on the intramolecular push-pull system. $327[329]$ To examine the effect of metal-binding on the rotational rate, the here investigated model compound $\mathbf{B}_{\text {Azacrown }}{ }^{\mathbf{E t}}$ was synthesized on the basis of the already characterized compound $\mathbf{B}^{\mathbf{E t}}(\mathbf{3 5})$, in order to have an easily comparable reference. The coordination of various metals, including alkali and earth alkali metals but also some transition metals, were investigated for their influence on the dynamic motion by UV-vis, NMR and VT NMR experiments. Additionally, as a reference for the assumed maximal electron-withdrawing effect, the protonation of the 
azacrown nitrogen was examined as well. NMR provided evidence for the interaction of all investigated metals with the azacrown ether, although the observable effect ranged from almost non-existing to clearly visible. However, an explicit indication for a decrease in rotation upon metal coordination stayed off under experimental conditions. Whereas the presence of strong acids led to the protonation of the azacrown nitrogen, what showed a clear impact the systems' electronics and the first time a deceleration in rotation rate could be observed qualitatively. It is noteworthy that an excess of nickel and copper perchlorate led to protonation of the rotating cyano acetate ester group in both compounds $\left(\mathbf{B}^{\mathbf{E t}}\right.$ and $\mathbf{B}_{\text {Azacrown }}{ }^{\mathbf{E t}}$ ), but could only be shown in $\mathrm{NMR}$ for the $\mathbf{B}^{\mathbf{E t}}$ compound. A possible explanation for this unusual behavior could be the usage of the nickel and copper perchlorate hexa-hydrate $\left(\mathrm{M}\left(\mathrm{ClO}_{4}\right)_{2} \times 6 \mathrm{H}_{2} \mathrm{O}\right)$, what possibly led to the in situ generation and simultaneously release of protons. But to be sure, further investigation are necessary. Since experiments under standard condition did not lead to the proof of concept. examination of the azacrown compound in presence of $\mathrm{Mg}\left(\mathrm{ClO}_{4}\right)_{2}$ with help of VT NMR measurements were carried out. The resulting NMR spectra revealed a notable change in the sensitivity of rotational rate towards the temperature, compared to the reference compound. Unfortunately, due to overall signal broadening and the lack of control experiments, these findings are not complete yet and can only be considered as promising indications for the successful control of rotational rate by an external stimuli. In further studies additional control experiments have to made to get a clear proof of principle. Furthermore, the change to another coordination site, e.g. terpyridine, 330 . with an stronger influence on the push-pull system electronics or better matching crown ether-metal pair $320 \mid 331$ as well as a fine tuning of the experimental conditions, could make the system being promising for either molecular sensor applications, dynamic stimuli responsive supramolecular systems, catalytic cavities or functional materials. 

Experimental Section
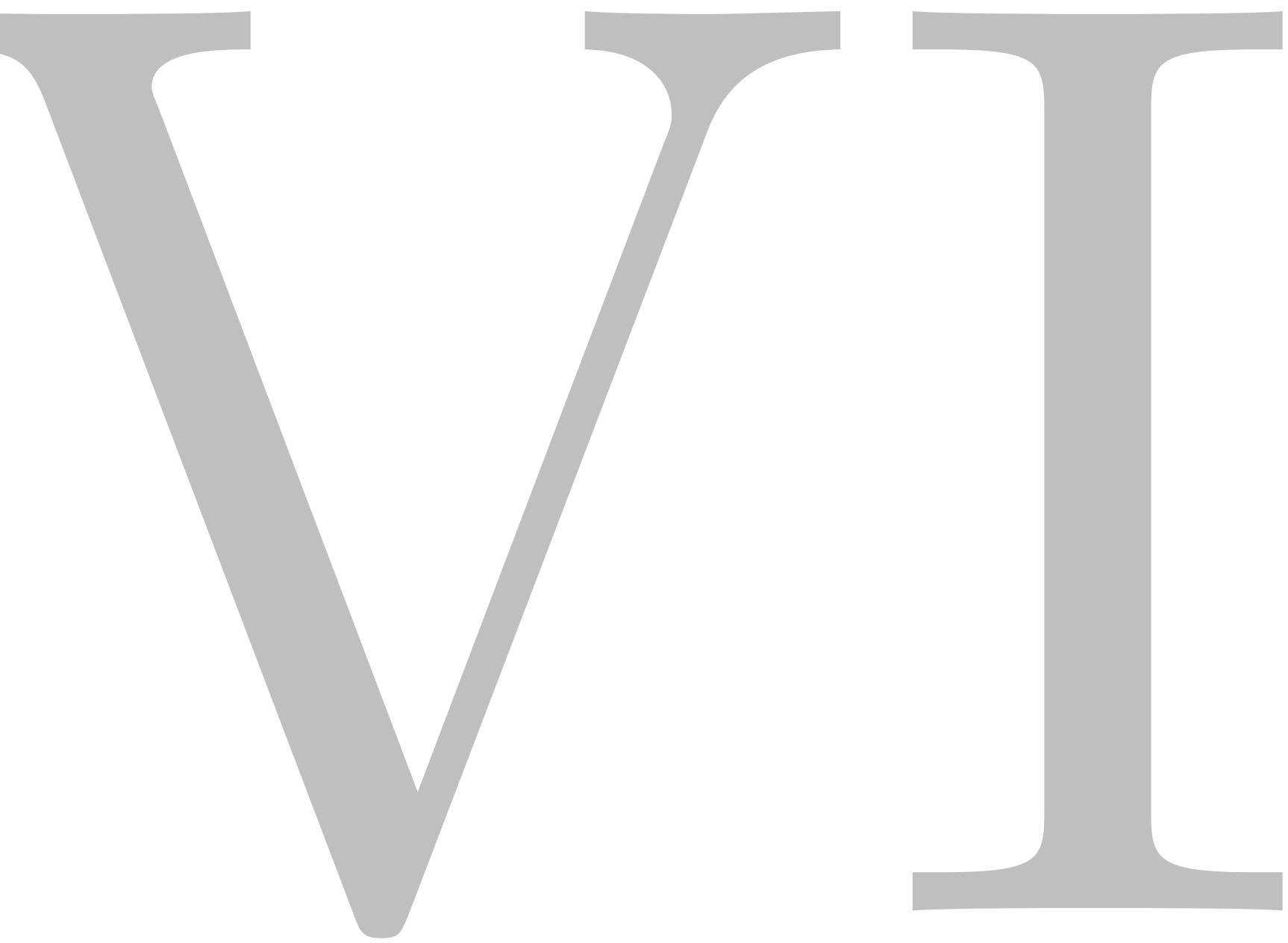


\section{Chapter}

\section{Syntheses of Olignucleotide Incorporable Ligands}

\section{Contents}

VI.1.1 Synthesis of the Acridone-based Ligand . . . . . . . . . 195

VI.1.1.1 Compound $\mathbf{3}$. . . . . . . . . . . . . . . . . . . . . . . . . . . . . 196

VI.1.1.2 Compound 4 . . . . . . . . . . . . . . . . . . . . . . . . . . . . . . . 198

VI.1.1.3 Compound $\mathbf{5}$. . . . . . . . . . . . . . . . . . . . . . . . . 200

VI.1.1.4 Compound 6 . . . . . . . . . . . . . . . . . . . . . 202

VI.1.1.5 Compound 7 . . . . . . . . . . . . . . . . . . . . . . 204

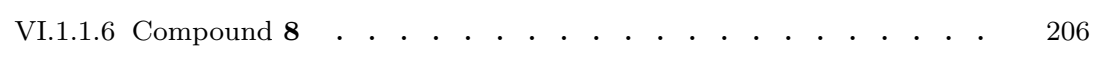

VI.1.1.7 Compound $\mathbf{9}$. . . . . . . . . . . . . . . . . . . . . . . . . . . . 208

VI.1.1.8 Compound 10 . . . . . . . . . . . . . . . . . . . 210

VI.1.1.9 Compound 11 . . . . . . . . . . . . . . . . . . . . . . . . . . 212

VI.1.2 Syntheses of the Methoxybenzylamine-based Ligand . . 214

VI.1.2.1 Compound 12 . . . . . . . . . . . . . . . . . . . . . . . . . . 214

VI.1.2.2 Compound 13 . . . . . . . . . . . . . . . . . . . . . . . 216

VI.1.2.3 Compound 14 . . . . . . . . . . . . . . . . . . . . . . . . 218

VI.1.2.4 Compound 15 . . . . . . . . . . . . . . . . . . . . . . . . 220

VI.1.2.5 Compound 16 . . . . . . . . . . . . . . . . . . . . . 222

VI.1.2.6 Compound 17 . . . . . . . . . . . . . . . . . . . . . . 224

VI.1.2.7 Compound $\mathbf{1 8}$. . . . . . . . . . . . . . . . . . . . . . . . . . . 226

VI.1.2.8 Compound 19 . . . . . . . . . . . . . . . . . . . . . 228

VI.1.2.9 Compound 20 - BTT . . . . . . . . . . . . . . . . . . . . . 230 


\section{VI.1.1 Syntheses of the Acridone-based Ligand}

The precursor 2,7-Diiodo-10-(2-(2-methoxyethoxy)ethyl)acridin-9(10H)-one $\mathbf{2}$ was prepared in a two step synthesis from acridone. In a first step the methyl-ethylenglycol chain was intorduced in a substitution reaction with 2-Methoxyethoxy-ethylbromide to the nitrogen postiton of the acridone (Cmpd. 1). Subsequently, iodine was regioseletivly introduced at the 2- and 7-position of the acridone via an trifluoroacetic acid catalysed electrophilic aromatic substitution.<smiles>O=c1c2ccccc2[nH]c2ccccc12</smiles><smiles>COCCOCCOCCOC</smiles><smiles>COCCOCCn1c2ccccc2c(=O)c2ccccc21</smiles>

1
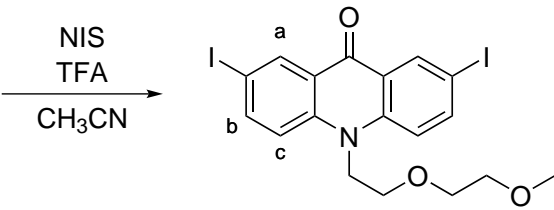

2

Scheme VI.1.1.12: Synthesis of compound 2.

${ }^{1} \mathbf{H}-\mathbf{N M R}\left(500 \mathrm{MHz}, 298 \mathrm{~K}, \mathrm{CD}_{3} \mathrm{Cl}\right): \delta(\mathrm{ppm})=8.70\left(\mathrm{~d},{ }^{4} J=2.4 \mathrm{~Hz}, 2 \mathrm{H}, \mathrm{a}\right), 7.85(\mathrm{dd}$, $\left.{ }^{3} J=9.1 \mathrm{~Hz},{ }^{4} J=2.3 \mathrm{~Hz}, 2 \mathrm{H}, \mathrm{b}\right), 7.30\left(\mathrm{~d},{ }^{3} J=9.2 \mathrm{~Hz}, 2 \mathrm{H}, \mathrm{c}\right), 4.46\left(\mathrm{t},{ }^{3} J=6.3 \mathrm{~Hz}, 2 \mathrm{H}\right.$, $\left.\mathrm{NCH} 2 \mathrm{CH}_{2}\right), 3.88\left(\mathrm{t},{ }^{3} \mathrm{~J}=6.3 \mathrm{~Hz}, 2 \mathrm{H}, \mathrm{NCH}_{2} \underline{\mathrm{CH}}\right)$, 3.63-3.52 (m, 2H, $\left.\underline{\mathrm{CH}} 2 \mathrm{CH}_{2} \mathrm{OCH}_{3}\right)$, 3.49-3.38 (m, 2H, $\left.\mathrm{CH}_{2} \underline{\mathrm{CH} 2} \mathrm{OCH}_{3}\right), 3.28\left(\mathrm{~s}, 3 \mathrm{H}, \mathrm{OCH}_{3}\right)$.

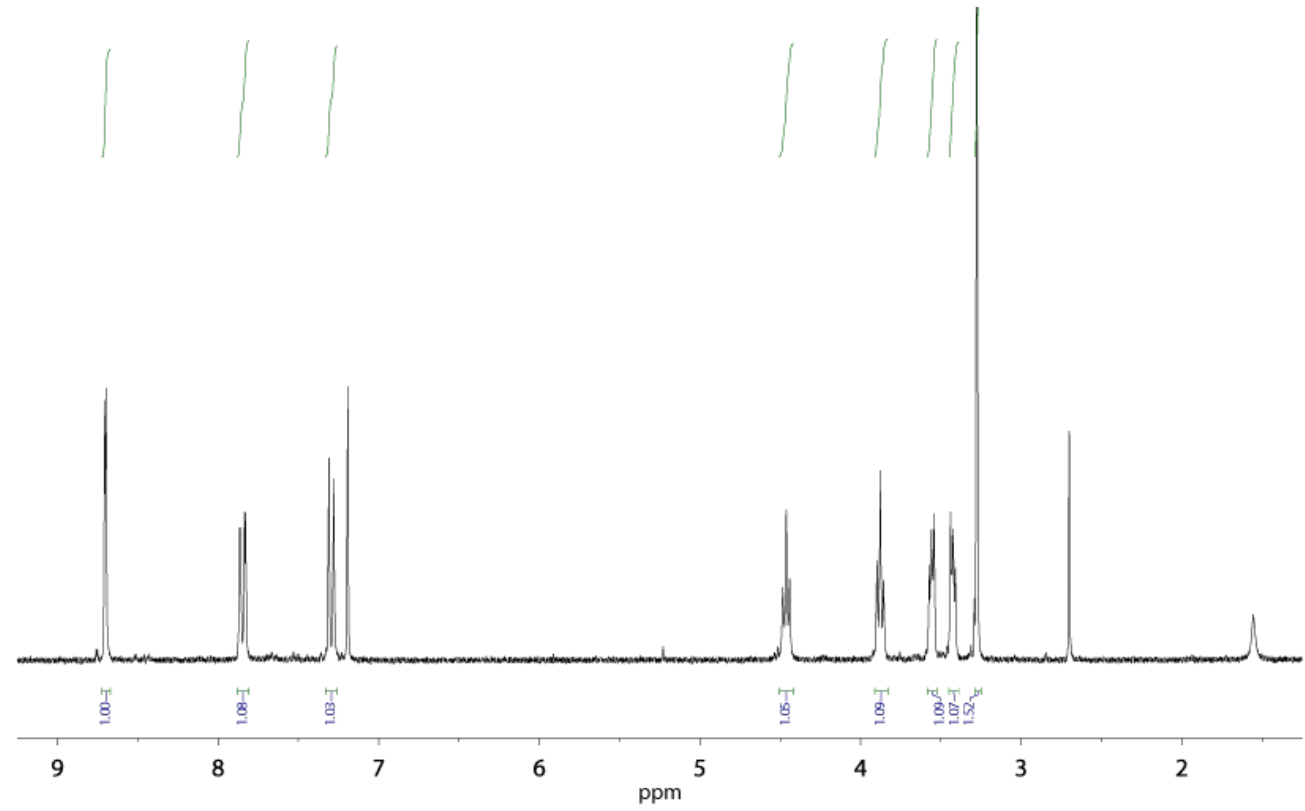

ESI-HRMS $\left(\left[\mathrm{C}_{18} \mathrm{H}_{17} \mathrm{NO}_{3} \mathrm{I}_{2} \mathrm{jj}+\mathrm{Na}\right]^{+}\right)$: measured: $\quad 571.9171$

calculated: $\quad 571.9190$ 
VI.1.1.1 2-(2-((Tetrahydro-2H-pyran-2-yl)oxy)ethoxy)ethan-1-ol (3)<smiles>OCCOCCO</smiles>

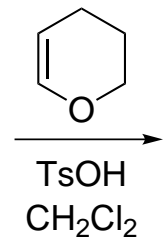

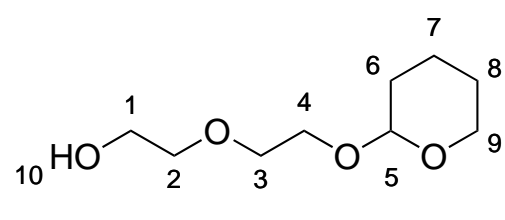

3

Scheme VI.1.1.13: Synthesis of compound 3 .

To a solution of diethylene glycol (25.37 mL, $265 \mathrm{mmol}, 5.00$ equiv.) and 4methylbenzenesulfonic acid (0.91 g, $5.31 \mathrm{mmol}, 0.10$ equiv.) in $\mathrm{CH}_{2} \mathrm{Cl}_{2}$ (200 mL), 3,4-dihydro- $2 H$-pyran (4.46 g, $53.1 \mathrm{mmol}, 1.00$ equiv.) was added dropwise at 0 řC. The mixture was stirred for $1.5 \mathrm{~h}$ at room temperature. After, $\mathrm{H}_{2} \mathrm{O}(200 \mathrm{~mL})$ was added and the aq. phase was extracted with $\mathrm{CH}_{2} \mathrm{Cl}_{2}(3 \times 50 \mathrm{~mL})$. The combined organic phases were dried over $\mathrm{MgSO}_{4}$ and the solvent was removed in vacuo. The residue was purified by means of column chromatography $\left(\mathrm{SiO}_{2}\right.$, diethyl ether/ ethyl acetate 1:1). The product 37 (7.50 g, $39.4 \mathrm{mmol}, 74 \%$ ) was obtained as a clear oil.

${ }^{1}$ H-NMR $\left(300 \mathrm{M} \mathrm{Hz}, 298 \mathrm{~K}, \mathrm{CD}_{3} \mathrm{CN}\right): \delta(\mathrm{ppm})=4.67-4.55(\mathrm{~m}, 2 \mathrm{H}, 5,10), 3.82-3.44(\mathrm{~m}$, $10 \mathrm{H}, 1-4,9), 1.83-1.42(\mathrm{~m}, 6 \mathrm{H}, 6-8)$.

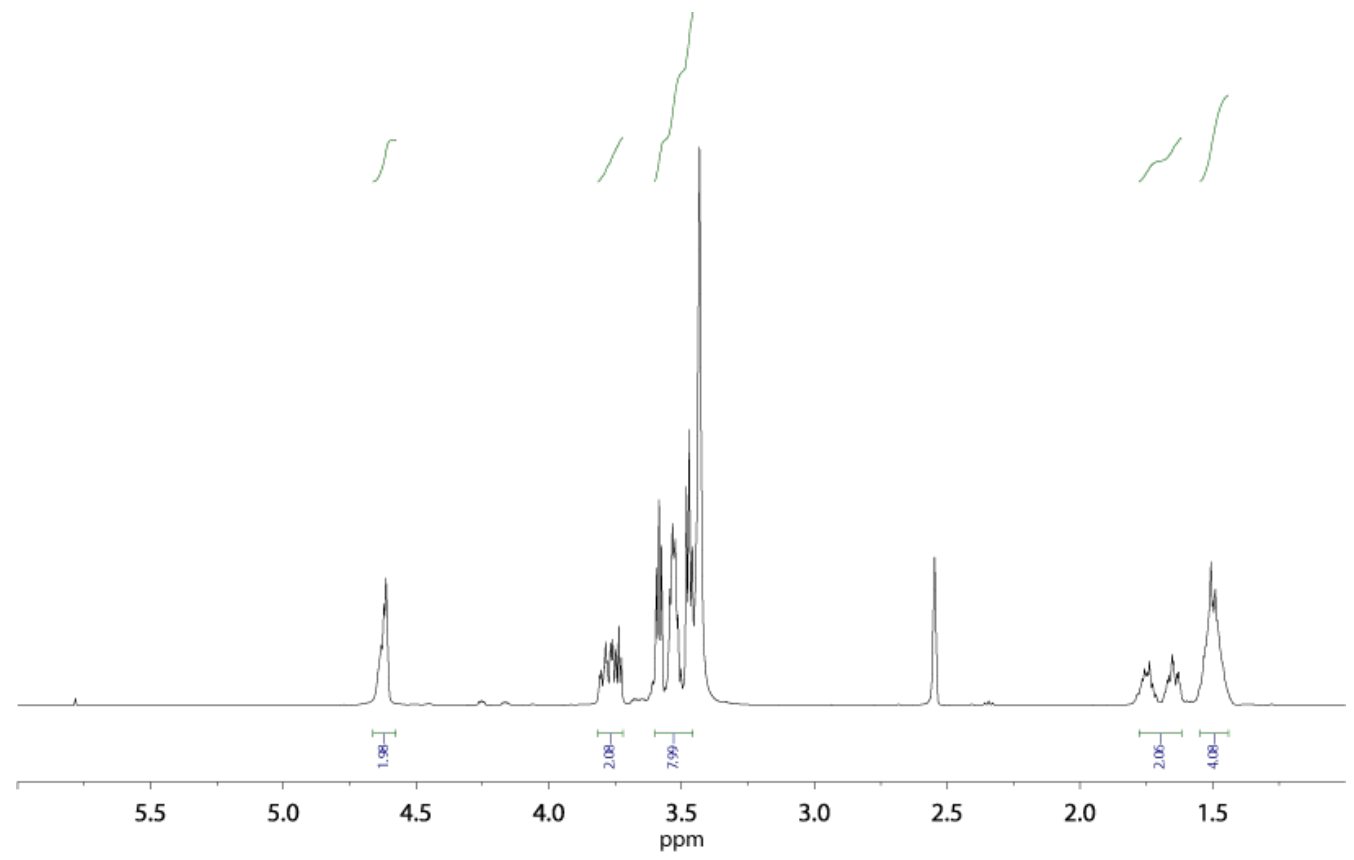

${ }^{13}$ C-NMR $\left(176 \mathrm{MHz}, 298 \mathrm{~K}, \mathrm{CD}_{3} \mathrm{CN}\right): \delta(\mathrm{ppm})=99.6,72.8,70.2,66.6,61.8,60.7,30.7$, 25.5, 19.6 . 


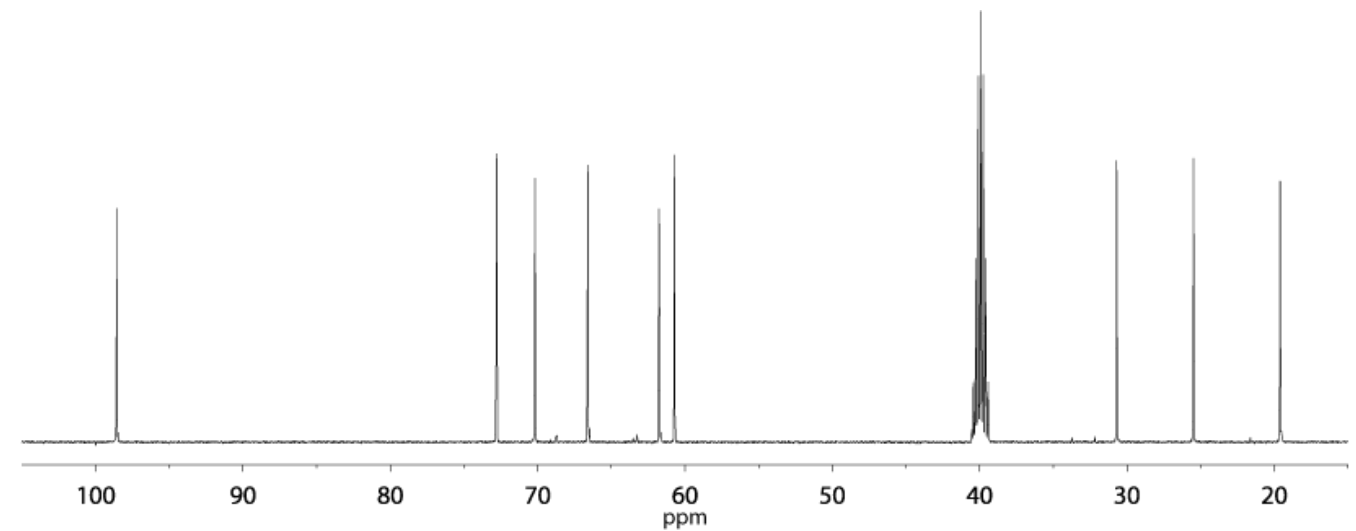

ESI-HRMS $\left(\left[\mathrm{C}_{9} \mathrm{H}_{18} \mathrm{O}_{4}+\mathrm{H}\right]^{+}\right)$:

measured:

191.1279

calculated:

191.1278 


\section{VI.1.1.2 2-(2-((Tetrahydro-2H-pyran-2-yl)oxy)ethoxy)ethyl} 4-methylbenzenesulfonate (4)

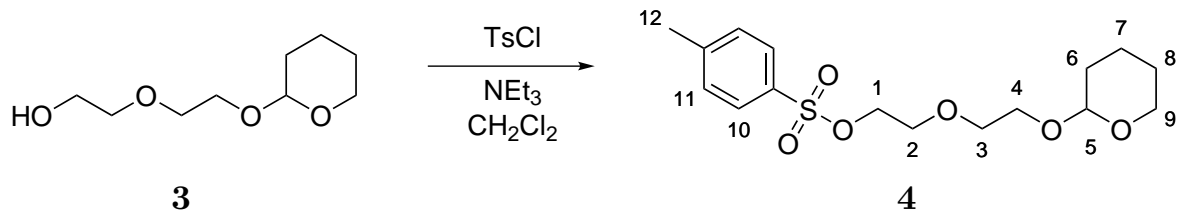

Scheme VI.1.1.14: Synthesis of compound 4.

To a solution of diethylene glycol (25.37 mL, $265 \mathrm{mmol}, 5.00$ equiv.) and 4methylbenzenesulfonic acid (0.91 g, $5.31 \mathrm{mmol}, 0.10$ equiv.) in $\mathrm{CH}_{2} \mathrm{Cl}_{2}$ (200 mL), 3,4-dihydro-2 $H$-pyran (4.46 g, $53.1 \mathrm{mmol}, 1.00$ equiv.) was added dropwise at 0r̆C. The mixture was stirred for $1.5 \mathrm{~h}$ at room temperature. After, $\mathrm{H}_{2} \mathrm{O}(200 \mathrm{~mL})$ was added and the aq. phase was extracted with $\mathrm{CH}_{2} \mathrm{Cl}_{2}(3 \times 50 \mathrm{~mL})$. The combined organic phases were dried over $\mathrm{MgSO}_{4}$ and the solvent was removed in vacuo. The residue was purified by means of column chromatography $\left(\mathrm{SiO}_{2}\right.$, diethyl ether/ ethyl acetate 1:1). The product $\mathbf{3 7}$ (7.50 g, $39.4 \mathrm{mmol}, 74 \%$ ) was obtained as a clear oil.

${ }^{1}$ H-NMR $\left(300 \mathrm{M} \mathrm{Hz}, 298 \mathrm{~K}, \mathrm{CD}_{3} \mathrm{CN}\right): \delta(\mathrm{ppm})=7.80\left(\mathrm{~d},{ }^{3} J=8.3 \mathrm{~Hz}, 2 \mathrm{H}, 10\right), 7.33$ $\left(\mathrm{d},{ }^{3} J=7.8 \mathrm{~Hz}, 2 \mathrm{H}, 11\right), 4.58\left(\mathrm{t},{ }^{3} J=3.5 \mathrm{~Hz}, 1 \mathrm{H}, 5\right), 4.22-4.10(\mathrm{~m}, 2 \mathrm{H}, 9), 3.91-3.72(\mathrm{~m}$, $2 \mathrm{H}, 1)$, 3.91-3.72 (m, 2H, 3), 3.91-3.72 (m, 2H, 4), 3.91-3.72 (m, 2H, 2), $2.44(\mathrm{~s}, 3 \mathrm{H}, 12)$, 1.88-141 (m, 6H, 6-8).

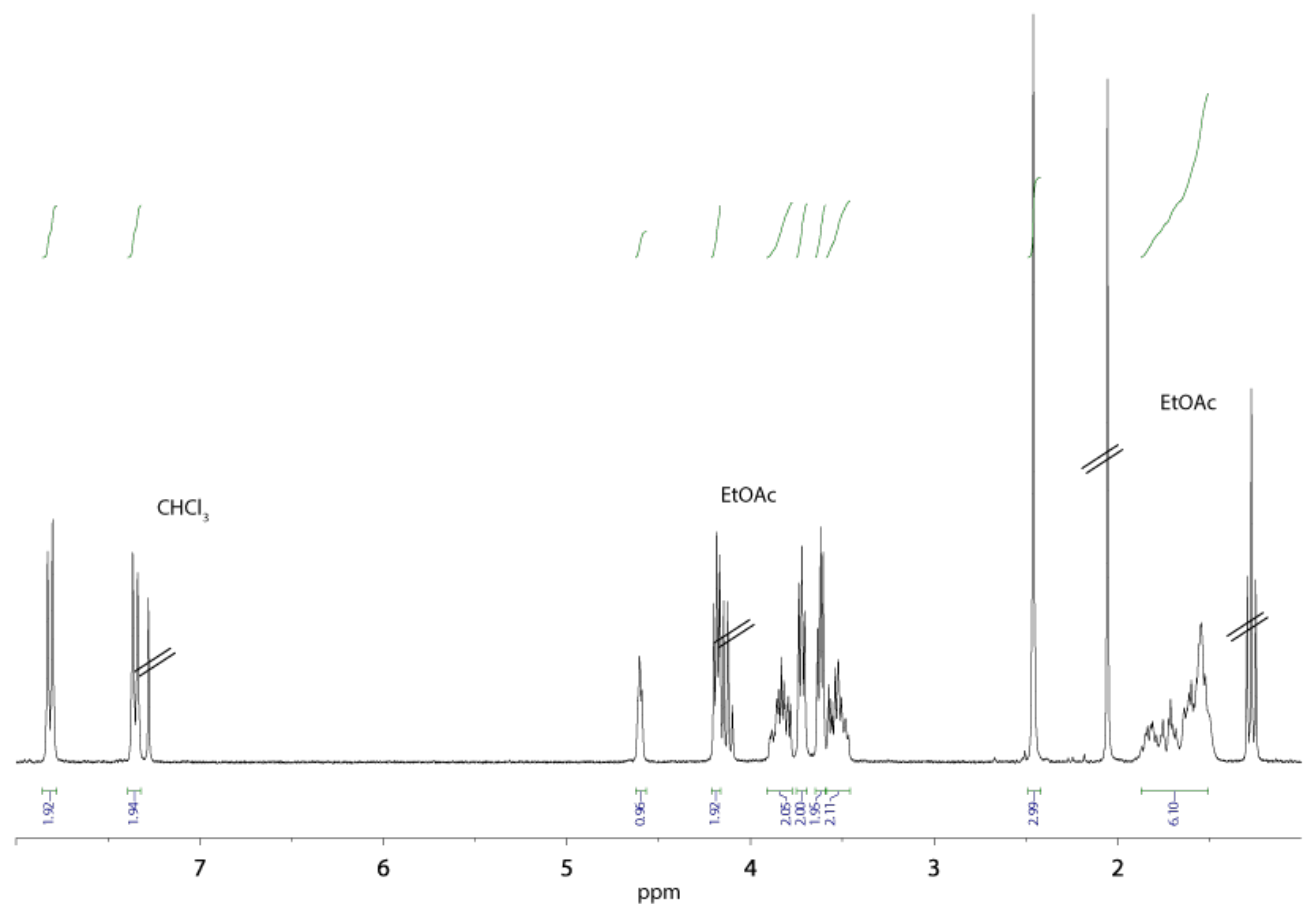


ESI-HRMS $\left(\left[\mathrm{C}_{9} \mathrm{H}_{18} \mathrm{O}_{4}+\mathrm{H}\right]^{+}\right)$: $\quad$ measured: $\quad 345.1359$

calculated: $\quad 345.1366$ 
VI.1.1.3 3-Bromo-5-(2-(2-((tetrahydro-2H-pyran-2yl)oxy)ethoxy)ethoxy)pyridine (5)<smiles>Cc1ccc(S(=O)(=O)OCCOCCOC2CCCCO2)cc1</smiles>

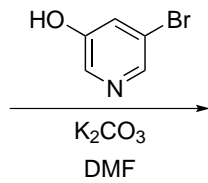

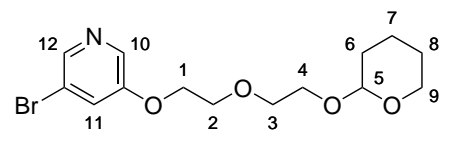

5

Scheme VI.1.1.15: Synthesis of compound 5.

${ }^{1} \mathbf{H}-\mathbf{N M R}\left(300 \mathrm{M} \mathrm{Hz}, 298 \mathrm{~K}, \mathrm{CD}_{3} \mathrm{CN}\right): \delta(\mathrm{ppm})=8.30\left(\mathrm{~d},{ }^{4} J=2.6 \mathrm{~Hz}, 1 \mathrm{H}, 12\right), 8.27(\mathrm{~d}$, $\left.{ }^{4} J=1.9 \mathrm{~Hz}, 1 \mathrm{H}, 10\right), 7.74-7.71(\mathrm{~m}, 1 \mathrm{H}, 11), 4.56\left(\mathrm{t},{ }^{3} J=3.6 \mathrm{~Hz}, 1 \mathrm{H}, 5\right), 4.26-4.17(\mathrm{~m}$, $2 \mathrm{H}, 1), 3.80-3.66(\mathrm{~m}, 4 \mathrm{H}, 2,3), 3.60\left(\mathrm{t},{ }^{3} \mathrm{~J}=4.8 \mathrm{~Hz}, 9\right), 3.54-3.45$ (m, 2H, 4), $1.72-1.35$ (m, 6H, 6-8).

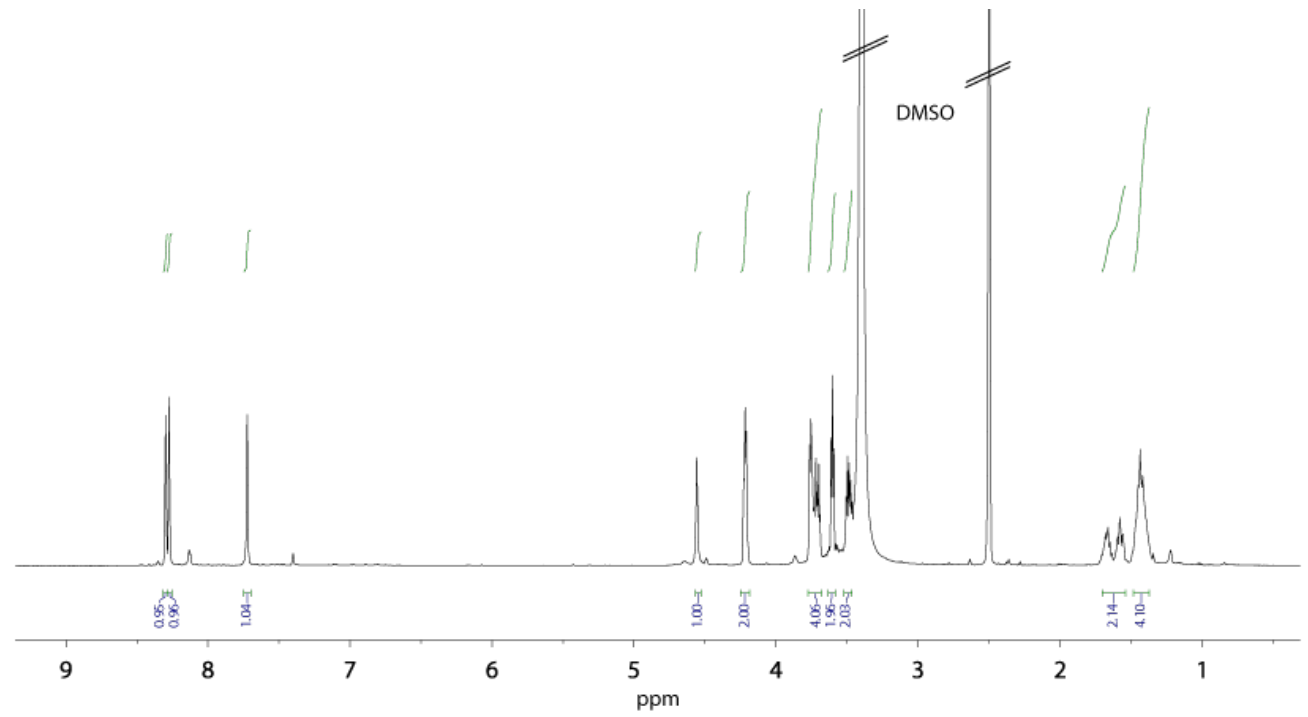

${ }^{13}$ C-NMR $\left(176 \mathrm{MHz}, 298 \mathrm{~K}, \mathrm{CD}_{3} \mathrm{CN}\right): \delta(\mathrm{ppm})=155.4,142.2,137.1,123.8,120.1,92.1$, $69.9,68.8,68.2,66.1,61.3,30.2,25.1,19.1$.

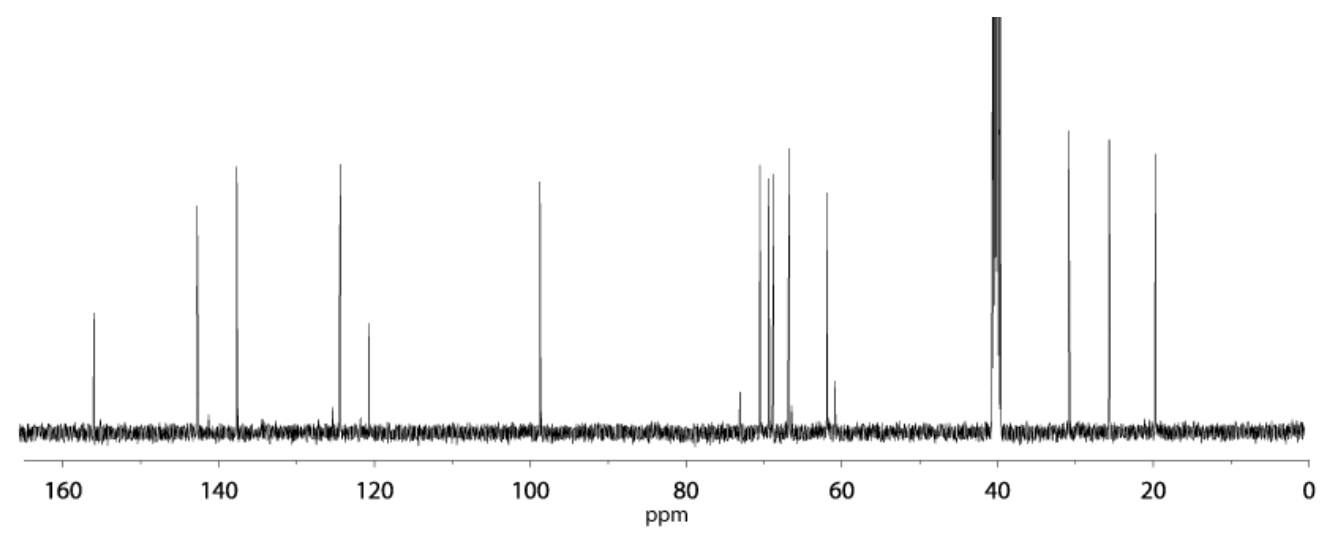


ESI-HRMS $\left(\left[\mathrm{C}_{14} \mathrm{H}_{20} \mathrm{BrNO}_{4}+\mathrm{H}\right]^{+}\right)$: $\quad$ measured: $\quad 346.0649$

calculated: $\quad 346.0648$ 
VI.1.1.4 3-(2-(2-((Tetrahydro-2H-pyran-2-yl)oxy)ethoxy)ethoxy)-5((trimethylsilyl)ethynyl)pyridine (6)<smiles>Brc1cncc(OCCOCCOC2CCCCO2)c1</smiles>

5

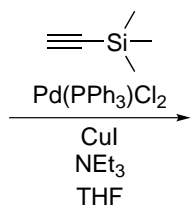

THF

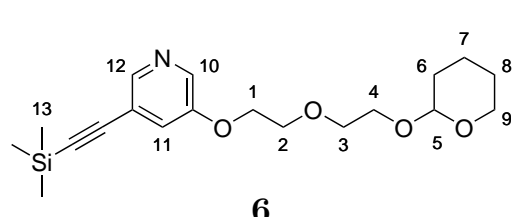

6

Scheme VI.1.1.16: Synthesis of compound 6.

${ }^{1} \mathbf{H}-\mathbf{N M R}\left(300 \mathrm{M} \mathrm{Hz}, 298 \mathrm{~K}, \mathrm{CD}_{3} \mathrm{CN}\right): \delta(\mathrm{ppm})=8.29(\mathrm{~s}, 1 \mathrm{H}, 12), 8.26(\mathrm{~s}, 1 \mathrm{H}, 10), 7.26(\mathrm{~s}$, $1 \mathrm{H}, 11), 4.63(\mathrm{t}, 1 \mathrm{H}, 5), 4.21-4.13(\mathrm{~m}, 2 \mathrm{H}, 1), 3.96-3.82(\mathrm{~m}, 4 \mathrm{H}, 2,3), 3.79-6.69$ (m, 2H, 4), 3.68-3.42 (m, 2H, 9), 1.93-1.44 (m, 6H, 6-8), 0.25 (s, 9H, 13)

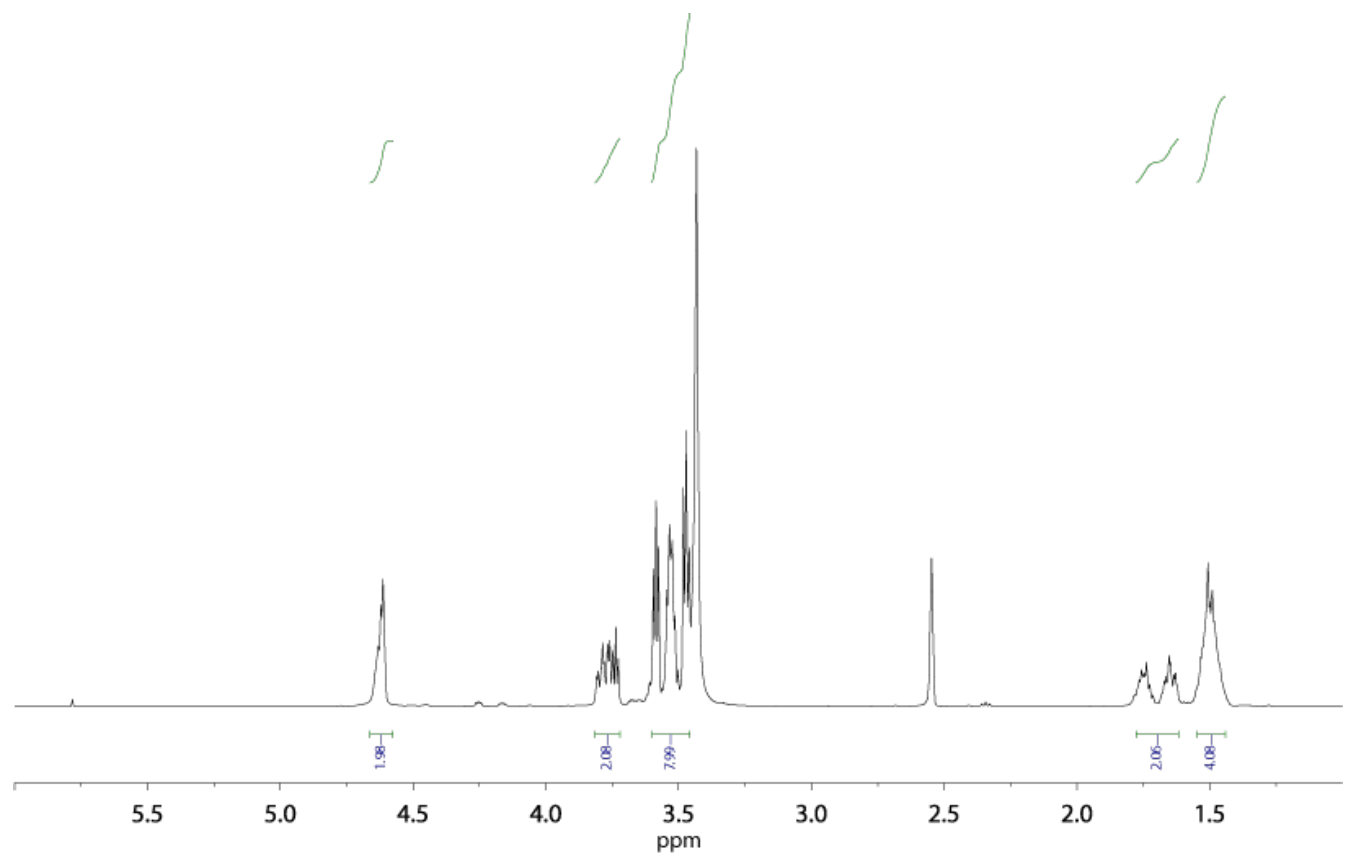

${ }^{13}$ C-NMR $\left(176 \mathrm{MHz}, 298 \mathrm{~K}, \mathrm{CD}_{3} \mathrm{CN}\right): \delta(\mathrm{ppm})=154.0,143.9,138.3,123.0,118.9,97.9$, 83.7, 80.1, 69.6, 68.6, 67.6, 65.9, 30.0, 24.8, 18.8, 1.4. 


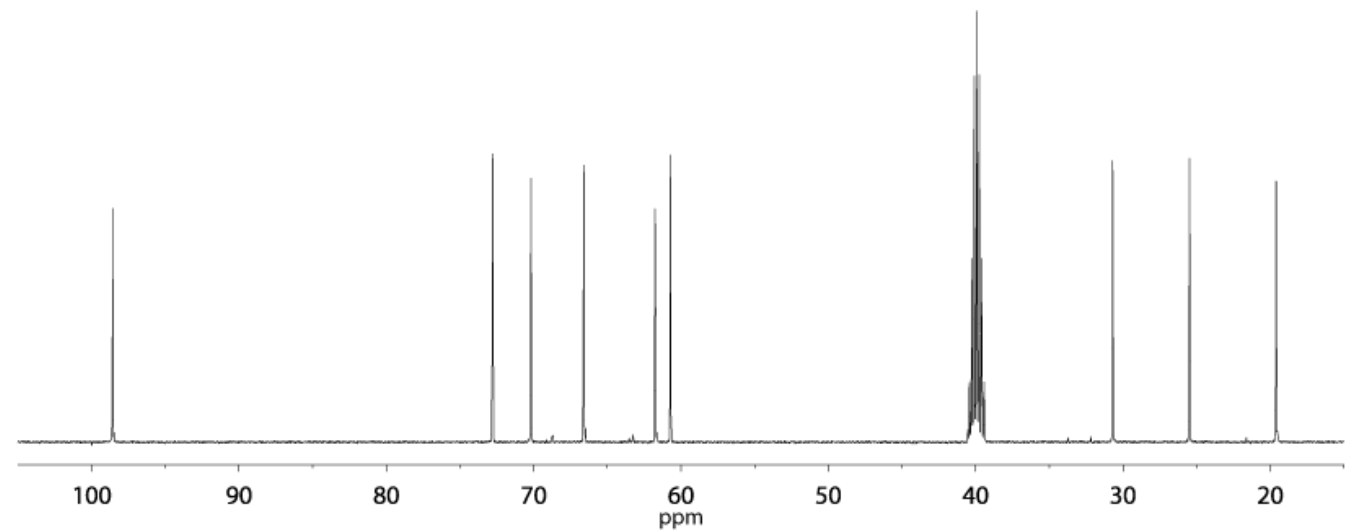

$\begin{array}{lll}\text { ESI-HRMS }\left(\left[\mathrm{C}_{19} \mathrm{H}_{29} \mathrm{NO}_{4} \mathrm{Si}+\mathrm{H}\right]^{+}\right): & \text {measured: } & 364.1940 \\ & \text { calculated: } & 364.1939\end{array}$ 
VI.1.1.5 3-ethynyl-5-(2-(2-((tetrahydro-2H-pyran-2yl)oxy)ethoxy)ethoxy)pyridine (7)<smiles>C[Si](C)(C)C#Cc1cncc(OCCOCCOC2CCCCO2)c1</smiles>

6

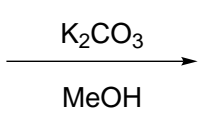

$\mathrm{MeOH}$

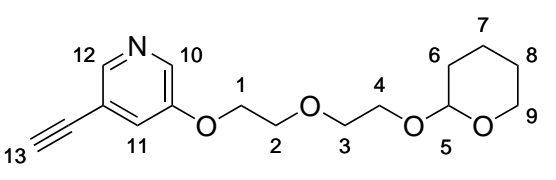

7

Scheme VI.1.1.17: Synthesis of compound 7 .

6 and $\mathrm{K}_{2} \mathrm{CO}_{3}$ in $\mathrm{MeOH}(90 \mathrm{~mL})$ were stirred at room temperature for $3.5 \mathrm{~h}$. The solvent was removed in vacuo and the residue was resolved in $\mathrm{CH}_{2} \mathrm{Cl}_{2}(150 \mathrm{~mL})$. The mixture was washed with $\mathrm{H}_{2} \mathrm{O}(2 \times 75 \mathrm{~mL})$ and brine $(50 \mathrm{~mL})$. The organic phase was dried over $\mathrm{MgSO}_{4}$ and the solvent was remove in vacuo. The product 7 (2.57 g, $\left.8.72 \mathrm{mmol}, 99 \%\right)$ was obtained as a brownish oil.

${ }^{\mathbf{1}} \mathbf{H}-\mathbf{N M R}\left(300 \mathrm{M} \mathrm{Hz}, 298 \mathrm{~K}, \mathrm{CD}_{3} \mathrm{CN}\right): \delta(\mathrm{ppm})=8.25\left(\mathrm{~d},{ }^{4} J=2.8 \mathrm{~Hz}, 12\right), 8.20\left(\mathrm{~d},{ }^{4} J\right.$ $=1.6 \mathrm{~Hz}, 10), 7.46\left(\mathrm{dd},{ }^{4} J=2.9,1.6 \mathrm{~Hz}, 11\right), 4.51\left(\mathrm{t},{ }^{3} J=3.6 \mathrm{~Hz}, 1 \mathrm{H}\right), 4.36(\mathrm{~s}, 1 \mathrm{H}, 13)$, $4.15\left(\mathrm{dd},{ }^{3} J=5.3,3.7 \mathrm{~Hz}, 4 \mathrm{H}, 2,3\right), 3.74-3.62(\mathrm{~m}, 2 \mathrm{H}, 4), 3.57-3.53(\mathrm{~m}, 2 \mathrm{H}, 1), 3.47-3-40$ $(\mathrm{m}, 2 \mathrm{H}, 9), 1.67-1.30(\mathrm{~m}, 6 \mathrm{H}, 6-8)$.

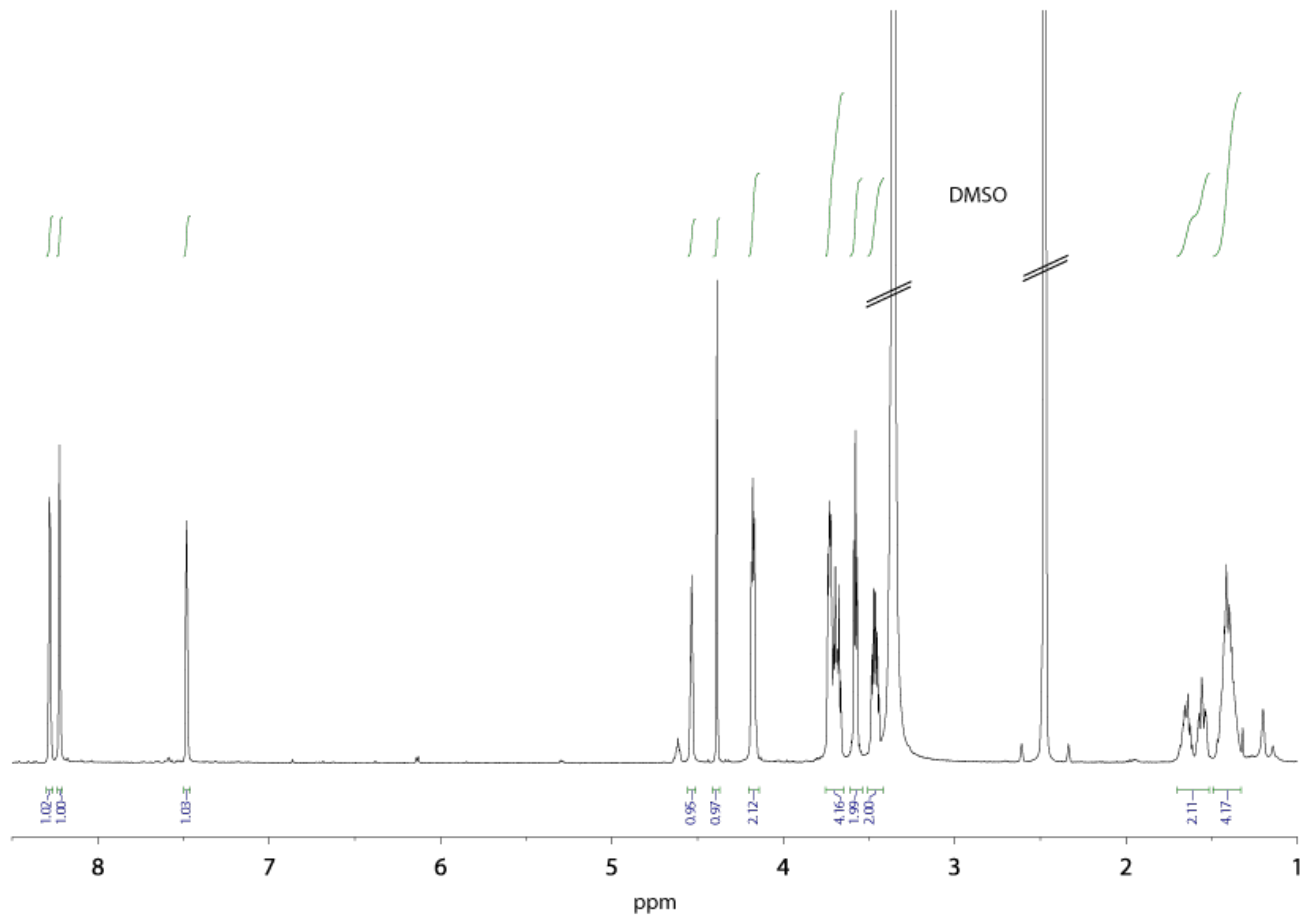

${ }^{13}$ C-NMR $\left(176 \mathrm{MHz}, 298 \mathrm{~K}, \mathrm{CD}_{3} \mathrm{CN}\right): \delta(\mathrm{ppm})=154.7,144.6,138.9,123.7,119.5,98.53$, 84.4, 80.7, 70.3, 69.2, 68.3, 66.6, 61.7, 30.7, 25.5, 19.6 . 


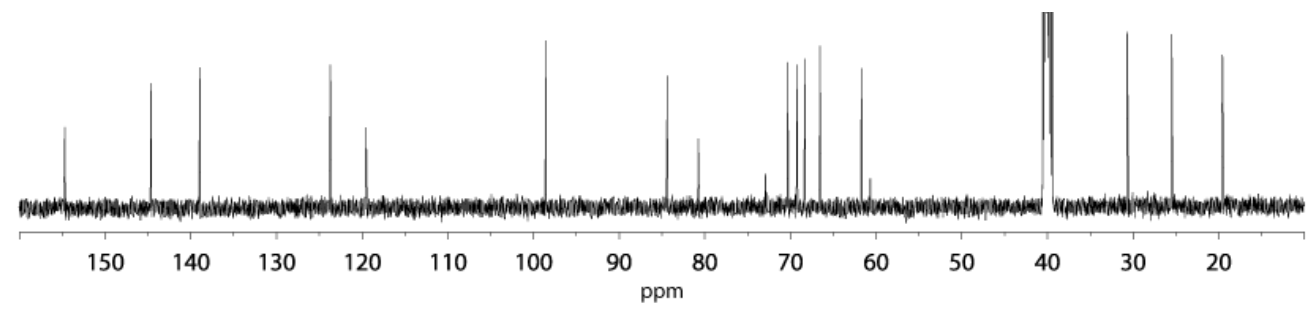

$\begin{array}{lll}\text { ESI-HRMS }\left(\left[\mathrm{C}_{16} \mathrm{H}_{21} \mathrm{NO}_{4}+\mathrm{H}\right]^{+}\right): & \text {measured: } & 292.1515 \\ & \text { calculated: } & 292.1549\end{array}$ 


\section{VI.1.1.6 10-(2-(2-methoxyethoxy)ethyl)-2,7-bis((5-(2-(2-((tetrahydro-2H- pyran-2-yl)oxy)ethoxy)ethoxy)pyridin-3-yl)ethynyl)acridin-9(10H)- one (8)}

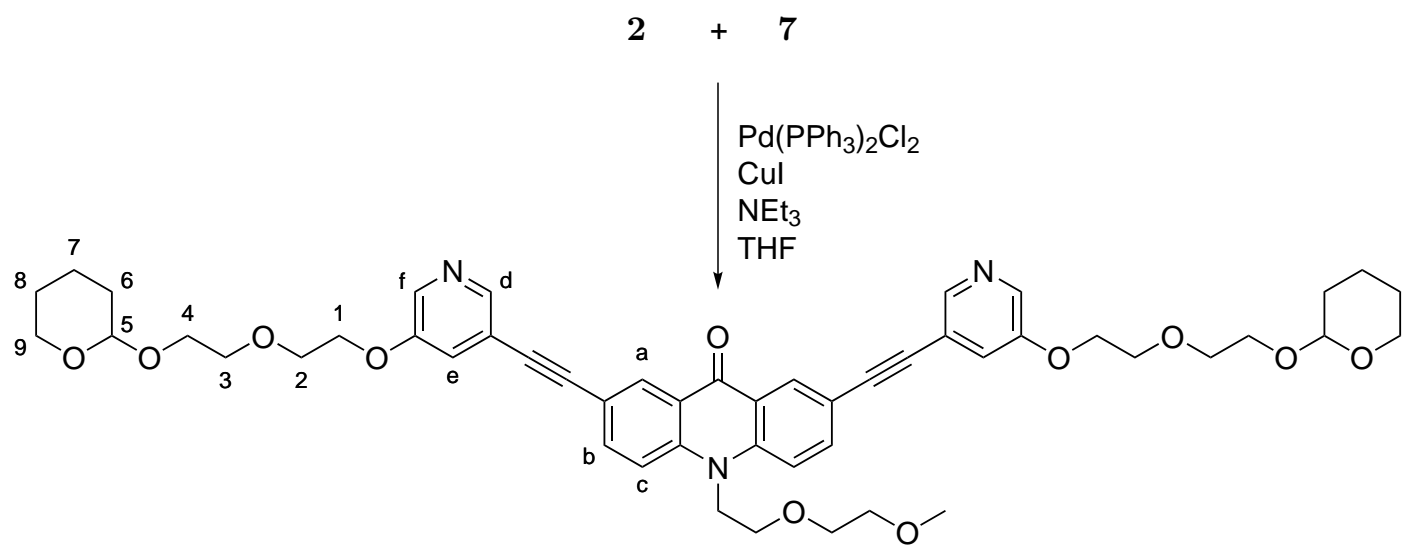

8

Scheme VI.1.1.18: Synthesis of compound 8 .

2 (2.15 g, $3.92 \mathrm{mmol}, 1.00$ equiv.), 7 (2.40 g, $8.22 \mathrm{mmol}, 2.10$ equiv.), $\mathrm{Pd}\left(\mathrm{PPh}_{3}\right)_{2} \mathrm{Cl}_{2}$ (275 mg, $0.39 \mathrm{mmol}, 0.10$ equiv.) and $\mathrm{CuI}(74.6 \mathrm{mg}, 0.39 \mathrm{mmol}, 0.10$ equiv.) were solved in dry THF $(15 \mathrm{~mL})$. Triethylamine $(2.00 \mathrm{~mL}, 15.7 \mathrm{mmol}, 4.00$ equiv.) was added and the reaction mixture was stirred at $70{ }^{\circ} \mathrm{C}$ over night. The cooled down reaction mixture was filtered through celite and the residue was washed with $\mathrm{CH}_{2} \mathrm{Cl}_{2}(3 \times 10 \mathrm{~mL})$. The solvent of the combined organic phases was removed in vacuo and the residue was purifed by means of column chromatography $\left(\mathrm{SiO}_{2}\right.$, chloroform / methanol 50:1 $\rightarrow$ 10:1). The product 8 was obtained as a yellow-brownish resin.

${ }^{1}$ H-NMR $(300 \mathrm{MHz}, 298 \mathrm{~K}, \mathrm{DMSO}): \delta(\mathrm{ppm})=8.88-8.14(\mathrm{br}, 4 \mathrm{H}, \mathrm{d}, \mathrm{f}), 8.47\left(\mathrm{~d},{ }^{4} \mathrm{~J}=\right.$ $2.1 \mathrm{~Hz}, \mathrm{a}), 7.99\left(\mathrm{~d},{ }^{3} J=9.2 \mathrm{~Hz}, 2 \mathrm{H}, \mathrm{c}\right), 7.94\left(\mathrm{dd},{ }^{3} J=9.2 \mathrm{~Hz},{ }^{4} J=2.1 \mathrm{~Hz}, 2 \mathrm{H}, \mathrm{b}\right), 7.66$ (s, 2H, e), $4.76\left(\mathrm{t},{ }^{3} \mathrm{~J}=6.0 \mathrm{~Hz}, 2 \mathrm{H}, \mathrm{NCH}_{2} \mathrm{CH}_{2}\right), 4.58\left(\mathrm{~d},{ }^{3} J=3.6 \mathrm{~Hz}, 2 \mathrm{H}, 5\right), 4.25$ (t, $\left.{ }^{3} J=4.4 \mathrm{~Hz}, 4 \mathrm{H}, 1\right), 3.93\left(\mathrm{t},{ }^{3} J=5.5 \mathrm{~Hz}, 2 \mathrm{H}, \mathrm{NCH}_{2} \mathrm{CH}_{2}\right), 3.80$ (t, $\left.{ }^{3} J=4.5 \mathrm{~Hz}, 4 \mathrm{H}, 2\right)$, 3.79-3.70 (m, 4H, 9), $3.64\left(\mathrm{dd},{ }^{3} J=4.8 \mathrm{~Hz}, 4 \mathrm{H}, \mathrm{CH}_{2} \mathrm{CH}_{2} \mathrm{OCH}_{3}\right), 3.56-3.48(\mathrm{~m}, 4 \mathrm{H}, 3)$, $3.35-3.28(\mathrm{~m}, 4 \mathrm{H}, 4), 3.12\left(\mathrm{~s}, 3 \mathrm{H}, \mathrm{OCH}_{3}\right)$. 


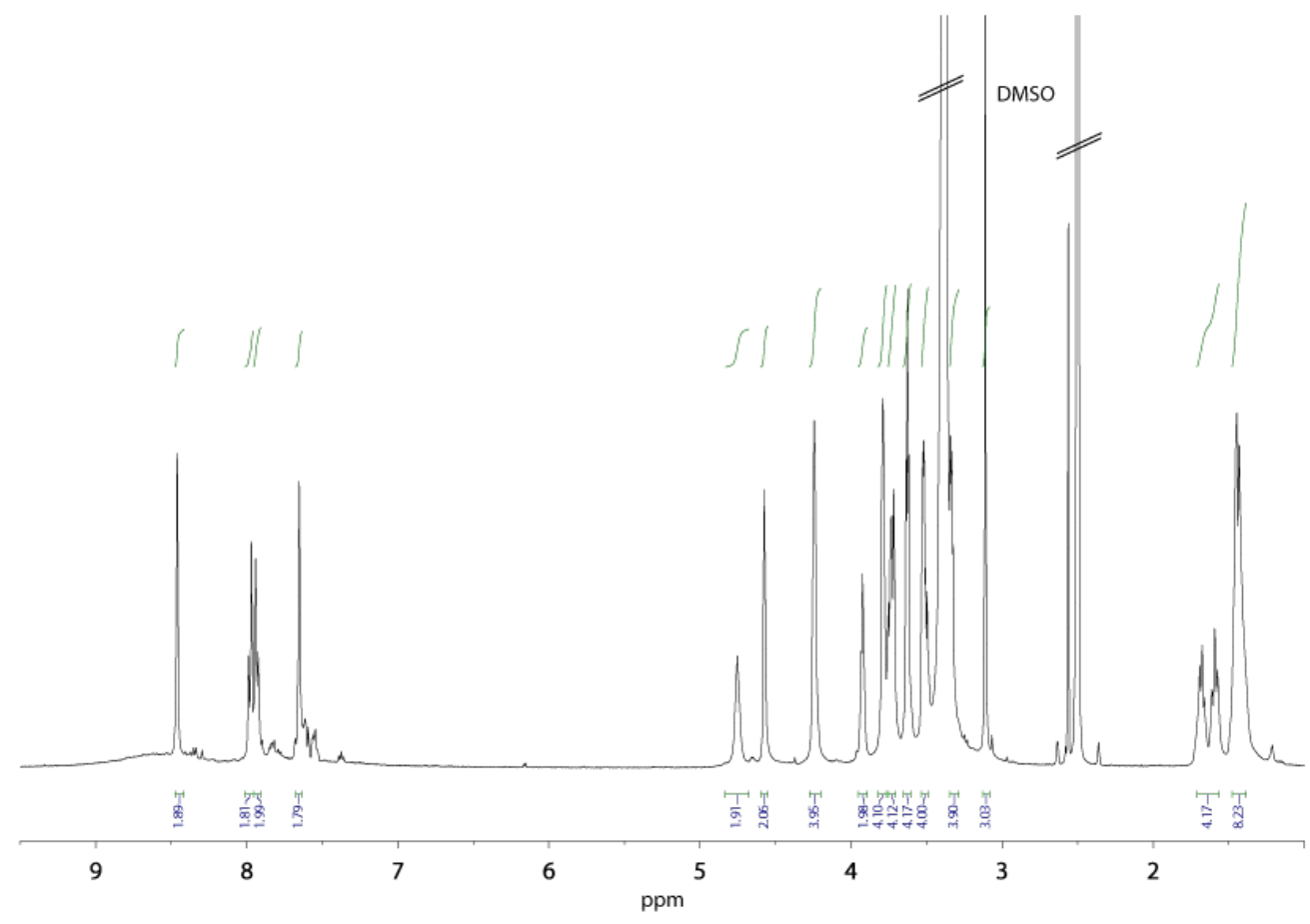

${ }^{13}$ C-NMR (176 MHz, $\left.298 \mathrm{~K}, \mathrm{DMSO}\right): \delta(\mathrm{ppm})=179.5,175.2,142.9,136.2,131.5,130.1$, 128.8, 121.6, 118.0, 115.0, 92.1, 91.5, 71.3, 70.2, 69.9, 68.8, 68.1, 67.9, 66.1, 61.3, 58.1, j46.3, $30.2,29.5,25.1,19.1$.

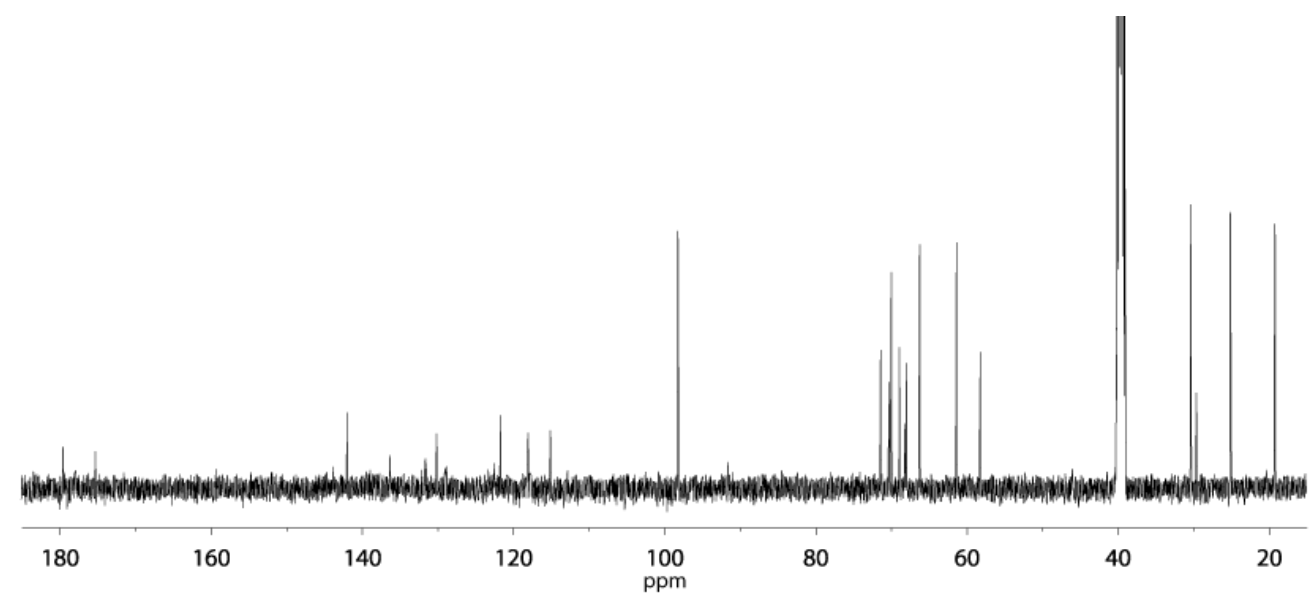

ESI-HRMS $\left(\left[\mathrm{C}_{50} \mathrm{H}_{57} \mathrm{~N}_{3} \mathrm{O}_{11}+\mathrm{H}\right]^{+}\right): \quad$ measured: $\quad 876.4050$

calculated: $\quad 876.4066$ 
VI.1.1.7 2,7-bis((5-(2-(2-hydroxyethoxy)ethoxy)pyridin-3yl)ethynyl)-10-(2-(2-methoxyethoxy)ethyl)acrid-9-one (9)<smiles>COCCOCCn1c2ccc(C#Cc3cncc(OCCOCCOC4CCCCO4)c3)cc2c(=O)c2cc(C#Cc3cncc(OCCOCCOC4CCCCO4)c3)ccc21</smiles>

8 $\mid \begin{aligned} & \mathrm{TsOH} \\ & \mathrm{CH}_{2} \mathrm{Cl}_{2}\end{aligned}$<smiles>COCCOCCn1c2ccc(C#Cc3cncc(OCCOCCO)c3)cc2c(=O)c2cc(C#Cc3cncc(OCCOCCO)c3)ccc21</smiles>

9

Scheme VI.1.1.19: Synthesis of compound 9.

8 (850 mg, 1.00 mmol, 1 equiv.) was solved in $\mathrm{EtOH}(100 \mathrm{~mL})$ and $\mathrm{HCl}(1 \mathrm{M}, 10 \mathrm{~mL}$, 10.0 mmol, 10.0 equiv.) was added. The mixture was heated to under reflux for $1 \mathrm{~h}$. After cooling down the solvent volume was reduced to ca. $10 \mathrm{~mL}$, then $\mathrm{CH}_{2} \mathrm{Cl}_{2}$ (50 mL) was added. The mixture was washed with sat. $\mathrm{NaHCO}_{3}$ solution $(50 \mathrm{~mL})$ and brine $(30 \mathrm{~mL})$ and the organic phase was dried over $\mathrm{MgSO}_{4}$. The solvent was removed in vacuo. The product $9(0.65 \mathrm{~g}, 0.92 \mathrm{mmol}, 92 \%)$ was obtained as a yellow-brownish solid.

${ }^{1} \mathbf{H}-\mathbf{N M R}\left(300 \mathrm{M} \mathrm{Hz}, 298 \mathrm{~K}, \mathrm{CD}_{3} \mathrm{Cl}\right): \delta(\mathrm{ppm})=8.68\left(\mathrm{~d},{ }^{4} J=2.0 \mathrm{~Hz}, 2 \mathrm{H}, \mathrm{a}\right), 8.49(\mathrm{~s}$, $2 \mathrm{H}, \mathrm{f}), 8.31(\mathrm{~s}, 2 \mathrm{H}, \mathrm{d}), 7.83\left(\mathrm{dd},{ }^{3} J=9.0 \mathrm{~Hz},{ }^{4} J=2.0 \mathrm{~Hz}, 2 \mathrm{H}, \mathrm{b}\right), 7.64\left(\mathrm{~d},{ }^{3} J=9.0 \mathrm{~Hz}\right.$, $2 \mathrm{H}, \mathrm{c}), 7.36(\mathrm{~s}, 2 \mathrm{H}, \mathrm{e}), 4.64\left(\mathrm{t},{ }^{3} J=6.3 \mathrm{~Hz}, 2 \mathrm{H}, \mathrm{NCH} 2\right), 4.25-4.20(\mathrm{~m}, 4 \mathrm{H}, 1), 4.01$ (t, $\left.{ }^{3} J=6.3 \mathrm{~Hz}, 2 \mathrm{H}, \mathrm{NCH}_{2} \underline{\mathrm{CH}_{2}}\right), 3.94-3.89(\mathrm{~m}, 4 \mathrm{H}, 2), 3.82-3.77(\mathrm{~m}, 4 \mathrm{H}, 3), 3.72-3.68(\mathrm{~m}$, $4 \mathrm{H}, 4), 3.68-3.64\left(\mathrm{~m}, 2 \mathrm{H}, \underline{\mathrm{CH}_{2}} \mathrm{CH}_{2} \mathrm{OCH}_{3}\right), 3.54-3.50\left(\mathrm{~m}, 2 \mathrm{H}, \mathrm{CH}_{2} \underline{\mathrm{CH}_{2}} \mathrm{OCH}_{3}\right), 3.36(\mathrm{~s}, 3 \mathrm{H}$, OCH3). 


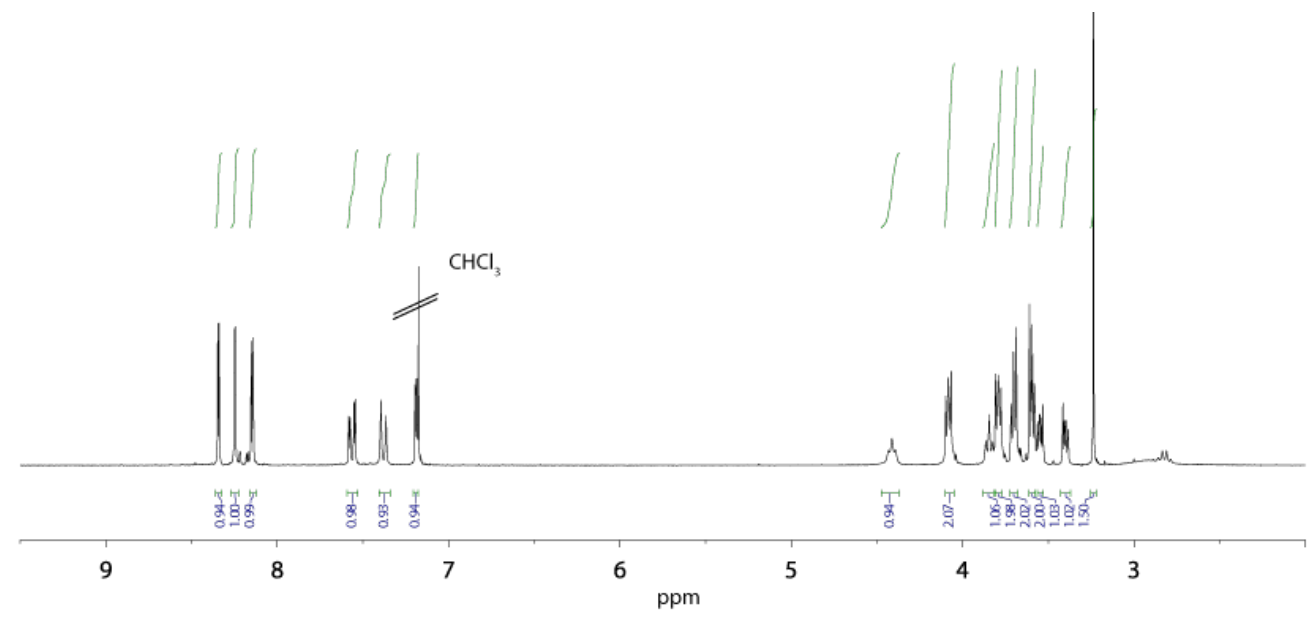

${ }^{13} \mathrm{C}-\mathrm{NMR}\left(176 \mathrm{MHz}, 298 \mathrm{~K}, \mathrm{CD}_{3} \mathrm{CN}\right): \delta(\mathrm{ppm})=176.4,144.7,141.8,137.9,136.5,131.6$, $122.9,122.4,116.1,115.8,91.6,86.4,77.2,72.7,72.0,71.2,69.4,68.1,67.9,61.7,59.2,59.1$, 46.4 .

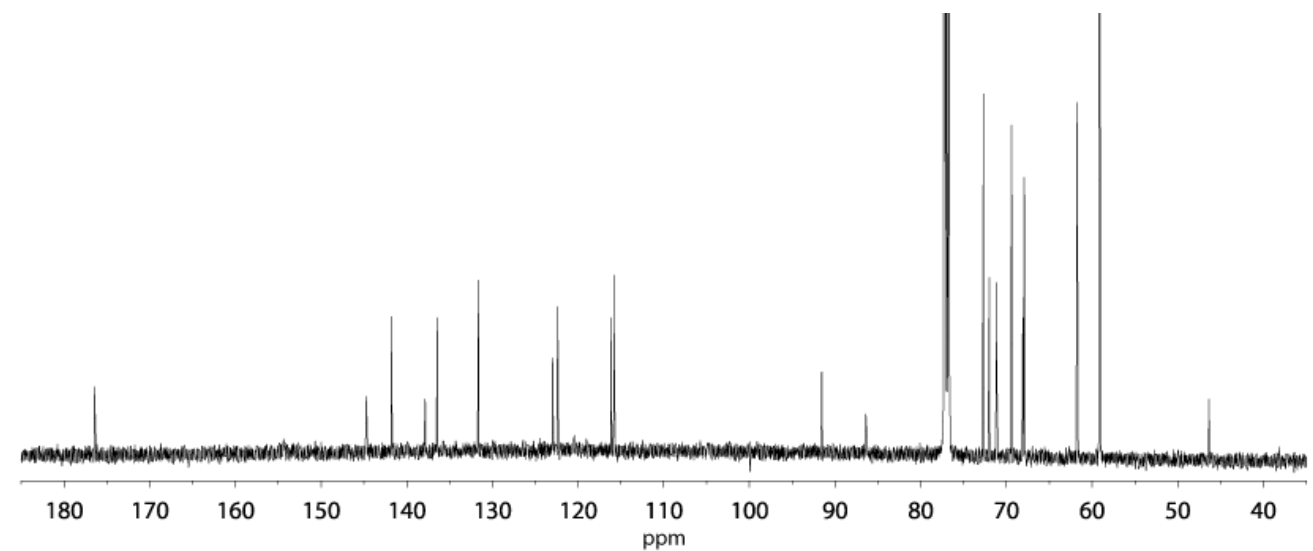

ESI-HRMS $\left(\left[\mathrm{C}_{40} \mathrm{H}_{11} \mathrm{~N}_{3} \mathrm{O}_{9}+\mathrm{H}\right]^{+}\right)$: $\quad$ measured: $\quad 708.2899$

calculated: $\quad 708.2916$ 
VI.1.1.8 2-((5-(2-(2-(Bis) $4-$

methoxyphenyl)(phenyl)methoxy)ethoxy)ethoxy)pyridin-3-

yl)ethynyl)-7-((5-(2-(2-hydroxyethoxy)ethoxy)pyridin-3-yl)ethynyl)-

10-(2-(2-methoxyethoxy)ethyl)acridin-9(10H)-one (10)<smiles>COCCOCCn1c2ccc(C#Cc3cncc(OCCOCCO)c3)cc2c(=O)c2cc(C#Cc3cncc(OCCOCCO)c3)ccc21</smiles>

9

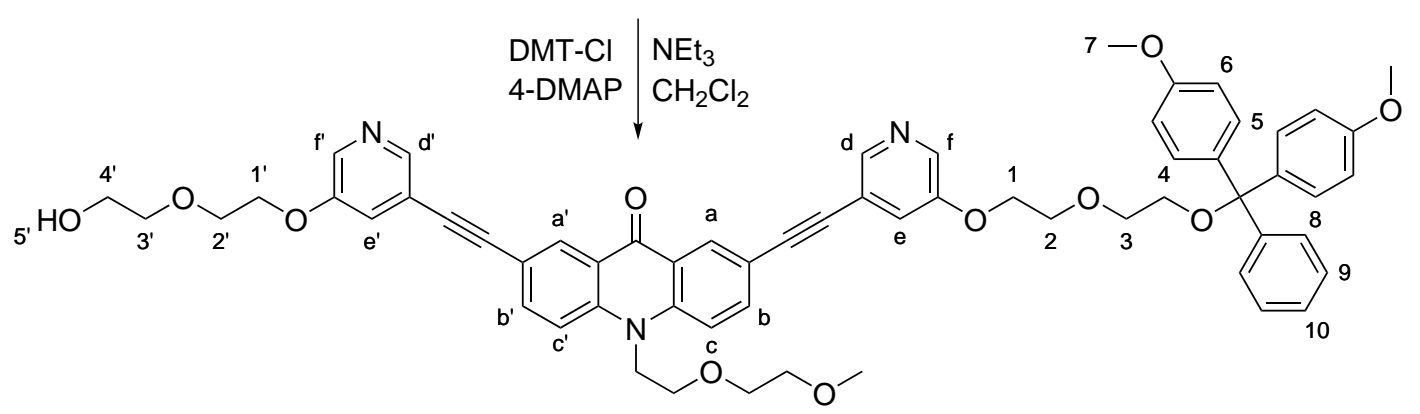

10

Scheme VI.1.1.20: Synthesis of compound 10.

4,4'-Dimethoxytrityl chloride (522 mg, $1.54 \mathrm{mmol}, 1.05$ equiv.), 4-DMAP (9.00 mg, $0.07 \mathrm{mmol}, 0.05$ equiv.) und $\mathbf{9}$ (1.04 g, $1.47 \mathrm{mmol}, 1.00$ equiv.) was solved in $\mathrm{CH}_{2} \mathrm{Cl}_{2}$ $(50 \mathrm{~mL})$ and triethylamine $(0.31 \mathrm{~mL}, 2.20 \mathrm{mmol}, 1.50$ equiv. $)$ was added dropwise at $0{ }^{\circ} \mathrm{C}$. The reaction mixture was stirred at room temperature over night, whereby the mixture warmed to room temperature. The mixture was diluted with $\mathrm{CH}_{2} \mathrm{Cl}_{2}$ and the organic phase was washed with $5 \% \mathrm{NaHCO}_{3}$ solution $(25 \mathrm{~mL})$ and brine $\left.25 \mathrm{~mL}\right)$. The organic phase was dired over $\mathrm{MgSO}_{4}$ and the solvent was removed in vacuo. The residue was purified by means of column chromatography ( $\mathrm{SiO}_{2}$, chloroform / methanol 50:1 $\rightarrow$ 10:1, with $1 \%$ TEA). The product was obtained $\mathbf{1 0}$ was obtained as an orange foam.

${ }^{1} \mathbf{H}-\mathbf{N M R}\left(300 \mathrm{M} \mathrm{Hz}, 298 \mathrm{~K}, \mathrm{CD}_{3} \mathrm{Cl}\right): \delta(\mathrm{ppm})=8.65\left(\mathrm{dd},{ }^{4} J=2.1 \mathrm{~Hz}, 1 \mathrm{H}, \mathrm{a}\right), 8.63$ $\left(\mathrm{dd},{ }^{4} J=2.1 \mathrm{~Hz}, 1 \mathrm{H}, \mathrm{a}\right) 8.38\left(\mathrm{~d},{ }^{3} J=1.8 \mathrm{~Hz}, 2 \mathrm{H}, \mathrm{f}, \mathrm{f}^{\prime}\right), 8.29\left(\mathrm{dd},{ }^{4} J=2.8 \mathrm{~Hz}, 2 \mathrm{H}\right.$, d), $8.27\left(\mathrm{dd},{ }^{4} J=2.8 \mathrm{~Hz}, 2 \mathrm{H}, \mathrm{d}\right), 7.79\left(\mathrm{dd},{ }^{3} J=4.1 \mathrm{~Hz},{ }^{4} J=2.2 \mathrm{~Hz}, 1 \mathrm{H}, \mathrm{b}\right), 7.76$ $\left(\mathrm{dd},{ }^{3} J=4.1 \mathrm{~Hz},{ }^{4} J=2.2 \mathrm{~Hz}, 1 \mathrm{H}, \mathrm{b}\right), 7.61$ (d, $\left.{ }^{3} J=3.4 \mathrm{~Hz}, 1 \mathrm{H}, \mathrm{c}\right), 7.58$ (d, ${ }^{3} J=3.4$ $\mathrm{Hz}, 1 \mathrm{H}, \mathrm{c}), 7.48-7.44$ (m, 2H, 8), 7.38-7.30 (m, 6H, 5, e, e'), 7.30-7.13 (m, 3H, 9, 10), 6.84-6.76 (m, 4H, 6), $4.60\left(\mathrm{t},{ }^{3} J=6.2 \mathrm{~Hz}, 2 \mathrm{H}, \mathrm{NCH}_{2} \mathrm{CH}_{2}\right), 4.25-4.16\left(\mathrm{~m}, 4 \mathrm{H}, 1,1^{\prime}\right), 3.99$ (t, $\left.{ }^{3} \mathrm{~J}=6.2 \mathrm{~Hz}, 2 \mathrm{H}, \mathrm{NCH}_{2} \mathrm{CH}_{2}\right), 3.95-3.86$ (m, $4 \mathrm{H}, 2,2$ '), 3.83-3.62 (m, 14H, 3, 3', 4, 7, $\left.\mathrm{CH}_{2} \mathrm{CH}_{2} \mathrm{OCH}_{3}\right), 3.53-3.47\left(\mathrm{~m}, 2 \mathrm{H}, 4^{\prime}\right), 3.34\left(\mathrm{~s}, 3 \mathrm{H}, \mathrm{OCH}_{3}\right), 3.27$ (t, ${ }^{3} J=5.1 \mathrm{~Hz}, 2 \mathrm{H}$, $\mathrm{CH}_{2} \mathrm{CH}_{2} \mathrm{OCH}_{3}$ ). 


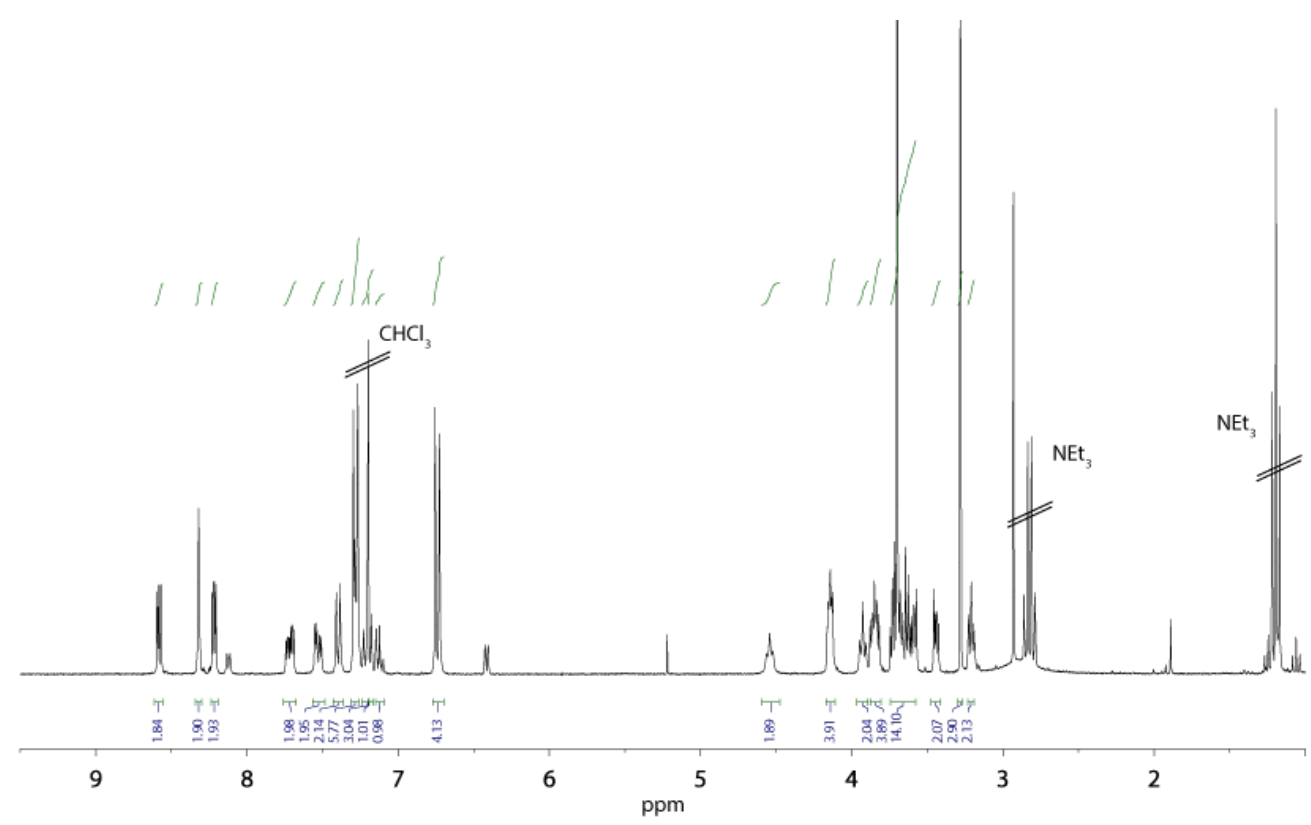

${ }^{13}$ C-NMR $\left(176 \mathrm{MHz}, 298 \mathrm{~K}, \mathrm{CD}_{3} \mathrm{Cl}\right): \delta(\mathrm{ppm})=176.6,158.5,155.0,154.4,145.1,144.9$, 144.8, 142.0, 138.1, 136.7, 136.6, 136.4, 135.5, 131.9, 131.8, 130.2, 129.0, 128.5, 128.4, 128.3, $127.9,126.8,123.1,123.0,122.6,120.6,120.5,116.4,116.0,115.9,113.2,91.7,91.6,86.7$, $86.6,72.8,72.2,71.3,71.2,69.7,69.6,68.3,68.2,68.1,67.2,63.3,62.0,59.3,55.4,55.0$, 46.5 .

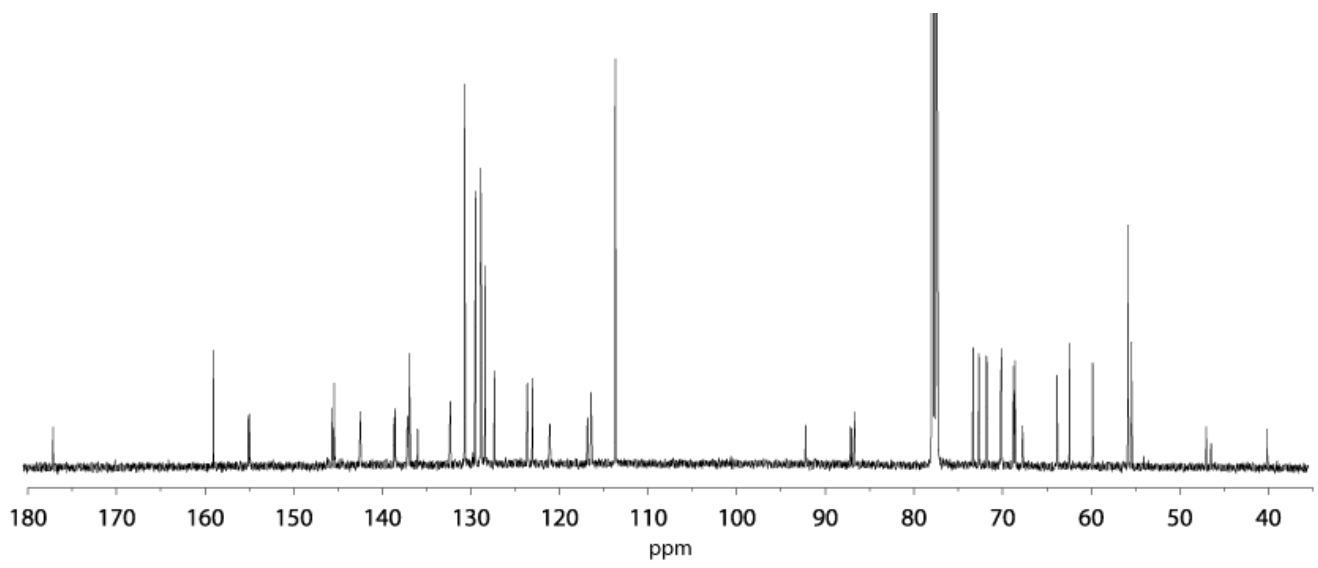

ESI-HRMS $\left(\left[\mathrm{C}_{61} \mathrm{H}_{59} \mathrm{~N}_{3} \mathrm{O}_{11}+\mathrm{H}\right]^{+}\right)$: $\quad$ measured: $\quad 1010.4217$ calculated: $\quad 1010.4222$ 
VI.1.1.9 2-(2-((5-)( 7-((5-)(2-(2-)(Bis) $4-$ methoxyphenyl)(phenyl)methoxy)ethoxy)ethoxy)pyridin-3yl)ethynyl)-10-(2-(2-methoxyethoxy)ethyl)-9-oxo-9,10dihydroacridin-2-yl)ethynyl)pyridin-3-yl)oxy)ethoxy)ethyl (2-cyanoethyl) diisopropylphosphoramidite (11)<smiles>COCCOCCn1c2ccc(C#Cc3cncc(OCCOCCO)c3)cc2c(=O)c2cc(C#Cc3cncc(OCCOCCOC(c4ccccc4)(c4ccc(OC)cc4)c4ccc(OC)cc4)c3)ccc21</smiles>

10

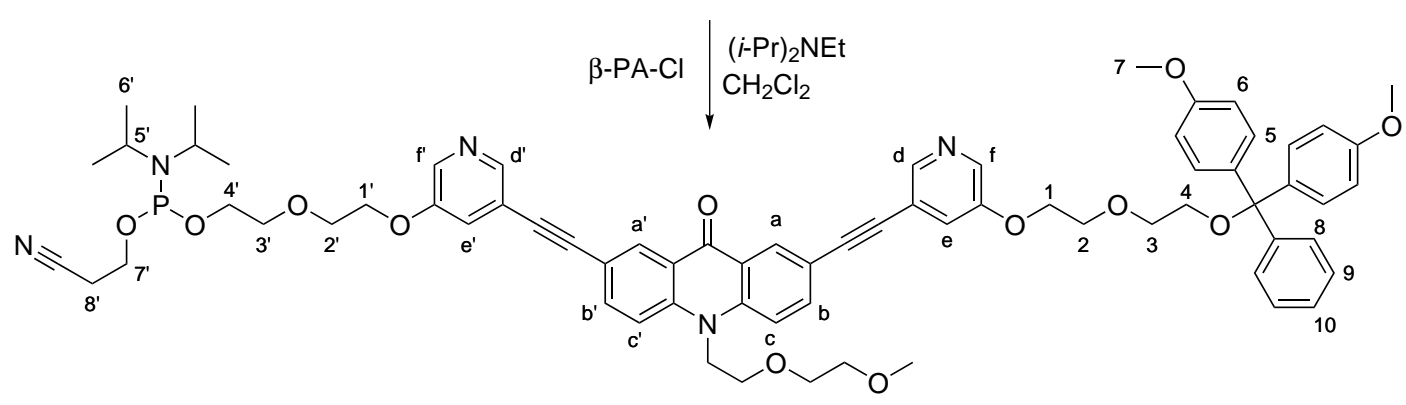

11

Scheme VI.1.1.21: Synthesis of compound 11.

10 (0.81 g, $0.80 \mathrm{mmol}, 1.00$ equiv.) and DIPEA (0.75 mL, $2.43 \mathrm{mmol}, 3.00$ equiv.) were solved in dry THF $(5 \mathrm{~mL})$ and stirred for $0.5 \mathrm{~h}$ at room temperature. 2-Cyanoethyl- $N, N$ diisopropylchlorophosphoramidite $(0.23 \mathrm{~mL}, 1.04 \mathrm{mmol}, 1.30$ equiv. $)$ was added dropwise and the reaction was followed by thin layer chromatography. After completion, the solvent was removed in vacuo and the residue was resolved in dry acetontrile. The mixture was filtered under inert conditions and the solvent was removed again in vacuo. The product 11 was obtained as an orange foam and was used immedeatly for DNA synthesis without further purification.

${ }^{31} \mathbf{P}$-NMR $\left(300 \mathrm{MHz}, 298 \mathrm{~K}, \mathrm{CDCl}_{3}\right): \delta(\mathrm{ppm})=148.0$. (peaks at 14 or $8 \mathrm{ppm}$ are oxidized or hydrolyzed side products) 


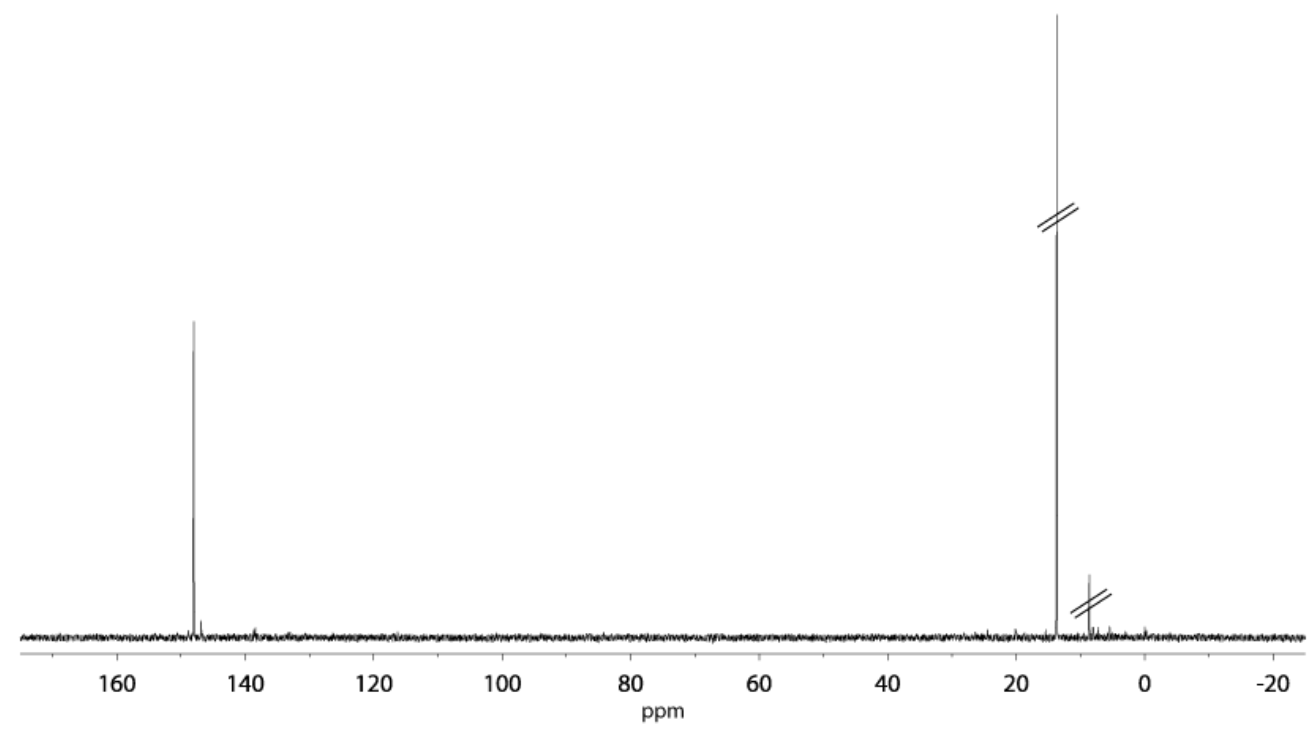




\section{VI.1.2 Syntheses of the Methoxybenzylamine-based Ligand}

\section{VI.1.2.1 4-Hydroxy-3,5-diiodobenzaldehyde (12)}<smiles>O=Cc1ccc(O)cc1</smiles><smiles>CC(C)(O)[Mg]O[Na]</smiles><smiles>O=Cc1cc(I)c(O)c(I)c1</smiles>

12

Scheme VI.1.2.22: Synthesis of compound 12 .

To a stirred solution of 4-hydroxybenzaldehyde (5.00 g, $40.9 \mathrm{mmol}, 1.00$ equiv.), potassium iodate ( $8.76 \mathrm{~g}, 40.9 \mathrm{mmol}, 1.00$ equiv) and potassium chloride ( $6.10 \mathrm{~g}, 81.9 \mathrm{mmol}, 2.00$ equiv.) in acetic acid/water $(9: 1,150 \mathrm{~mL})$, potassium iodide $(13.6 \mathrm{~g}, 81.9 \mathrm{mmol}, 2.00$ equiv.) was added at room temperature. After $6 \mathrm{~h}$, EtOAc $(500 \mathrm{~mL})$ was $10 \% \mathrm{NaS}_{2} \mathrm{O}_{3}$ solution $(500 \mathrm{~mL})$ were added. The organic layer was separated and the aq. phase was extracted with EtOAc $(2 \times 250 \mathrm{~mL})$. The combined organic layers were washed with $10 \% \mathrm{NaS}_{2} \mathrm{O}_{3}$ solution $(250 \mathrm{~mL})$ and brine $(250 \mathrm{~mL})$ and dried over $\mathrm{MgSO}_{4}$. The solvent was removed in vacuo. The product $12(14.8 \mathrm{~g}, 39.7 \mathrm{mmol}, 97 \%)$ was obtained as yellowish solid and could be taken for next synthesis without further purification.

${ }^{1} \mathbf{H}-\mathbf{N M R}\left(300 \mathrm{M} \mathrm{Hz}, 298 \mathrm{~K}, \mathrm{CD}_{3} \mathrm{Cl}_{3}\right): \delta(\mathrm{ppm})=9.74(\mathrm{~s}, 1 \mathrm{H}, \mathrm{b}), 8.20(\mathrm{~s}, 2 \mathrm{H}, \mathrm{a}), 6.28$ (br, 1H, c). 


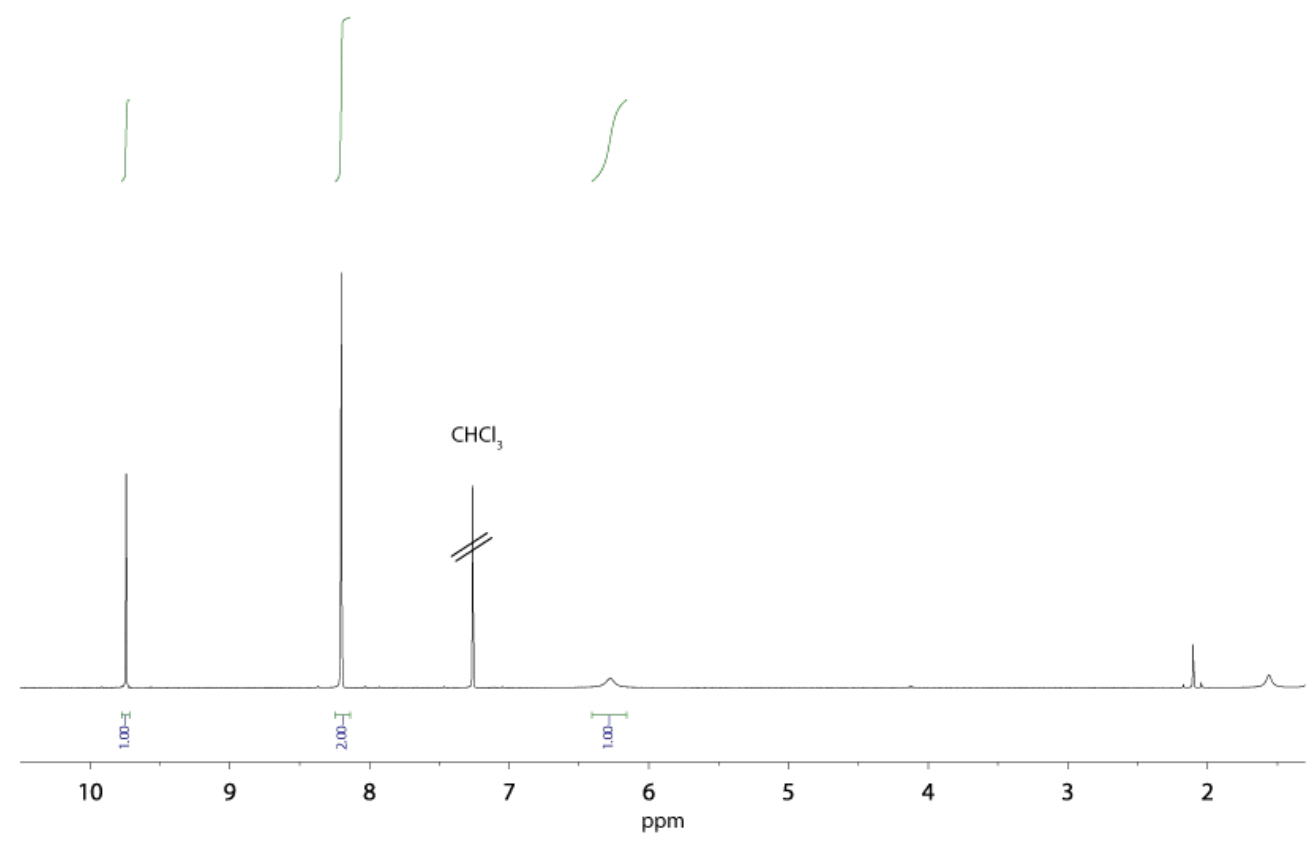

$\begin{array}{lll}\text { ESI-HRMS }\left(\left[\mathrm{C}_{7} \mathrm{H}_{4} \mathrm{I}_{2} \mathrm{O}_{2}+\mathrm{H}\right]^{+}\right): & \text {measured: } & 374.8369 \\ & \text { calculated: } & 374.8373\end{array}$ 


\section{VI.1.2.2 3,5-Diiodo-4-methoxybenzaldehyde (13)}<smiles>Cc1cc(C=O)cc(I)c1O</smiles>

12

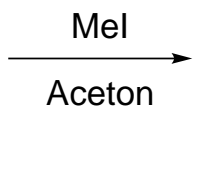

Scheme VI.1.2.23: Synthesis of compound 13.

To 12 (7.50 g, 20.1 mmol, 1.00 equiv.) solved in Actone (100 mL) $\mathrm{K}_{2} \mathrm{CO}_{3}$ (6.93 g, $50.2 \mathrm{mmol}$, 2.50 equiv.) was added and the suspension was stirred $15 \mathrm{~min}$ at room temperature. MeI (6.47 mL, $100 \mathrm{mmol}, 5.00$ equiv.) was added and the reaction mixture was heated over night under reflux. The cooled down mixture was washed with $\mathrm{H}_{2} \mathrm{O}(100 \mathrm{~mL})$ and brine $(50 \mathrm{~mL})$ and the organic layer was dried over $\mathrm{MgSO}_{4}$. After, the solvent was removed in vacuo. The product 13 (7.40 g, $19.1 \mathrm{mmol}, 95 \%)$ was obtained as brownish white solid.

${ }^{1}$ H-NMR $\left(300 \mathrm{M} \mathrm{Hz}, 298 \mathrm{~K}, \mathrm{CD}_{3} \mathrm{Cl}\right): \delta(\mathrm{ppm})=9.81$ (s, 1H, b), 8.27 (s, 2H, a), 3.93 (s, $3 \mathrm{H}, \mathrm{c})$.

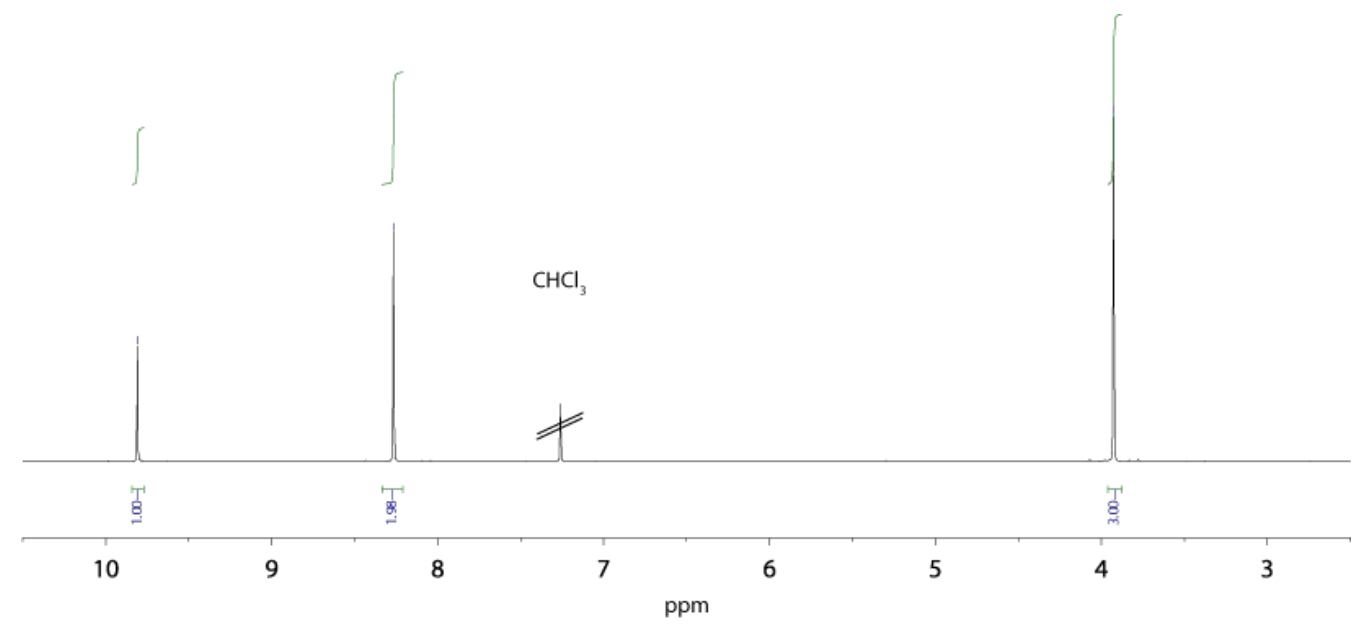

${ }^{13}$ C-NMR $\left(176 \mathrm{MHz}, 298 \mathrm{~K}, \mathrm{CD}_{3} \mathrm{Cl}\right): \delta(\mathrm{ppm})=188.2,163.9,141.4,135.5,91.4$, 61.0. 


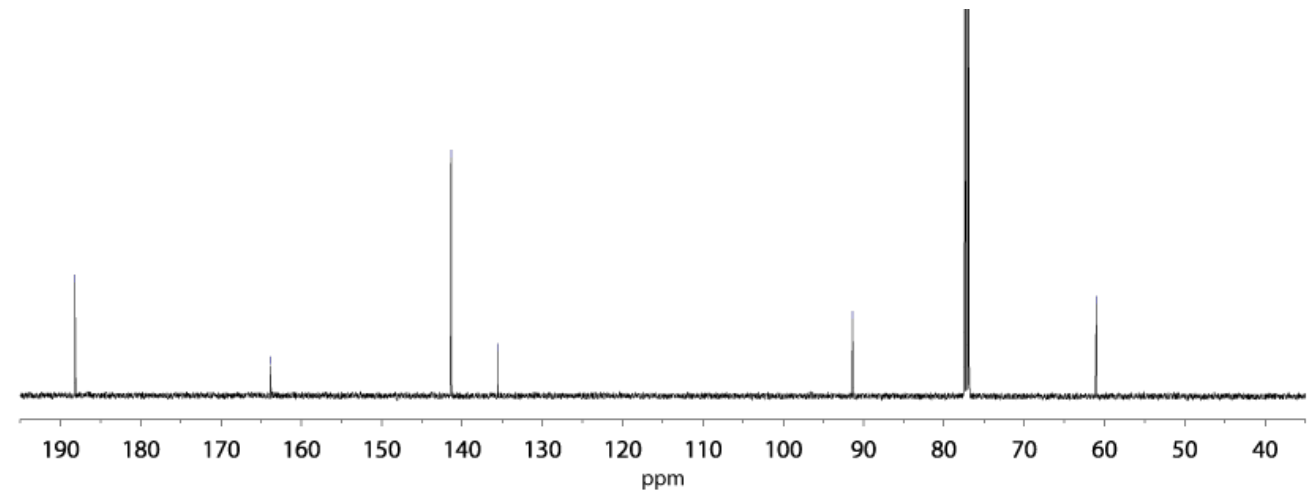

$\begin{array}{lll}\text { ESI-HRMS }\left(\left[\mathrm{C}_{8} \mathrm{H}_{6} \mathrm{I}_{2} \mathrm{O}_{2}+\mathrm{H}\right]^{+}\right): & \text {measured: } & 388.8345 \\ & \text { calculated: } & 388.8350\end{array}$ 


\section{VI.1.2.3 (3,5-Diiodo-4-methoxyphenyl)methanol (14)}<smiles>COc1c(I)cc(C=O)cc1I</smiles>

13

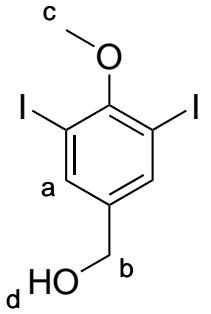

14

Scheme VI.1.2.24: Synthesis of compound 14.

To a well stirring mixture containing 13 (4.00 g, $10.3 \mathrm{mmol}$, 1 equiv.) in $\mathrm{CH} 2 \mathrm{Cl} 2$ (35 $\mathrm{mL})$ and $\mathrm{CH} 3 \mathrm{OH}(35 \mathrm{~mL})$ at $0{ }^{\circ} \mathrm{C}$ was added portionwise $\mathrm{NaBH} 4(0.41 \mathrm{~g}, 10.8 \mathrm{mmol}$, 1.05 equiv.) and the mixture was stirred at $\mathrm{rt}$ for $3 \mathrm{~h}$. Saturated $\mathrm{NH}_{4} \mathrm{Cl}(10 \mathrm{~mL})$ was then added. After removal of most of the solvents, the residue was taken into Et2O (100 mL), washed with $\mathrm{H} 2 \mathrm{O}(2 \times 50 \mathrm{~mL})$ and the organic layer was dried over $\mathrm{MgSO}_{4}$. The solvent was removed in vacuo. The prodcut 14 (4.00 g, $10.2 \mathrm{mmol}, 99 \%)$ was obtained as white solid.

${ }^{1}$ H-NMR $(300 \mathrm{M} \mathrm{Hz}, 298 \mathrm{~K}, \mathrm{DMSO}): \delta(\mathrm{ppm})=7.75(\mathrm{~s}, 2, \mathrm{a}), 5.31\left(\mathrm{t},{ }^{3} J=5.9 \mathrm{~Hz}, 1 \mathrm{H}\right.$, d), $4.40\left(\mathrm{~d},{ }^{3} J=5.8 \mathrm{~Hz}, 2 \mathrm{H}, \mathrm{b}\right), 3.73$ (s, 3H, c).

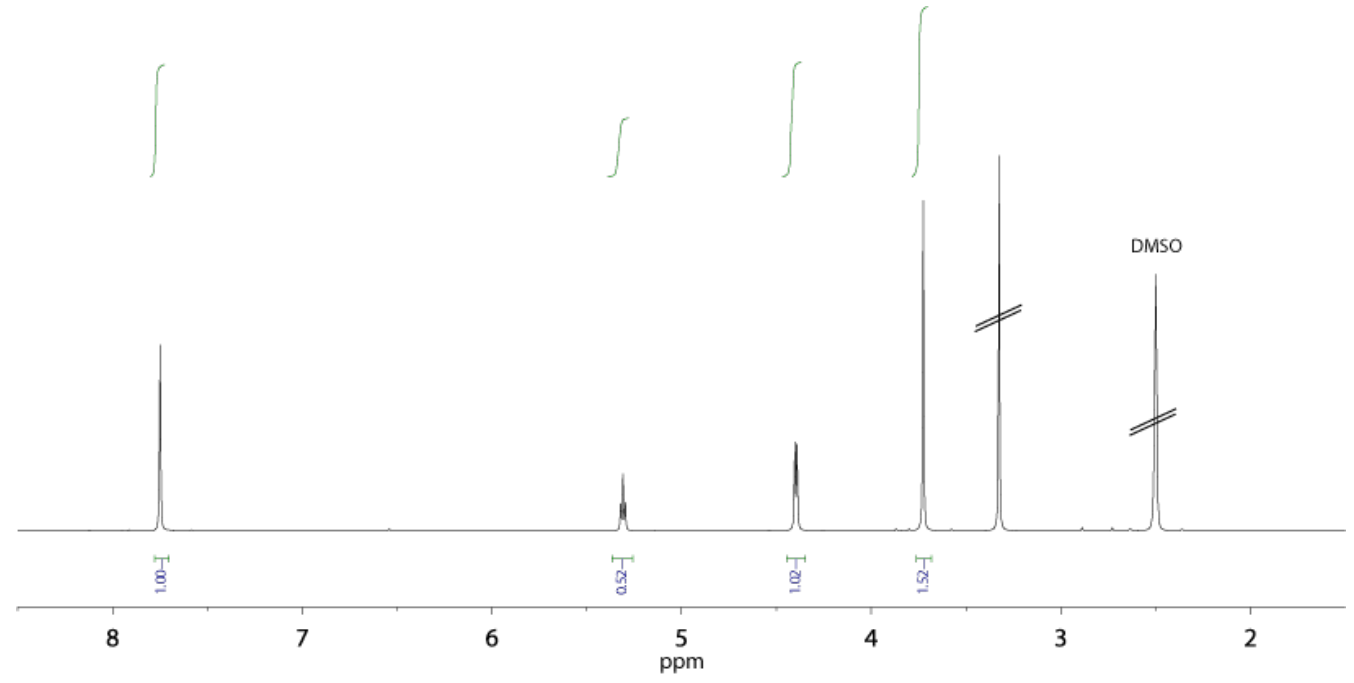

${ }^{13}$ C-NMR (176 MHz, $\left.298 \mathrm{~K}, \mathrm{DMSO}\right): \delta(\mathrm{ppm})=157.0,142.8,137.4,91.0,60.7$, 60.3 . 


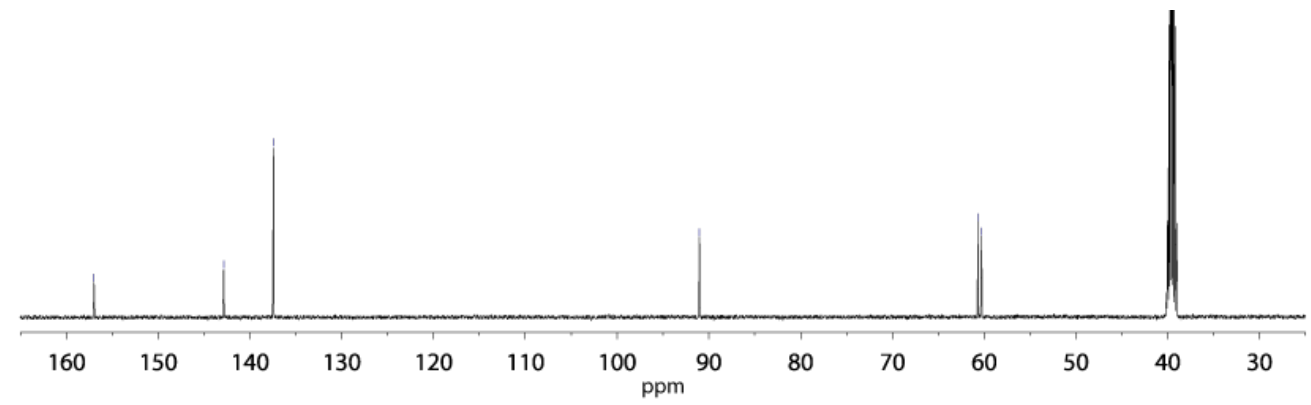

$\begin{array}{lll}\text { ESI-HRMS }\left(\left[\mathrm{C}_{28} \mathrm{H}_{26} \mathrm{~N}_{2} \mathrm{O}_{2}+\mathrm{H}\right]^{+}\right): & \text {measured: } & 390.8690 \\ & \text { calculated: } & 390.8686\end{array}$ 


\section{VI.1.2.4 5-(chloromethyl)-1,3-diiodo-2-methoxybenzene (15)}<smiles>COc1c(C)cc(CO)cc1I</smiles>

14

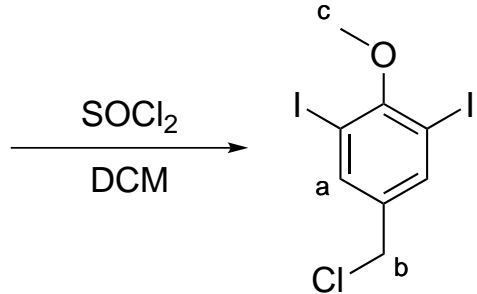

15

Scheme VI.1.2.25: Synthesis of compound 15.

To a solution of $14(3.90 \mathrm{~g}, 10.0 \mathrm{mmol}, 1.00$ equiv. $)$ and triethylamine $(1.39 \mathrm{~mL}, 10.0 \mathrm{mmol}$, 1.00 equiv.) in dichloromethane $(20 \mathrm{~mL})$ was slowly added thionyl chloride $(0.87 \mathrm{~mL}$, $12.0 \mathrm{mmol}, 1.20$ equiv.) at 0 r $\mathrm{C}$. The solution was stirred at $0 \stackrel{\mathrm{r} C}{\mathrm{C}}$ for $15 \mathrm{~min}$ then at $\mathrm{rt}$ for $3 \mathrm{~h}$. Dichloromethane $(80 \mathrm{~mL})$ was added and the solution was washed with water $(50 \mathrm{~mL})$, sat. $\mathrm{NaHCO}_{3}$ solution $(50 \mathrm{~mL})$ then brine $(50 \mathrm{~mL})$. The organic layer was dried $\mathrm{MgSO}_{4}$ and the solvent was removed in vacuo. The product 15 (3.95 g, $9.68 \mathrm{mmol}$, $97 \%$ ) was obtained as a brown oil, which was used in the next step without further purification.

${ }^{1} \mathbf{H}-\mathbf{N M R}\left(300 \mathrm{M} \mathrm{Hz}, 298 \mathrm{~K}, \mathrm{CD}_{3} \mathrm{Cl}\right): \delta(\mathrm{ppm})=7.78(\mathrm{~s}, 2 \mathrm{H}, \mathrm{a}), 4.44(\mathrm{~s}, 2 \mathrm{H}, \mathrm{b}), 3.86(\mathrm{~s}$, $3 \mathrm{H}, \mathrm{c})$.

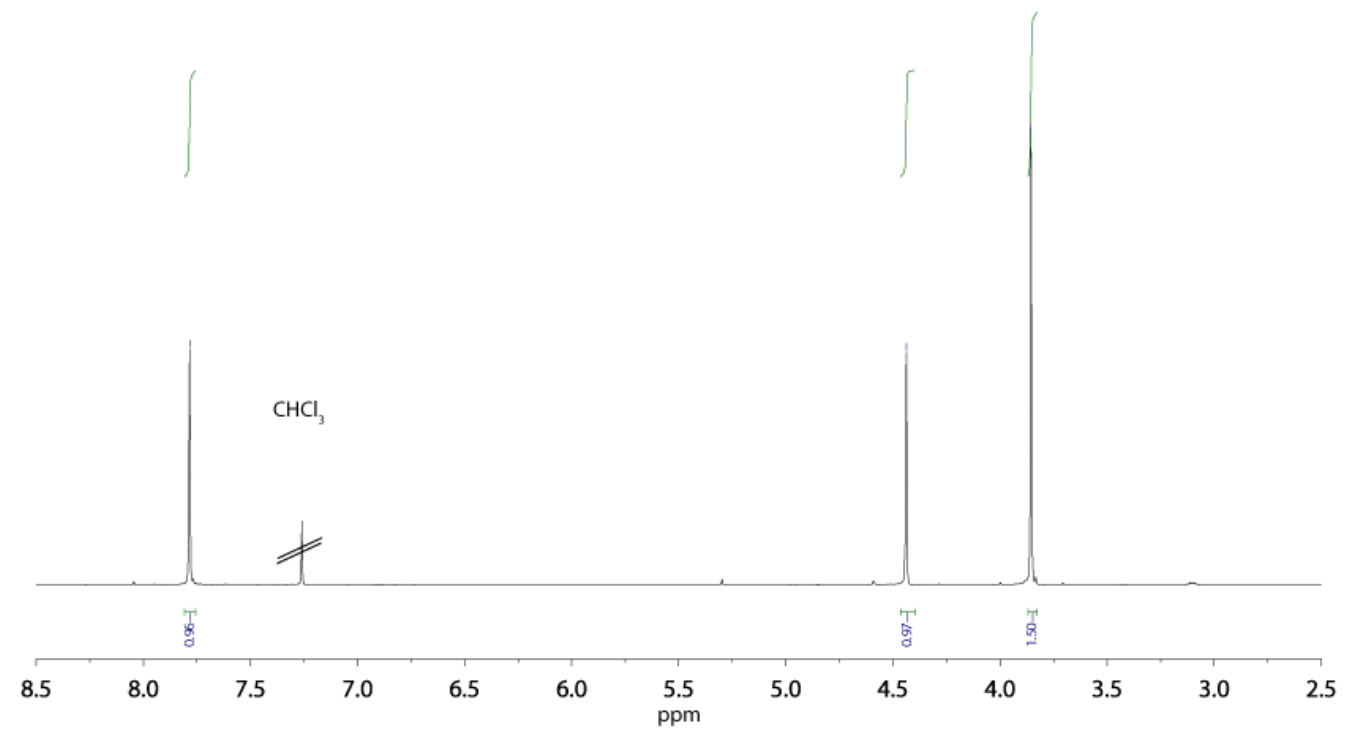

${ }^{13}$ C-NMR $\left(176 \mathrm{MHz}, 298 \mathrm{~K}, \mathrm{CD}_{3} \mathrm{CN}\right): \delta(\mathrm{ppm})=159.1,140.0,137.1,90.5,60.9$, 43.6 . 


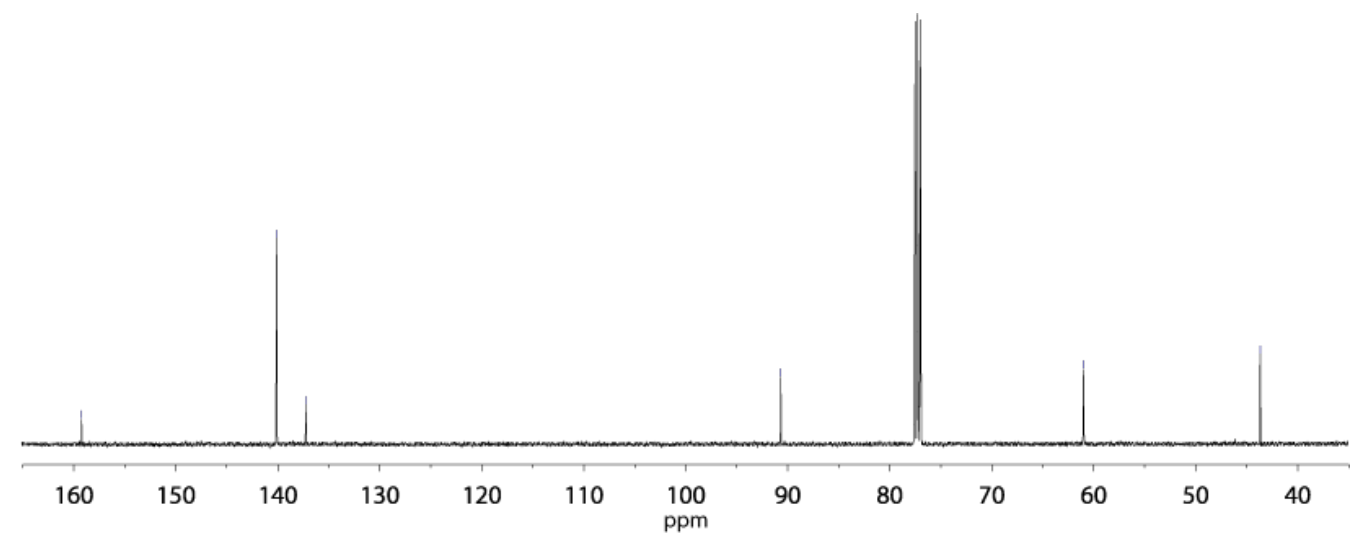

$\begin{array}{lll}\text { ESI-HRMS }\left(\left[\mathrm{C}_{8} \mathrm{H}_{8} \mathrm{ClI}_{2} \mathrm{O}+\mathrm{H}\right]^{+}\right): & \text {measured: } & 408.8345 \\ & \text { calculated: } & 408.8343\end{array}$ 
VI.1.2.5 2,2'-((3,5-diiodo-4-methoxybenzyl)azanediyl)bis(ethan-1-ol) (16)

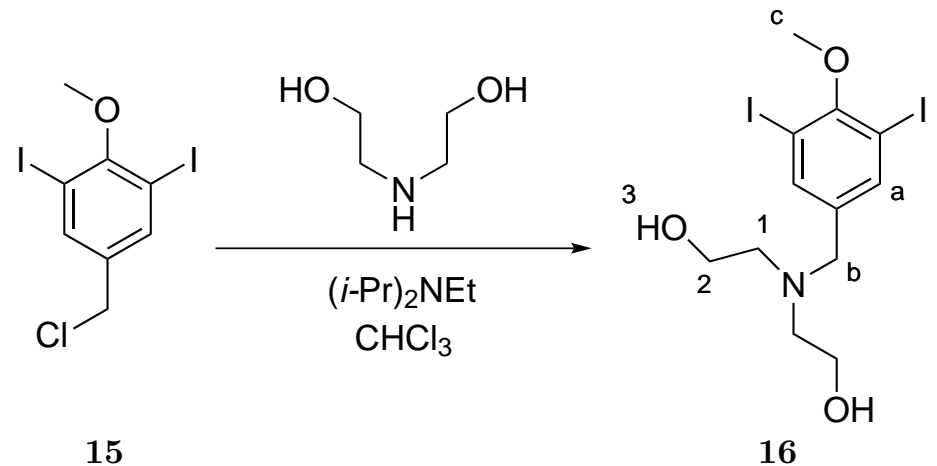

Scheme VI.1.2.26: Synthesis of compound 16.

A mixture of 15 (2.30 g, $5.63 \mathrm{mmol}, 1.00$ equiv.), diethanolamine (0.59 mL, $6.19 \mathrm{mmol}$, 1.10 equiv.) and DIPEA (1.25 mL, $7.05 \mathrm{mmol}, 1.25$ equiv.) in $\mathrm{CHCl}_{3}(50 \mathrm{~mL})$ was heated under reflux over night. After cooling down, $\mathrm{CHCl}_{3}(50 \mathrm{~mL})$ was added and the organic layer was washed with $\mathrm{H}_{2} \mathrm{O}(50 \mathrm{~mL})$ and brine $(50 \mathrm{~mL})$. The organic phase was dried over $\mathrm{MgSO}_{4}$ and the solvent was removed. The product 16 (2.40 g, $\left.5.01 \mathrm{mmol}, 89 \%\right)$ was obtained as a yellowish oil.

${ }^{1}$ H-NMR $\left(300 \mathrm{M} \mathrm{Hz}, 298 \mathrm{~K}, \mathrm{CD}_{3} \mathrm{CN}\right): \delta(\mathrm{ppm})=7.83(\mathrm{~s}, 2 \mathrm{H}, \mathrm{a}), 3.80(\mathrm{~s}, 3 \mathrm{H}, \mathrm{c}), 3.57(\mathrm{~s}$, $2 \mathrm{H}, \mathrm{b}), 3.50\left(\mathrm{t},{ }^{3} \mathrm{~J}=5.6 \mathrm{~Hz}, 3 \mathrm{H}, 2\right), 2.57\left(\mathrm{t},{ }^{3} \mathrm{~J}=5.6 \mathrm{~Hz}, 1\right)$.

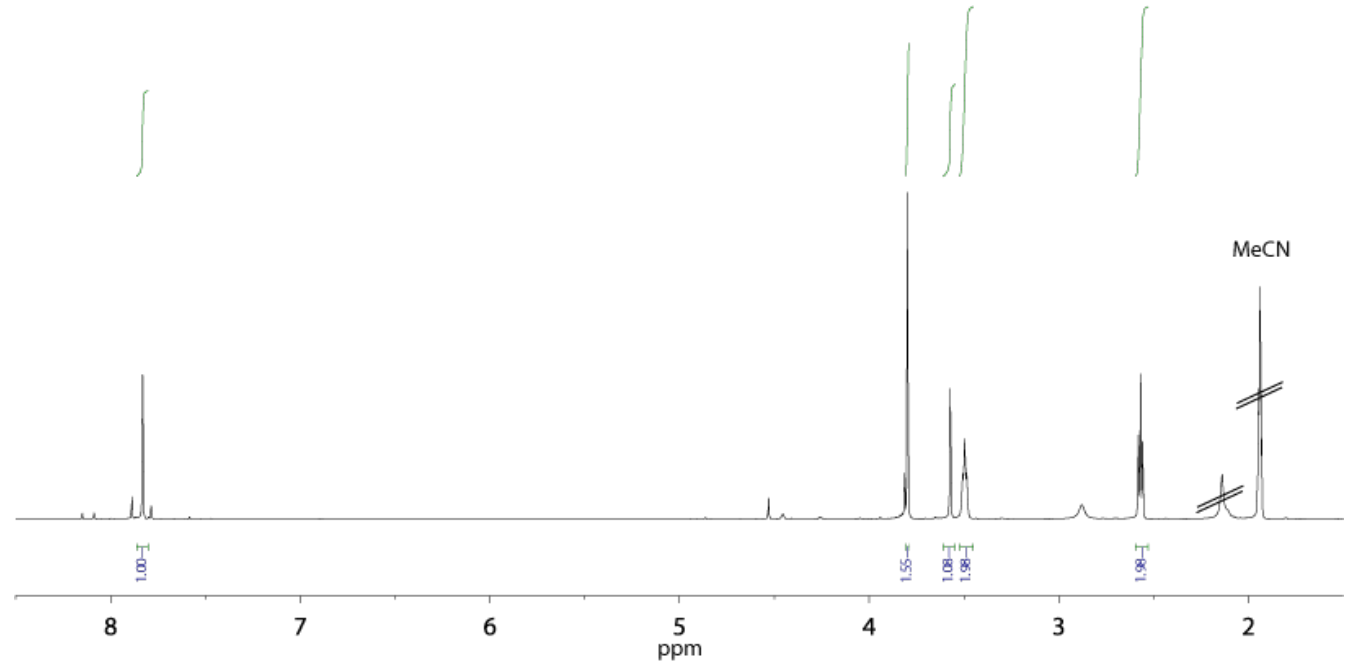

${ }^{13} \mathrm{C}-\mathrm{NMR}\left(176 \mathrm{MHz}, 298 \mathrm{~K}, \mathrm{CD}_{3} \mathrm{CN}\right): \delta(\mathrm{ppm})=158.5,141.3,141.1,90.6,61.3,60.2$, $57.8,56.7$. 


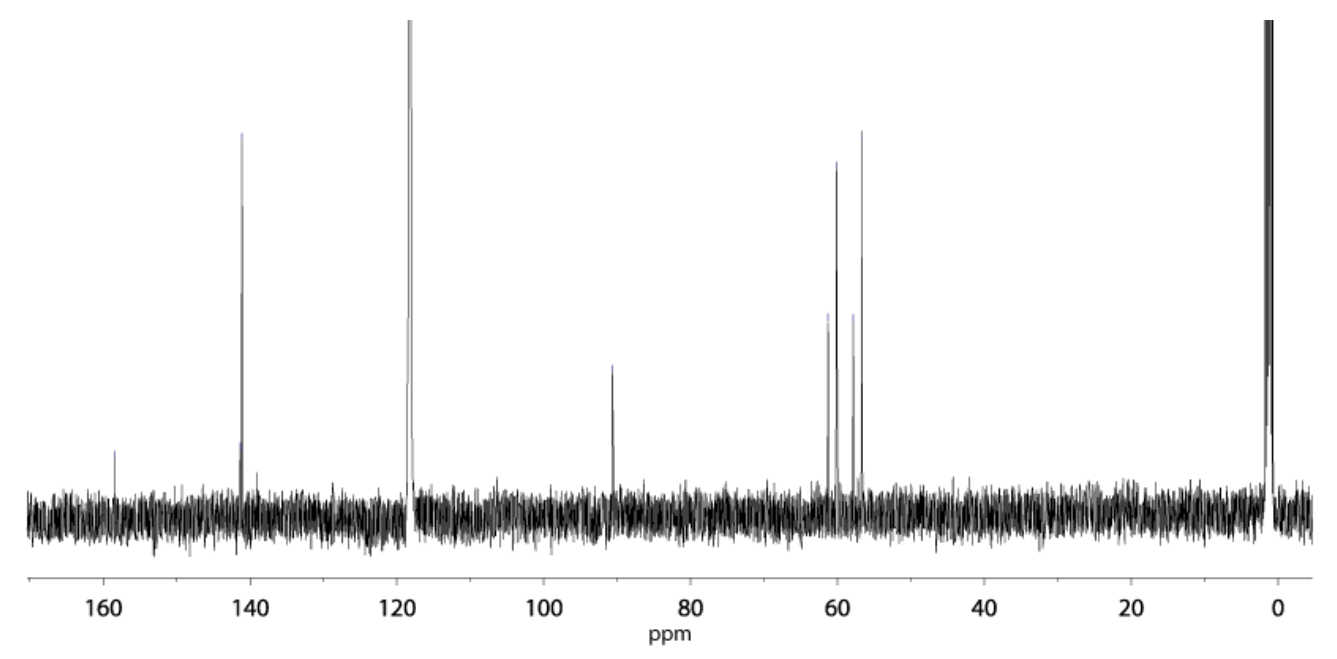

ESI-HRMS $\left(\left[\mathrm{C}_{12} \mathrm{H}_{1} \mathrm{I}_{2} \mathrm{NO}_{3}+\mathrm{H}\right]^{+}\right)$: $\quad$ measured: 477.9368

calculated: $\quad 477.9371$


VI.1.2.6 (2,2'-((4-Methoxy-3,5-bis(pyridin-3ylethynyl)benzyl)azanediyl)bis(ethan-1-ol) (17)<smiles>COc1c(I)cc(CN(CCO)CCO)cc1I</smiles>

16
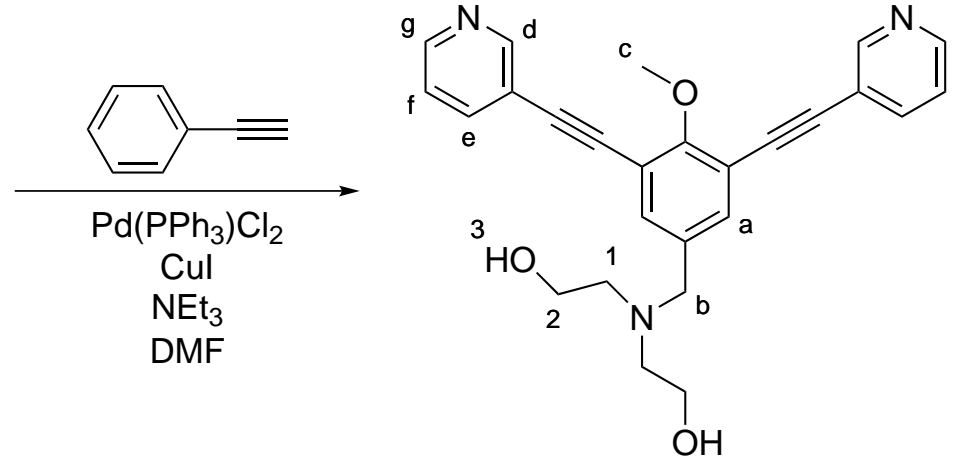

17

Scheme VI.1.2.27: Synthesis of compound 17 .

To a mixture of $\mathbf{1 6}$ (1.00 g, $2.10 \mathrm{mmol}, 1.00$ equiv.), 3-ethynyl pyridine (0.64 g, $6.29 \mathrm{mmol}$, 3.00 equiv.), $\mathrm{Pd}\left(\mathrm{PPh}_{3}\right)_{2} \mathrm{Cl}_{2}$ (0.15 g, $0.21 \mathrm{mmol}, 0.10$ equiv.) and $\mathrm{CuI}(0.40 \mathrm{~g}, 0.21 \mathrm{mmol}$, 0.10 equiv) in dry DMF $(10 \mathrm{~mL})$, triethylamine $(0.65 \mathrm{~mL}, 4.61 \mathrm{mmol}, 2.20$ equiv.) was added. The mixture was stirred at $75{ }^{\circ} \mathrm{C}$ over night. After, the DMF was removed in vacuo and the residue was resolved in $\mathrm{CH}_{2} \mathrm{Cl}_{2}(50 \mathrm{~mL})$ and filtered through celtie. The filtrate was washed with sat. aq. $\mathrm{NaHCO}_{3}(30 \mathrm{~mL})$ and brine $(30 \mathrm{~mL})$. The organic layer was dried over $\mathrm{MgSO}_{4}$ and the solvent was removed in vacuo. The residue was purified by means of column chromatography $\left(\mathrm{SiO}_{2}, \mathrm{CH}_{2} \mathrm{Cl}_{2} /\right.$ acetone $\left.2: 1\right)$. The product $\mathbf{1 7}(2.1 \mathrm{~g}$, $4.91 \mathrm{mmol}, 78 \%$ ) was obtained as yellowish brown resin.

${ }^{1} \mathbf{H}-\mathbf{N M R}\left(300 \mathrm{M} \mathrm{Hz}, 298 \mathrm{~K}, \mathrm{CD}_{3} \mathrm{CN}\right): \delta(\mathrm{ppm})=8.36\left(\mathrm{dd},{ }^{3} J=8.3 \mathrm{~Hz},{ }^{4} J=1.4 \mathrm{~Hz}, 2 \mathrm{H}\right.$, a/a'), $7.72\left(\mathrm{ddd},{ }^{3} J=8.5,6.8 \mathrm{~Hz},{ }^{4} J=1.5 \mathrm{~Hz}, 2 \mathrm{H}, \mathrm{c} / \mathrm{c}^{\prime}\right), 7.67\left(\mathrm{dd},{ }^{3} J=8.9 \mathrm{~Hz},{ }^{4} J=1.2\right.$ $\left.\mathrm{Hz}, 2 \mathrm{H}, \mathrm{d} / \mathrm{d}^{\prime}\right), 7.43-7.38$ (m, 2H, f), 7.31 (ddd, ${ }^{3} J=8.2,6.9 \mathrm{~Hz},{ }^{4} J=1.2 \mathrm{~Hz}, 2 \mathrm{H}, \mathrm{b} / \mathrm{b}$ ), $7.26\left(\mathrm{tt},{ }^{3} \mathrm{~J}=7.5 \mathrm{~Hz},{ }^{4} \mathrm{~J}=1.3 \mathrm{~Hz}, 1 \mathrm{H}, \mathrm{g}\right), 7.16-7.10$ (m, $\left.2 \mathrm{H}, \mathrm{e}\right), 4.43-4.35\left(\mathrm{~m}, 2 \mathrm{H}, \mathrm{NCH}_{2}\right.$ ), $1.95-1.86\left(\mathrm{~m}, 2 \mathrm{H}, \mathrm{NCH}_{2} \mathrm{CH}_{2}\right), 1.54-1.44(\mathrm{~m}, 2 \mathrm{H}, \mathrm{NCH} 2 \mathrm{CH} 2 \mathrm{CH} 2), 1.42-1.28(\mathrm{~m}, 4 \mathrm{H}$, $\left.\mathrm{CH}_{2} \mathrm{CH}_{2} \mathrm{CH}_{3}\right), 0.90\left(\mathrm{t},{ }^{3} \mathrm{~J}=7.1 \mathrm{~Hz}, 3 \mathrm{H}, \mathrm{CH}_{2} \mathrm{CH}_{2} \mathrm{CH}_{3}\right.$ ). 


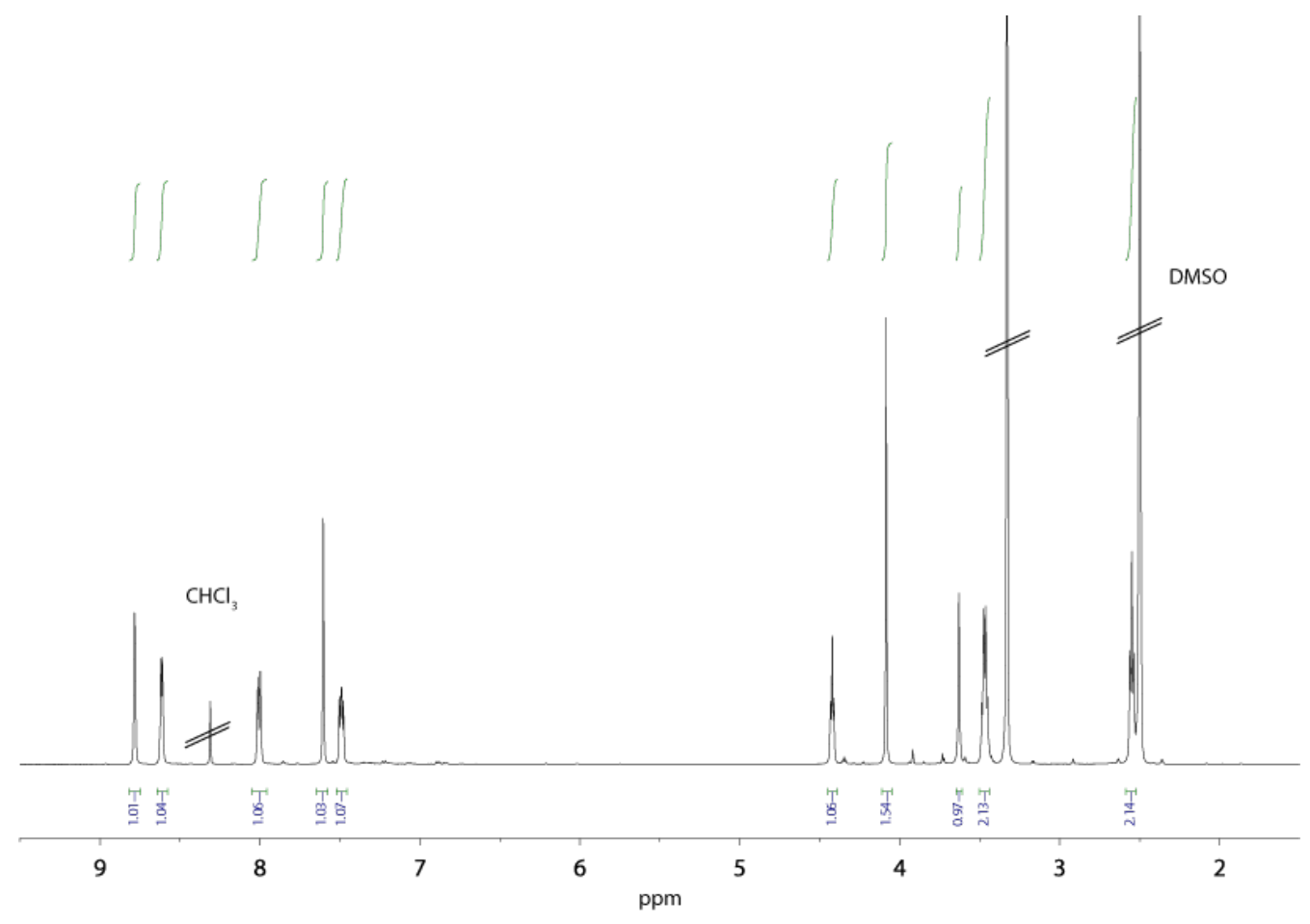

${ }^{13}$ C-NMR $\left(176 \mathrm{MHz}, 298 \mathrm{~K}, \mathrm{CD}_{3} \mathrm{CN}\right): \delta(\mathrm{ppm})=165.5,155.7,151.7,140.0,134.0$, $130.4,129.3,126.9,122.7,122.4,121.1,120.5,116.0,86.6,48.6,32.1,27.5,27.0,23.3$, 14.3 .

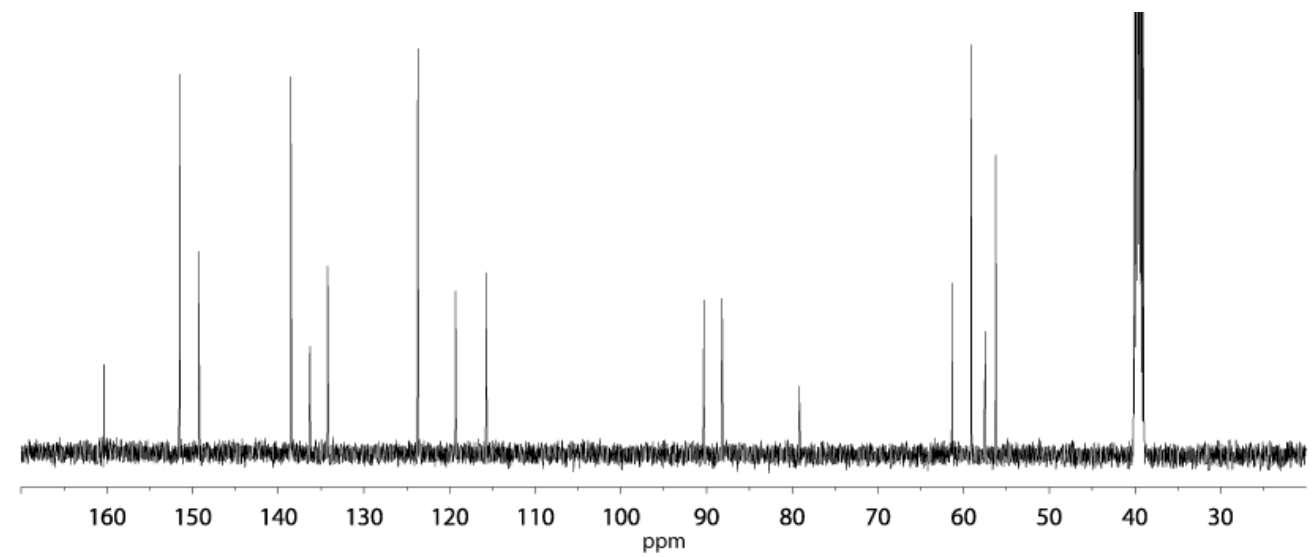

Melting point: $169-170{ }^{\circ} \mathrm{C}$.

ESI-HRMS $\left(\left[\mathrm{C}_{28} \mathrm{H}_{26} \mathrm{~N}_{2} \mathrm{O}_{2}\right]^{+}\right): \quad$ measured: $\quad 423.2051$ 


\section{VI.1.2.7 (2-((2-(bis(4-methoxyphenyl)(phenyl)methoxy)ethyl)(4-methoxy-3,5- bis(pyridin-3-ylethynyl)benzyl)amino)ethan-1-ol (18)}<smiles>COc1c(C#Cc2cccnc2)cc(CN(CCO)CCO)cc1C#Cc1cccnc1</smiles>

17
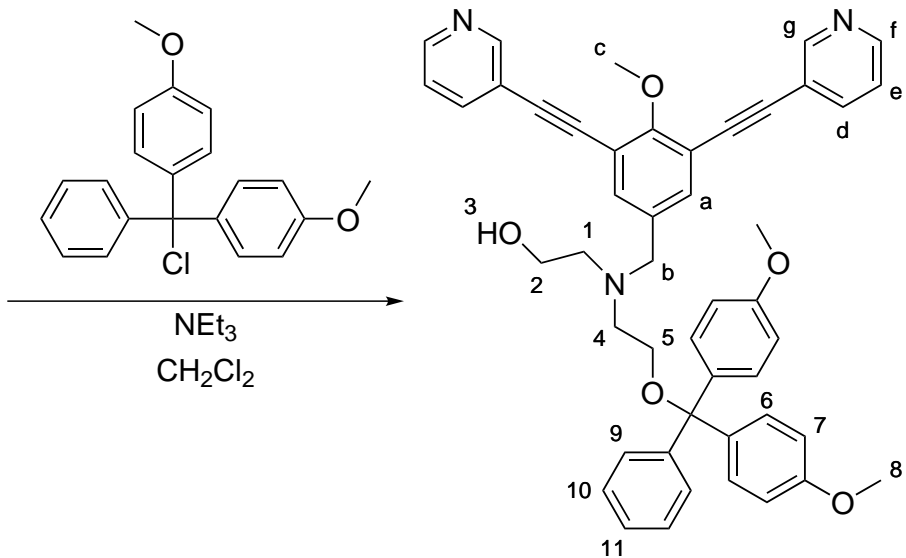

18

Scheme VI.1.2.28: Synthesis of compound 18 .

$\mathrm{TiCl}_{4}$ (255 mg, $1.34 \mathrm{mmol}, 1.25$ equiv.) was slowly added to $N$-hexyl-acridone (300 $\mathrm{mg}$, $1.07 \mathrm{mmol}, 1.00$ equiv.) solved in dry $\mathrm{CH}_{2} \mathrm{Cl}_{2}(15 \mathrm{~mL})$ and stirred for $15 \mathrm{~min}$ at room temperature. 36 (346 mg, $2.15 \mathrm{mmol}, 2.00$ equiv.) and triehtylamine (2 mL) were added consecutively to the reaction mixture and heated under reflux for $24 \mathrm{~h}$. The cooled down reaction mixture was filtered through celite and the residue was washed with $\mathrm{CH}_{2} \mathrm{Cl}_{2}(2 \mathrm{x}$ $5 \mathrm{~mL})$. The solvent was removed in vacuo and the residue was purified by means of column chromatography $\left(\mathrm{SiO}_{2}\right.$, chloroform/methanol 40:1 $\rightarrow$ 20:1). The product 37 (271 mg, 0.64 mmol, $60 \%$ ) was obtained as a dark purple solid.

${ }^{1}$ H-NMR $\left(300 \mathrm{M} \mathrm{Hz}, 298 \mathrm{~K}, \mathrm{CD}_{3} \mathrm{CN}\right): \delta(\mathrm{ppm})=8.74(\mathrm{~d}, 1.2 \mathrm{~Hz}, 2 \mathrm{H}), 8.55(\mathrm{dd}, 4.9$ $\mathrm{Hz}, 1.7 \mathrm{~Hz}, 2 \mathrm{H}), 7.79$ (dt, $7.9 \mathrm{~Hz}, 2.0 \mathrm{~Hz}, 2 \mathrm{H}), 7.48$ (s, 2H), 7.44 (m, 8.5 Hz, $1.4 \mathrm{~Hz}, 2 \mathrm{H})$, 7.36-7.26 (m, 8H), 7.21-7.15 (m, 1H), $6.83(\mathrm{~d}, 8.9 \mathrm{~Hz}, 4 \mathrm{H}), 4.14(\mathrm{~s}, 3 \mathrm{H}), 3.73(\mathrm{~s}, 6 \mathrm{H}), 3.61$ $(\mathrm{s}, 2 \mathrm{H}), 3.56(\mathrm{t}, 5.3 \mathrm{~Hz}, 2 \mathrm{H}), 3.19(\mathrm{t}, 5.5 \mathrm{~Hz}, 2 \mathrm{H}), 2.77$ (t, $5.5 \mathrm{~Hz}, 2 \mathrm{H}), 2.66$ (t, $5.5 \mathrm{~Hz}, 2 \mathrm{H})$. The proton of the hydroxy group was not detectable. 


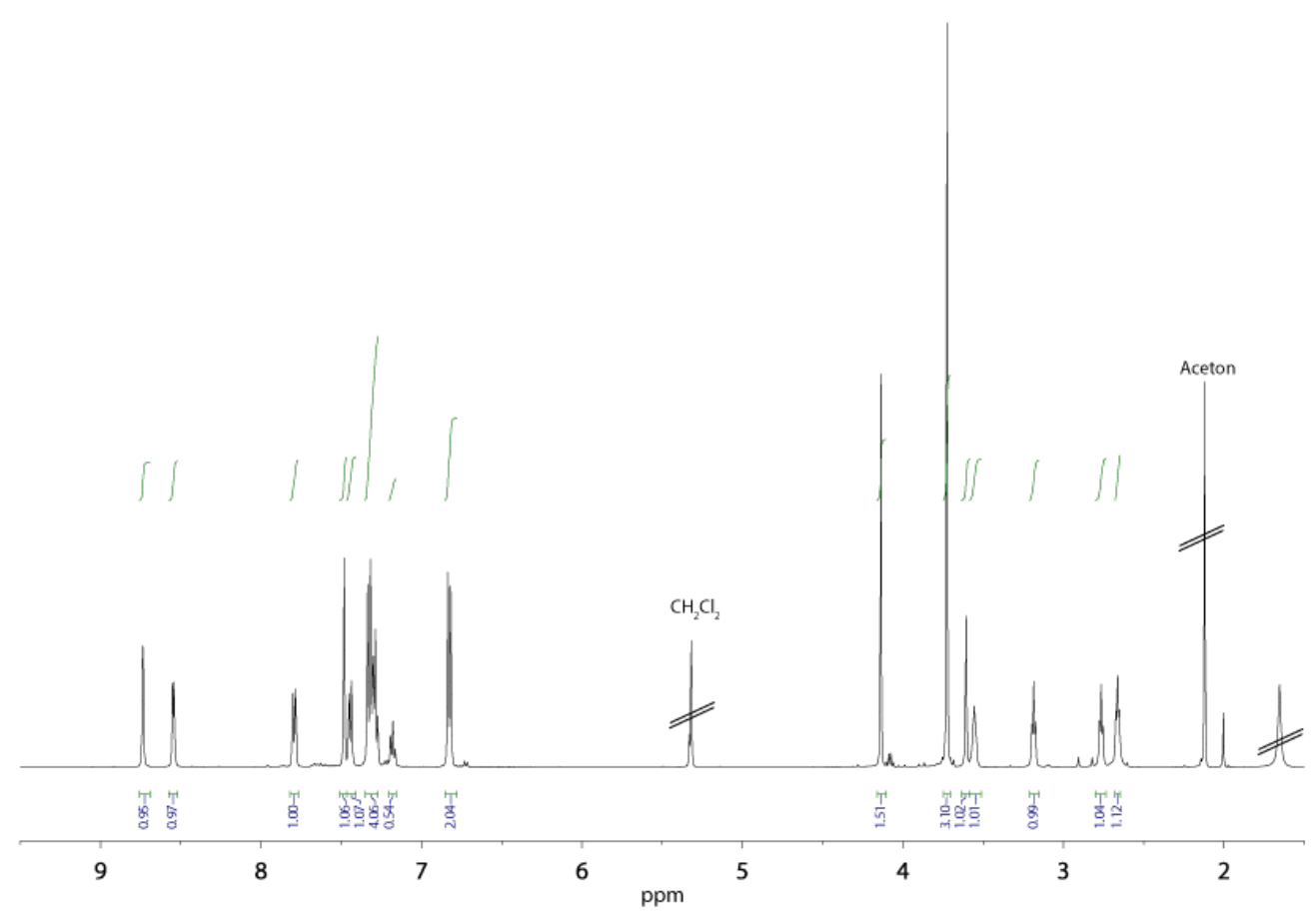

${ }^{13}$ C-NMR $\left(176 \mathrm{MHz}, 298 \mathrm{~K}, \mathrm{CD}_{3} \mathrm{CN}\right): \delta(\mathrm{ppm})=161.6,159.1,152.7,149.41,145.8$, 138.9, 136.8, 135.9, 134.9, 130.5, 128.6, 128.4, 127.2, 123.6, 120.71, 117.3, 113.6, 113.5, 91.0, $88.8,86.9,62.3,62.1,59.4,58.5,56.6,55.7$.

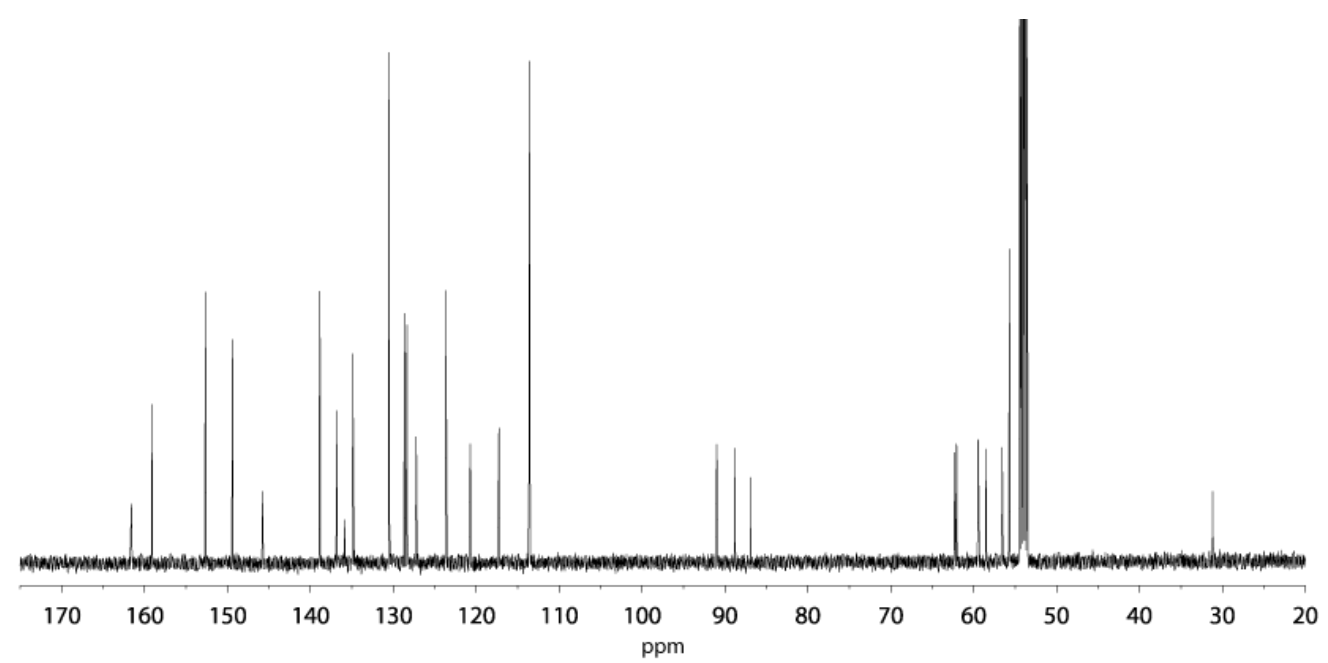

ESI-HRMS $\left(\left[\mathrm{C}_{28} \mathrm{H}_{26} \mathrm{~N}_{2} \mathrm{O}_{2}\right]^{+}\right)$:

measured: $\quad 423.2051$

calculated: $\quad 423.2067$ 
VI.1.2.8 2-((2-(Bis(4-methoxyphenyl)(phenyl)methoxy)ethyl)(4-methoxy-3,5bis(pyridin-3-ylethynyl)benzyl)amino)ethyl (2-cyanoethyl)

diisopropylphosphoramidite (19)

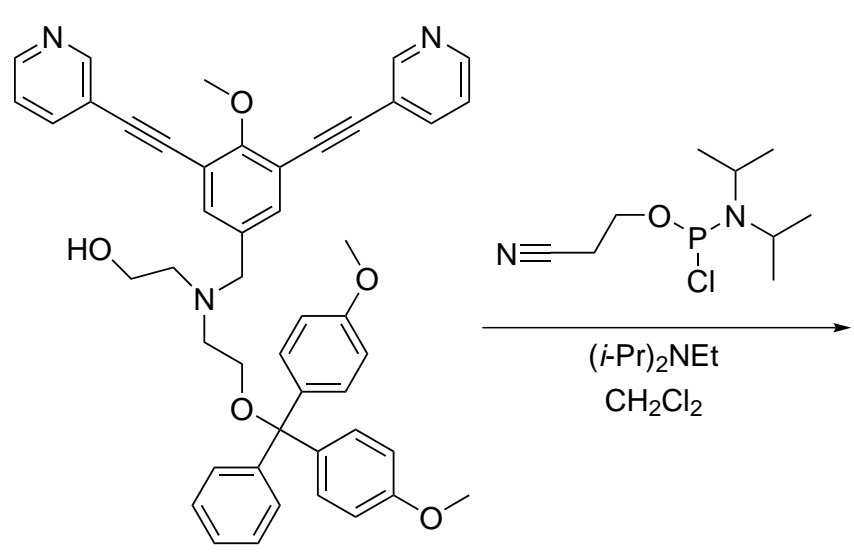

18

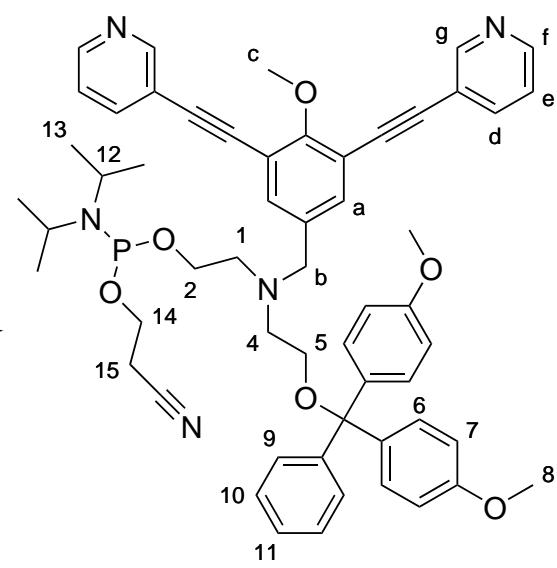

19

Scheme VI.1.2.29: Synthesis of compound 19.

${ }^{1} \mathbf{H}-\mathbf{N M R}\left(300 \mathrm{M} \mathrm{Hz}, 298 \mathrm{~K}, \mathrm{CD}_{3} \mathrm{CN}\right): \delta(\mathrm{ppm})=8.74(\mathrm{~d}, 1.2 \mathrm{~Hz}, 2 \mathrm{H}), 8.55(\mathrm{dd}, 4.9$ $\mathrm{Hz}, 1.7 \mathrm{~Hz}, 2 \mathrm{H}), 7.79$ (dt, $7.9 \mathrm{~Hz}, 2.0 \mathrm{~Hz}, 2 \mathrm{H}), 7.48$ (s, 2H), 7.44 (m, $8.5 \mathrm{~Hz}, 1.4 \mathrm{~Hz}, 2 \mathrm{H})$, 7.36-7.26 (m, 8H), 7.21-7.15 (m, 1H), $6.83(\mathrm{~d}, 8.9 \mathrm{~Hz}, 4 \mathrm{H}), 4.14(\mathrm{~s}, 3 \mathrm{H}), 3.73(\mathrm{~s}, 6 \mathrm{H}), 3.61$ (s, 2H), 3.56 (t, $5.3 \mathrm{~Hz}, 2 \mathrm{H}), 3.19$ (t, $5.5 \mathrm{~Hz}, 2 \mathrm{H}), 2.77$ (t, $5.5 \mathrm{~Hz}, 2 \mathrm{H}), 2.66$ (t, $5.5 \mathrm{~Hz}, 2 \mathrm{H})$. The proton of the hydroxy group was not detectable.

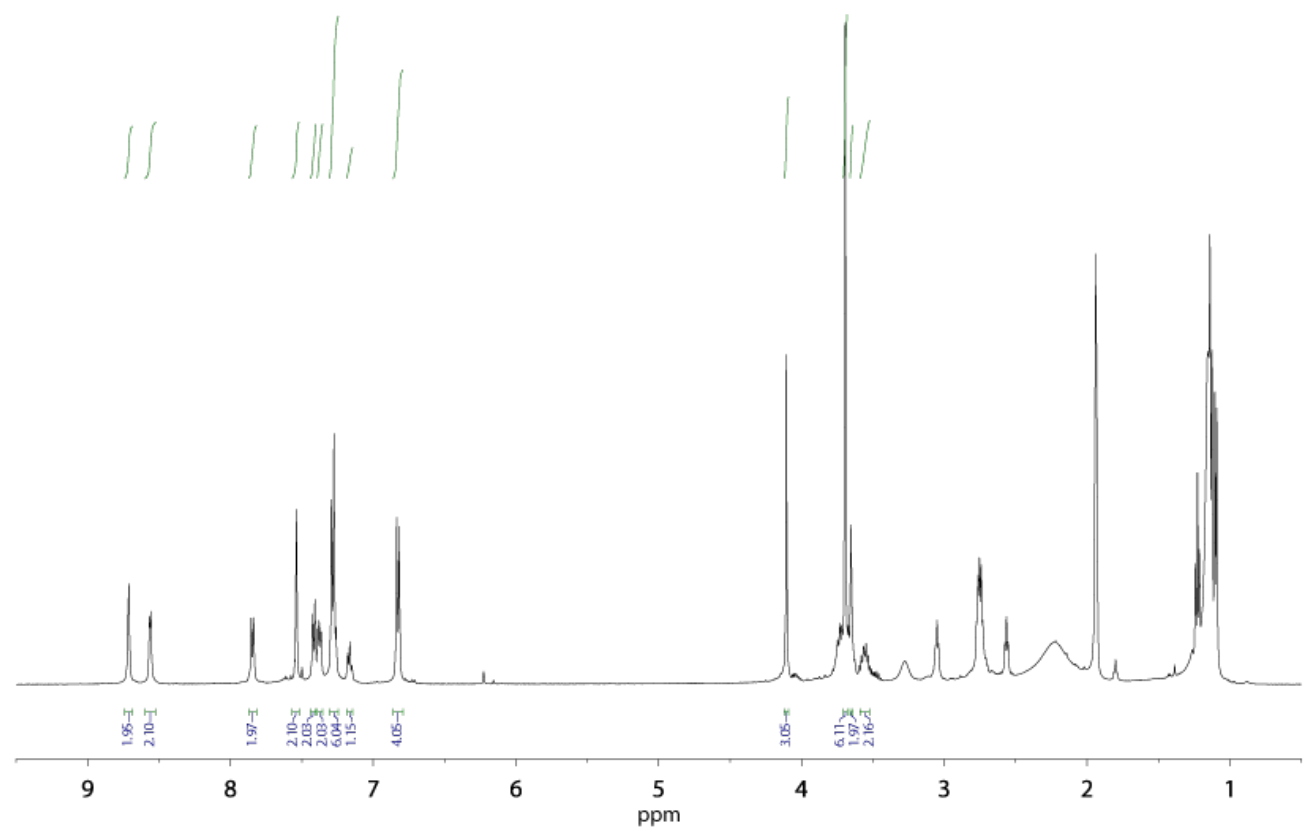




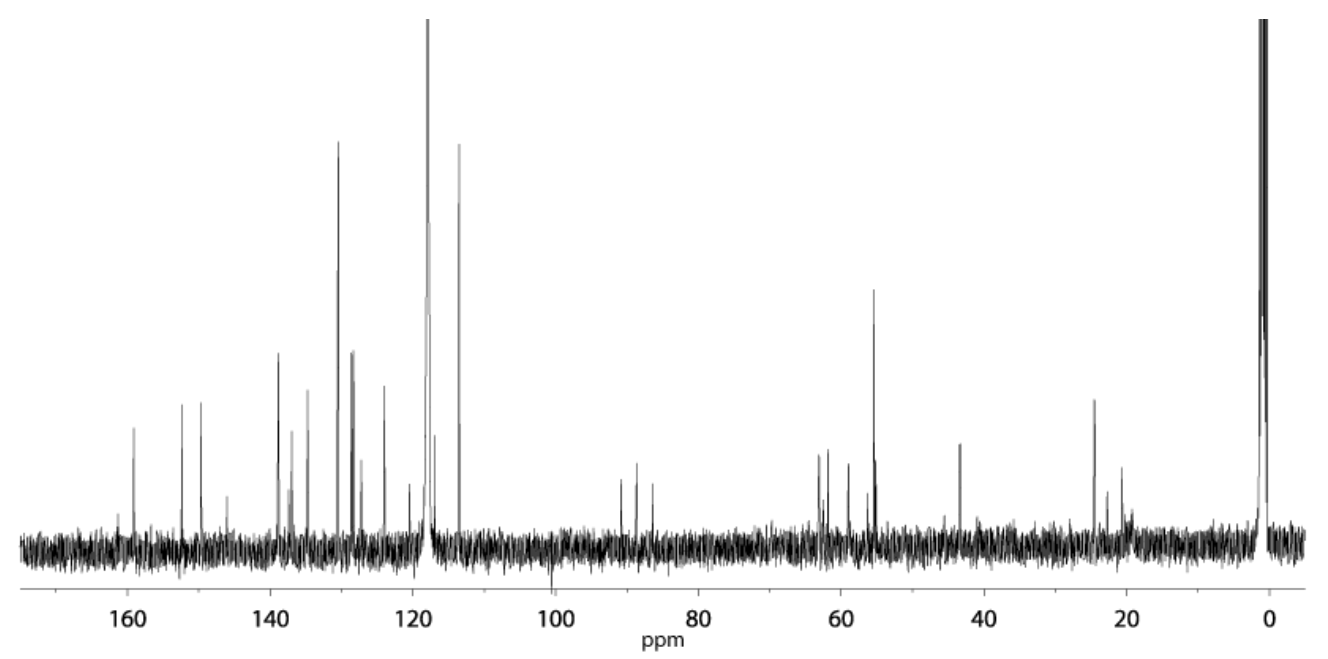

${ }^{31}$ P-NMR $\left(300 \mathrm{MHz}, 298 \mathrm{~K}, \mathrm{CD}_{3} \mathrm{CN}\right): \delta(\mathrm{ppm})=146.4$. (peak of unreacted phosphoamidite at $14 \mathrm{ppm}$. )

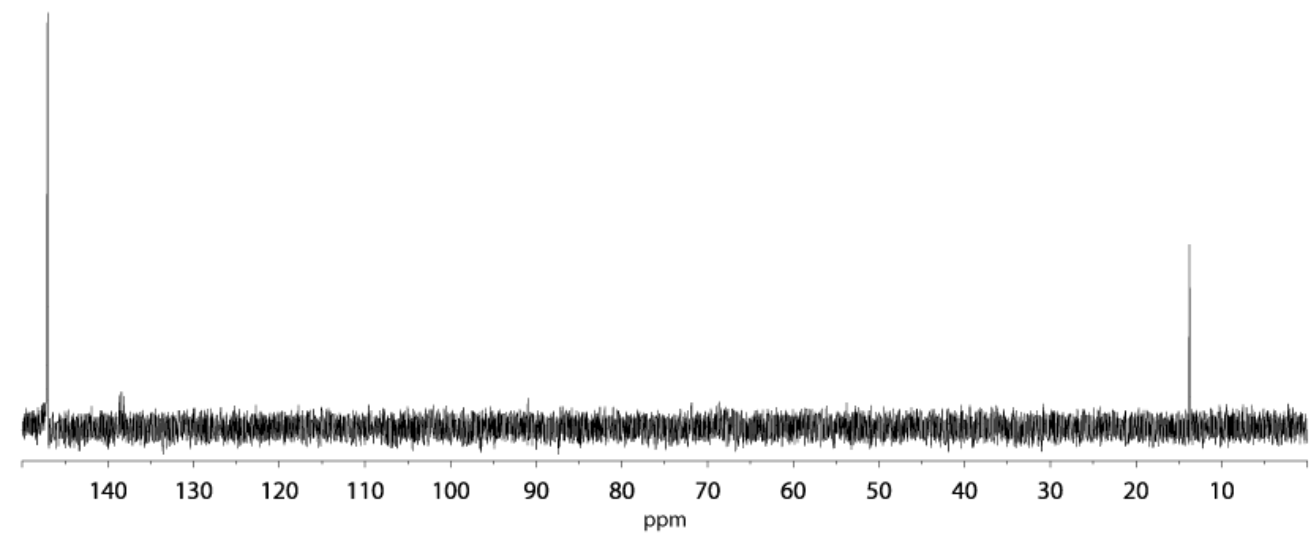




\section{VI.1.2.9 5-(Benzylthio)-1H-tetrazole (20)}

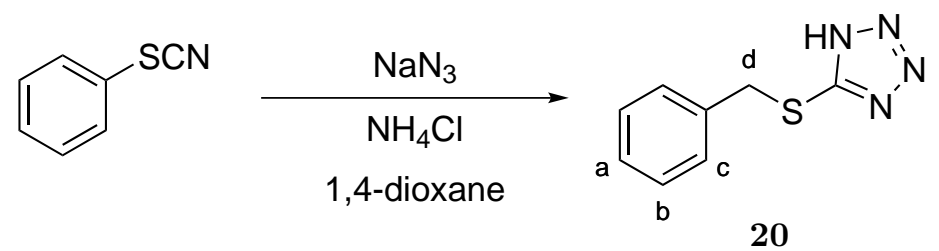

Scheme VI.1.2.30: Synthesis of compound 20 .

To a solution of Benzyl thiocyanate (35.5 g, $0.24 \mathrm{~mol}, 1.00$ equiv.) in 1,4-dioxane (200 $\mathrm{mL}$ ) was added an aq. $\mathrm{NH}_{4} \mathrm{Cl}$ solution $(5 \mathrm{M}, 50.0 \mathrm{~mL}, 0.25 \mathrm{~mol}, 1.05$ equiv.). The mixture was degassed under reduced pressure with help of an ultra-sonic bath. To the resulting reaction mixture sodium azide ( $15.5 \mathrm{~g}, 0.24 \mathrm{~mol}, 1.00$ equiv.) was added portionwise and the suspension was stirred at $90{ }^{\circ} \mathrm{C}$ for $6 \mathrm{~h}$. After the reaction has been cooled down, the solvent volume was reduced in vacuo to approx. $50 \mathrm{~mL}$. Ice was added to the remaining solution was acidified subsequently with hydrochloric acid until $\mathrm{pH} 2$ was reached. The resulting precipitation was filtered off and resuspended in toluene $(150 \mathrm{~mL})$. The heavily stirring suspension was heated to $110^{\circ} \mathrm{C}$ for $1 \mathrm{~h}$. The aqueous layer was removed and aceotonitrile $(10 \mathrm{~mL})$ was added to the remaining organic layer. The solution was kept heating for another $30 \mathrm{~min}$ at $110^{\circ} \mathrm{C}$. After, the solution was filtered off hot and the filtrate was allowed to cool down slowly, whereupon the product starts to crystallize from solution. The crystalline precipitation was collected and dried in vacuo. The product 20 (28.8 g, 0.15 mol, $63 \%$ ) was obtained as a white crystalline solid. 332

${ }^{1}$ H-NMR $(500 \mathrm{MHz}, 298 \mathrm{~K}, \mathrm{DMSO}): \delta(\mathrm{ppm})=7.40\left(\mathrm{~d},{ }^{3} \mathrm{~J}=7.2 \mathrm{~Hz}, 2 \mathrm{H}, \mathrm{c}\right), 7.30$ $\left.\left(\mathrm{t},{ }^{3} J=7.4 \mathrm{~Hz}, 2 \mathrm{H}, \mathrm{b}\right), 7.24{ }^{3} J=7.3 \mathrm{~Hz}, 1 \mathrm{H}, \mathrm{a}\right), 4.51$ (s, 2H, d), (NH not observable).

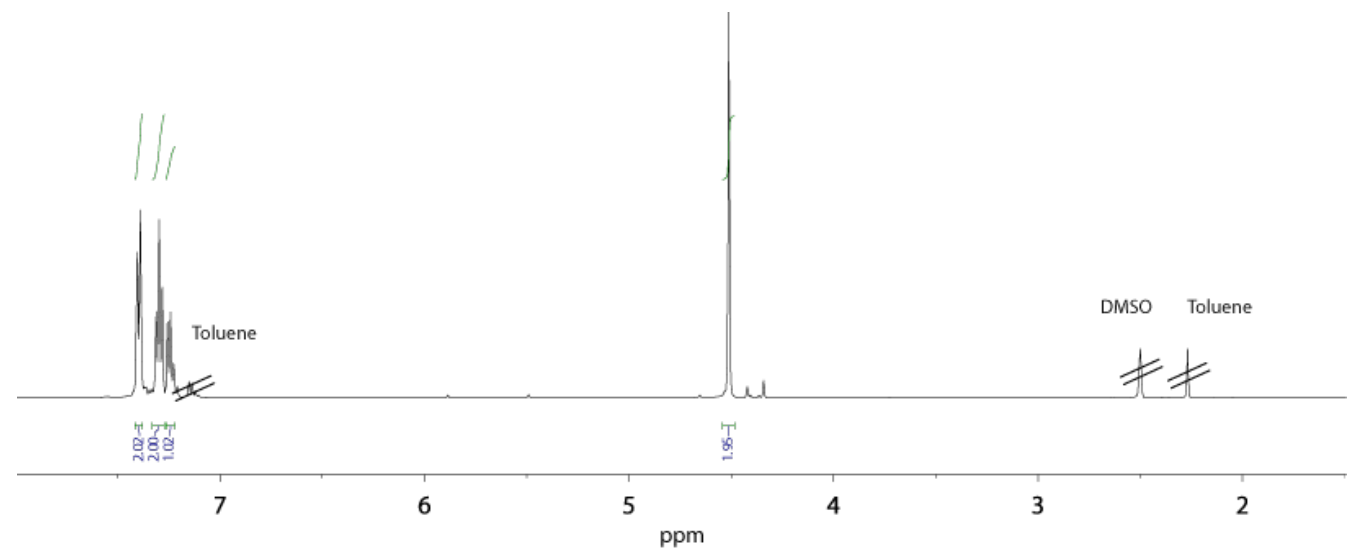

${ }^{13}$ C-NMR $(125 \mathrm{MHz}, 298 \mathrm{~K}, \mathrm{DMSO}): \delta(\mathrm{ppm})=153.8,136.7,129.0,128.6,127.7$, 36.1 . 


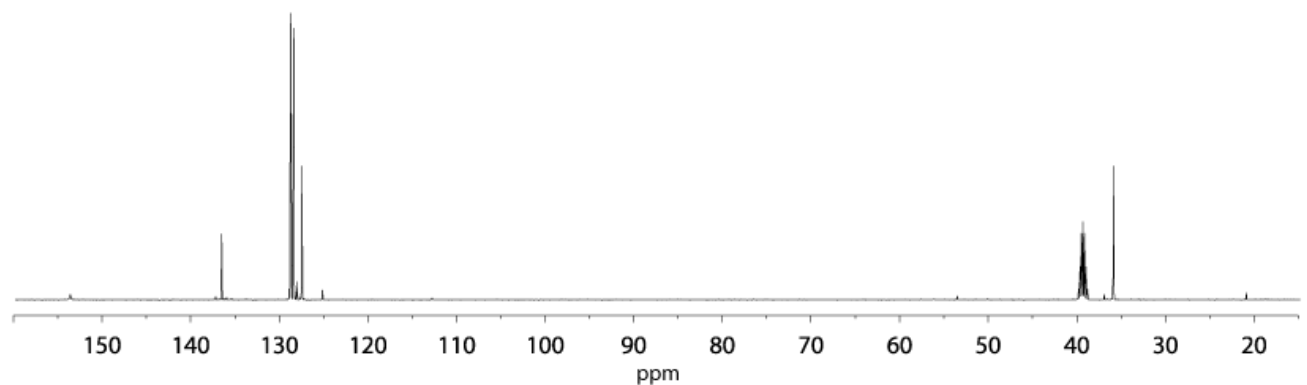

$\begin{array}{lll}\text { ESI-HRMS }\left(\left[\mathrm{C}_{8} \mathrm{H}_{9} \mathrm{~N}_{4} \mathrm{~S}+\mathrm{H}\right]^{+}\right): & \text {measured: } & 193.0533 \\ & \text { calculated: } & 193.0542\end{array}$




\section{Chapter}

\section{Syntheses of Water-Soluble Ligands}

\section{Contents}

VI.2.1 Syntheses of Sulfobetaine Ligand . . . . . . . . . . . . 232

VI.2.1.1 Compound 22 . . . . . . . . . . . . . . . . . . . . . . . 232

VI.2.1.2 Compound 23 . . . . . . . . . . . . . . . . . . . . . . . . . 235

VI.2.1.3 Compound 24 . . . . . . . . . . . . . . . . . . . . . . . . . 237

VI.2.2 Syntheses of Hydroxybenzylamine-based Ligand . . . . 239

VI.2.2.1 Compound 26 . . . . . . . . . . . . . . . . . . . . . . . 239

VI.2.2.2 Compound 27 $\ldots \ldots \ldots$

VI.2.2.3 Compound 28 . . . . . . . . . . . . . . . . . . . . . . . . 243

VI.2.2.4 Compound 29 . . . . . . . . . . . . . . . . . . . . . . . . . . . . 245

VI.2.2.5 Compound $\mathbf{3 0}$. . . . . . . . . . . . . . . . . . . . . . 247

VI.2.2.6 Compound 31 . . . . . . . . . . . . . . . . . . . . . . . . 249

VI.2.2.7 Compound 32 . . . . . . . . . . . . . . . . . . . . . . 251

\section{VI.2.1 Syntheses of Sulfobetaine Ligand}

\section{VI.2.1.1 1-(3-iodo-4-methoxy-5-methylphenyl)-N,N- dimethylmethanamine (22)}

To a solution of $\mathbf{1 5}$ (1.00 g, $2.45 \mathrm{mmol}, 1.00$ equiv.) in THF ( $5 \mathrm{~mL})$, an aq. solution of $\mathrm{Me}_{2} \mathrm{NH}(40 \%$ (w/v), $1.40 \mathrm{~mL}, 12.2 \mathrm{mmol}, 5.00$ equiv) was added. The mixture was stirred at room temperature until the completion of the reaction was observed by TLC. The solvent was removed and the residue was redissolved in EtOAc $(50 \mathrm{~mL})$. The organic layer was washed with sat. aq. $\mathrm{NaHCO}_{3}$ solution $(25 \mathrm{~mL})$ and brine $(25 \mathrm{~mL})$. The organic phase was 


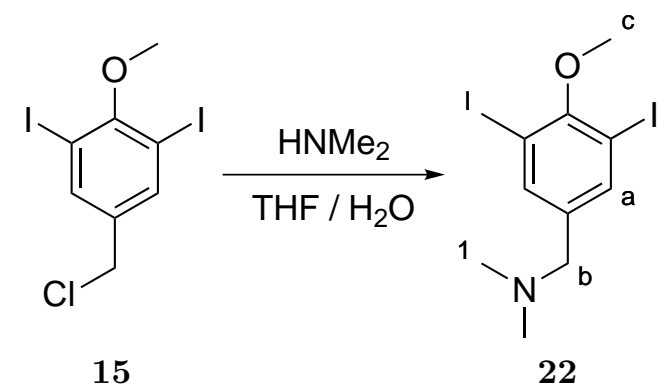

Scheme VI.2.1.31: Synthesis of compound 22 .

dried over $\mathrm{MgSO}_{4}$ and the solvent was removed in vacuo. The residue was purified by means of column chromatography $\left(\mathrm{SiO}_{2}\right.$, pentane / ethyl acetate 10:1 $\left.\rightarrow 2: 1\right)$. The product 22 (0.95 g, $2.28 \mathrm{mmol}, 93 \%$ ) was obtained as a brownish solid.

${ }^{1}$ H-NMR $(300 \mathrm{M} \mathrm{Hz}, 298 \mathrm{~K}, \mathrm{DMSO}): \delta(\mathrm{ppm})=7.74(\mathrm{~s}, 2 \mathrm{H}, \mathrm{a}), 3.74(\mathrm{~s}, 3 \mathrm{H}, \mathrm{c}), 3.29$ (s, $2 \mathrm{H}, \mathrm{b}), 2.13(\mathrm{~s}, 6 \mathrm{H}, 1)$.

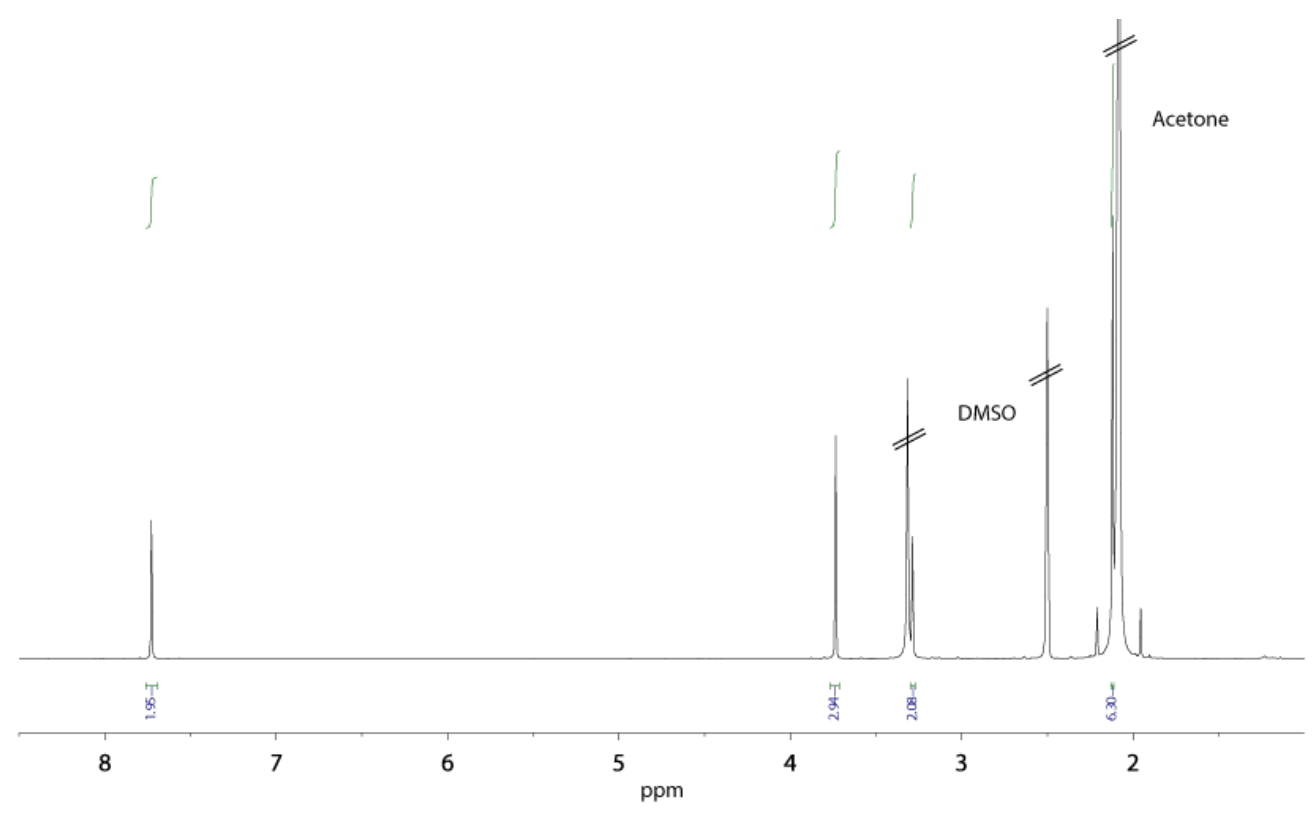

${ }^{13}$ C-NMR $(176 \mathrm{MHz}, 298 \mathrm{~K}, \mathrm{DMSO}): \delta(\mathrm{ppm})=157.2,139.5,139.4,91.0,60.9,60.2$, 44.8 . 


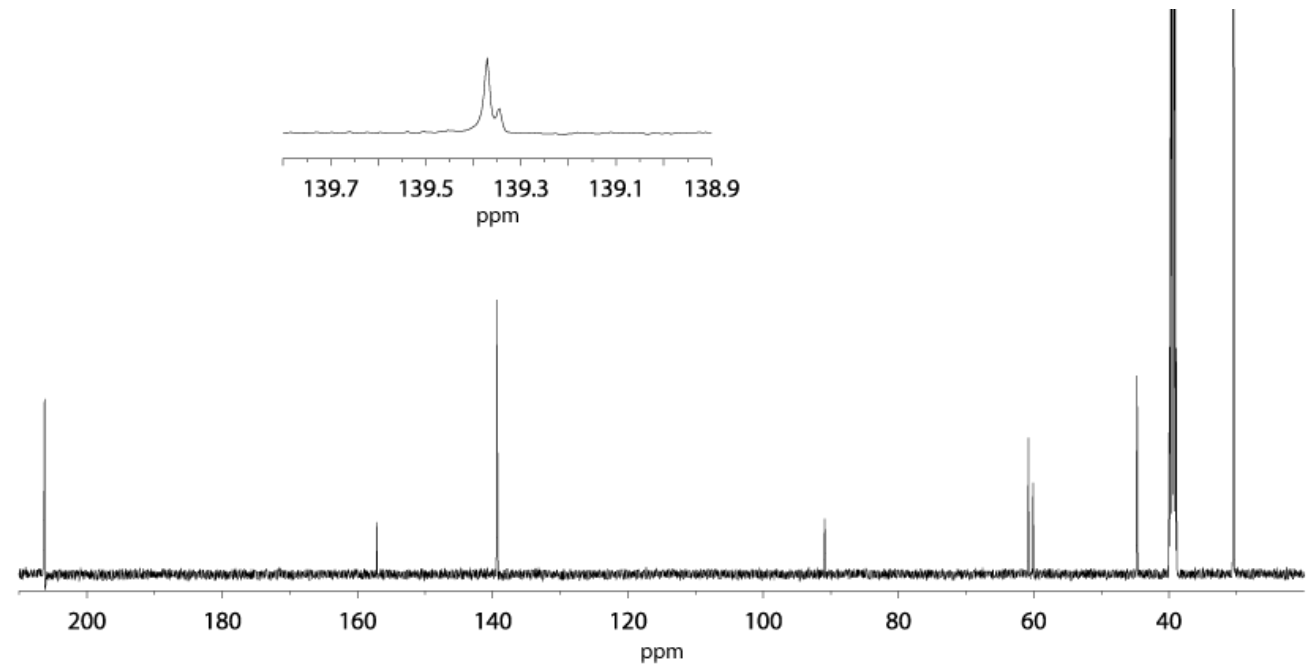

ESI-HRMS $\left(\left[\mathrm{CH}_{26} \mathrm{~N}_{2} \mathrm{O}_{2}\right]^{+}\right)$:

measured: $\quad 416.9079$

calculated: $\quad 416.9087$ 


\section{VI.2.1.2 3-((3,5-Diiodo-4-methoxybenzyl)dimethylammonio)propane-1- sulfonate (23)}<smiles>COc1c(I)cc(CN(C)C)cc1I</smiles>

22

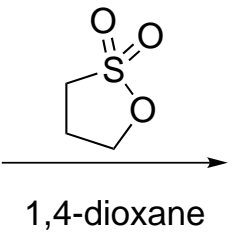

Scheme VI.2.1.32: Synthesis of compound 23 .

To a solution of 1,3-propane sultone $(0.15 \mathrm{~mL}, 1.71 \mathrm{mmol}, 1.00$ equiv. $)$ in 1,4-dioxane (3 mL), a solutiion of 22 (750 mg, $1.80 \mathrm{mmol}, 1.05$ equiv.) in 1,4-dioxan (3 mL) was added at room temperature, over a period of $15 \mathrm{~min}$. The reaction mixture was heated for $4 \mathrm{~h}$ under reflux. The milky suspension was cooled down with an ice-bath, subsequently the suspension was filtered and the collected solid was washed with acetone $(2 \times 10 \mathrm{~mL})$, after, the solid was dried in a vacuum. The prodcut 23 (700 mg, $1.30 \mathrm{mmol}, 76 \%)$ was obtained as a white solid.

${ }^{1} \mathbf{H}-\mathbf{N M R}(300 \mathrm{M} \mathrm{Hz}, 298 \mathrm{~K}, \mathrm{MeOH}): \delta(\mathrm{ppm})=8.08(\mathrm{~s}, 2 \mathrm{H}, \mathrm{a}), 4.45(\mathrm{~s}, 2 \mathrm{H}, \mathrm{b}), 3.90(\mathrm{~s}$, $3 \mathrm{H}, \mathrm{c}), 3.60-3.49(\mathrm{~s}, 2 \mathrm{H}, 2), 3.05(\mathrm{~s}, 6 \mathrm{H}, 1), 2.90\left(\mathrm{t},{ }^{3} \mathrm{~J}=6.8 \mathrm{~Hz}, 2 \mathrm{H}, 4\right), 2.36-2.31(\mathrm{~m}, 2 \mathrm{H}$, $3)$.

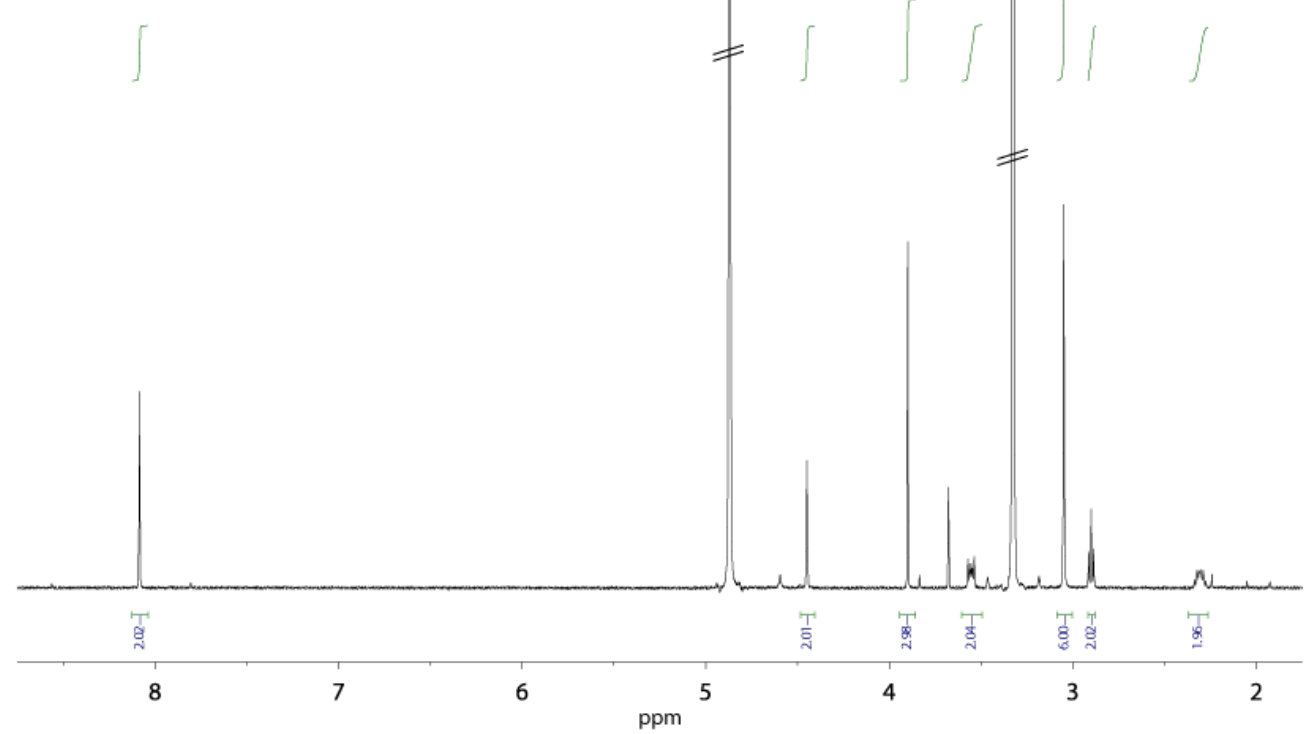


ESI-HRMS $\left(\left[\mathrm{C}_{13} \mathrm{H}_{19} \mathrm{I}_{2} \mathrm{NO}_{4} \mathrm{~S}+\mathrm{H}\right]^{+}\right)$: $\quad$ measured: $\quad 539.9215$

calculated: $\quad 539.9197$ 


\section{VI.2.1.3 3-((4-Methoxy-3,5-bis(pyridin-3-}

\section{ylethynyl)benzyl)dimethylammonio)propane-1-sulfonate (24)}<smiles>COc1c(I)cc(C[N+](C)(C)CCCS(=O)(=O)O)cc1I</smiles>

23

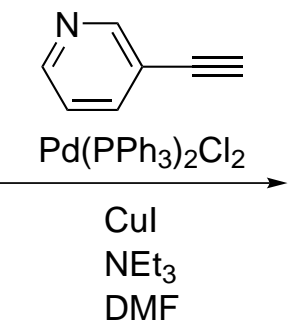

DMF

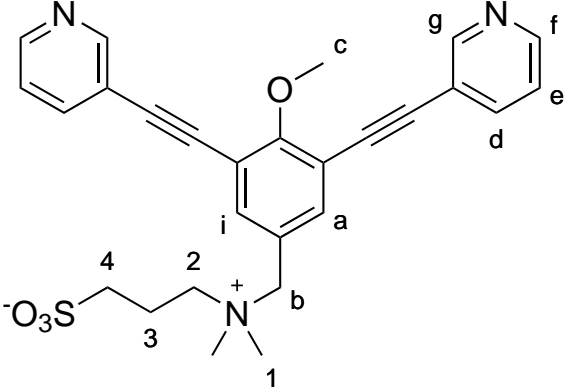

24

Scheme VI.2.1.33: Synthesis of compound 24 .

23 (250 mg, $0.46 \mathrm{mmol}, 1.00$ equiv), 3-ethynyl pyridine (120 mg, $1.16 \mathrm{mmol}, 2.50$ equiv.), $\mathrm{Pd}\left(\mathrm{PPh}_{3}\right)_{2} \mathrm{Cl}_{2}$ (20.4 mg, $0.05 \mathrm{mmol}, 0.10$ equiv) and $\mathrm{CuI}$ (13.3 $\mathrm{mg}, 0.07 \mathrm{mmol}, 0.15$ equiv.) were solved in dry DMF $(5 \mathrm{~mL})$. To the stirred reaction triethylamine $(0.38 \mathrm{~mL}, 2.32 \mathrm{mmol}$, 5.00 equiv.) was added and the reaction mixture was stirred at $80{ }^{\circ} \mathrm{C}$ over night. The solvent was removed in vacuo and the residue was purified by means of column chromatography (reversed phase RP-C18 with Isolera, $\mathrm{H}_{2} \mathrm{O} / \mathrm{MeCN}$ 1:1). The product 24 (52 mg, $0.11 \mathrm{mmol}$, $23 \%$ ) was obtained as a greyish white solid.

${ }^{1} \mathbf{H}-\mathbf{N M R}(300 \mathrm{M} \mathrm{Hz}, 298 \mathrm{~K}, \mathrm{MeOH}): \delta(\mathrm{ppm})=8.74\left(\mathrm{~d},{ }^{4} J=1.9 \mathrm{~Hz}, 2 \mathrm{H}, \mathrm{g}\right), 8.57(\mathrm{dd}$, $\left.{ }^{3} J=5.1 \mathrm{~Hz},{ }^{4} J=1.7 \mathrm{~Hz}, 2 \mathrm{H}, \mathrm{f}\right), 8.04\left(\mathrm{dt},{ }^{3} J=7.9 \mathrm{~Hz},{ }^{4} J=1.9 \mathrm{~Hz}, 2 \mathrm{H}, \mathrm{d}\right), 7.84(\mathrm{~s}, 2 \mathrm{H}, \mathrm{a})$, 7.51 (dd, $\left.{ }_{3} J=8.0,5.0 \mathrm{~Hz}, 2 \mathrm{H}, \mathrm{e}\right), 4.54$ (s, 2H, b), 4.26 (s, 3H, c), 3.64-3.55 (m, 2H, 2), 3.10 $(\mathrm{s}, 6 \mathrm{H}, 1), 2.92\left(\mathrm{t},{ }^{3} \mathrm{~J}=6.8 \mathrm{~Hz}, 2 \mathrm{H}, 4\right), 2.40-2.27(\mathrm{~m}, 2 \mathrm{H}, 3)$

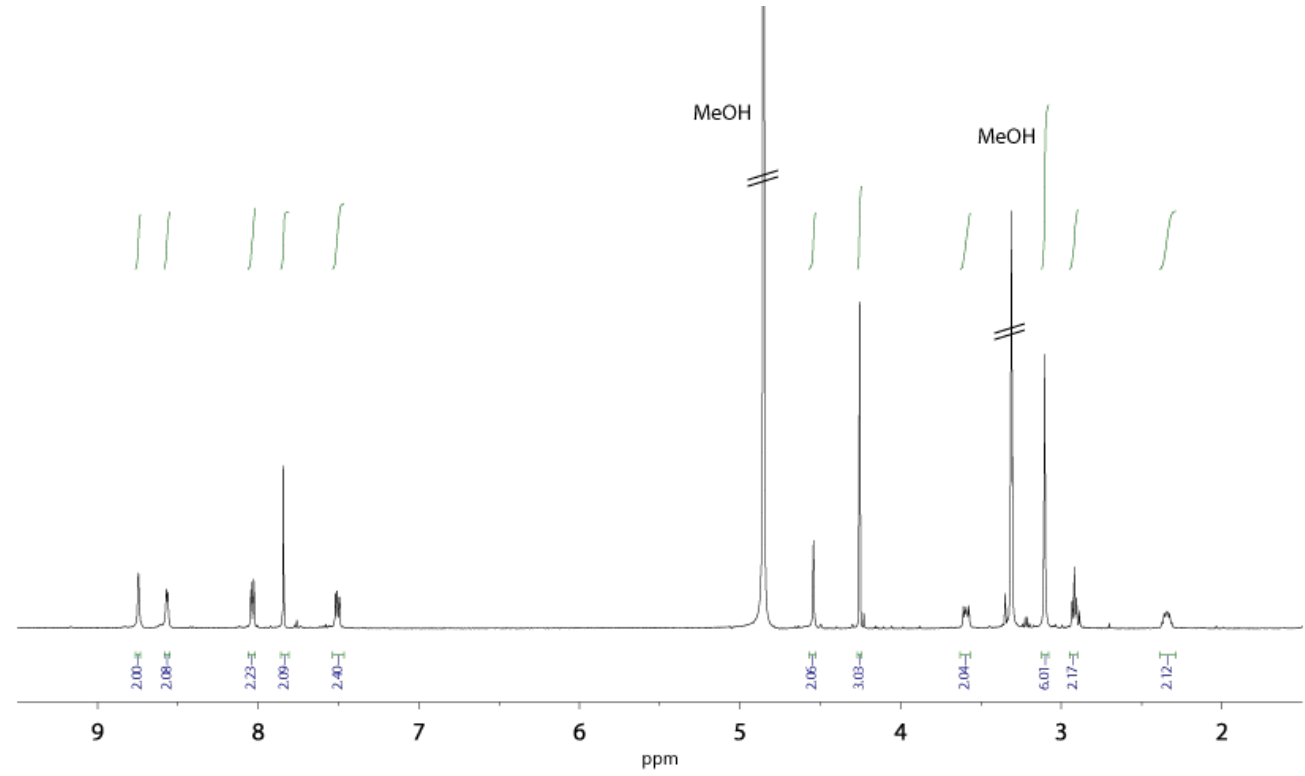


VI.2. SYNTH. WAT.-SOL. LIG.

ESI-HRMS $\left(\left[\mathrm{C}_{27} \mathrm{H}_{27} \mathrm{~N}_{3} \mathrm{O}_{4} \mathrm{~S}+\mathrm{H}\right]^{+}\right)$: $\quad$ measured: 490.1803

calculated: $\quad 490.1795$ 


\section{VI.2.2 Syntheses of Hydroxybenzylamine-based Ligand}

\section{VI.2.2.1 (3,5-Diiodo-4-((trimethylsilyl)oxy)benzaldehyde) (26)}

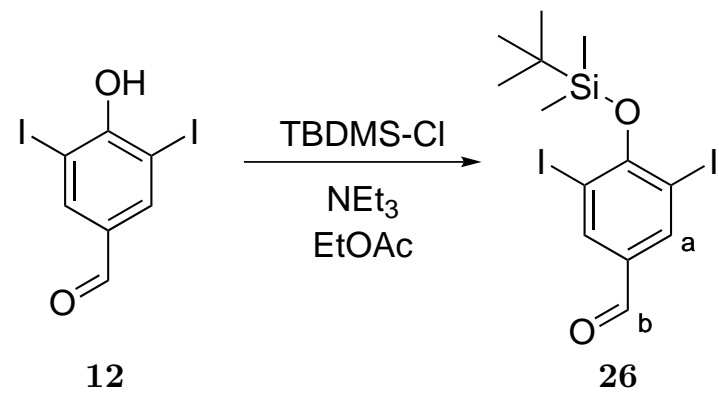

Scheme VI.2.2.34: Synthesis of compound 26.

To a solution of 12 (9.90 g, $26.5 \mathrm{mmol}, 1.00$ equiv.) and tert-butyldimethylsilyl chloride (4.79 g, $31.8 \mathrm{mmol}, 1.20$ equiv.) in ethyl acetate $(50 \mathrm{~mL})$, triethylamine $(7.34 \mathrm{~mL}, 53.0 \mathrm{mmol}$, 2.00 equiv.) was added. The reaction mixture was stirred over night at room temperature. The solids were filtered off and the solvent volume was reduced to the half. The solution was allowed to stand for $2 \mathrm{~h}$. After, the solids were removed again by filtration and the solvent of the filtrate was removed in vacuo. The product 26 (11,9 g, $24.4 \mathrm{mmol}, 92 \%)$ was obtained as a white solid.

${ }^{1} \mathbf{H}-\mathbf{N M R}(300 \mathrm{M} \mathrm{Hz}, 298 \mathrm{~K}, \mathrm{DMSO}): \delta(\mathrm{ppm})=9.72(\mathrm{~s}, 1 \mathrm{H}, \mathrm{b}), 8.24$ (s, 2H, a), 0.83 (s, $\left.9 \mathrm{H}, \mathrm{Si}\left(\mathrm{CH}_{2}\right)_{2} \mathrm{C}\left(\underline{\mathrm{CH}_{3}}\right)_{3}\right),-0.05\left(\mathrm{~s}, 6 \mathrm{H}, \mathrm{Si}\left(\underline{\mathrm{CH}_{2}}\right)_{2} \mathrm{C}\left(\mathrm{CH}_{3}\right)_{3}\right)$.

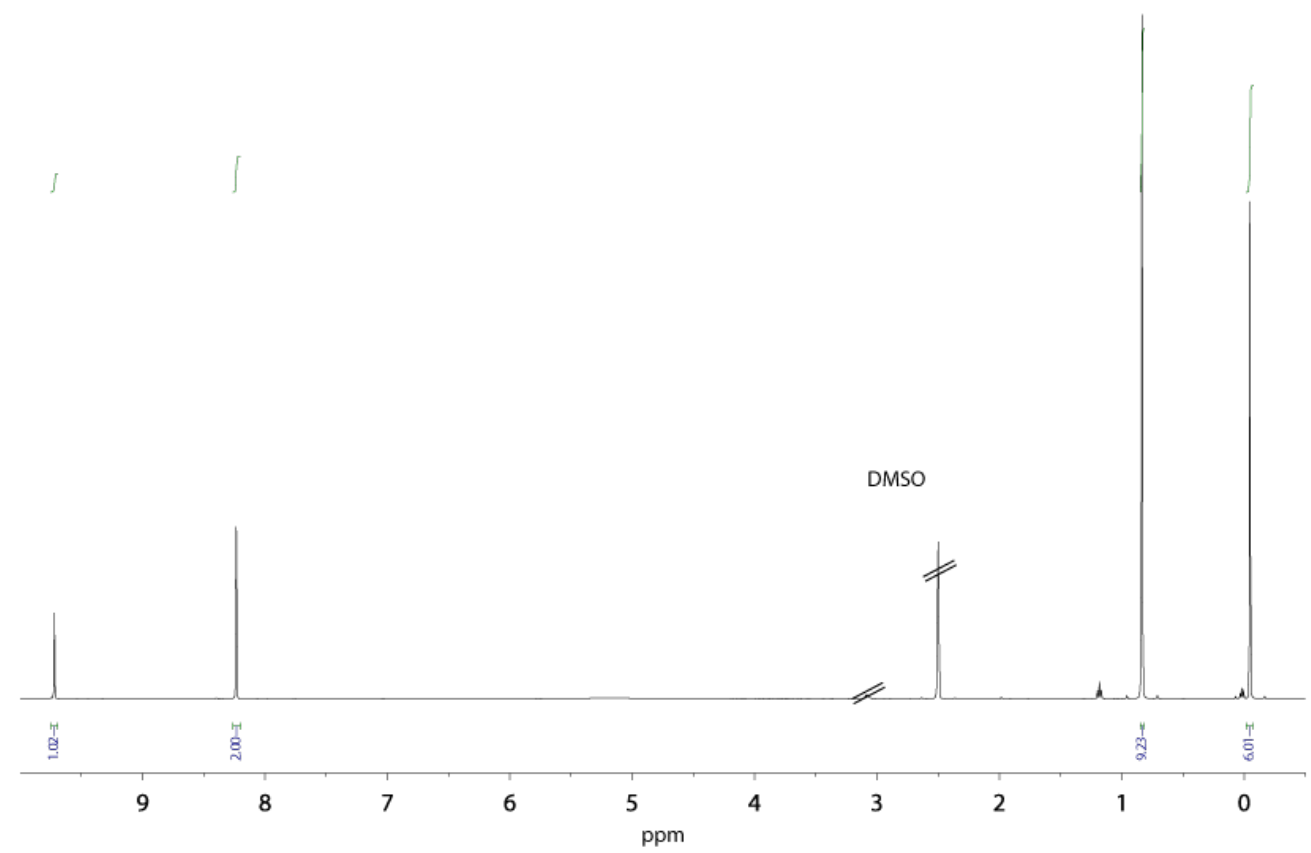


${ }^{13}$ C-NMR $(176 \mathrm{MHz}, 298 \mathrm{~K}, \mathrm{DMSO}): \delta(\mathrm{ppm})=189.3,160.7,140.7,132.1,86.6,25.8$, 17.8, -3.20.

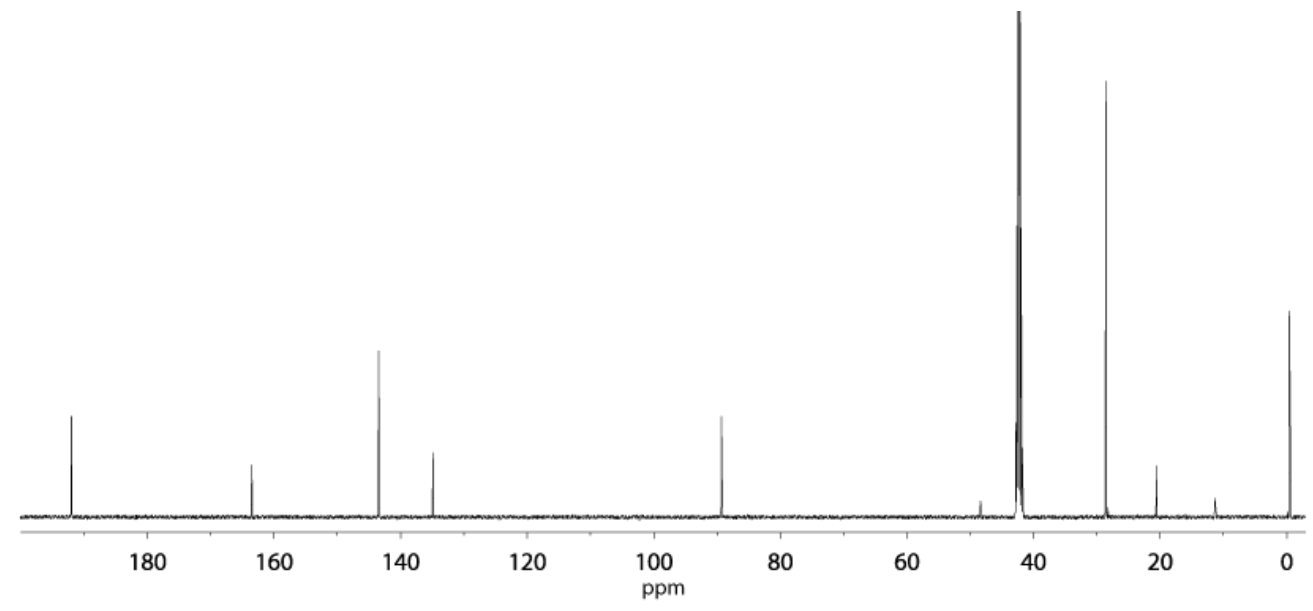

$\begin{array}{lll}\text { ESI-HRMS }\left(\left[\mathrm{C}_{13} \mathrm{H}_{18} \mathrm{I}_{2} \mathrm{O}_{2} \mathrm{Si}+\mathrm{H}\right]^{+}\right): & \text {measured: } & 488.9235 \\ & \text { calculated: } & 488.9238\end{array}$ 


\section{VI.2.2.2 ((3,5-Diiodo-4-((trimethylsilyl)oxy)phenyl)methanol) (27)}<smiles>Cc1cc(C=O)cc(I)c1O[Si](C)(C)C(C)(C)C</smiles>

26

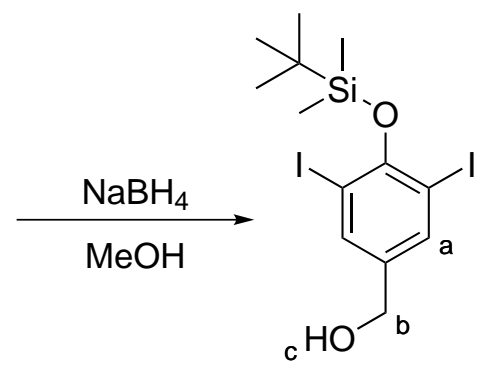

27

Scheme VI.2.2.35: Synthesis of compound 27 .

To a well stirring mixture containg 26 (4.50 g, 9.21 mmol, 1.00 equiv.) $\mathrm{CH}_{2} \mathrm{Cl}_{2}$ (40 mL) and $\mathrm{MeOH}(40 \mathrm{~mL})$ at $0{ }^{\circ} \mathrm{C}$ was added portionwise $\mathrm{NaBH}_{4}(0.37 \mathrm{~g}, 9.68 \mathrm{mmol}, 1.05$ equiv. $)$ and the mixture was stirred for further $3 \mathrm{~h}$. After, sat. $\mathrm{NH}_{4} \mathrm{Cl}$ solution $(5 \mathrm{~mL})$ was added and the solvent was reduced to the half. $\mathrm{Et}_{2} \mathrm{O}(50 \mathrm{~mL})$ was added and the organic layer was washed with $\mathrm{H}_{2} \mathrm{O}(25 \mathrm{~mL})$ and brine $(25 \mathrm{~mL})$. The organic phase was dried over $\mathrm{MgSO}_{4}$ and the solvent was removed in vacuo. The product 27 (4.42 g, $9.02 \mathrm{mmol}, 98 \%$ ) was obtained as a yellowish solid.

${ }^{1}$ H-NMR $(300 \mathrm{M} \mathrm{Hz}, 298 \mathrm{~K}, \mathrm{DMSO}): \delta(\mathrm{ppm})=7.78$ (s, 2H, a), $5.29(\mathrm{~s}, 1 \mathrm{H}, \mathrm{c}), 4.41(\mathrm{~s}, 2 \mathrm{H}$, b), 1.07 (s, $\left.9 \mathrm{H}, \mathrm{Si}\left(\mathrm{CH}_{2}\right)_{2} \mathrm{C}\left(\underline{\mathrm{CH}_{3}}\right)_{3}\right), 0.48\left(\mathrm{~s}, 6 \mathrm{H}, \mathrm{Si}\left(\underline{\mathrm{CH}_{2}}\right)_{2} \mathrm{C}\left(\mathrm{CH}_{3}\right)_{3}\right)$.

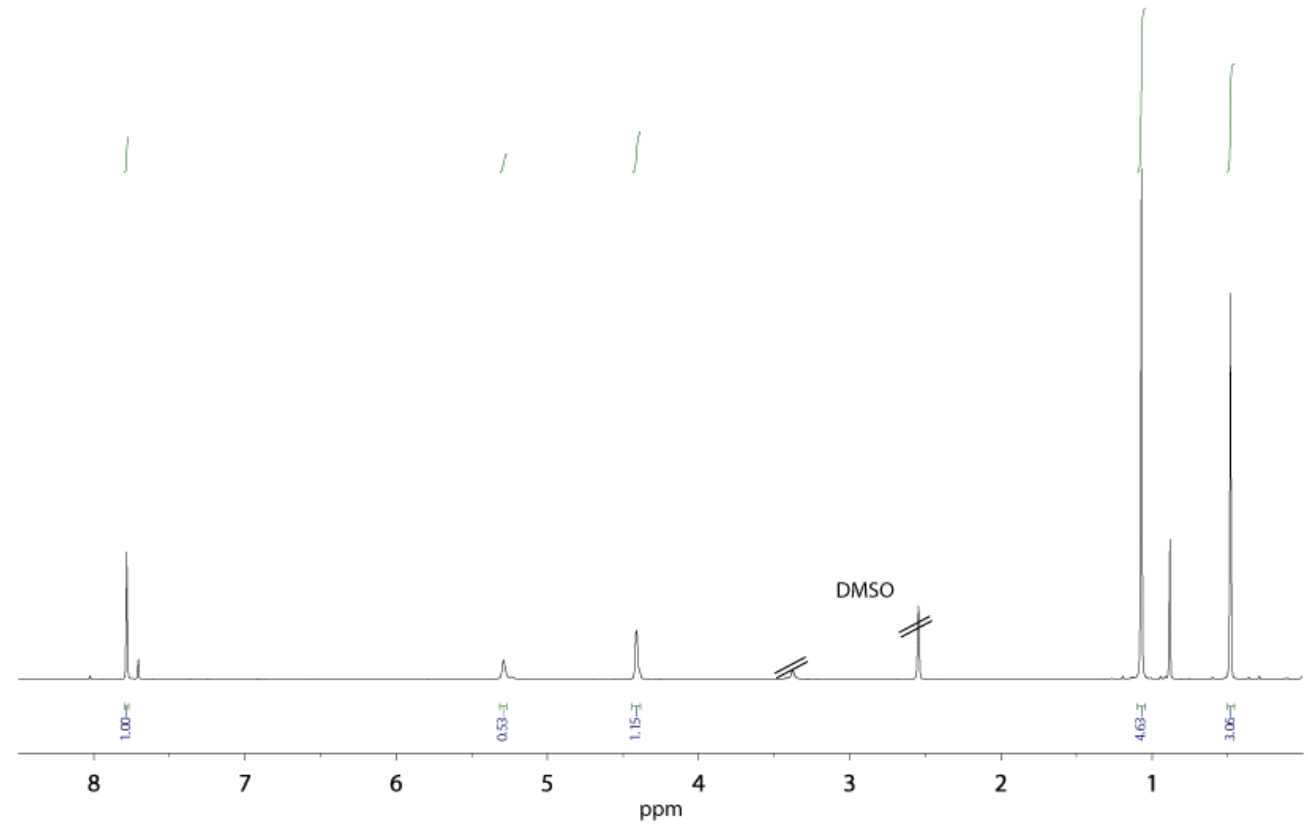

${ }^{13}$ C-NMR $(176 \mathrm{MHz}, 298 \mathrm{~K}, \mathrm{DMSO}): \delta(\mathrm{ppm})=153.2,139.8,138.0,89.2,60.7,26.4$, $18.6,-0.2$. 


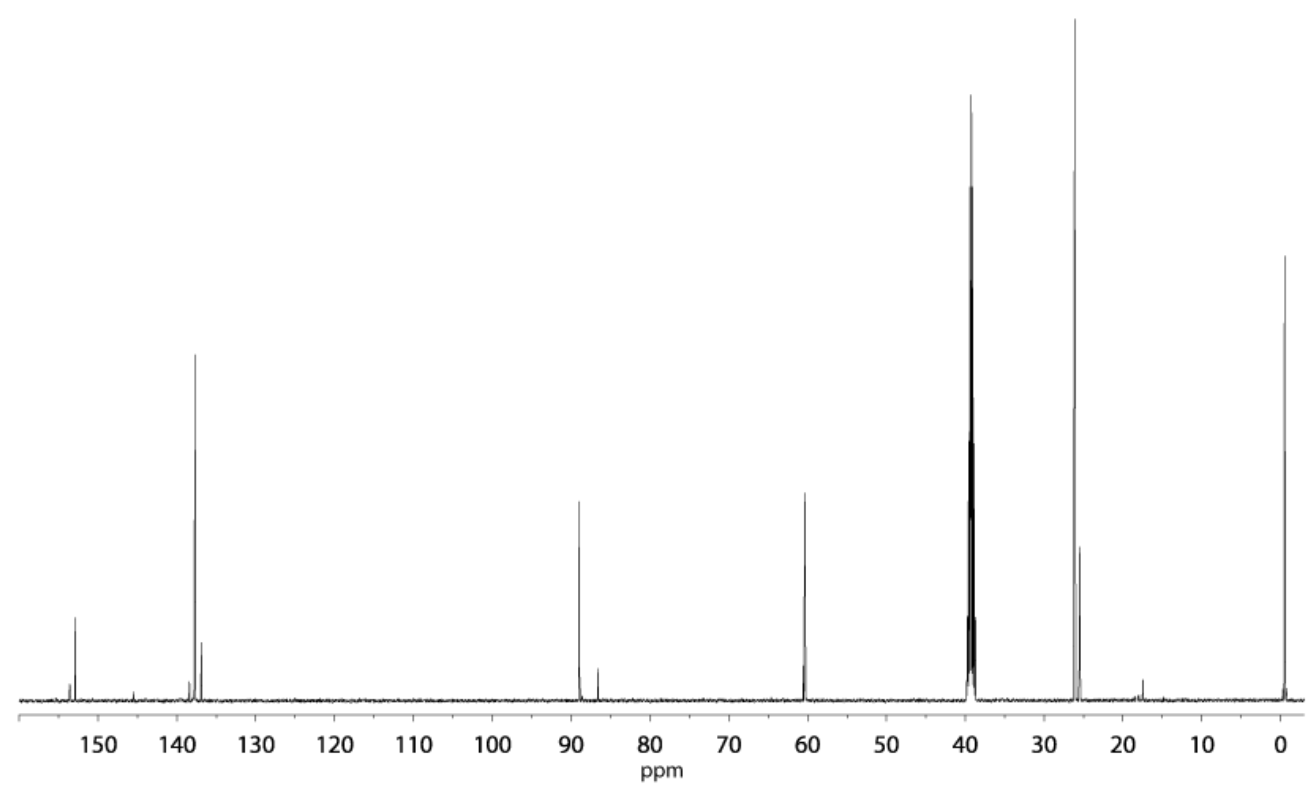

ESI-HRMS $\left(\left[\mathrm{C}_{13} \mathrm{H}_{20} \mathrm{I}_{2} \mathrm{O}_{2} \mathrm{Si}+\mathrm{H}\right]^{+}\right)$: $\quad$ measured: 490.9387
calculated:
490.9395 


\section{VI.2.2.3 (4-(Chloromethyl)-2,6-diiodophenoxy)trimethylsilane) (28)}

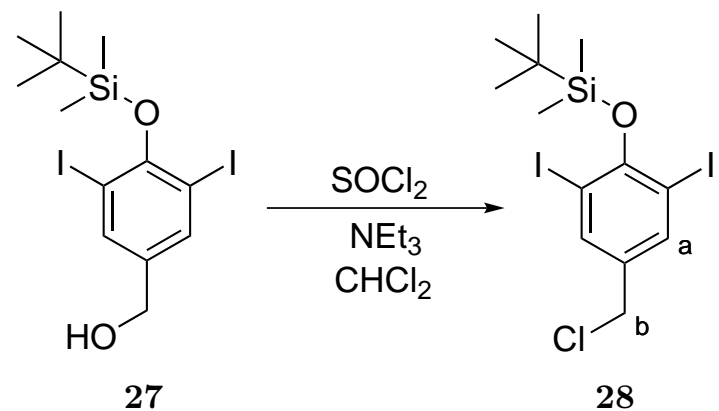

Scheme VI.2.2.36: Synthesis of compound 28 .

To a solution of $\mathbf{2 7}$ (4.20 g, $8.57 \mathrm{mmol}, 1.00$ equiv.) and triethylamine (1.32 mL, $9.41 \mathrm{mmol}$, 1.10 equiv.) in $\mathrm{CH}_{2} \mathrm{Cl}_{2}(10 \mathrm{~mL})$, thionyl chloride ( $0.75 \mathrm{~mL}, 10.3 \mathrm{mmol}, 1.20$ equiv.) was slowly added at $0{ }^{\circ} \mathrm{C}$. The solution was stirred for $3 \mathrm{~h}$, while slowly warming to room temperature. $\mathrm{CH}_{2} \mathrm{Cl}_{2}(90 \mathrm{~mL})$ was added and the organic layer was washed subsequently with $\mathrm{H}_{2} \mathrm{O}(30 \mathrm{~mL})$, sat. aq. $\mathrm{NaHCO}_{3}(30 \mathrm{~mL})$ and brine $(30 \mathrm{~mL})$. The organic phase was dried over $\mathrm{MgSO}_{4}$ and the solvent was removed in vacuo. The product 28 (3.82 g, $7.51 \mathrm{mmol}, 88 \%$ ) was obtained as a brown oil and was used for the next step without further purification.

${ }^{1} \mathbf{H}-\mathbf{N M R}\left(300 \mathrm{M} \mathrm{Hz}, 298 \mathrm{~K}, \mathrm{CD}_{3} \mathrm{CN}\right): \delta(\mathrm{ppm})=7.81(\mathrm{~s}, 2 \mathrm{H}, \mathrm{a}), 4.65(\mathrm{~s}, 2 \mathrm{H}, \mathrm{b}), 1.07$ (s, $\left.9 \mathrm{H}, \mathrm{Si}\left(\mathrm{CH}_{2}\right)_{2} \mathrm{C}\left(\mathrm{CH}_{3}\right)_{3}\right), 0.48\left(\mathrm{~s}, 6 \mathrm{H}, \mathrm{Si}\left(\mathrm{CH}_{2}\right)_{2} \mathrm{C}\left(\mathrm{CH}_{3}\right)_{3}\right)$.

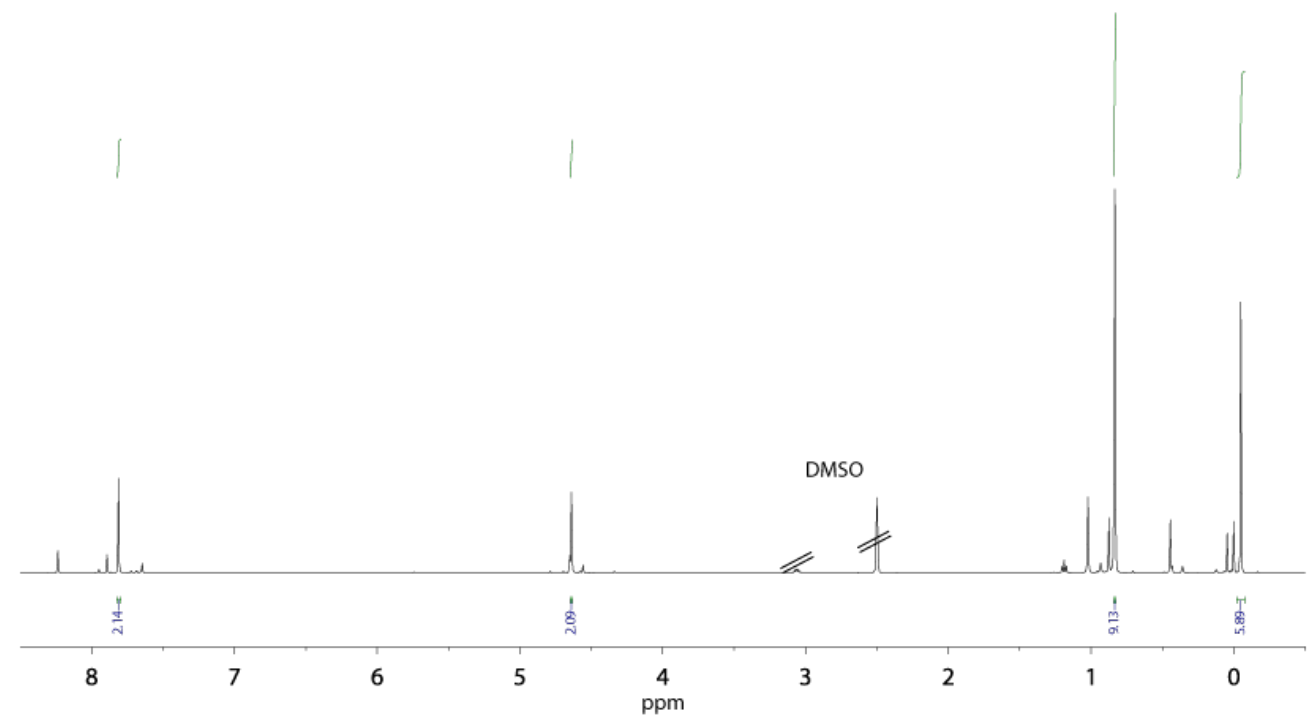

${ }^{13}$ C-NMR $\left(176 \mathrm{MHz}, 298 \mathrm{~K}, \mathrm{CD}_{3} \mathrm{CN}\right): \delta(\mathrm{ppm})=155.5,139.6,133.6,86.7,43.8,25.8$, $17.8,-3.2$. 


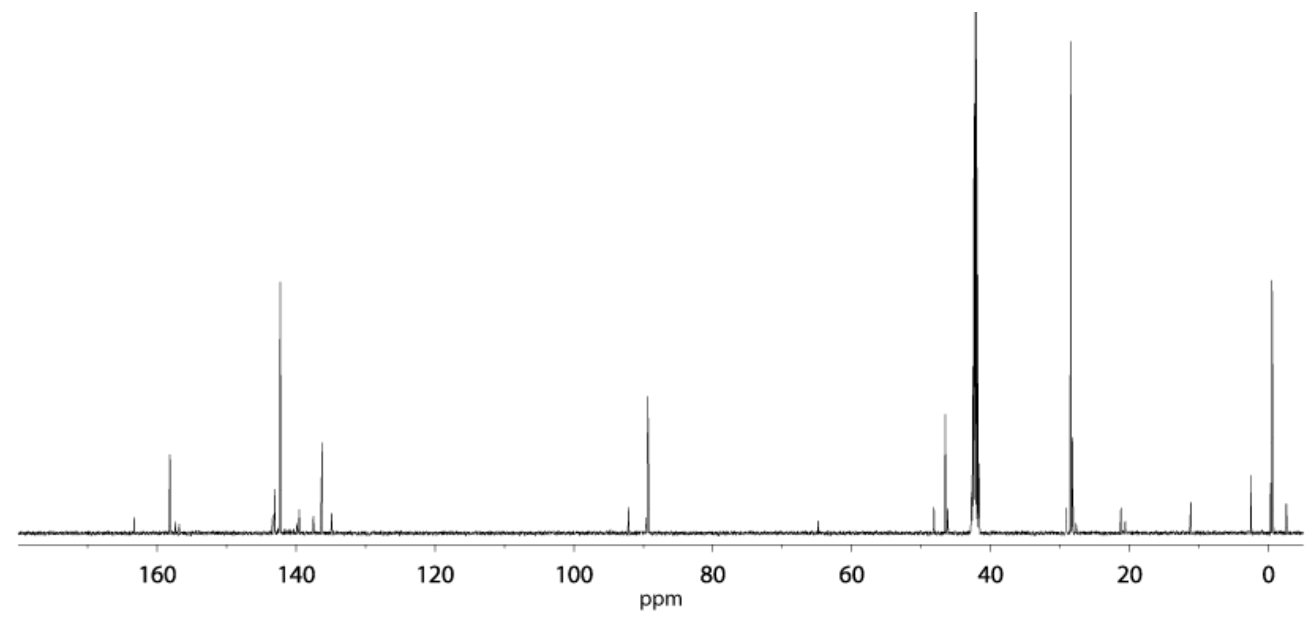

ESI-HRMS $\left(\left[\mathrm{C}_{13} \mathrm{H}_{19} \mathrm{ClI}_{2} \mathrm{OSi}+\mathrm{H}\right]^{+}\right)$: $\quad$ measured: $\quad 508.9011$

calculated: $\quad 508.9056$ 


\section{VI.2.2.4 2,2'-((3,5-Diiodo-4-((trimethylsilyl)oxy)benzyl)azanediyl)bis(ethan-1- ol) (29)}<smiles>CC(C)(C)[Si](C)(C)Oc1c(I)cc(CCl)cc1I</smiles>

28

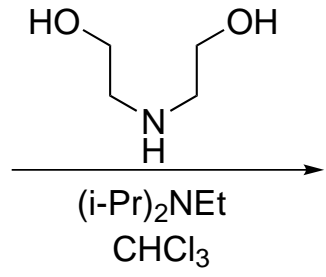

$\mathrm{CHCl}_{3}$

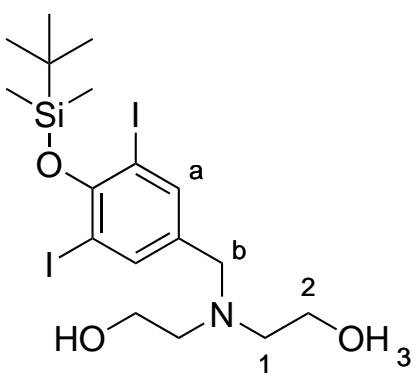

29

Scheme VI.2.2.37: Synthesis of compound 29.

A mixture of $28(2.00 \mathrm{~g}, 3.93 \mathrm{mmol}, 1.00$ equiv. $)$, diethanolamine (0.75 mL, $7.86 \mathrm{mmol}$, 2.00 equiv.) and DIPEA (1.34 mL, $7.86 \mathrm{mmol}, 2.00$ equiv.) in $\mathrm{CHCl}_{3}(50 \mathrm{~mL})$ was heated under reflux over night. After cooling down, $\mathrm{CHCl}_{3}(50 \mathrm{~mL})$ was added and the organic layer was washed with sat. aqueous $\mathrm{NaHCO}_{3}(50 \mathrm{~mL})$ and brine $(50 \mathrm{~mL})$. The organic phase was dried over $\mathrm{MgSO}_{4}$ and the solvent was removed. The residue was purifed by means of column chromatography (Biotage, $\mathrm{SiO}_{2}, \mathrm{CH}_{2} \mathrm{Cl}_{2} /$ Acetone 92:8 $\rightarrow$ 34:66) The product 29 (1.36 g, $2.35 \mathrm{mmol}, 60 \%$ ) was obtained as a yellowish oil.

${ }^{1} \mathbf{H}-\mathbf{N M R}\left(300 \mathrm{M} \mathrm{Hz}, 298 \mathrm{~K}, \mathrm{CD}_{3} \mathrm{CN}\right): \delta(\mathrm{ppm})=7.77(\mathrm{~s}, 2 \mathrm{H}, \mathrm{a}), 4.41(\mathrm{~s}, 2 \mathrm{H}, 3), 3.53(\mathrm{~s}$, $2 \mathrm{H}, \mathrm{b}), 3.49-3.37$ (s, 4H, 2), 2.56-2.47 (m, 4H, 1), 1.03 (s, 9H, $\left.\mathrm{Si}\left(\mathrm{CH}_{2}\right)_{2} \mathrm{C}\left(\underline{\mathrm{CH}_{3}}\right)_{3}\right), 0.44$ (s, $\left.6 \mathrm{H}, \mathrm{Si}\left(\underline{\mathrm{CH}_{2}}\right)_{2} \mathrm{C}\left(\mathrm{CH}_{3}\right)_{3}\right)$.

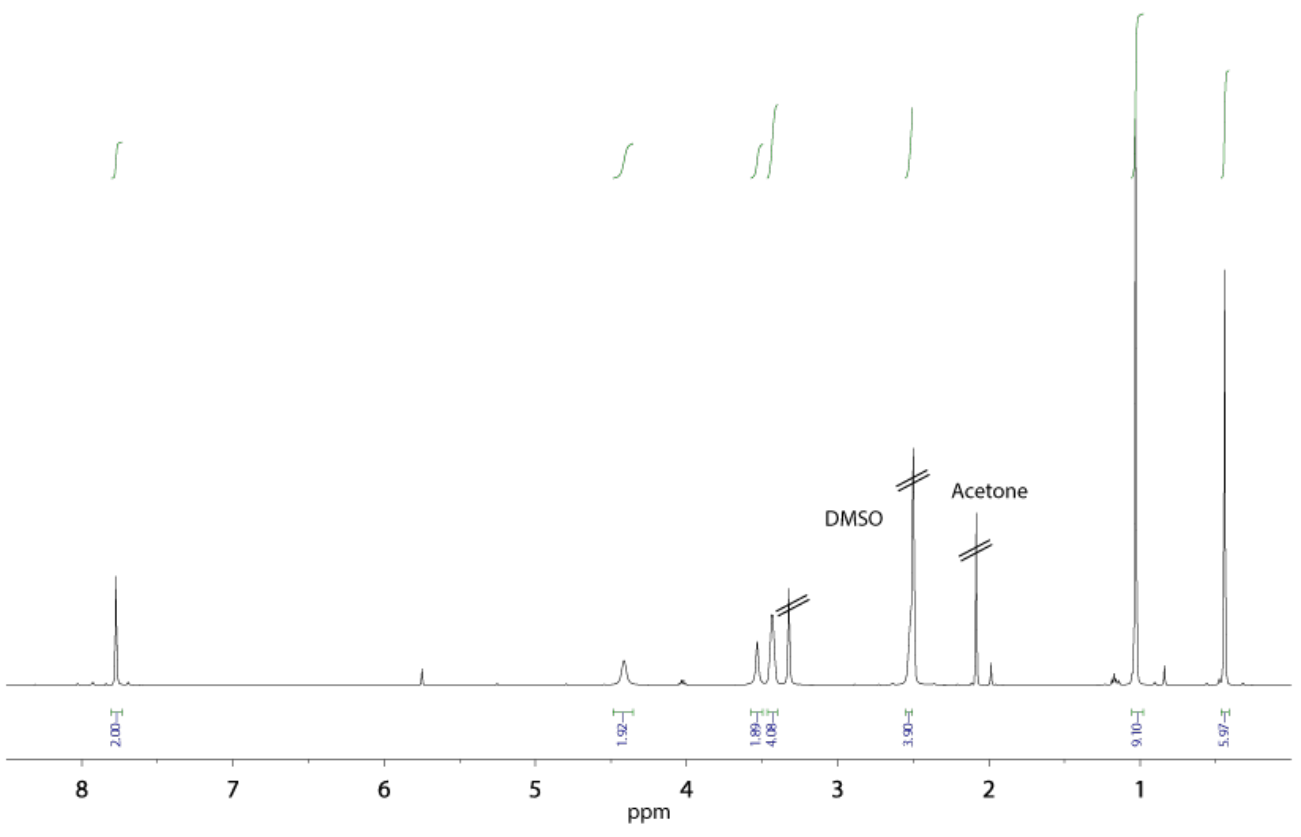


${ }^{13}$ C-NMR $\left(176 \mathrm{MHz}, 298 \mathrm{~K}, \mathrm{CD}_{3} \mathrm{CN}\right): \delta(\mathrm{ppm})=153.2,139.9,137.3,89.3,59.0,56.7$, $56.1,30.7,26.4,-0.1$.

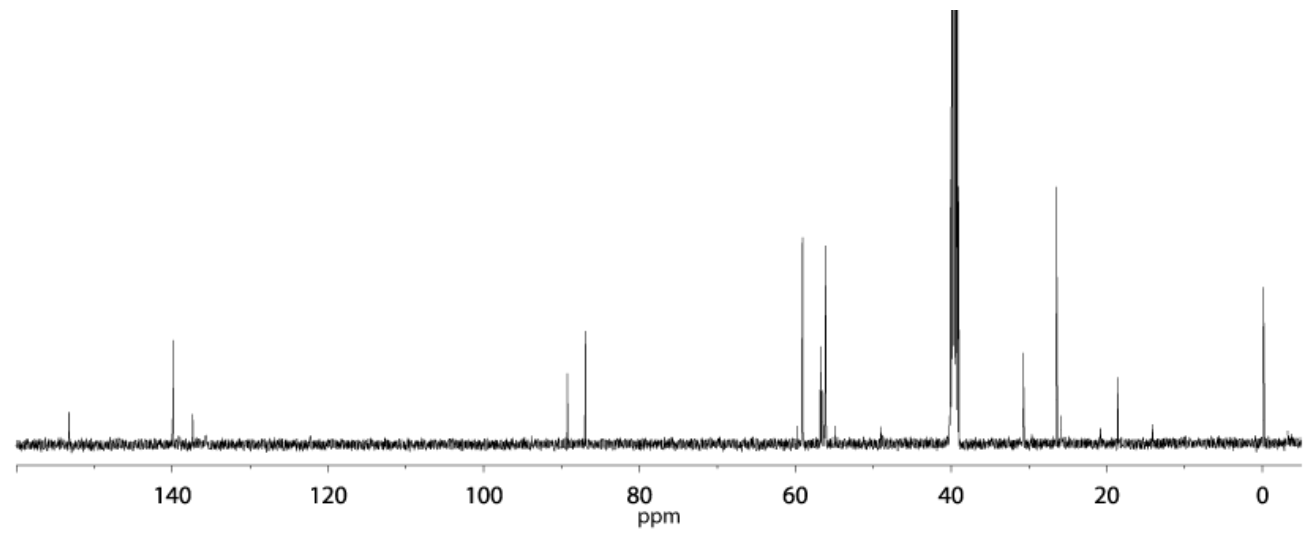

$\begin{array}{lll}\text { ESI-HRMS }\left(\left[\mathrm{C}_{17} \mathrm{H}_{29} \mathrm{I}_{2} \mathrm{NO}_{3} \mathrm{Si}+\mathrm{H}\right]^{+}\right): & \text {measured: } & 578.0053 \\ \text { calculated: } & 578.0079\end{array}$ 


\section{VI.2.2.5 2,2'-((3,5-Bis(pyridin-3-ylethynyl)-4-}

((trimethylsilyl)oxy)benzyl)azanediyl)bis(ethan-1-ol)) (30)

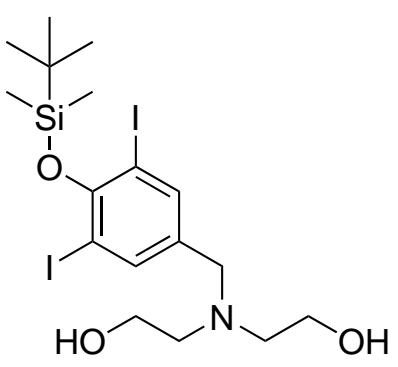

29
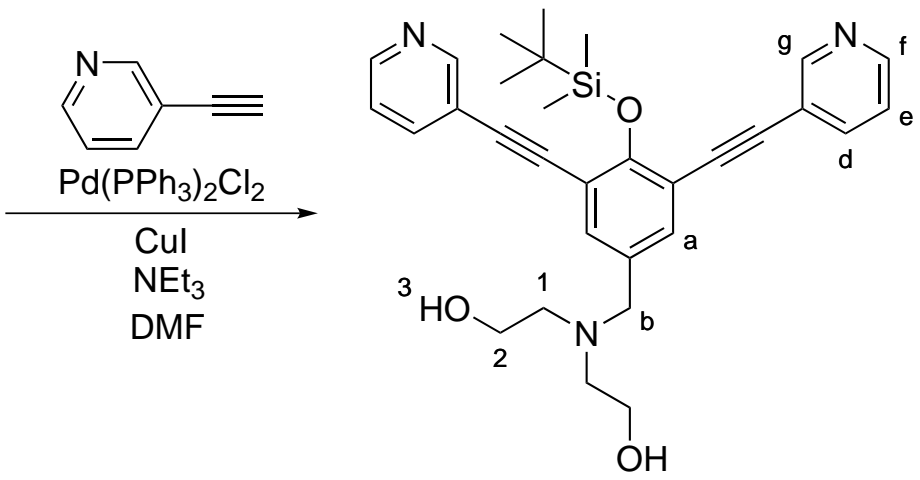

30

Scheme VI.2.2.38: Synthesis of compound 61a.

23 (1.40 g, $2.43 \mathrm{mmol}, 1.00$ equiv), 3-ethynyl pyridine ( $0.55 \mathrm{~g}, 5.34 \mathrm{mmol}, 2.20$ equiv.), $\mathrm{Pd}\left(\mathrm{PPh}_{3}\right)_{2} \mathrm{Cl}_{2}$ (0.17 g, $0.25 \mathrm{mmol}, 0.10$ equiv) and $\mathrm{CuI}$ (0.05 g, $0.25 \mathrm{mmol}, 0.10$ equiv.) were solved in dry DMF $(10 \mathrm{~mL})$. To the stirred reaction triethylamine $(1.34 \mathrm{~mL}, 9.70 \mathrm{mmol}$, 4.00 equiv.) was added and the reaction mixture was stirred at $80^{\circ} \mathrm{C}$ over night. The solvent was removed in vacuo and the residue was purified by means of column chromatography $\left(\mathrm{SiO}_{2}\right.$, dichloromethane 20:1 $\rightarrow$ 10:1). The product $24(0.71 \mathrm{~g}, 1.35 \mathrm{mmol}, 55 \%)$ was obtained as a yellowish resin.

${ }^{1}$ H-NMR $(300 \mathrm{M} \mathrm{Hz}, 298 \mathrm{~K}, \mathrm{DMSO}): \delta(\mathrm{ppm})=8.73(\mathrm{~s}, 2 \mathrm{H}, \mathrm{g}), 8.59\left(\mathrm{~d},{ }^{3} \mathrm{~J}=4.6\right.$ $\mathrm{Hz}, 2 \mathrm{H}, \mathrm{f}), 7.91\left(\mathrm{dt},{ }^{3} J=7.9 \mathrm{~Hz},{ }^{4} J=2.1 \mathrm{~Hz}, 2 \mathrm{H}, \mathrm{d}\right), 7.58(\mathrm{~s}, 2 \mathrm{H}),, 7.46\left(\mathrm{dd},{ }^{3} J=\right.$ 8.0, $4.8 \mathrm{~Hz}, 2 \mathrm{H}, \mathrm{e}), 4.46\left(\mathrm{t},{ }^{3} J=5.1 \mathrm{~Hz}, 2 \mathrm{H}, 3\right), 3.60(\mathrm{~s}, 2 \mathrm{H}, \mathrm{b}), 3.49$ (q, ${ }^{3} J=5.1$ $\mathrm{Hz}, 4 \mathrm{H}, 2), 2.57\left(\mathrm{t},{ }^{3} \mathrm{~J}=6.2 \mathrm{~Hz}, 4 \mathrm{H}, 1\right), 0.98$ (s, 9H, $\left.\mathrm{Si}\left(\mathrm{CH}_{2}\right)_{2} \mathrm{C}\left(\underline{\mathrm{CH}_{3}}\right)_{3}\right), 0.27$ (s, 6H, $\left.\mathrm{Si}\left(\mathrm{CH}_{2}\right)_{2} \mathrm{C}\left(\mathrm{CH}_{3}\right)_{3}\right)$. 


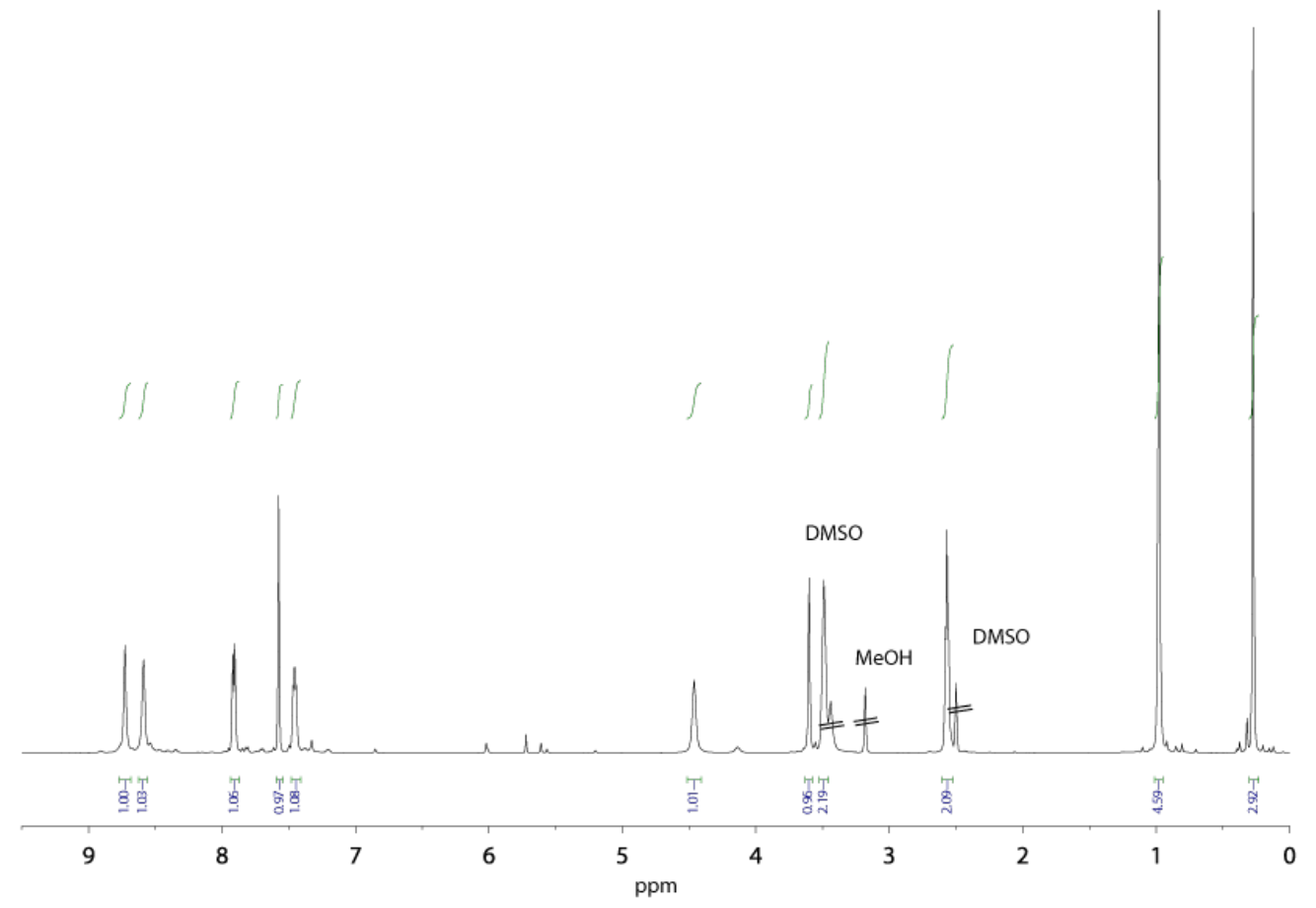




\section{VI.2.2.6 2,2'-((4-Hydroxy-3,5-bis(pyridin-3-} ylethynyl)benzyl)azanediyl)bis(ethan-1-ol) (31)

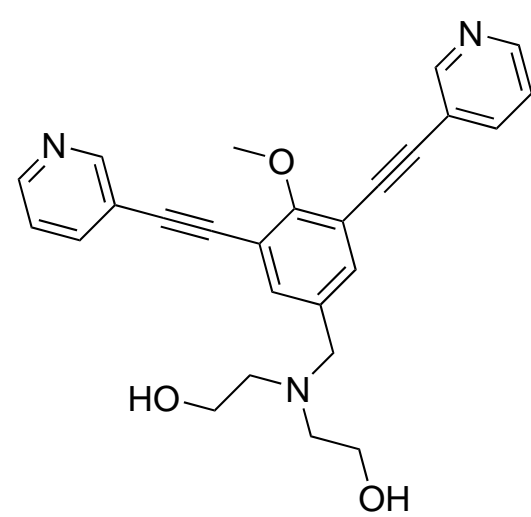

17

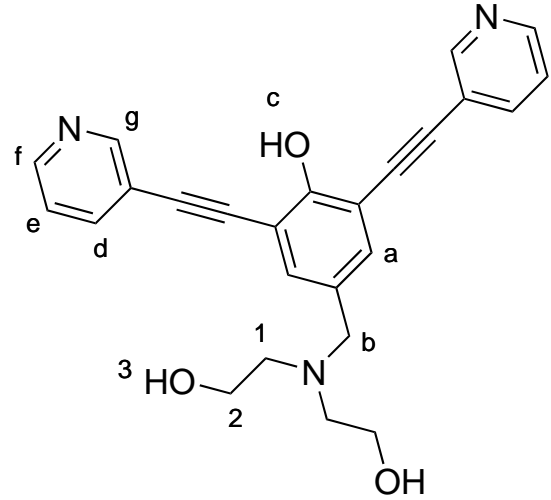

31

Scheme VI.2.2.39: Synthesis of compound 31.

To a solution of 17 (100 mg, $0.23 \mathrm{mmol}, 1.00$ equiv.) was solved in dry $\mathrm{CH}_{2} \mathrm{Cl}_{2}$ (5 mL) $\mathrm{BBr}_{3}$ ( $1 \mathrm{M}, 0.35 \mathrm{~mL}, 0.35 \mathrm{mmol}, 1.50$ equiv.) was added dropwise at $0{ }^{\circ} \mathrm{C}$. The reaction mixture was allowed to warm to room temperature and was stirred over night. After, $\mathrm{H}_{2} \mathrm{O}$ was added and the mixture was extracted with $\mathrm{CH}_{2} \mathrm{Cl}_{2}$. The combined organic phases were dried over $\mathrm{NaSO}_{4}$ and the solvent was removed in vacuo. The residue was purified by means of column chromatography ( $\mathrm{SiO} 2$, dichloromethane / methanol 20:1 $\rightarrow$ 10:1). The product 31 (41.4 mg, $0.10 \mathrm{mmol}, 43 \%$ ) was obatined as yellowish solid.

${ }^{1} \mathbf{H}-\mathbf{N M R}\left(300 \mathrm{M} \mathrm{Hz}, 298 \mathrm{~K}, \mathrm{~d}^{4}-\mathrm{MeOH}\right): \delta(\mathrm{ppm})=8.74\left(\mathrm{~d},{ }^{4} J=2.4 \mathrm{~Hz}, 2 \mathrm{H}, \mathrm{g}\right), 8.51$ $\left(\mathrm{dt},{ }^{3} J=4.9 \mathrm{~Hz},{ }^{4} J=2.2 \mathrm{~Hz}, 2 \mathrm{H}, \mathrm{f}\right), 8.02\left(\mathrm{dt},{ }^{3} J=7.8 \mathrm{~Hz},{ }^{4} J=2.0 \mathrm{~Hz}, 2 \mathrm{H}, \mathrm{d}\right), 7.52(\mathrm{~s}$, $2 \mathrm{H}, \mathrm{a}), 7.47\left(\mathrm{dd},{ }^{3} J=8.1 \mathrm{~Hz},{ }^{4} J=4.9 \mathrm{~Hz}, 2 \mathrm{H}, \mathrm{e}\right), 4.58(\mathrm{~s}, 2 \mathrm{H}, 3), 3.69$ (s, 2H, b), 3.65 (t, $\left.{ }^{3} J=5.9 \mathrm{~Hz}, 4 \mathrm{H}, 2\right), 2.73\left(\mathrm{t},{ }^{3} J=5.8 \mathrm{~Hz}, 4 \mathrm{H}, 1\right)$. 


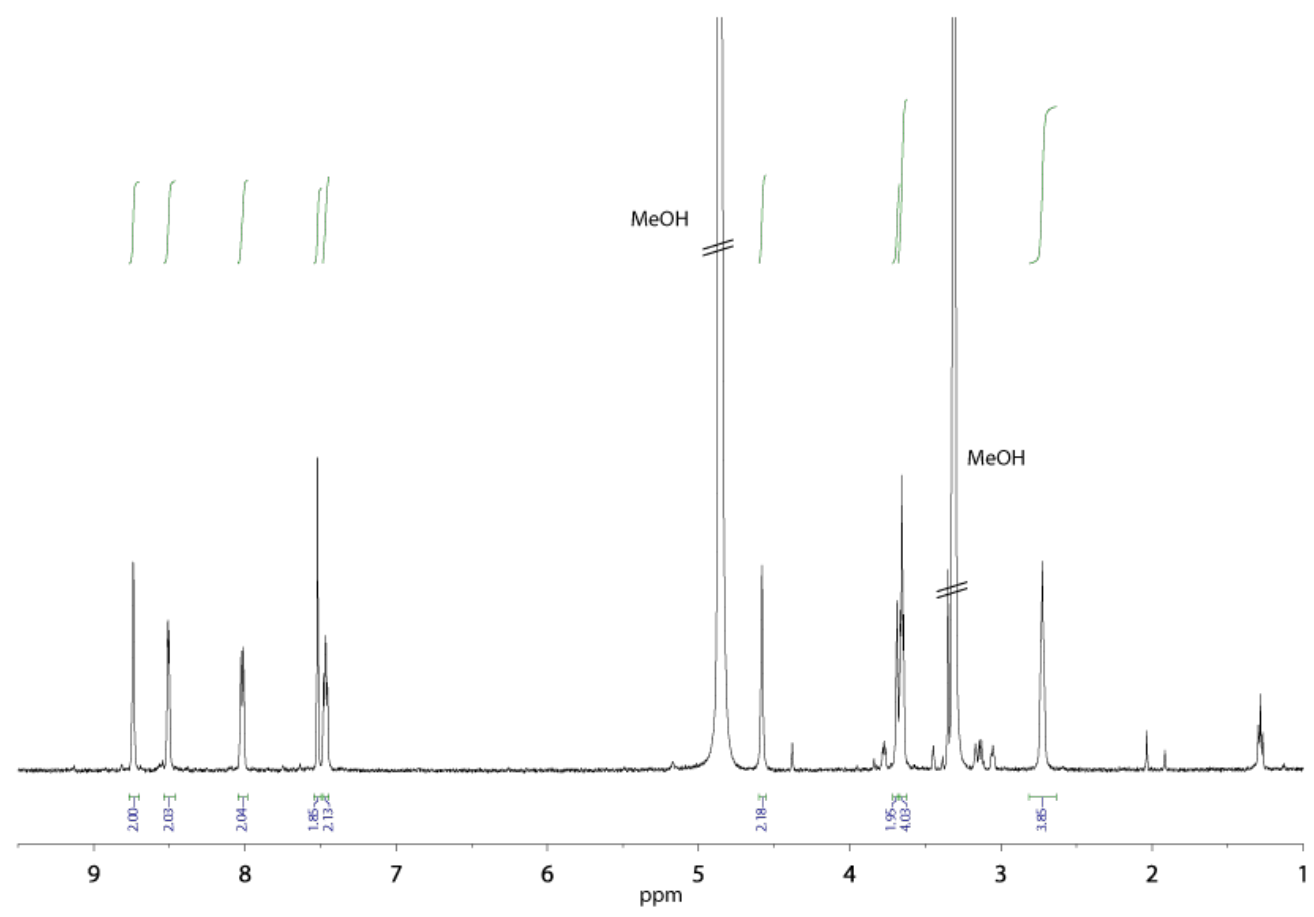

$\begin{array}{lll}\text { ESI-HRMS }\left(\left[\mathrm{C}_{25} \mathrm{H}_{23} \mathrm{~N}_{3} \mathrm{O}_{3}+\mathrm{H}\right]^{+}\right): & \text {measured: } & 414.1825 \\ & \text { calculated: } & 414.1812\end{array}$ 


\section{VI.2.2.7 2,2'-(((2-(Pyridin-3-yl)-7-(pyridin-3-ylethynyl)benzofuran-5- yl)methyl)azanediyl)bis(ethan-1-ol) (32)}

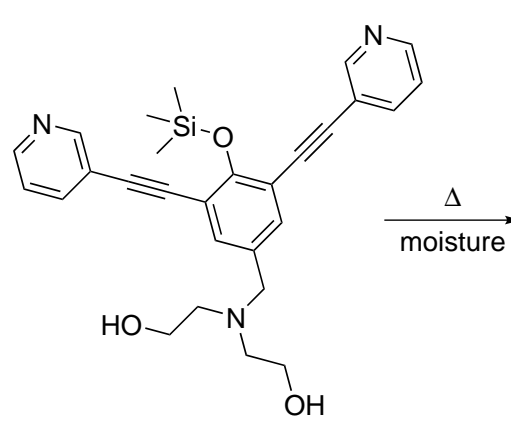

30

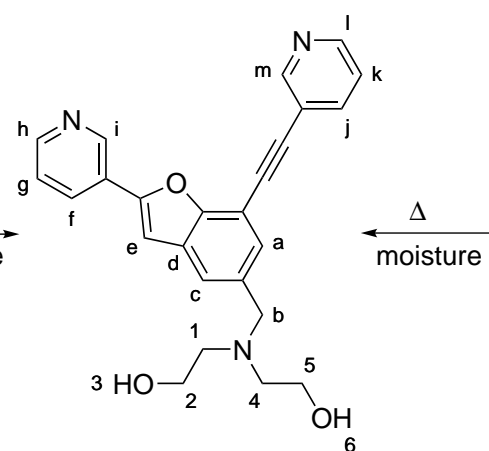

32<smiles>OCCN(CCO)Cc1cc(C#Cc2cccnc2)c(O)c(C#Cc2cccnc2)c1</smiles>

31

Scheme VI.2.2.40: Synthesis of compound 32 .

30 or 31 are placed in an open flask, either as solid or solved in wet polar solvents (like DMSO, $\mathrm{MeOH}$ or $\mathrm{MeCN}$ ) and heated for several ours at 60 řC. After, the solvent was removed and the residue was purified by means of column chromatography ( $\mathrm{SiO} 2$, dichloromethane / methanol 30:1 $\rightarrow$ 10:1) and subsequent purification with help of GPC The product 32 was obtained as a yellow solid.

${ }^{1} \mathbf{H}-\mathbf{N M R}\left(300 \mathrm{M} \mathrm{Hz}, 298 \mathrm{~K}, \mathrm{~d}^{3}-\mathrm{MeCN}\right): \delta(\mathrm{ppm})=9.16\left(\mathrm{~d},{ }^{3} J=2.4 \mathrm{~Hz}, 1 \mathrm{H}, \mathrm{i}\right), 8.86$ $\left(\mathrm{d},{ }^{3} J=2.3 \mathrm{~Hz}, 1 \mathrm{H}, \mathrm{m}\right), 8.60\left(\mathrm{dt},{ }^{3} J=4.7 \mathrm{~Hz},{ }^{4} J=2.1 \mathrm{~Hz}, 2 \mathrm{H}, \mathrm{h}, \mathrm{l}\right), 8.27\left(\mathrm{dt},{ }^{3} J=7.9\right.$ $\left.\mathrm{Hz},{ }^{4} J=2.0 \mathrm{~Hz}, 1 \mathrm{H}, \mathrm{f}\right), 8.01\left(\mathrm{dt},{ }^{3} J=7.9 \mathrm{~Hz},{ }^{4} J=2.0 \mathrm{~Hz}, 1 \mathrm{H}, \mathrm{j}\right), 7.72\left(\mathrm{~d},{ }^{4} J=1.7 \mathrm{~Hz}\right.$, $1 \mathrm{H}, \mathrm{c}), 7.57$ (d, $\left.{ }^{4} J=1.7 \mathrm{~Hz}, 1 \mathrm{H}, \mathrm{a}\right), 7.48\left(\mathrm{dd},{ }^{3} J=8.0,4.7 \mathrm{~Hz}, 1 \mathrm{H}, \mathrm{g}\right), 7.43\left(\mathrm{dd},{ }^{3} J=8.0\right.$, $4.7 \mathrm{~Hz}, 1 \mathrm{H}, \mathrm{k}), 7.35(\mathrm{~s}, 1 \mathrm{H}, \mathrm{e}), 3.79(\mathrm{~s}, 2 \mathrm{H}, \mathrm{b}), 3.54(\mathrm{~s}, 4 \mathrm{H}, 2,5), 2.66\left(\mathrm{t},{ }^{3} J=5.6 \mathrm{~Hz}, 4 \mathrm{H}\right.$, $1,4)$. 


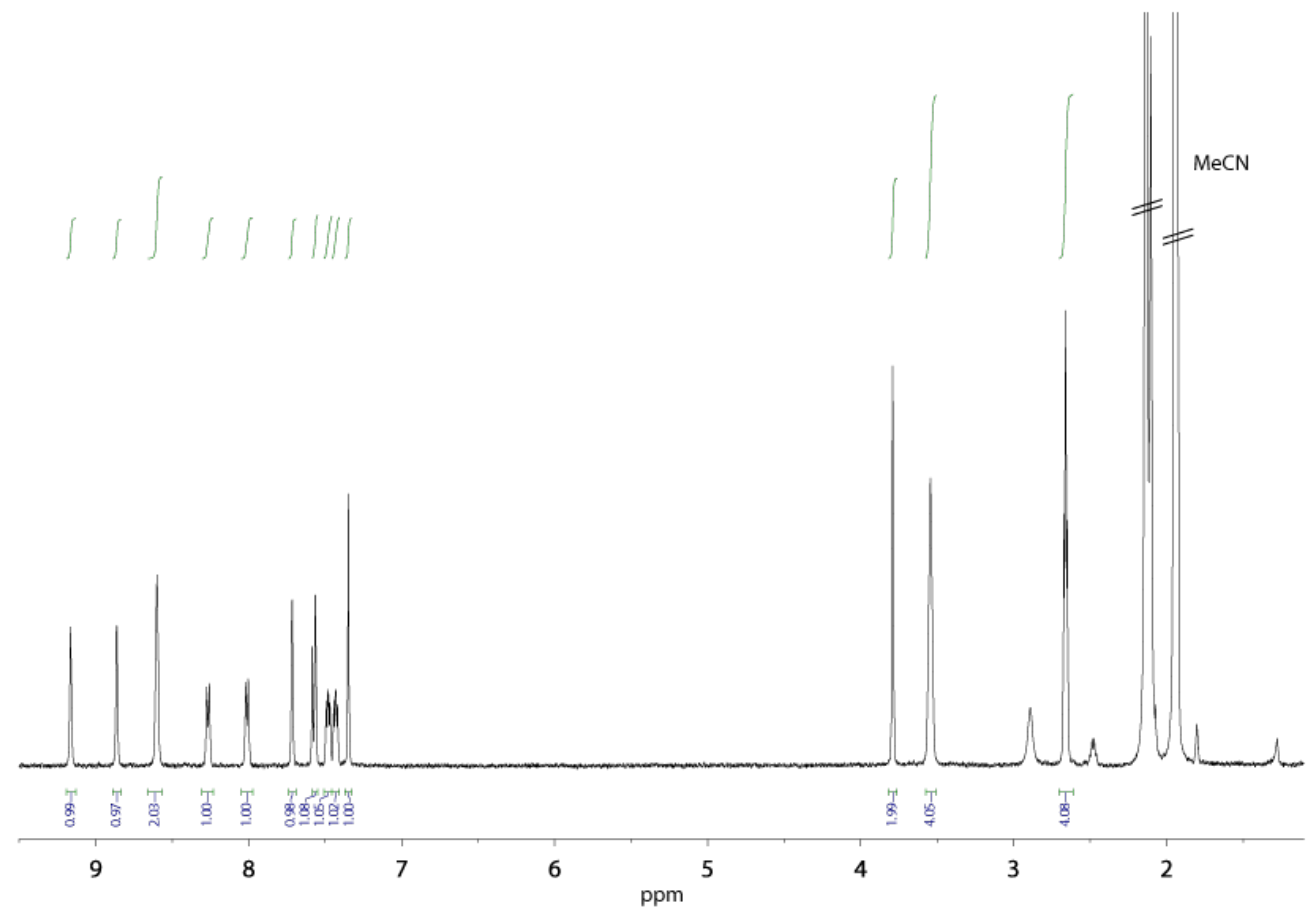

$\begin{array}{lll}\text { ESI-HRMS }\left(\left[\mathrm{C}_{25} \mathrm{H}_{23} \mathrm{~N}_{3} \mathrm{O}_{3}+\mathrm{H}\right]^{+}\right): & \text {measured: } & 414.1825 \\ & \text { calculated: } & 414.1812\end{array}$ 


\title{
Chapter
}

\section{VI.3}

\section{Syntheses of Acridinylidene Derivatives}

\author{
Contents
}

VI.3.1 Syntheses of Backbone Derivatives $\ldots . . . . . . .254$

VI.3.1.1 Compound $34-\mathrm{B}^{\mathrm{tBu}} \ldots \ldots \ldots \ldots$. . . . . . . . 254

VI.3.1.2 Compound $35-\mathrm{B}^{\mathrm{Et}} \ldots \ldots \ldots$

VI.3.1.3 Compound 36 . . . . . . . . . . . . . . . . . . . . . 259

VI.3.1.4 Compound $37-\mathrm{B}^{\mathrm{Ph}} \ldots \ldots \ldots$

VI.3.2 Syntheses of Ligand Derivatives . . . . . . . . . . . . 262

VI.3.2.1 Compound $40-\mathrm{L}^{\mathrm{CN}}$. . . . . . . . . . . . . . . . . . 262

VI.3.2.2 Compound $41-\mathrm{L}^{\mathrm{tBu}} \ldots \ldots \ldots$. . . . . . . . 265

VI.3.2.3 Compound $42-\mathrm{L}^{\mathrm{Et}} \ldots \ldots \ldots$

VI.3.2.4 Compound $43-\mathrm{L}^{\mathrm{Ph}} \ldots \ldots \ldots$

VI.3.3 Syntheses of Cage Derivatives . . . . . . . . . . . . . . 274

VI.3.3.1 Compound $44-\mathrm{C}^{\mathrm{CN}}$. . . . . . . . . . . . . . . 2 275

VI.3.3.2 Compound $45-\mathrm{C}^{\mathrm{tBu}}$. . . . . . . . . . . . . . 2777

VI.3.3.3 Compound $46-\mathrm{C}^{\mathrm{Et}} \ldots \ldots \ldots \ldots$

VI.3.3.4 Compound $47-\mathrm{C}^{\mathrm{PH}} \ldots \ldots \ldots$

VI.3.4 Syntheses of Azacrown Ethyl Acridinylidine . . . . . . 281

VI.3.4.1 Compound 48 . . . . . . . . . . . . . . . . . . . . . . . 281

VI.3.4.2 Compound 49 . . . . . . . . . . . . . . . . . . . . . . 283

VI.3.4.3 Compound 50 . . . . . . . . . . . . . . . . . . . . 286

VI.3.4.4 Compound 51 . . . . . . . . . . . . . . . . . . . . . . 288

VI.3.4.5 Compound 52 . . . . . . . . . . . . . . . . . . . . 291 
<smiles>CCCCCCn1c2ccccc2c(=O)c2ccccc21</smiles>

33

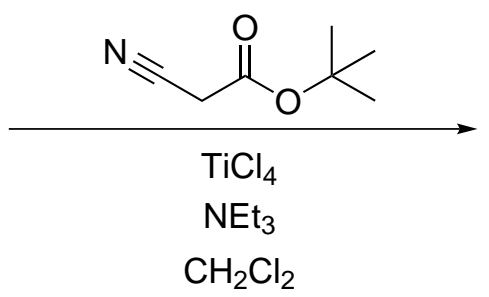

$\mathrm{CH}_{2} \mathrm{Cl}_{2}$<smiles>CCCCCCN1c2ccccc2C(=C(C#N)C(=O)OC(C)(C)C)c2ccccc21</smiles>

34

Scheme VI.3.1.41: Synthesis of compound 34 .

\section{VI.3.1 Syntheses of Backbone Derivatives}

\section{VI.3.1.1 2-Cyano-2-(10-hexylacrid-9-inylidene)tert-butylacetate (34)}

$\mathrm{TiCl}_{4}$ (255 mg, $1.34 \mathrm{mmol}, 1.25$ equiv.) was slowly added to $N$-hexyl-acridone (300 $\mathrm{mg}$, $1.07 \mathrm{mmol}, 1.00$ equiv.) solved in dry $\mathrm{CH}_{2} \mathrm{Cl}_{2}(15 \mathrm{~mL})$ and stirred for $15 \mathrm{~min}$ at room temperature. 2-cyano-tert-butylacetate (304 mg, $2.15 \mathrm{mmol}, 2.00$ equiv.) and triethylamine $(2 \mathrm{~mL})$ were added consecutively to the reaction mixture and heated under reflux for $24 \mathrm{~h}$. The cooled down reaction mixture was filtered through celite and the residue was washed with $\mathrm{CH}_{2} \mathrm{Cl}_{2}(2 \times 5 \mathrm{~mL})$. The solvent was removed in vacuo and the residue was purified by means of column $\mathrm{SiO}_{2}$, chloroform/methanol 40:1 $\rightarrow$ 20:1). The product 34 (288 mg, $0.72 \mathrm{mmol}, 67 \%$ )was obtained as a yellow brownish solid.

${ }^{1} \mathbf{H}-\mathbf{N M R}\left(300 \mathrm{M} \mathrm{Hz}, 298 \mathrm{~K}, \mathrm{CD}_{3} \mathrm{CN}\right): \delta(\mathrm{ppm})=8.03\left(\mathrm{~s}, 2 \mathrm{H}, \mathrm{a} / \mathrm{a}^{\prime}\right), 7.62\left(\mathrm{ddd},{ }^{3} J=8.6\right.$, $\left.7.0 \mathrm{~Hz},{ }^{4} J=1.6 \mathrm{~Hz}, 2 \mathrm{H}, \mathrm{c} / \mathrm{c}^{\prime}\right), 7.53\left(\mathrm{dd},{ }^{3} J=8.5 \mathrm{~Hz},{ }^{4} J=0.9 \mathrm{~Hz}, 2 \mathrm{H}, \mathrm{d} / \mathrm{d}\right.$ '), 7.20 (ddd, $\left.{ }^{3} J=8.1,7.0 \mathrm{~Hz},{ }^{4} J=1.1 \mathrm{~Hz}, 2 \mathrm{H}, \mathrm{b} / \mathrm{b}\right), 4.36-4.19(\mathrm{~m}, 2 \mathrm{H}, \mathrm{NCH} 2), 1.86\left(\mathrm{p},{ }^{3} J=7.7\right.$ $\left.\mathrm{Hz}, 2 \mathrm{H}, \mathrm{NCH}_{2} \mathrm{CH}_{2}\right), 1.47-1.41\left(\mathrm{~m}, 2 \mathrm{H}, \mathrm{NCH}_{2} \mathrm{CH}_{2} \mathrm{CH}_{2}\right), 1.42(\mathrm{~s}, 9 \mathrm{H}, \mathrm{e}), 1.40-1.28(\mathrm{~m}$, $4 \mathrm{H}, \mathrm{CH}_{2} \mathrm{CH}_{2} \mathrm{CH}_{3}$ ), $0.90\left(\mathrm{t},{ }^{3} \mathrm{~J}=7.1 \mathrm{~Hz}, 3 \mathrm{H}, \mathrm{CH}_{2} \mathrm{CH}_{2} \mathrm{CH}_{3}\right.$ ). 


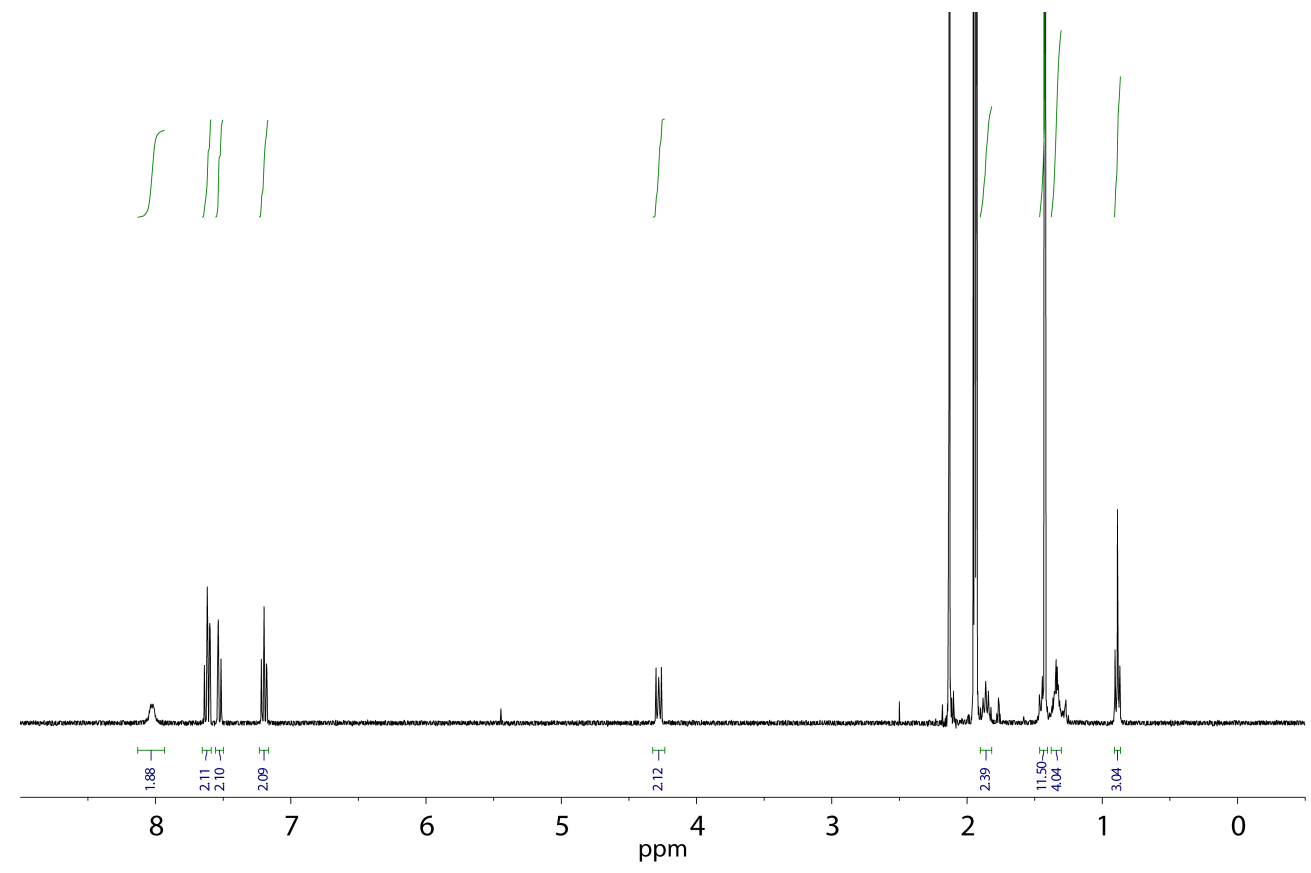

${ }^{13}$ C-NMR $\left(176 \mathrm{MHz}, 298 \mathrm{~K}, \mathrm{CD}_{3} \mathrm{CN}\right): \delta(\mathrm{ppm})=165.20,151.63,140.17,132.85,128.53$, $121.79,120.33,120.13,115.41,94.60,83.51,47.87,32.10,28.01,27.20,27.03,23.34$, 14.27 .

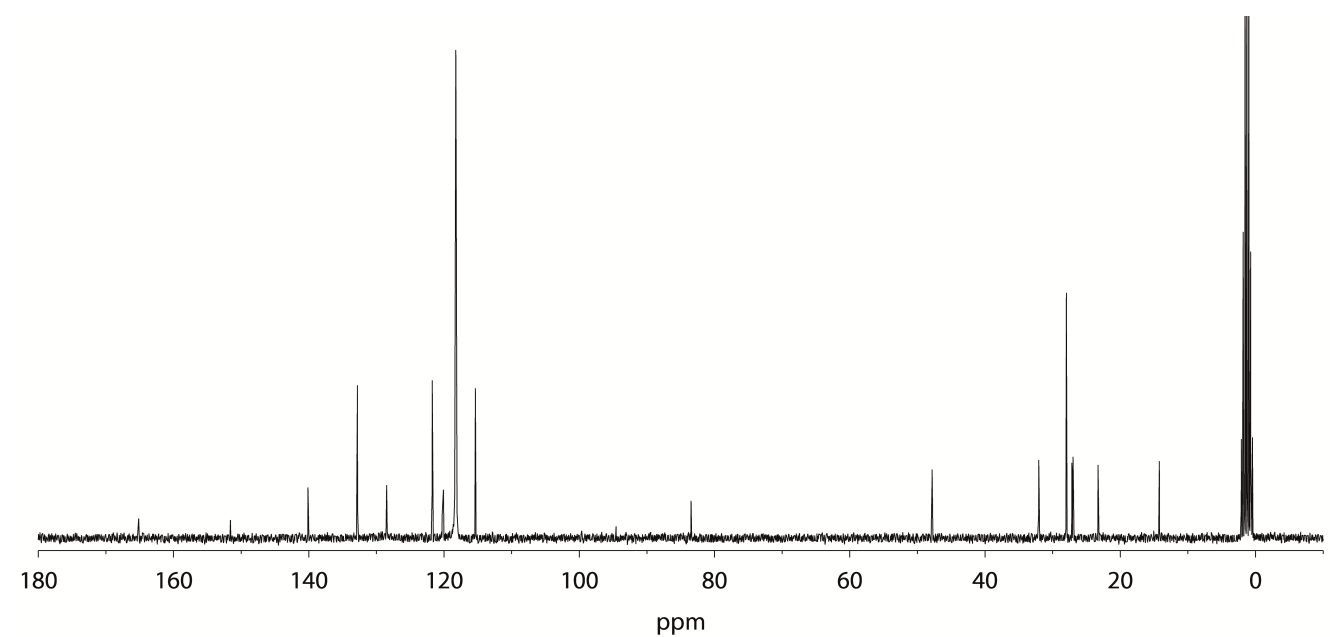

IR (ATR): $\tilde{\nu}\left(\mathrm{cm}^{-1}\right)=2924,2868,2193,1709,1601,1577,1507,1460,1377,1263,1231$, 1103, 751, 659 .

Melting point: $94-95{ }^{\circ} \mathrm{C}$

ESI-HRMS $\left(\left[\mathrm{C}_{24} \mathrm{H}_{26} \mathrm{~N}_{2} \mathrm{O}_{2}\right]^{+}\right): \quad$ measured: $\quad 375.2064$ 
<smiles>CCCCCCn1c2ccccc2c(=O)c2ccccc21</smiles>

33<smiles>CCCCCCN1c2ccccc2C(=C(C#N)C(=O)OCC)c2ccccc21</smiles>

35

Scheme VI.3.1.42: Synthesis of compound 35.

\section{VI.3.1.2 2-Cyano-2-(10-hexyl-9-acridinylidene)ethylacetate (35)}

$\mathrm{TiCl}_{4}(255 \mathrm{mg}, 1.34 \mathrm{mmol}, 1.25$ equiv.) was slowly added to $N$-hexyl-acridone (300 $\mathrm{mg}$, $1.07 \mathrm{mmol}, 1.00$ equiv.) solved in dry $\mathrm{CH}_{2} \mathrm{Cl}_{2}(15 \mathrm{~mL})$ and stirred for $15 \mathrm{~min}$ at room temperature. 2-cyanoethylacetate (243 mg, $2.15 \mathrm{mmol}, 2.00$ equiv.) and triethylamine (2 $\mathrm{mL}$ ) were added consecutively to the reaction mixture and heated under reflux for $24 \mathrm{~h}$. The cooled down reaction mixture was filtered through celite and the residue was washed with $\mathrm{CH}_{2} \mathrm{Cl}_{2}(2 \times 5 \mathrm{~mL})$. The solvent was removed in vacuo and the residue was purified by means of column chromatography $\left(\mathrm{SiO}_{2}\right.$, chloroform/methanol 40:1 $\rightarrow$ 20:1). The product 35 (316 mg, $0.84 \mathrm{mmol}, 79 \%$ ) was obtained as an orange solid.

${ }^{1} \mathbf{H}-\mathbf{N M R}\left(300 \mathrm{MHz}, 298 \mathrm{~K}, \mathrm{CD}_{3} \mathrm{CN}\right): \delta(\mathrm{ppm})=8.02\left(\mathrm{~d},{ }^{3} J=8.1 \mathrm{~Hz}, 2 \mathrm{H}, \mathrm{a} / \mathrm{a}^{\prime}\right), 7.62$ $\left(\mathrm{ddd},{ }^{3} J=8.6,7.0 \mathrm{~Hz},{ }^{4} J=1.5 \mathrm{~Hz}, 2 \mathrm{H}, \mathrm{c} / \mathrm{c}^{\prime}\right), 7.53\left(\mathrm{dd},{ }^{3} J=8.7 \mathrm{~Hz},{ }^{4} J=0.7 \mathrm{~Hz}, 2 \mathrm{H}\right.$, b/b'), $7.20\left(\mathrm{ddd},{ }^{3} J=8.1,7.0 \mathrm{~Hz},{ }^{4} J=1.1 \mathrm{~Hz}, 2 \mathrm{H}, \mathrm{d} / \mathrm{d}^{\prime}\right), 4.36-4.25\left(\mathrm{~m}, 2 \mathrm{H}, \mathrm{NCH}_{2}\right)$, $4,30\left(\mathrm{q},{ }^{3} \mathrm{~J}=7.1 \mathrm{~Hz}, 2 \mathrm{H}, \mathrm{OCH}_{2}\right), 1.92-1.81(\mathrm{~m}, 2 \mathrm{H}, \mathrm{NCH} 2 \mathrm{CH} 2), 1.49-1.41(\mathrm{~m}, 2 \mathrm{H}$, $\left.\mathrm{NCH}_{2} \mathrm{CH}_{2} \mathrm{CH}_{2}\right), 1.40-1.28\left(\mathrm{~m}, 4 \mathrm{H}, \mathrm{CH}_{2} \mathrm{CH}_{2} \mathrm{CH}_{3}\right), 1.17$ (t, $\left.{ }^{3} \mathrm{~J}=7.1 \mathrm{~Hz}, 3 \mathrm{H}, \mathrm{OCH}_{2} \mathrm{CH}_{3}\right)$, $0.91\left(\mathrm{t},{ }^{3} \mathrm{~J}=7.1 \mathrm{~Hz}, 3 \mathrm{H}, \mathrm{CH}_{2} \mathrm{CH}_{2} \mathrm{CH}_{3}\right)$.

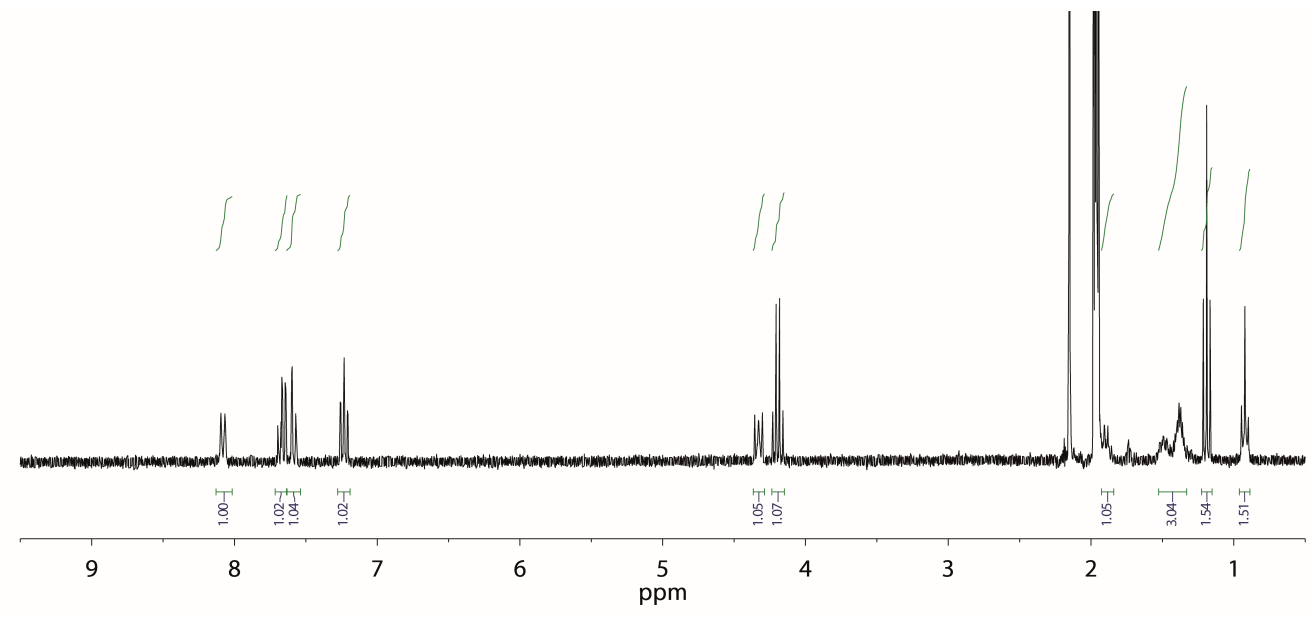


${ }^{13}$ C-NMR $\left(176 \mathrm{MHz}, 298 \mathrm{~K}, \mathrm{CD}_{3} \mathrm{CN}\right): \delta(\mathrm{ppm})=166.2,152.9,140.1,133.5$, $128.8,121.8,120.3,119.9,118.3,115.4,62.5,48.0,32.0,27.2,27.0,23.3,14.3$, 14.2 .

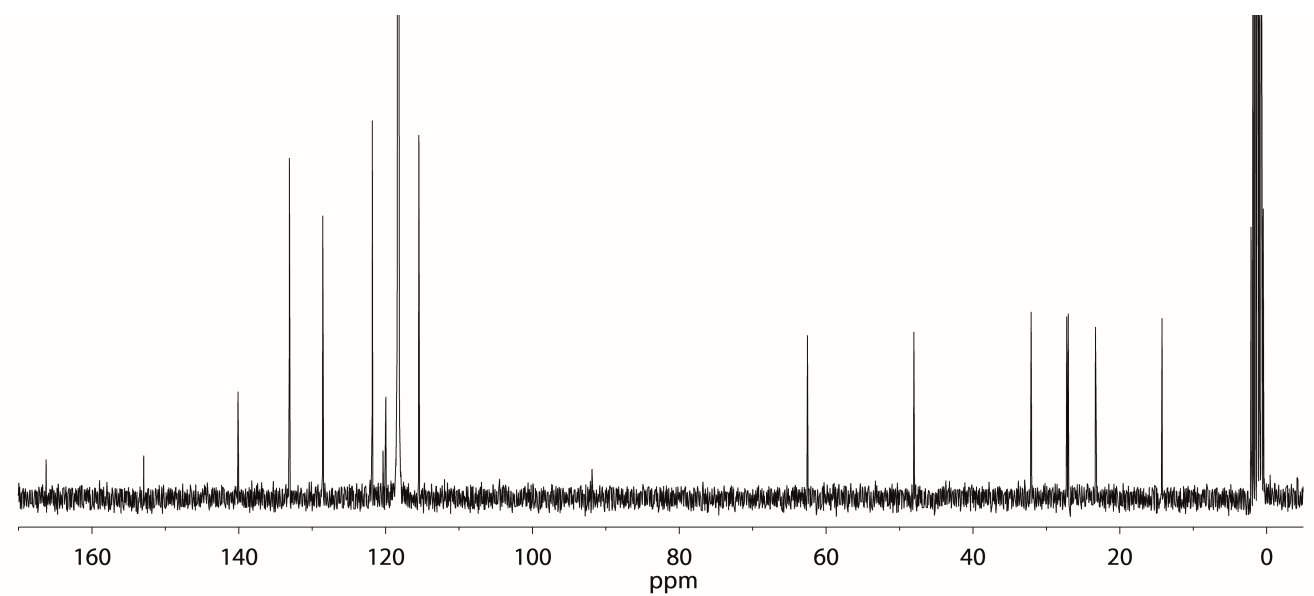

IR (ATR): $\tilde{\nu}\left(\mathrm{cm}^{-1}\right)=2924,2868,2193,1709,1601,1577,1507,1460,1377,1263,1231$, 1103, 751, 659 .

Melting point: $94-95{ }^{\circ} \mathrm{C}$

ESI-HRMS $\left(\left[\mathrm{C}_{24} \mathrm{H}_{26} \mathrm{~N}_{2} \mathrm{O}_{2}\right]^{+}\right): \quad$ measured: $\quad 375.2064$

calculated: $\quad 375.2067$ 
<smiles>N#CCC(=O)O</smiles>

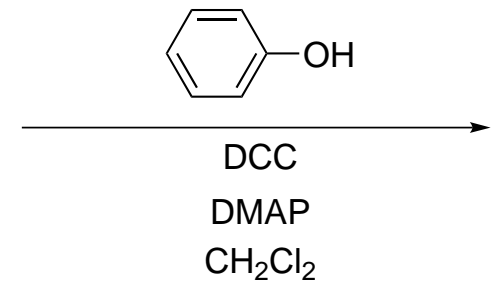<smiles>N#CCC(=O)Oc1ccccc1</smiles>

36

Scheme VI.3.1.43: Synthesis of compound 36.

\section{VI.3.1.3 2-Cyanophenylacetate (36)}

2-Cyanoacetic acid (1.00 g, $11.76 \mathrm{mmol}, 1.00$ equiv.), phenol (1.22 g, $12.93 \mathrm{mmol}, 1.10$ equiv.) 4,4'-dimethylaminpyridine (143 mg, $1.18 \mathrm{mmol}, 0.10$ equiv.) and $N, N$ '-dicyclohexyl carbodiimide (2.67 g, $12.93 \mathrm{mmol}, 1.10$ equiv.) in $\mathrm{CH}_{2} \mathrm{Cl}_{2}(15 \mathrm{~mL})$ were stirred at $0{ }^{\circ} \mathrm{C}$. The reaction mixture was allowed to warm up to room temperature over $12 \mathrm{~h}$. After that the reaction mixture was filtered through celite and the solvent was removed in vacuo. The residue was purified by means of column chromatography $\left(\mathrm{SiO}_{2}\right.$, pentane /ethyl acetate $6: 1 \rightarrow 2: 1)$. The product $(1.53 \mathrm{~g}, 9.50 \mathrm{mmol}, 81 \%)$ was obtained as red oil.

${ }^{1} \mathbf{H}-\mathbf{N M R}\left(300 \mathrm{MHz}, 298 \mathrm{~K}, \mathrm{CD}_{3} \mathrm{CN}\right): \delta(\mathrm{ppm})=7.47-7.38(\mathrm{~m}, 2 \mathrm{H}, \mathrm{c}), 7.35-7.29(\mathrm{~m}$, $1 \mathrm{H}, \mathrm{d}), 7.19-7.13$ (m, 2H, b), 3.73 (s, 2H, a).

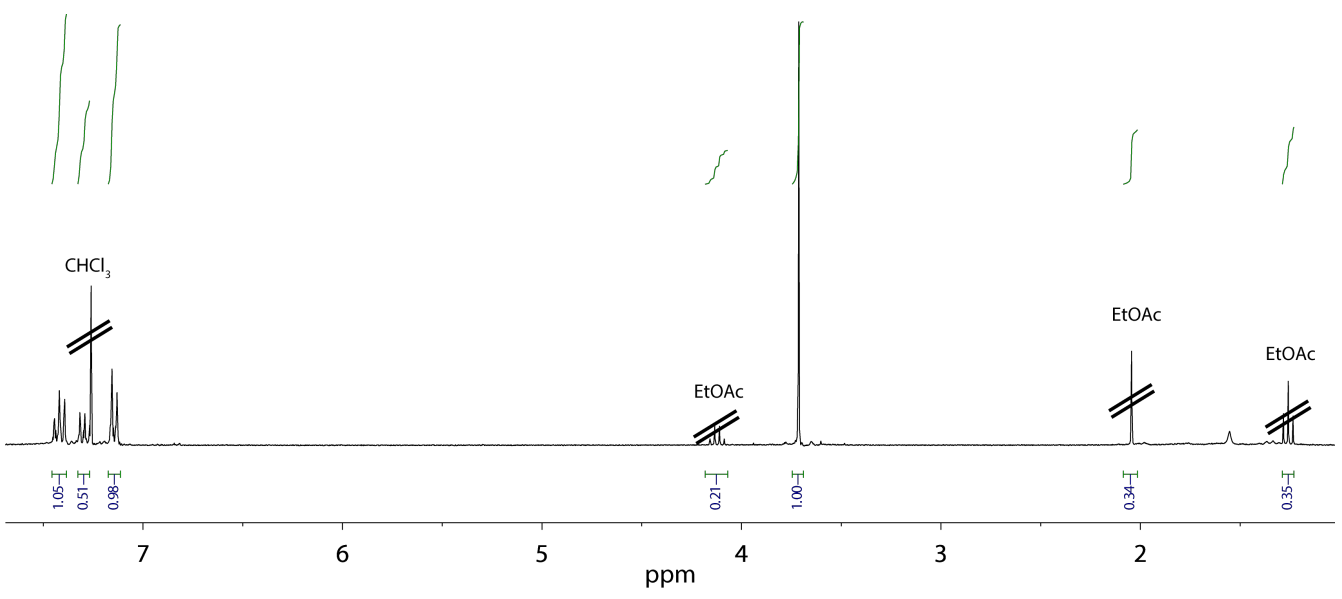

$\begin{array}{lll}\text { ESI-HRMS }\left(\left[\mathrm{C}_{28} \mathrm{H}_{26} \mathrm{~N}_{2} \mathrm{O}_{2}\right]^{+}\right): & \text {measured: } & 161.0423 \\ & \text { calculated: } & 161.0477\end{array}$


<smiles>CCCCCCn1c2ccccc2c(=O)c2ccccc21</smiles>

33

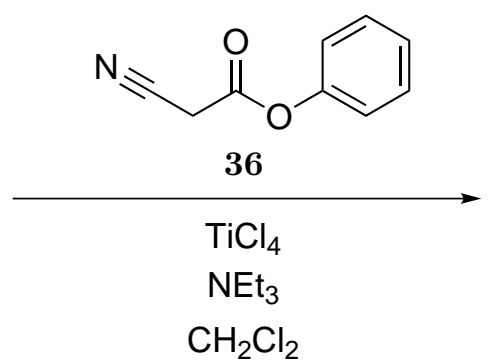

$\mathrm{CH}_{2} \mathrm{Cl}_{2}$<smiles>CCCCCCN1c2ccccc2C(=C(C#N)C(=O)Oc2ccccc2)c2ccccc21</smiles>

37

Scheme VI.3.1.44: Synthesis of compound 37 .

\section{VI.3.1.4 2-Cyano-2-(10-hexyl-9-acridinylidene)phenylacetate (37)}

$\mathrm{TiCl}_{4}$ (255 mg, $1.34 \mathrm{mmol}, 1.25$ equiv.) was slowly added to $N$-hexyl-acridone (300 $\mathrm{mg}$, $1.07 \mathrm{mmol}, 1.00$ equiv.) solved in dry $\mathrm{CH}_{2} \mathrm{Cl}_{2}(15 \mathrm{~mL})$ and stirred for $15 \mathrm{~min}$ at room temperature. 36 (346 mg, $2.15 \mathrm{mmol}, 2.00$ equiv.) and triehtylamine ( $2 \mathrm{~mL})$ were added consecutively to the reaction mixture and heated under reflux for $24 \mathrm{~h}$. The cooled down reaction mixture was filtered through celite and the residue was washed with $\mathrm{CH}_{2} \mathrm{Cl}_{2}(2 \mathrm{x}$ $5 \mathrm{~mL})$. The solvent was removed in vacuo and the residue was purified by means of column chromatography $\left(\mathrm{SiO}_{2}\right.$, chloroform/methanol 40:1 $\rightarrow$ 20:1). The product 37 (271 mg, 0.64 mmol, $60 \%$ ) was obtained as a dark purple solid.

${ }^{1} \mathbf{H}-\mathbf{N M R}\left(300 \mathrm{M} \mathrm{Hz}, 298 \mathrm{~K}, \mathrm{CD}_{3} \mathrm{CN}\right): \delta(\mathrm{ppm})=8.36\left(\mathrm{dd},{ }^{3} J=8.3 \mathrm{~Hz},{ }^{4} J=1.4 \mathrm{~Hz}, 2 \mathrm{H}\right.$, a/a'), $7.72\left(\mathrm{ddd},{ }^{3} J=8.5,6.8 \mathrm{~Hz},{ }^{4} J=1.5 \mathrm{~Hz}, 2 \mathrm{H}, \mathrm{c} / \mathrm{c}^{\prime}\right), 7.67\left(\mathrm{dd},{ }^{3} J=8.9 \mathrm{~Hz},{ }^{4} J=1.2\right.$ $\left.\mathrm{Hz}, 2 \mathrm{H}, \mathrm{d} / \mathrm{d}^{\prime}\right), 7.43-7.38(\mathrm{~m}, 2 \mathrm{H}, \mathrm{f}), 7.31$ (ddd, ${ }^{3} J=8.2,6.9 \mathrm{~Hz},{ }^{4} J=1.2 \mathrm{~Hz}, 2 \mathrm{H}, \mathrm{b} / \mathrm{b}$ '), $7.26\left(\mathrm{tt},{ }^{3} \mathrm{~J}=7.5 \mathrm{~Hz},{ }^{4} J=1.3 \mathrm{~Hz}, 1 \mathrm{H}, \mathrm{g}\right), 7.16-7.10(\mathrm{~m}, 2 \mathrm{H}, \mathrm{e}), 4.43-4.35$ (m, 2H, $\mathrm{NCH}_{2}$ ), $1.95-1.86\left(\mathrm{~m}, 2 \mathrm{H}, \mathrm{NCH}_{2} \mathrm{CH}_{2}\right), 1.54-1.44(\mathrm{~m}, 2 \mathrm{H}, \mathrm{NCH} 2 \mathrm{CH} 2 \mathrm{CH} 2), 1.42-1.28(\mathrm{~m}, 4 \mathrm{H}$, $\left.\mathrm{CH}_{2} \mathrm{CH}_{2} \mathrm{CH}_{3}\right), 0.90\left(\mathrm{t},{ }^{3} \mathrm{~J}=7.1 \mathrm{~Hz}, 3 \mathrm{H}, \mathrm{CH}_{2} \mathrm{CH}_{2} \mathrm{CH}_{3}\right.$ ). 


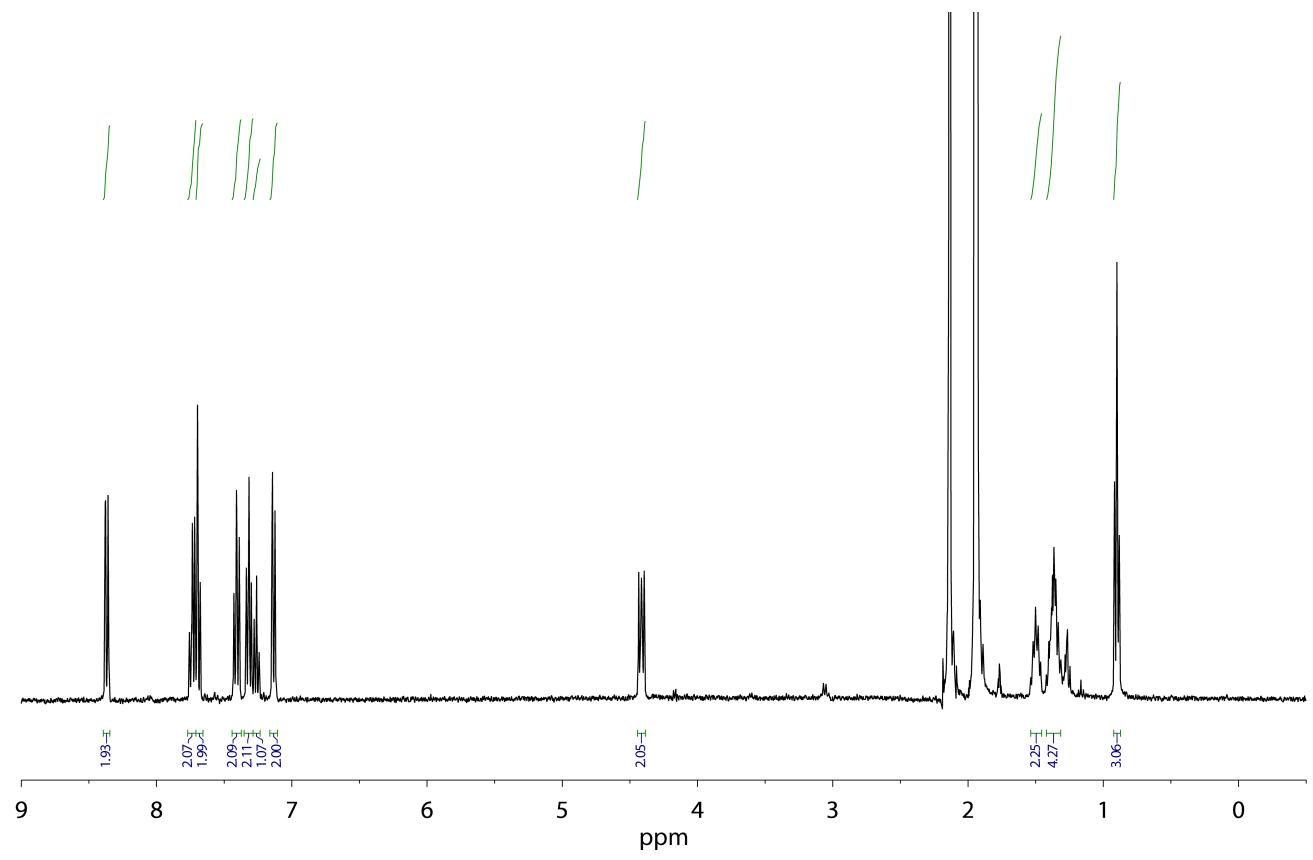

${ }^{13}$ C-NMR $\left(176 \mathrm{MHz}, 298 \mathrm{~K}, \mathrm{CD}_{3} \mathrm{CN}\right): \delta(\mathrm{ppm})=165.5,155.7,151.7,140.0,134.0$, 130.4, 129.3, 126.9, 122.7, 122.4, 121.1, 120.5, 116.0, 86.6, 48.6, 32.1, 27.5, 27.0, 23.3, 14.3 .

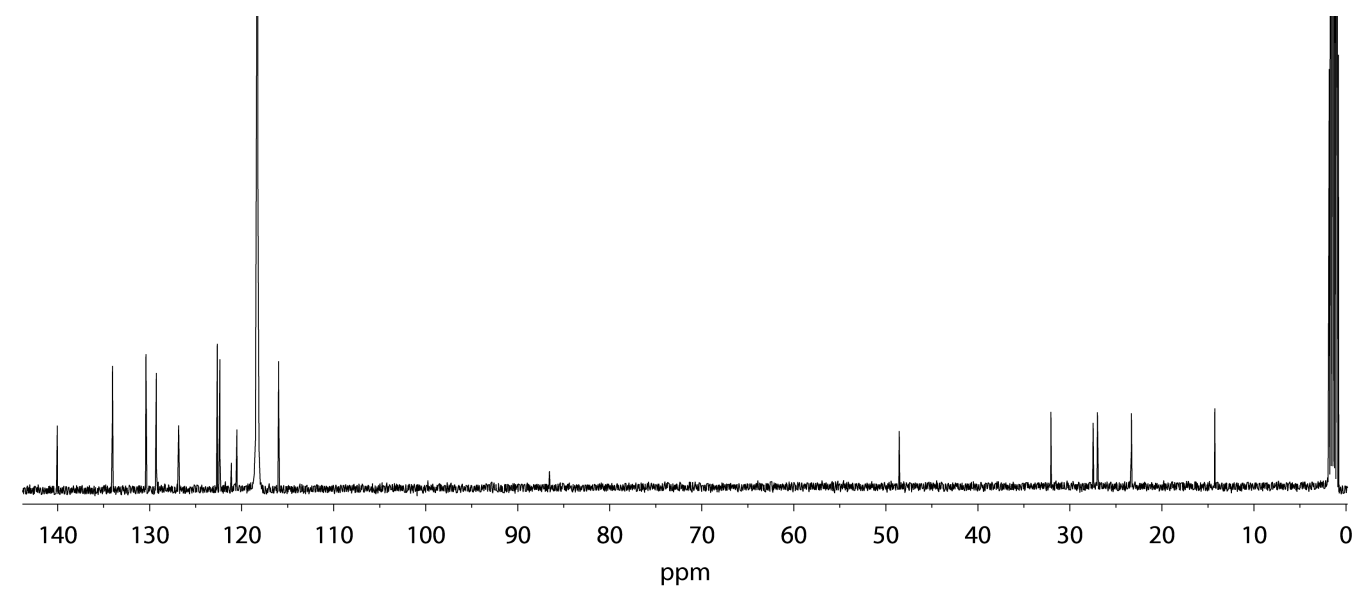

IR (ATR): $\tilde{\nu}\left(\mathrm{cm}^{-1}\right)=2172,1747,1669,1570,1487,1407,1364,1266,1178,1044,896$ $742,619,532$.

Melting point: $169-170{ }^{\circ} \mathrm{C}$.

ESI-HRMS $\left(\left[\mathrm{C}_{28} \mathrm{H}_{26} \mathrm{~N}_{2} \mathrm{O}_{2}\right]^{+}\right)$: $\quad$ measured: $\quad 423.2051$

calculated: $\quad 423.2067$ 


\section{VI.3.2 Syntheses of Ligand Derivatives}

\section{VI.3.2.1 2-(2,7-Di[(pyridin-3-yl)ethynyl)]10-hexyl-9- acridinylidene)malononitrile(40)}

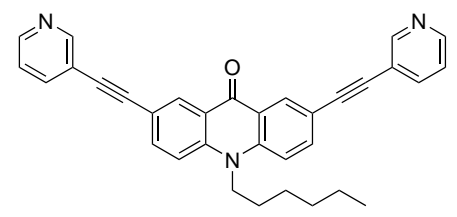

39

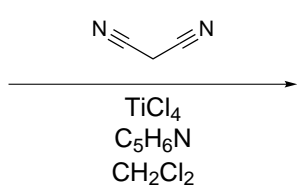

$\mathrm{CH}_{2} \mathrm{Cl}_{2}$

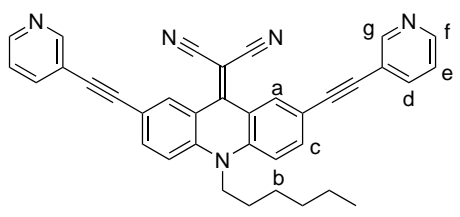

40

Scheme VI.3.2.45: Synthesis of compound 40.

$\mathrm{TiCl}_{4}$ (255 mg, $1.34 \mathrm{mmol}, 1.25$ equiv.) was slowly added to 39 (300 mg, $1.07 \mathrm{mmol}$, 1.00 equiv.) dissolved in dry $\mathrm{CH}_{2} \mathrm{Cl}_{2}(15 \mathrm{~mL})$ and stirred for $15 \mathrm{~min}$ at room temperature. Malononitrile (142 mg, $2.15 \mathrm{mmol}, 2.00$ equiv.) and triethylamine (2 mL) were added consecutively to the reaction mixture and heated to $70{ }^{\circ} \mathrm{C}$ under reflux for $24 \mathrm{~h}$. The cooled down reaction mixture was filtered through celite and the residue was washed with $\mathrm{CH}_{2} \mathrm{Cl}_{2}(2 \times 50 \mathrm{~mL})$. The solvent was removed in vacuo and the residue was purified by column chromatography $\left(\mathrm{SiO}_{2}\right.$, chloroform / methanol 40:1 $\rightarrow$ 20:1) followed by purification via GPC. The product (482 $\mathrm{mg}, 0.91 \mathrm{mmol}, 85 \%$ ) was obtained as an orange solid.

${ }^{1} \mathbf{H}-\mathbf{N M R}\left(300 \mathrm{M} \mathrm{Hz}, 298 \mathrm{~K}, \mathrm{CD}_{3} \mathrm{CN}\right): \delta(\mathrm{ppm})=8.77(\mathrm{dd}, 4 \mathrm{~J}=2.2 \mathrm{~Hz}, 2 \mathrm{H}, \mathrm{g}), 8.61(\mathrm{~d}$, $4 \mathrm{~J}=1.9 \mathrm{~Hz}, 2 \mathrm{H}, \mathrm{a}), 8.57(\mathrm{dd}, 3 \mathrm{~J}=4.9,4 \mathrm{~J}=1.7 \mathrm{~Hz}, 2 \mathrm{H}, \mathrm{f}), 7.92(\mathrm{dt}, 3 \mathrm{~J}=7.9,4 \mathrm{~J}=2.0$ $\mathrm{Hz}, 2 \mathrm{H}, \mathrm{d}), 7.90(\mathrm{dd}, 3 \mathrm{~J}=3.7,4 \mathrm{~J}=1.9 \mathrm{~Hz}, 2 \mathrm{H}, \mathrm{c}), 7.72(\mathrm{~d}, 3 \mathrm{~J}=9.1 \mathrm{~Hz}, 2 \mathrm{H}, \mathrm{b}), 7.40$ $(\mathrm{dd}, 3 \mathrm{~J}=7.9,4 \mathrm{~J}=4.9,2 \mathrm{H}, \mathrm{e}), 4.44-4.31(\mathrm{~m}, 2 \mathrm{H}, \mathrm{NCH} 2), 1.95-1.83(\mathrm{~m}, 2 \mathrm{H}, \mathrm{CH} 2)$ $1.61-1.44(\mathrm{~m}, 2 \mathrm{H}, \mathrm{CH} 2), 1.43-1.32(\mathrm{~m}, 4 \mathrm{H}, \mathrm{CH} 2 \mathrm{CH} 2 \mathrm{CH} 3)$, ), $0.94(\mathrm{t}, \mathrm{J}=7.0 \mathrm{~Hz}, 3 \mathrm{H}$, CH3). 


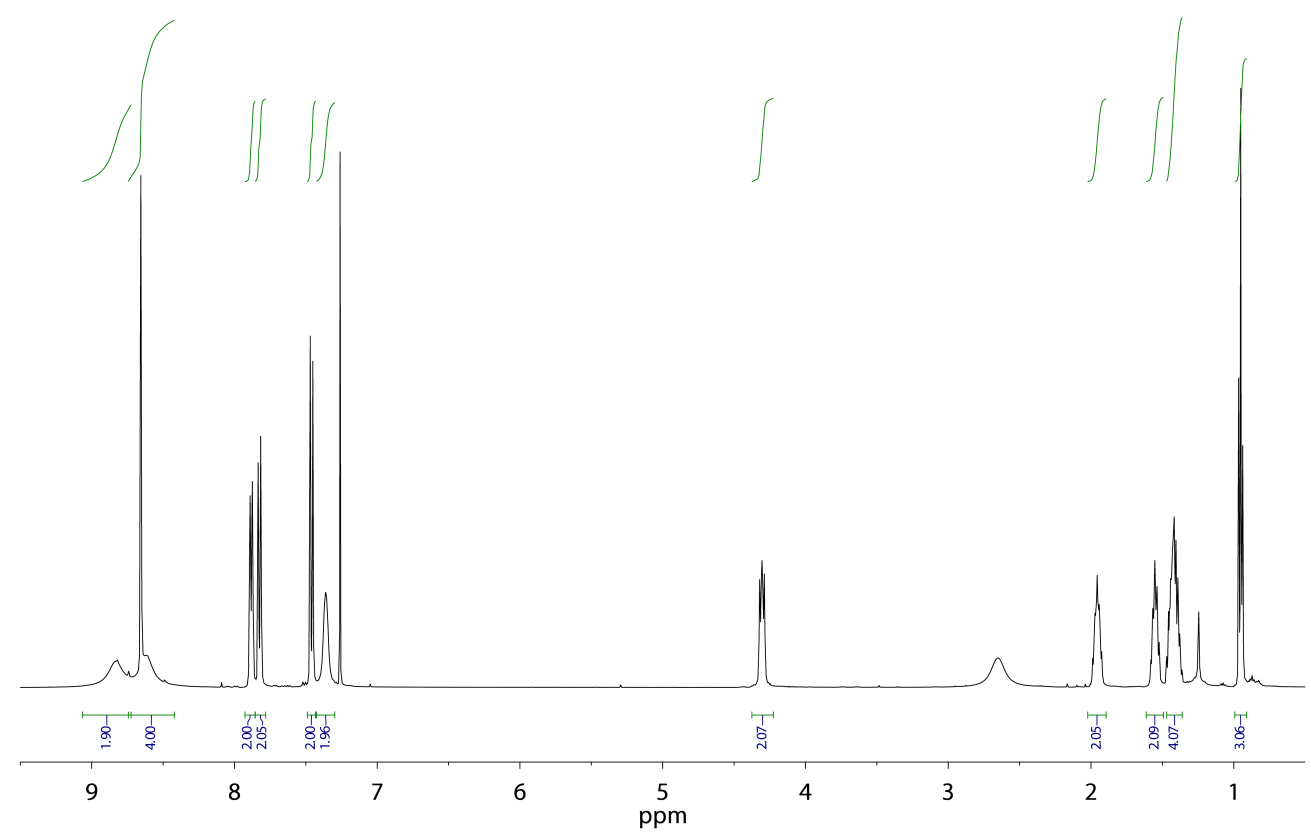

${ }^{13}$ C-NMR $\left(176 \mathrm{MHz}, 298 \mathrm{~K}, \mathrm{CD}_{3} \mathrm{CN}\right): \delta(\mathrm{ppm})=154.4,151.8,148.3,139.2,138.5,136.5$, 130.3, 123.5, 118.2, 116.8, 116.6, 114.9, 91.33, 87.49, 77.4, 70.7, 48.4, 31.5, 26.9, 26.7, 22.7, 14.1.

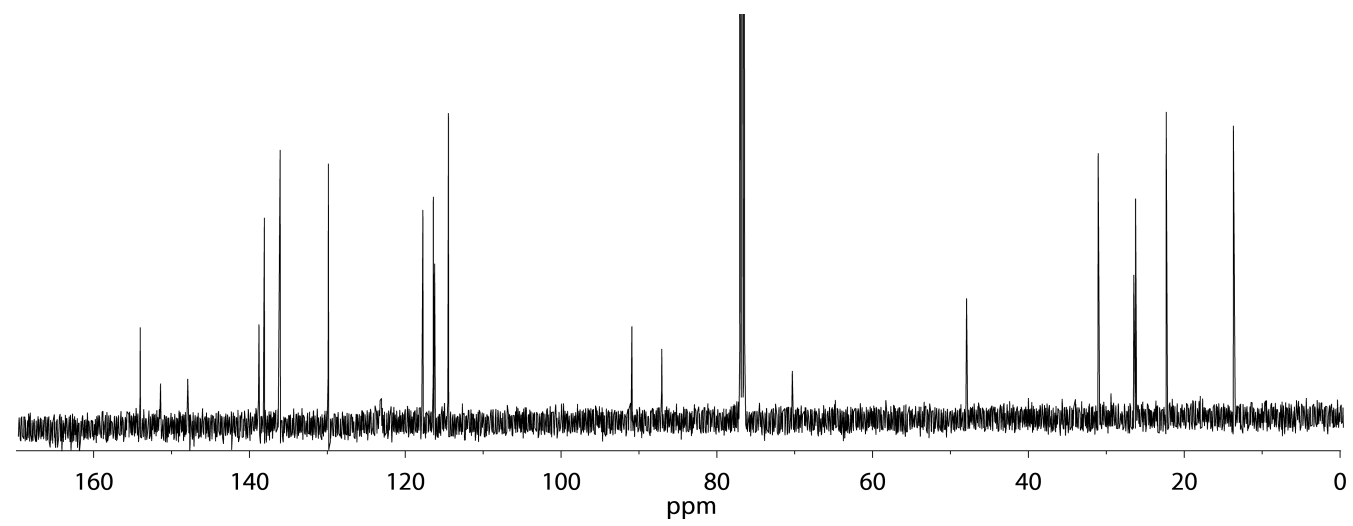

IR (ATR): $\tilde{\nu}\left(\mathrm{cm}^{-1}\right)=2924,2854,2206,1562,1480,1465,1368,1263,1186,1021,895$, $820,806,702,627,588$.

UV/Vis $(\mathbf{M e C N}): \lambda_{\max }(\mathrm{nm})\left[\varepsilon\left(\mathrm{dm} \mathrm{mol}^{-3} \mathrm{~cm}^{-1}\right)\right]=287$ [48500], 347 [62300], 505 [16000].

ESI-HRMS $\left(\left[\mathrm{C}_{38} \mathrm{H}_{32} \mathrm{~N}_{4} \mathrm{O}_{2}\right]^{+}\right)$: $\quad$ measured: $\quad 530.2326$ 
calculated: $\quad 530.2339$

EA: $\left(\mathrm{C}_{38} \mathrm{H}_{32} \mathrm{~N}_{4} \mathrm{O}_{2} \times \mathrm{H}_{2} \mathrm{O}\right) \quad$ calculated: $\quad \% \mathrm{C} 79.0 \quad \% \mathrm{H} 5.4 \quad \% \mathrm{~N} 12.8$

found: $\quad \% \mathrm{C} 79.8 \quad \% \mathrm{H} 5.8 \quad \% \mathrm{~N} 12.2$ 


\section{VI.3.2.2 2-Cyano-2-(2,7-di[(pyridin-3-yl)ethynyl)]10-hexyl-9- acridinylidene)tert-butylacetate (41)}

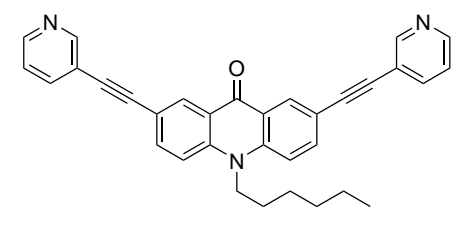

39

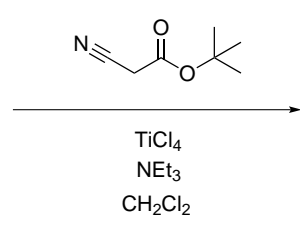

$\mathrm{CH}_{2} \mathrm{Cl}_{2}$

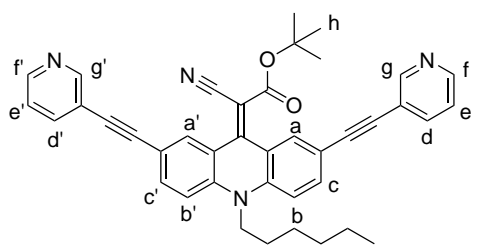

41

Scheme VI.3.2.46: Synthesis of compound 41.

$\mathrm{TiCl}_{4}$ (255 mg, $1.34 \mathrm{mmol}, 1.25$ equiv.) was slowly added to 39 (300 mg, $1.07 \mathrm{mmol}$, 1.00 equiv.) dissolved in dry $\mathrm{CH}_{2} \mathrm{Cl}_{2}(15 \mathrm{~mL})$ and stirred for $15 \mathrm{~min}$ at room temperature. 2-Cyano-tert-butylacetate (304 mg, $2.15 \mathrm{mmol}, 2.00$ equiv.) and triethylamine (2 mL) were added consecutively to the reaction mixture and heated to $70{ }^{\circ} \mathrm{C}$ under reflux for $24 \mathrm{~h}$. The cooled down reaction mixture was filtered through celite and the residue was washed with $\mathrm{CH}_{2} \mathrm{Cl}_{2}(2 \times 50 \mathrm{~mL})$. The solvent was removed in vacuo and the residue was purified by means of column chromatography $\left(\mathrm{SiO}_{2}\right.$, chloroform / methanol 40:1 $\rightarrow$ 20:1) followed by purification via GPC. The product $41(214 \mathrm{mg}, 0.36 \mathrm{mmol}, 33 \%)$ was obtained as an orange solid.

${ }^{1}$ H-NMR $\left(300 \mathrm{M} \mathrm{Hz}, 298 \mathrm{~K}, \mathrm{CD}_{3} \mathrm{CN}\right): \delta(\mathrm{ppm})=8.74(\mathrm{~s}, 2 \mathrm{H}, \mathrm{g} / \mathrm{g}$ '), 8.71 (s, 1H, a'), $8.54\left(\mathrm{dd},{ }^{3} J=5.0 \mathrm{~Hz},{ }^{4} J=1.7 \mathrm{~Hz}, 2 \mathrm{H}, \mathrm{f} / \mathrm{f}^{\prime}\right), 7.80\left(\mathrm{dt},{ }^{3} J=7.9 \mathrm{~Hz},{ }^{4} J=2.0 \mathrm{~Hz}, 2 \mathrm{H}\right.$, d/d'), 7.76 (s, 1H, a), 7.69 (dd, $\left.{ }^{3} J=9.0 \mathrm{~Hz},{ }^{4} J=2.0 \mathrm{~Hz}, 2 \mathrm{H}, \mathrm{c} / \mathrm{c}^{\prime}\right), 7.29$ (d, ${ }^{3} J=8.9$ $\mathrm{Hz}, 2 \mathrm{H}, \mathrm{b} / \mathrm{b}$ ), 7.28 (dt, $\left.{ }^{3} J=7.9 \mathrm{~Hz},{ }^{4} J=4.8 \mathrm{~Hz}, 2 \mathrm{H}, \mathrm{e} / \mathrm{e}^{*}\right), 4.23-4.09\left(\mathrm{~m}, 2 \mathrm{H}, \mathrm{NCH}_{2}\right.$ ), $1.90-1.81\left(\mathrm{~m}, 2 \mathrm{H}, \mathrm{CH}_{2}\right), 1.48(\mathrm{~s}, 9 \mathrm{H}, \mathrm{h}), 1.51-1.45\left(\mathrm{~m}, 2 \mathrm{H}, \mathrm{CH}_{2}\right), 1.43-1.32(\mathrm{~m}, 4 \mathrm{H}$, $\left.\mathrm{CH}_{2} \mathrm{CH}_{2} \mathrm{CH}_{3}\right), 0.93\left(\mathrm{t},{ }^{3} \mathrm{~J}=7.0 \mathrm{~Hz}, 3 \mathrm{H}, \mathrm{CH}_{3}\right)$. 


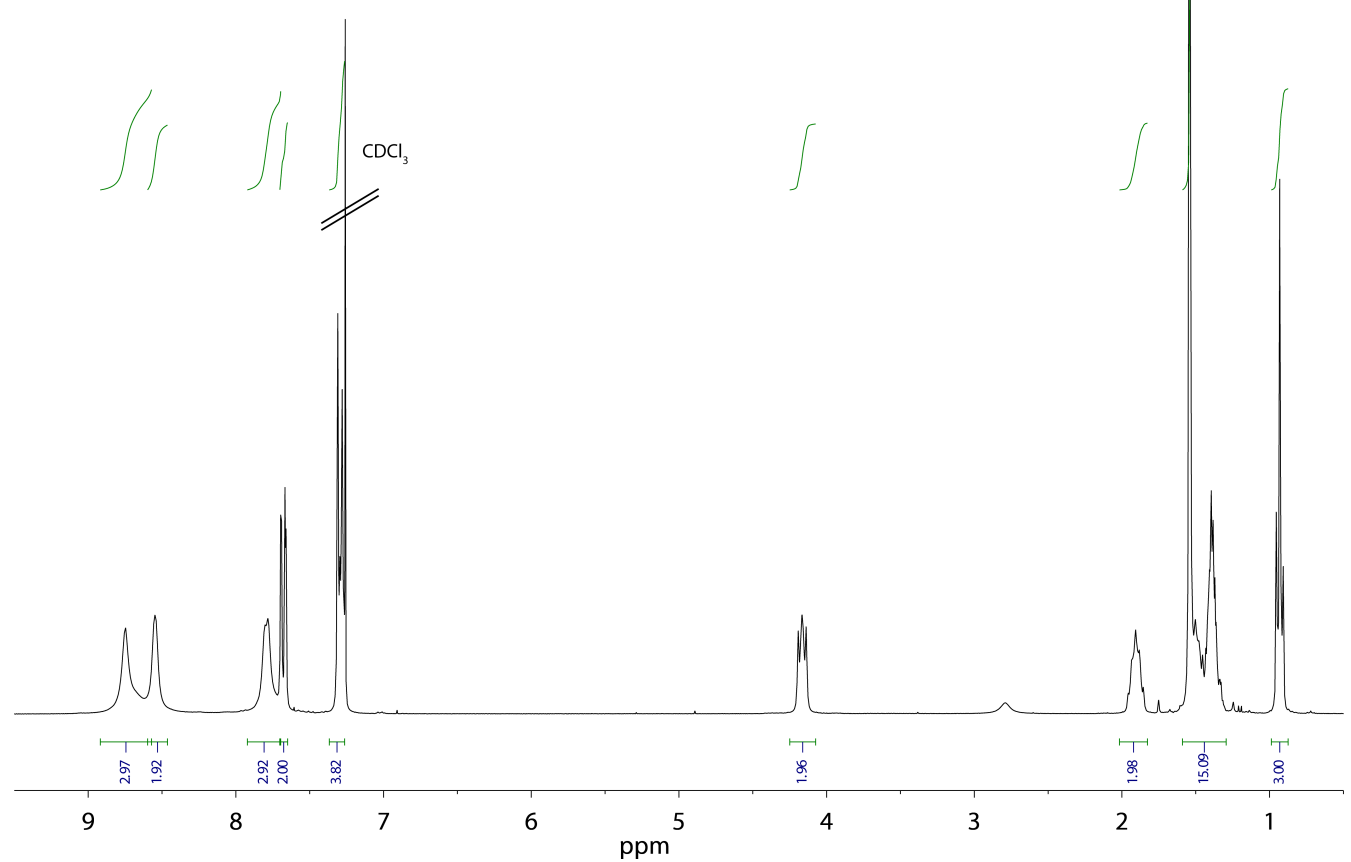

${ }^{13}$ C-NMR $\left(176 \mathrm{MHz}, 298 \mathrm{~K}, \mathrm{CD}_{3} \mathrm{CN}\right): \delta(\mathrm{ppm})=163.7,151.7,148.2,147.7,139.1,138.7$, 134.7, 132.7, 129.9, 123.6, 118.4, 114.2, 97.3, 92.1, 86.6, 83.9, 47.8, 31.5, 29.8, 27.9, 26.7, $26.6,22.8,14.1$.

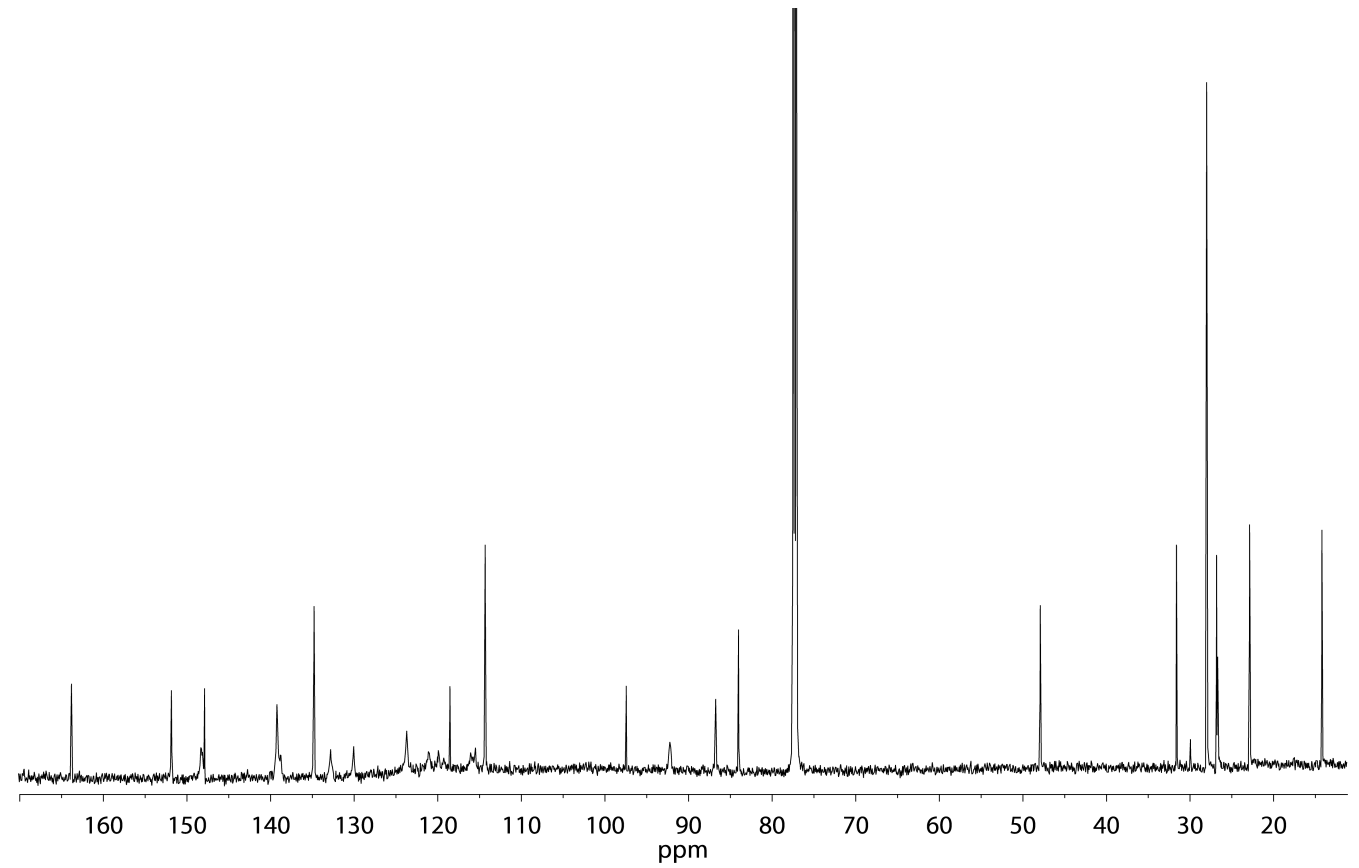

IR (ATR): $\tilde{\nu}\left(\mathrm{cm}^{-1}\right)=2932,2198,1719,1575,1481,1460,1370,1250,1163,1144,1105$, 1022, 840, 801, 702 . 
UV/Vis (MeCN): $\lambda_{\max }(\mathrm{nm})\left[\left(\mathrm{dm}^{3} \mathrm{~mol}^{-1} \mathrm{~cm}^{-1}\right)\right]=273$ [45900], 367 [38800], 466 [9800].

Melting point: $169-170{ }^{\circ} \mathrm{C}$.

ESI-HRMS $\left(\left[\mathrm{C}_{42} \mathrm{H}_{33} \mathrm{~N}_{4} \mathrm{O}_{2}\right]^{+}\right)$: $\quad$ measured: $\quad 605.2838$

calculated: $\quad 605.2822$

EA: $\left(\mathrm{C}_{42} \mathrm{H}_{32} \mathrm{~N}_{4} \mathrm{O}_{2} \times \mathrm{H}_{2} \mathrm{O}\right) \quad$ calculated: $\quad \% \mathrm{C} 77.2 \quad \% \mathrm{H} 6.2 \quad \% \mathrm{~N} 9.0$

found: $\quad$ \% $77.6 \quad \% \mathrm{H} 6.1 \quad \% \mathrm{~N} 8.9$ 


\section{VI.3.2.3 2-Cyano-2-(2,7-di[(pyridin-3-yl)ethynyl)]10-hexyl-9- acridinylidene)ethylacetate (42)}

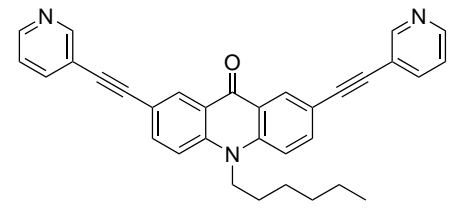

39

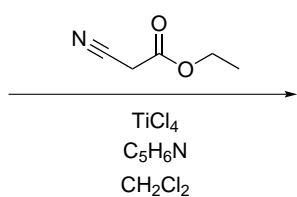

$\mathrm{CH}_{2} \mathrm{Cl}_{2}$

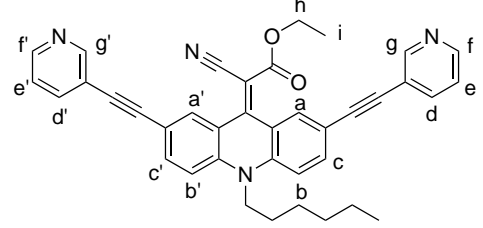

42

Scheme VI.3.2.47: Synthesis of compound 42.

$\mathrm{TiCl}_{4}$ (255 mg, $1.34 \mathrm{mmol}, 1.25$ equiv.) was slowly added to 39 (300 mg, $1.07 \mathrm{mmol}$, 1.00 equiv.) dissolved in dry $\mathrm{CH}_{2} \mathrm{Cl}_{2}(15 \mathrm{~mL})$ and stirred for $15 \mathrm{~min}$ at room temperature. 2-Cyanoethylacetate (244 mg, $2.15 \mathrm{mmol}, 2.00$ equiv.) and triethylamine (2 mL) were added consecutively to the reaction mixture and heated to $70{ }^{\circ} \mathrm{C}$ under reflux for $24 \mathrm{~h}$. The cooled down reaction mixture was filtered through celite and the residue was washed with $\mathrm{CH}_{2} \mathrm{Cl}_{2}(2 \times 50 \mathrm{~mL})$. The solvent was removed in vacuo and the residue was purified by column chromatography $\left(\mathrm{SiO}_{2}\right.$, chloroform / methanol 40:1 $\rightarrow$ 20:1) followed by purification via GPC. The product $42(481 \mathrm{mg}, 0.83 \mathrm{mmol}, 78 \%)$ was obtained as a red solid.

${ }^{1} \mathbf{H}-\mathbf{N M R}\left(300 \mathrm{M} \mathrm{Hz}, 298 \mathrm{~K}, \mathrm{CD}_{3} \mathrm{CN}\right): \delta(\mathrm{ppm})=8.78\left(\mathrm{~s}, 2 \mathrm{H}, \mathrm{g} / \mathrm{g}\right.$ '), $8.60\left(\mathrm{dd},{ }^{3} J=5.0\right.$ $\left.\mathrm{Hz},{ }^{4} J=1.7 \mathrm{~Hz}, 2 \mathrm{H}, \mathrm{f} / \mathrm{f}^{\prime}\right), 7.86\left(\mathrm{dt},{ }^{3} J=7.9 \mathrm{~Hz},{ }^{4} J=2.0 \mathrm{~Hz}, 2 \mathrm{H}, \mathrm{d} / \mathrm{d}^{\prime}\right), 7.72\left(\mathrm{dd},{ }^{3} J=\right.$ $\left.9.0 \mathrm{~Hz},{ }^{4} J=2.0 \mathrm{~Hz}, 2 \mathrm{H}, \mathrm{c} / \mathrm{c}^{\prime}\right), 7.34\left(\mathrm{~d},{ }_{3} J=8.9 \mathrm{~Hz}, 2 \mathrm{H}, \mathrm{b} / \mathrm{b}\right), 7.34\left(\mathrm{dt},{ }^{3} J=7.9 \mathrm{~Hz},{ }^{4} J\right.$ $\left.=4.8 \mathrm{~Hz}, 2 \mathrm{H}, \mathrm{e} / \mathrm{e}^{*}\right), 4.30\left(\mathrm{q},{ }^{3} J=7.2 \mathrm{~Hz}, 2 \mathrm{H}, \mathrm{h}\right), 4.24-4.18\left(\mathrm{~m}, 2 \mathrm{H}, \mathrm{NCH}_{2}\right), 2.01-1.88$ $\left(\mathrm{m}, 2 \mathrm{H}, \mathrm{CH}_{2}\right), 1.59-1.48\left(\mathrm{~m}, 2 \mathrm{H}, \mathrm{CH}_{2}\right), 1.47-1.33\left(\mathrm{~m}, 4 \mathrm{H}, \mathrm{CH}_{2} \mathrm{CH}_{2} \mathrm{CH}_{3}\right), 1.31\left(\mathrm{t},{ }^{3} \mathrm{~J}=\right.$ $7.1 \mathrm{~Hz}, 3 \mathrm{H}, \mathrm{i}), 0.94\left(\mathrm{t},{ }^{3} \mathrm{~J}=7.0 \mathrm{~Hz}, 3 \mathrm{H}, \mathrm{CH}_{3}\right)$. 


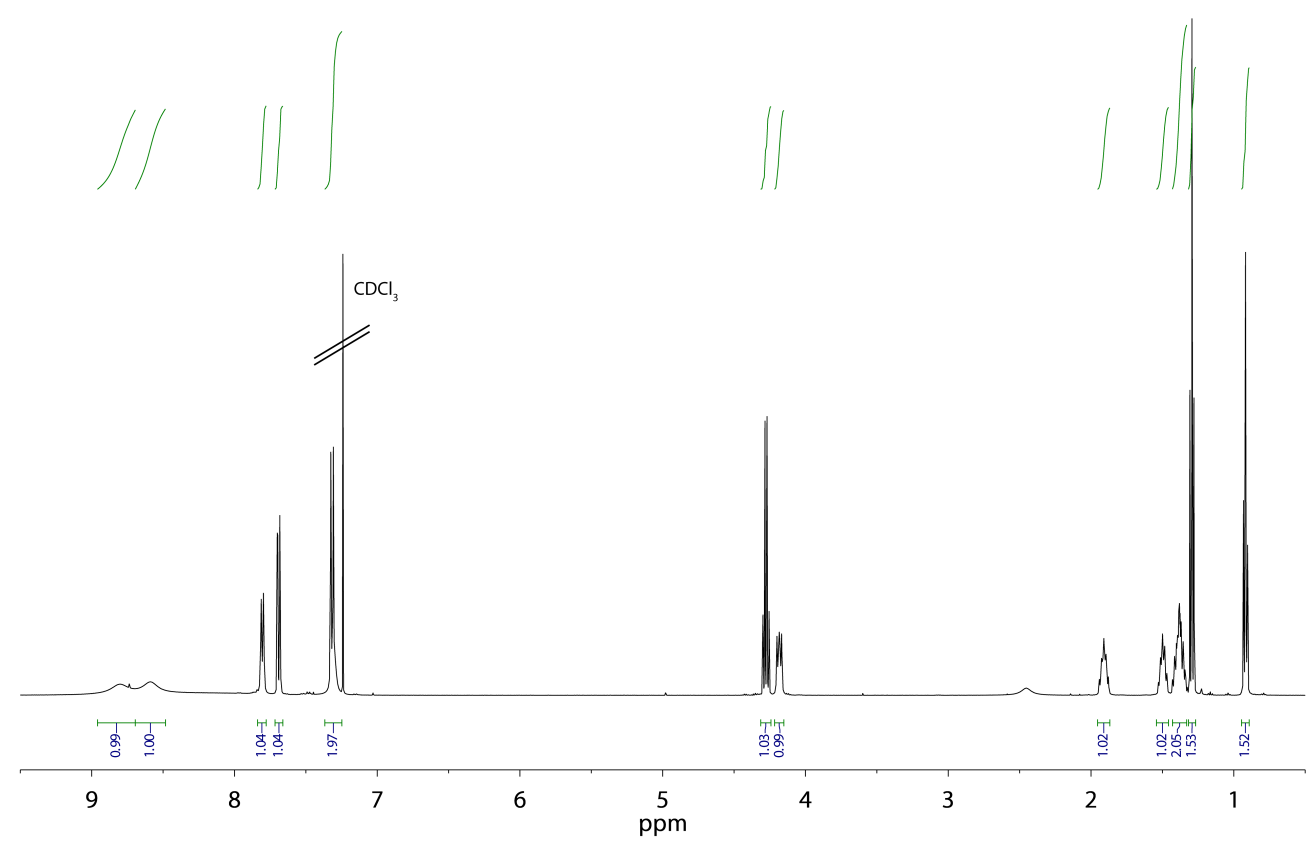

${ }^{13}$ C-NMR $\left(176 \mathrm{MHz}, 298 \mathrm{~K}, \mathrm{CD}_{3} \mathrm{CN}\right): \delta(\mathrm{ppm})=164.6,151.6,149.7,147.9,139.5,138.9$, $134.9,123.5,118.3,115.6,114.2,94.7,91.9,86.6,77.25,62.3,47.8,31.4,26.6,26.5,24.6$, $22.6,14.1,14.0$.

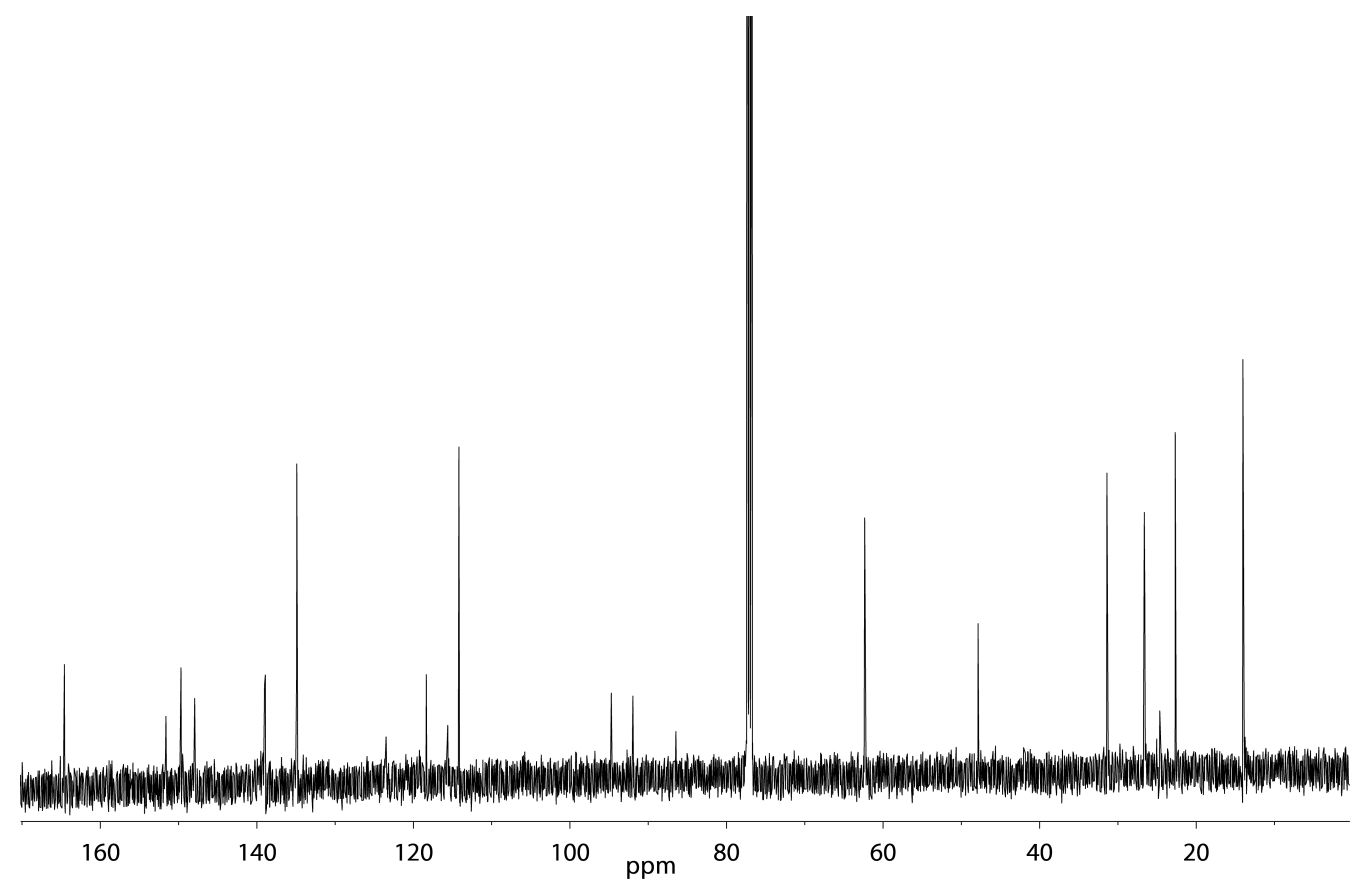

IR (ATR): $\tilde{\nu}\left(\mathrm{cm}^{-1}\right)=2926,2207,1716,1522,1478,1465,1372,1235,1183,1102,1021$, $823,804,702$. 
UV/Vis (MeCN): $\lambda_{\max }(\mathrm{nm})\left[\left(\mathrm{dm}^{3} \mathrm{~mol}^{-1} \mathrm{~cm}^{-1}\right)\right]=272$ [48000], 340 [44600], 472 [10600]

Melting point: $148-149{ }^{\circ} \mathrm{C}$.

ESI-HRMS $\left(\left[\mathrm{C}_{38} \mathrm{H}_{32} \mathrm{~N}_{4} \mathrm{O}_{2}\right]^{+}\right)$: $\quad$ measured: $\quad 577.2577$

calculated: $\quad 577.2598$

EA: $\left(\mathrm{C}_{38} \mathrm{H}_{32} \mathrm{~N}_{4} \mathrm{O}_{2} \times \mathrm{H}_{2} \mathrm{O}\right) \quad$ calculated: $\quad \% \mathrm{C} 76.7 \quad \% \mathrm{H} 5.8 \quad \% \mathrm{~N} 9.4$

found: $\quad \% \mathrm{C} 76.3 \quad \% \mathrm{H} 5.9 \quad \% \mathrm{~N} 9.2$ 


\section{VI.3.2.4 2-Cyano-2-(2,7-di[(pyridin-3-yl)ethynyl)]10-hexyl-9- acridinylidene)phenylacetate(43)}

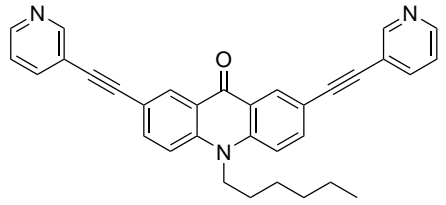

39

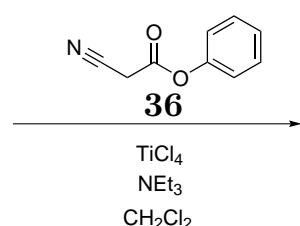

$\mathrm{CH}_{2} \mathrm{Cl}_{2}$

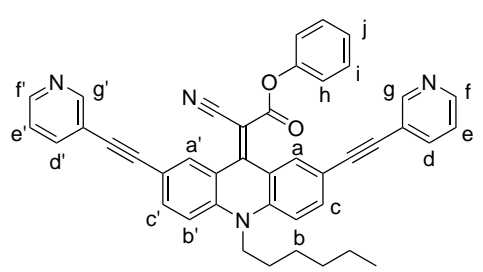

43

Scheme VI.3.2.48: Synthesis of compound 43.

$\mathrm{TiCl}_{4}$ (255 mg, $1.34 \mathrm{mmol}, 1.25$ equiv.) was slowly added to 39 (300 mg, $1.07 \mathrm{mmol}, 1.00$ equiv.) solved in dry $\mathrm{CH}_{2} \mathrm{Cl}_{2}(15 \mathrm{~mL})$ and stirred for $15 \mathrm{~min}$ at room temperature. 36 (346 mg, $2.15 \mathrm{mmol}, 2.00$ equiv.) and triethylamine $(2 \mathrm{~mL})$ were added consecutively to the reaction mixture and heated to $70{ }^{\circ} \mathrm{C}$ under reflux for $24 \mathrm{~h}$. The cooled down reaction mixture was filtered through celite and the residue was washed with $\mathrm{CH}_{2} \mathrm{Cl}_{2}$ (2 $\mathrm{x} 50 \mathrm{~mL}$ ). The solvent was removed in vacuo and the residue was purified by means of column chromatography $\left(\mathrm{SiO}_{2}\right.$, chloroform / methanol 40:1 $\rightarrow$ 20:1) followed by purification via GPC. The product (153 $\mathrm{mg}, 0.25 \mathrm{mmol}, 23 \%$ ) was obtained as a dark red solid.

${ }^{1} \mathbf{H}-\mathbf{N M R}\left(300 \mathrm{M} \mathrm{Hz}, 298 \mathrm{~K}, \mathrm{CD}_{3} \mathrm{CN}\right): \delta(\mathrm{ppm})=8.76\left(\mathrm{~s}, 2 \mathrm{H}, \mathrm{g} / \mathrm{g}\right.$ '), $8.57\left(\mathrm{dd},{ }^{3} J=5.0\right.$ $\left.\mathrm{Hz},{ }^{4} J=1.7 \mathrm{~Hz}, 2 \mathrm{H}, \mathrm{f} / \mathrm{f}^{\prime}\right), 8.42\left(\mathrm{~d},{ }^{4} J=2.0 \mathrm{~Hz}, 2 \mathrm{H}, \mathrm{a} / \mathrm{a}\right), 7.90\left(\mathrm{dt},{ }^{3} J=7.9 \mathrm{~Hz},{ }^{4} J=\right.$ $\left.2.0 \mathrm{~Hz}, 2 \mathrm{H}, \mathrm{d} / \mathrm{d}^{\prime}\right), 7.82\left(\mathrm{dd},{ }^{3} J=9.0 \mathrm{~Hz},{ }^{4} J=2.0 \mathrm{~Hz}, 2 \mathrm{H}, \mathrm{c} / \mathrm{c}^{\prime}\right), 7.65\left(\mathrm{~d},{ }^{3} J=8.9 \mathrm{~Hz}, 2 \mathrm{H}\right.$, b/b'), $7.41-7.35$ (m, 4H, e/e*, i), $7.27-7.23$ (m, 1H, j), $7.22-7.18$ (m, 2H, h), $4.37-$ $4.30\left(\mathrm{~m}, 2 \mathrm{H}, \mathrm{NCH}_{2}\right), 1.92-1.83\left(\mathrm{~m}, 2 \mathrm{H}, \mathrm{CH}_{2}\right), 1.52-1.45\left(\mathrm{~m}, 2 \mathrm{H}, \mathrm{CH}_{2}\right), 1.42-1.30(\mathrm{~m}$, $\left.4 \mathrm{H}, \mathrm{CH}_{2} \mathrm{CH}_{2} \mathrm{CH}_{3}\right), 0.90$ (t, ${ }^{3} \mathrm{~J}=7.0 \mathrm{~Hz}, 3 \mathrm{H}, \mathrm{CH}_{3}$ ). 


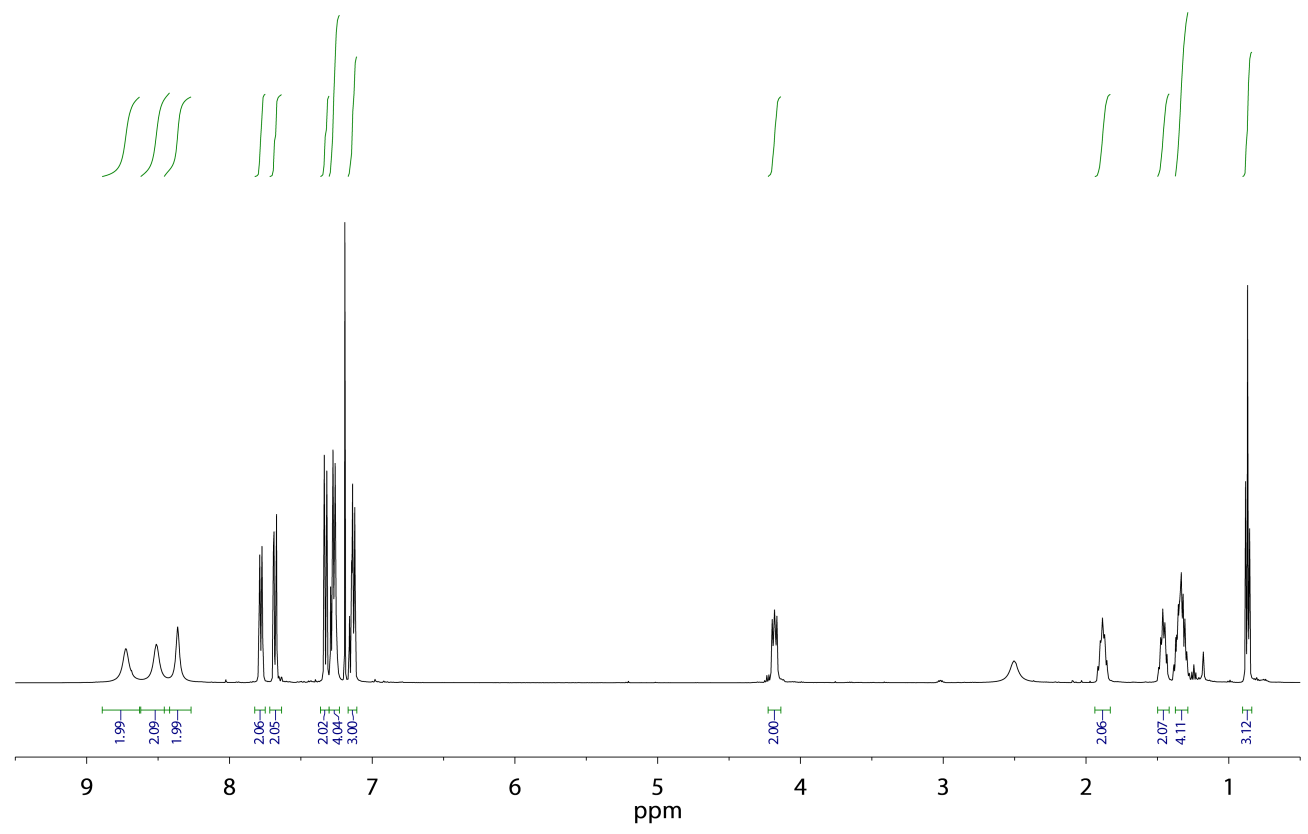

${ }^{13}$ C-NMR $\left(176 \mathrm{MHz}, 298 \mathrm{~K}, \mathrm{CD}_{3} \mathrm{CN}\right): \delta(\mathrm{ppm})=163.7,151.7,148.2,147.7,139.1,138.7$, 134.7, 132.7, 129.9, 123.6, 118.4, 114.2, 97.3, 92.1, 86.6, 83.9, 47.8, 31.5, 29.8, 27.9, 26.7, $26.6,22.8,14.1$.

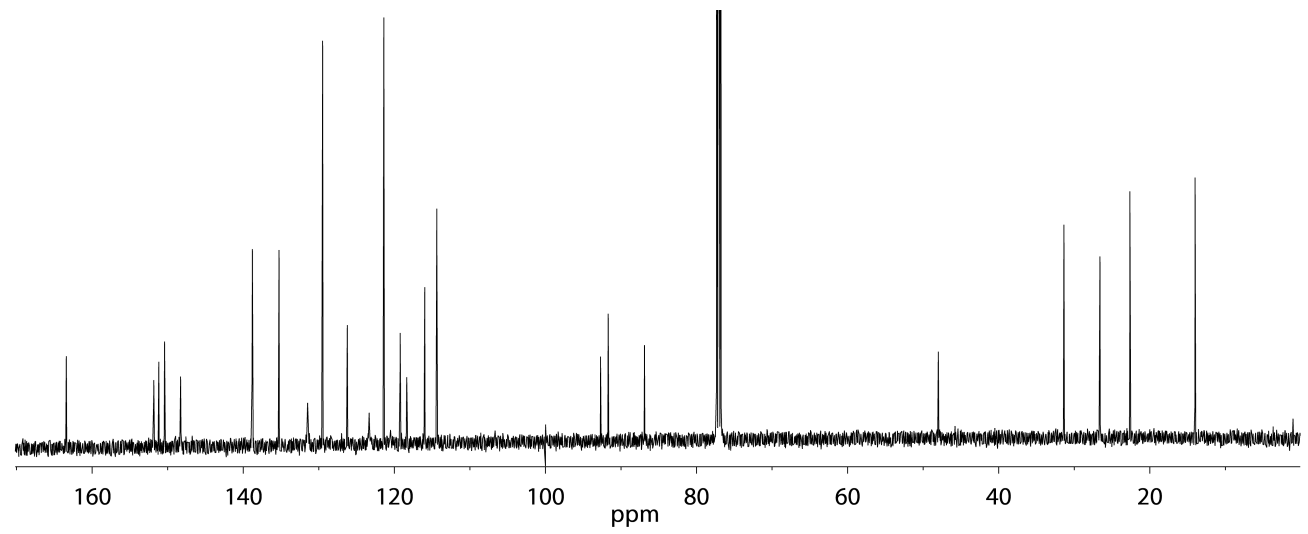

IR $($ ATR $): \tilde{\nu}\left(\mathrm{cm}^{-1}\right)=2931,2183,1716,1564,1480,1468,1410,1370,1267,1176,1165$, 1079, 810, 748, 703 .

UV/Vis (MeCN): $\lambda_{\max }(\mathrm{nm})\left[\left(\mathrm{dm}^{3} \mathrm{~mol}^{-1} \mathrm{~cm}^{-1}\right)\right]=273$ [38500], 345 [42900], 489 [9600].

ESI-HRMS $\left(\left[\mathrm{C}_{42} \mathrm{H}_{33} \mathrm{~N}_{4} \mathrm{O}_{2}\right]^{+}\right)$: $\quad$ measured: $\quad 625.2583$ 
calculated: $\quad 625.2525$

$\begin{array}{lllll}\text { EA: }\left(\mathrm{C}_{42} \mathrm{H}_{32} \mathrm{~N}_{4} \mathrm{O}_{2} \times \mathrm{H}_{2} \mathrm{O}\right) & \text { calculated: } & \% \mathrm{C} 78.5 & \% \mathrm{H} 5.4 & \% \mathrm{~N} 8.7 \\ & \text { found } & \% \mathrm{C} 78.3 & \% \mathrm{H} 5.8 & \% \mathrm{~N} 9.0\end{array}$




\section{VI.3.3 Syntheses of Cage Derivatives}

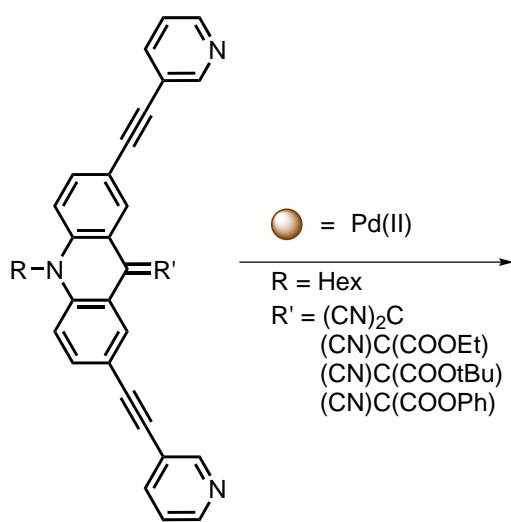

$40,41,42$ and 43

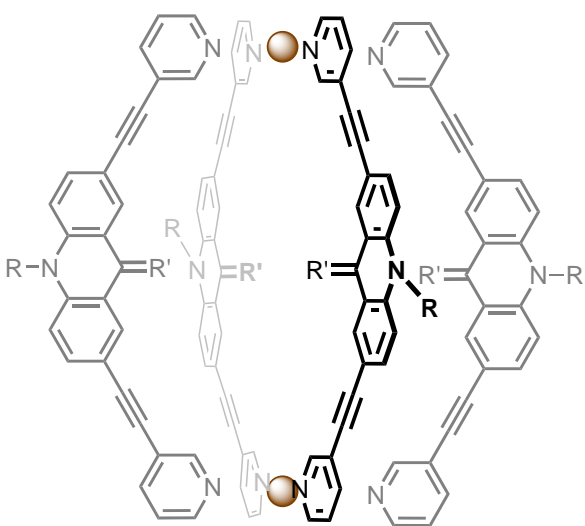

$44,45,46$ and 47

Scheme VI.3.3.49: Synthesis of palladium-mediated coordination cages 44, 45, 46 and 47 fro ligands $40,41,42$ and 43.

To a solution of the respective ligand $\mathbf{4 0 , 4 1 , 4 2}$ and $\mathbf{4 3}(0.5 \mathrm{~mL}, 2.8 \mathrm{mM}, 2.0$ equiv.) in $\mathrm{MeCN}$, a solution of $\left[\mathrm{Pd}(\mathrm{MeCN})_{4}\right]\left(\mathrm{BF}_{4}\right)_{2}(50 \mathrm{uL}, 15 \mathrm{mM}, 1.1$ equiv. $)$ in MeCN was added. Subsequently the mixed solutions were heated between 1-8 $\mathrm{h}$. The completion of the cage assembly was monitored by ${ }^{\mathbf{1}} \mathbf{H} \mathbf{N M R}$. 


\section{VI.3.3.1 $\left.\left[\mathrm{Pd}_{2}\left(\mathrm{LCN}_{4}\right)_{4}\right]\left(\mathrm{BF}_{4}\right)_{2}\right](44)$}

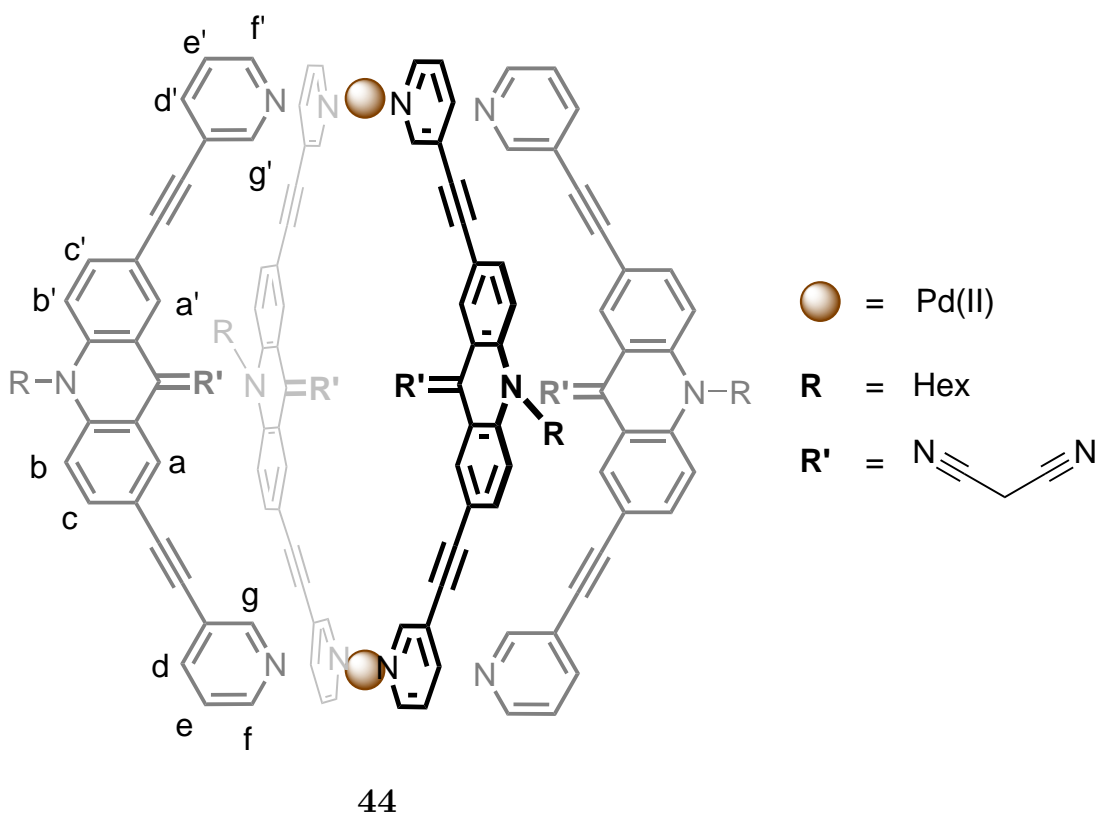

Scheme VI.3.3.50: Synthesis of compound 44.

${ }^{1} \mathbf{H}-\mathbf{N M R}\left(300 \mathrm{MHz}, 298 \mathrm{~K}, \mathrm{CD}_{3} \mathrm{CN}\right): \delta(\mathrm{ppm})=9.32\left(\mathrm{dd},{ }^{4} J=2.2 \mathrm{~Hz}, 2 \mathrm{H}, \mathrm{g}\right), 9.02$ $\left(\mathrm{dd},{ }^{3} J=4.9 \mathrm{~Hz},{ }^{4} J=1.7 \mathrm{~Hz}, 2 \mathrm{H}, \mathrm{f}\right), 8.80\left(\mathrm{~d},{ }^{4} J=1.9 \mathrm{~Hz}, 2 \mathrm{H}, \mathrm{a}\right), 8.09\left(\mathrm{dt},{ }^{3} J=7.9 \mathrm{~Hz}\right.$, $\left.{ }^{4} J=2.0 \mathrm{~Hz}, 2 \mathrm{H}, \mathrm{d}\right), 7.89\left(\mathrm{dd},{ }^{3} J=3.7 \mathrm{~Hz},{ }^{4} J=1.9 \mathrm{~Hz}, 2 \mathrm{H}, \mathrm{c}\right), 7.71\left(\mathrm{~d},{ }^{3} J=9.1 \mathrm{~Hz}, 2 \mathrm{H}\right.$, b), $7.62\left(\mathrm{dd},{ }^{3} J=7.9 \mathrm{~Hz},{ }^{4} J=4.9 \mathrm{~Hz}, 2 \mathrm{H}, \mathrm{e}\right), 4.47-4.24\left(\mathrm{~m}, 2 \mathrm{H}, \mathrm{NCH}_{2}\right), 1.92-1.78(\mathrm{~m}$, $\left.2 \mathrm{H}, \mathrm{CH}_{2}\right) 1.51-1.40\left(\mathrm{~m}, 2 \mathrm{H}, \mathrm{CH}_{2}\right), 1.37-1.24\left(\mathrm{~m}, 4 \mathrm{H}, \mathrm{CH}_{2} \mathrm{CH}_{2} \mathrm{CH}_{3}\right), 0.84\left(\mathrm{t},{ }^{3} J=7.0\right.$ $\left.\mathrm{Hz}, 3 \mathrm{H}, \mathrm{CH}_{3}\right)$.

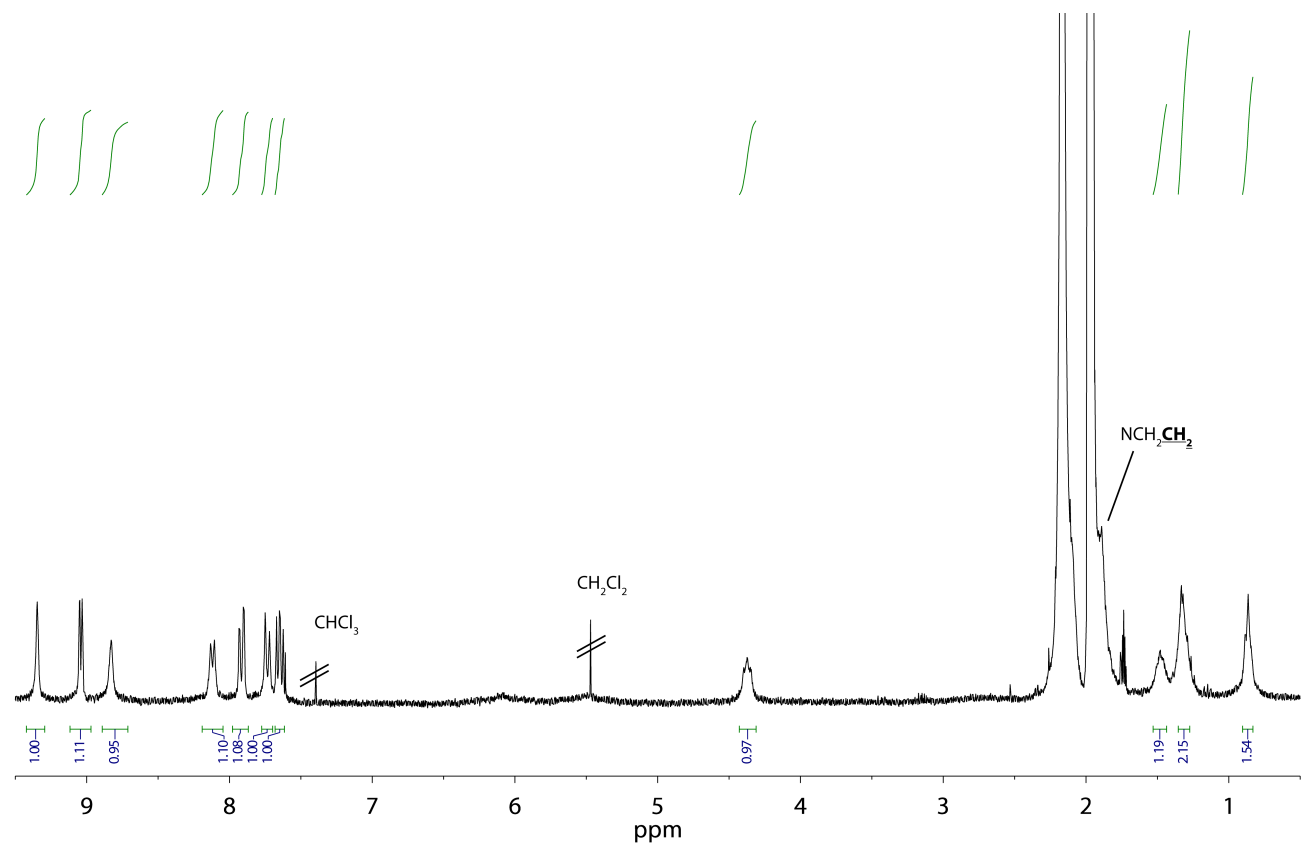


ESI-HRMS $\left(\left[\mathrm{C}_{144} \mathrm{H}_{108} \mathrm{~N}_{20} \mathrm{Pd}_{2}\right]^{4+}\right)$ : $\quad$ measured: $\quad 581.4256$

calculated: $\quad 581.4287$ 


\section{VI.3.3.2 $\left.\left[\mathrm{Pd}_{2}\left(\mathrm{~L}^{t \mathrm{Bu}}\right)_{4}\right]\left(\mathrm{BF}_{4}\right)_{2}\right](45)$}

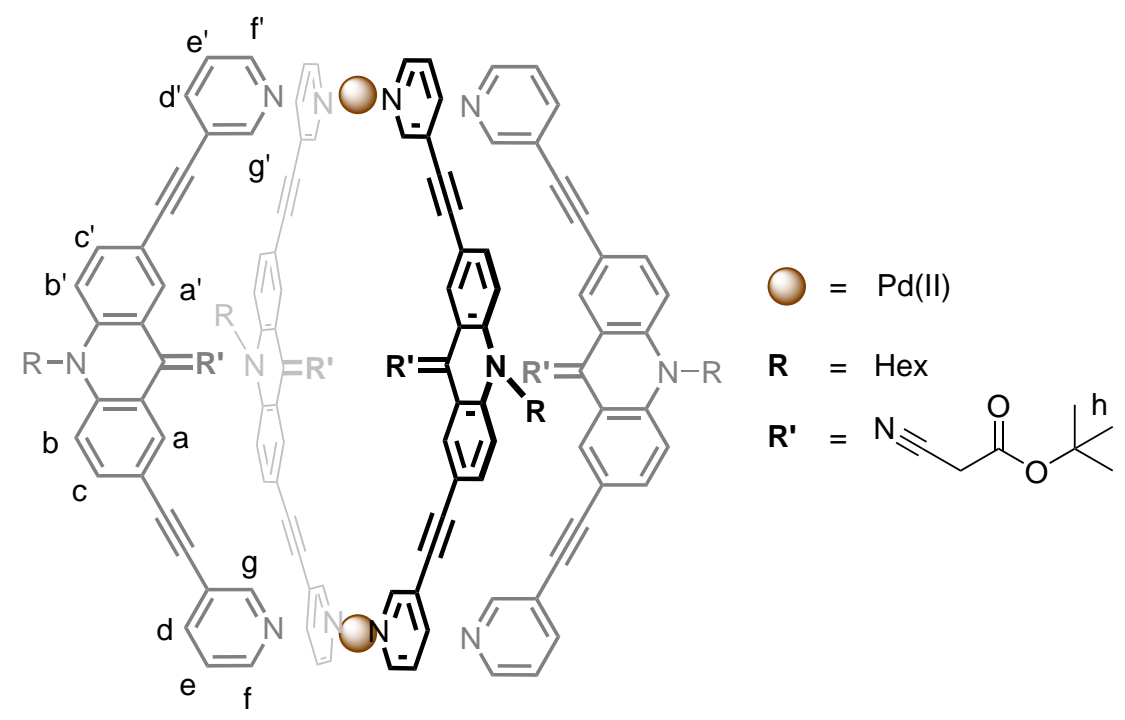

45

Scheme VI.3.3.51: Synthesis of compound 45.

${ }^{1} \mathbf{H}-\mathbf{N M R}\left(300 \mathrm{MHz}, 298 \mathrm{~K}, \mathrm{CD}_{3} \mathrm{CN}\right): \delta(\mathrm{ppm})=9.17(\mathrm{~s}, 2 \mathrm{H}, \mathrm{g} / \mathrm{g}), 8.94\left(\mathrm{dd},{ }^{3} J=5.0\right.$ $\mathrm{Hz},{ }^{4} J=1.7 \mathrm{~Hz}, 2 \mathrm{H}, \mathrm{f} / \mathrm{f}$ '), $8.09\left(\mathrm{dt},{ }^{3} J=7.9 \mathrm{~Hz},{ }^{4} J=2.0 \mathrm{~Hz}, 2 \mathrm{H}, \mathrm{d} / \mathrm{d}\right.$ '), 7.79 (dd, ${ }^{3} J=$ $\left.9.0 \mathrm{~Hz},{ }^{4} J=2.0 \mathrm{~Hz}, 2 \mathrm{H}, \mathrm{c} / \mathrm{c}^{\prime}\right), 7.63\left(\mathrm{dt},{ }^{3} J=7.9 \mathrm{~Hz},{ }^{4} J=4.8 \mathrm{~Hz}, 2 \mathrm{H}, \mathrm{e} / \mathrm{e}^{*}\right), 7.56\left(\mathrm{~d},{ }^{3} J\right.$ $\left.=8.9 \mathrm{~Hz}, 2 \mathrm{H}, \mathrm{b} / \mathrm{b}^{\prime}\right), 4.32-4.13\left(\mathrm{~m}, 2 \mathrm{H}, \mathrm{NCH}_{2}\right), 1.88-1.74\left(\mathrm{~m}, 2 \mathrm{H}, \mathrm{CH}_{2}\right), 1.42-1.28(\mathrm{~m}$, $\left.2 \mathrm{H}, \mathrm{CH}_{2}\right), 1.31-1.18\left(\mathrm{~m}, 4 \mathrm{H}, \mathrm{CH}_{2} \mathrm{CH}_{2} \mathrm{CH}_{3}\right), 1.01(\mathrm{~s}, 9 \mathrm{H}, \mathrm{h}), 0.92\left(\mathrm{t},{ }^{3} J=7.0 \mathrm{~Hz}, 3 \mathrm{H}\right.$, $\mathrm{CH}_{3}$ ).

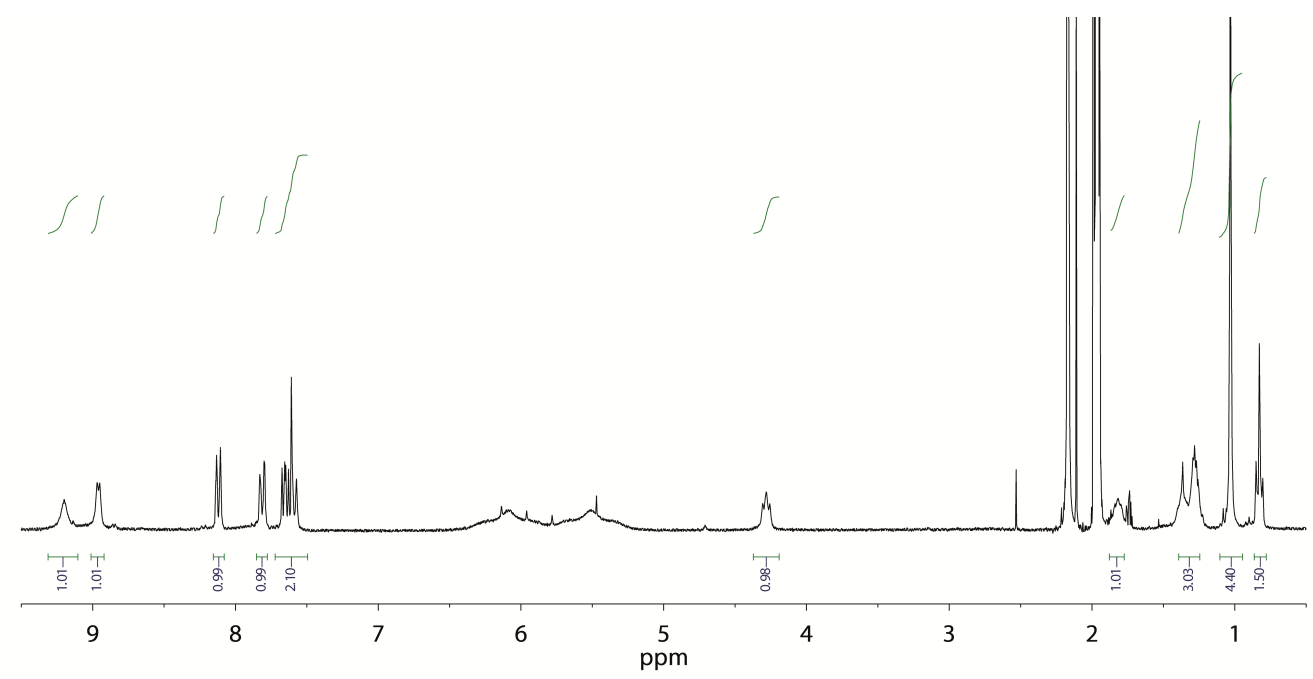

ESI-HRMS $\left(\left[\mathrm{C}_{160} \mathrm{H}_{144} \mathrm{~N}_{16} \mathrm{O}_{8} \mathrm{Pd}_{2}\right]^{4+}\right)$ : measured: $\quad 656.2363$

calculated: $\quad 656.2359$ 


\section{VI.3.3.3 $\left.\left[\mathrm{Pd}_{2}\left(\mathrm{~L}^{\mathrm{Et}}\right)_{4}\right]\left(\mathrm{BF}_{4}\right)_{2}\right](46)$}

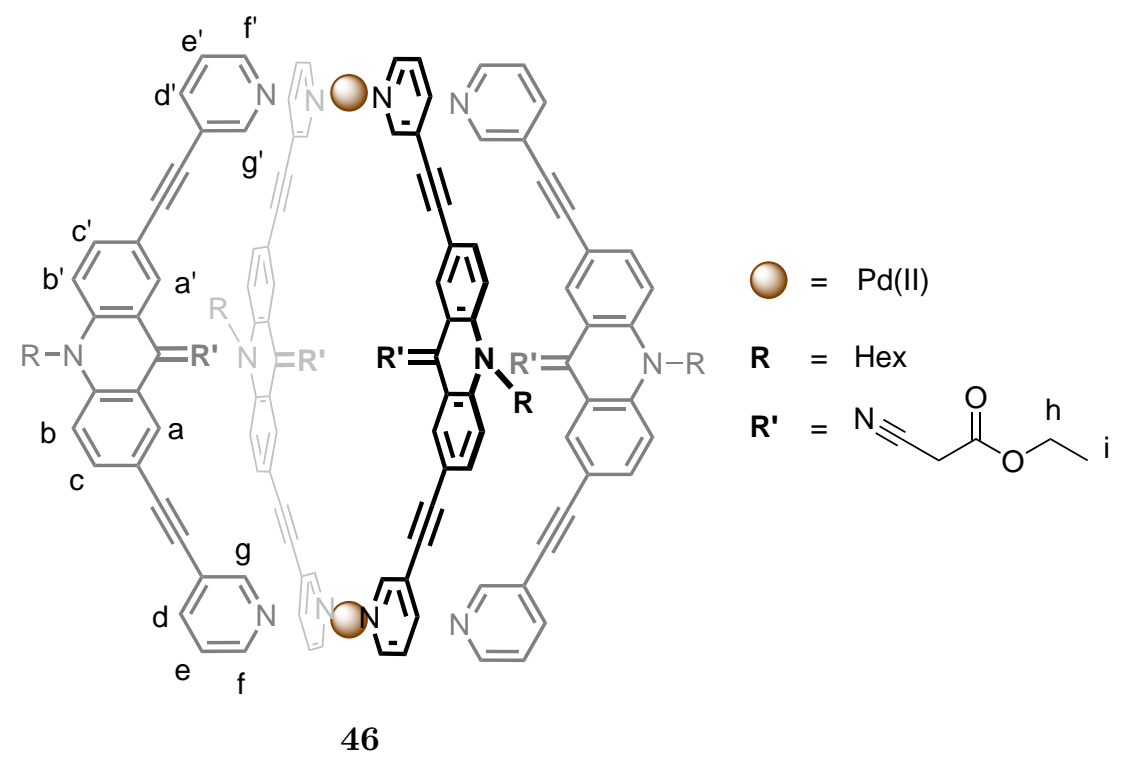

Scheme VI.3.3.52: Synthesis of compound 46.

${ }^{1} \mathbf{H}-\mathbf{N M R}\left(300 \mathrm{MHz}, 298 \mathrm{~K}, \mathrm{CD}_{3} \mathrm{CN}\right): \delta(\mathrm{ppm})=9.19\left(\mathrm{~s}, 2 \mathrm{H}, \mathrm{g} / \mathrm{g}^{\prime}\right), 8.95\left(\mathrm{dd},{ }^{3} J=5.0 \mathrm{~Hz}\right.$, ${ }^{4} J=1.7 \mathrm{~Hz}, 2 \mathrm{H}, \mathrm{f} / \mathrm{f}$ '), 8.35 (s, 2H, a/a'), 8.10 (dt, $\left.{ }^{3} J=7.9 \mathrm{~Hz},{ }^{4} J=2.0 \mathrm{~Hz}, 2 \mathrm{H}, \mathrm{d} / \mathrm{d}^{\prime}\right)$, $7.78\left(\mathrm{dd},{ }^{3} J=9.0 \mathrm{~Hz},{ }^{4} J=2.0 \mathrm{~Hz}, 2 \mathrm{H}, \mathrm{c} / \mathrm{c}^{\prime}\right), 7.63\left(\mathrm{dt},{ }^{3} J=7.9 \mathrm{~Hz},{ }^{4} J=4.8 \mathrm{~Hz}, 2 \mathrm{H}\right.$, e/e $\mathrm{e}^{*}, 7.57$ (d, ${ }^{3} J=8.9 \mathrm{~Hz}, 2 \mathrm{H}, \mathrm{b} / \mathrm{b}$ ), $4.30-4.21\left(\mathrm{~m}, 2 \mathrm{H}, \mathrm{NCH}_{2}\right.$ ), 4.00 (q, ${ }^{3} J=7.2 \mathrm{~Hz}, 2 \mathrm{H}$, h), $1.86-1.77\left(\mathrm{~m}, 2 \mathrm{H}, \mathrm{CH}_{2}\right), 1.45-1.37\left(\mathrm{~m}, 2 \mathrm{H}, \mathrm{CH}_{2}\right), 1.36-1.23\left(\mathrm{~m}, 4 \mathrm{H}, \mathrm{CH}_{2} \mathrm{CH}_{2} \mathrm{CH}_{3}\right)$, $0.87\left(\mathrm{t},{ }^{3} J=7.1 \mathrm{~Hz}, 3 \mathrm{H}\right.$, i) $0.83\left(\mathrm{t},{ }^{3} J=7.0 \mathrm{~Hz}, 3 \mathrm{H}, \mathrm{CH}_{3}\right)$.

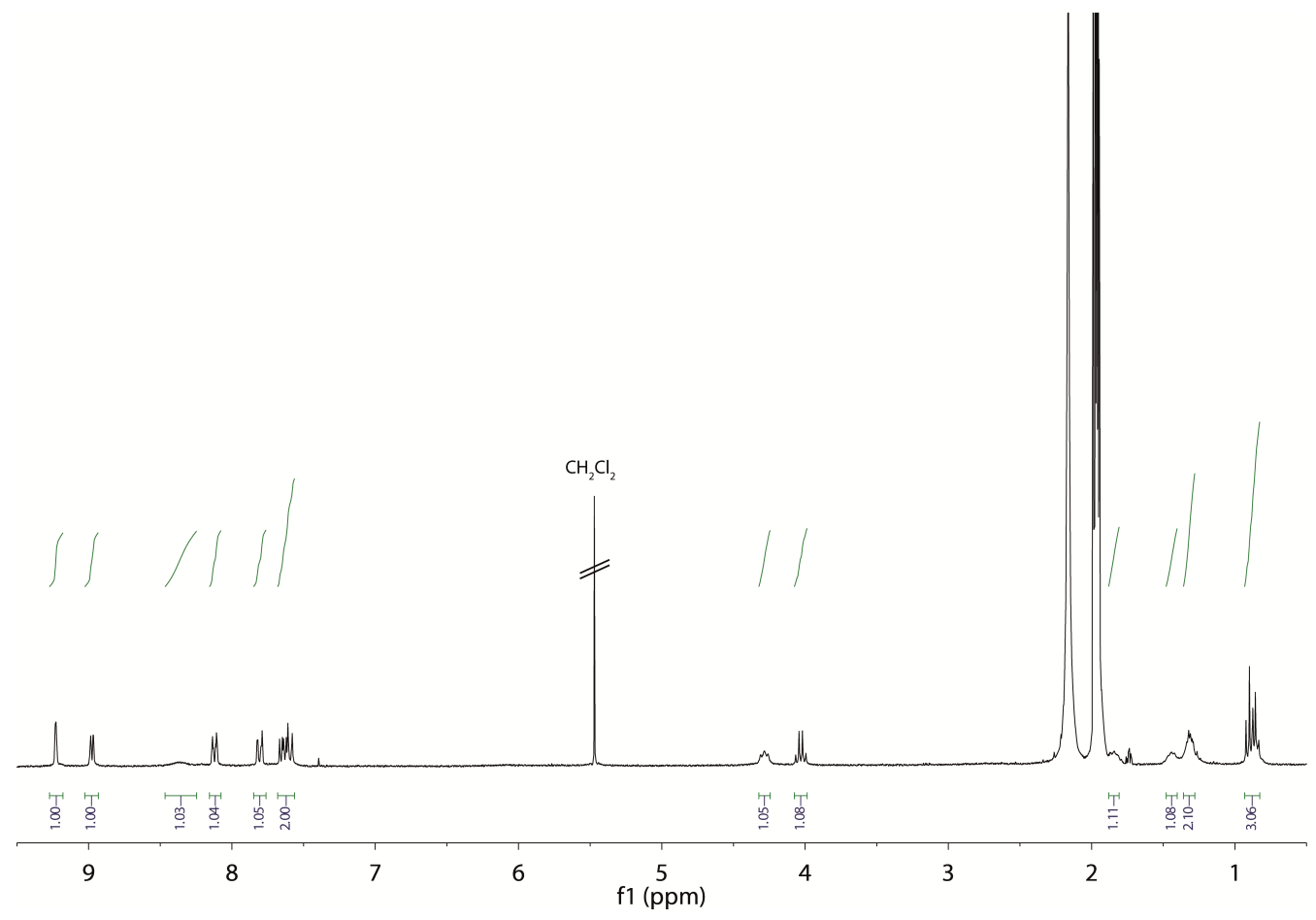


ESI-HRMS $\left(\left[\mathrm{C}_{152} \mathrm{H}_{128} \mathrm{~N}_{16} \mathrm{O}_{8} \mathrm{Pd}_{2}\right]^{4+}\right)$ : measured: $\quad 628.2029$

calculated: $\quad 628.2046$ 


\section{VI.3.3.4 $\left.\left[\mathrm{Pd}_{2}\left(\mathrm{~L}^{\mathrm{Ph}}\right)_{4}\right]\left(\mathrm{BF}_{4}\right)_{2}\right](47)$}

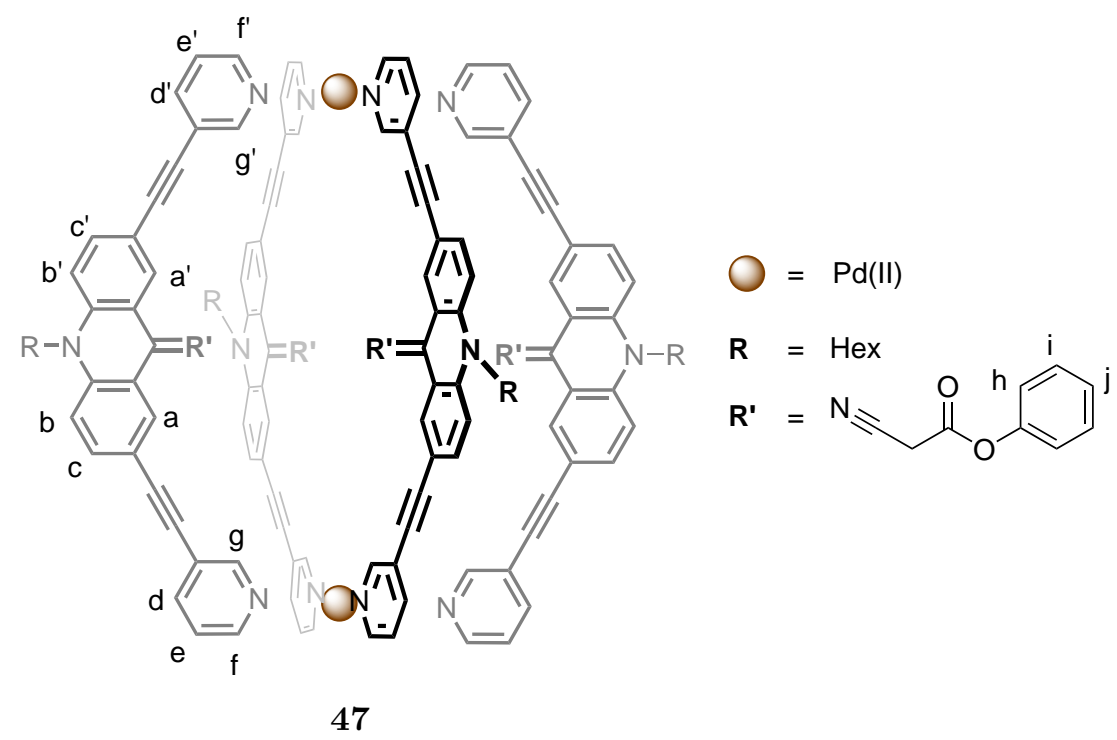

Scheme VI.3.3.53: Synthesis of compound 47.

${ }^{1}$ H-NMR $\left(300 \mathrm{MHz}, 298 \mathrm{~K}, \mathrm{CD}_{3} \mathrm{CN}\right): \delta(\mathrm{ppm})=9.18\left(\mathrm{~s}, 2 \mathrm{H}, \mathrm{g} / \mathrm{g}\right.$ '), $9.01\left(\mathrm{dd},{ }^{3} J=5.0\right.$ $\left.\mathrm{Hz},{ }^{4} J=1.7 \mathrm{~Hz}, 2 \mathrm{H}, \mathrm{f} / \mathrm{f}^{\prime}\right), 8.37$ (d, ${ }^{4} J=2.0 \mathrm{~Hz}, 2 \mathrm{H}, \mathrm{a} / \mathrm{a}$ '), 8.11 (dt, ${ }^{3} J=7.9 \mathrm{~Hz},{ }^{4} J=2.0$ $\left.\mathrm{Hz}, 2 \mathrm{H}, \mathrm{d} / \mathrm{d}^{\prime}\right), 7.71\left(\mathrm{dd},{ }^{3} J=9.0 \mathrm{~Hz},{ }^{4} J=2.0 \mathrm{~Hz}, 2 \mathrm{H}, \mathrm{c} / \mathrm{c}^{\prime}\right), 7.65\left(\mathrm{dt},{ }^{3} J=7.9 \mathrm{~Hz},{ }^{4} J=4.8\right.$ $\left.\mathrm{Hz}, 2 \mathrm{H}, \mathrm{e} / \mathrm{e}^{*}\right), 7.55$ (d, $\left.{ }^{3} J=8.9 \mathrm{~Hz}, 2 \mathrm{H}, \mathrm{b} / \mathrm{b}\right), 7.08-6.97$ (m, 3H, h,j), $6.86-6.80(\mathrm{~m}, 2 \mathrm{H}$, i), $4.35-4.16\left(\mathrm{~m}, 2 \mathrm{H}, \mathrm{NCH}_{2}\right), 1.84-1.66\left(\mathrm{~m}, 2 \mathrm{H}, \mathrm{CH}_{2}\right), 1.45-1.31\left(\mathrm{~m}, 2 \mathrm{H}, \mathrm{CH}_{2}\right), 1.31-$ $1.19\left(\mathrm{~m}, 4 \mathrm{H}, \mathrm{CH}_{2} \mathrm{CH}_{2} \mathrm{CH}_{3}\right), 0.83\left(\mathrm{t},{ }^{3} \mathrm{~J}=7.0 \mathrm{~Hz}, 3 \mathrm{H}, \mathrm{CH}_{3}\right)$.

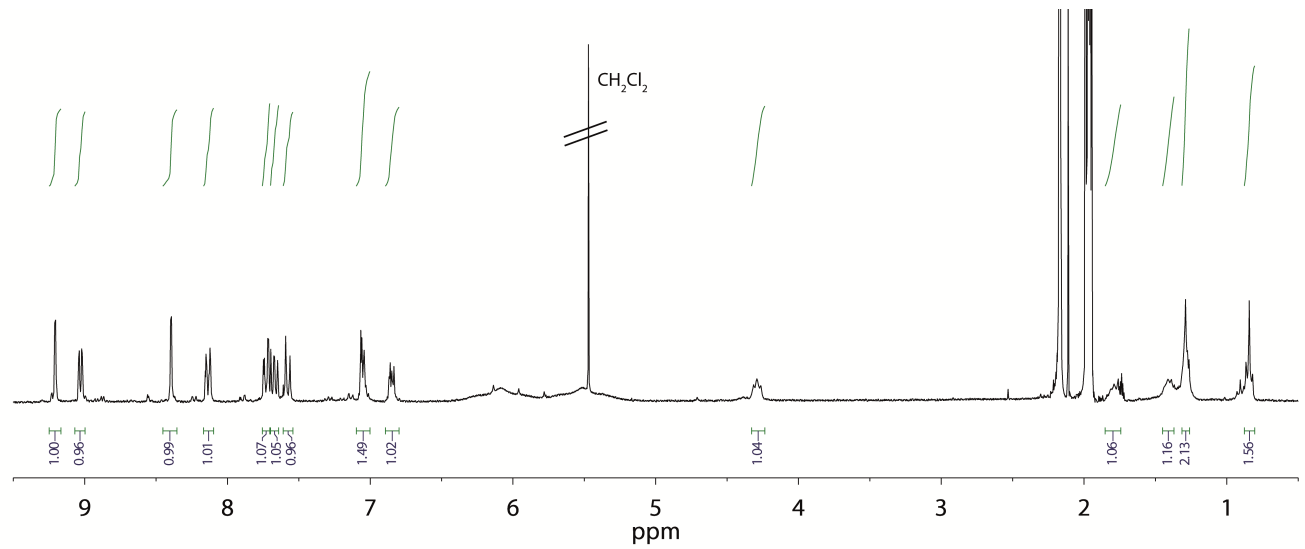

ESI-HRMS $\left(\left[\mathrm{C}_{168} \mathrm{H}_{128} \mathrm{~N}_{16} \mathrm{O}_{8} \mathrm{Pd}_{2}\right]^{4+}\right)$ : measured:

676.4550

calculated:

676.4547 


\section{VI.3.4 Syntheses of Azacrown Ethyl Acridinylidine}

\section{VI.3.4.1 (Ethane-1,2-diylbis(oxy))bis(ethane-2,1-diyl)} bis(4-methylbenzenesulfonate)) (48)

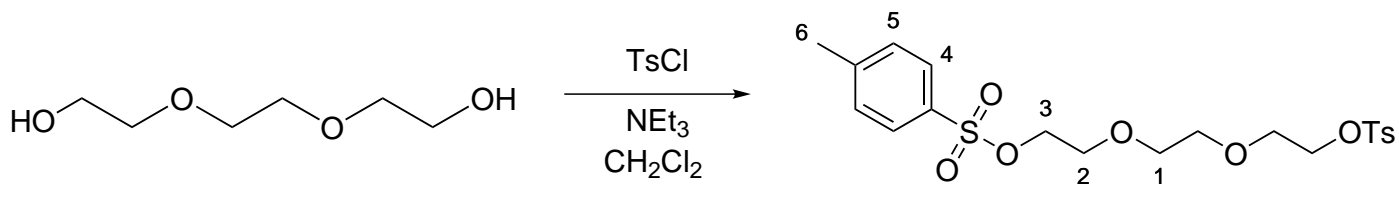

48

Scheme VI.3.4.54: Synthesis of compound 48 .

To a solution of triethylene glycol $(6.68 \mathrm{~mL}, 50.0 \mathrm{mmol}, 1.00$ equiv.) and triethylamine (27.4 mL, $200 \mathrm{mmol}, 4.00$ equiv.) in $\mathrm{CH}_{2} \mathrm{Cl}_{2}(100 \mathrm{~mL})$, p-tosyl chloride (28.6 g, $150 \mathrm{mmol}$, 3.00 equiv.) was added. The reaction mixture was treated for $30 \mathrm{~min}$ at $50{ }^{\circ} \mathrm{C}$ in the ultrasonic bath. After cooling down, the white solid was filtered off and the solvent was removed in vacuo. After recrystalization (2x) from methanol the product 48 (16.9 g, $36.8 \mathrm{mmol}, 74 \%$ ) was obtained as a white solid.

${ }^{1} \mathbf{H}-\mathbf{N M R}\left(300 \mathrm{MHz}, 298 \mathrm{~K}, \mathrm{CD}_{3} \mathrm{Cl}\right): \delta(\mathrm{ppm})=7.78\left(\mathrm{~d},{ }^{3} J=8.1 \mathrm{~Hz}, 4 \mathrm{H}, 4\right), 7.34(\mathrm{~d}$, $\left.{ }^{3} J=8.1 \mathrm{~Hz}, 4 \mathrm{H}, 5\right), 4.13(\mathrm{~m}, 4 \mathrm{H}, 3), 3.65\left(\mathrm{t},{ }^{3} J=4.8 \mathrm{~Hz}, 4 \mathrm{H}, 2\right), 3.52(\mathrm{~s}, 4 \mathrm{H}, 1), 2.44(\mathrm{~s}$, $6 \mathrm{H}, 6)$.

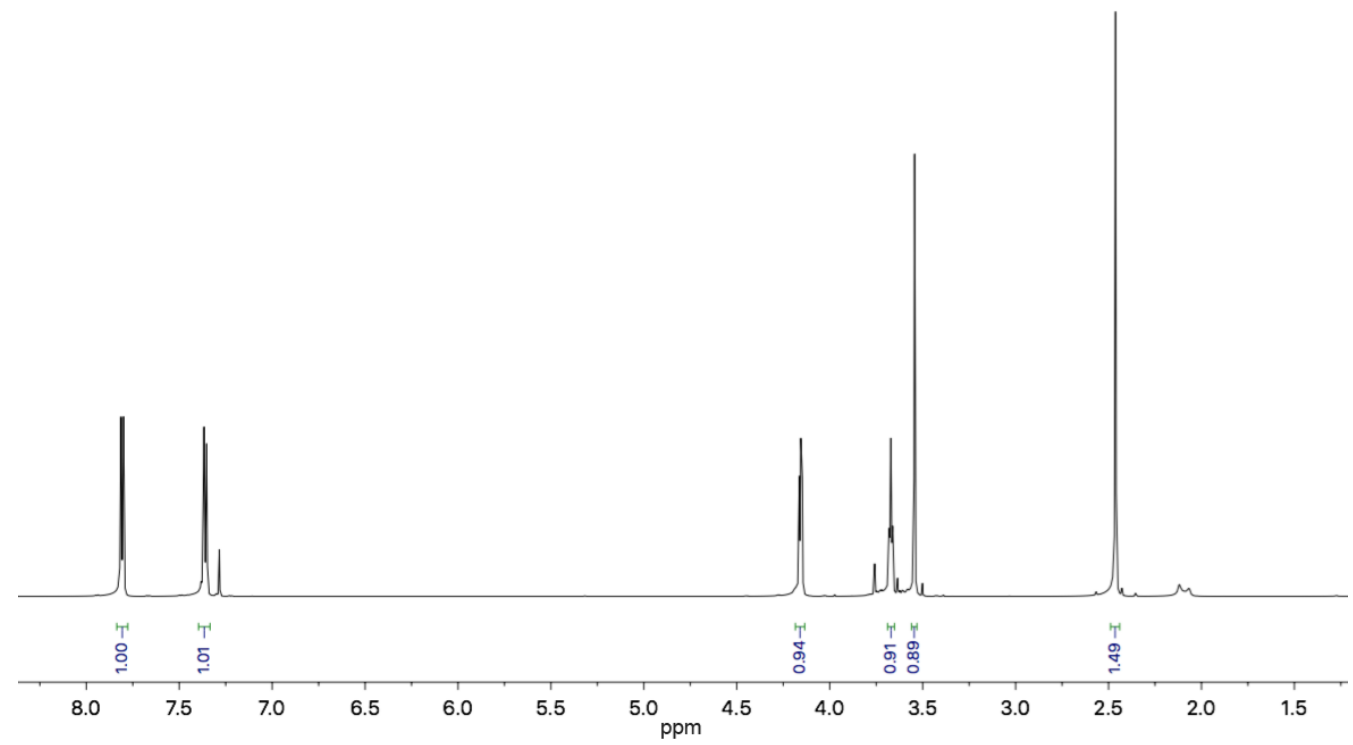

${ }^{13}$ C-NMR $\left(176 \mathrm{MHz}, 298 \mathrm{~K}, \mathrm{CD}_{3} \mathrm{Cl}\right): \delta(\mathrm{ppm})=145.01,132.97,129.98,128.08,70.80$, $69.33,68.86,21.76$. 


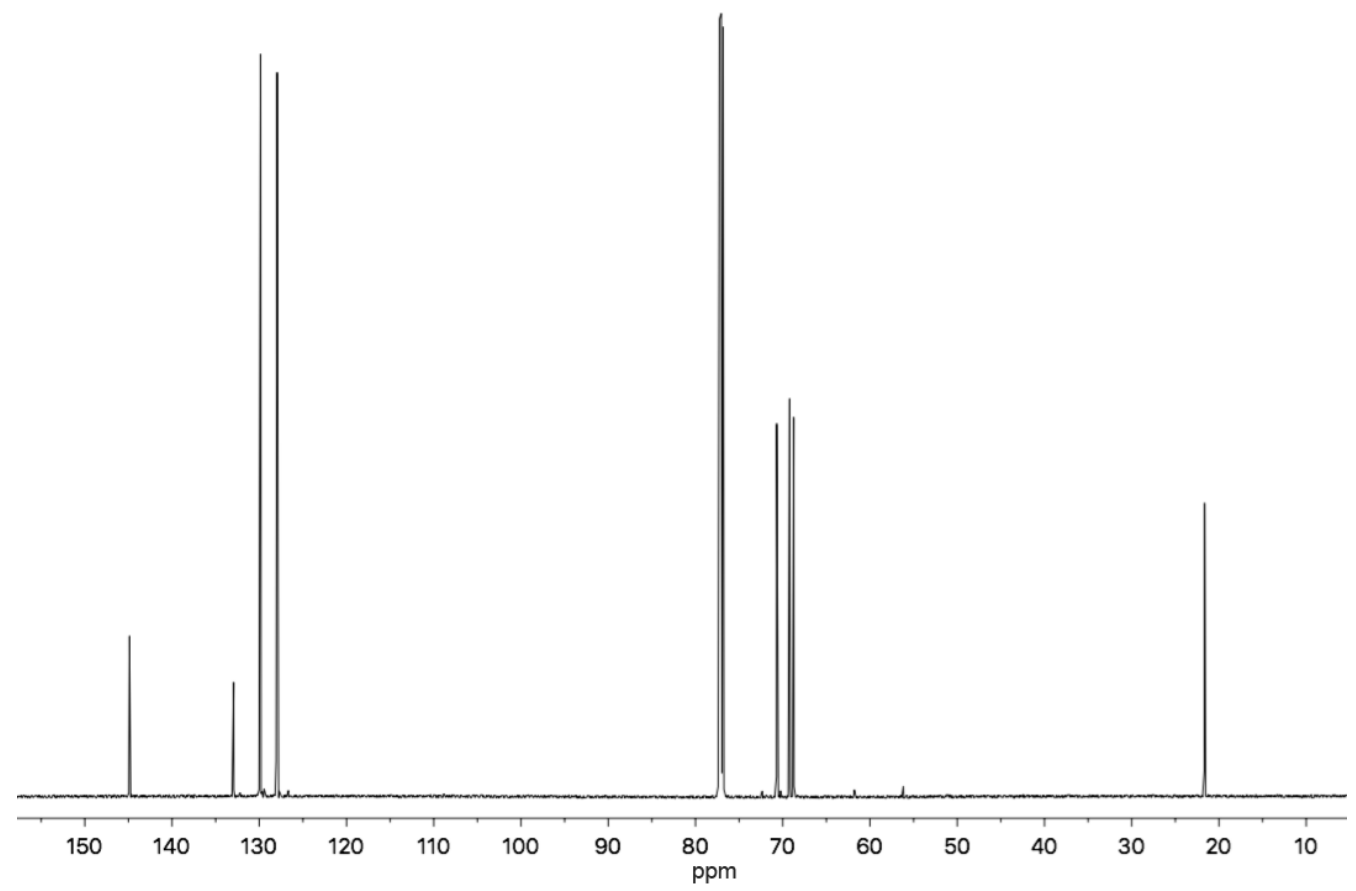

IR (ATR): $\tilde{\nu}\left(\mathrm{cm}^{-1}\right)=2898,1597,1439,1348,1170,1132,1015,981,909,804,777,663$, $582,552,488,443$.

$\begin{array}{lll}\text { ESI-HRMS }\left(\left[\mathrm{C}_{24} \mathrm{H}_{26} \mathrm{~N}_{2} \mathrm{O}_{2}\right]^{+}\right): & \text {measured: } & 459.1139 \\ & \text { calculated: } & 459.1147\end{array}$ 


\section{VI.3.4.2 (N-Phenyl-1-aza-5-crown-15)) (49)}

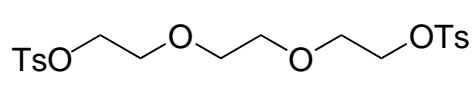

48

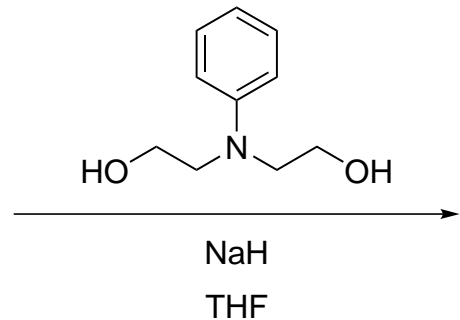

THF

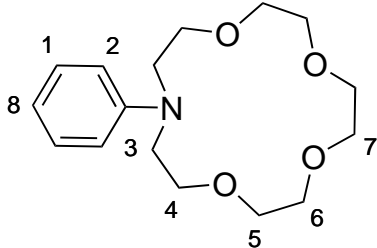

49

Scheme VI.3.4.55: Synthesis of compound 49.

Hexane $(20 \mathrm{~mL})$ was added to $\mathrm{NaH}(60 \%$ in mineral oil, $4.81 \mathrm{~g}, 120 \mathrm{mmol}, 3.63$ equiv.) and stirred at room temperature for $15 \mathrm{~min}$. After the hexane was removed, dry THF ( $480 \mathrm{~mL}$ ) was added. To this suspension phenyldiethanolamine (6.00 g, $33.1 \mathrm{mmol}, 1.00$ equiv.) and 48 (15.2 g, $33.1 \mathrm{mmol}, 1.00$ equiv.), both in THF (60 mL), were added dropwise within $80 \mathrm{~min}$. The reaction mixture was heated under reflux over night. After cooling down, the mixture was filtered and the solvent was removed in vacuo. The residue was resolved in $\mathrm{MeOH}(50 \mathrm{~mL})$ and $\mathrm{NaClO}_{4} \times \mathrm{H}_{2} \mathrm{O}$ (4.64 g, $37.8 \mathrm{mmol}, 1.15$ equiv.) in $\mathrm{MeOH}$ $(50 \mathrm{~mL})$ and the mixture was heated under reflux for $10 \mathrm{~min}$. The solvent was removed in vacuo and EtOAc $(30 \mathrm{~mL})$ stirred for $5 \mathrm{~min}$ and removed again. The residue was recrystalized from ethyl acetate $(350 \mathrm{~mL})$. The sodium crown-ether complex was stirred in $\mathrm{CH}_{2} \mathrm{Cl}_{2} / \mathrm{H}_{2} \mathrm{O}(1: 1,60 \mathrm{~mL})$ for $30 \mathrm{~min}$. The aquoeus phase was washed with $\mathrm{CH}_{2} \mathrm{Cl}_{2}$ $(2 \times 30 \mathrm{~mL})$, the combined organic phases were dried over $\mathrm{Na}_{2} \mathrm{SO}_{4}$ and the solvent was removed in vacuo. The product 49 (3.55 g, $12.0 \mathrm{mmol}, 36 \%)$ was obtained as a transparent oil.

${ }^{1}$ H-NMR $(300 \mathrm{MHz}, 298 \mathrm{~K}, \mathrm{DMSO}): \delta(\mathrm{ppm})=7.13\left(\mathrm{dd},{ }^{3} J=8.7,7.3 \mathrm{~Hz}, 2 \mathrm{H}, 1\right), 6.61$ $\left(\mathrm{d},{ }^{3} J=8.2 \mathrm{~Hz}, 2 \mathrm{H}, 2\right), 6.56\left(\mathrm{t},{ }^{3} J=7.2 \mathrm{~Hz}, 1 \mathrm{H}, 8\right), 3.62\left(\mathrm{t},{ }^{3} J=6.2 \mathrm{~Hz}, 4 \mathrm{H}, 3\right), 3.56-3.48$ (m, 16H, 4-7). 


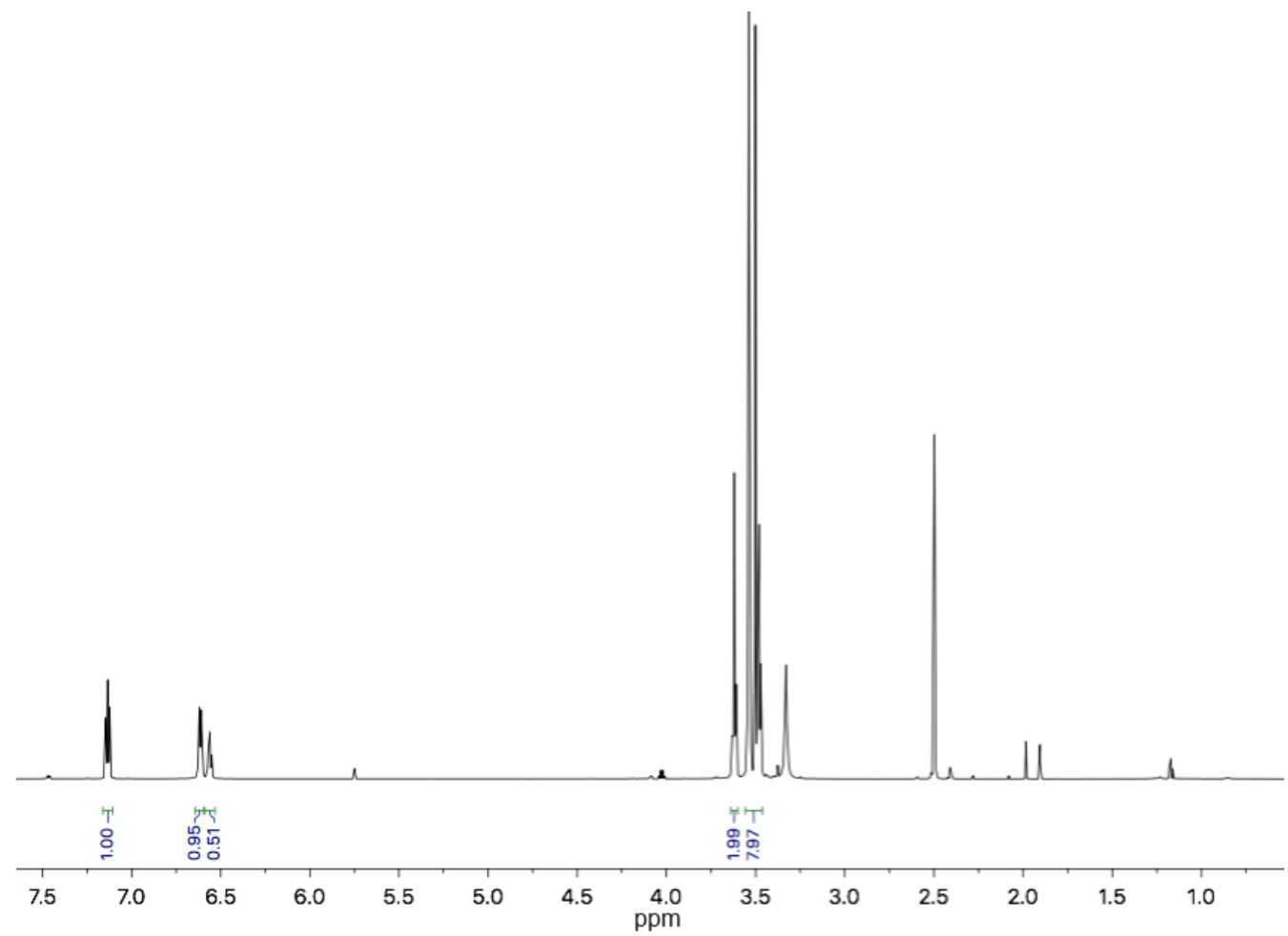

${ }^{13}$ C-NMR $(176 \mathrm{MHz}, 298 \mathrm{~K}, \mathrm{DMSO}): \delta(\mathrm{ppm})=147.32,129.13,115.15,111.09,70.35$, $69.46,69.07,67.91,51.86$.

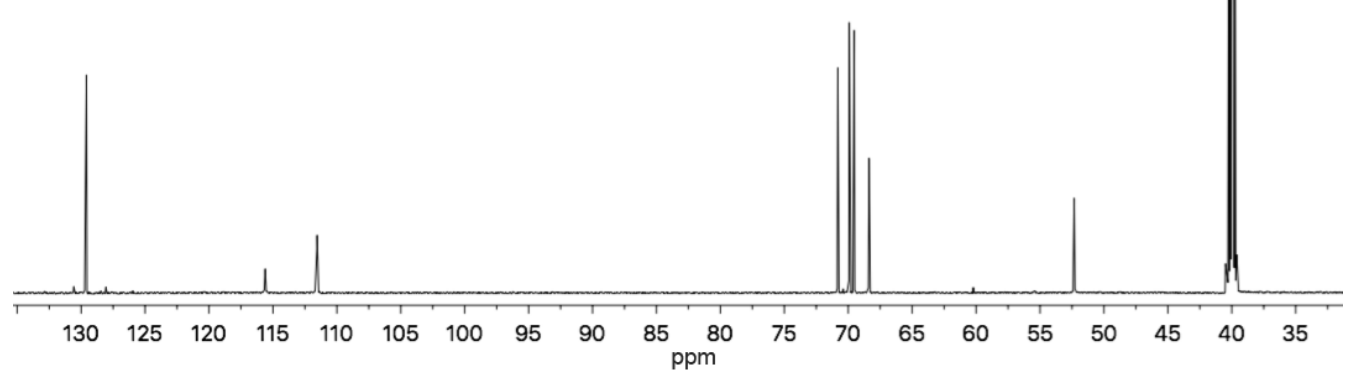

IR $($ ATR $): \tilde{\nu}\left(\mathrm{cm}^{-1}\right)=2862,1597,1505,1345,1117,988,938,745,693,622$, 511.

ESI-HRMS $\left(\left[\mathrm{C}_{24} \mathrm{H}_{26} \mathrm{~N}_{2} \mathrm{O}_{2}\right]^{+}\right)$: $\quad$ measured: 318.1700 
VI.3.4 SYN AZACROWN-ACR.

calculated:

318.1681 


\section{VI.3.4.3 p-lodo-N-phenyl-1-aza-5-crown-15(50)}<smiles>c1ccc(N2CCOCCOCCOCCOCC2)cc1</smiles>
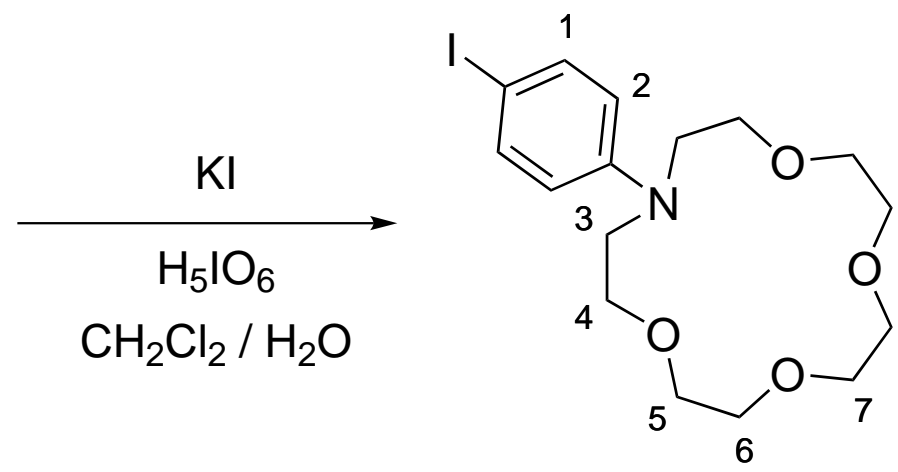

Scheme VI.3.4.56: Synthesis of compound 50.

To a solution of 49 (1.00 g, $3.39 \mathrm{mmol}, 1.00$ equiv.) in $\mathrm{CH}_{2} \mathrm{Cl}_{2}(30 \mathrm{~mL})$, KI (674 mg, $4.06 \mathrm{mmol}, 1.20$ equiv.) and $\mathrm{H}_{5} \mathrm{IO}_{6}$, both solved in $\mathrm{H}_{2} \mathrm{O}(30 \mathrm{~mL})$, were added and the mixture was stirred for $3.5 \mathrm{~h}$ at room temperature. After, $\mathrm{Na}_{2} \mathrm{~S}_{2} \mathrm{O}_{3}$ solution $(10 \%(\mathrm{w} / \mathrm{v})$, $50 \mathrm{~mL})$ was added and the aq. phase was washed with $\mathrm{CH}_{2} \mathrm{Cl}_{2}(3 \times 30 \mathrm{~mL})$. The combined organic phases were dried over $\mathrm{Na}_{2} \mathrm{SO}_{4}$ and the solvent was removed in vacuo. The product 50 (945 mg, $2.24 \mathrm{mmol}, 66 \%$ ) was obtained as brown oil.

${ }^{1} \mathbf{H}-\mathbf{N M R}(300 \mathrm{MHz}, 298 \mathrm{~K}, \mathrm{DMSO}): \delta(\mathrm{ppm})=7.39\left(\mathrm{~d},{ }^{3} J=8.9 \mathrm{~Hz}, 2 \mathrm{H}, 1\right), 6.48(\mathrm{~d}$, $\left.{ }^{3} J=8.9 \mathrm{~Hz}, 2 \mathrm{H}, 2\right), 3.61-3.43(\mathrm{~m}, 20 \mathrm{H}, 3-7)$.

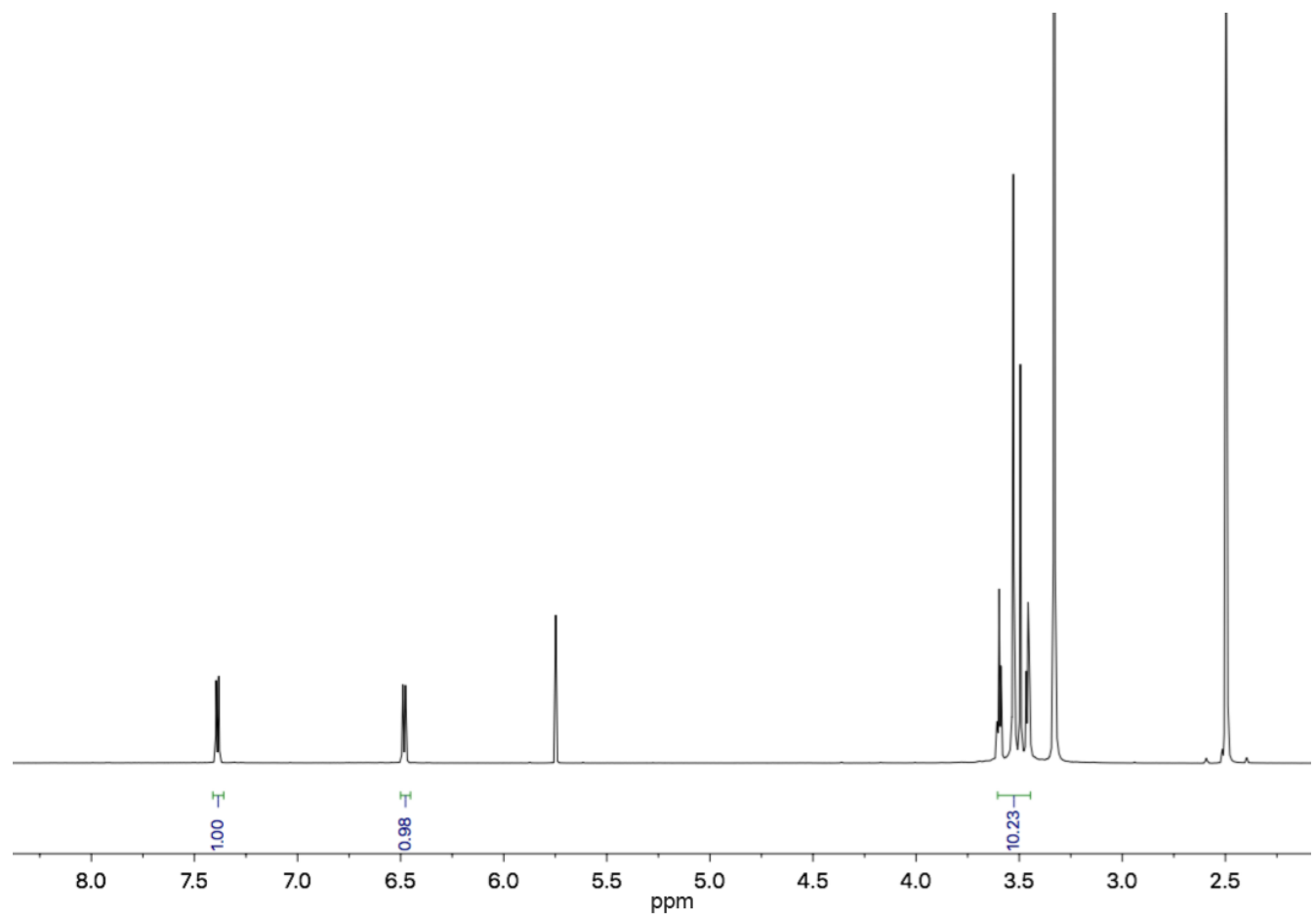


${ }^{13}$ C-NMR (176 MHz, $\left.298 \mathrm{~K}, \mathrm{DMSO}\right): \delta(\mathrm{ppm})=147.01,137.28,114.02,75.92,70.34$, $69.48,69.07,67.66,54.92,51.86$.

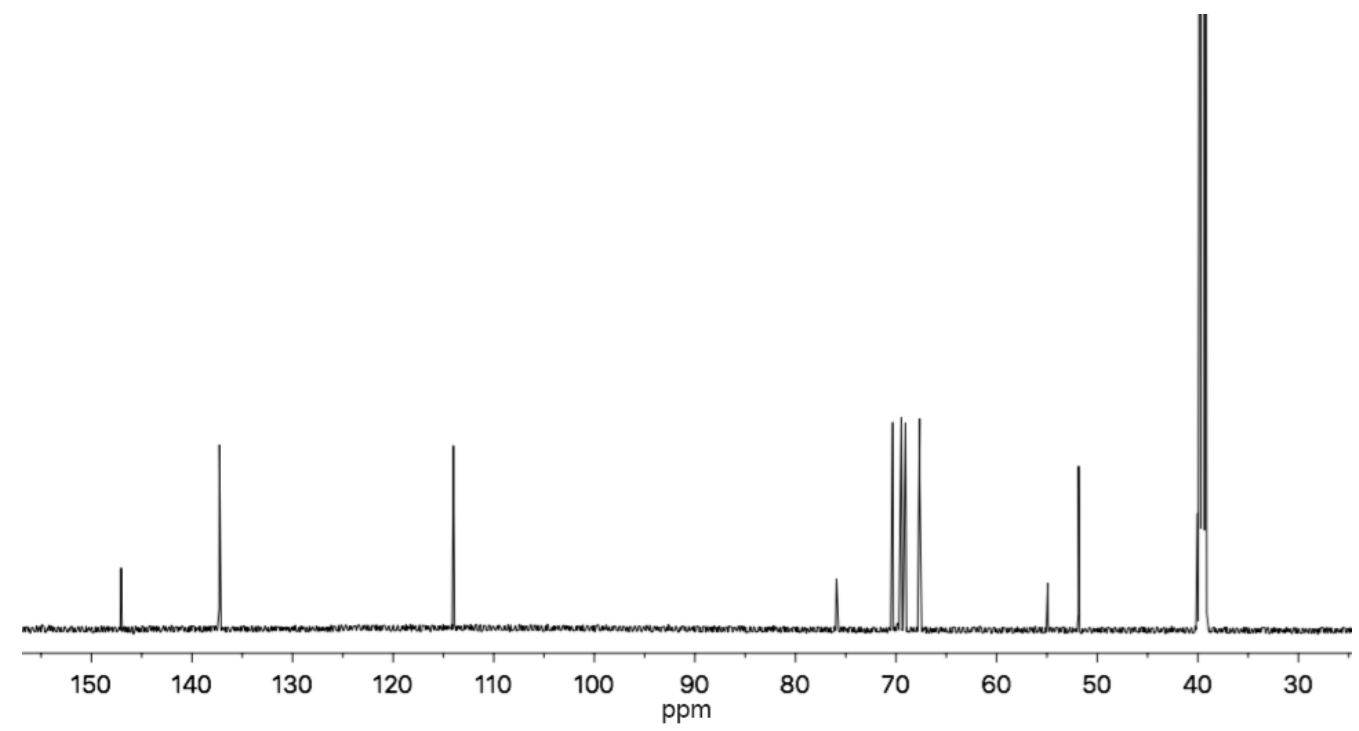

IR (ATR): $\tilde{\nu}\left(\mathrm{cm}^{-1}\right)=2863,1586,1493,1352,1115,984,937,802,745,694$, 506.

ESI-HRMS $\left(\left[\mathrm{C}_{24} \mathrm{H}_{26} \mathrm{~N}_{2} \mathrm{O}_{2}\right]^{+}\right): \quad$ measured: $\quad 422.1000$

calculated: $\quad 422.0828$ 
VI.3.4.4 N-(N-phenyl-1-aza-5-crown-15)-acridin-9(10H)-one (51)

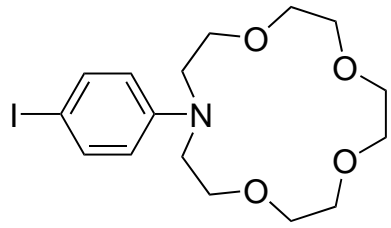

50

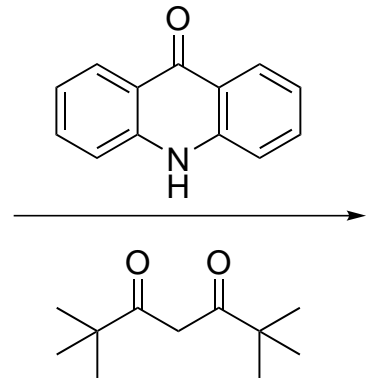

$\mathrm{N}(n \mathrm{Bu})_{4} \mathrm{OH}$

Cul DMF

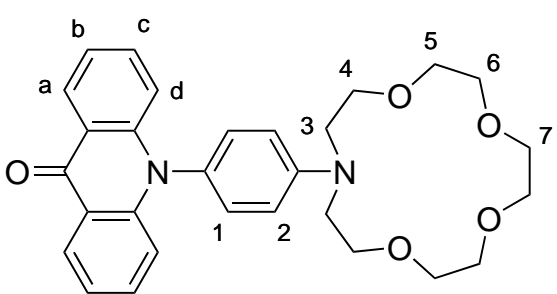

51

Scheme VI.3.4.57: Synthesis of compound 51.

50 (500 mg, $1.19 \mathrm{mmol}, 1.00$ equiv.), acrid-9(10H)-one (278 mg, $1.42 \mathrm{mmol}, 1.20$ equiv.) and $\mathrm{CuI}$ (11.3 mg, $0.06 \mathrm{mmol}, 0.05$ equiv.) were solved in dry DMF (40 ml). After addition of dipivaloylmethane $(0.05 \mathrm{~mL}, 0.24 \mathrm{mmol}, 0.20$ equiv.) and tetra- $n$-butyl ammoniumhydroxide ( $40 \%$ in $\mathrm{MeOH}, 1.15 \mathrm{~mL}, 1.78 \mathrm{mmol}, 1.50$ Äq.), the reaction mixture was heated $48 \mathrm{~h}$ under reflux. $\mathrm{HCl}(50 \mathrm{~mL}, 3 \mathrm{M})$ and $\mathrm{CH}_{2} \mathrm{Cl}_{2}(100 \mathrm{~mL})$ were added to the cooled-down reaction mixture. The organic phase was washed with aq. sat. $\mathrm{NaHCO}_{3}$, aq. sat. $\mathrm{NH}_{4} \mathrm{Cl}$ and brine. The organic phase was dried over $\mathrm{Na}_{2} \mathrm{SO}_{4}$ and the solvent was removed in vacuo. The residue was purified by means of column chromatography $\left(\mathrm{SiO}_{2}\right.$, pentane/ethyl acetate 1:1) and subsequent purification via GPC. The product 35 (106 mg, $0.22 \mathrm{mmol}, 18 \%$ ) was obtained as a yellowish solid.

${ }^{1} \mathbf{H}-\mathbf{N M R}\left(300 \mathrm{MHz}, 298 \mathrm{~K}, \mathrm{~d}^{6}-\mathrm{DMSO}\right): \delta(\mathrm{ppm})=8.35\left(\mathrm{dd},{ }^{3} J=8.0 \mathrm{~Hz},{ }^{4} J=1.4 \mathrm{~Hz}\right.$, $2 \mathrm{H}), 7.65\left(\mathrm{ddd},{ }^{3} J=8.6,6.9 \mathrm{~Hz},{ }^{4} J=1.7 \mathrm{~Hz}, 2 \mathrm{H}\right), 7.31\left(\mathrm{ddd},{ }^{3} J=7.9,6.9 \mathrm{~Hz},{ }^{4} J=0.9\right.$ $\mathrm{Hz}, 2 \mathrm{H}), 7.24\left(\mathrm{~d},{ }^{3} J=8.7 \mathrm{~Hz} 2 \mathrm{H}\right), 6.94\left(\mathrm{~d},{ }^{3} J=9.0 \mathrm{~Hz}, 2 \mathrm{H}\right), 6.90\left(\mathrm{~d},{ }^{3} J=8.6 \mathrm{~Hz}, 2 \mathrm{H}\right)$, $3.74\left(\mathrm{t},{ }^{3} \mathrm{~J}=6.1 \mathrm{~Hz}, 4 \mathrm{H}\right), 3.64-3.57(\mathrm{~m}, 16 \mathrm{H})$. 


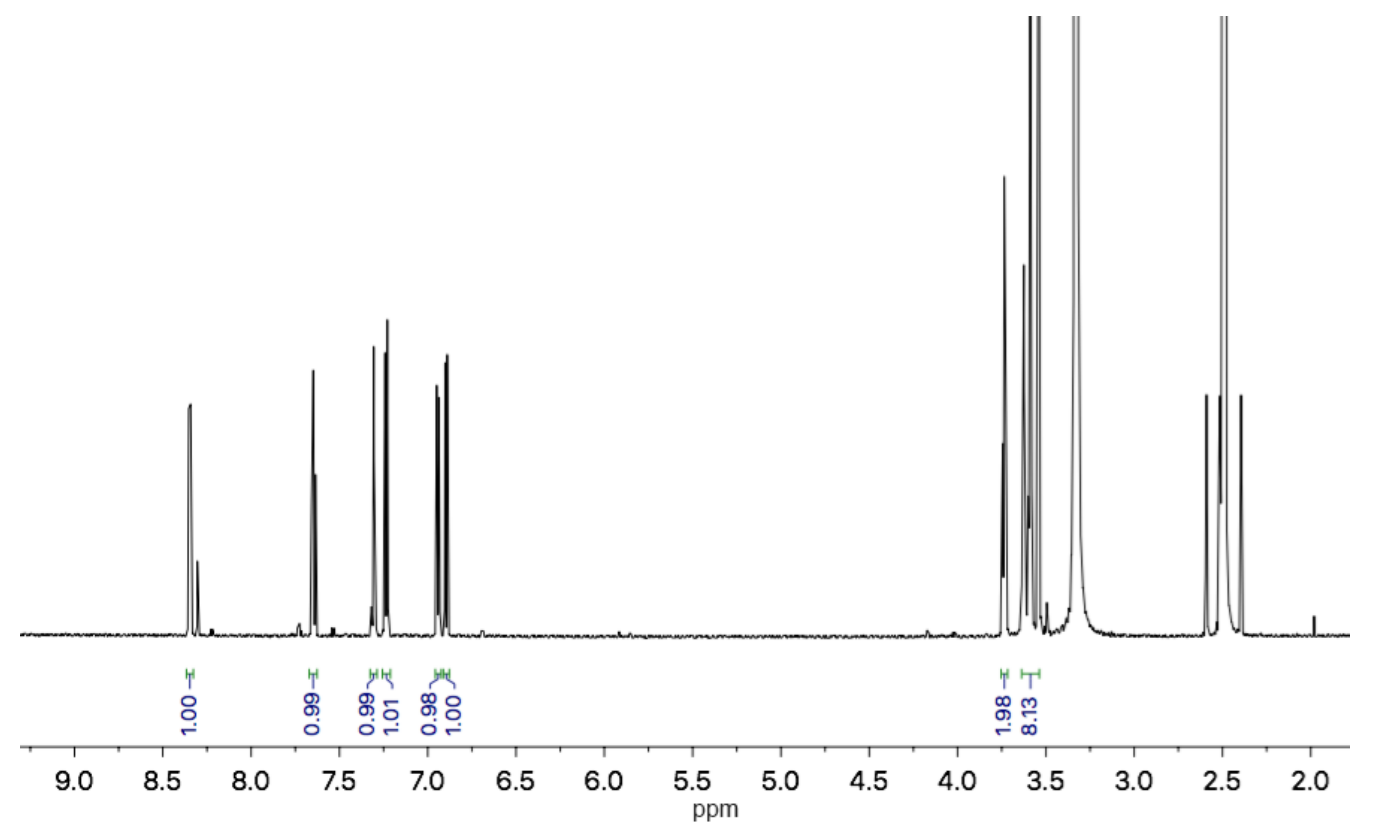

${ }^{13}$ C-NMR (176 MHz, $\left.298 \mathrm{~K}, \mathrm{~d}^{6}-\mathrm{DMSO}\right): \delta(\mathrm{ppm})=176.82,148.11,143.60,133.82$, $130.28,126.37,125.70,121.52$, 121.20, 117.48, 112.89, 70.46, 69.70, 69.05, 67.96, 52.48 .

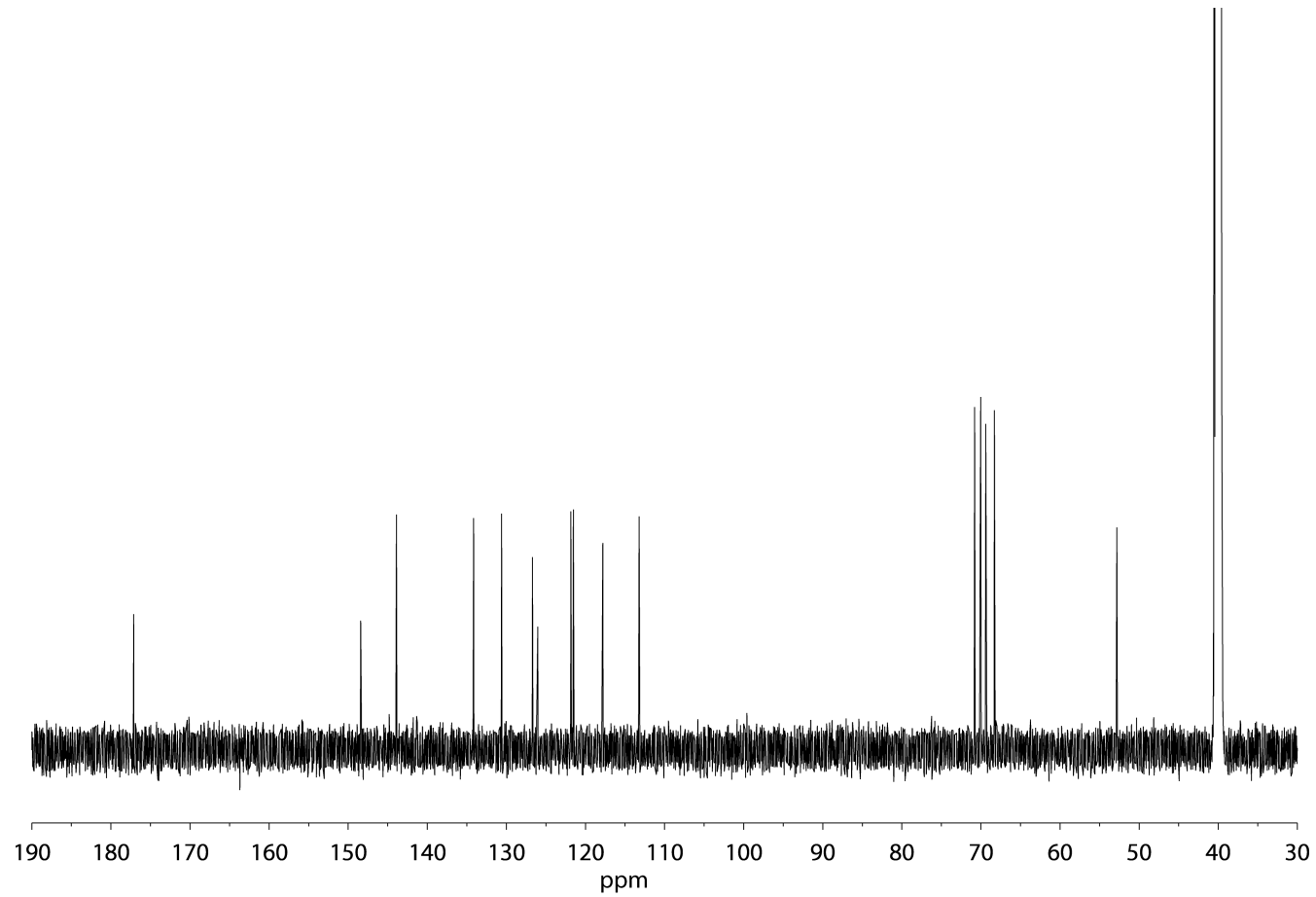

IR (ATR): $\tilde{\nu}\left(\mathrm{cm}^{-1}\right)=1631,1597,1518,1482,1455,1358,1112,760,677,621$, 547. 
ESI-HRMS $\left(\left[\mathrm{C}_{24} \mathrm{H}_{26} \mathrm{~N}_{2} \mathrm{O}_{2}\right]^{+}\right)$: $\quad$ measured: 489.2378

calculated: $\quad 489.2389$ 


\section{VI.3.4.5 Ethyl-2-(N-(N-phenyl-1-aza-5-crown-15)-acridin-9(10H)-ylidene-2- cyanoacetate (52)}<smiles>O=c1c2ccccc2n(-c2ccc(N3CCOCCOCCOCCOCC3)cc2)c2ccccc12</smiles>

51
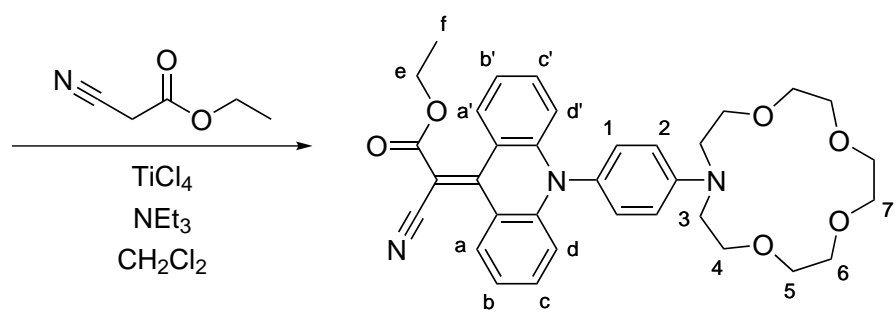

52

Scheme VI.3.4.58: Synthesis of compound $\mathbf{5 2}$.

$1 \mathrm{M} \mathrm{TiCl}_{4}$ (in $\mathrm{CH}_{2} \mathrm{Cl}_{2}$ ) (0.26 mL, $0.26 \mathrm{mmol}, 1.25$ equiv.) was slowly added to 51 (100 mg, $0.21 \mathrm{mmol}, 1.00$ equiv.) solved in dry $\mathrm{CH}_{2} \mathrm{Cl}_{2}(10 \mathrm{~mL})$ and stirred for $15 \mathrm{~min}$ at room temperature. 2-cyanoethylacetate (243 $\mathrm{mg}, 2.15 \mathrm{mmol}, 2.00$ equiv.) and triethylamine $(1.5 \mathrm{~mL})$ were added consecutively to the reaction mixture and heated at $70{ }^{\circ} \mathrm{C}$ under reflux for $24 \mathrm{~h}$. To the cooled down reaction mixture $\mathrm{H}_{2} \mathrm{O}(40 \mathrm{~mL})$ and $\mathrm{CH}_{2} \mathrm{Cl}_{2}(25 \mathrm{~mL})$ were added. The seperated organic phase was dried over $\mathrm{Na}_{2} \mathrm{SO}_{4}$ and the solvent was removed in vacuo. The residue was purified by means of column chromatography $\left(\mathrm{SiO}_{2}\right.$, dichloromethane/methanol 20:1) and subsequent purification via GPC. The product 35 (33.0 mg, $0.06 \mathrm{mmol}, 28 \%$ ) was obtained as a red solid.

${ }^{1} \mathbf{H}-\mathbf{N M R}\left(300 \mathrm{MHz}, 298 \mathrm{~K}, \mathrm{CD}_{3} \mathrm{Cl}\right): \delta(\mathrm{ppm})=8.22\left(\mathrm{~d},{ }^{3} J=6.6 \mathrm{~Hz}, 2 \mathrm{H}\right), 7.39(\mathrm{ddd}$, $\left.{ }^{3} J=8.5,7.0 \mathrm{~Hz},{ }^{4} J=1.4 \mathrm{~Hz}, 2 \mathrm{H}\right), 7.17\left(\mathrm{ddd},{ }^{3} \mathrm{~J}=8.2,7.0 \mathrm{~Hz},{ }^{4} \mathrm{~J}=1.1 \mathrm{~Hz}, 2 \mathrm{H}\right), 7.11$ $\left(\mathrm{d},{ }^{3} J=8.9 \mathrm{~Hz}, 2 \mathrm{H}\right), 6.88\left(\mathrm{~d},{ }^{3} J=8.1 \mathrm{~Hz}, 2 \mathrm{H}\right), 6.84\left(\mathrm{~d},{ }^{3} J=9.0 \mathrm{~Hz}, 2 \mathrm{H}\right), 4.26(\mathrm{q}$, $\left.{ }^{3} \mathrm{~J}=7.1 \mathrm{~Hz}, 2 \mathrm{H}\right), 3.84\left(\mathrm{t},{ }^{3} \mathrm{~J}=6.2 \mathrm{~Hz}, 4 \mathrm{H}\right), 3.71-3.65(\mathrm{~m}, 16 \mathrm{H}), 1.28\left(\mathrm{t},{ }^{3} \mathrm{~J}=7.1 \mathrm{~Hz}\right.$, $3 \mathrm{H})$. 


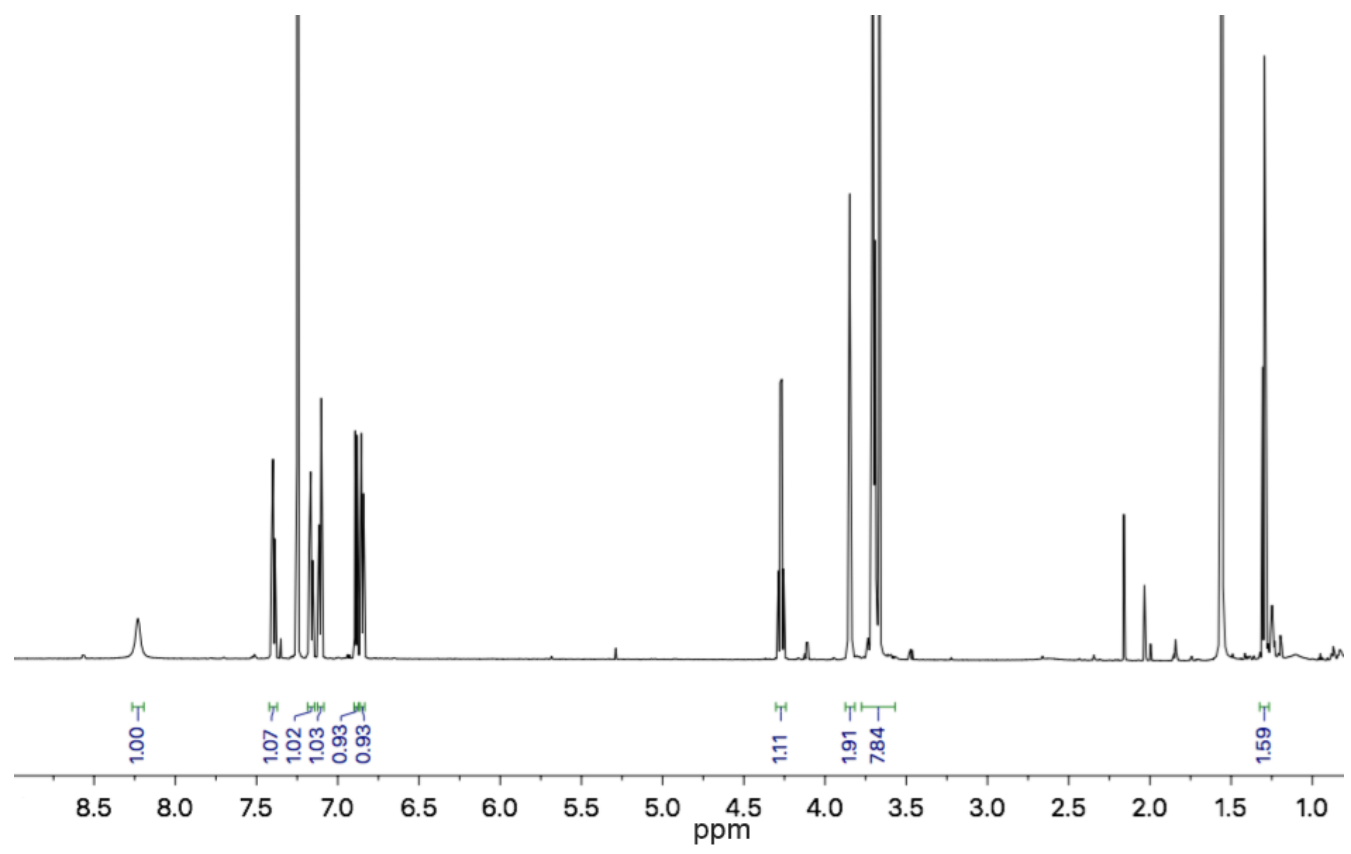

${ }^{13}$ C-NMR $\left(176 \mathrm{MHz}, 298 \mathrm{~K}, \mathrm{CD}_{3} \mathrm{Cl}\right): \delta(\mathrm{ppm})=166.10,153.02,148.20,141.11,131.96$, 130.65, 127.91, 126.31, 121.23, 120.68, 118.25, 116.28, 112.92, 89.34, 71.50, 70.47, 70.06, $68.53,61.76,53.09,14.32$.

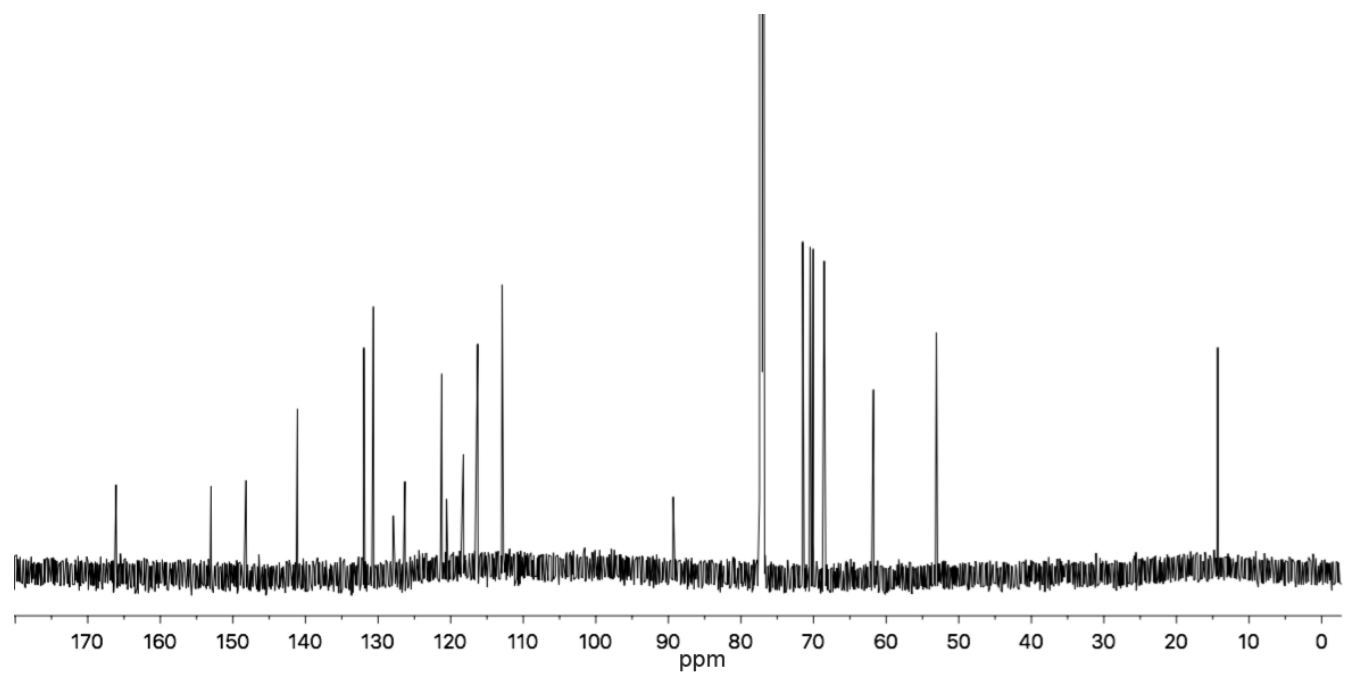

IR $($ ATR $): \tilde{\nu}\left(\mathrm{cm}^{-1}\right)=2863,2180,1703,1602,1505,1456,1354,1227,1093,740,622$, 542 .

ESI-HRMS $\left(\left[\mathrm{C}_{24} \mathrm{H}_{26} \mathrm{~N}_{2} \mathrm{O}_{2}\right]^{+}\right)$: $\quad$ measured: $\quad 584.2749$

calculated: $\quad 584.2761$ 
Appendix 


\section{Appendix}

\section{Further Analytical Data}

\section{Contents}

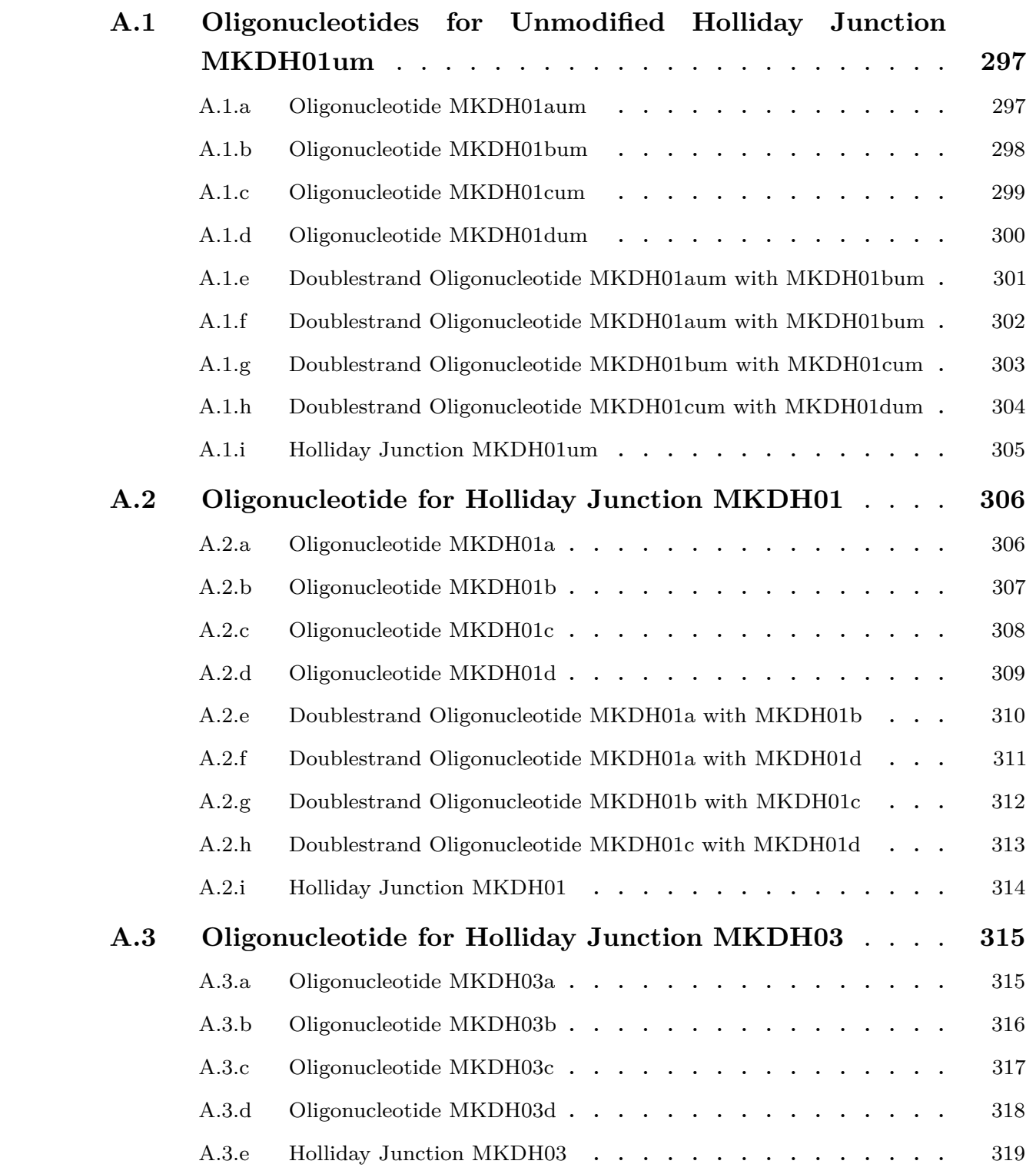




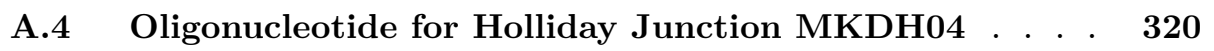

A.4.a Oligonucleotide MKDH04a . . . . . . . . . . . . . . . 320

A.4.b Oligonucleotide MKDH04b . . . . . . . . . . . . . . . 321

A.4.c Oligonucleotide MKDH04c . . . . . . . . . . . . . . . 322

A.4.d Oligonucleotide MKDH04d . . . . . . . . . . . . . . . . 323

A.4.e Holliday Junction MKDH04 … . . . . . . . . 324

A.5 Oligonucleotide for Holliday Junction MKDH05 … 325

A.5.a Oligonucleotide MKDH05a . . . . . . . . . . . . 325

A.5.b Oligonucleotide MKDH05b. . . . . . . . . . . . . 326

A.5.c Oligonucleotide MKDH05c . . . . . . . . . . . . . . . 327

A.5.d Oligonucleotide MKDH05d . . . . . . . . . . . . . . 328

A.5.e Holliday Junction MKDH05 . . . . . . . . . . . . . . . 329

\begin{tabular}{|ll}
\hline A.6 & Oligonucleotide for Holliday Junction MKDH06
\end{tabular} . . . 330

A.6.a Oligonucleotide MKDH06a . . . . . . . . . . . . . 330

A.6.b Oligonucleotide MKDH06b . . . . . . . . . . . . . . . 331

A.6.c Oligonucleotide MKDH06c . . . . . . . . . . . . . 332

A.6.d Oligonucleotide MKDH06d . . . . . . . . . . . . . . . 333

A.6.e Holliday Junction MKDH06 . . . . . . . . . . . . . . 334

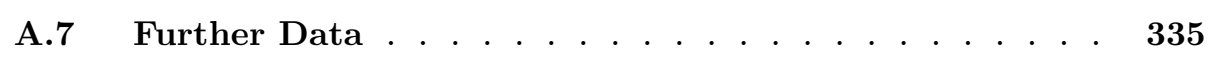

A.7.a Host-Guest Titration Diethanolamin-Methoxy Ligand . . . . . 335

\begin{tabular}{|l|l|l|}
\hline A.7.b & VT NMR experiments for the ligands $\mathbf{L}^{t \mathrm{Bu}}, \mathbf{L}^{\mathrm{Et}}, \mathbf{L}^{\mathrm{Ph}}$ and $\mathbf{L}^{\mathrm{CN}}$ \\
\hline
\end{tabular}

A.7.c Cyano-acridinylidine ethyl acetate titration data . . . . . . 337

A.7.d Aza-15-crown-cyano-acridinylidine ethyl acetate . . . . . . 342

A.8 Crystal Data and Structure Refinement . . . . . . . . 345 


\section{Oligonucleotides Analytical Data}

The here presented data represent the characterization of the synthesized modified and unmodified oligonucleotide strands used and described in this thesis. The measured data for the melting curves of the respective ONS system is shown as red solid line. The corresponding fit to determine the denaturation temperature is always represented as a black dashed line. Since there is no general method to determine the denaturation temperature $\left(\mathrm{T}_{\mathrm{M}}\right.$, all established methods, described in the publication of Lacroix et al.,160 were used. At first, from the measured data, the denaturation temperature was determined with help of the baseline method. Secondly, $T_{M}$ was determined from first derivative of the measured melting curve with help of a GaussAmp fit. Last denaturation temperature was received from the "fraction folded" method. From the newly generated curve the temperature was determined with a DoseResp fit, which is normally used for the determination of $\mathrm{EH}_{50}$ values. 333 


\section{A.1.a Oligonucleotide MKDH01aum}

\section{Sequence 5' $\rightarrow$ 3'}

GCC ATA GTT TGA GCA CG

\section{Sum Formula}

$$
\mathrm{C}_{166} \mathrm{H}_{209} \mathrm{~N}_{65} \mathrm{O}_{100} \mathrm{P}_{16}
$$

\section{$5207.91 \mathrm{~g} \mathrm{~mol}^{-1}$}

\section{HPLC Traces}

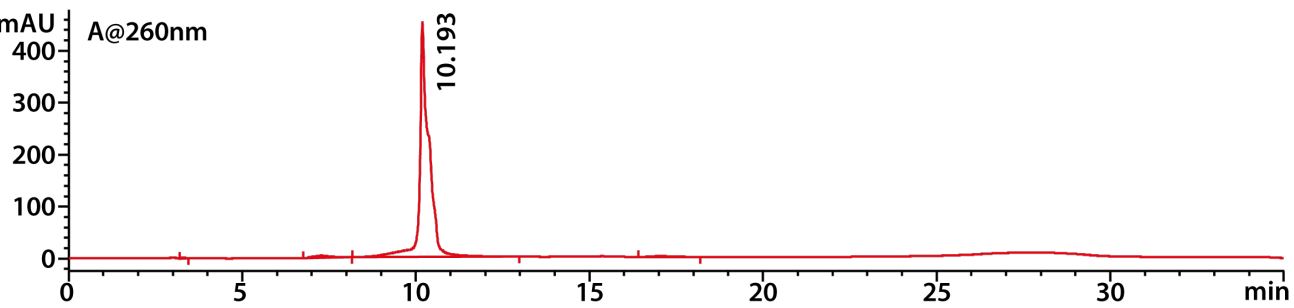

HPLC Traces

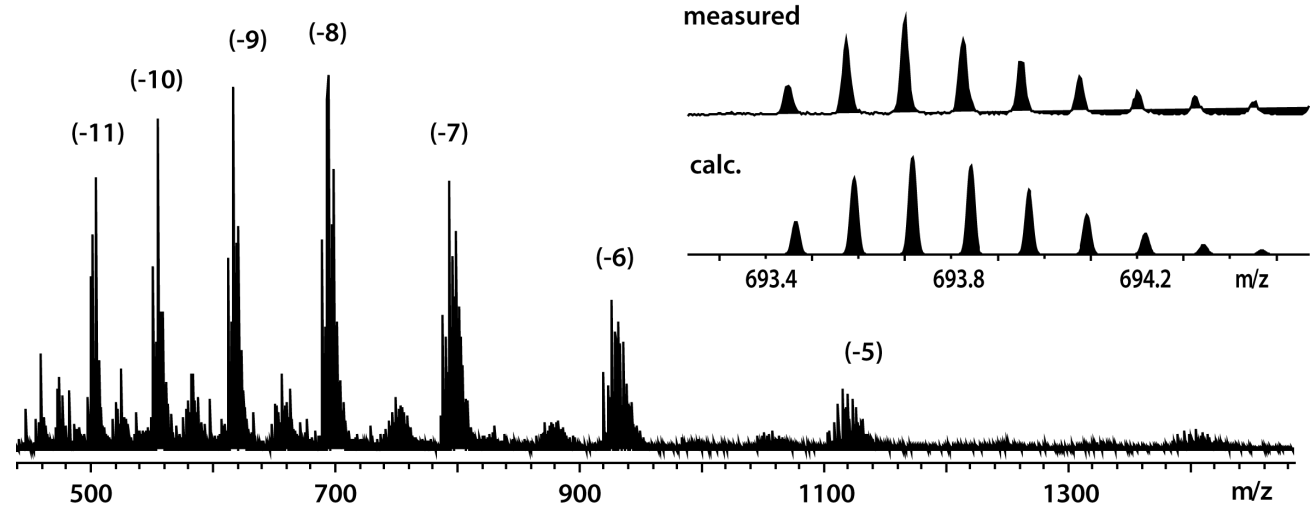




\section{A.1.b Oligonucleotide MKDH01bum}

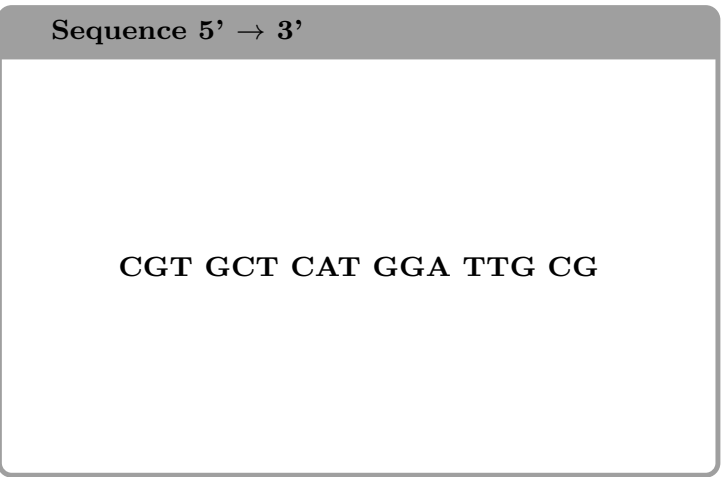

Sum Formula

$$
\mathrm{C}_{166} \mathrm{H}_{210} \mathrm{~N}_{62} \mathrm{O}_{103} \mathrm{P}_{16}
$$

Mol. Mass

$5214.89 \mathrm{~g} \mathrm{~mol}^{-1}$

\section{HPLC Traces}

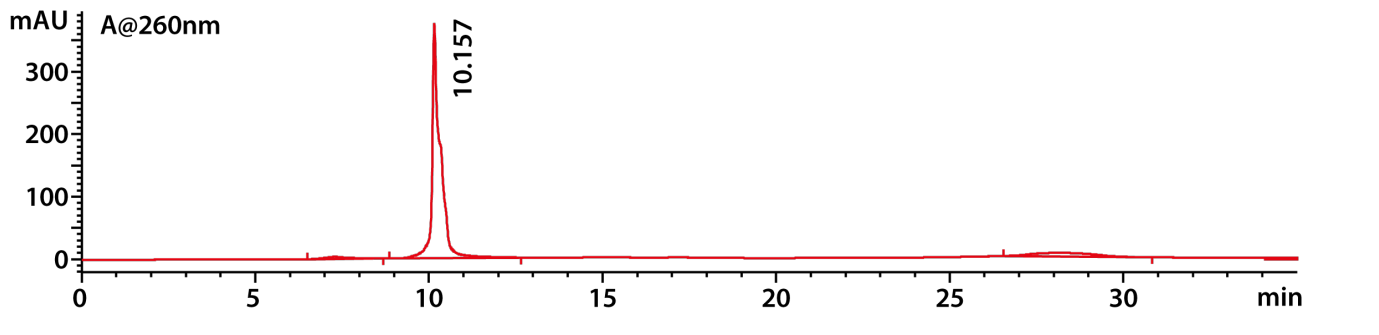

HPLC Traces

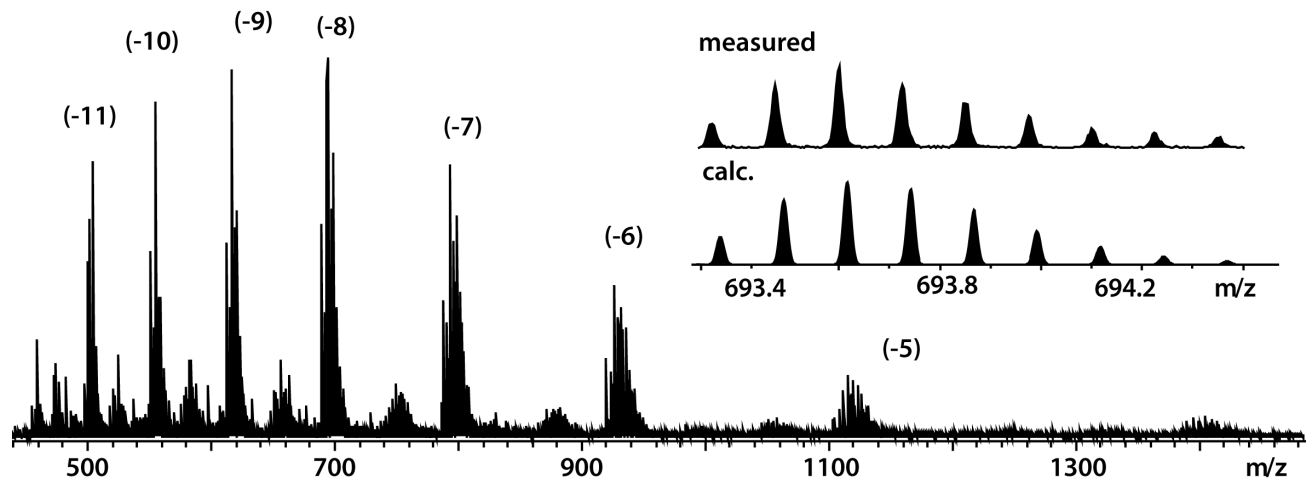




\section{A.1.c Oligonucleotide MKDH01cum}

\section{Sequence 5' $\rightarrow$ 3'}

CGC AAT CCT CCG AAT GC

\section{Sum Formula}

$$
\mathrm{C}_{163} \mathrm{H}_{208} \mathrm{~N}_{62} \mathrm{O}_{99} \mathrm{P}_{16}
$$

$$
5112.89 \mathrm{~g} \mathrm{~mol}^{-1}
$$

\section{HPLC Traces}

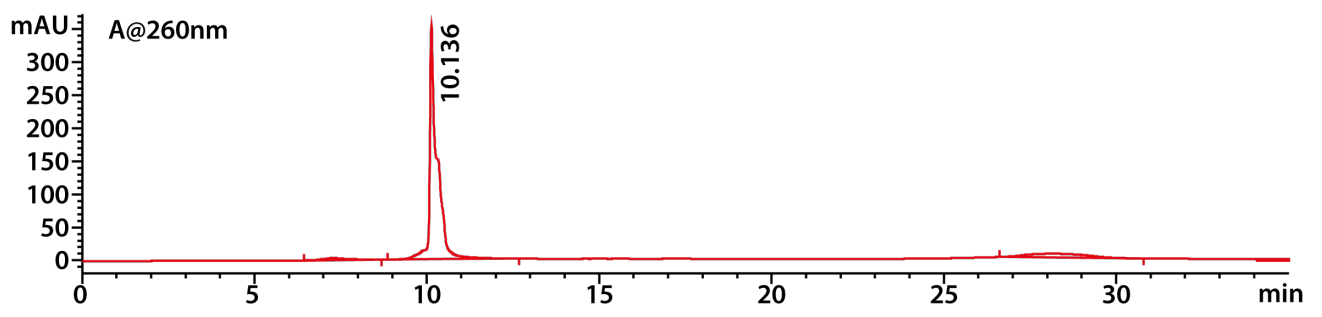

\section{HPLC Traces}

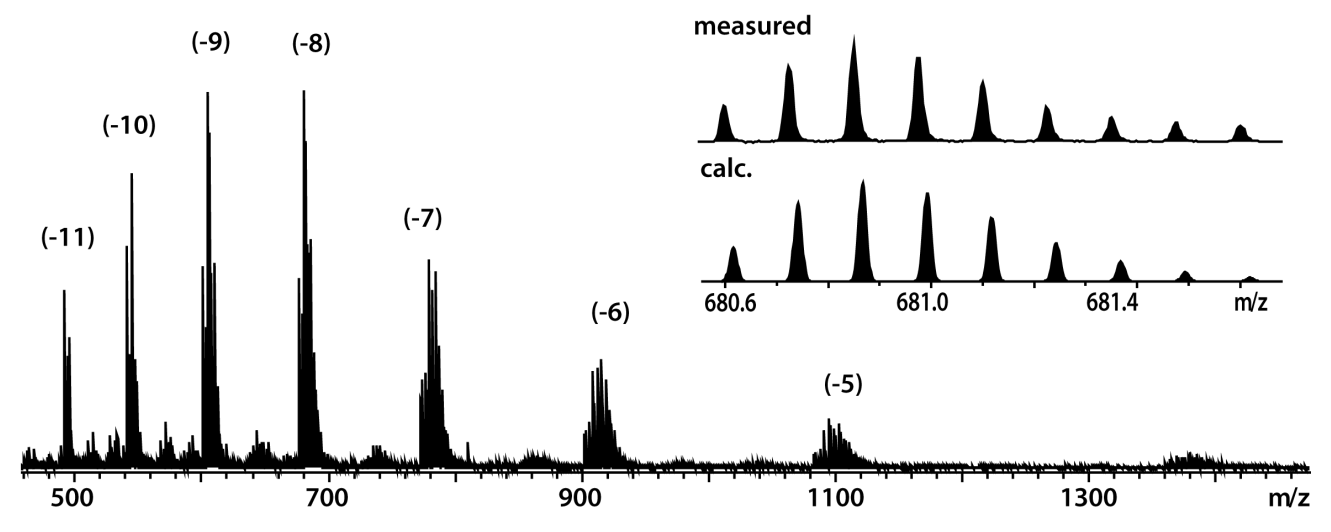




\section{A.1.d Oligonucleotide MKDH01dum}

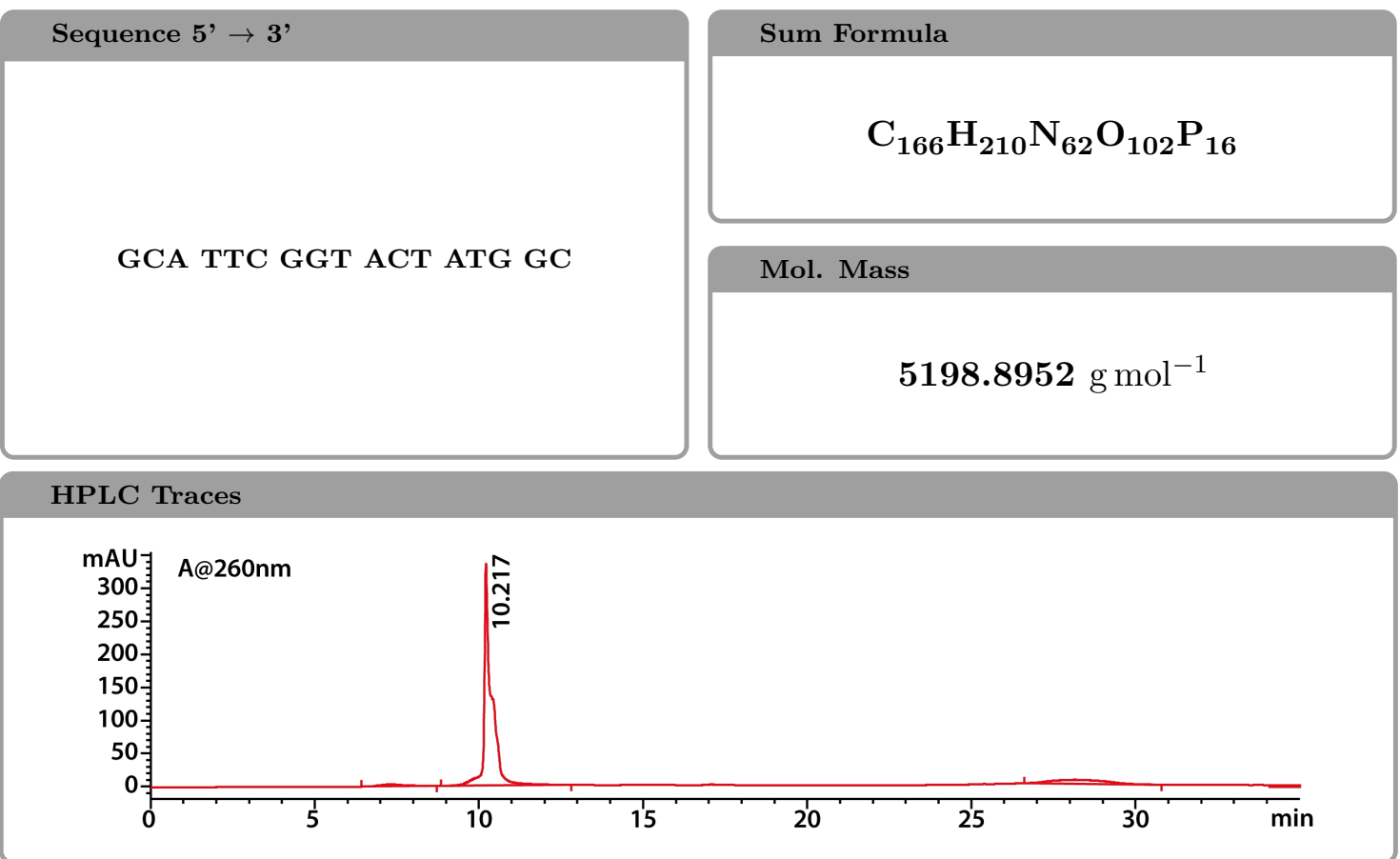

\section{HPLC Traces}

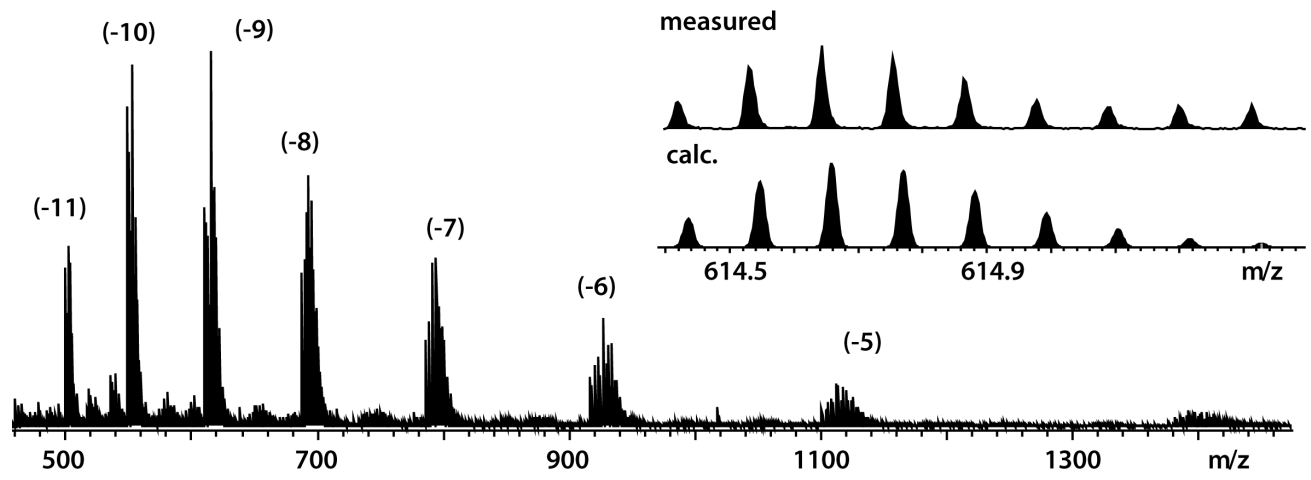




\section{A.1.e Doublestrand Oligonucleotide MKDH01aum with MKDH01bum}

\section{Sequences}

a: 5'-GCC ATA GTT TGA GCA CG-3'

b: 3'-GCG TTA GGT ACT CGT GC-5'

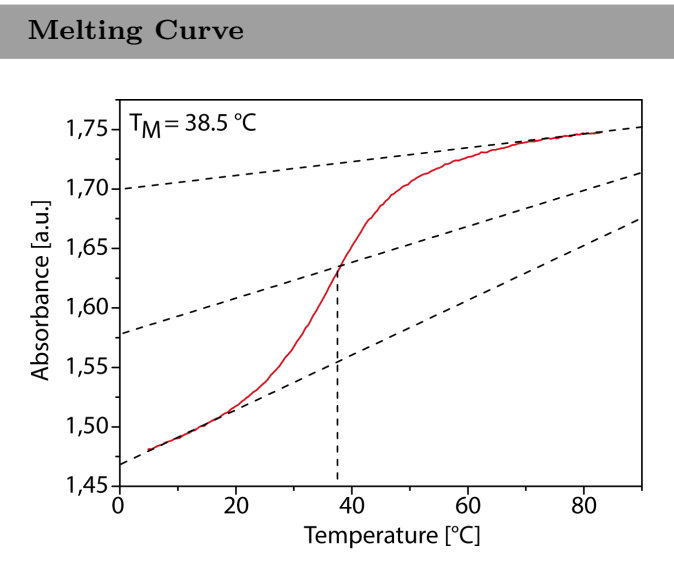

Melting Curve

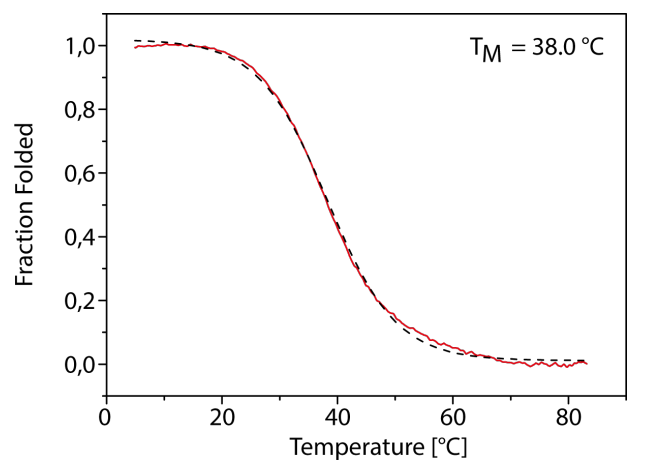

\section{Melting Curve}

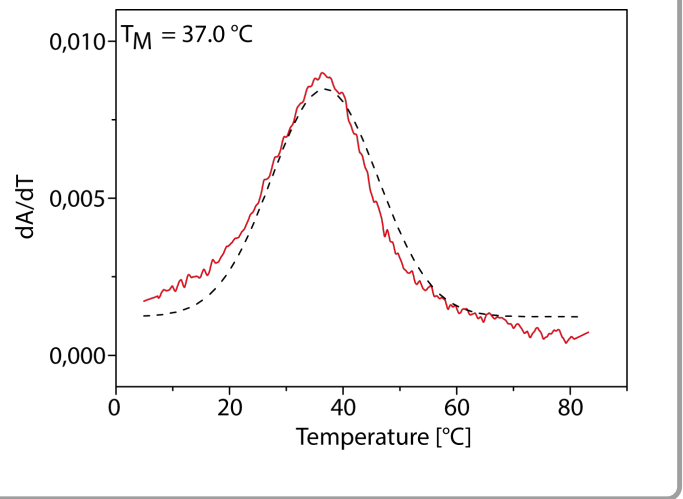

UV-vis

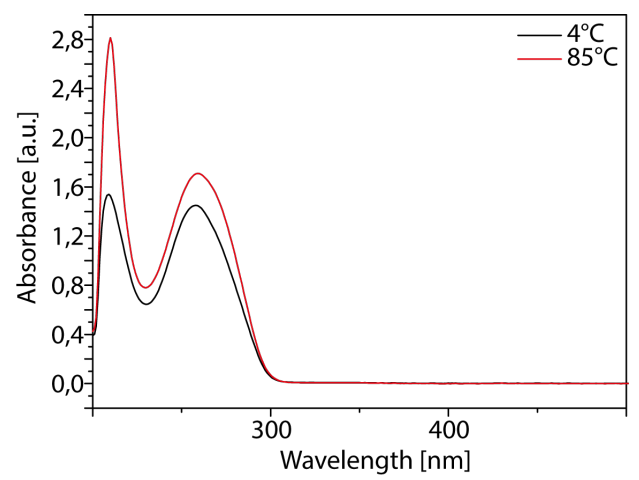




\section{A.1.f Doublestrand Oligonucleotide MKDH01aum with MKDH01bum}

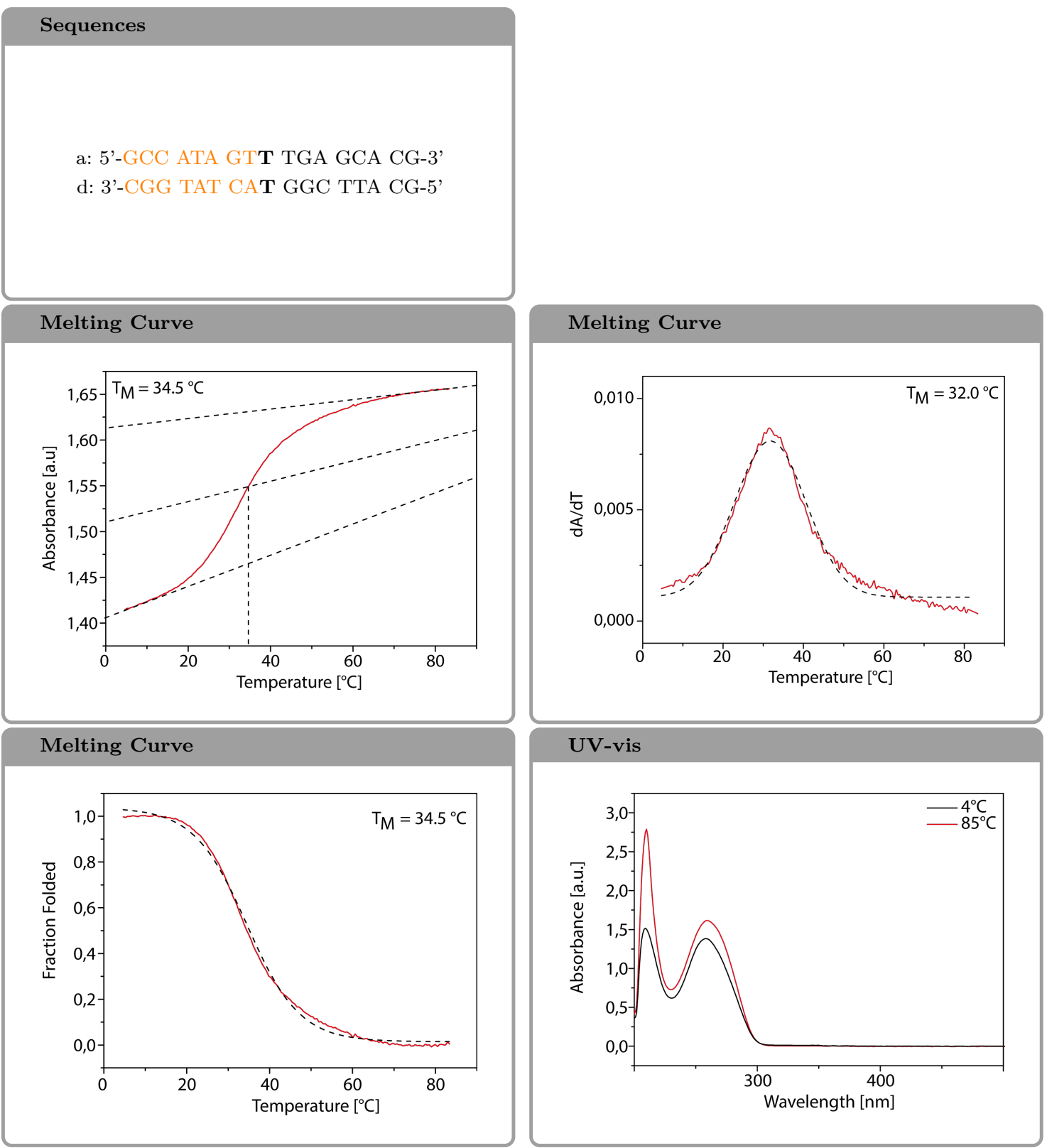




\section{A.1.g Doublestrand Oligonucleotide MKDH01bum with MKDH01cum}

\section{Sequences}

b: 3'-GCG TTA GGT ACT CGT GC-5'

c: 5'-CGC AAT CCT CCG AAT GC-3'

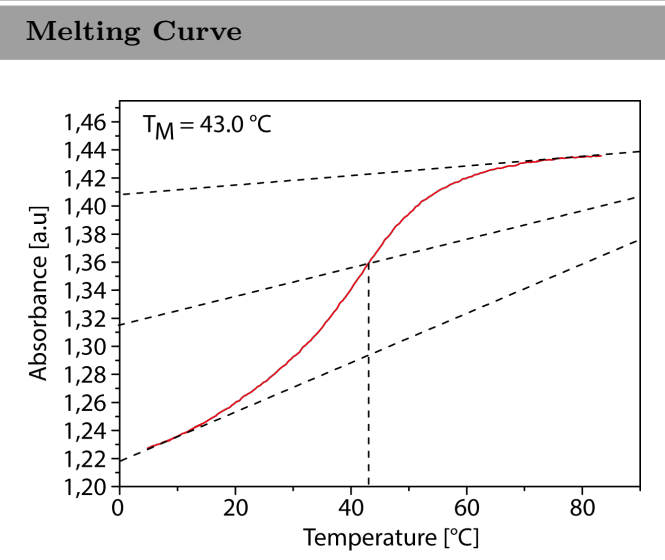

\section{Melting Curve}

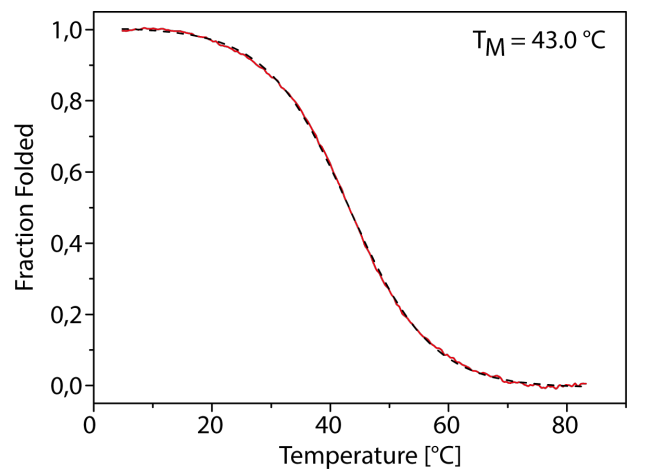

\section{Melting Curve}

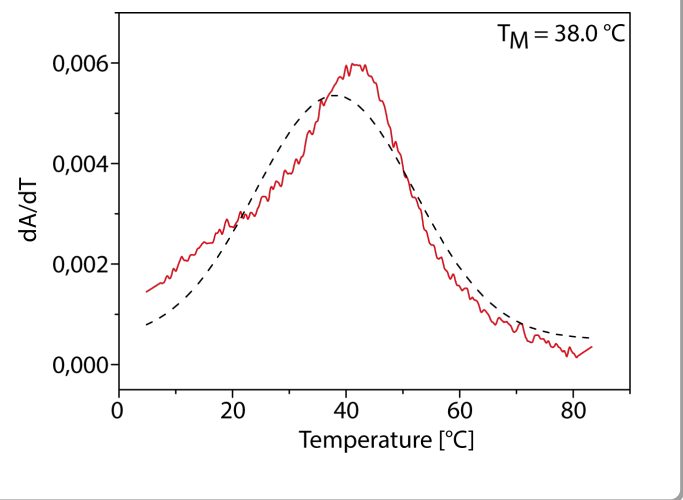

UV-vis

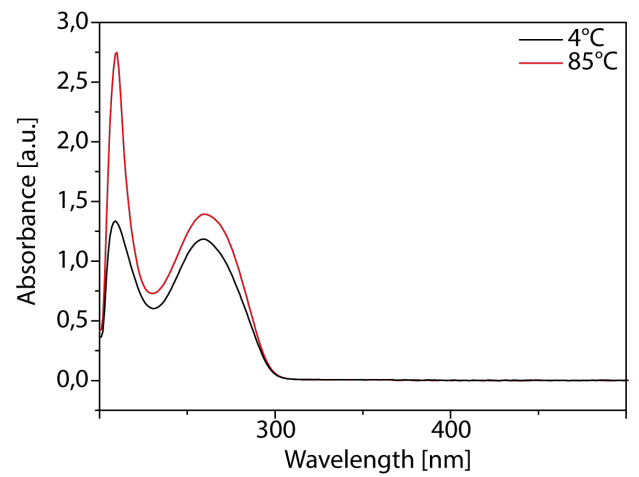




\section{A.1.h Doublestrand Oligonucleotide MKDH01cum with MKDH01dum}
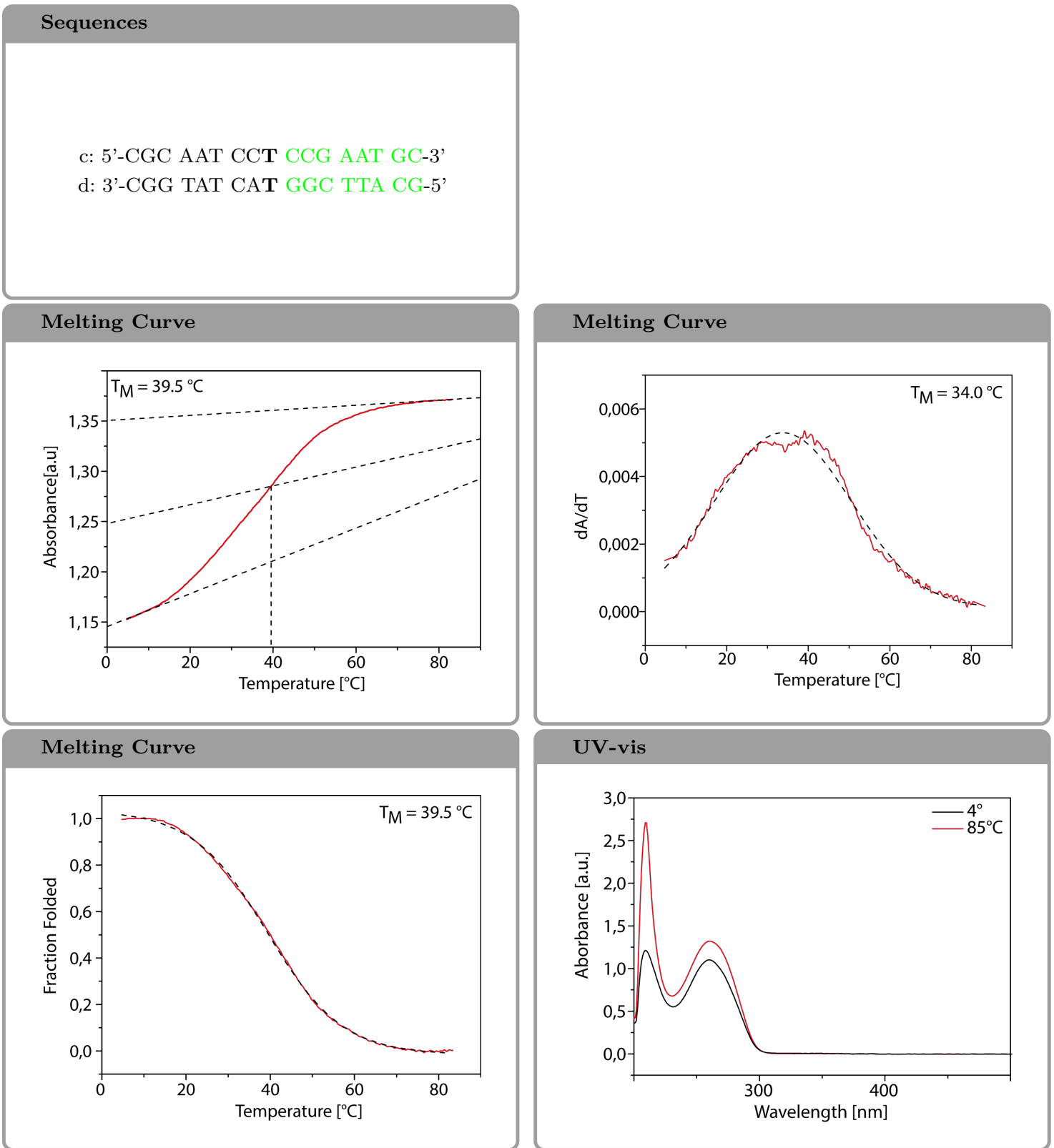

$\mathrm{UV}$-vis

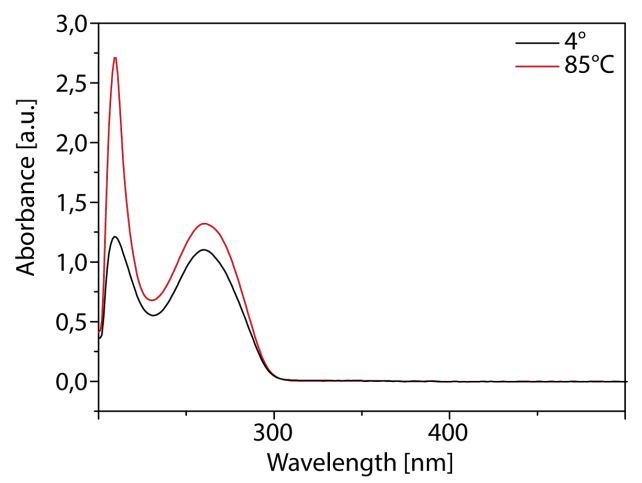




\section{A.1.i Holliday Junction MKDH01um}

\section{Sequences}

a: 5'-GCC ATA GTT TGA GCA CG-3'

b: 3'-GCG TTA GGT ACT CGT GC-5'

c: 5'-CGC AAT CCT CCG AAT GC-3'

d: 3'-CGG TAT CAT GGC TTA CG-5'
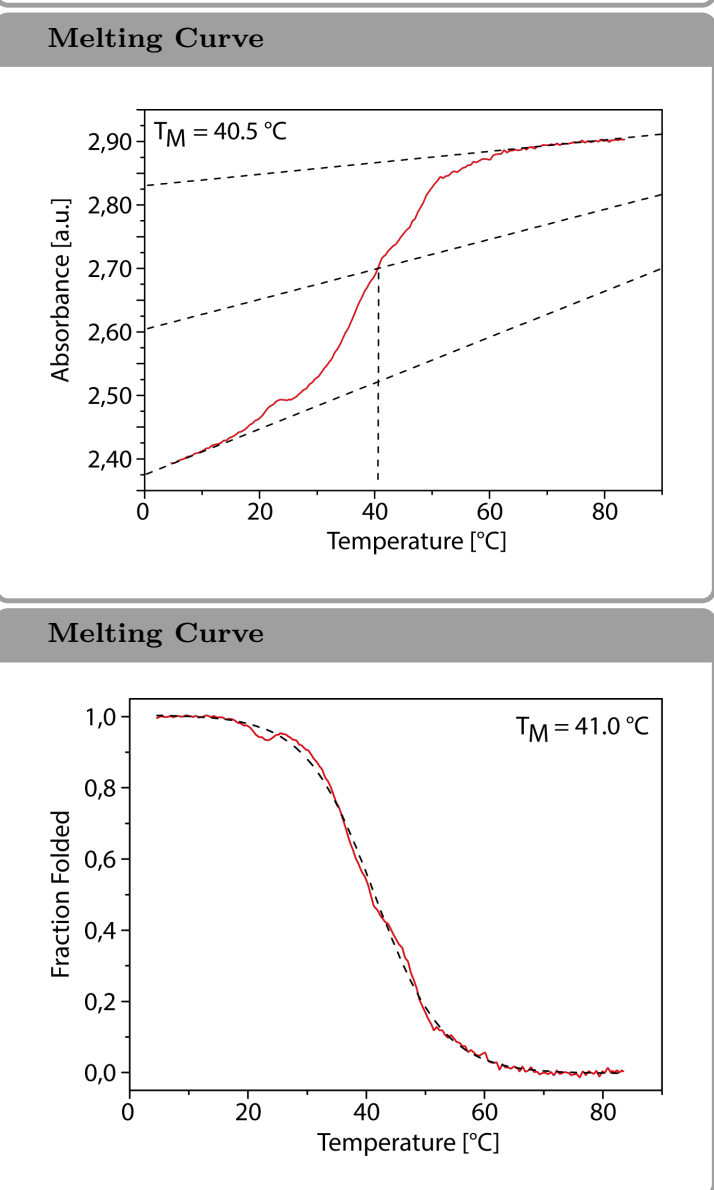

Circular Dichroism

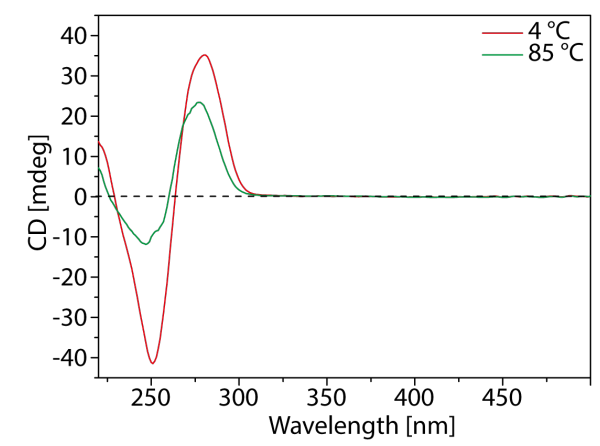

\section{Melting Curve}

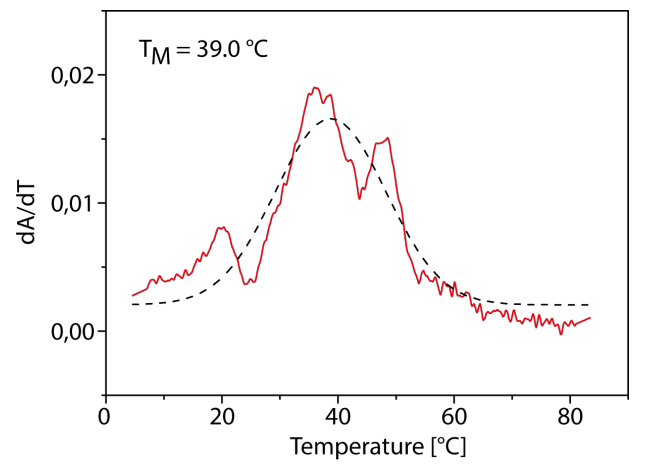

\section{UV-vis}

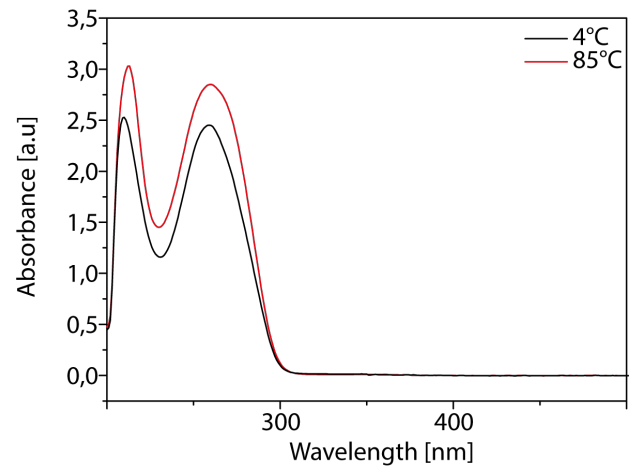




\section{A.2.a Oligonucleotide MKDH01a}

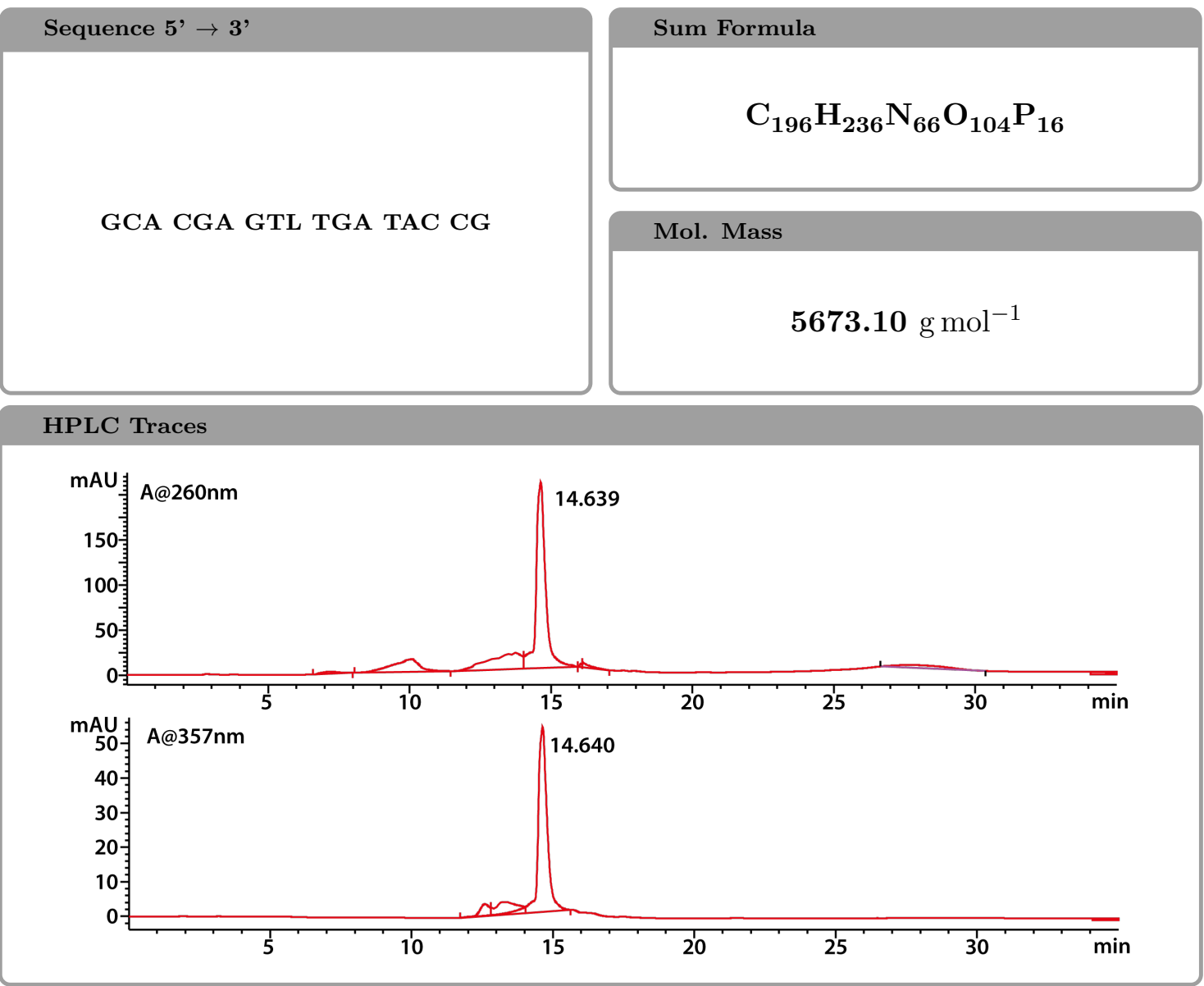

HPLC Traces

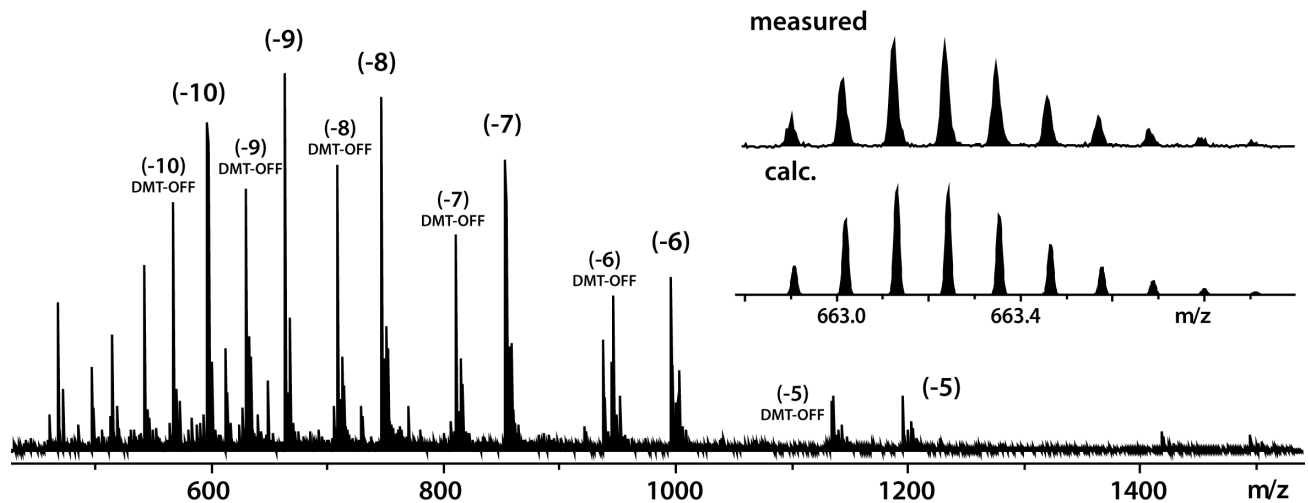




\section{A.2.b Oligonucleotide MKDH01b}

Sequence 5' $\rightarrow$ 3'

GCG TTA GGL ACT CGT GC
Sum Formula

$$
\mathrm{C}_{196} \mathrm{H}_{237} \mathrm{~N}_{63} \mathrm{O}_{107} \mathrm{P}_{16}
$$

$5680.0841 \mathrm{~g} \mathrm{~mol}^{-1}$

\section{HPLC Traces}
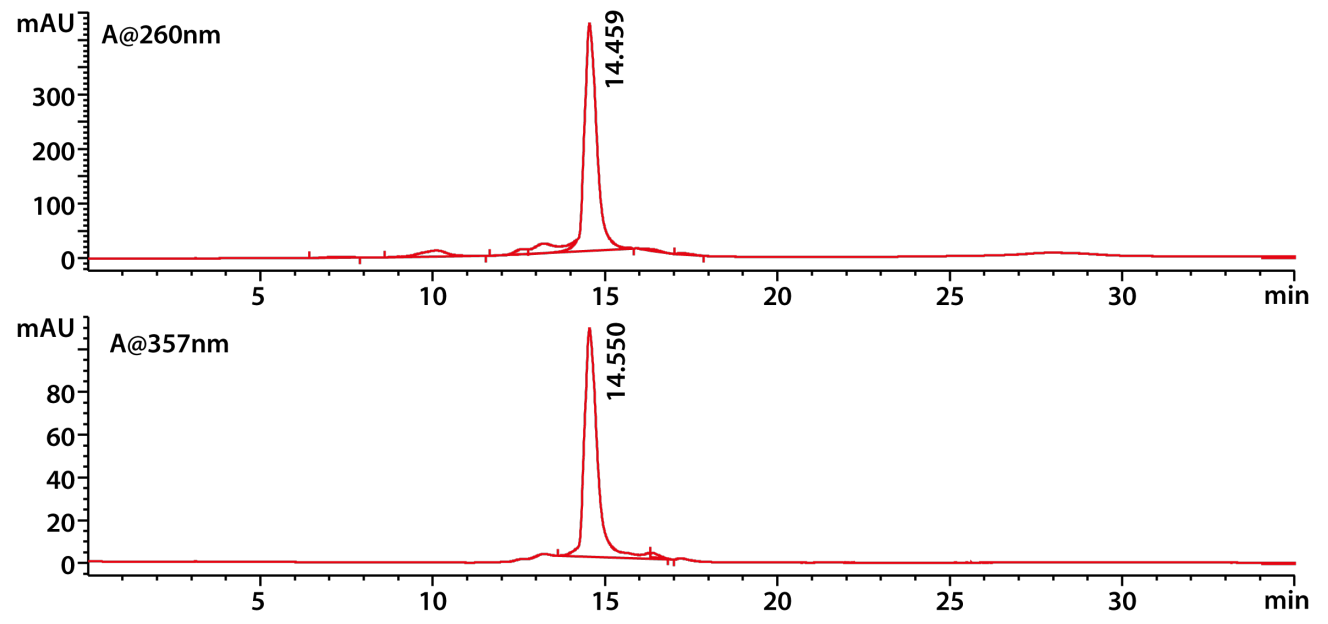

HPLC Traces

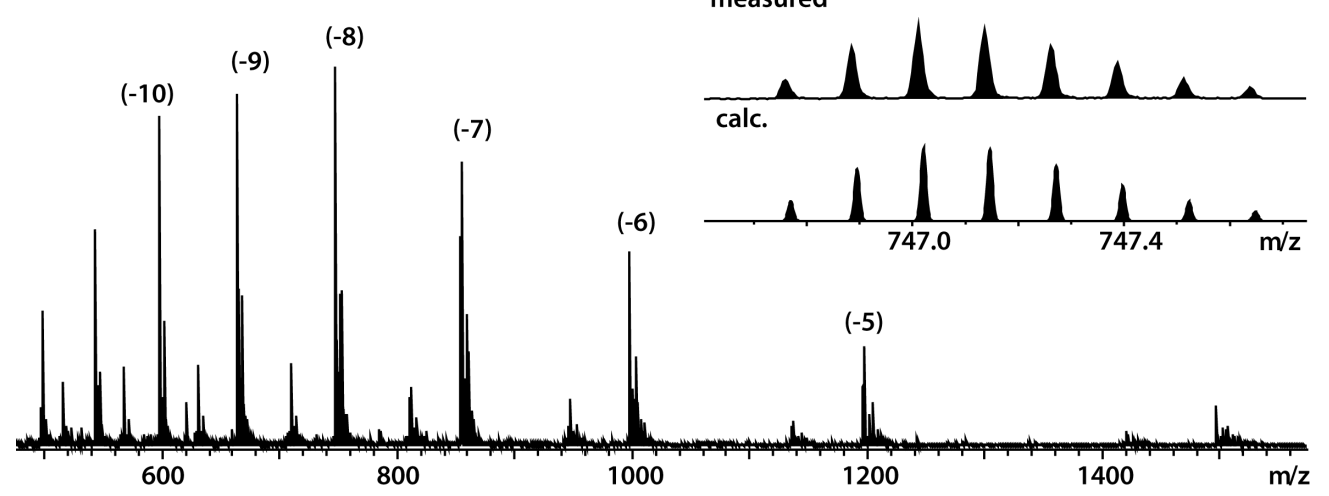




\section{A.2.c Oligonucleotide MKDH01c}

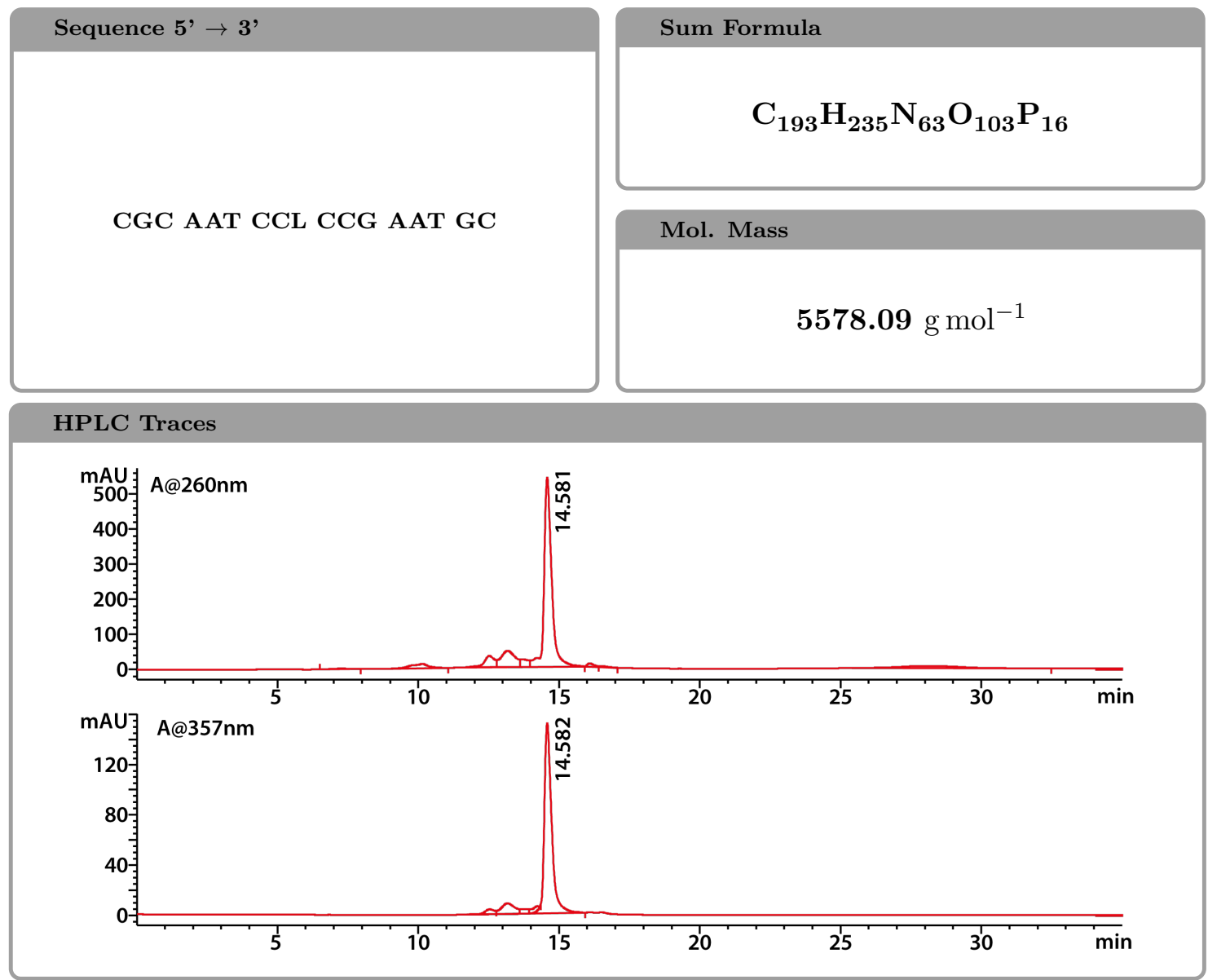

HPLC Traces

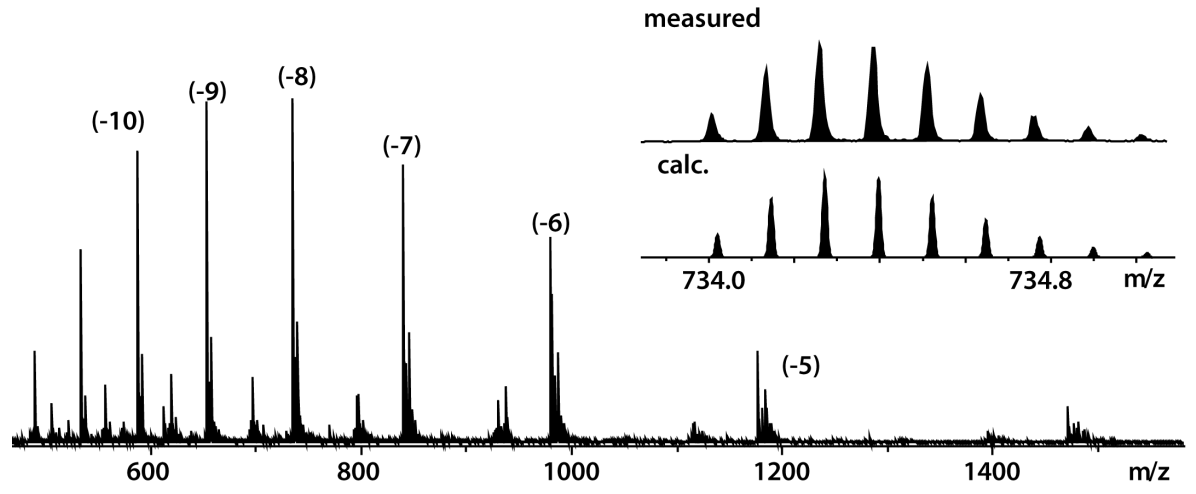




\section{A.2.d Oligonucleotide MKDH01d}

Sequence 5' $\rightarrow$ 3'

GCA TTC GGL ACT ATG GC
Sum Formula

$$
\mathrm{C}_{196} \mathrm{H}_{237} \mathrm{~N}_{63} \mathrm{O}_{106} \mathrm{P}_{16}
$$

\section{$5664.09 \mathrm{~g} \mathrm{~mol}^{-1}$}

\section{HPLC Traces}

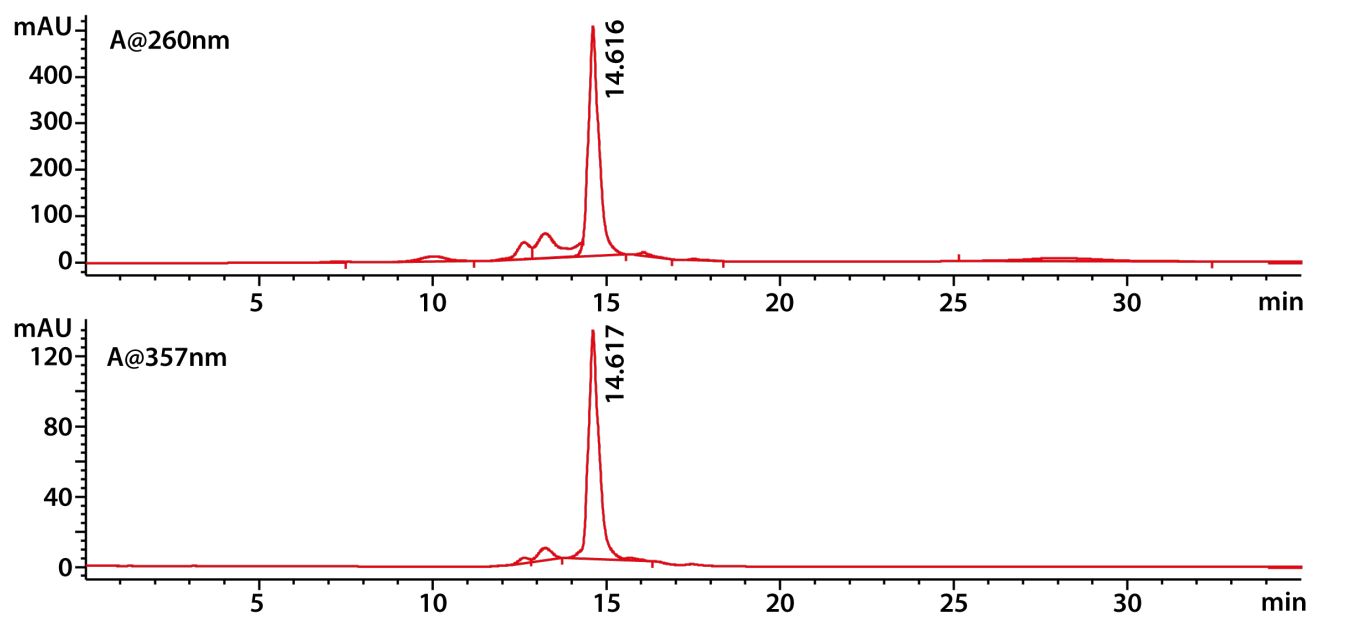

HPLC Traces

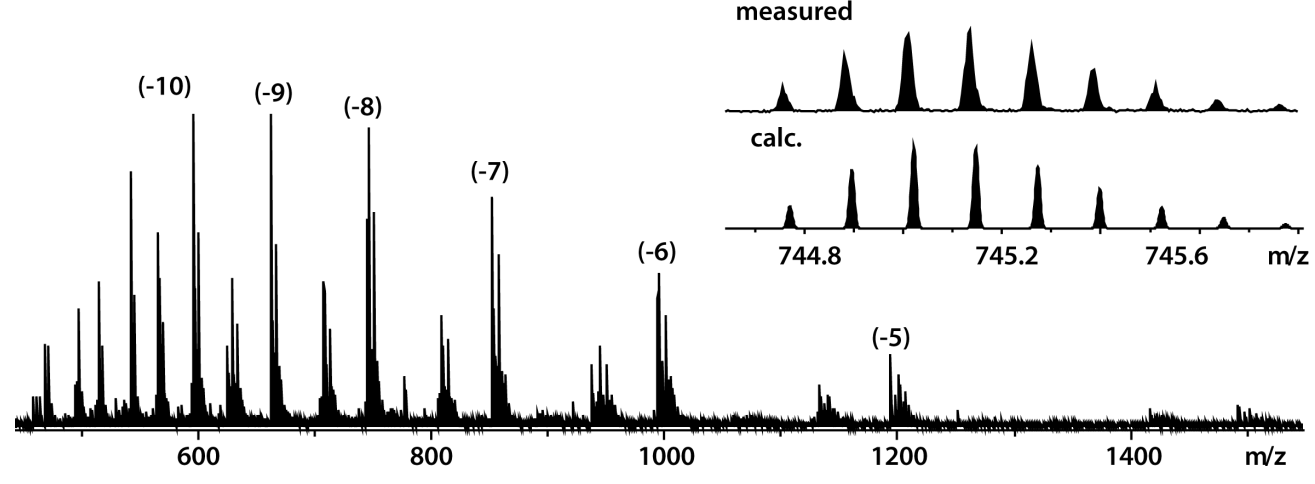




\section{A.2.e Doublestrand Oligonucleotide MKDH01a with MKDH01b}
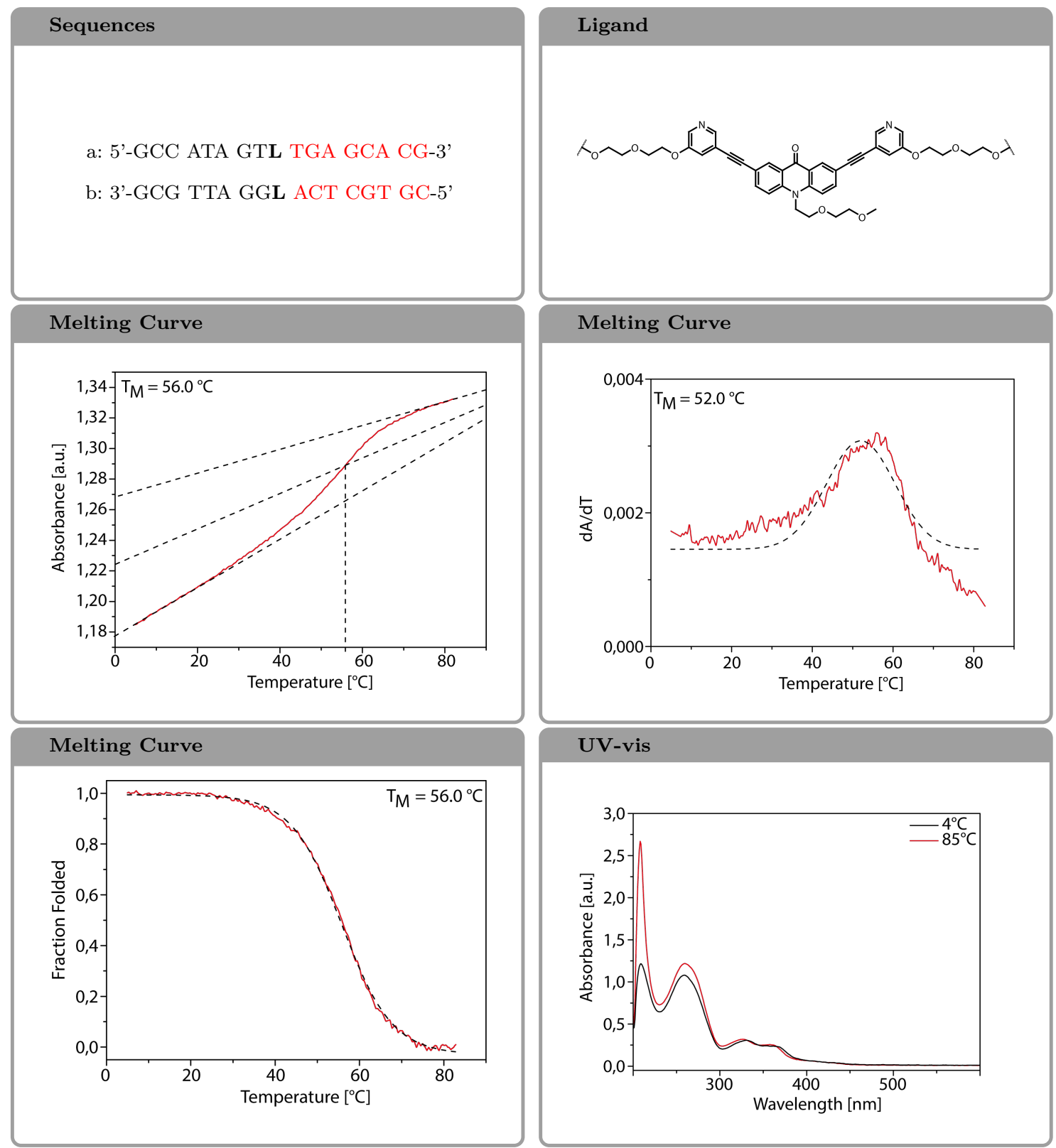

\section{Melting Curve}

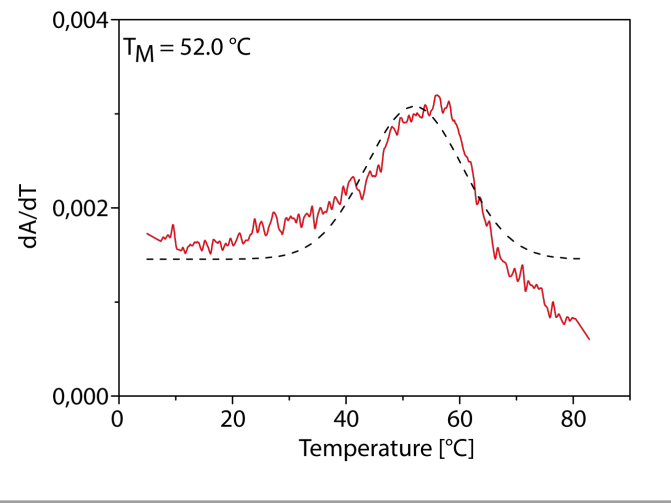

UV-vis

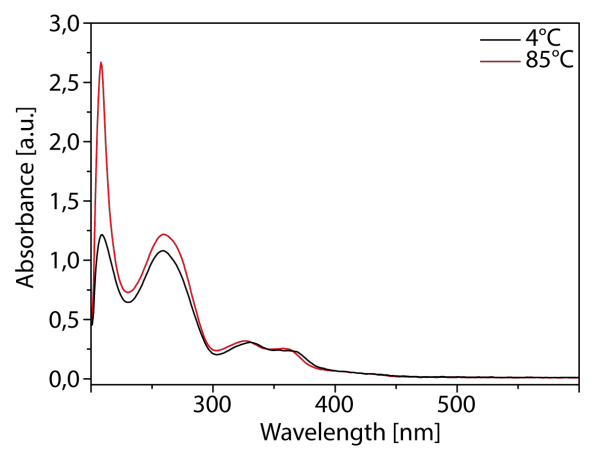




\section{A.2.f Doublestrand Oligonucleotide MKDH01a with MKDH01d}
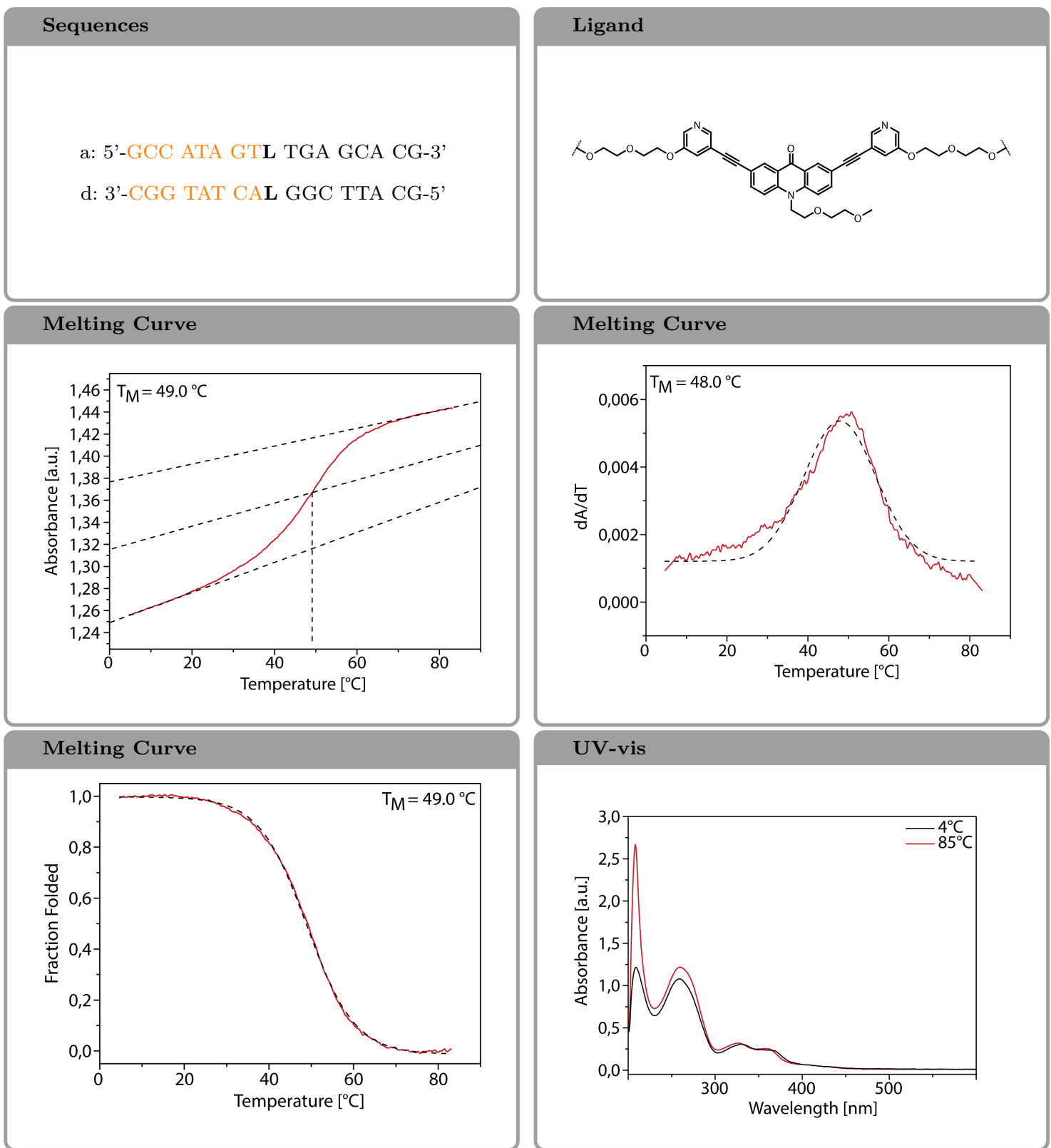

\section{Melting Curve}

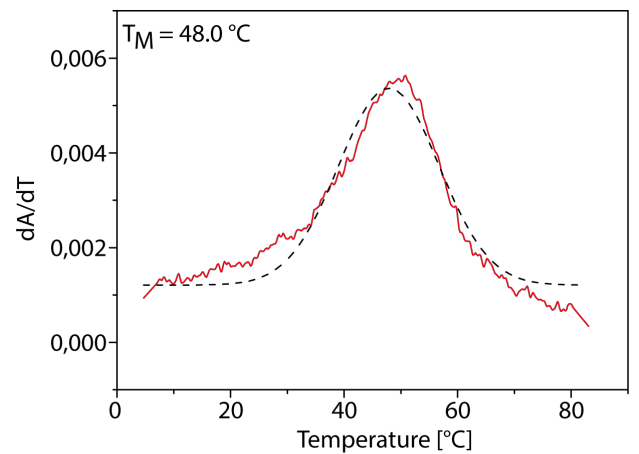

UV-vis

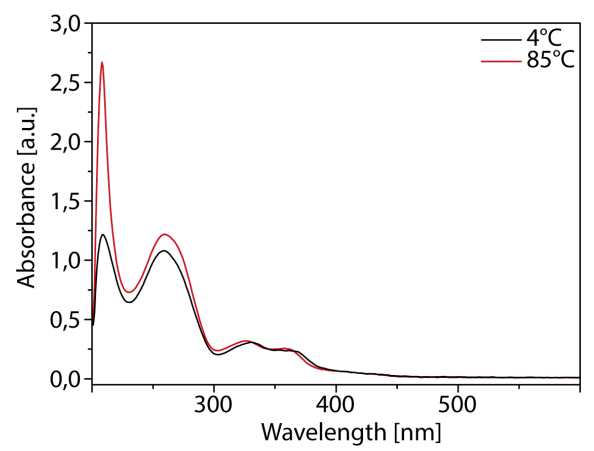




\section{A.2.g Doublestrand Oligonucleotide MKDH01b with MKDH01c}

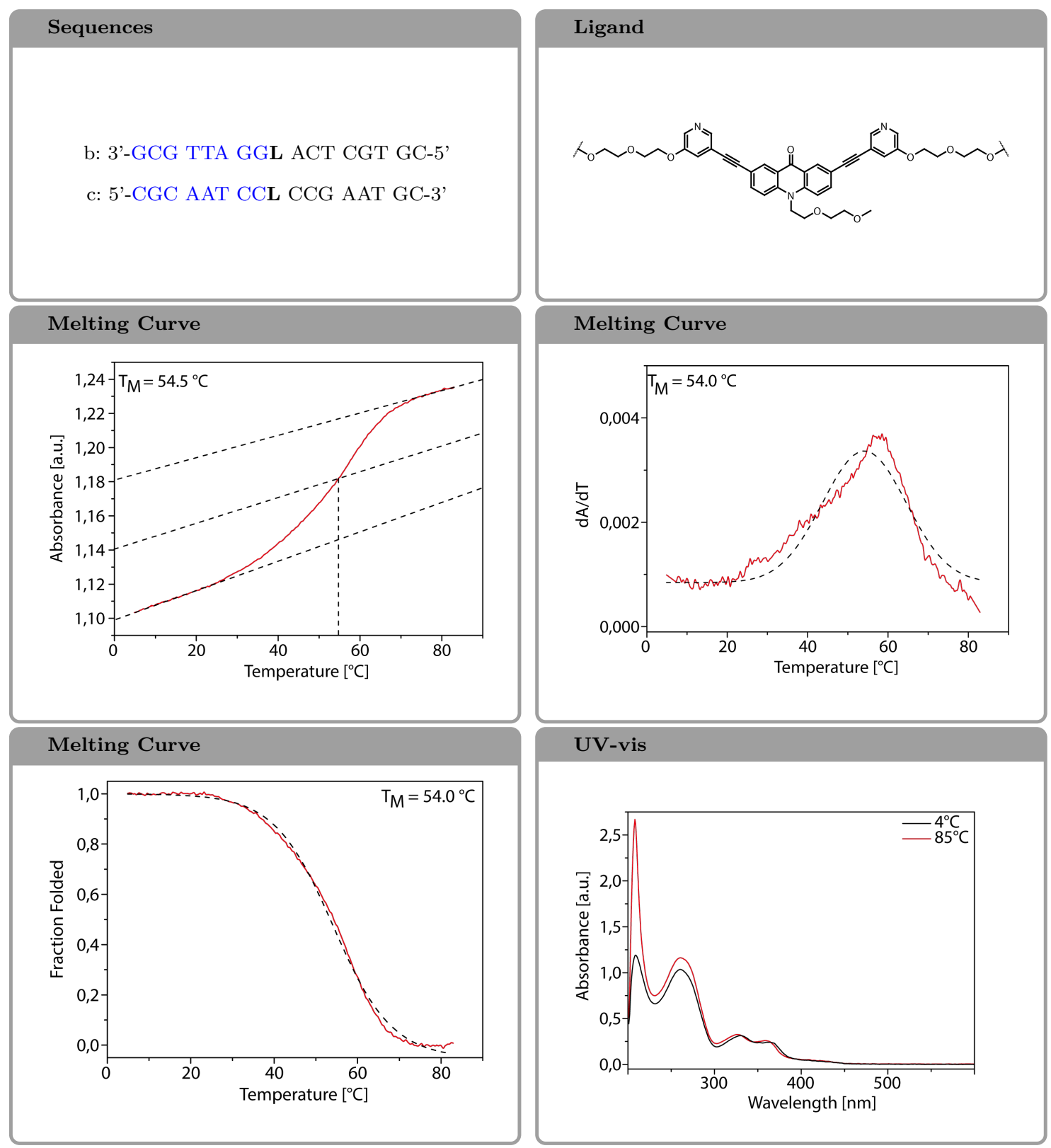




\section{A.2.h Doublestrand Oligonucleotide MKDH01c with MKDH01d}
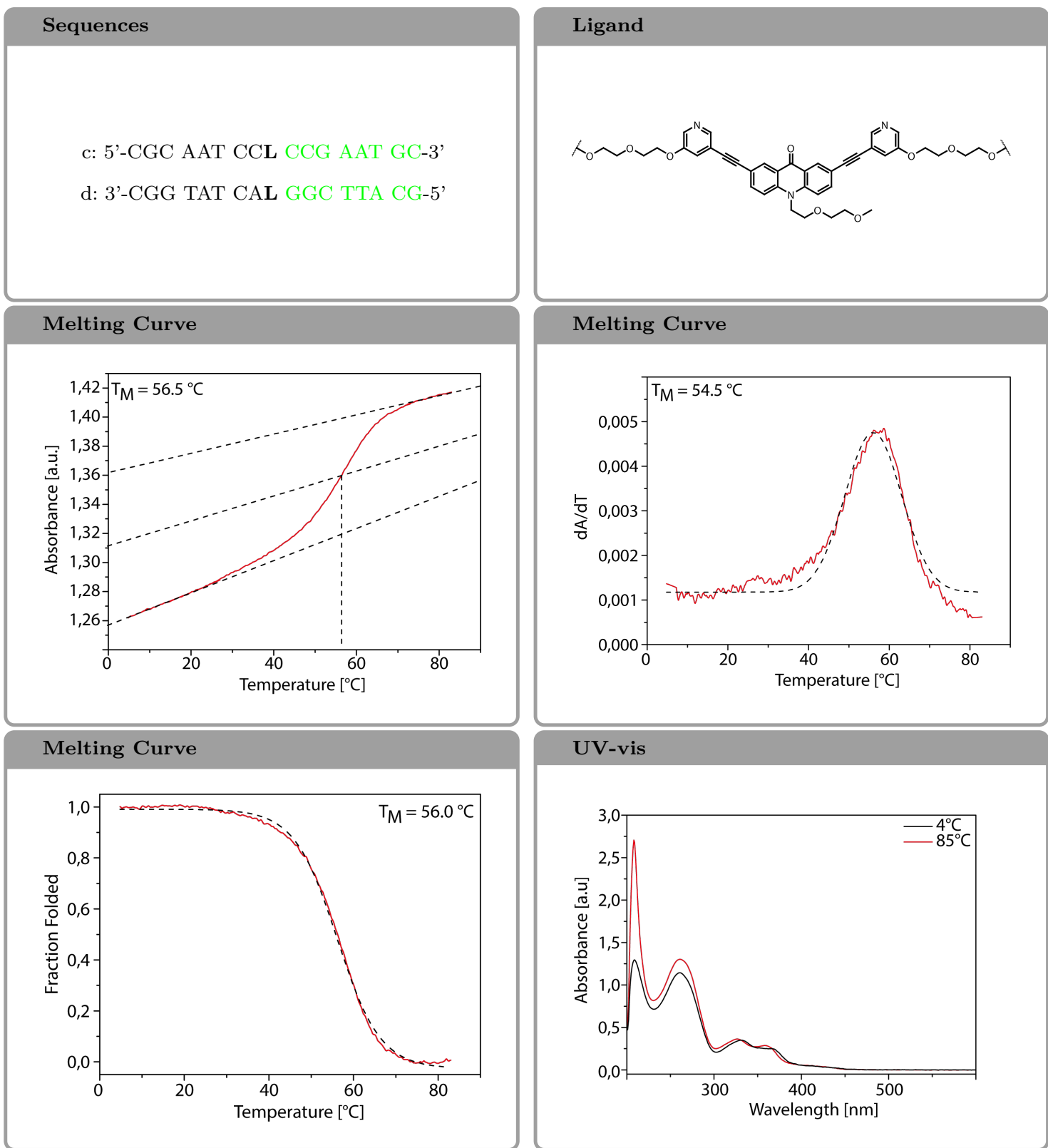

\section{Melting Curve}

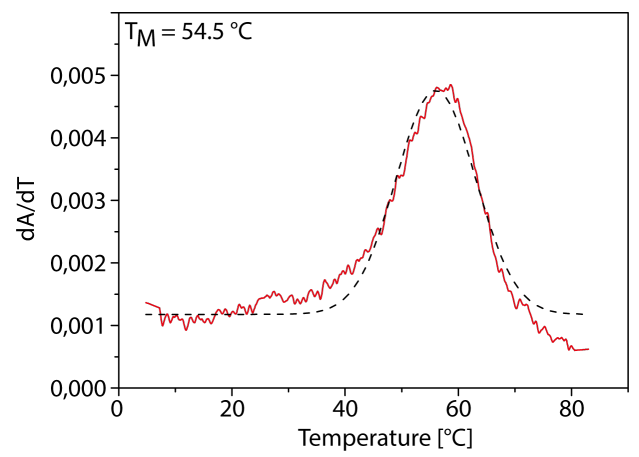

UV-vis

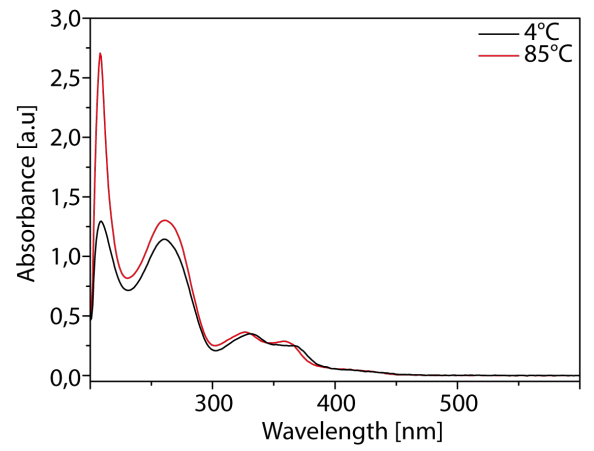




\section{A.2.i Holliday Junction MKDH01}

\begin{tabular}{l} 
Sequences \\
a: 5'-GCC ATA GTL TGA GCA CG-3' \\
b: 3'-GCG TTA GGL ACT CGT GC-5' \\
c: 5'-CGC AAT CCL CCG AAT GC-3' \\
d: 3'-CGG TAT CAL GGC TTA CG-5' \\
\hline
\end{tabular}
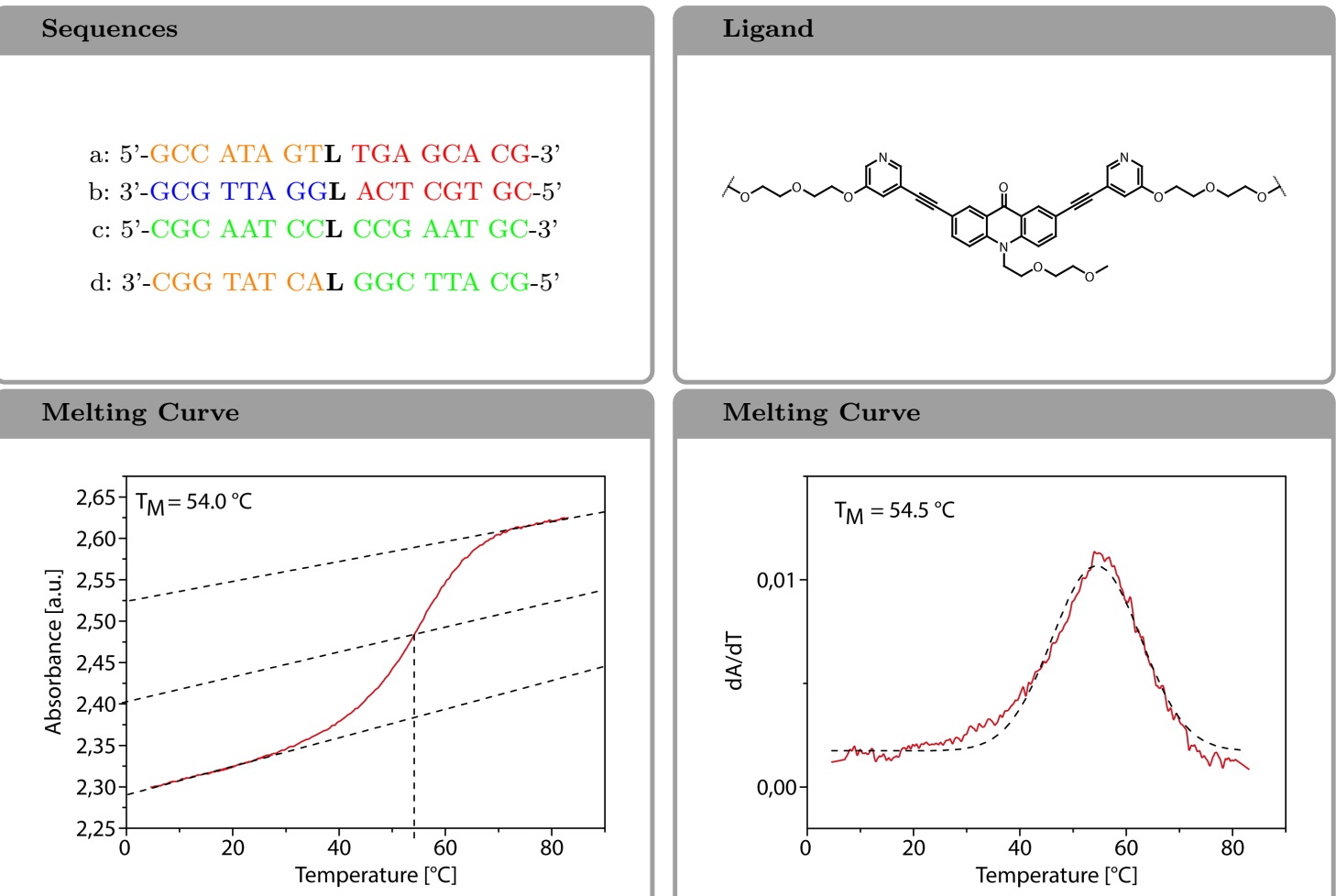

\section{Melting Curve}

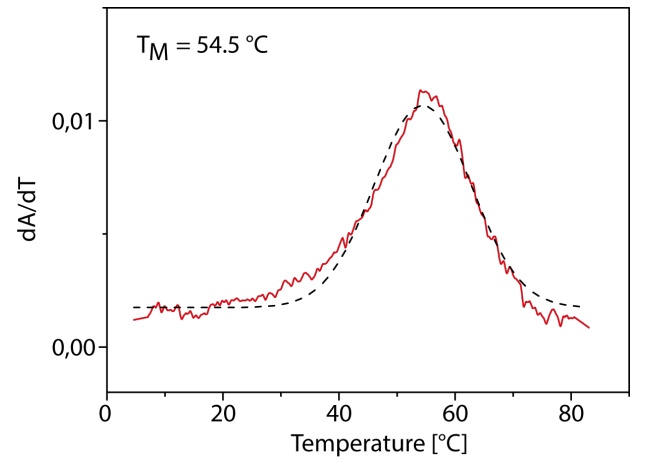

\section{Melting Curve}

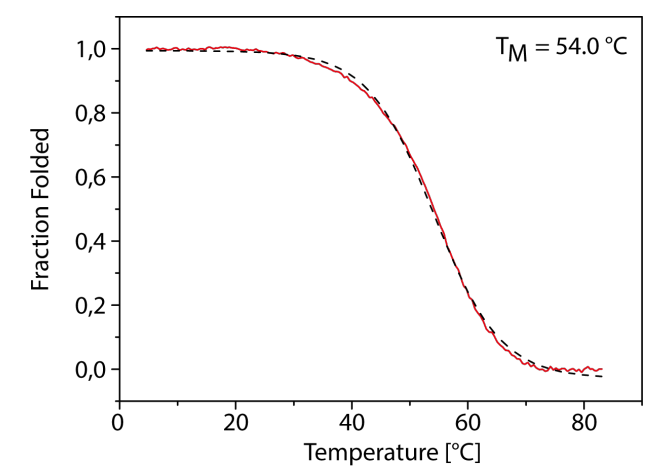

\section{Circular Dichroism}

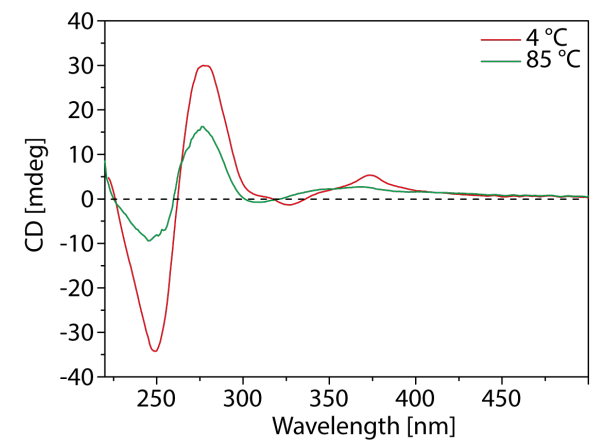

\section{UV-vis}

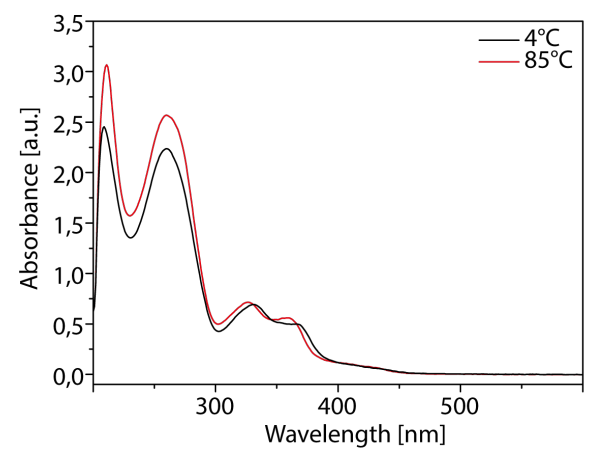

Fluoresence

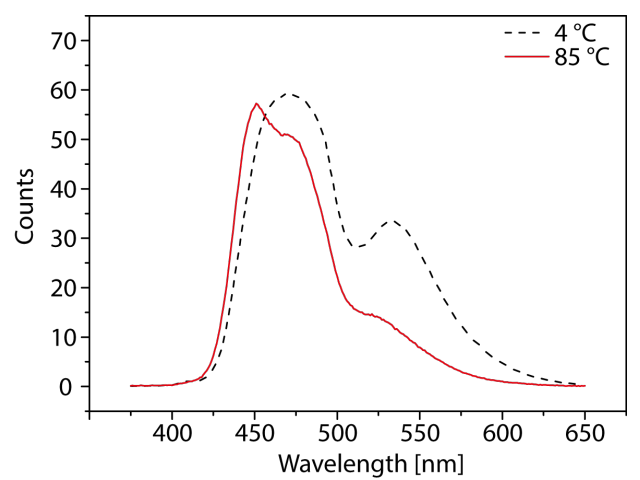




\section{A.3.a Oligonucleotide MKDH03a}

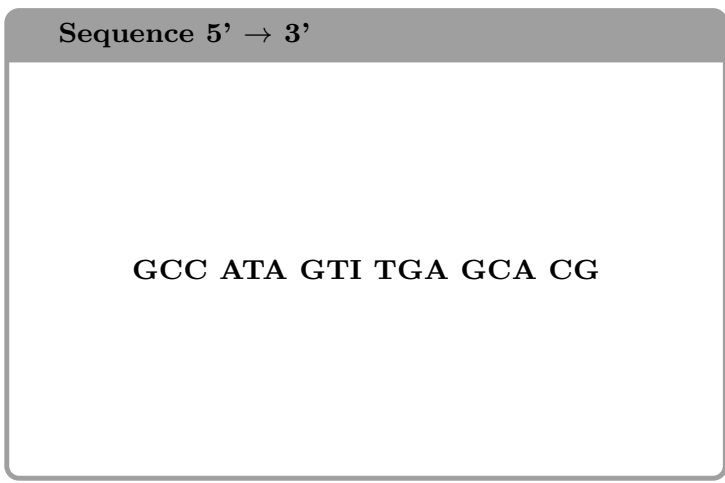

Sum Formula

$$
\mathrm{C}_{164} \mathrm{H}_{209} \mathrm{~N}_{65} \mathrm{O}_{98} \mathrm{P}_{16}
$$

Mol. Mass

$5152.03 \mathrm{~g} \mathrm{~mol}^{-1}$

\section{HPLC Traces}

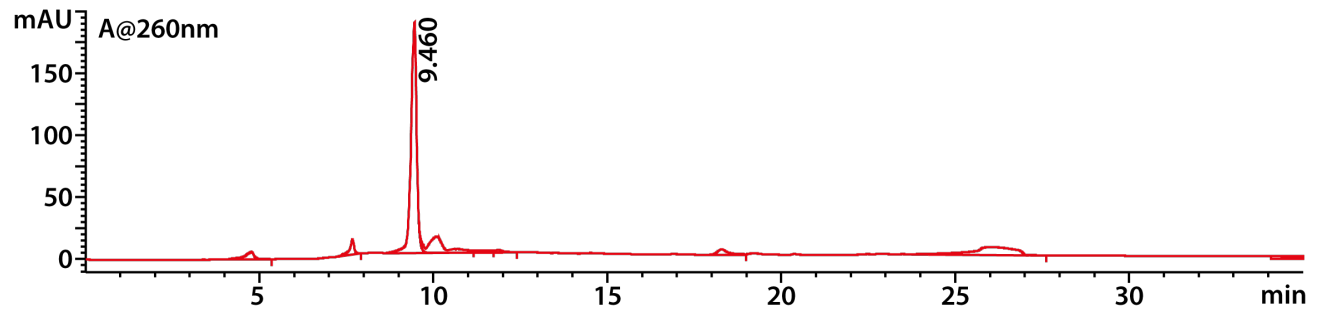

HPLC Traces

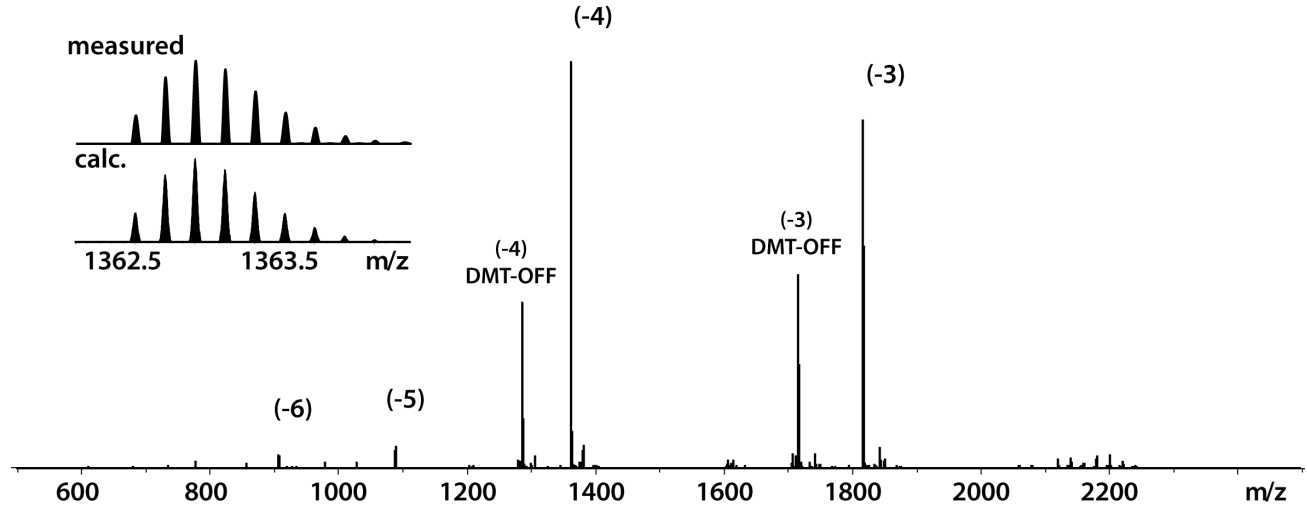




\section{A.3.b Oligonucleotide MKDH03b}

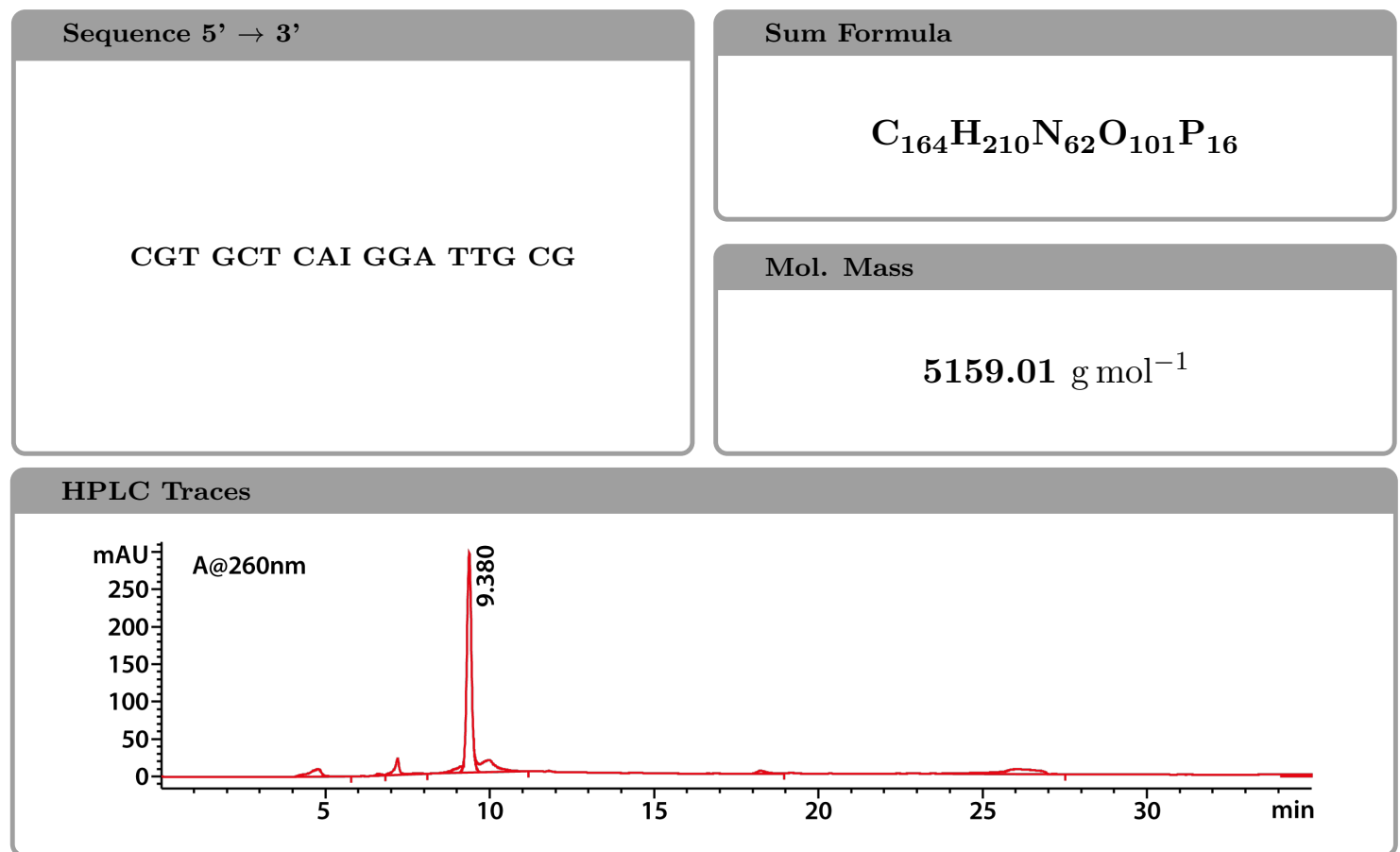

\section{HPLC Traces}

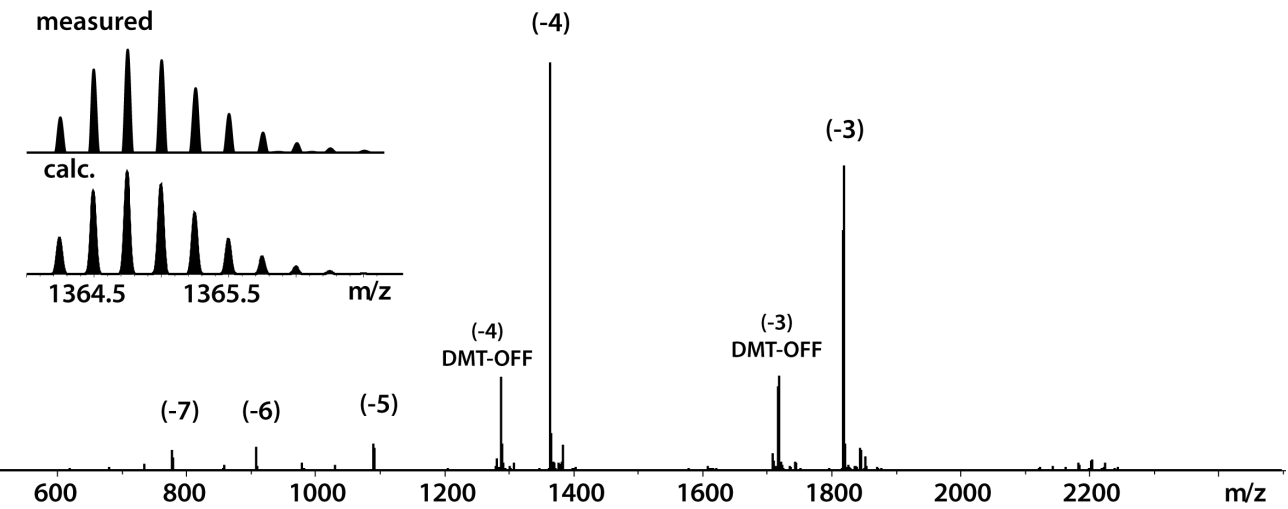




\section{A.3.c Oligonucleotide MKDH03c}

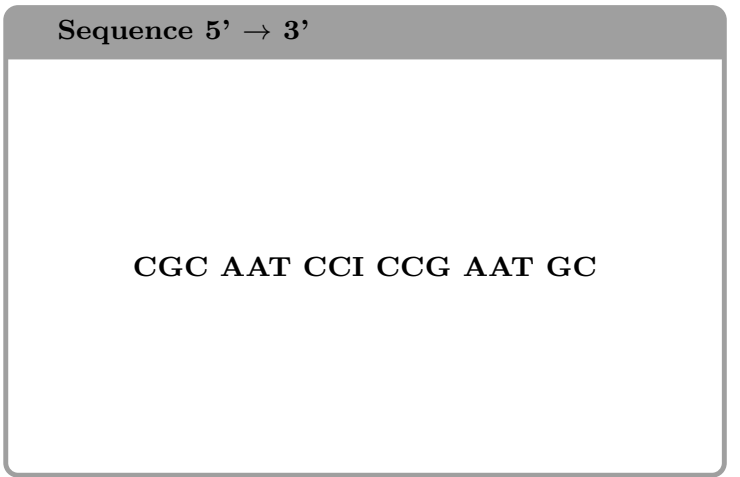

Sum Formula

$$
\mathrm{C}_{161} \mathrm{H}_{208} \mathrm{~N}_{62} \mathrm{O}_{97} \mathrm{P}_{16}
$$

Mol. Mass

$5057.02 \mathrm{~g} \mathrm{~mol}^{-1}$

\section{HPLC Traces}

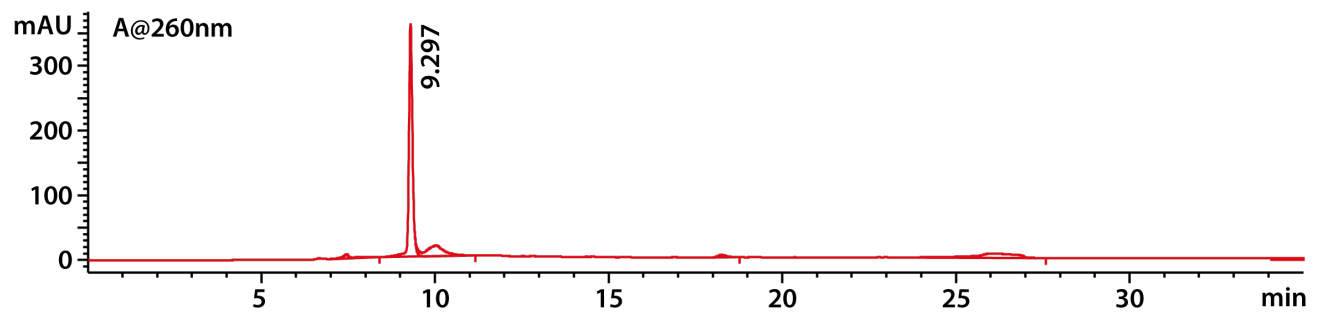

\section{HPLC Traces}

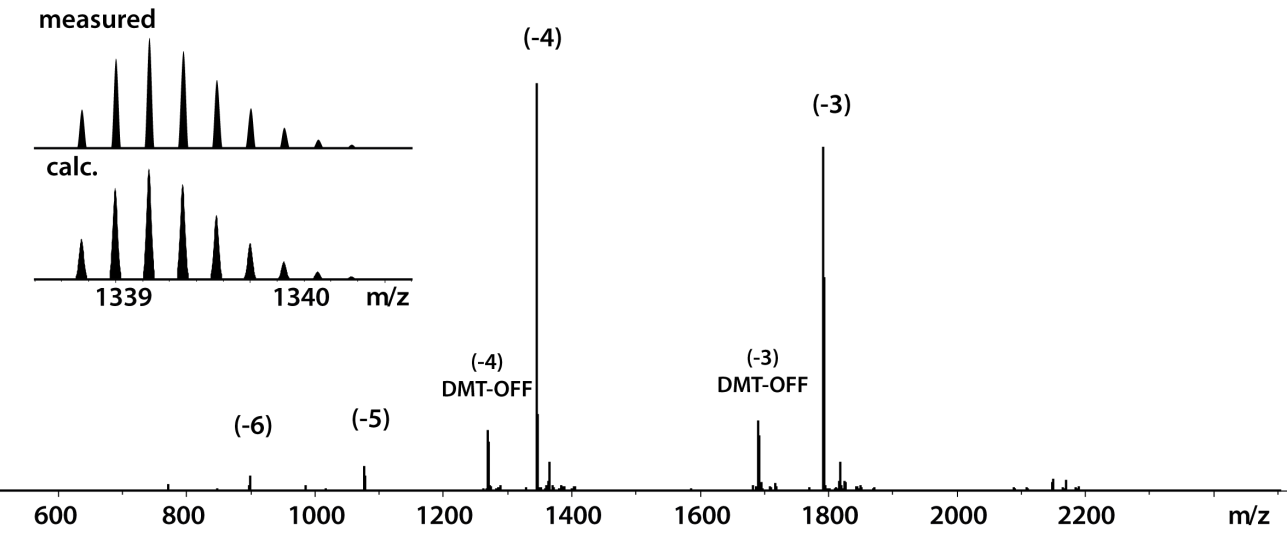




\section{A.3.d Oligonucleotide MKDH03d}

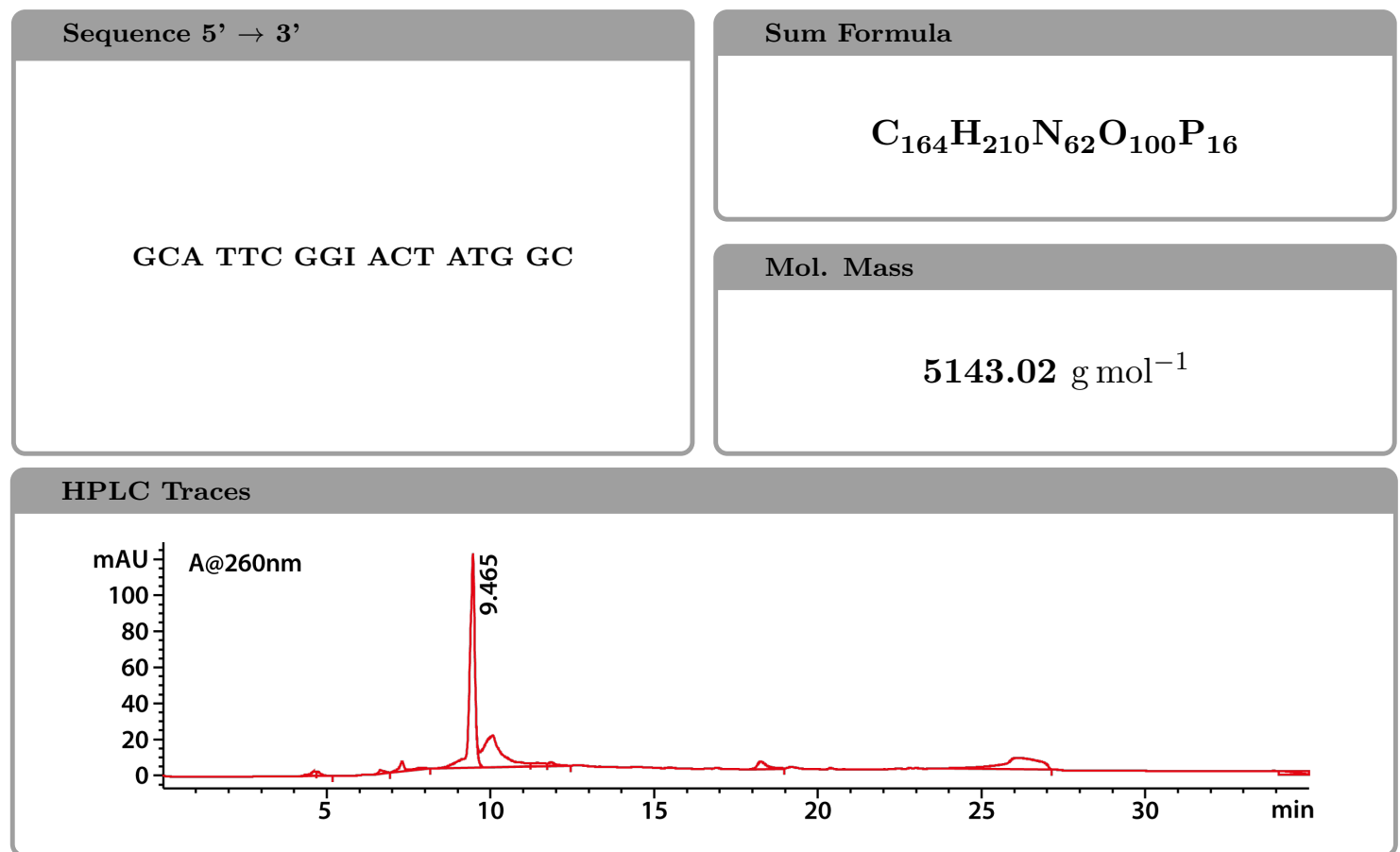

\section{HPLC Traces}

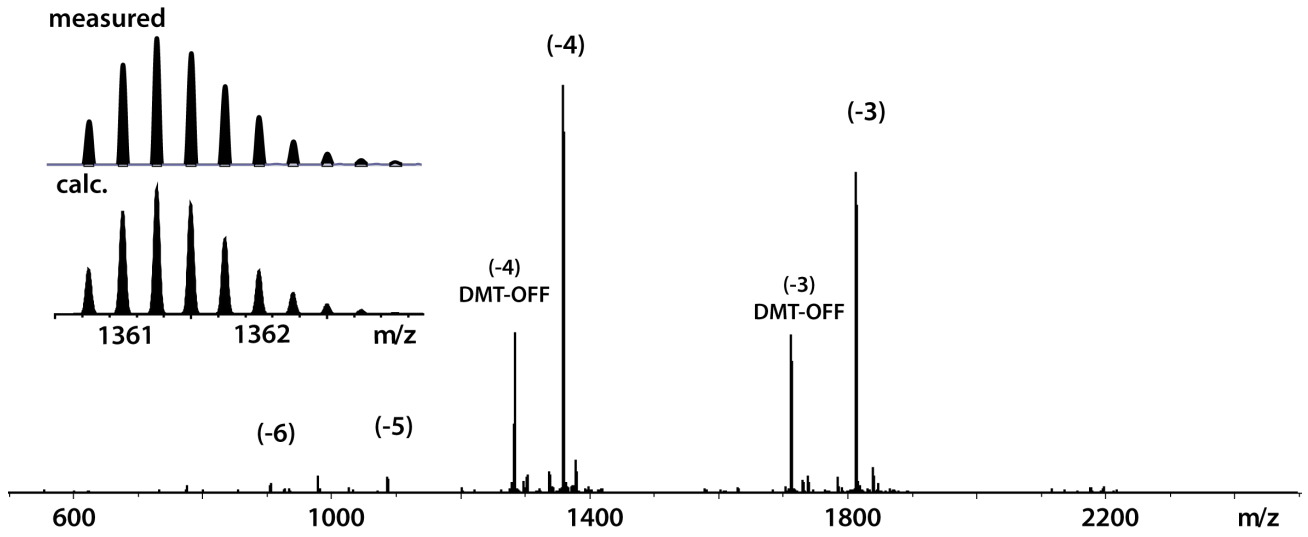




\section{A.3.e Holliday Junction MKDH03}
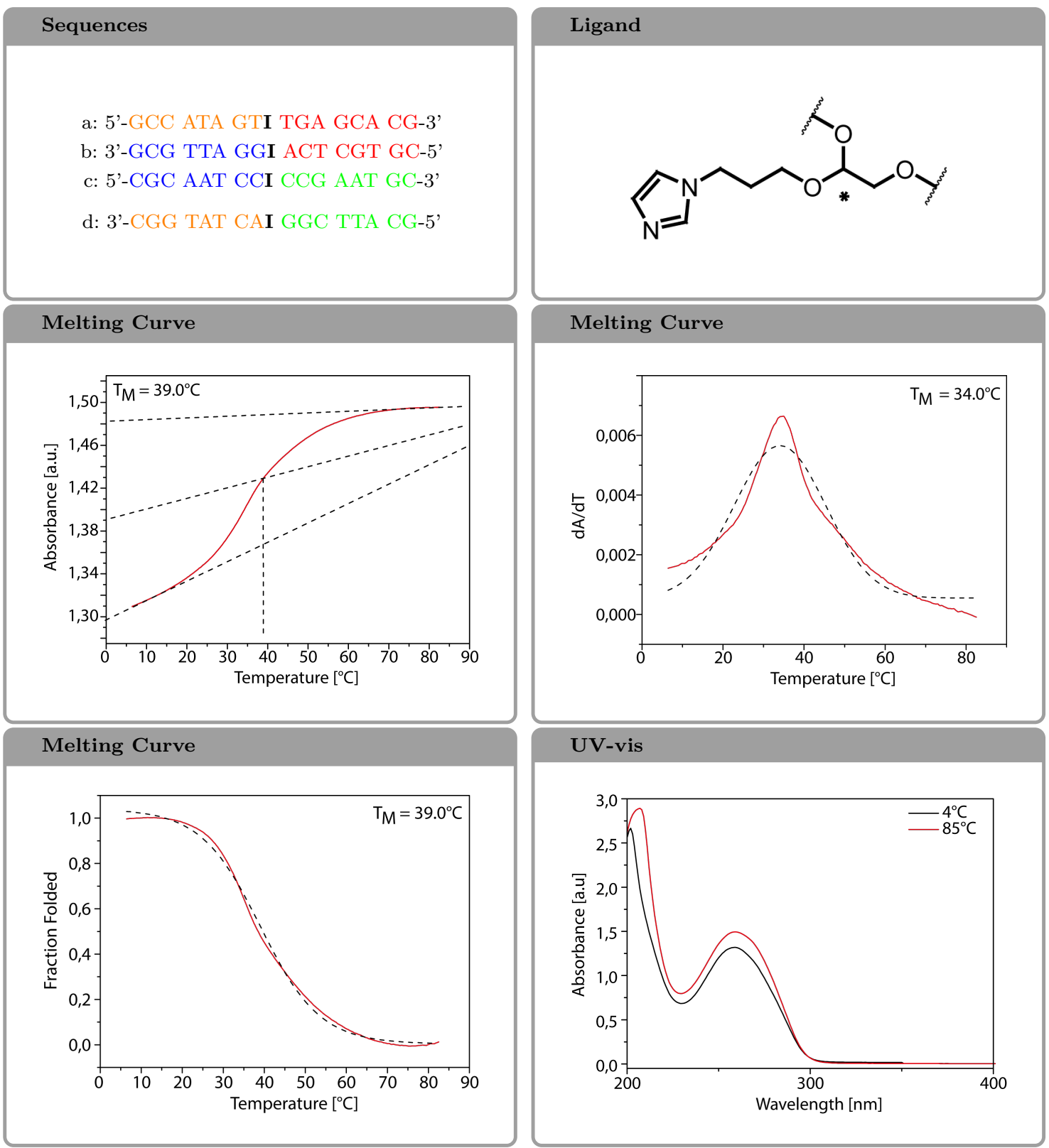

\section{Circular Dichroism}

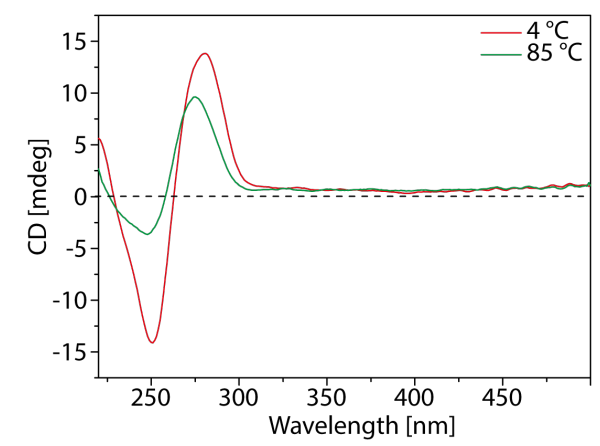

\section{Fluoresence}

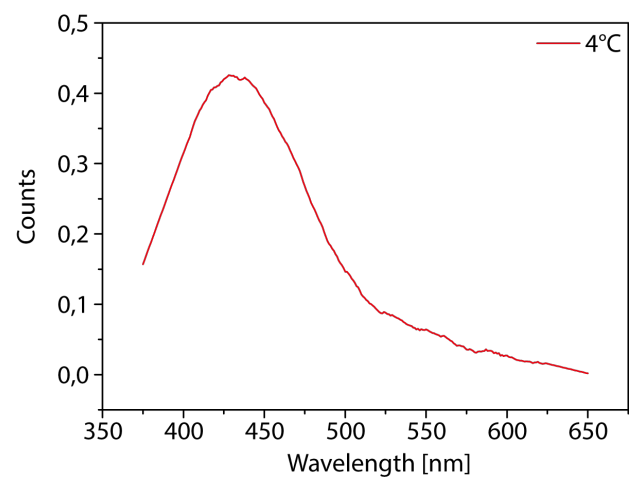




\section{A.4.a Oligonucleotide MKDH04a}

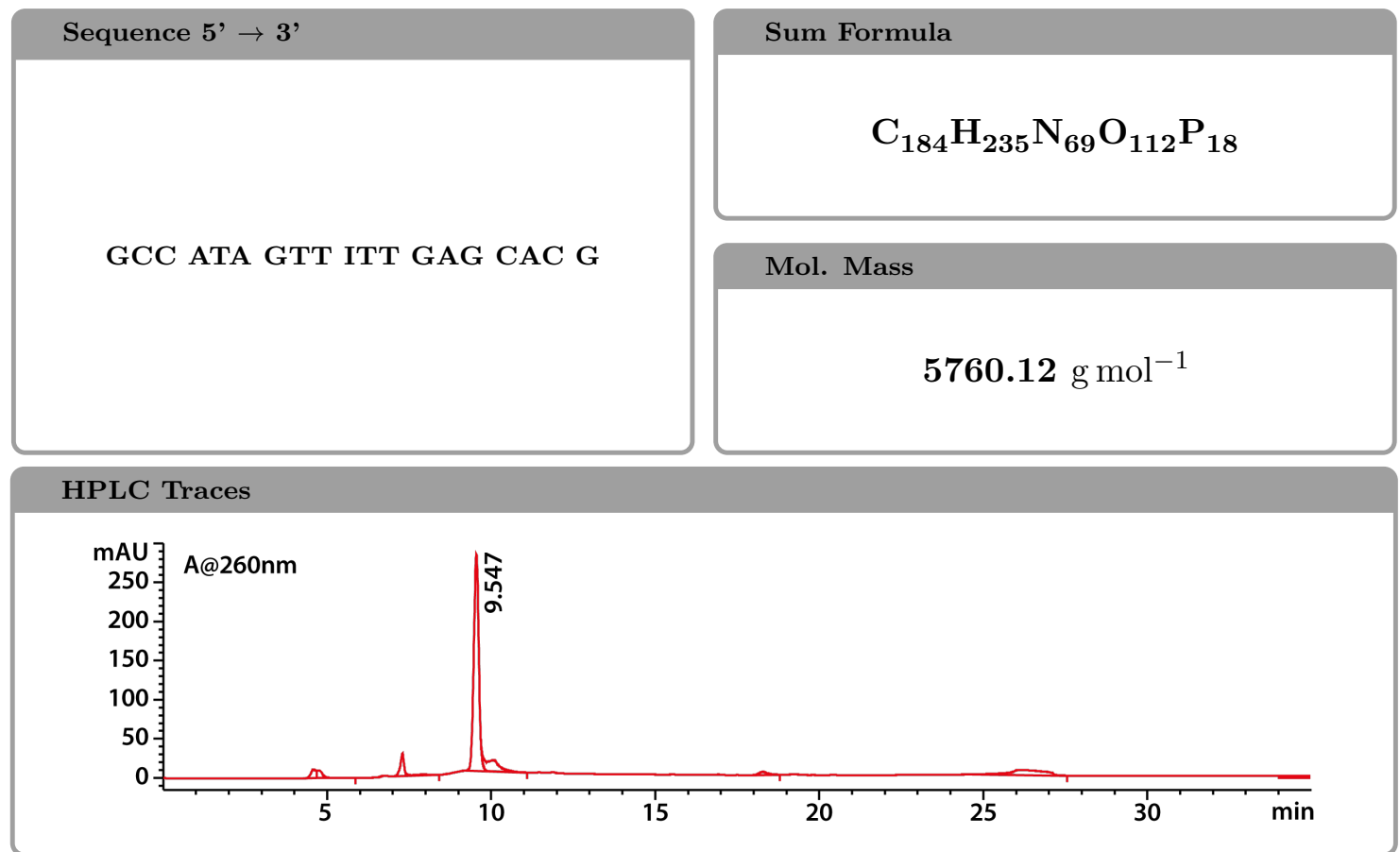

\section{HPLC Traces}

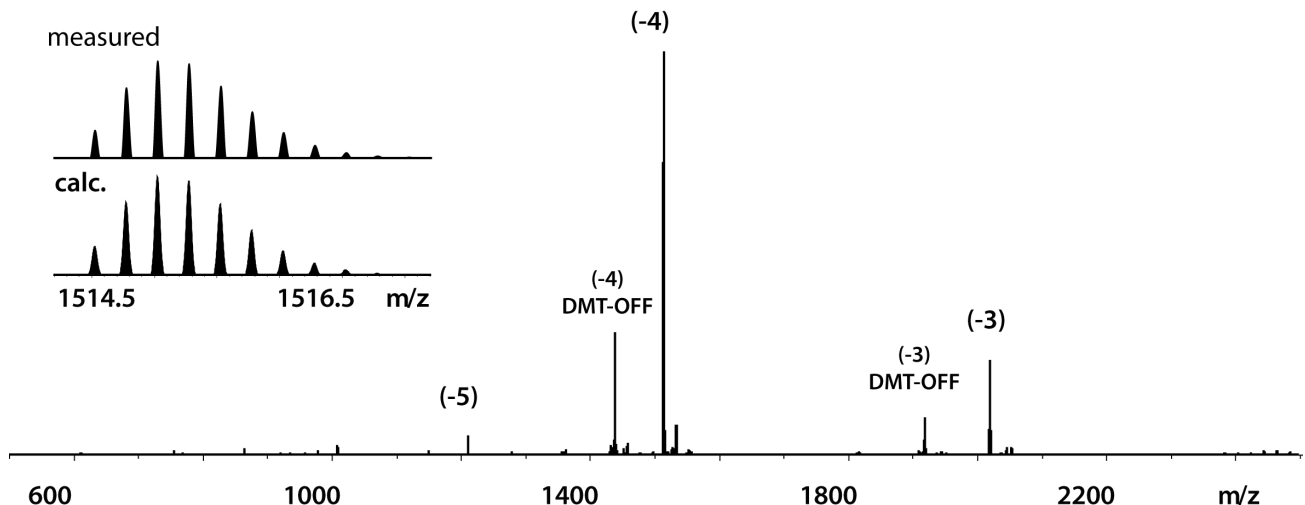




\section{A.4.b Oligonucleotide MKDH04b}

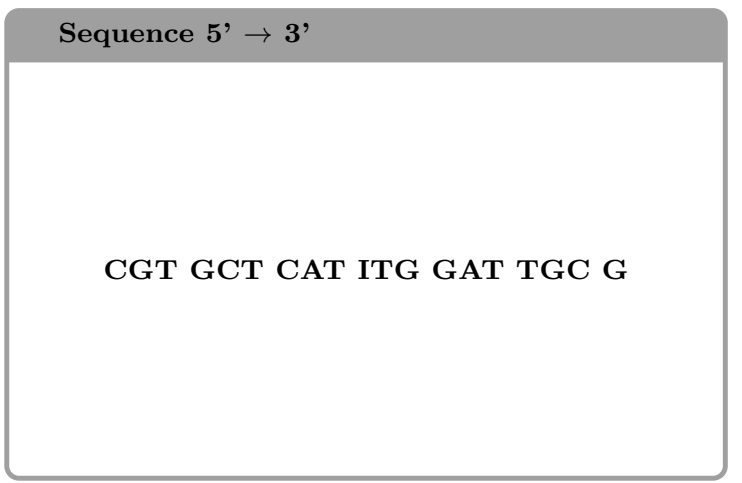

Sum Formula

$$
\mathrm{C}_{184} \mathrm{H}_{236} \mathrm{~N}_{66} \mathrm{O}_{115} \mathrm{P}_{18}
$$

Mol. Mass

$\mathbf{5 7 6 7 . 1 0} \mathrm{g} \mathrm{mol}^{-1}$

\section{HPLC Traces}

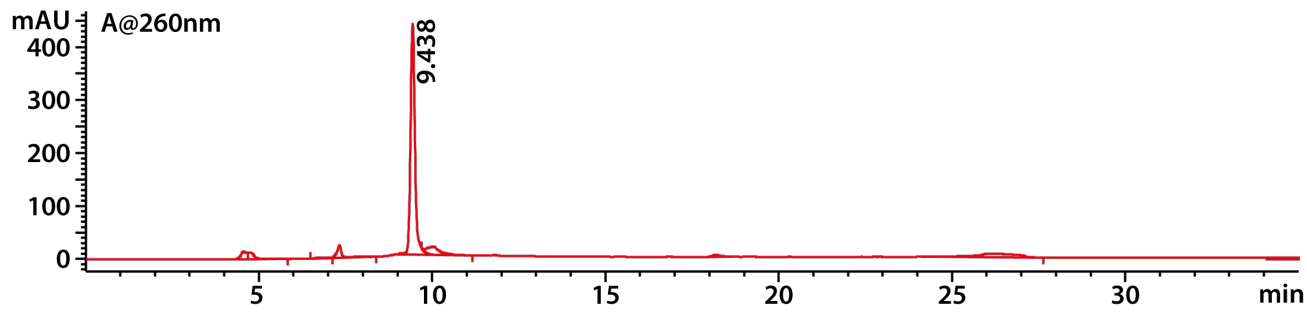

\section{HPLC Traces}

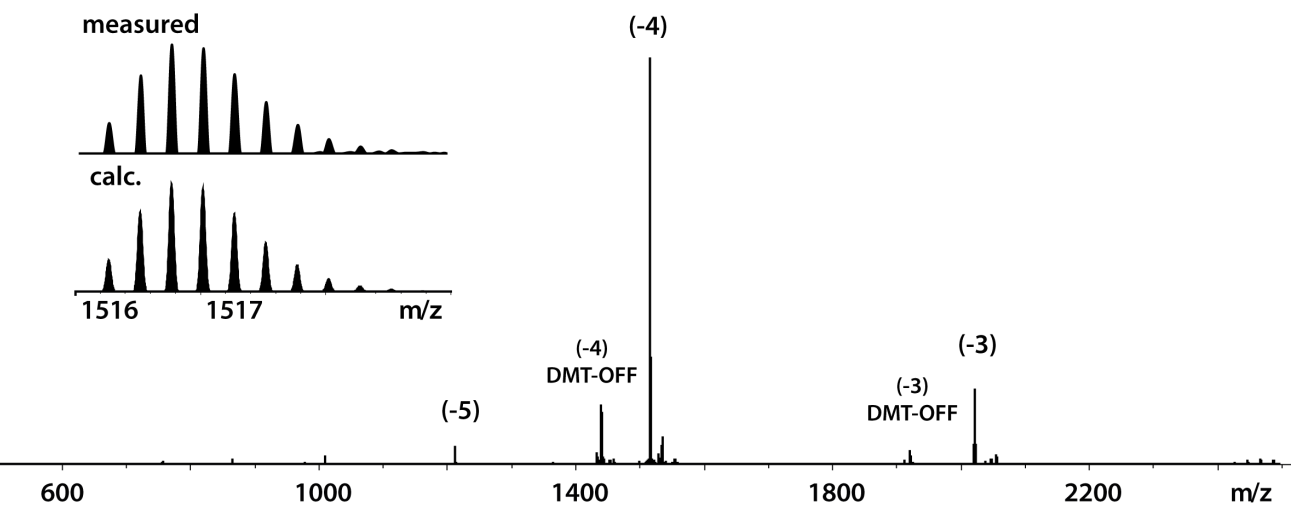




\section{A.4.c Oligonucleotide MKDH04c}

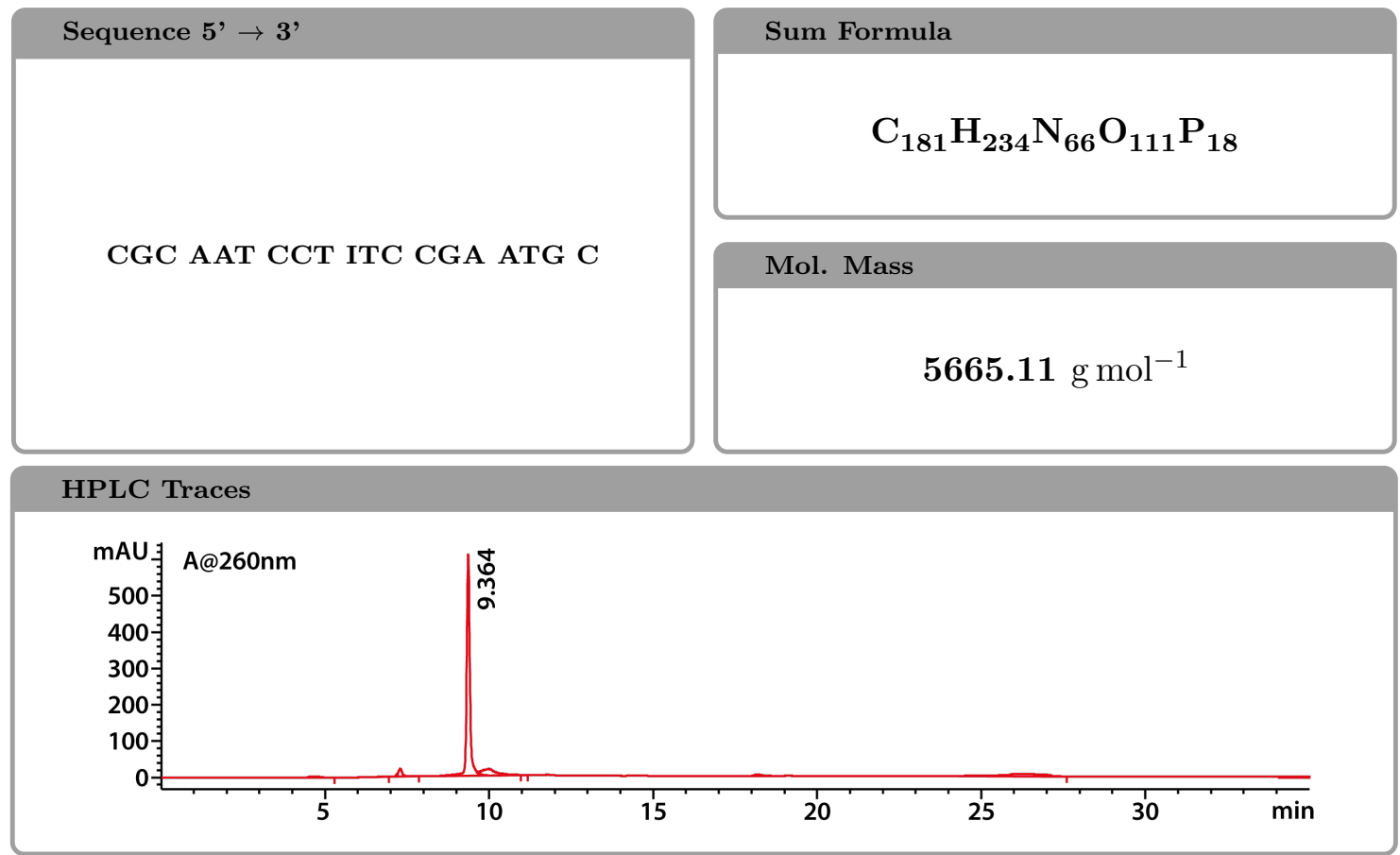

\section{HPLC Traces}

$(-4)$

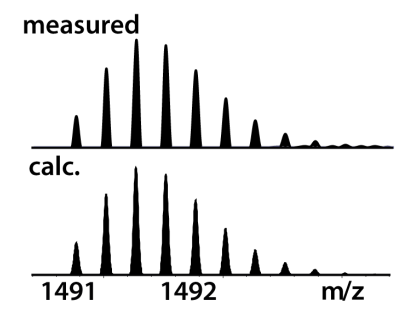

$(-3)$

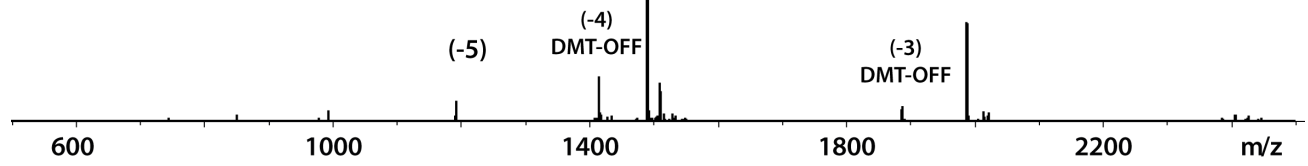




\section{A.4.d Oligonucleotide MKDH04d}

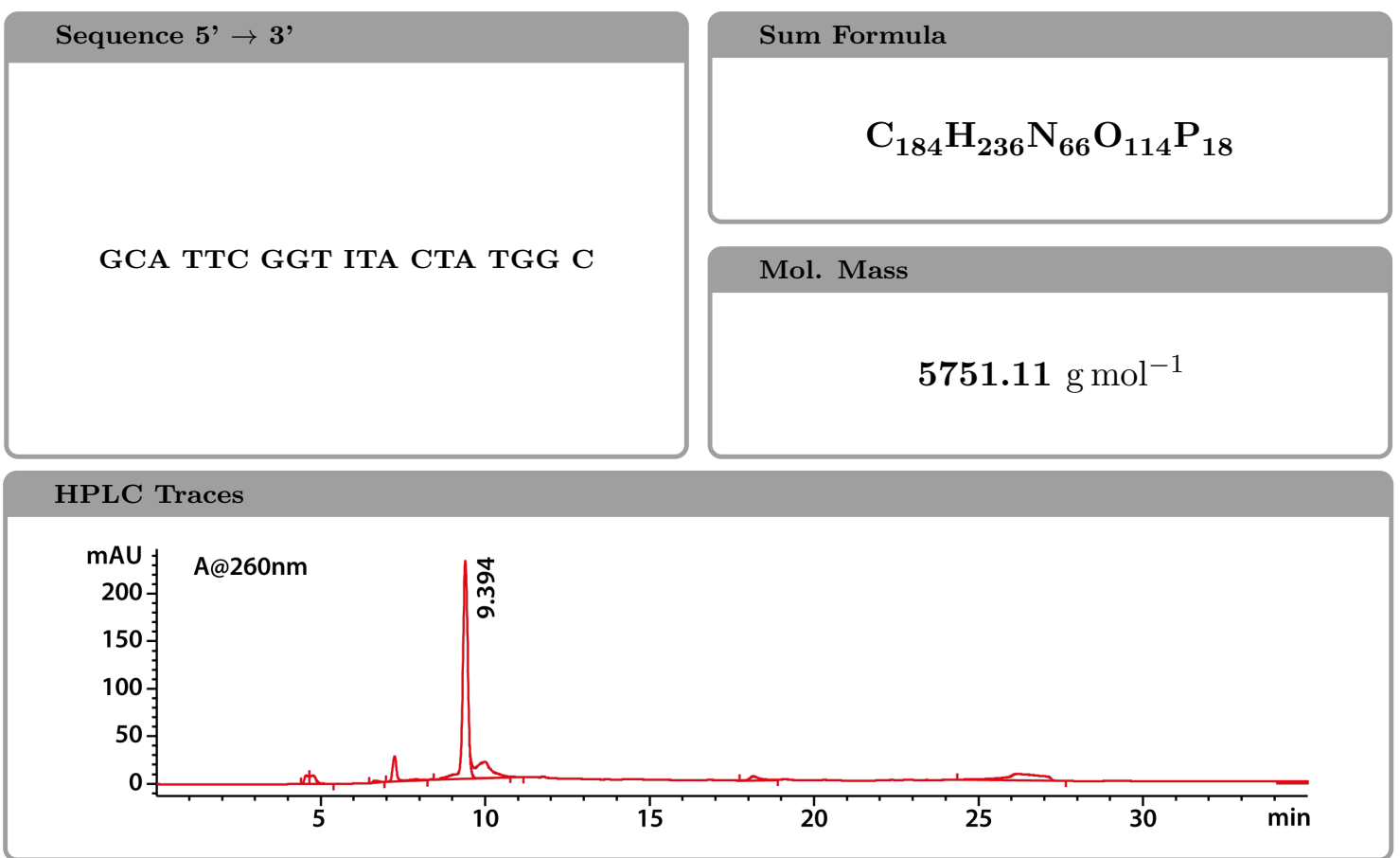

HPLC Traces

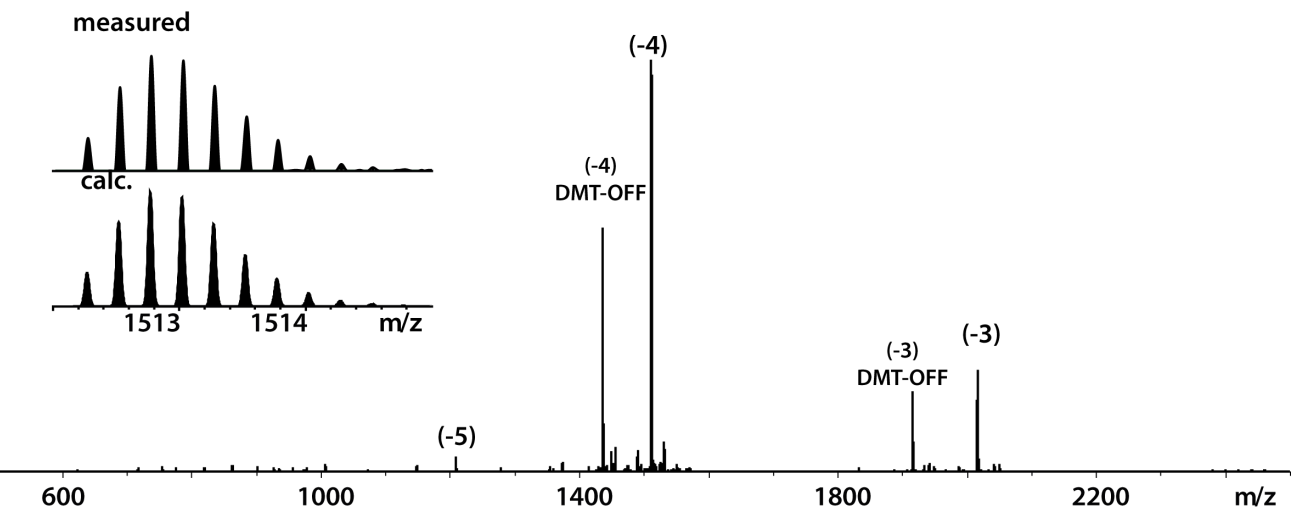




\section{A.4.e Holliday Junction MKDH04}
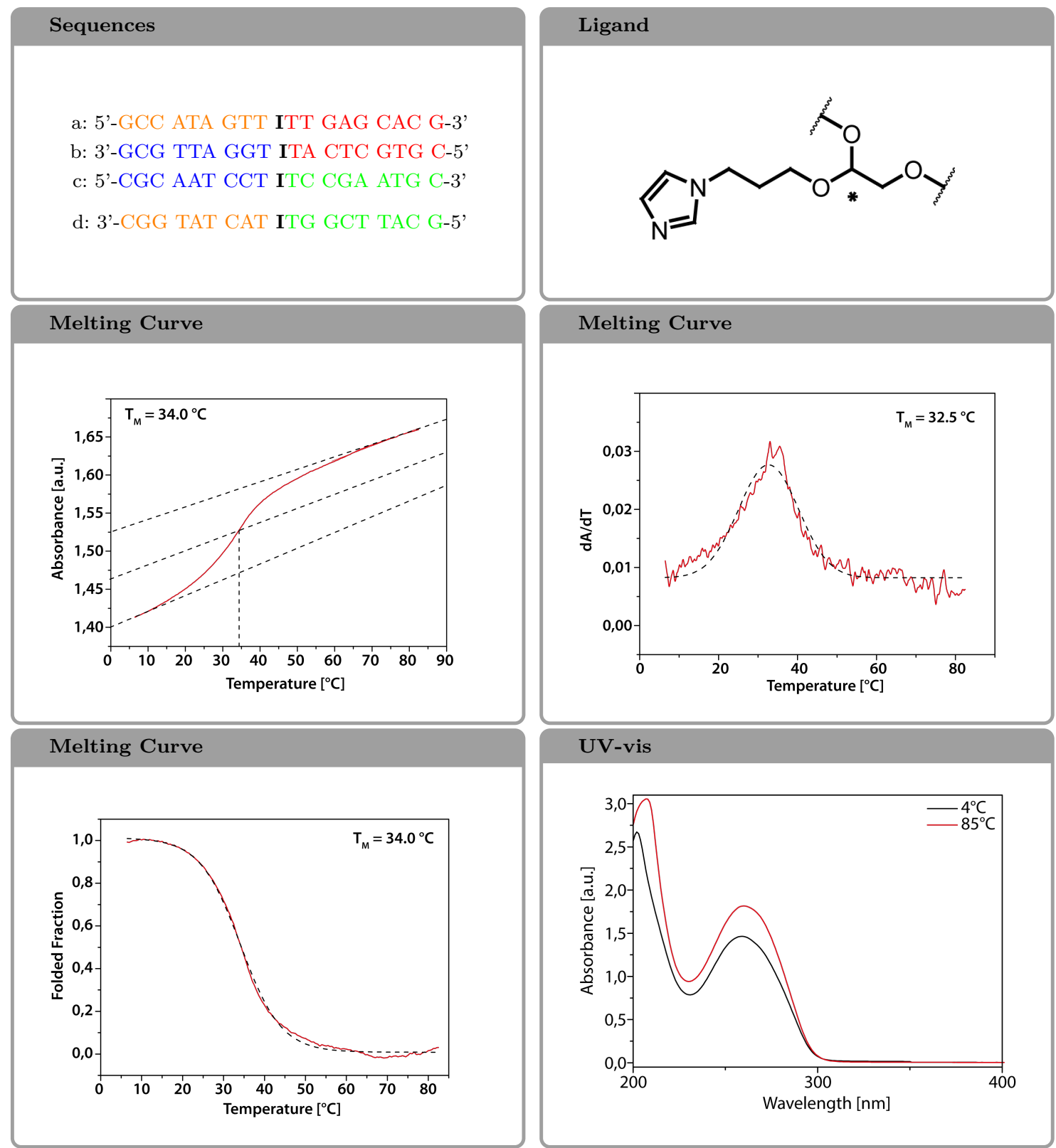

\section{Melting Curve}

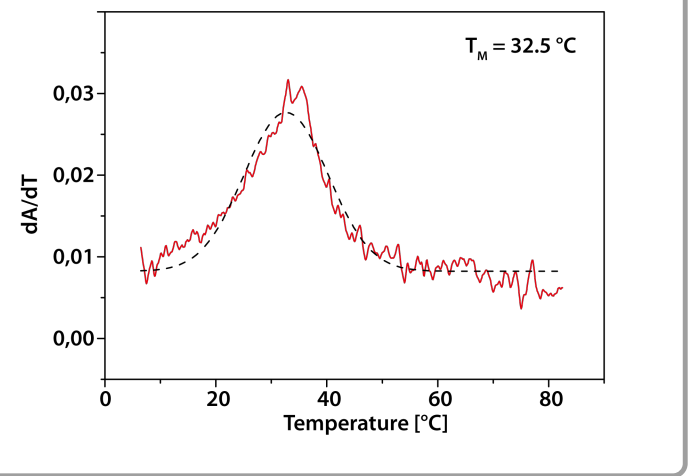

UV-vis

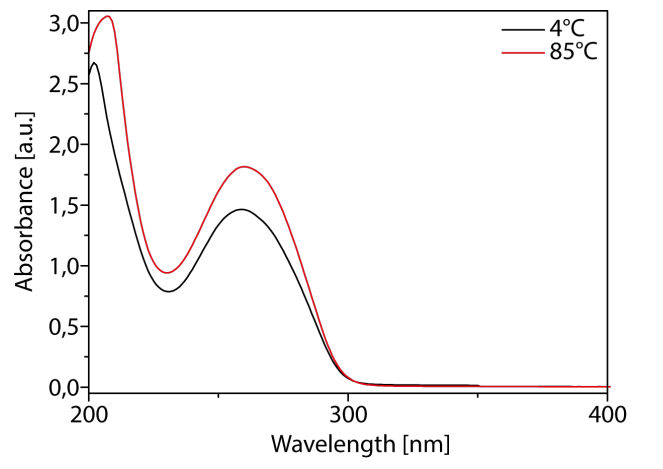

Circular Dichroism

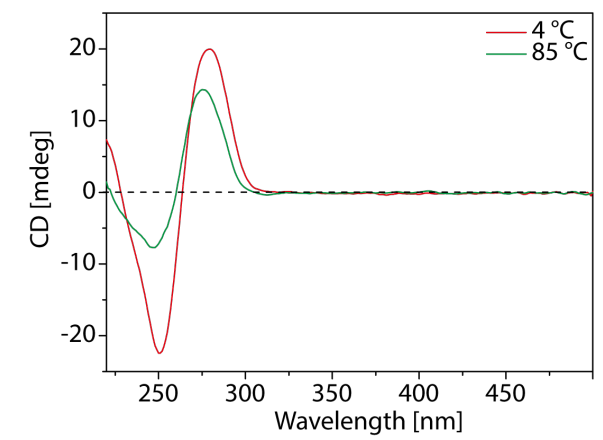




\section{A.5.a Oligonucleotide MKDH05a}

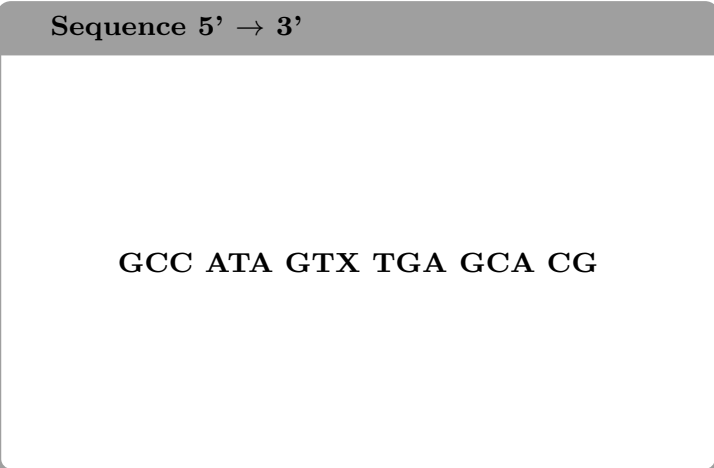

Sum Formula

$$
\mathrm{C}_{182} \mathrm{H}_{220} \mathrm{~N}_{66} \mathrm{O}_{98} \mathrm{P}_{16}
$$

Mol. Mass

$5393.32 \mathrm{~g} \mathrm{~mol}^{-1}$

\section{HPLC Traces}

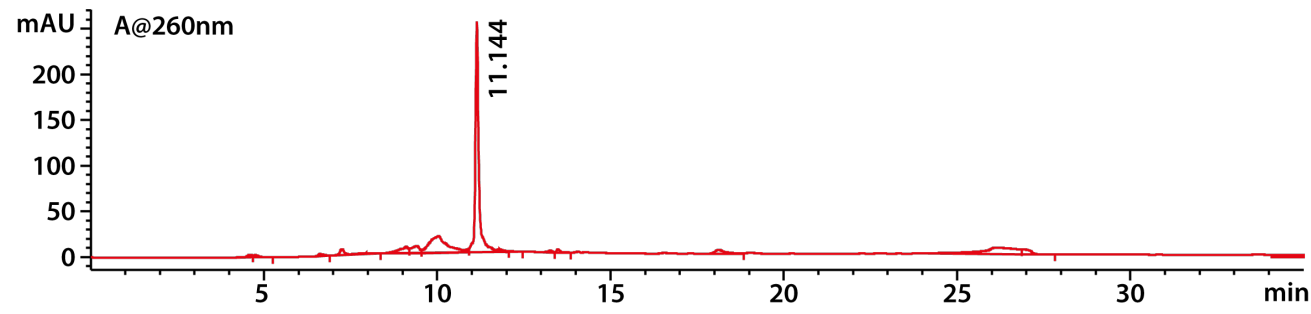

\section{HPLC Traces}

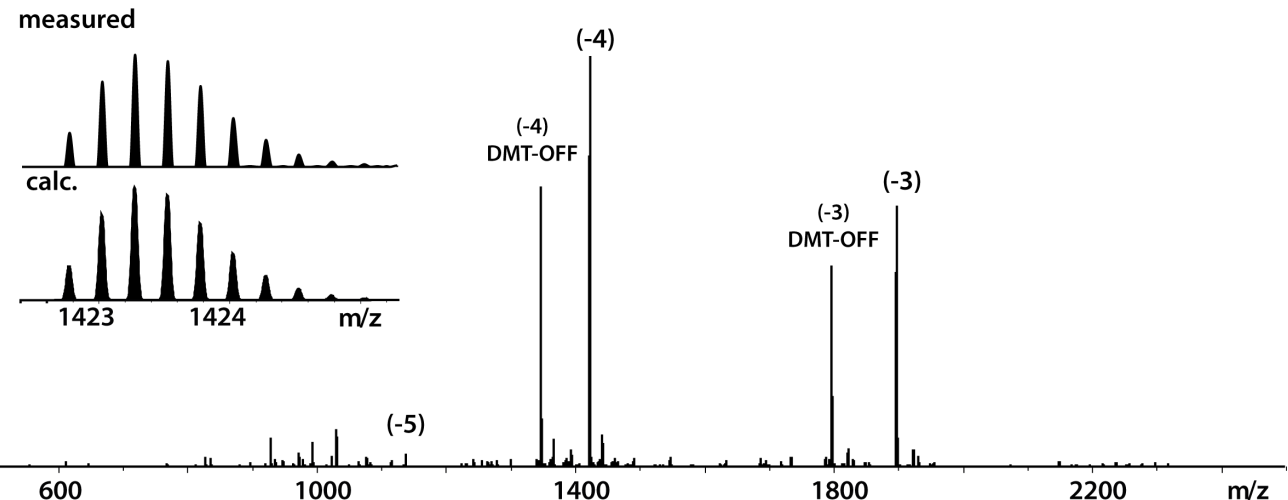




\section{A.5.b Oligonucleotide MKDH05b}

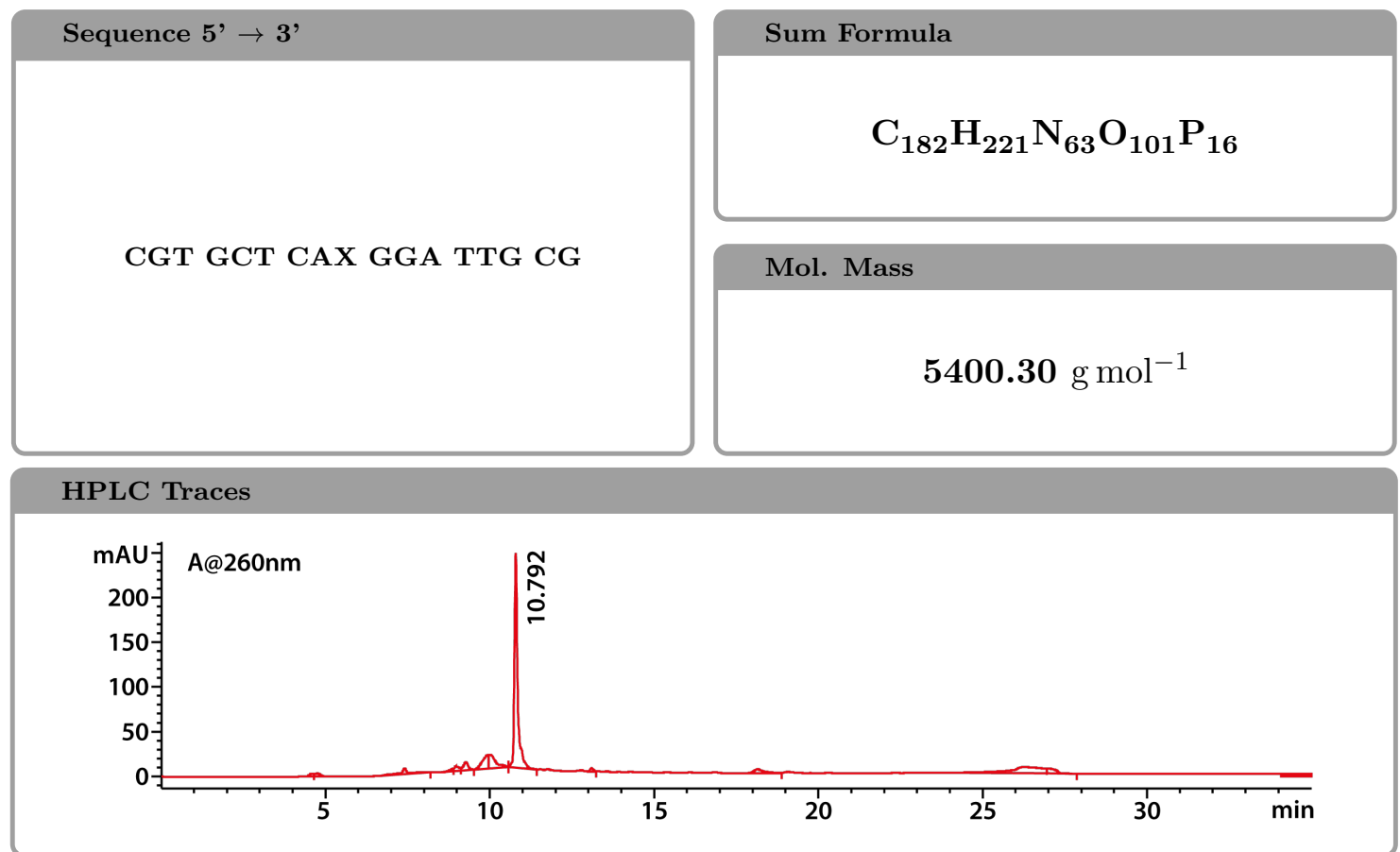

\section{HPLC Traces}

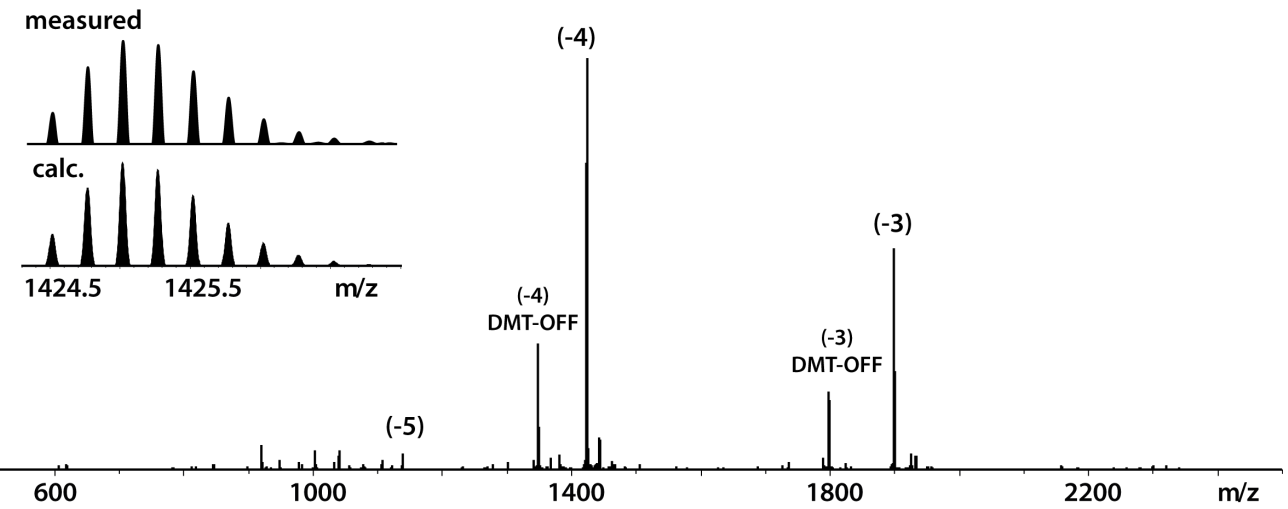




\section{A.5.c Oligonucleotide MKDH05c}

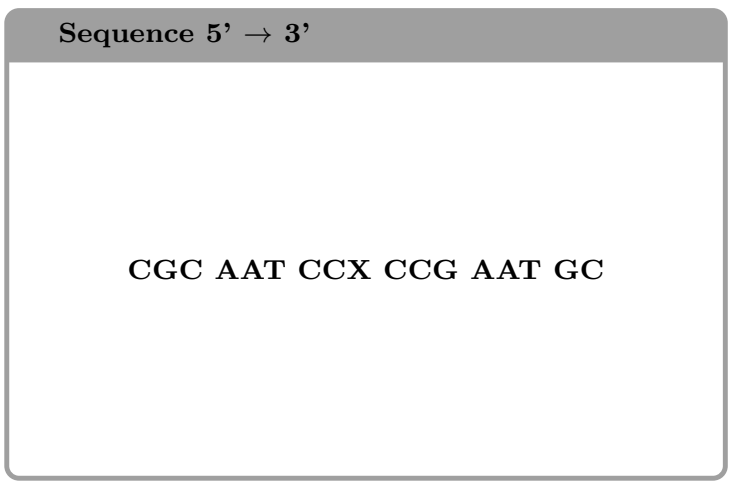

Sum Formula

$$
\mathrm{C}_{179} \mathrm{H}_{219} \mathrm{~N}_{63} \mathrm{O}_{97} \mathrm{P}_{16}
$$

Mol. Mass

$5298.31 \mathrm{~g} \mathrm{~mol}^{-1}$

\section{HPLC Traces}

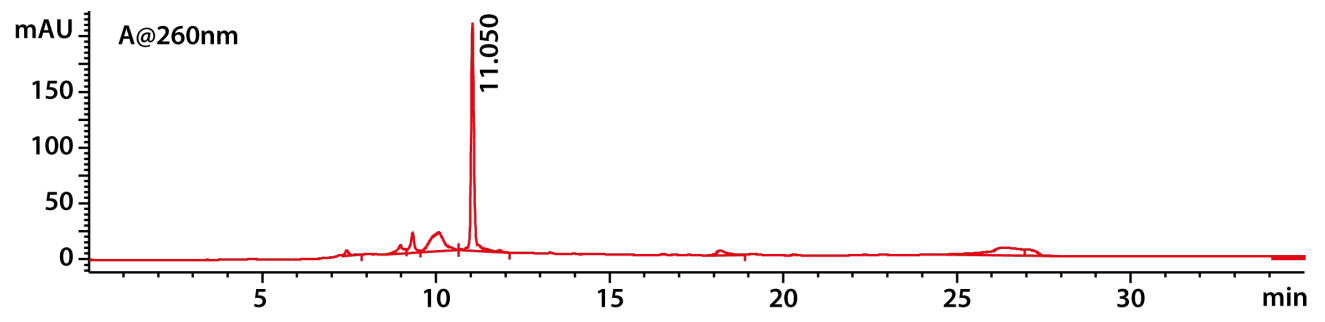

\section{HPLC Traces}

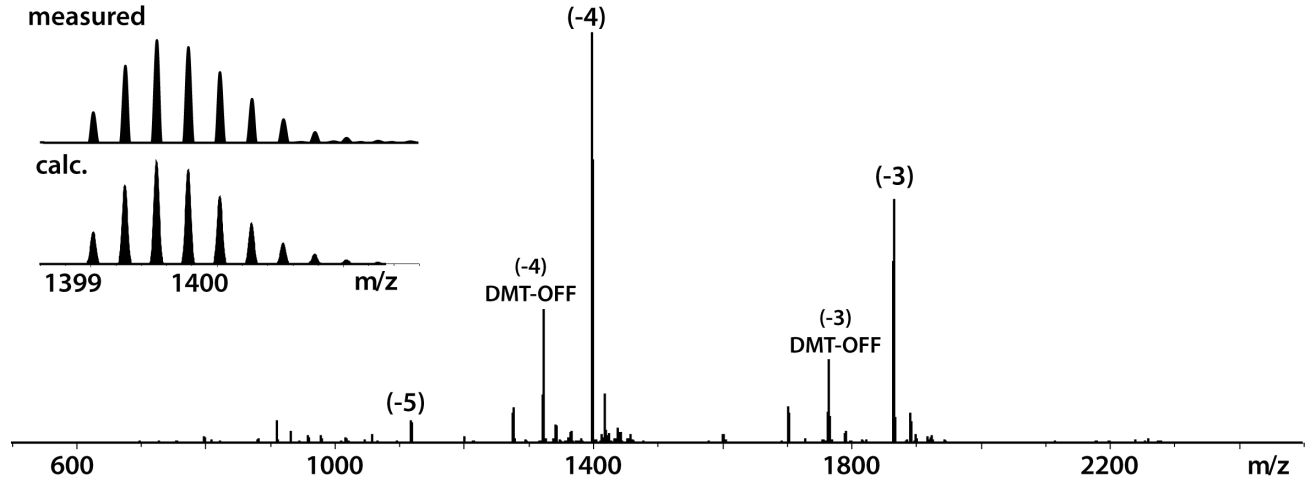




\section{A.5.d Oligonucleotide MKDH05d}

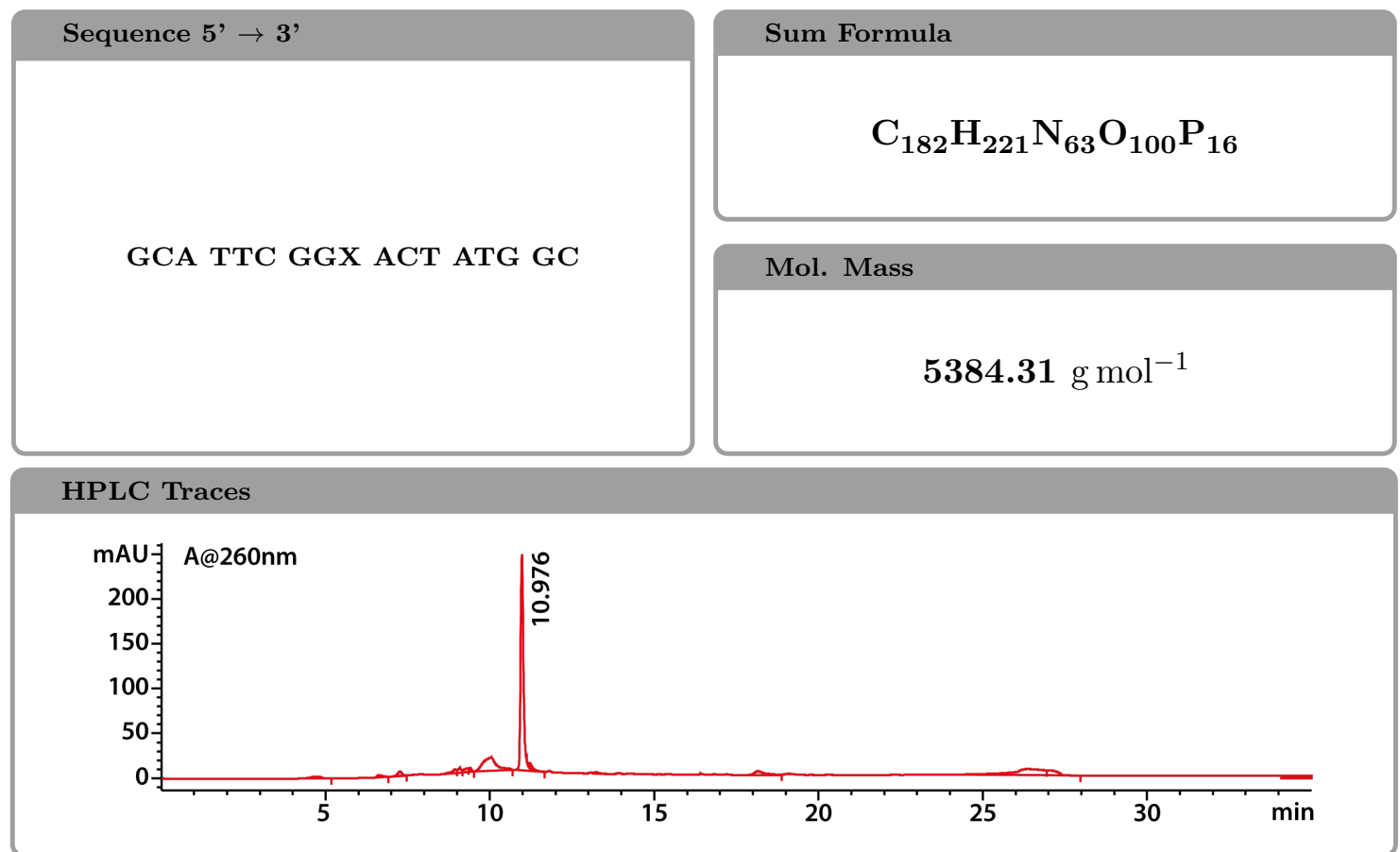

\section{HPLC Traces}

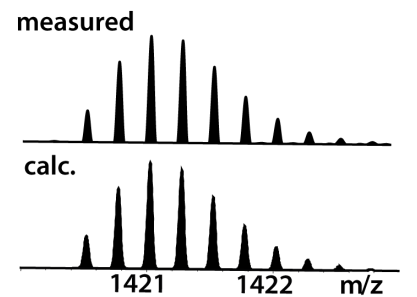

$(-4)$

$(-5)$

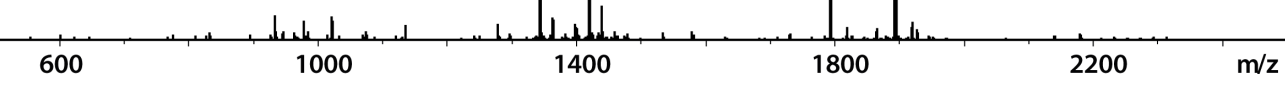




\section{A.5.e Holliday Junction MKDH05}
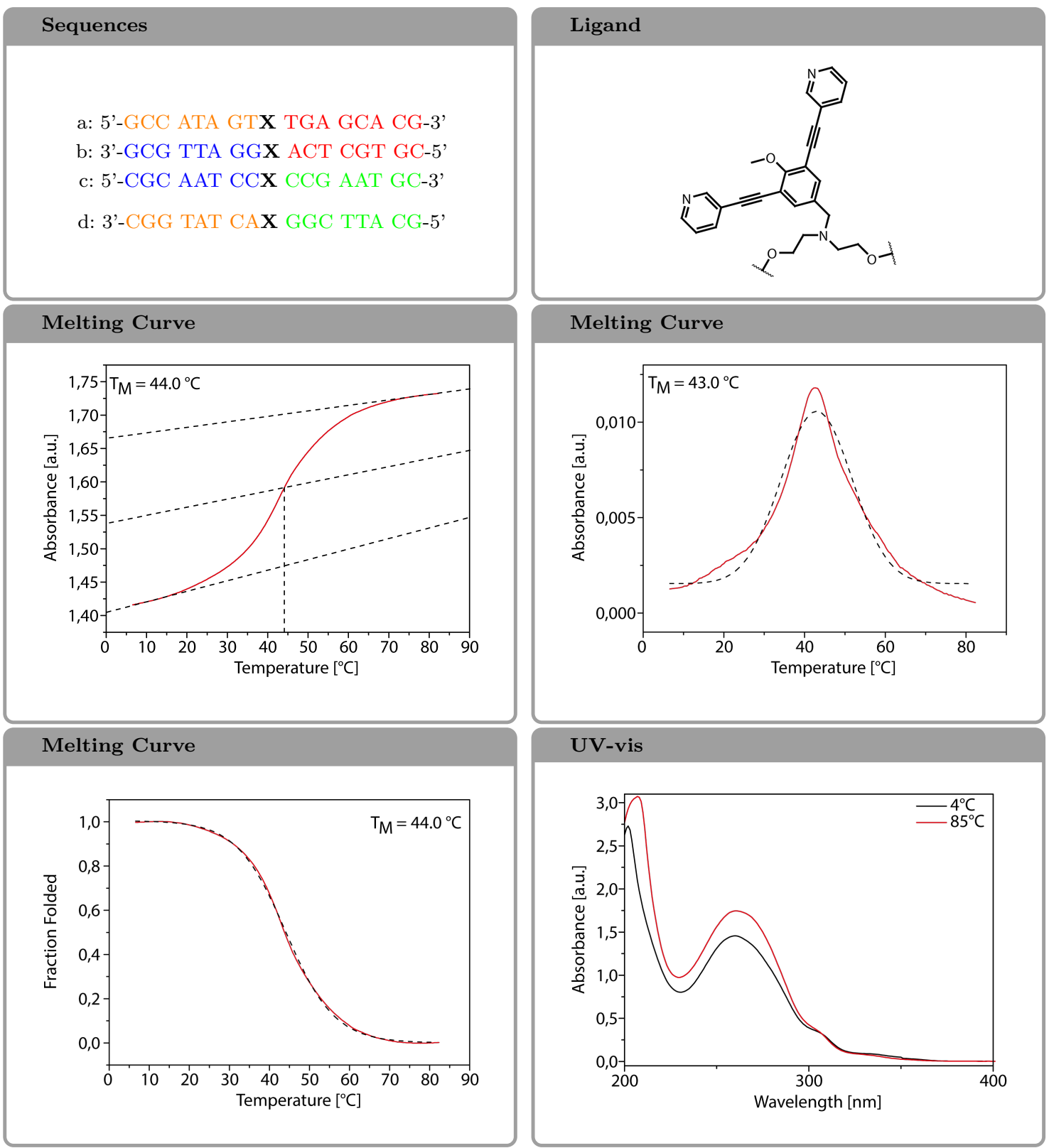

\section{Circular Dichroism}

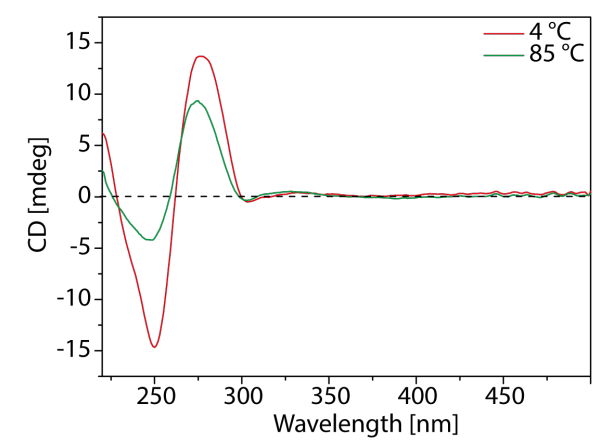

Fluoresence

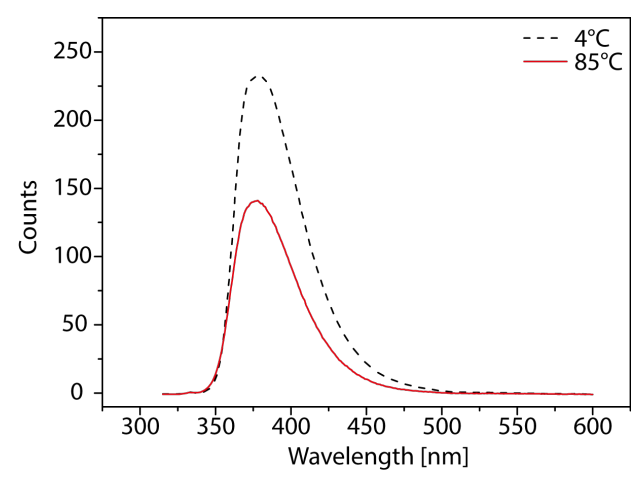




\section{A.6.a Oligonucleotide MKDH06a}

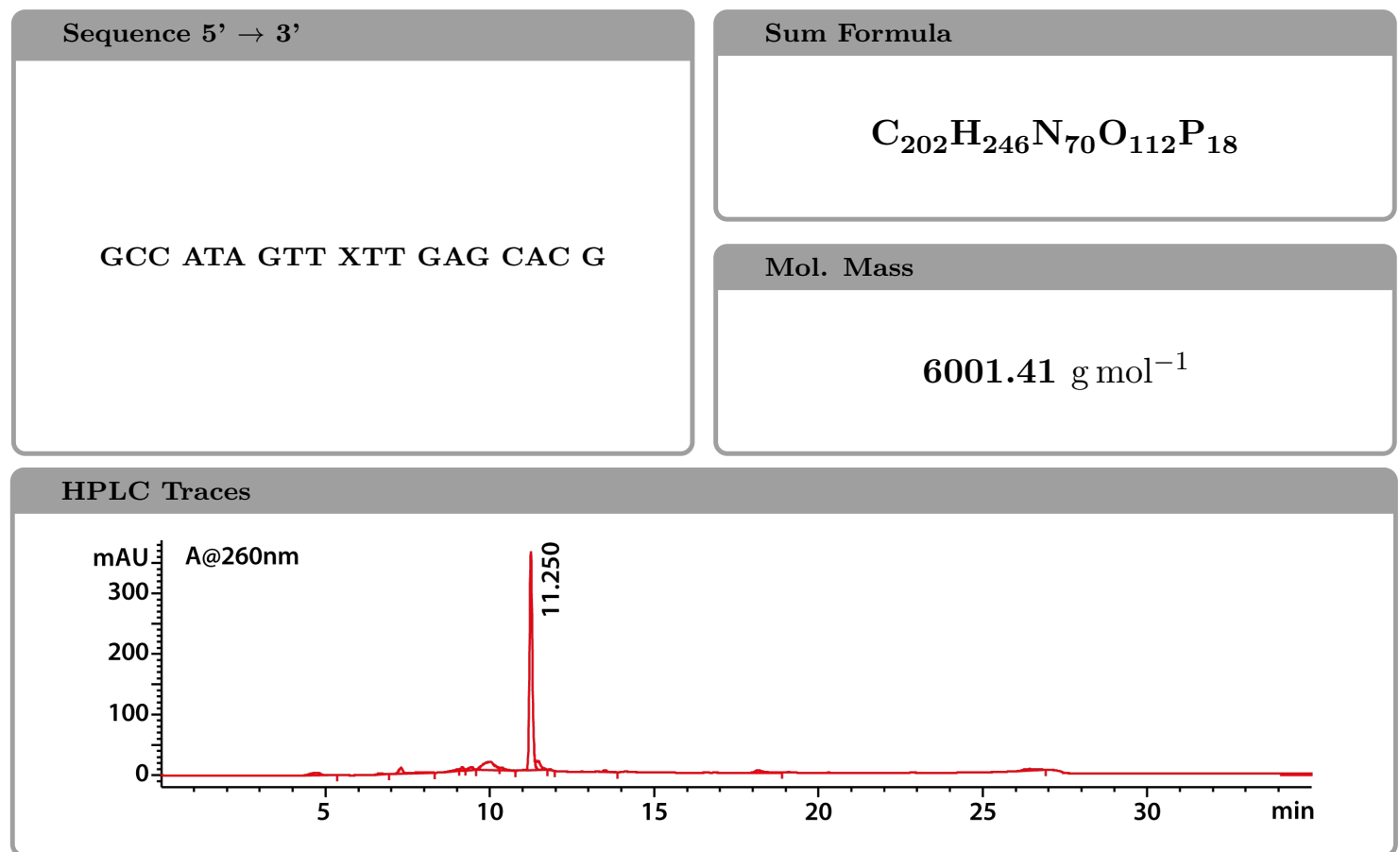

\section{HPLC Traces}

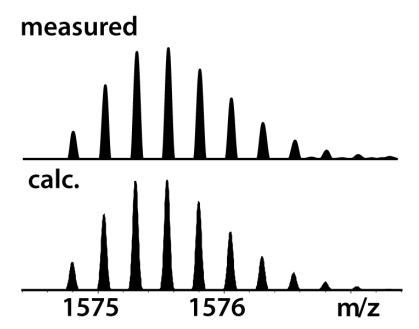

$(-4)$

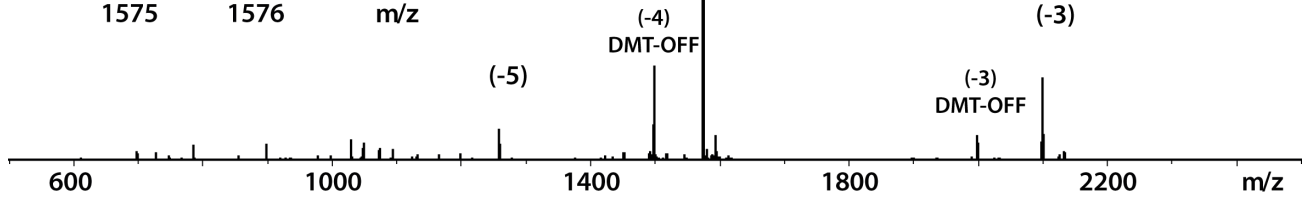




\section{A.6.b Oligonucleotide MKDH06b}

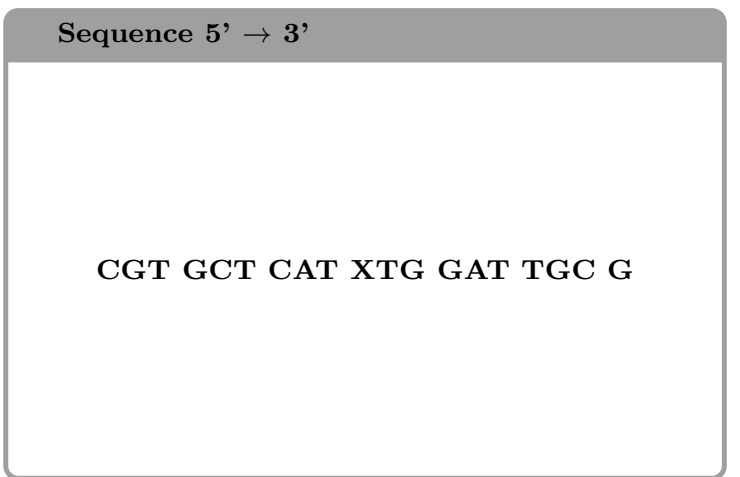

Sum Formula

$$
\mathrm{C}_{202} \mathrm{H}_{247} \mathrm{~N}_{67} \mathrm{O}_{115} \mathrm{P}_{18}
$$

Mol. Mass

$6008.40 \mathrm{~g} \mathrm{~mol}^{-1}$

\section{HPLC Traces}

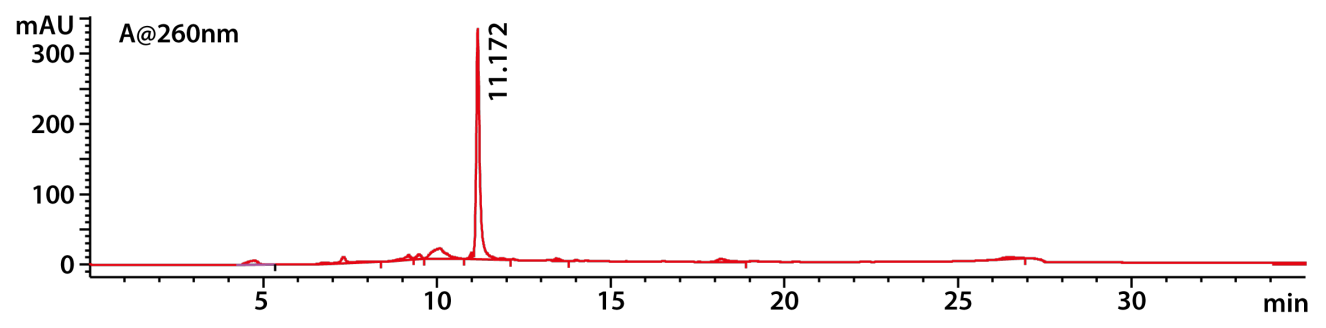

HPLC Traces

measured
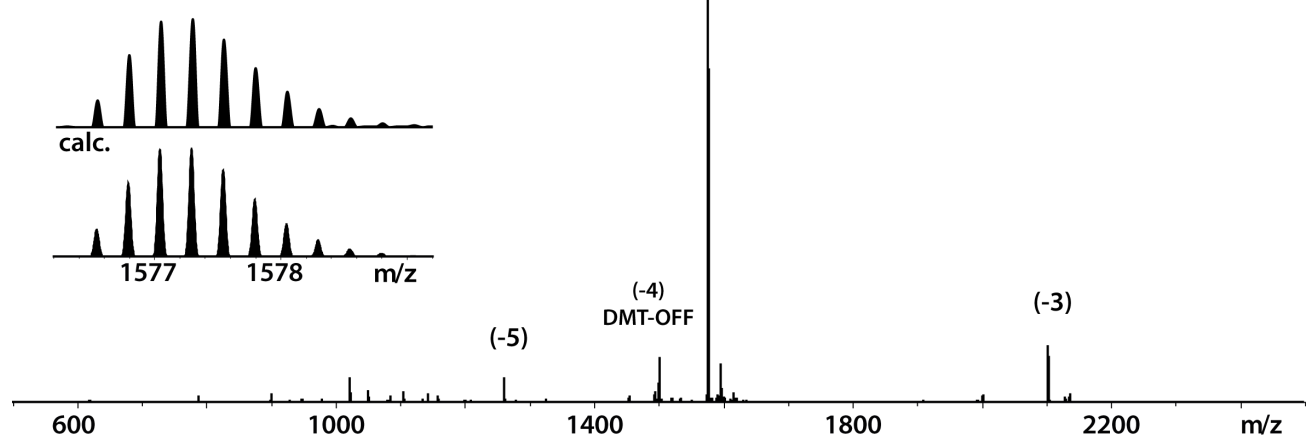


\section{A.6.c Oligonucleotide MKDH06c}

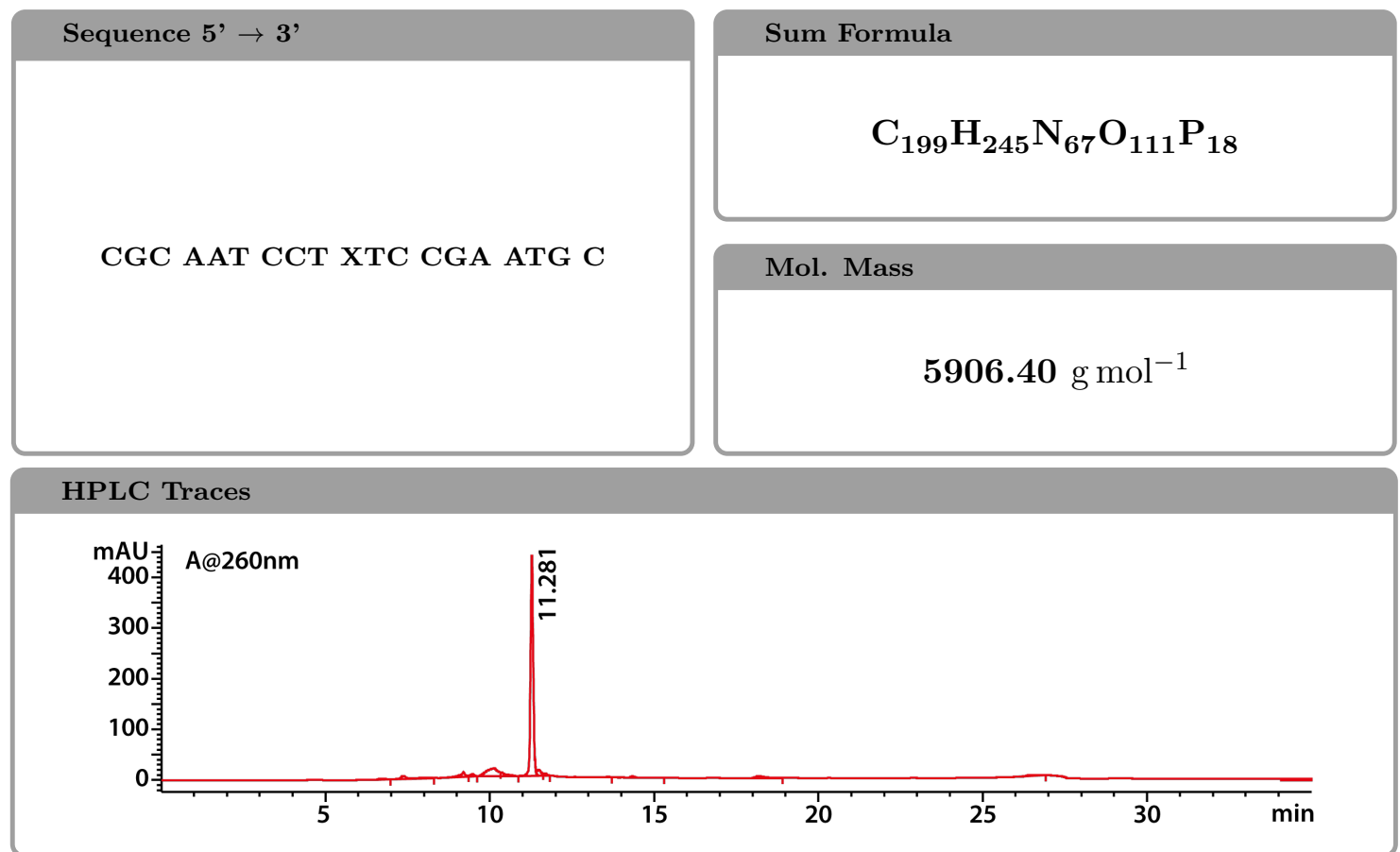

\section{HPLC Traces}

measured

(-4)
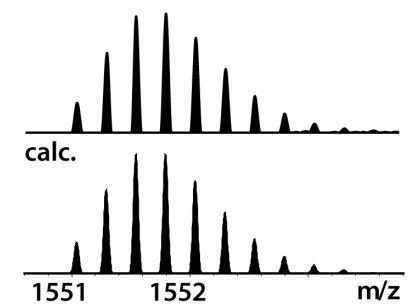

$(-3)$

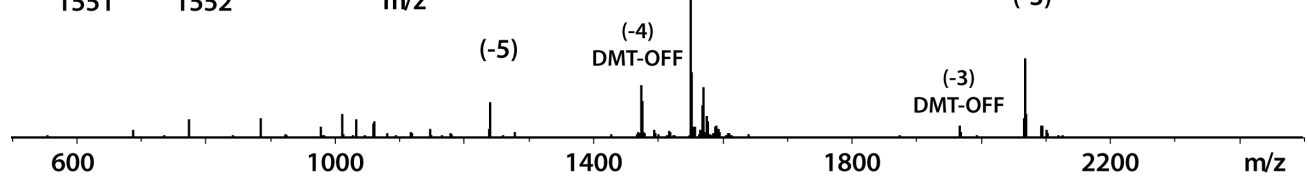




\section{A.6.d Oligonucleotide MKDH06d}

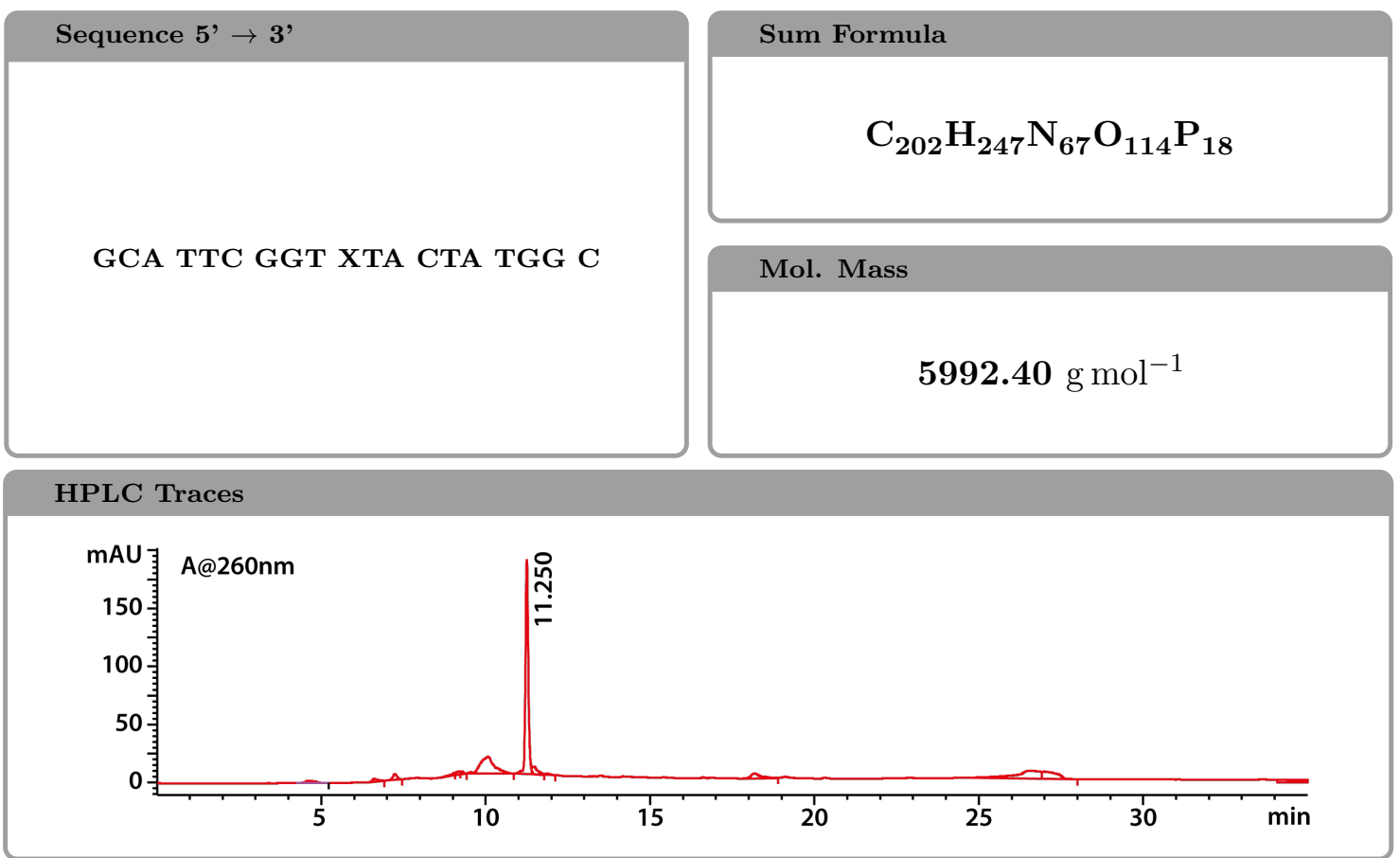

\section{HPLC Traces}

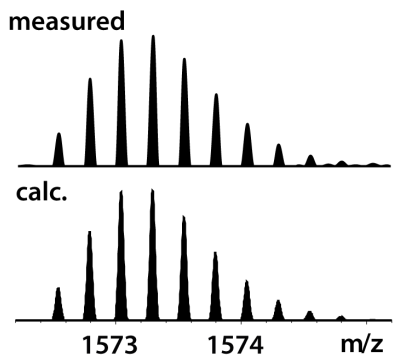

$(-4)$

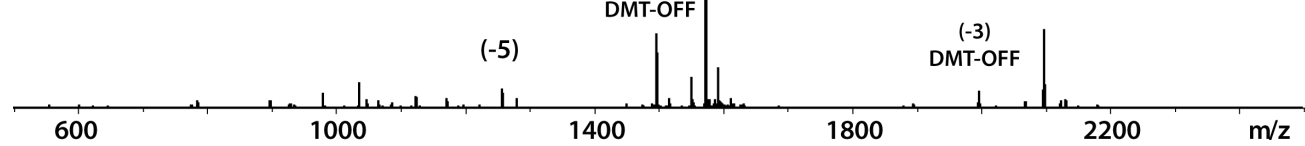




\section{A.6.e Holliday Junction MKDH06}
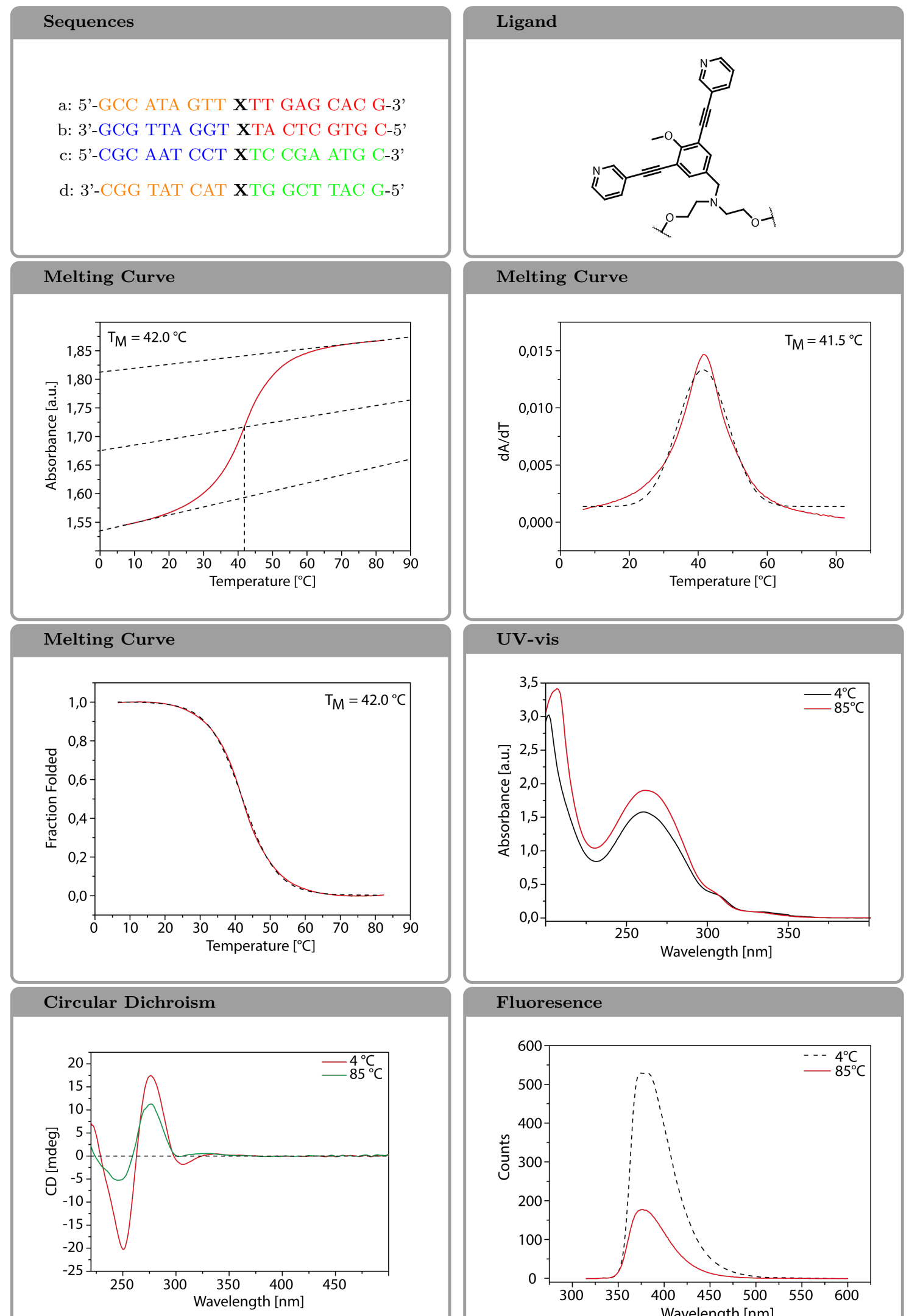

\section{Fluoresence}

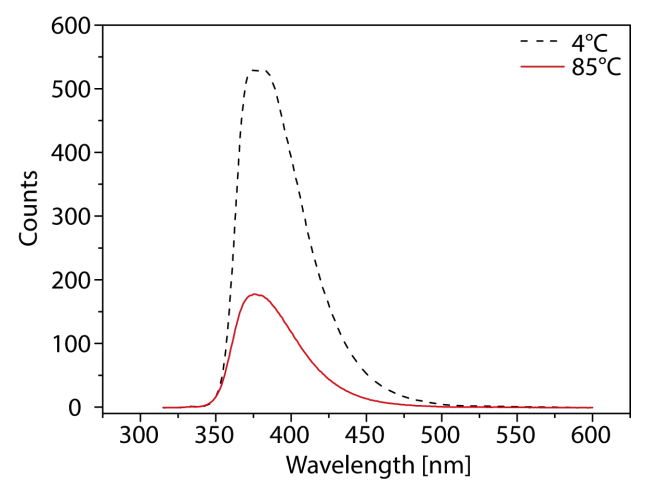




\section{A.7 Further Data}

\section{A.7.a Host-Guest Titration Diethanolamin-Methoxy Ligand}

(a)

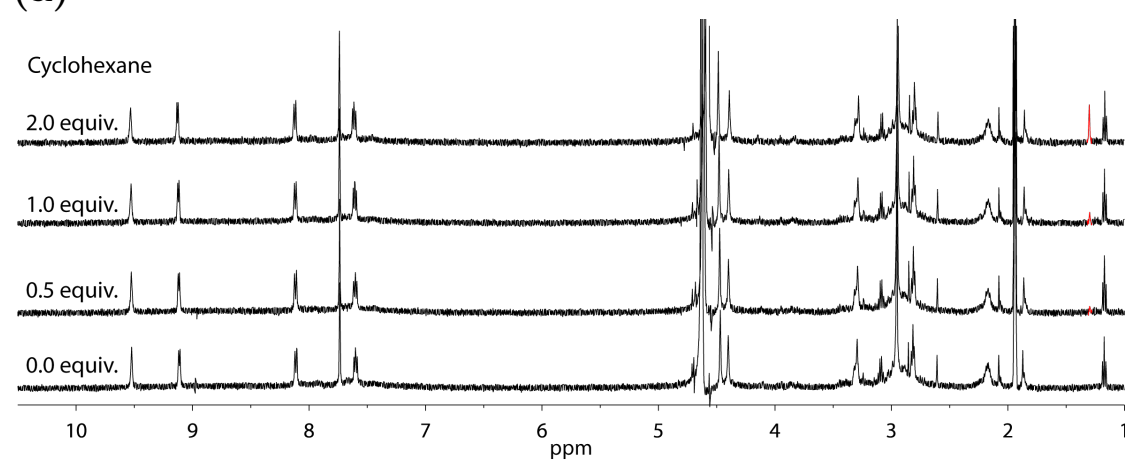

(b)

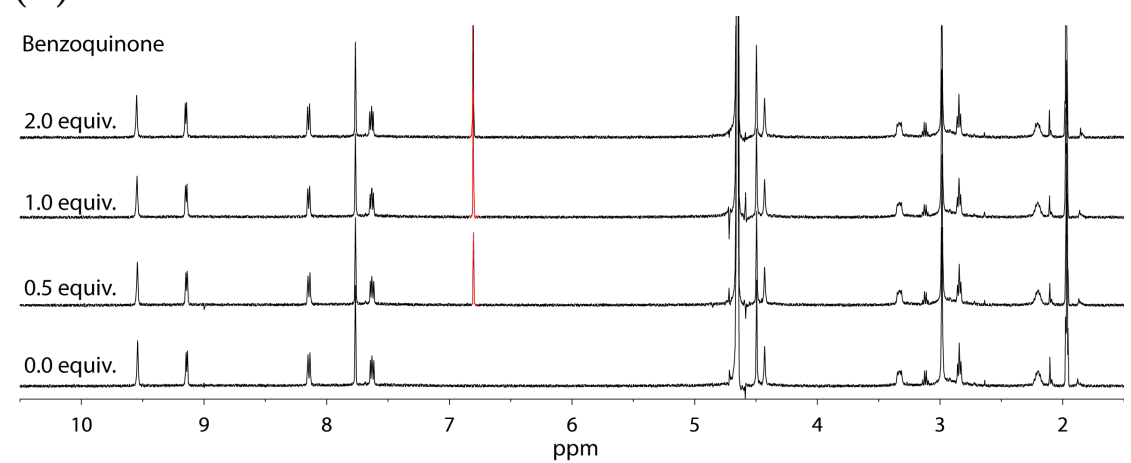

(c)

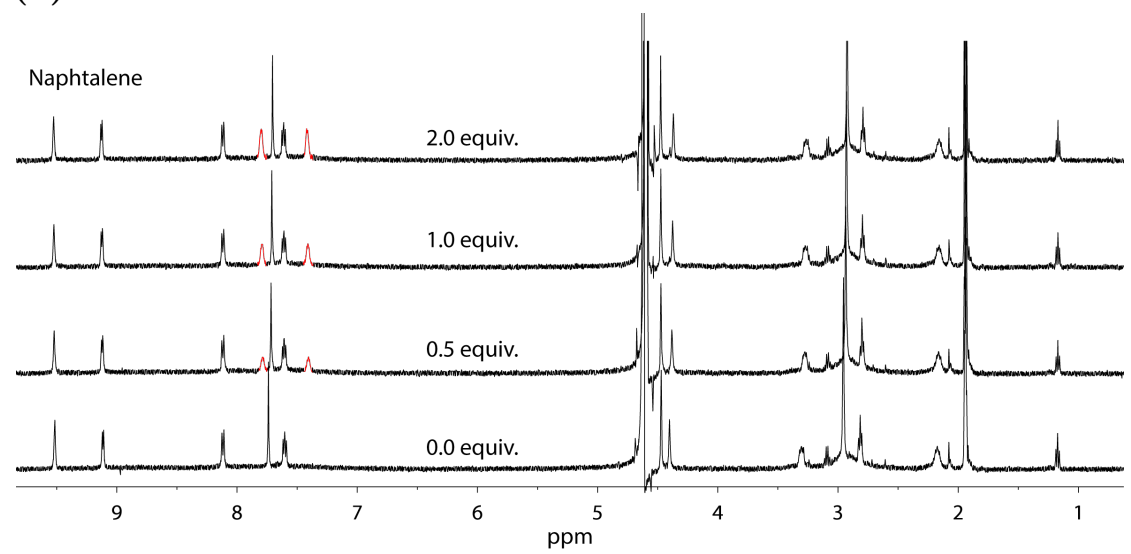

Figure VI.1.7.1: ${ }^{1} \mathrm{H}$ NMR (400 MHz, D $\left.2 \mathrm{O}\right)$ host guest titration experiments with 25 (0.7 mM and cyclohexane (a), 1,4-benzoquinone (b) and naphtalene (c). The signals of the corresponding guest molecules are highlighted in red. 
A.7.b VT NMR experiments for the ligands $\mathrm{L}^{t \mathrm{Bu}}, \mathrm{L}^{\mathrm{Et}}, \mathrm{L}^{\mathrm{Ph}}$ and $\mathrm{L}^{\mathrm{CN}}$

(a)

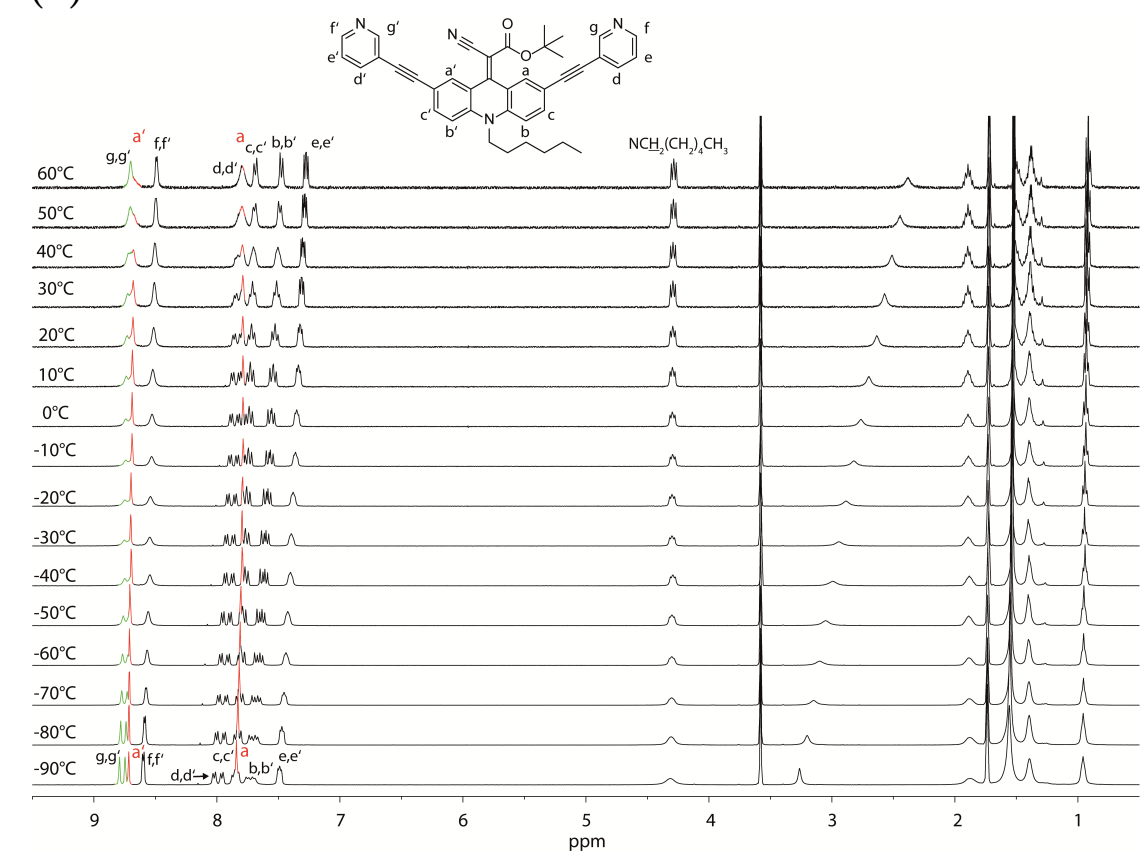

(b)

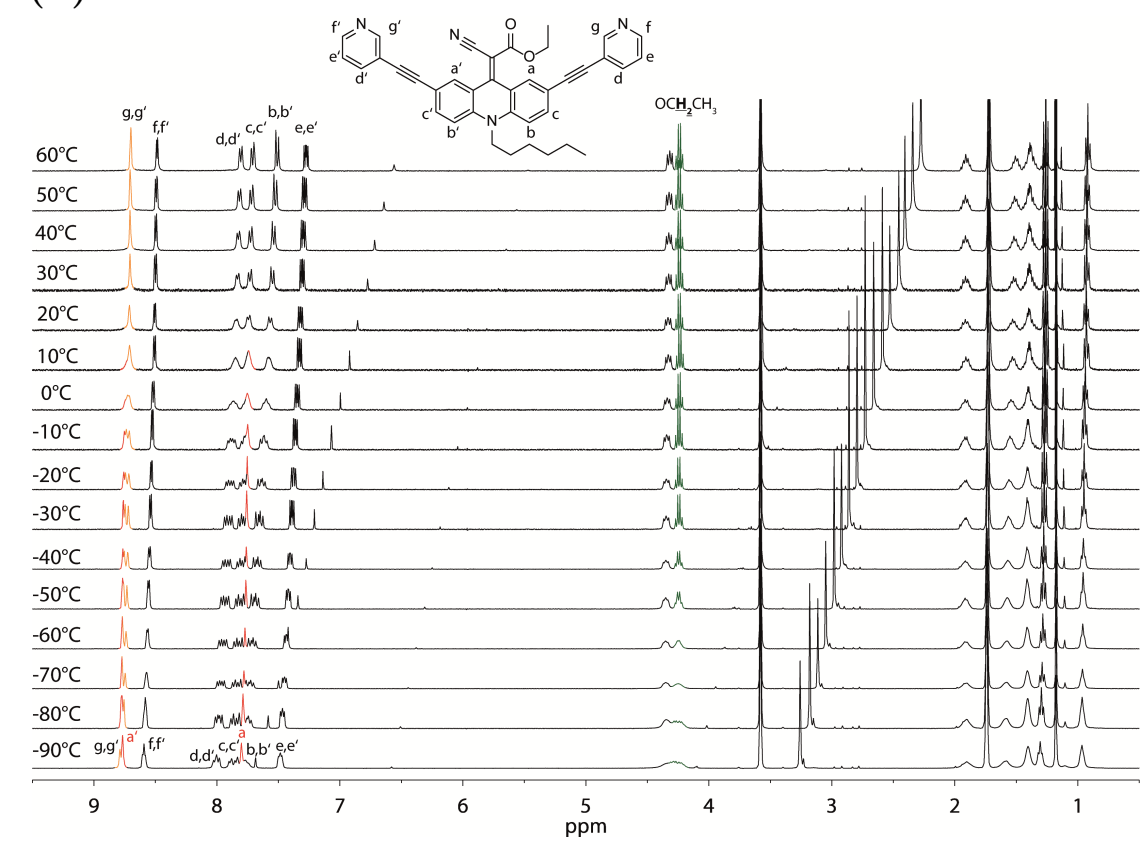


(c)

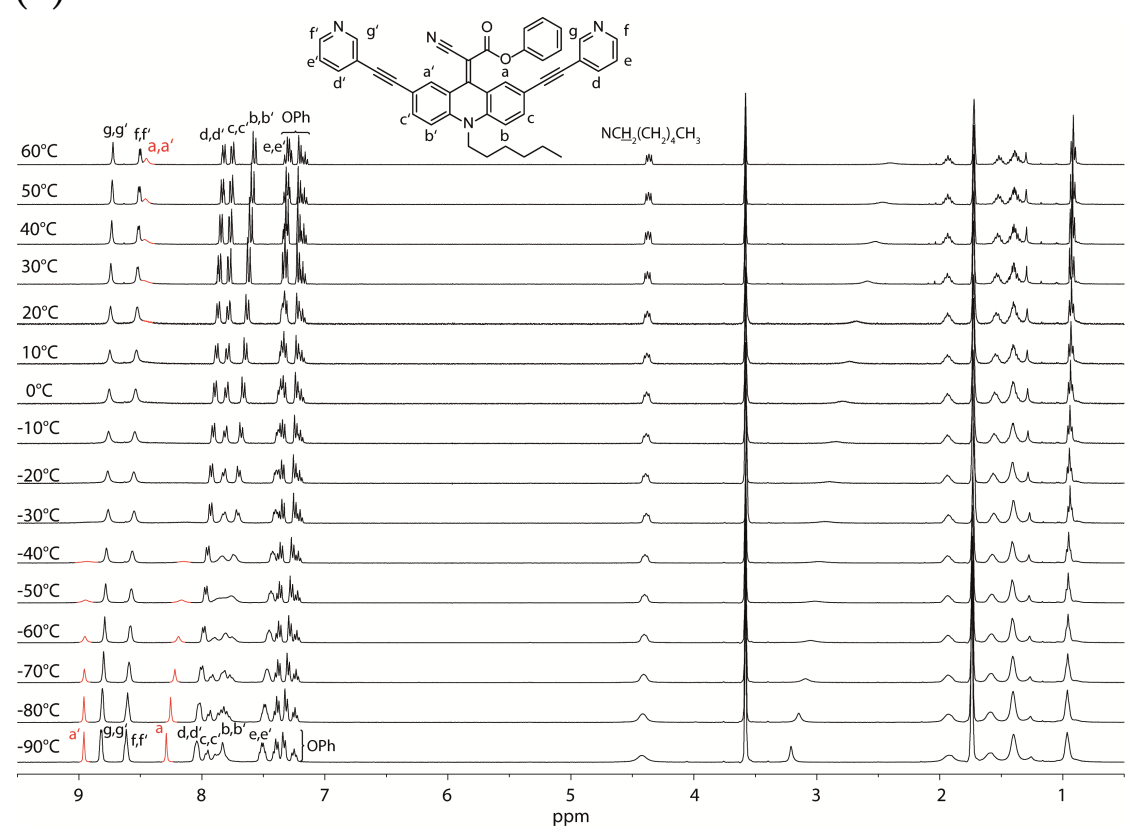

Figure VI.1.7.2: a) $\mathbf{V T -}{ }^{1} \mathbf{H}$ NMR spectra $\left(400 \mathrm{MHz}, d^{8}-\mathrm{THF}\right)$ of ligand $\mathbf{L}^{\boldsymbol{t} \mathbf{B u}}$. The rotation rate and the associated signal sharpness of a/a' (red) depend on the temperature. At low temperature two sets of signals are observable due to a low rotation rate. Higher temperatures increase the rotation rates and lead to one set of signals. The coalescence temperature is not reached in this experiment. b) VT ${ }^{\mathbf{1}} \mathbf{H} \mathbf{~ N M R}$ spectra $\left(400 \mathrm{MHz}, d^{8}\right.$-THF) of ligand $\mathbf{L}^{\mathbf{P h}}$. The rotation rate and the associated signal sharpness of a/a' (red) depend on the temperature. At low temperatures, two sets of signals are observable due to a low rotation rate. Higher temperatures increase the rotation rate and lead to one signal. The coalescence temperature is aprrox. $-10 \check{\mathrm{r} C}$

\section{A.7.c Cyano-acridinylidine ethyl acetate titration data}

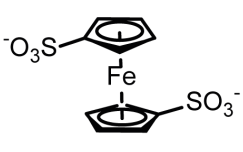

$\mathrm{G}^{1}$<smiles>O=[S+]([O-])c1ccc([S+](=O)[O-])cc1</smiles>

$\mathbf{G}^{2}$

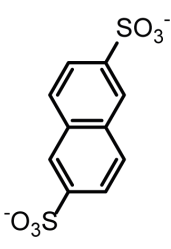

$\mathbf{G}^{3}$<smiles>O=S(=O)(O)c1ccc(-c2ccc(O)cc2)cc1</smiles>

$\mathbf{G}^{4}$<smiles>O=S(=O)(O)c1ccc2ccc([Se](=O)(=O)[O-])cc2c1</smiles>

$\mathbf{G}^{5}$

Scheme VI.1.7.59: Guest molecules $\mathrm{G}^{1}-\mathrm{G}^{5}$ for ${ }^{\mathbf{1}} \mathbf{H}-\mathbf{N M R}$ host-guest titration experiments. All guests come with two tetra-n-butylammonium cations $\left.(\mathrm{nBu})_{4} \mathrm{~N}^{+}\right)$as counter-ions. 


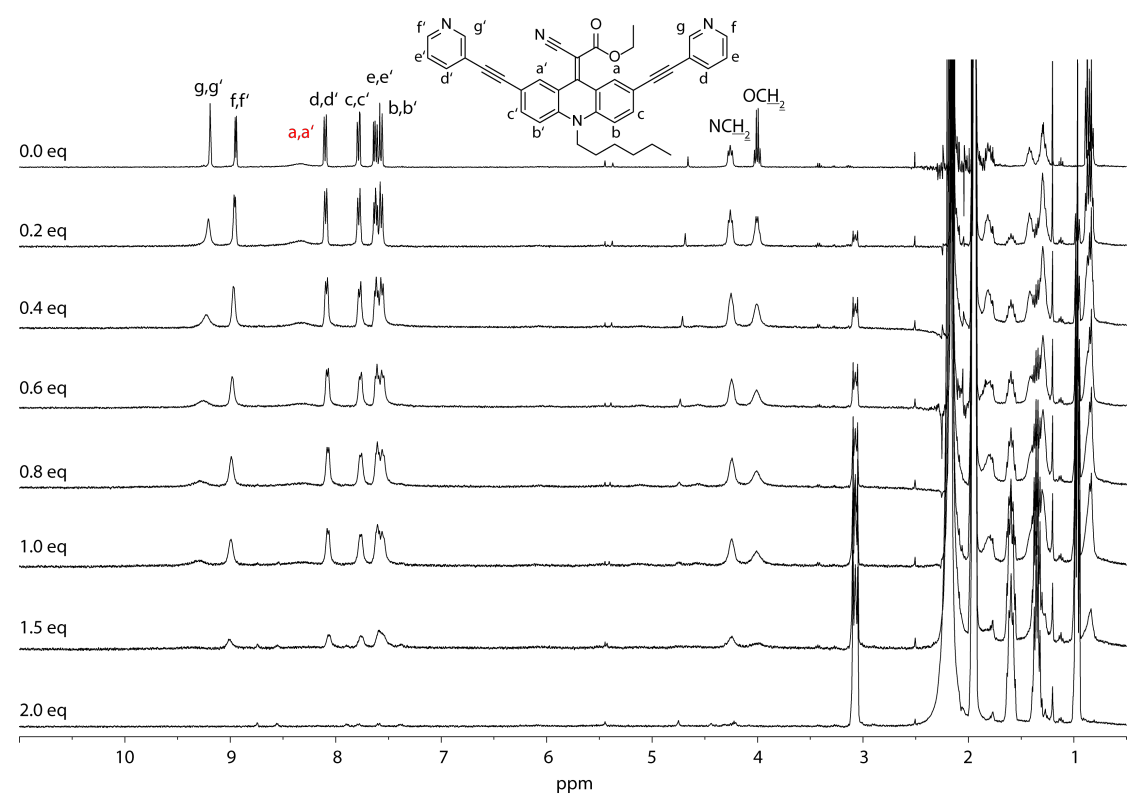

Figure VI.1.7.3: $1 \mathrm{H}$ NMR titration $\left(400 \mathrm{MHz}, 298 \mathrm{~K}, \mathrm{CD}_{3} \mathrm{CN}\right)$ of $\mathbf{C}^{\mathbf{E t}}\left(\left[\mathrm{Pd}_{2} \mathrm{~L}^{\mathrm{Et}}{ }_{4}\right]\right)$ with $\left(\mathrm{NBu}_{4}\right)_{2} \mathbf{G}^{\mathbf{1}}$. The chemical shifts of the inward pointing protons change gradually due to a fast exchange of the guest on the NMR time scale. Excess addition of $\mathbf{G}^{\mathbf{1}}$ leads to precipitation of the cage.

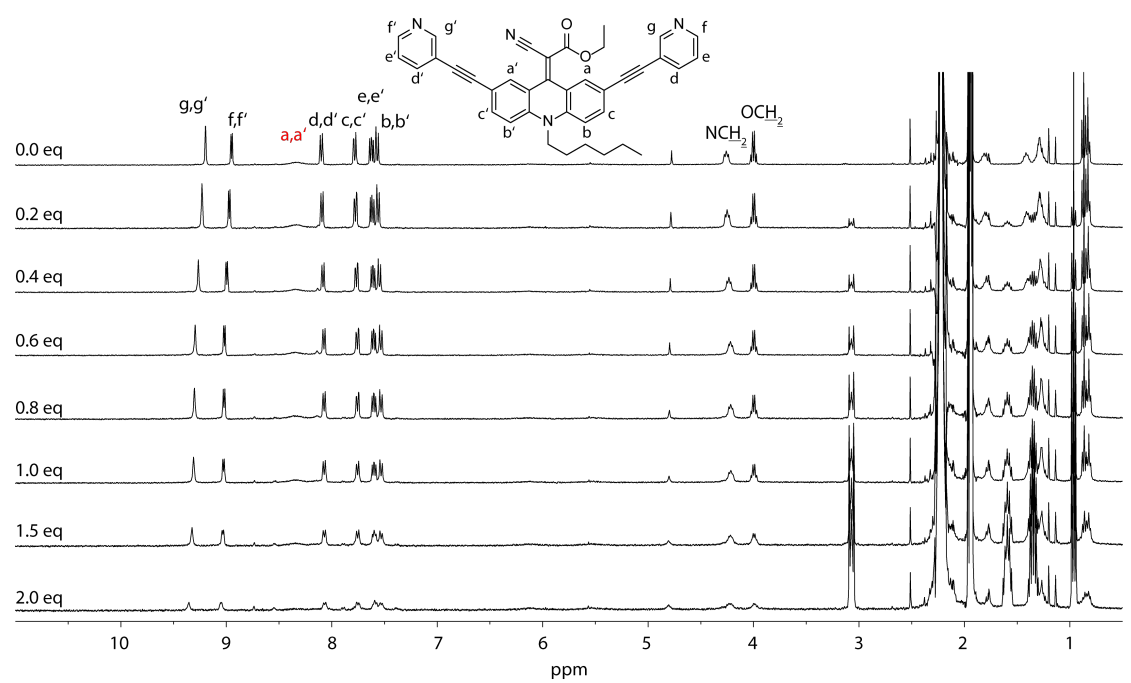

Figure VI.1.7.4: $1 \mathrm{H}$ NMR titration $\left(400 \mathrm{MHz}, 298 \mathrm{~K}, \mathrm{CD}_{3} \mathrm{CN}\right)$ of $\mathbf{C}^{\mathrm{Et}}\left(\left[\mathrm{Pd}_{2} \mathrm{~L}^{\mathrm{Et}}{ }_{4}\right]\right)$ with $\left(\mathrm{NBu}_{4}\right)_{2} \mathbf{G}^{\mathbf{2}}$. The chemical shifts of the inward pointing protons change gradually due to a fast exchange of the guest on the NMR time scale. Excess addition of $\mathbf{G}^{2}$ leads to precipitation of the cage. 


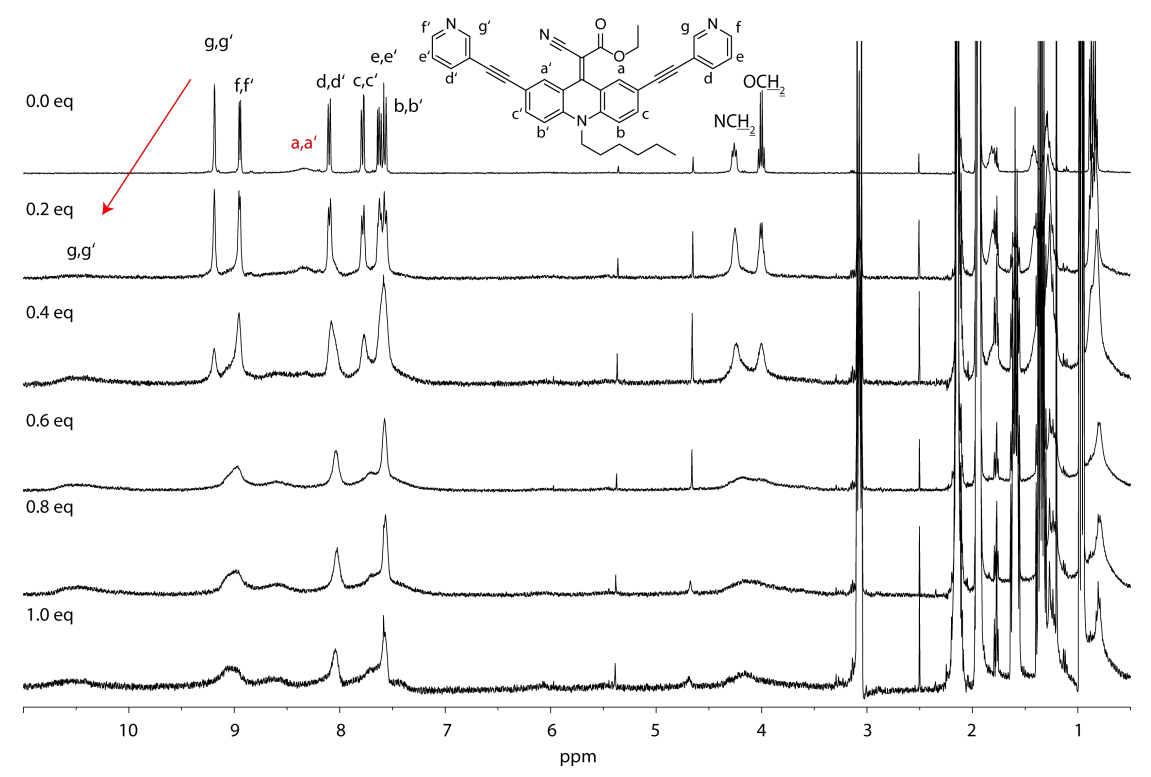

Figure VI.1.7.5: $1 \mathrm{H}$ NMR titration $\left(400 \mathrm{MHz}, 298 \mathrm{~K}, \mathrm{CD}_{3} \mathrm{CN}\right)$ of $\mathbf{C}^{\mathrm{Et}}\left(\left[\mathrm{Pd}_{2} \mathrm{~L}^{\mathrm{Et}}{ }_{4}\right]\right)$ with $\left(\mathrm{NBu}_{4}\right)_{2} \mathbf{G}^{\mathbf{3}}$. Upon addition of one equivalent of guest $\mathbf{G}^{3}$, the uprising of more downfield shifted signal $\mathbf{g} / \mathbf{g}$ ' from $\left[\mathrm{G}_{3} @ \mathrm{Pd}_{2} \mathrm{~L}^{\mathrm{Et}}{ }_{4}\right]$ species is observable due to slow exchange of the guest.

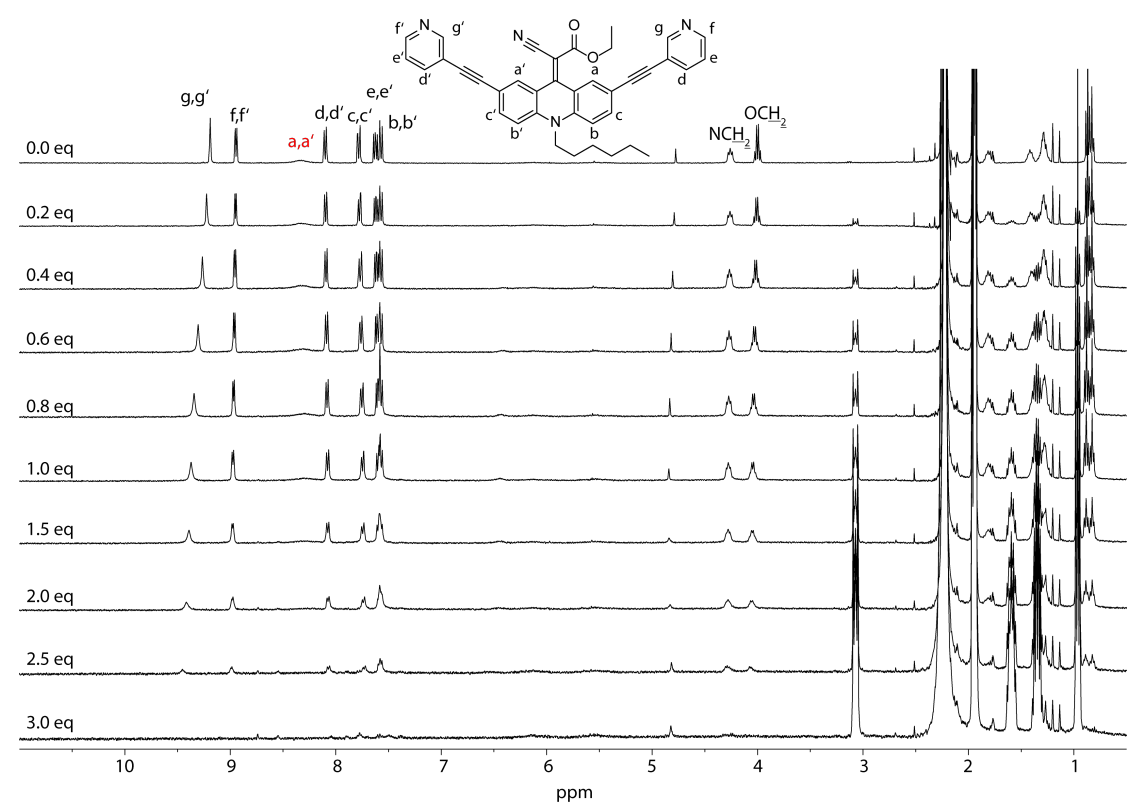

Figure VI.1.7.6: $1 \mathrm{H}$ NMR titration $(400 \mathrm{MHz}, 298 \mathrm{~K}, \mathrm{CD} 3 \mathrm{CN})$ of $\left[\mathrm{Pd}_{2} \mathrm{~L}^{\mathrm{Et}}{ }_{4}\right]$ with $\left(\mathrm{NBu}_{4}\right)_{2} \mathrm{G}^{4}$. The chemical shifts of the inward pointing protons change gradually due to a fast exchange of the guest on the NMR time scale. Excess addition of $\mathrm{G}^{4}$ leads to precipitation of the cage. 


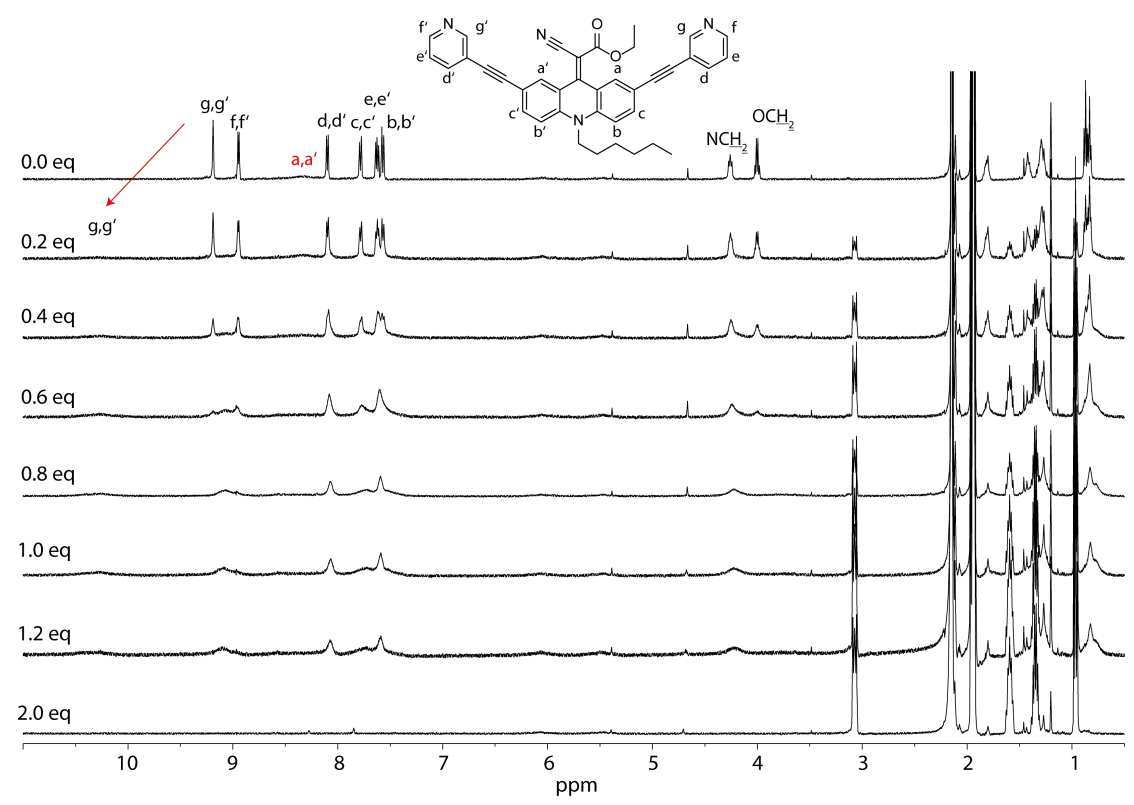

Figure VI.1.7.7: $1 \mathrm{H}$ NMR titration $(400 \mathrm{MHz}, 298 \mathrm{~K}, \mathrm{CD} 3 \mathrm{CN})$ of $\mathbf{C}^{\mathrm{Et}}\left(\left[\mathrm{Pd}_{2} \mathrm{~L}^{\mathrm{Et}}{ }_{4}\right]\right)$ with $\left(\mathrm{NBu}_{4}\right)_{2} \mathbf{G}^{\mathbf{5}}$. Upon addition of one equivalent of guest $\mathbf{G}^{5}$, the uprising of more downfield shifted signal $\mathrm{g} / \mathrm{g}$ ' from $\left[\mathbf{G}^{\mathbf{5}} @ \mathbf{P d}_{2} \mathbf{L}^{\mathbf{E t}}{ }_{4}\right]$ species is observable due to slow exchange of the guest. Excess addition of $\mathbf{G}_{\mathbf{5}}$ leads to precipitation of the cage.

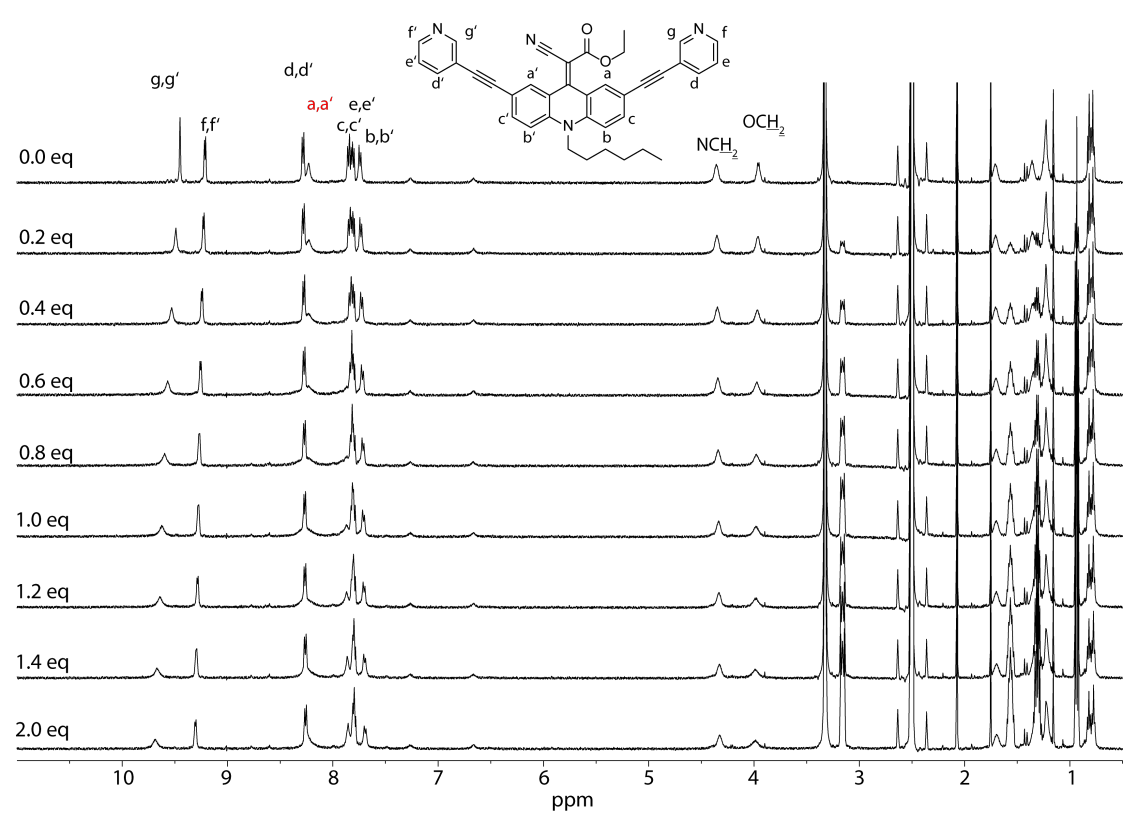

Figure VI.1.7.8: ${ }^{\mathbf{1}} \mathbf{H}$ NMR titration $\left(400 \mathrm{MHz}, 298 \mathrm{~K}, d^{6}\right.$-DMSO) of $\left[\mathrm{Pd}_{2} \mathrm{~L}^{\mathrm{Et}}{ }_{4}\right]$ with $\left(\mathrm{NBu}_{4}\right)_{2} \mathrm{G}^{5}$. The chemical shifts of the inward pointing protons change gradually due to a fast exchange of the guest on the NMR time scale. In contrast to the situation in $\mathrm{CD}_{3} \mathrm{CN}$, in DMSO a fast exchange is observable for $\mathbf{G}^{\mathbf{5}}$ with $\left[\mathrm{Pd}_{2} \mathrm{~L}^{\mathrm{Et}}{ }_{4}\right]$. 

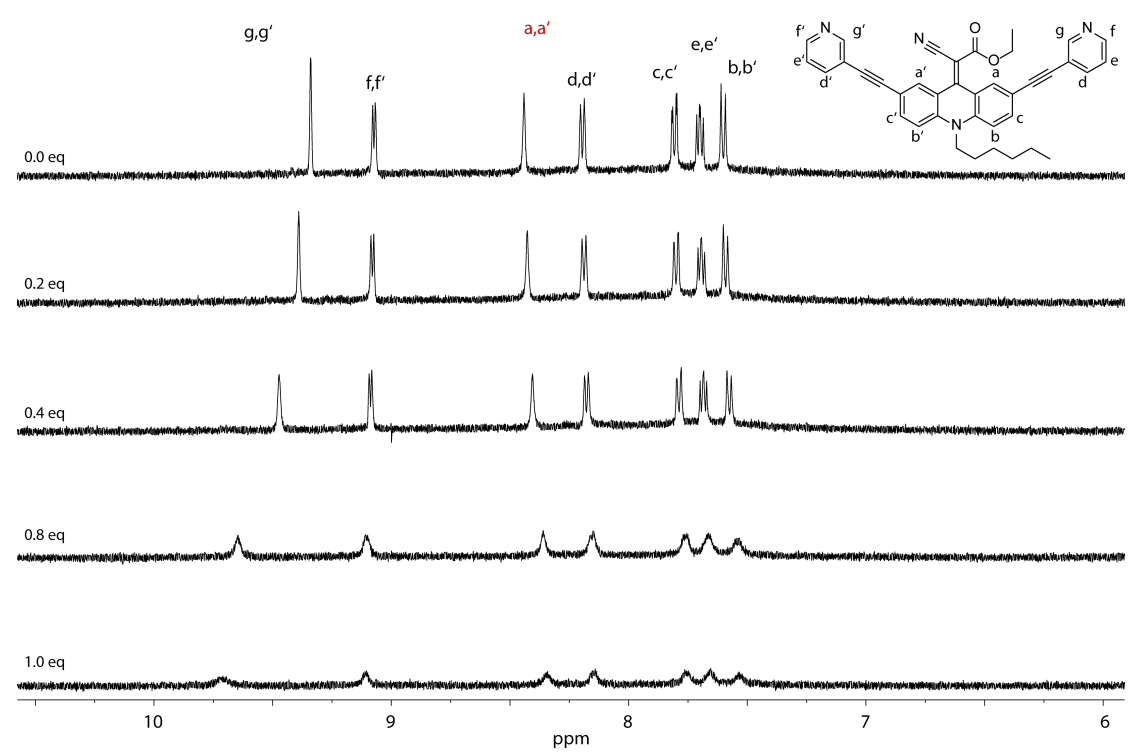

Figure VI.1.7.9: ${ }^{\mathbf{1}} \mathbf{H}$ NMR titration $\left(400 \mathrm{MHz}, 333 \mathrm{~K}, \mathrm{CD}_{3} \mathrm{OD}\right)$ of $\left[\mathrm{Pd}_{2} \mathrm{~L}^{\mathrm{Et}}{ }_{4}\right]$ with $\left(\mathrm{NBu}_{4}\right)_{2} \mathrm{G}^{5}$. The chemical shifts ofthe inward pointing protons change gradually due to a fast exchange of the guest on the NMR time scale. Addition of 0.4 eq of $\mathbf{G}^{\mathbf{5}}$ leads already to visible precipitation in the nmr tube.

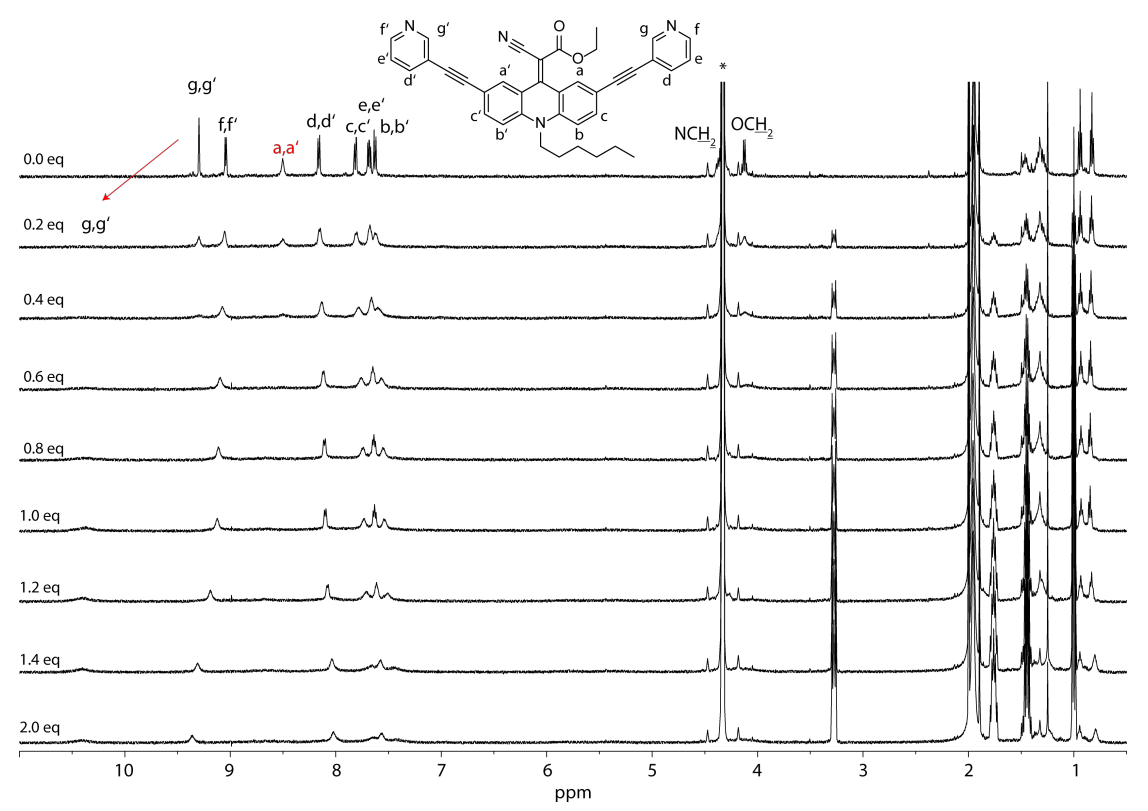

Figure VI.1.7.10: $1 \mathrm{H}$ NMR titration $\left(400 \mathrm{MHz}, 333 \mathrm{~K}, \mathrm{CD}_{3} \mathrm{NO}_{2}\right)$ of $\left[\mathrm{Pd}_{2} \mathrm{~L}^{\mathrm{Et}}{ }_{4}\right]$ with $\left(\mathrm{NBu}_{4}\right)_{2} \mathrm{G}^{5}$. Upon addition of one equivalent of guest $\mathbf{G}^{5}$ the $\left[\mathrm{Pd}_{2} \mathrm{~L}^{\mathrm{Et}}{ }_{4}\right]$ cage transforms into $\left[\mathrm{G}^{5} @ \mathrm{Pd}_{2} \mathrm{~L}^{\mathrm{Et}}{ }_{4}\right]$. Excess addition of $\mathbf{G}^{5}$ leads to precipitation of the cage. 


\section{A.7.d Aza-15-crown-cyano-acridinylidine ethyl acetate Titration Data}

(a)

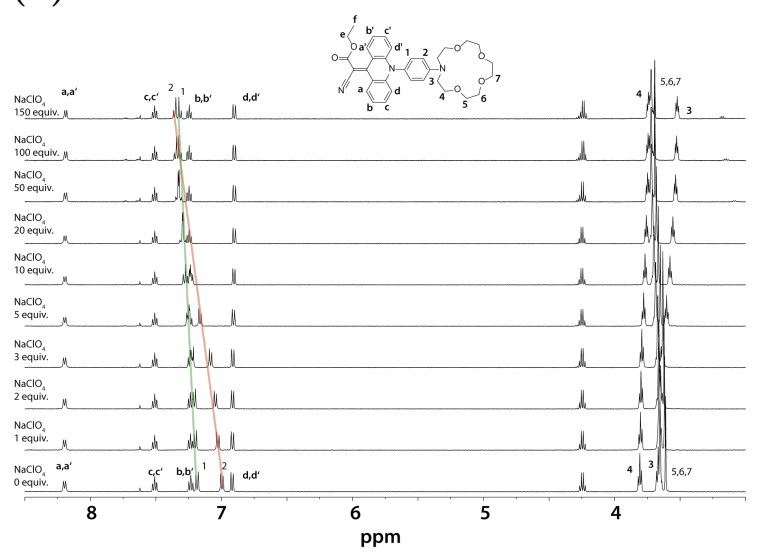

(b)

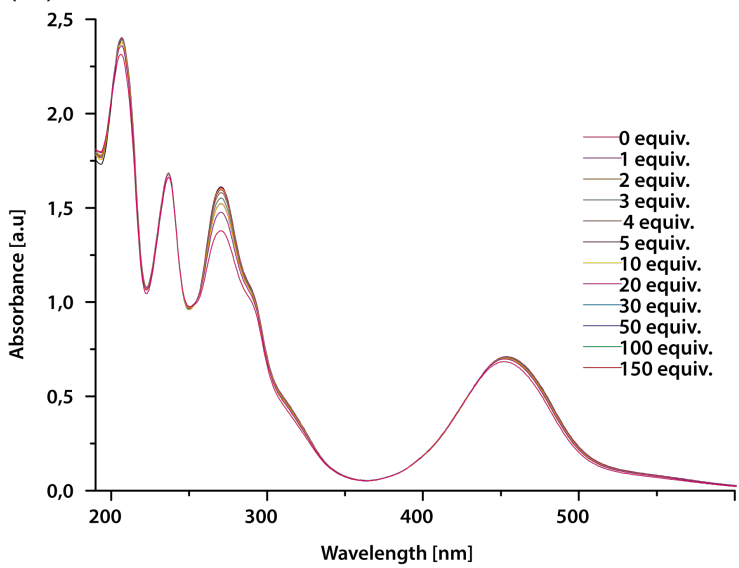

Figure VI.1.7.11: UV-vis and NMR titration experiments for $\mathbf{5 2}$ with $\mathrm{NaClO}_{4}$. a) ${ }^{1} \mathrm{H} \mathrm{NMR}\left(400 \mathrm{MHz}, \mathrm{d}^{3}-\right.$ $\mathrm{MeCN}$ ) titration experiments of $\mathbf{5 2}$ and $\mathrm{NaClO}_{4}$ ranging from 0 to 150 equiv. of added salt. As in previous titration experiments an downfield shifting of the bridging phenyl signals can be observed, which becomes more significant at higher salt concentrations. However, there is no significant change detectable for the a/a' signal, which turns out to be a characteristic indicator for a change in the push-pull system electronics. b) There is also just a minimal change in UV-vis titration experiment $(0.005 \mathrm{mM} \mathbf{5 2}$ in MeCN). There maximum observable blue-shift of $3 \mathrm{~nm}$, after adding 100 equiv of the corresponding sodium salt. 
(a)

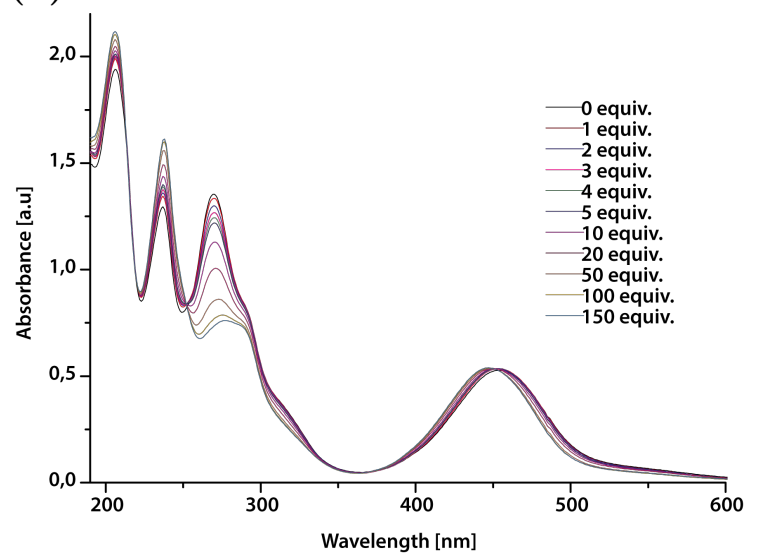

(b)

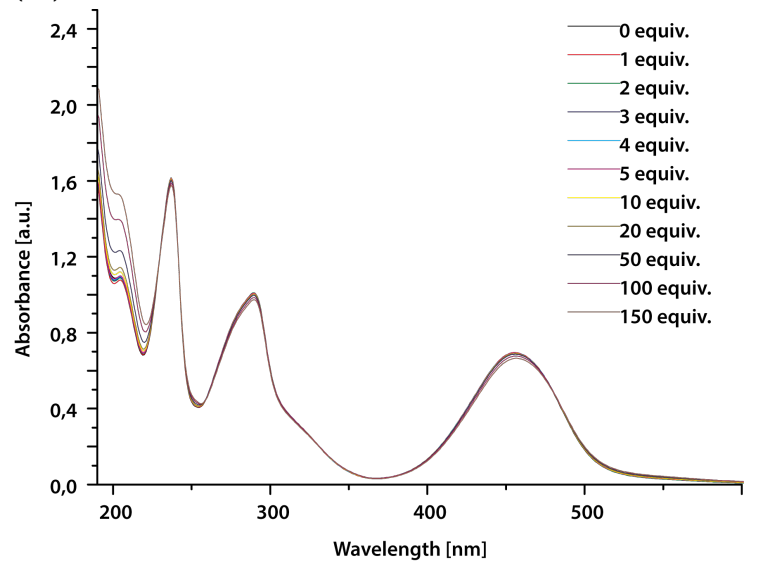

(c)

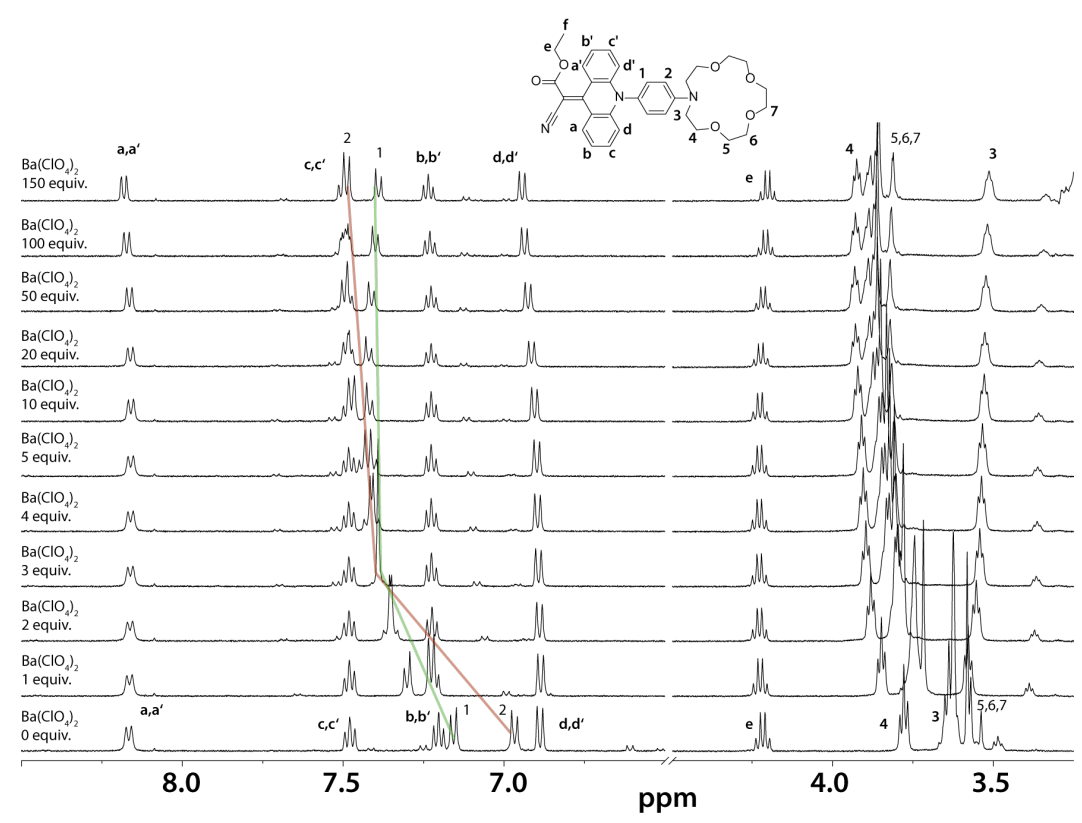

Figure VI.1.7.12: UV-vis and NMR titration experiments for $\mathbf{5 2}$ with $\mathrm{Ba}\left(\mathrm{ClO}_{4}\right)_{2}$. a) UV-vis experiment with $0.005 \mathrm{M} 52$ in $\mathrm{MeCN}$. $\mathrm{Ba}\left(\mathrm{ClO}_{4}\right)_{2}$ was added until no shift of the $455 \mathrm{~nm}$ was detected. A maximum hypsochromic shift of $7 \mathrm{~nm}$ could be observed after addition of 100 equiv. of metal salt. b) Control experiment. Titration of $\mathrm{Ba}\left(\mathrm{ClO}_{4}\right)_{2}$ to $\mathrm{B}^{\mathrm{Et}}(\mathbf{3 5})$. Except of a slight hypochromic shift, due to the salt addition caused dilution, no shift of the push-pull system characteristic $455 \mathrm{~nm}$ band was observed. c) NMR titration experiment (400 MHz, $\left.\mathrm{d}^{3}-\mathrm{MeCN}\right)$ of $\mathrm{B}^{\mathrm{Et}}$ Azacrown $(\mathbf{5 2}, 2.8 \mathrm{mM})$ with $\mathrm{Ba}\left(\mathrm{ClO}_{4}\right)_{2}$. A significant chemical shift for the phenyl hydrogens $\mathbf{1}$ and $\mathbf{2}$ is observable upon addition of 5 equiv. of the corresponding metal salt (shifting is highlighted by green and red line). Addison of further metal salt led to further slight shifting of those signals. It is worth to mention, that the signal 2 experience to highest downfield shift, what indicates an interaction between barium cation and azacrown, since that speaks for a shifting of electron-density towards the azacrown nitrogen. However, no significant change for the $\mathbf{a} / \mathbf{a}$ ' is observable. It seems that the signal becomes slightly sharper by increasing the amount of metal salt. This finding can be explained by an slight increase in stabilization of the charge-separated state, caused by the increased ion concentration within the sample solution. 
(a)

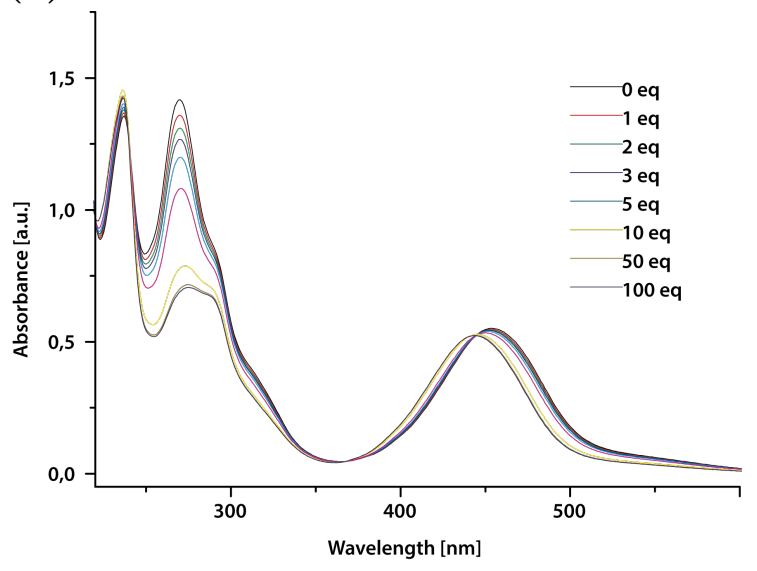

(c) (b)

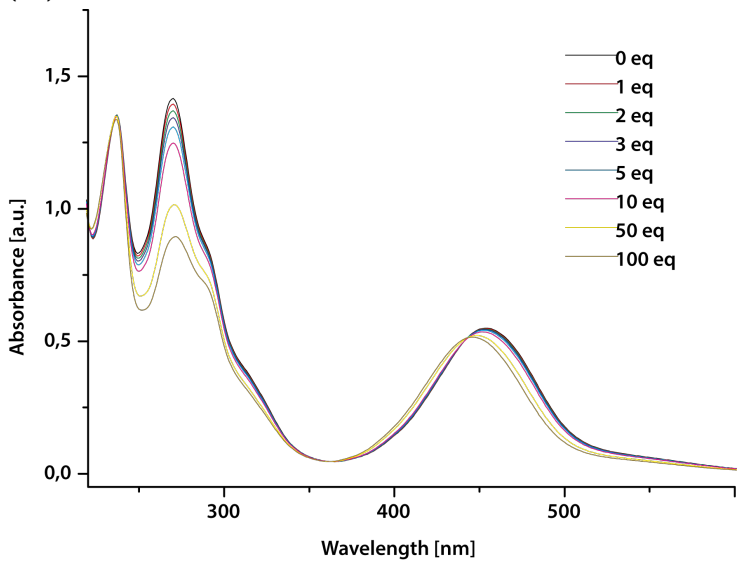

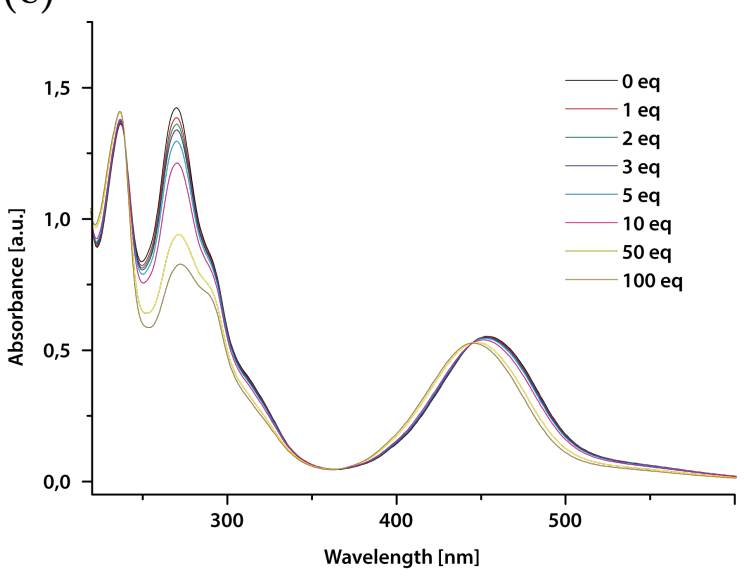

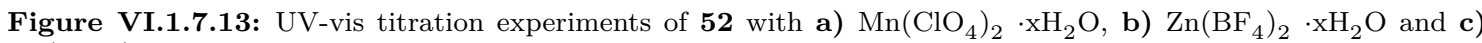
$\mathrm{Cd}\left(\mathrm{ClO}_{4}\right)_{2} \cdot \mathrm{xH}_{2} \mathrm{O}$. All samples show a slight blue-shift upon addition of the corresponding metal salts. For all samples 100 equiv. of the corresponding metal salt was added in total, leading to a maximum blue shift of: $12 \mathrm{~nm}$ for $\mathrm{Mn}\left(\mathrm{ClO}_{4}\right)_{2}, 10 \mathrm{~nm}$ for $\mathrm{Zn}\left(\mathrm{BF}_{4}\right)_{2}$ and $11 \mathrm{~nm}$ for $\mathrm{Cd}\left(\mathrm{ClO}_{4}\right)_{2}$.

(a)

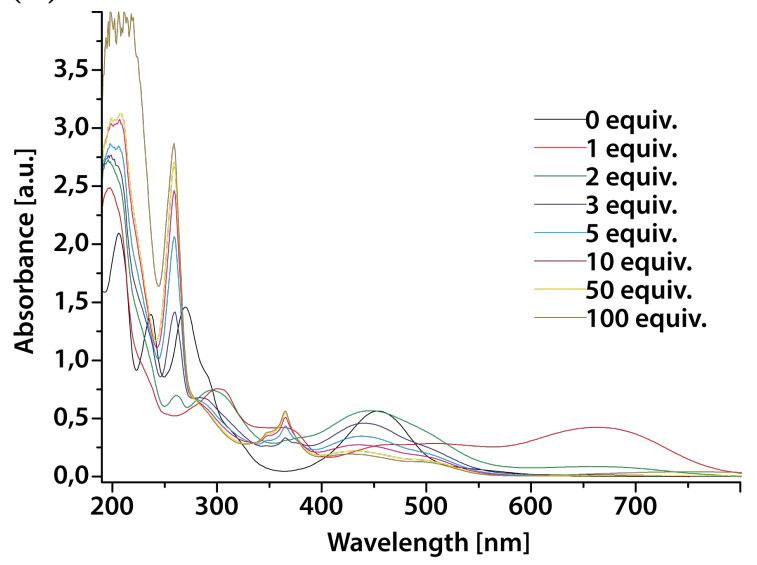

(b)

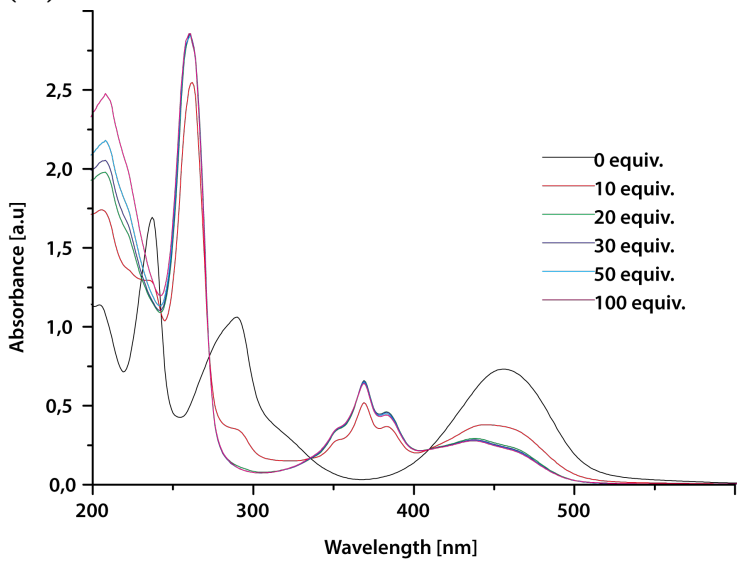

Figure VI.1.7.14: UV-vis titration experiments of a) $\mathbf{5 2}$ and b) $\mathbf{3 5}$ with $\mathrm{Cu}\left(\mathrm{BF}_{4}\right)_{2}$. a) Addition of 1.0 equiv. copper salt to compound $\mathbf{5 2}(0.005 \mathrm{M}$ in $\mathrm{MeCN})$ led to a broad peak at ca. $675 \mathrm{~nm}$, vanishing up addition of second equivalent of the copper salt. The behavior can often be observed for the formation of radical species. Further addition led to the acridinium ion specific absorption pattern. b) The titration of $\mathrm{Cu}\left(\mathrm{BF}_{4}\right)_{2}$ to compound 35 (0.005 M did not show this behavior upon addition of 1.0 or 2.0 equiv. copper salt, therefore these steps were neglected. However, the acridinium ion absorption pattern appeared as well for higher copper salt concentrations. 


\section{A.8 Crystal Data and Structure Refinement}

Table VI.1.8.1: Data from X-ray diffraction analysis for compounds $\mathrm{B}^{\mathrm{Et}}(\mathbf{3 5})$ and $\mathrm{I}_{2}-\mathrm{B}^{t \mathrm{Bu}}$. Both crystals were measured and refined by Dr. Julian Holstein.

\begin{tabular}{|c|c|c|}
\hline Compound & $\mathrm{B}^{\mathrm{Et}}(34)$ & $\mathrm{I}_{2}-\mathrm{B}^{t \mathrm{Bu}}$ \\
\hline CCDC number & 1542074 & - \\
\hline Empirical formula & C96 H104 N8 O8 & C26 H28 I2 N2 O2 \\
\hline Formula weight & 1497.87 & 654.30 \\
\hline Temperature & $100(2) \mathrm{K}$ & $100(2) \mathrm{K}$ \\
\hline Wavelength & $1.54178 \AA$ & $0.71073 \AA$ \\
\hline Crystal system & Monoclinic & Monoclinic \\
\hline Space group & $\mathrm{P} 21$ & $\mathrm{P} 2 / \mathrm{n}$ \\
\hline Unit cell dimensions & $\begin{array}{c}\mathrm{a}=10.8878(6) \AA \\
\mathrm{b}=26.8038(15) \AA \\
\mathrm{c}=14.9978(9) \AA \\
\alpha=90^{\circ} \\
\beta=110.114(3) \circ \\
\gamma=90^{\circ}\end{array}$ & $\begin{array}{c}\mathrm{a}=15.6998(12) \AA \\
\mathrm{b}=9.1687(7) \AA \\
\mathrm{c}=18.6041(14) \AA \\
\alpha=90^{\circ} \\
\beta=105.955(3) \circ \\
\gamma=90^{\circ}\end{array}$ \\
\hline Volume & $4109.9(4) \AA^{3}$ & $2574.8(3) \AA^{3}$ \\
\hline $\mathrm{Z}$ & 2 & 4 \\
\hline Density (calculated) & $1.210 \mathrm{Mg} \mathrm{m}^{-3}$ & $1.688 \mathrm{Mg} \mathrm{m}^{-3}$ \\
\hline Absorption coefficient & $0.609 \mathrm{~mm}$ & $2.467 \mathrm{~mm}$ \\
\hline $\mathrm{F}(000)$ & 1600 & 1280 \\
\hline Crystal size & $0.300 \times 0.200 \times 0.200 \mathrm{~mm}$ & $0.100 \times 0.100 \times 0.100 \mathrm{~mm}$ \\
\hline $\begin{array}{l}\text { Theta range for data } \\
\text { collection }\end{array}$ & 3.138 to $68.245^{\circ}$ & 2.983 to $35.019 \check{r}^{\circ}$ \\
\hline Index ranges & $\begin{array}{c}-9<=\mathrm{h}<=13,-31<=\mathrm{k}<=31 \\
-18<=\mathrm{l}<=18\end{array}$ & $\begin{array}{c}-25<=\mathrm{h}<=25,-14<=\mathrm{k}<=14, \\
-30<=\mathrm{l}<=30\end{array}$ \\
\hline Reflections collected & 33136 & 229857 \\
\hline Independent reflections & $14121[\mathrm{R}($ int $)=0.0612]$ & $11342[\mathrm{R}(\mathrm{int})=0.0666]$ \\
\hline $\begin{array}{c}\text { Completeness to theta }= \\
67.679^{\circ}\end{array}$ & $98.7 \%$ & $99.8 \%$ \\
\hline Absorption correction & $\begin{array}{l}\text { Semi-empirical from } \\
\text { equivalents }\end{array}$ & \\
\hline Max. and min. transmission & 0.7542 and 0.5472 & \\
\hline Refinement method & $\begin{array}{l}\text { Full-matrix least-squares on } \\
\qquad \mathrm{F}^{2}\end{array}$ & $\begin{array}{l}\text { Full-matrix least-squares on } \\
\qquad \mathrm{F}^{2}\end{array}$ \\
\hline $\begin{array}{c}\text { Data / restraints / } \\
\text { parameters }\end{array}$ & $14121 / 1 / 1017$ & $11342 / 0 / 293$ \\
\hline Goodness-of-fit on F2 & 1.031 & 1.118 \\
\hline Final R indices $[\mathrm{I}>2 \operatorname{sigma}(\mathrm{I})]$ & $\mathrm{R} 1=0.0763, \mathrm{wR} 2=0.2256$ & $\mathrm{R} 1=0.0264, \mathrm{wR} 2=0.0612$ \\
\hline $\mathrm{R}$ indices (all data) & $\mathrm{R} 1=0.0807, \mathrm{wR} 2=0.2303$ & $\mathrm{R} 1=0.0352, \mathrm{wR} 2=0.0679$ \\
\hline Absolute structure parameter & $0.40(11)$ & \\
\hline Extinction coefficient & $\mathrm{n} / \mathrm{a}$ & $\mathrm{n} / \mathrm{a}$ \\
\hline Largest diff. peak and hole & 0.542 and -0.406 e. $\AA^{3}$ & 1.917 and -1.934 e. $\AA^{3}$ \\
\hline
\end{tabular}


Table VI.1.8.2: Data from X-ray diffraction analysis for compounds 40, 42 and 43 . Compounds 40 and 42 were measured and refined by Dr. Julian Holstein. Compound $\mathbf{4 3}$ was measured by Dr. Christian Würtele and refined by Dr. Julian Holstein

\begin{tabular}{|c|c|c|c|}
\hline Compound & $\mathrm{L}^{\mathrm{CN}}(40)$ & $\mathrm{L}^{\mathrm{Et}}(42)$ & $\mathbf{L}^{\mathrm{Ph}}(43)$ \\
\hline CCDC number & & 1478914 & 1478915 \\
\hline Empirical formula & C36 H27 N5 & C38 H32 N4 O2 & C46 H32 D8 N4 O3 \\
\hline Formula weight & 529.62 & 576.67 & 704.87 \\
\hline Temperature & $100(2) \mathrm{K}$ & $100(2) \mathrm{K}$ & $100(2) \mathrm{K}$ \\
\hline Wavelength & $1.54178 \AA$ & $0.71073 \AA$ & $1.54178 \AA$ \\
\hline Crystal system & Monoclinic & Monoclinic & Triclinic \\
\hline Space group & $\mathrm{P} 2_{1 / \mathbf{n}}$ & $\mathrm{P} 2_{1 / \mathbf{n}}$ & $\mathrm{P}-1$ \\
\hline Unit cell dimensions & $\begin{array}{c}\mathrm{a}=10.9411(5) \AA \\
\mathrm{b}=7.5173(3) \AA \\
\mathrm{c}=31.9858(15) \AA \\
\alpha=90^{\circ} \\
\beta=95.053(2)^{\circ} \\
\gamma=92.206(2)^{\circ}\end{array}$ & $\begin{array}{c}\mathrm{a}=11.4777(5) \AA \\
\mathrm{b}=15.9325(7) \AA \\
\mathrm{c}=17.0968(8) \AA \\
\alpha=90^{\circ} \\
\beta=103.233(2)^{\circ} \\
\gamma=97.198(2)^{\circ}\end{array}$ & $\begin{array}{c}\mathrm{a}=9.7141(3) \AA \\
\mathrm{b}=13.7175(4) \AA \\
\mathrm{c}=13.8642(4) \AA \\
\alpha=90^{\circ} \\
\beta=110.114(3)^{\circ} \\
\gamma=98.744(2)^{\circ}\end{array}$ \\
\hline Volume & $2620.5(2) \AA^{3}$ & $3043.4(2) \AA^{3}$ & $1808.46(9) \AA^{3}$ \\
\hline $\mathrm{Z}$ & 4 & 4 & 2 \\
\hline Density (calculated) & $1.342 \mathrm{Mg} \mathrm{m}^{-3}$ & $1.259 \mathrm{Mg} \mathrm{m}^{-3}$ & $1.294 \mathrm{Mg} \mathrm{m}^{-3}$ \\
\hline Absorption coefficient & $0.629 \mathrm{~mm}$ & $0.079 \mathrm{~mm}$ & $0.638 \mathrm{~mm}$ \\
\hline $\mathrm{F}(000)$ & 1112 & 1216 & 736 \\
\hline Crystal size & $\begin{array}{c}0.100 \times 0.100 \times \\
0.100 \mathrm{~mm}\end{array}$ & $\begin{array}{c}0.844 \times 0.744 \times \\
0.542 \mathrm{~mm}\end{array}$ & $\begin{array}{c}0.300 \times 0.116 \times \\
0.072 \mathrm{~mm}\end{array}$ \\
\hline $\begin{array}{c}\text { Theta range for data } \\
\text { collection }\end{array}$ & 2.774 to $63.685^{\circ}$ & 2.331 to $33.141^{\circ}$ & 3.22 to $74.61^{\circ}$ \\
\hline Index ranges & $\begin{array}{c}-12<=\mathrm{h}<=12, \\
-8<=\mathrm{k}<=8 \\
-37<=\mathrm{l}<=37\end{array}$ & $\begin{array}{c}-17<=\mathrm{h}<=17 \\
-23<=\mathrm{k}<=24 \\
-26<=\mathrm{l}<=23\end{array}$ & $\begin{array}{l}-12<=\mathrm{h}<=12 \\
-17<=\mathrm{k}<=17 \\
-17<=\mathrm{l}<=17\end{array}$ \\
\hline Reflections collected & 35316 & 114976 & 21094 \\
\hline $\begin{array}{c}\text { Independent } \\
\text { reflections }\end{array}$ & $\begin{array}{c}4275[\mathrm{R}(\text { int })= \\
0.0529]\end{array}$ & $\begin{array}{c}11572[\mathrm{R}(\text { int })= \\
0.0375]\end{array}$ & $\begin{array}{c}7238[\mathrm{R}(\text { int })= \\
0.0382]\end{array}$ \\
\hline $\begin{array}{c}\text { Completeness to } \\
\text { theta }=\end{array}$ & $63.685^{\circ} / 99.0 \%$ & $25.242^{\circ} / 99.5 \%$ & $74.61^{\circ} / 97.7 \%$ \\
\hline Absorption correction & $\begin{array}{c}\text { Semi-empirical from } \\
\text { equivalents }\end{array}$ & $\begin{array}{c}\text { Semi-empirical from } \\
\text { equivalents }\end{array}$ & $\begin{array}{c}\text { Semi-empirical from } \\
\text { equivalents }\end{array}$ \\
\hline $\begin{array}{l}\text { Max. and min. } \\
\text { transmission }\end{array}$ & 0.7542 and 0.5472 & 0.7485 and 0.6842 & 0.75 and 0.68 \\
\hline Refinement method & $\begin{array}{c}\text { Full-matrix } \\
\text { least-squares on } \mathrm{F}^{2}\end{array}$ & $\begin{array}{c}\text { Full-matrix } \\
\text { least-squares on } \mathrm{F}^{2}\end{array}$ & $\begin{array}{c}\text { Full-matrix } \\
\text { least-squares on } \mathrm{F}^{2}\end{array}$ \\
\hline $\begin{array}{c}\text { Data / restraints / } \\
\text { parameters }\end{array}$ & 4275 / 0 / 371 & 11572 / 0 / 399 & 7238 / 1 / 499 \\
\hline Goodness-of-fit on F2 & 1.186 & 1.116 & 1.047 \\
\hline $\begin{array}{c}\text { Final } \mathrm{R} \text { indices } \\
{[\mathrm{I}>2 \operatorname{sigma}(\mathrm{I})]}\end{array}$ & $\begin{array}{c}\mathrm{R} 1=0.0584, \mathrm{wR} 2= \\
0.1631\end{array}$ & $\begin{array}{c}\mathrm{R} 1=0.0411, \mathrm{wR} 2= \\
0.1192\end{array}$ & $\begin{array}{c}\mathrm{R} 1=0.0477, \mathrm{wR} 2= \\
0.1094\end{array}$ \\
\hline $\mathrm{R}$ indices (all data) & $\begin{array}{c}\mathrm{R} 1=0.0605, \mathrm{wR} 2= \\
0.1639\end{array}$ & $\begin{array}{c}\mathrm{R} 1=0.0473, \mathrm{wR} 2= \\
0.1246\end{array}$ & $\begin{array}{c}\mathrm{R} 1=0.0643, \mathrm{wR} 2= \\
0.1183\end{array}$ \\
\hline Extinction coefficient & $\mathrm{n} / \mathrm{a}$ & $\mathrm{n} / \mathrm{a}$ & $\mathrm{n} / \mathrm{a}$ \\
\hline $\begin{array}{l}\text { Largest diff. peak } \\
\text { and hole }\end{array}$ & 0.267 and -0.294 e. $\AA^{3}$ & 0.829 and -0.284 e. $\AA^{3}$ & 0.243 and -0.275 e. $\AA^{3}$ \\
\hline
\end{tabular}




\section{Appendix}

\section{Methods}

\section{Instruments for analytics}

\section{NMR (Nuclear magnetic resonance spectroscopy)}

The NMR data was measured on the spectrometers Bruker AV 500 Advance NEO, Bruker AV 400 Advance III HD NanoBay, AV 500 Advance III HD, AV 600 Advance III HD, AV 700 Advance III HD and Agilent Technologies DD2. The chemical shifts were calibrated on tetramethylsilane $(1 \mathrm{H}, 0 \mathrm{ppm}), \mathrm{CFCl}_{3}\left({ }^{19} \mathrm{~F}, 0 \mathrm{ppm}\right), 85 \%$ phosphoric acid $\left({ }^{31} \mathrm{P}, 0 \mathrm{ppm}\right)$. For the ${ }^{13} \mathrm{C}$ NMR spectra the signals of the solvents were used as the internally standard $\left(\mathrm{CD}_{3} \mathrm{CN}: 1.32,118.26 \mathrm{ppm}, \mathrm{C}_{6} \mathrm{D}_{6}: 128.06 \mathrm{ppm}, \mathrm{CD}_{2} \mathrm{Cl}_{2}: 54.00\right.$ ppm, $\mathrm{CDCl}_{3}: 77.00 \mathrm{ppm}, \mathrm{CD}_{3} \mathrm{OD}: 49.00 \mathrm{ppm}$, more). The chemical shift $\delta$ is given in ppm, the coupling constants $J$ in $\mathrm{Hz}$. All spectra were recorded at $25^{\circ} \mathrm{C}$, otherwise it is mentioned. 334

\section{Electronspray ionization mass spectrometry}

ESI-MS measurements were performed on a Bruker maXis ESI-TOF MS spectrometer on a Bruker timsTOF mass spectrometer including electron spray ionisation (ESI) (positive / negative mode). The timsTOF is a Hybrid Quadrupole / Atmospheric Pressure Ionization orthogonal accelerated Time-Of-Flight mass spectrometer using Trapped Ion Mobility Spectrometry (TIMS). For calibration of the TIMS and TOF devices an Agilent ESILow Concentration Tuning Mix was used. The quotients of mass to charge $(\mathrm{m} / \mathrm{z})$ are given and the relative intensity related to the basis peak $(I=100)$ are given in brackets. $240[335$ 


\section{Ultraviolet-visible spectroscopy}

Standard UV-vis data was obtained from Agilent 8453 DAD UV-Visible Spectrophotometer, using standard quartz glass cuvettes suitable for the ultra-violet and visible light spectra. Thermal denaturation experiments and thermal difference spectra were measured with the JASCO V-650 and JASCO V-750 UV-Vis Spectrophotometer, both equipped with the PAC-743 Automatic 6/8-position Peltier Cell Changer.

\section{Fluorescence spectroscopy}

FS measurements were performed on the JASCO FP-8300 Spectrofluorometer with standard $10 \mathrm{~mm}$ quartz glass cuvettes suitable for fluorescence measurements in the UV-vis region. Additionally, the Unisoku Coolspek temperature-controlled cuvette holder was used for various temperature measurements above or below room temperature. 336

\section{Cyclic voltammertry}

CV was measured on a Metrohm Autolab PGSTat101 CV setup. Measurements were performed in custom made glass cell, with air tight inlets for the platinum working electrode $(\mathrm{WE})$, platinum wire counter electrode (CE) and the reference electrode (RE). Two additional Teflon valves were available for the degassing process.

\section{Circular dichroism}

CD measurements were performed on the qCD Chirascan CD-spectrometer from Applied Photophysics with temperature-controlled cuvette holder and an external nitrogen generator to ensure constant flushing of the whole machine, in order to avoid an accumulation of damaging ozone, generated during the active operation of the machine. 
For all steps oligonucleotide strands (ONSs) are involved, ultra pure water (type 1, $18.2 \mathrm{M} \Omega \mathrm{cm}$ ) was used, received from Puranity TU-3 UV VWR International GmbH, if not mentioned otherwise. Furthermore in connection with $\mathrm{ONS}$ containing solution, solvent removing steps in vacuo are always performed in a vacuum centrifuge (S-Concentrator BA-VC-300H, H. Saur) at $60{ }^{\circ} \mathrm{C}$.

\section{B.1 DNA Solid State Synthesis}

The synthesis of the oligonucleotide strands ONSs were performed on a K\&A DNA Synthesizer H- 8 by phosphoramidite solid phase synthesis on a $1 \mu \mathrm{mol}$ scale using standard methods for phosphoramidite synthesis on controlled pore glass (CPG). All used solution table VI.2.1.1 were prepared with anhydrous solvent and additionally stored over molecular sieves prior to synthesis for more than 12 hours.

Table VI.2.1.1: Solution used during solid-phase synthesis of the oligonucleotides.

\begin{tabular}{lll}
\hline Purpose & Label & Composition \\
\hline \hline Detritylation & DCA & $3 \%(v / v)$ dichloro acetic acid in anhydrous DCM \\
Activator & ACT & $0.3 \mathrm{M}$ BTT in anhydrous MeCN \\
Capping Reagent A & Cap A & $6.5 \%(w / v)$ DMAP in anhydrous THF \\
Capping Reagent B & Cap B & 2,6 -lutidine $/$ acetic anhydride / anhydrous THF \\
& & $1: 1: 8(v / v / v)$ \\
Oxidizer & OXI & $0.02 \mathrm{M}$ iodine in THF / pyridine $/ \mathrm{H}_{2} \mathrm{O}$ \\
& & $7: 2: 1(v / v / v)$ \\
\hline
\end{tabular}

The compound BTT (20) used as activation agent was synthesized as described in scheme VI.1.2.30. As unmodified nucleotides commercially available DMT and CEP protected DNA phosphoramidites (DMT-dT-CEP, DMT-dG $(i \mathrm{Bu})$-CEP, DMT-dA(Bz)CEP, DMT-dC(Bz)-CEP). As the solid support commercially availabe CPG was used with the respective starting nucleoside already immobilized on the CPG and DMT protected. The cartridges for the synthesis were manually packed. The synthesis itself was performed with a slightly modified version of the built-in methods. Following steps were performed during coupling process, after each step the cartridge was washed with anhydrous MeCN, followed by drying step with argon.

1. The CPG was flushed three times with DCA solution. 
2. The respective phosphoramidite and the activator were mixed with the ratio $1: 1$ $(v / v)$. Coupling time for normal nucleoside was $0.5 \mathrm{~min}$. For the ligands the coupling time was increased to $3.5 \mathrm{~min}$.

3. For the capping process Cap A and Cap B were mixed 1:1 $(v / v)$

4. The CPG was treated with the oxidizer solution

5. An extra washing step with anhydrous MeCN was introduced to remove as much water as possible, which interfere the coupling process.

The cycle was repeated for every coupled nucleobase. The progress and success of coupling steps was monitored by internal trityl absorption measurements. All syntheses were performed in DMT-ON mode, means the last deprotection step is omitted. After synthesis the CPG was removed from the cartridge and suspended in concentrated aqueous ammonia solution $(33 \%)$. The mixture was heated over night at $55^{\circ} \mathrm{C}$ to cleaver the ONS from the solid support and in addition deprotected the nucleobases. The supernatant was transferred and the left over CPG was washed twice with $\mathrm{H}_{2} \mathrm{O}(200 \mathrm{\mu l})$. A sample for ESI-MS was taken and subsequently the collected solutions were reduced to a volume of $300 \mathrm{pl}$ in vacuo, followed by the purification process.

\section{B.2 Oligonucleotide Purification}

\section{B.2.a Reversed Phase - HPLC}

ONSs samples were purified DMT-ON on a Agilent 1260 Infinity I or II system. Analytic HPLC measurements were performed on a Macherny Nagel 250 Nucleodor 100-5 C18 ec analytic column $(\varnothing 4.6 \mathrm{~mm})$. The separation of ONSs were realized on a Macherny Nagel 250 Nucleodor 100-5 C18 ec semi-preparative column ( $\varnothing 10 \mathrm{~mm}$ ) The used methods are presented in table VI.2.2.2. Initially, 0.1 molar triethylammonium acetate (TEAA) solution was used, but the high buffer concentration led to column problems and was therefore reduced to $50 \mathrm{mM}$, what gave very similar separation resolutions. All measurements and separation were at $60{ }^{\circ} \mathrm{C}$ column temperature, to avoid unwanted assemblies of the ONSs. The used methods for the HPLC purification and analysis can be found in table VI.2.2.2, 
Table VI.2.2.2: HPLC methods used in for analytic investigation and ONS separation.

\begin{tabular}{ccccc}
\hline Method & Solvent & Gradient & Flowrate & Time \\
\hline \hline \multirow{2}{*}{ Analytic } & A: $0.05 \mathrm{M}$ & B 0-80\% & $1 \mathrm{~mL} / \mathrm{min}$ & $35 \mathrm{~min}$ \\
& TEAA, pH 7.0 & & & \\
& B: $70: 30$ & & & \\
MeCN / A & & $3 \mathrm{~mL} / \mathrm{min}$ & $35 \mathrm{~min}$ \\
Analytic & A: $0.05 \mathrm{M}$ & B 0-80\% & & \\
& TEAA, pH 7.0 & & & \\
& B: $70: 30$ & & & \\
& $\mathrm{MeCN} / \mathbf{A}$ & & & \\
\hline
\end{tabular}

The HPLC traces were always recorded at $260 \mathrm{~nm}$, additionally for the ONSs containing ligand 39 the specific absorption at $357 \mathrm{~nm}$ was also observed. The traces shown in chapter $\mathrm{A}$ were recorded after detritylation and desalting of the samples.

\section{B.2.b Solid Phase Extraction}

After HPLC purification the isolated ONS was desalted and the DMT-protecting group was removed via Solid Phase Extraction on Waters Sep-Pak C18 cartridges. After preconditioning the Sep-Pak material by washing with 2 × $2 \mathrm{~mL} \mathrm{MeCN,} 2 \mathrm{~mL}$ 70:30 MeCN / 0.1 M TEAA, pH 7.0 and $2 \times 2 \mathrm{~mL} 0.1 \mathrm{M}$ TEAA, the ONS solution was 3 times slowly passed through the cartridge. The absorbed ONS were subsequently washed with $2 \times 2 \mathrm{~mL} 0.1 \mathrm{M}$ TEAA and treated with $3 \%(v / v)$ TFA for at least $1 \mathrm{~min}$. A successful detritylation was indicated by temporary red colored solution. Afterwards, the bound ONS were washed with $2 \times 2 \mathrm{~mL} \mathrm{H} \mathrm{H}_{2} \mathrm{O}$. The deprotected $\mathrm{ONSs}$ were eluated from the $\mathrm{C} 18$ material with $\mathrm{MeCN} / \mathrm{H}_{2} \mathrm{O}$ 1:1 $(v / v)$ and the solvent was removed in vacuo to a volume of roughly $500 \mathrm{\mu l}$. Less solvent has led to precipitation which was hardly resoluble. The ONS solution was filled up with $\mathrm{H}_{2} \mathrm{O}$ to $1 \mathrm{ml}$. This solution was used for the characterization and all prepared samples used in all further described experiments. 


\section{B.3 Oligonuecleotide Characterization}

\section{B.3.a Oligonucleotide Mass Analysis}

The molecular mass of the ONSs were determined with help of ESI-MS. The samples were measured on Bruker maXis with TOF analysator or Bruker timsTOF, likewise equipped with a TOF analysator. The samples were prepared from $1 \mu \mathrm{lONS}$, directly taken from stock solution and diluted 9:1 with $\mathrm{H}_{2} \mathrm{O} / \mathrm{MeOH}$ or $\mathrm{H}_{2} \mathrm{O} / \mathrm{MeCN}$.

The mass of the synthesized ONS was determined three times before purification and after purification and again after the desalting/deprotection step. Before purification the crude solution checked if the synthesis was successful and it contains the desired strand. After purification every collected fraction was analyzed for the containing ONS and once again after desalting and deprotection, to confirm an successful deprotection.

\section{B.3.b Oligonucleotide Concentration Determination}

The concentration of each ONSs stock solution was determined by UV-vis absorbance at $260 \mathrm{~nm}$ at $25{ }^{\circ} \mathrm{C}$ using the revised extinction coefficients for each nucleoside. 159 Since the integrated ligands are aromatic systems, they also show an absorption at $260 \mathrm{~nm}$, that has to be taken into account. Therefore the extinction coefficient of each used ligand was determined by simple UV-vis dilution experiments. For that reason a solution with a known concentration of ligand was gradually diluted and the absorbance of each dilution step was record at $260 \mathrm{~nm}$. From the plot absorbance vs. concentration the determined absorbances were fitted by an linear regression, where the slope represents the extinction coefficient $\varepsilon_{260 \mathrm{~nm}}{ }^{25^{\circ} \mathrm{C}}$ of the respective ligand for $260 \mathrm{~nm}$ at $25^{\circ} \mathrm{C}$. 
(a)

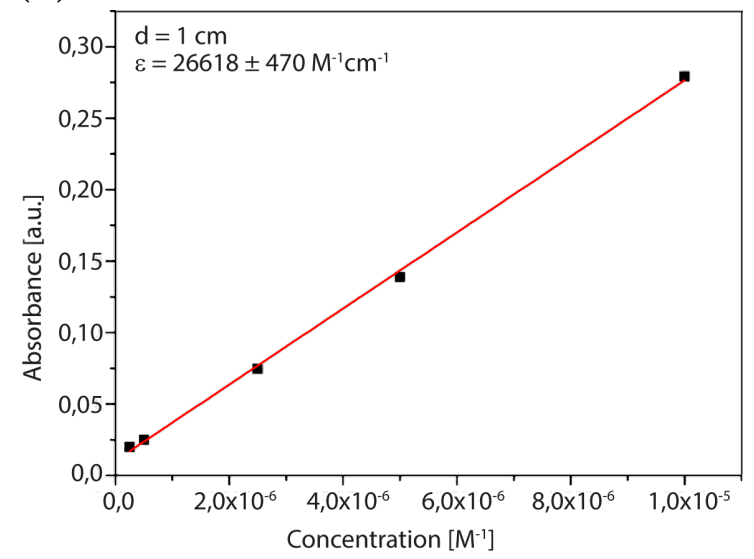

(c) (b)

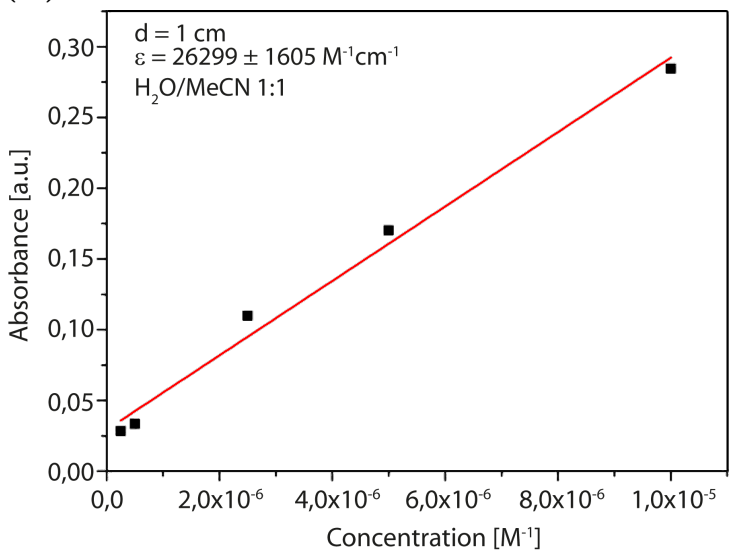

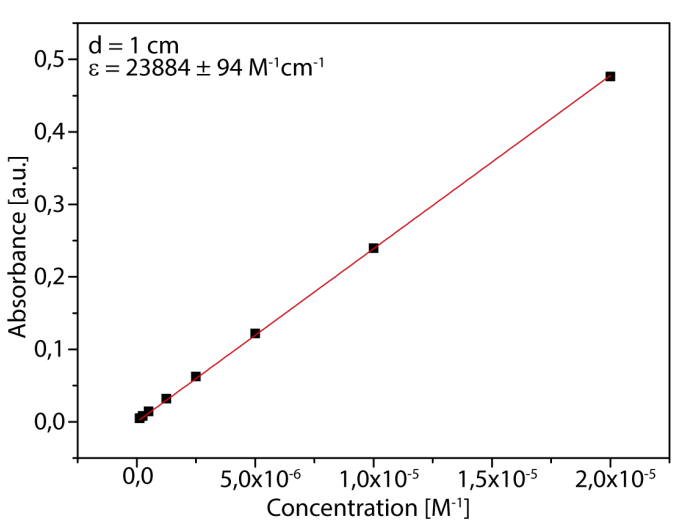

Figure VI.2.3.1: Determination of the ligands extinction coefficient by UV-vis dilution experiments

The determination of concentration was initially performed by UV-vis measurement on the Agilent DAD HP-8453 UV-Vis spectrometer. For that reason $1 \mathrm{\mu l}$ of each ONSs sample was diluted with water to a total volume of $400 \mu \mathrm{l}$ and its absorbance was measured. With the help of the individual extinction coefficient for each ONS with simultaneous consideration of the dilution, the concentration of each stock solution could be obtained. Later the concentration was elucidated with a Thermo Scientific Nanodrop One, where the absorbance could be directly measured from a single drop (1 $\mathrm{\mu l}$ ) of respective stock solution. For a better comparison, the previously determined ONS stock solution concentrations were checked again on the Nanodrop machine, but were matching with the old ones. 


\section{B.4 Spectroscopic Methods}

\section{B.4.a ONS sample preparation}

DNA samples were prepared with $10 \mathrm{mM}$ sodium cacodylate $(\mathrm{pH}=7.3)$ or sodium phosphate $(\mathrm{pH}=7.2)$ buffer, $100 \mathrm{mM} \mathrm{NaCl}$ or $\mathrm{NaClO}_{4}$ and $2 \mathrm{mM} \mathrm{MgCl}_{2}$ or $\mathrm{Mg}\left(\mathrm{ClO}_{4}\right)_{2}$. Each ONS belonging to a Holliday junction systems was added to obtain concentration of $2 \mu \mathrm{M}$ per strand, so $8 \mu \mathrm{M}$ in total, in order to end up with $2 \mu \mathrm{M}$ of the desired Holliday junction structure and filled up with water to end up with a total volume of $800 \mathrm{\mu l}$. The samples were heated to $90{ }^{\circ} \mathrm{C}$ for $5 \mathrm{~min}$ and subsequently cooled down to $4{ }^{\circ} \mathrm{C}$ in $0.5{ }^{\circ} \mathrm{C} \mathrm{min}-1$ steps. The samples were stored in the fridge at ca. $3{ }^{\circ} \mathrm{C}$, when not used immediately. The respective metal salts were also prepared in ultra pure water with a concentration of $800 \mu \mathrm{M}$, so that one equivalent of metal salt could be added in a $22 \mu \mathrm{l}$ portion.

\section{B.4.b UV-vis Spectroscopy}

Except of the determination of concentration all UV-vis measurements on ONSs were performed on the JASCO V-650 or Jasco V-750 UV-vis spectrometer, both equipped with a PAC-743 temperature-controlled 6-cell cuvette changer slide. The actual temperature was contentiously measured during experiments in water-filled cuvette, which in turn reduced the amount of max. simultaneously measured samples to 5 . The UV-vis bandwidth was set to $2.0 \mathrm{~nm}$, the data interval to $1 \mathrm{~nm}$. For the measurement the $800 \mu \mathrm{l}$ of ONS samples were filled in Hellma Analytics 114-QS, quartz glass cuvettes with $10 \mathrm{~mm}$ path length, to ensure the that the beam complete transpasses the solution. To avoid condensation of moisture on the cuvettes outside during the cooling process, the cell holder were continuously flushed with dried air. Additionally, to avoid concentration change caused by unwanted evaporation during the heating process, each sample were topped with a thin layer of silicon oil before the cuvette was tightly closed with a PTFE lid. The volume of afterwards additions was kept as small as possible in comparison to the sample volume, in order to minimize the change in absorption due to dilution. Before and after melting curve measurements normal UV-vis spectra were recorded. Before the melting process a spectra at $4{ }^{\circ} \mathrm{C}$ was recorded and compared with the spectrum recorded at $4{ }^{\circ} \mathrm{C}$ after the melting curve measurements. Furthermore, after the melting curve experiment, spectra were recorded for $4{ }^{\circ} \mathrm{C}$ and $85{ }^{\circ} \mathrm{C}$, from which the TDS was generated. All measured spectra were background corrected (cuvette with buffer and electrolyte).

In case of thermal denaturation experiments, absorption of the samples were record at a wavelength of $260 \mathrm{~nm}$ with an increase or decrease in temperature of $0.5{ }^{\circ} \mathrm{C} \mathrm{min}{ }^{-1}$. For 
initial thermal denaturation measurements two full cycles were measured. After addition of a metal salt, three complete cycles were measured. The denaturation temperature $\left(\mathrm{T}_{\mathrm{M}}\right)$ was determined, if possible, in three ways: 160

1. Forming the midrule between the baselines of the disassembled and assembled strands. The crossing of the midrule with the recorded spectrum shows the melting temperature.

2. Calculating the first derivative of the recorded melting curve. The obtained plot was fitted with a Gaussian function. This method can become quite inaccurate, as soon as the denaturation process happened over a larger temperature range

3. Calculating the fraction folded values and determine the $\mathrm{T}_{\mathrm{M}}$ at the $\mathrm{y}$-value $=0.5$ (or $\alpha=0.5$, where $\alpha$ represent the fraction folded).

The most used method throughout the literature is to calculate the first derivative of the melting curve (method 2) and then to determine the its maximum either manually by estimating it in the graphic or by fitting the curve with a suitable regression function and extract the value from the fitting. It has the advantage that it is very easy to perform and no other data processing is necessary. The disadvantage of this method becomes clear, as soon as the signal-to-noise ratio is lowered, since this becomes even more dominant in the derivative curve, making it more difficult to locate the position of the maximum and results in a less accurate determination of $\mathrm{T}_{\mathrm{M}}$. Method 1 still comes from the time, when computer-assisted data analysis was not daily routine. However, this method is still valid an gives quite accurate values for $\mathrm{T}_{\mathrm{M}}$. In order to determine $\mathrm{T}_{\mathrm{M}}$ the bisectrix between the lower (assembled DNA) and upper (disassembled DNA) baseline is graphically assigned and $\overline{T_{M}}$ is obtained from the intersection of bisectrix and melting curve. In the third method, $\mathrm{T}_{\mathrm{M}}$ can be received from the fraction folded value $\alpha=0.5$, where it is assumed that $50 \%$ of the oligonucleotide is still assembled, or $50 \%$ is disassembled. For that reason, the recorded melting curve based on absorption is converted to a fraction folded curve, ranging from completely unfolded $(\alpha=0)$ to completely folded $(\alpha=1)$ oligonucleotidic structure, so that $=0.5$ can be easily assigned. This method can give more accurate results than the derivative method, however, the assignment of the corresponding baselines is rather a subjective approach. The needed fraction folded curve is plotted from the fraction folded values, which are calculated for the measured absorption values at the corresponding temperatures $\left(A_{260}\right)$ and the linear regressions of the upper $\left(A_{\text {high }}\right)$ and lower $\left(A_{\text {low }}\right)$ baselines section B.4.b. 160

$$
\alpha=\frac{A_{260}-A_{\text {high }}}{A_{\text {low }}-A_{\text {high }}}
$$


Besides the denaturation temperature, further thermodynamic characteristics could be obtained from melting curves. A relative thermodynamic stability can be deduced from the slope of the recorded melting curves. A broad transition indicates a rather weak temperature dependency on the affinity constants, giving rise to the assumption the structure has a lower stability, or a less favorable $\Delta G^{\circ}$, compared to a system with the same $\mathrm{T}_{\mathrm{M}}$ but a steeper slope. By implication, a sharp transition (displayed by a steep slope, meaning a transition in a small temperature range) is indicating a strong dependency of temperature on the reaction free enthalpy, accompanied by a higher affinity constant. As an example, a system with a higher melting temperature but a broader transition can thus have the a lower relative thermodynamic stability, compared to another system with lower melting temperature but a sharper transition. Therefore, a simple correlation between $\mathrm{T}_{\mathrm{M}}$ and $\Delta G^{\circ}$ is improper and other factors as i.e. the shape of the melting curve have to be considered as well, in order to compare the relative thermodynamic stability of different systems. 160.

\section{B.4.c CD Spectroscopy}

CD measurement of ONSs were performed on a Applied Photophysics qCD Chirascan machine with a temperature-controlled cuvette holder. For all measurements samples were taken from previous melting curve experiments, to ensure the existence of the Holliday junction species within the sample. All spectra were measured from $500 \mathrm{~nm}$ to $220 \mathrm{~nm}$, with a bandwidth of $1 \mathrm{~nm}$ and a data interval of $1 \mathrm{~nm}$ with standard sensitivity. The cell chamber was continuously flushed with a nitrogen stream to avoid condensation on the outer cuvette during cooling process. Due to the low signal intensity the measurement was repeated three times and data was accumulated. The ONSs, were measured at $4{ }^{\circ} \mathrm{C}$ and $80{ }^{\circ} \mathrm{C}$, each. All spectra were background corrected (cuvette + buffer + electrolyte) and smoothed (10pt, Savitzky-Golay).

\section{B.4.d Fluorescence Spectroscopy}

Fluorescence spectra were measured on a Jasco FP-8300 fluorescence spectrometer. The samples were directly taken from the $\mathrm{CD}$ measurement, heated to $90{ }^{\circ} \mathrm{C}$ and cooled down to $4{ }^{\circ} \mathrm{C}$ in $0.5{ }^{\circ} \mathrm{C} \mathrm{min}{ }^{-1}$. For measurement including ONSs an Unisoku Coolspek temperature-controlled cuvette holder was used, to measure the samples at $4{ }^{\circ} \mathrm{C}$ and $80{ }^{\circ} \mathrm{C}$. To determine the the the best excitation wavelength for the respective samples, 
3D fluorescence measurement, as shown in section V.1.3.4 were made, with excitation wavelength range from $220 \mathrm{~nm}$ to $400 \mathrm{~nm}$ in $5 \mathrm{~nm}$ steps and an emission wavelength range from $400 \mathrm{~nm}$ to $700 \mathrm{~nm}$ with a scan rate of $500 \mathrm{~nm} \mathrm{~min}^{-1}$. For the actual fluorescence measurements, the determined best excitation wavelength for the respective samples was used with a bandwidth of $2.5 \mathrm{~nm}$ and a bandwidth of $5 \mathrm{~nm}$ for the recorded emission wavelength. The spectra were recorded at a medium sensitivity and a scan rate of $200 \mathrm{~nm} \mathrm{~min}^{-1}$.

\section{B.5 PAGE Gel Electrophoresis}

PAGE experiments were carried out under non-denaturating conditions on a CBS Scientific Dual-Vertical Mini-Gel system MGV-402. All PAGE gels were run in constant voltage mode at $200 \mathrm{~V}$ for $3.5 \mathrm{~h}$ with running TBM buffer containing $89 \mathrm{mM}$ Tris-borate, $\mathrm{pH} 8.3$, with $2 \mathrm{mM} \mathrm{Mg}\left(\mathrm{ClO}_{4}\right)_{2}$. Gels were prepared in $15 \times 20(\mathrm{w} \times \mathrm{h})$ size and preconditioned with . Separating gel consists of $8 \%(w / v), 0.28 \%(w / v)$ bisacrylamide topped with a $4 \%(w / v), 0.14 \%(w / v)$ bisacrylamide stacking gel. For gel polymerization in both cases ammonium peroxodisulfate (APS) was used as radical starter, with N,N,N',N'tetramethylethylendiamine (TEMED) as stabilizing agent. Furthermore running buffer was already added to gel solutions, conditioning of the gels with running buffer after polymerization led to bad separation and massively blurred signals. Samples were prepared as with $10 \mathrm{mM}$ TB buffer $2 \mathrm{mM} \mathrm{Mg}\left(\mathrm{ClO}_{4}\right)_{2}$ and $12.5 \mathrm{ng}$ DNA filled up to $10 \mu \mathrm{l}$ with water. All samples were heated to $90^{\circ} \mathrm{C}$ for 5 min and then cooled down to $4{ }^{\circ} \mathrm{C}$ in $0.5{ }^{\circ} \mathrm{C} / \mathrm{min}$ steps. For loading the samples were mixed with $10 \mu \mathrm{l} 40 \%(w / v)$ sucrose solution. For a better visualization of the running front some samples were prepared with a dye containing loading buffer, containing additionally $0.05 \%(w / v)$ bromphenol blue and $0.05 \%(w / v)$ xylene cyanol. For size comparison the O'RangeRuler 10 bp DNA Ladder of Thermo Scientific was used, containing DNA double strands differing in length by 10 base pairs from 10 to 150 base pairs. The gels were run at constant $40{ }^{\circ} \mathrm{C}$, when not mentioned otherwise. After completion of the separation step, the gel was carefully removed and incubated in $250 \mathrm{~mL}$ TBM buffer containing $25 \mathrm{\mu l}$ Invitrogen SYBR Gold Nucleic Acid Gel Stain for 20 min. After, the pictures were made with the Intas Gel IX 20 gel documentation system, illuminated with an extinction wavelength of $312 \mathrm{~nm}$. All pictures were converted to black and white and the brightness and contrast was adjusted. 


\section{B.6 NMR Line-Shape Analysis}

The line shape analysis of the measured ${ }^{1} \mathrm{H}-\mathrm{NMR}$ spectra was made with help of the line fitting function in the program MestreLab Research S.L MestreNova NMR, with what the FWHM of the respective proton signals could be easily determined. All further values such as $\Delta \nu_{0}$ and $\Delta \nu_{e}$ could also be extracted from the respective NMR spectra. Subsequently, the values were used to calculate the experimental rotation constants with the suitable functions given in section V.1.2.4.

The simulated NMR spectra were made with the program Spinworks 4.2.4. Unfortunately the simulation of DNMR is limited to five nuclei, therefore only two exchanging proton pairs could be simulated at the same time. Since the signals (a/a') and (b/b') showed the most distinctive effect, these both signals were chosen for the simulation. First of all the chemical shift and coupling constants for all four signals ( $a$, a', b and b') were determined from low temperature measured NMR spectra. These values were used with the assumption that virtually no exchange between the proton pairs a/a' and b/b' occurs. The four signals were then simulated with the given values. Since the shape of signals caused by coupling could not be obtained with only the four protons, one dummy signal for both protons c/c' was added in the simulation, since it was only possible to add one more nucleus to the DNMR simulation. However, due to the fact that the signals c/c' does not exhibit such a big shifting caused by the exchange in the VT NMR, the use of this dummy signals gives a nice peak pattern for the signals of interest (a/a' and b/b') without having a big effect on the simulation outcome. Next, the simulated NMR spectra were superimposed with the measured one and the values of the simulated signals were adjusted with an iterative fitting function (NUMMRIT).

After, the parameters for the DNMR3 simulation was set up. First of all $\mathrm{T}_{2}$, this parameter defines the linewidth in the absence of exchange. Since it is known from the experiments that the linewidth at very low temperatures equals almost $1 \mathrm{~Hz}$, the default value for $\mathrm{T}_{2}=$ 1.0 was used. Since the chemical exchange in the investigated system is mutual exchange, mutual exchange was activated and the number of species was set to 2, including both enantiomeric structures. Since in the mutual exchange only the rate constant $\mathrm{RC}(1,2)$ is available, it was set to 0 , since we assumed the no rotation, or chemical exchange, for initial simulated spectrum. The population factors can be omitted in the case of mutual exchange. As last the permuation vectors have to be set. For that reason the order of the simulated nuclei has to be known. In the here described case, signal a $=$ nucleus 1 , signal a' $=$ nucleus $2, \mathrm{~b}=$ nucleus $3, \mathrm{~b}^{\prime}=$ nucleus 4 and the dummy signal $\mathrm{c}=$ nucleus 5 . Considering that the exchange occurs between a and a' or b and b' and $c$ is mapping itself the values inserting for the Permutation Vector 2 are 2, 1, 4, 3, 5. These values do result in following permutation matrix: 


$$
\left(\begin{array}{lllll}
0 & 1 & 0 & 0 & 0 \\
1 & 0 & 0 & 0 & 0 \\
0 & 0 & 0 & 1 & 0 \\
0 & 0 & 1 & 0 & 0 \\
0 & 0 & 0 & 0 & 1
\end{array}\right)
$$

With those settings, the rotation constant was varied till the simulated signals overlapped with the measured signals. The procedure was repeated for every recorded spectrum at the different temperatures and the determined rotations constants were afterwards plotted against the temperature likewise to those from experiments. 


\section{Appendix}

\section{Overview}

\section{C.1 List of Figures}

Figure II.1.1.1: Famous molecules of the noble price winners: Pederson, Cram, Lehn 9

Figure II.1.1.2: $\quad$ Stoddart, (a) molecular muscle and (b) molecular elevator . . . . 10

Figure II.1.1.3: $\quad$ Supramolecular nanocar by Feringa . . . . . . . . . . . . . . . . 11

Figure II.1.2.4: $\quad$ Non-covalent interactions in supramolecular chemistry. . . . . . . 12

Figure II.1.2.5: $\quad$ Strict vs. directed self-assembly . . . . . . . . . . . . . . . . 15

Figure II.1.2.6: Host-guest complex . . . . . . . . . . . . . . . . . . 15

Figure II.1.3.7: $\quad$ Various metal coordination environments . . . . . . . . . . . . . 17

Figure II.1.3.8: $\quad$ Possible three-dimensional structures for supramolecular metalcoodination assemblies . . . . . . . . . . . . 18

Figure II.1.3.9: $\quad$ Comparison of $\mathbf{L}^{\mathbf{E t}}$ and $\mathbf{C}^{\mathbf{E t}}$ in various solvents . . . . . . . . . 20

Figure II.1.3.10: Schematic representation of the assembly process for palladiummediated heteroleptic cages . . . . . . . . . . . . 21

Figure II.1.3.11: Concepts of self-sorting and designed sorting. . . . . . . . . . . . 24

Figure II.2.1.1: $\quad$ General structure of nucleotides, DNA nucleobases and WatsonCrick base pairs. . . . . . . . . . . . . . 27

Figure II.2.1.2: $\quad$ Comparison of DNA double strand structures. . . . . . . . . . . . 28

Figure II.2.1.3: $\quad$ Schematic representation of various canonical scondary non B-DNA structures . . . . . . . . . . . . . . . . 29

Figure II.2.1.4: $\quad$ Crystal structures of non-canonical secondary DNA structures. . . 30

Figure II.2.1.5: $\quad$ Modifications of the individual nucleotide constituents . . . . . . 32

Figure II.2.1.6: $\quad$ Ligandoside vs. incorporated ligands . . . . . . . . . . . . . . . 35

Figure II.2.2.7: $\quad$ Holliday Junction Isomers . . . . . . . . . . . . . . . . . . 36

Figure II.2.2.8: $\quad$ Crystal structures of open-X and stacked-X Holliday junctions . . 38 
Figure III.1.0.1: Triangular DNA architercuture by Han et al. . . . . . . . . . . 41

Figure III.1.0.2: $\quad$ Terpyridine-DNA structure by Krämer et al. . . . . . . . . . . . 42

Figure III.1.0.3: $\quad$ Mixed coordination DNA structure by Sleiman et al. . . . . . . . 43

Figure III.1.0.4: Chiral single stranded Metal-DNA fourway junction by Sleiman $e t$ al. .......................... 43

Figure III.1.0.5: $\quad$ Statistically mixtured coordination cage problematic. . . . . . . . 44

Figure III.1.0.6: Approach of a controlled assembly of a metal-mediated coordination cage, supported by a preassembled Holliday junction scaffold 45

Figure III.2.2.1: $\quad$ MMFF model of 1st generation DNA-cage hybrid structure . . . 49

Figure III.2.2.2: $\quad$ Fraction folded plots for the unmodified $\mid$ HJ|MKDH01um in presence and absence of magnesium cations. . . . . . . . . . 55

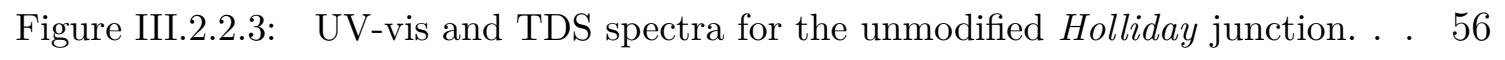

Figure III.2.2.4: $\mid$ CD $\mid$ spectra for the unmodified $\mid$ HJ|MKDH01um. . . . . . . . . . 58

\begin{tabular}{|l|l|l|l|l|}
\hline Figure III.2.2.5: & PAGE & gel for the unmodified & Holliday junction & MKDH01um . . . 59 \\
\hline
\end{tabular}

Figure III.2.2.6: Fraction folded plots for the modified $/$ HJ $/$ MKDH01 in presence and absence of magnesium cations. . . . . . . . . . . 60 60

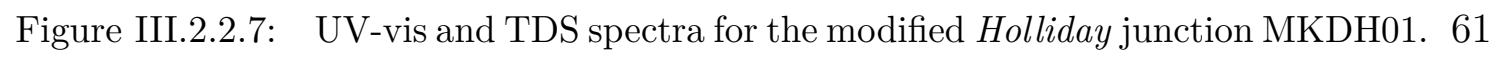

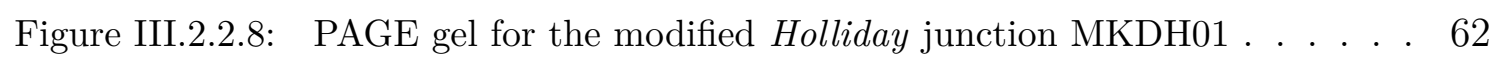

Figure III.2.2.9: $\mid$ CD $\mid$ spectra for the modified $\mid$ HJ $\mid$ MKDH01. . . . . . . . . . . . . 63

Figure III.2.2.10: Fluorescence emission spectra for the modified HJ MKDH01. . . 64

Figure III.2.2.11: $\mid$ UV-vis $\mid$ melting experiments for MKDH05 in presence of transition metals. . . . . . . . . . . . . . . 65 65

Figure III.2.3.12: MMFF model of 2nd generation DNA-cage hybrid structure . . . 67

Figure III.2.3.13: Fraction folded plots for the modified $\mid$ HJ|MKDH05. . . . . . . . 71

Figure III.2.3.14: $\mid$ UV-vis|and|TDS|spectra for the modified|Holliday junction|MKDH05. 72

Figure III.2.3.15: $\mid$ CD $\mid$ spectra for the unmodified $\mid$ HJ|MKDH05. . . . . . . . . . . . 73

Figure III.2.3.16: Fluorescence emission spectra for the modified|HJ|MKDH05. . 74

Figure III.2.3.17: $\mid$ PAGE| gel for the modified|Holliday junction|MKDH05 . . . . . . 75

Figure III.2.3.18: Fraction folded plots for the modified HJ|MKDH06. . . . . . . . 76

Figure III.2.3.19: $\mid$ UV-vis|and |TDS|spectra for the modified|Holliday junction|MKDH06. 76 Figure III.2.3.20: $\mid$ CD $\mid$ spectra for the unmodified $\mid$ HJ|MKDH06. . . . . . . . . . . . 77

Figure III.2.3.21: Fluorescence emission spectra for the modified|HJ|MKDH06. . . 78

Figure III.2.3.22: $\mid$ PAGE|gel for the modified|Holliday junction|MKDH06... .78

\begin{tabular}{|l|l|l|l|}
\hline Figure III.2.3.23: & UV-vis & melting experiments for MKDH05 in presence of transition \\
\hline
\end{tabular} metals. . . . . . . . . . . . . . . 80

\begin{tabular}{|l|l|l|l|}
\hline Figure III.2.3.24: & UV-vis & melting experiments for MKDH06 in presence of transition \\
\hline
\end{tabular} metals. . . . . . . . . . . . . . . . . . 81

Figure IV.1.0.1: Raymonds' gallium-mediated coordination cage . . . . . . . . . . 87

Figure IV.1.0.2: Highly charged cages by Ward and Fujita . . . . . . . . . . . . . 88 
Figure IV.1.0.3: Cages by Nitschke, Fujita and Yoshizawa. . . . . . . . . . . . . . 89

Figure IV.2.1.1: $\quad$ Formation of cage from ligand 17 . . . . . . . . . . . . . . . . . 92

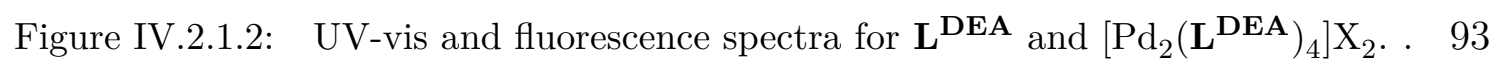

Figure IV.2.1.3: $\quad$ PM6 model of cage $\left[\mathrm{Pd}_{2}\left(\mathbf{L}^{\text {DEA }}\right)_{4}\right] \ldots \ldots \ldots$. . . . . . . . . . . 94

Figure IV.2.2.4: Comparison of compound $\mathbf{3 0}$ and $\mathbf{3 2}$ by NMR. . . . . . . . . . . 96

Figure IV.2.2.5: Conversion of $\mathbf{3 1}$ to $\mathbf{3 2}$ tracked by NMR. . . . . . . . . . . . . . 97

Figure IV.2.3.6: $\quad$ Formation of cage from ligand 24 . . . . . . . . . . . . . . . . . 101

Figure IV.2.3.7: Comparison PM6 model of sulfobetaine cage with acridone interpenetrad double cage crystal structure. . . . . . . . . . . 103

Figure V.1.1.1: $\quad$ Comparison of NMR spectra for ligands $\mathbf{L}^{\mathbf{C N}}$ and $\mathbf{L}^{\mathrm{Et}}$...... . 110

Figure V.1.2.2: $\quad$ Protonation of $\mathbf{3 5}$ - NMR . . . . . . . . . . . . . . . . . . . . 112

Figure V.1.2.3: $\quad$ Protonation of $\mathbf{3 5}$ - NMR . . . . . . . . . . . . . . . . . . . . . 113

Figure V.1.2.4: Acridinylidene Derivatives - NMR comparison . . . . . . . . . . 114

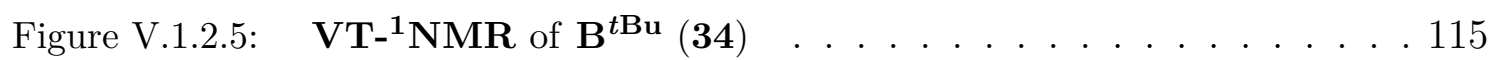

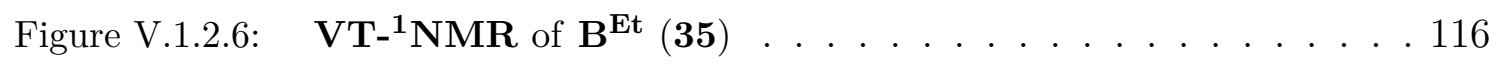

Figure V.1.2.7: $\quad$ VT- $^{1}{ }^{1} \mathbf{H}$ NMR of $\mathbf{B}^{\mathrm{Ph}}(\mathbf{3 7})$. . . . . . . . . . . . . . . . . 117

Figure V.1.2.8: $\quad$ Influence of solvent polarity on $\mathbf{3 4}$ - NMR and UV . . . . . . . . 118

Figure V.1.2.9: $\quad$ Influence of solvent polarity on 35 - NMR and UV . . . . . . . . 119

Figure V.1.2.10: $\quad$ Influence of solvent polarity on $\mathbf{3 7}$ - NMR and UV . . . . . . . . 120

Figure V.1.2.11: Determination of rotations constatns from NMR Data of $\mathbf{B}^{t \mathbf{B u}}$. . 122

Figure V.1.2.12: $\quad$ Determination of rotation constants from NMR Data of $\mathbf{B}^{\mathbf{E t}}$. . . 124

Figure V.1.2.13: $\quad$ Determination of rotations constatns from NMR Data of $\mathbf{B}^{\mathbf{P h}}$. . 125

Figure V.1.2.14: $\quad$ Cyclic voltamogramms of 34, 35 and 37. . . . . . . . . . . . . . 127

Figure V.1.2.15: Comparison of cyclic voltamogramms for 34, 35 and 37. . . . . . . 128

Figure V.1.2.16: Full cyclic voltammogramms for determination of oxidation- and reduction-offsets. . . . . . . . . . . . . . . . . . . 129

Figure V.1.2.17: Comparison crystal structures of the acridinylidene derivatives . . 130

Figure V.1.2.18: Angles of bended backbones extracted from the obtained crystal structures . . . . . . . . . . . . . . . . . . . . . . . . 131

Figure V.1.2.19: Asymetric unit of $\mathbf{B}^{\mathrm{Et}}$ measured crystal . . . . . . . . . . . . . 132

Figure V.1.2.20: $\quad$ Existing enantiomers extracted from $\mathbf{B}^{\mathrm{Et}}$ asymetric unit . . . . . 132

Figure V.1.2.21: TD-DFT calculated UV-vis simulations . . . . . . . . . . . . . . 134

Figure V.1.2.22: $\quad$ Calculated frontier Orbitals for the derivatives 34, 35 and 37 . . 135

Figure V.1.3.23: Protonation of the acridinylidene derivatives . . . . . . . . . . 137

Figure V.1.3.24: Protonation of 35 - NMR comparison . . . . . . . . . . . . . . . 138

Figure V.1.3.25: Determination of the protonated position in the acridinylidene derivative 35 by NMR . . . . . . . . . . . . . . . . 139

Figure V.1.3.26: Protonation of $\mathbf{3 5}$ - NMR . . . . . . . . . . . . . . . . . . . . 140

Figure V.1.3.27: NMR titration experiment of $\mathbf{3 4}$ with|TFA $\ldots$. . . . . . . . . 141 
Figure V.1.3.28: NMR titration experiment of 35 with|TFA $\ldots$. . . . . . . . . . 141

Figure V.1.3.29: $\quad$ Proof for reversibility of protonation in compound 35. . . . . . . 142

Figure V.1.3.30: $\quad$ NMR titration experiment of $\mathbf{3 7}$ with|TFA $\ldots$. . . . . . . . . . . 142

Figure V.1.3.31: $\quad$ UV-vis titration experiment with $\mid$ TFA $\mid$ for $\mathbf{B}^{t \mathbf{B u}} \ldots$. . . . . . . . 143

Figure V.1.3.32: UV-vis titration experiment with $\mid \mathrm{TFA}$ for $\mathbf{B}^{\mathbf{E t}}$. . . . . . . . . . 144

\begin{tabular}{|lll|l|l|}
\hline Figure V.1.3.33: & UV-vis titration experiment with & $\mathrm{TFA}$ & for $\mathbf{B}^{\mathbf{P h}}$ & . . . . . . . . . . . 144 \\
\hline
\end{tabular}

\begin{tabular}{|ll|l|l|l|}
\hline Figure V.1.3.34: & Fluorescence measurements of & TFA & titration experiments with $\mathbf{B}^{\text {tBu }}$ & 146 \\
\hline
\end{tabular}

\begin{tabular}{|ll|l|l|l|}
\hline Figure V.1.3.35: & Fluorescence measurements of & TFA & titration experiments with $\mathbf{B}^{\mathbf{E t}}$ & 1477 \\
\hline
\end{tabular}

\begin{tabular}{|ll|l|l|}
\hline Figure V.1.3.36: & Fluorescence measurements of & TFA & titration experiments with $\mathbf{B}^{\mathbf{P h}}$ \\
\hline
\end{tabular}

Figure V.1.3.37: Comparision $\mid$ TFA titration UV and fluorescence experiments. . . 149

Figure V.2.1.1: $\quad$ Model structure of Freye's endohedrally modified suberone based cage . . . . . . . . . . . . . . . . . . 153

Figure V.2.1.2: $\quad$ Crystal structure polynorbonene-Cage by Johnstone et al. . . . . . 154

Figure V.2.1.3: $\quad$ Crystal structure adamantyl cage by Löffler et al. . . . . . . . . 155

Figure V.2.2.4: $\quad$ Comparison NMR of the ligands $\mathbf{L}^{t \mathbf{B u}}, \mathbf{L}^{\mathrm{Et}}, \mathbf{L}^{\mathbf{P h}}$ and $\mathbf{L}^{\mathrm{CN}}$ in

MeCN at $298 \mathrm{~K}$. . . . . . . . . . . . . . . . . 157

Figure V.2.2.5: $\quad$ Comparison $\mathrm{B}^{\mathrm{Et}} 35$ and $\mathrm{L}^{\mathrm{Et}} 35$. . . . . . . . . . . . . . . . . . 159

Figure V.2.2.6: Comparison of $\mathbf{L}^{\mathbf{E t}}$ and $\mathbf{C}^{\mathbf{E t}}$ in various solvents . . . . . . . . . 160

Figure V.2.2.7: $\quad$ Comparison ${ }^{\mathbf{1}} \mathbf{H}$ NMR ligand and assembled cage. . . . . . . . . . 161

Figure V.2.2.8: $\quad$ Cage ESI-MS . . . . . . . . . . . . . . . . . . . . . . . . . . . 162

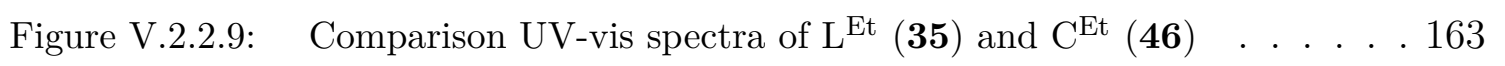

Figure V.2.2.10: Comparison crystal structures of ligand derivatives . . . . . . . . 164

\begin{tabular}{|l|l|l|}
\hline Figure V.2.2.11: & UV-vis & titration experiemts of all cyano acetate ligand derivatives \\
\hline
\end{tabular} with TFA. . . . . . . . . . . . . . . . 167

Figure V.2.2.12: NMR titration experiment of $\mathbf{L}^{\mathbf{E t}}$ with TFA . . . . . . . . . . . 168

\begin{tabular}{|l|l|l|l|}
\hline Figure V.2.2.13: & UV-vis & comparison of $\mathbf{L}^{\mathbf{E t}}$ and $\mathbf{C}^{\mathbf{E t}}$ in presence and abscence of \\
\hline
\end{tabular} TFA . . . . . . . . . . . . . . . . 169

Figure V.2.2.14: NMR titration experiment of $\mathbf{C}^{\mathbf{E t}}$ with TFA. . . . . . . . . . . . 170

Figure V.2.2.15: $\quad$ Comparison of ${ }^{\mathbf{1}} \mathbf{H}$ NMR spectra of cage 46 and ligand 42 in presence of TFA . . . . . . . . . . . . . . . 171

Figure V.2.2.16: Screening of proper conditions for host-guest experiemtens with

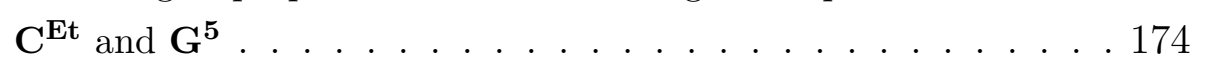

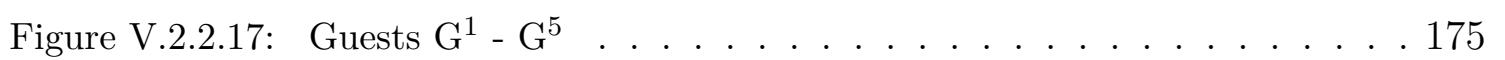

Figure V.2.2.18: Guests $\mathrm{G}^{1}-\mathrm{G}^{5}$. . . . . . . . . . . . . . . . . . . . 175

Figure V.3.2.1: Comparison VT-NMR for 52 with 35. . . . . . . . . . . . . . . 182

Figure V.3.2.2: $\quad$ Eyring plots of experimental determined and simulated rotation rates. . . . . . . . . . . . . . . . . 183

Figure V.3.2.3: $\quad$ Comparison $\mid \mathrm{UV}$-vis $\mid$ of $\mathbf{B}_{\text {Azacrown }}{ }^{\mathbf{E t}}$ and $\mathbf{B}^{\mathbf{E t}}$. . . . . . . . . . . 184 
\begin{tabular}{|l|l|l|l|l|l|}
\hline Figure V.3.2.4: & NMR & and & UV-vis & titration experiments of compound $\mathbf{5 2}$ and \\
\hline
\end{tabular} $\mathrm{Mg}\left(\mathrm{ClO}_{4}\right)_{2}$. . . . . . . . . . . . . . . . . 185

\begin{tabular}{|l|l|l|l|l|}
\hline Figure V.3.2.5: & NMR & and & UV-vis & titration experiments of compound $\mathbf{5 2}$ and \\
\hline
\end{tabular} $\mathrm{Ca}\left(\mathrm{ClO}_{4}\right)_{2} \ldots \ldots \ldots \ldots \ldots \ldots$. . . . . . . . . . . . . . . . . .

\begin{tabular}{|l|l|l|l|l|}
\hline Figure V.3.2.6: & NMR & and & UV-vis & titration experiments of compound $\mathbf{5 2}$ and TFA 187 \\
\hline
\end{tabular} Figure V.3.2.7: $\quad$ UV and NMR titration experiments with $\mathbf{B}_{\text {Azacrown }}{ }^{\mathbf{E t}} / \mathbf{B}^{\mathbf{E t}}$ and $\mathrm{Ni}\left(\mathrm{ClO}_{4}\right)_{2} . \ldots \ldots \ldots \ldots$. . . . . . . . . . . . . . . . . .

Figure V.3.2.8: $\quad$ VT-NMR comparison $\mathbf{B}_{\text {Azacrown }}{ }^{\mathrm{Et}}(2.8 \mathrm{mM})$ in $\mathrm{MeCN}$ and $\mathbf{B}_{\text {Azacrown }}{ }^{\text {Et }}(2.8 \mathrm{mM})+150$ eq. $\mathrm{Mg}\left(\mathrm{ClO}_{4}\right)_{2}$ in $\mathrm{MeCN}$. . . . . . 190

Figure VI.1.7.1: $\quad$ NMR host guest titration experiments with 25. . . . . . . . . . . 335

Figure VI.1.7.2: Cage ESI-MS . . . . . . . . . . . . . . . . . . . . . 337

Figure VI.1.7.3: $\quad$ Titration $\mathbf{C}^{\mathbf{E t}}$ with $\mathbf{G}^{\mathbf{1}}$. . . . . . . . . . . . . . . . . . . . . . 338

Figure VI.1.7.4: Titration $\mathbf{C}^{\mathbf{E t}}$ with $\mathbf{G}^{2}$. . . . . . . . . . . . . . . . . . . . . . 338

Figure VI.1.7.5: Titration $\mathbf{C}^{\mathbf{E t}}$ with $\mathbf{G}^{3}$. . . . . . . . . . . . . . . . . . . . . 339

Figure VI.1.7.6: $\quad$ Titration $\mathbf{C}^{\mathbf{E t}}$ with $\mathbf{G}^{4}$. . . . . . . . . . . . . . . . . . . . .339

Figure VI.1.7.7: Titration $\mathbf{C}^{\mathbf{E t}}$ with $\mathbf{G}^{5}$. . . . . . . . . . . . . . . . . . 340

Figure VI.1.7.8: Titration $\mathbf{C}^{\mathbf{E t}}$ with $\mathbf{G}^{5}$ in DMSO . . . . . . . . . . . . . . . . . 340

Figure VI.1.7.9: Titration $\mathbf{C}^{\mathbf{E t}}$ with $\mathbf{G}^{\mathbf{5}}$ in $\mathrm{MeOH}$ at $60{ }^{\circ} \mathrm{C}$. . . . . . . . . . . . 341

Figure VI.1.7.10: Titration $\mathbf{C}^{\mathbf{E t}}$ with $\mathbf{G}^{\mathbf{5}}$ in $\mathrm{MeNO}_{2}$ at $60{ }^{\circ} \mathrm{C}$. . . . . . . . . . . . 341

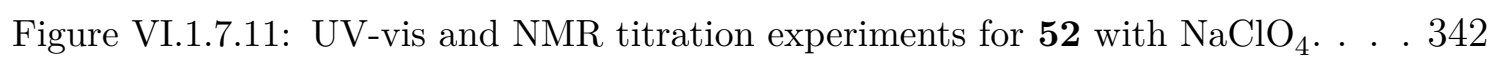

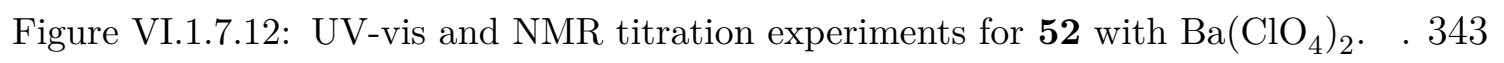

\begin{tabular}{|l|l|l|l|}
\hline Figure VI.1.7.13: & UV-vis & titration experiments of $\mathbf{5 2}$ with $\mathrm{Mn}\left(\mathrm{ClO}_{4}\right)_{2}, \mathrm{Zn}_{(}\left(\mathrm{BF}_{4}\right)_{2}$ \\
\hline
\end{tabular} and $\mathrm{Cd}\left(\mathrm{ClO}_{4}\right)_{2} \ldots \ldots \ldots \ldots \ldots \ldots$

\begin{tabular}{|l|l|l|l|}
\hline Figure VI.1.7.14: & $\mathrm{UV}$-vis & titration experiments of $\mathbf{5 2}$ and $\mathbf{3 5}$ with $\mathrm{Cu}\left(\mathrm{BF}_{4}\right)_{2} \ldots$ \\
\hline
\end{tabular}

Figure VI.2.3.1: Determination extinction coefficient by $\mid$ UV-vis . . . . . . . . . . 353 


\section{C.2 List of Schemes}

Scheme III.2.2.1: Synthetic path for ligand 11 . . . . . . . . . . . . . . . . . 50

Scheme III.2.3.2: $\quad$ Synthetic path for ligand 17 . . . . . . . . . . . . . . . . . . . 68

Scheme IV.2.2.3: $\quad$ Synthetic path for ligand 30. . . . . . . . . . . . . . . . . . . . 95

Scheme IV.2.2.4: $\quad$ Deprotection of $\mathbf{1 7}$ and subsequent intramolecualr cyclisation. . 97

Scheme IV.2.3.5: Synthetic path for ligand 24. . . . . . . . . . . . . . . . . 100

Scheme V.1.2.6: General synthesis of the acridinylidene derivatives . . . . . . . . 111

Scheme V.1.3.7: Protonation of $\mathbf{3 5}$. . . . . . . . . . . . . . . . . . . . . . . 138

Scheme V.2.2.8: $\quad$ Synthesis ligand derivatives 41, 42 and 43 . . . . . . . . . . . . 156

Scheme V.2.2.9: Guests $\mathrm{G}^{1}-\mathrm{G}^{5} \ldots \ldots \ldots$. . . . . . . . . . . . . . . . . . . 171

Scheme V.3.2.10: Retrosynthetic analysis of the intended azacrown ether system. . 179

Scheme V.3.2.11: Synthetic path for compound 52. . . . . . . . . . . . . . . . . 180

Scheme VI.1.1.12: Synthesis of compound 2. . . . . . . . . . . . . . . . . 195

Scheme VI.1.1.13: Synthesis of compound 3. . . . . . . . . . . . . . . . . . . . 196

Scheme VI.1.1.14: Synthesis of compound 4. . . . . . . . . . . . . . . . . . . . 198

Scheme VI.1.1.15: Synthesis of compound 5. . . . . . . . . . . . . . . . . . . . 200

Scheme VI.1.1.16: Synthesis of compound 6. . . . . . . . . . . . . . . . . . . . 202

Scheme VI.1.1.17: Synthesis of compound 7. . . . . . . . . . . . . . . . . . . . . 204

Scheme VI.1.1.18: Synthesis of compound 8. . . . . . . . . . . . . . . . . . . 206

Scheme VI.1.1.19: Synthesis of compound 9. . . . . . . . . . . . . . . . . . . 208

Scheme VI.1.1.20: Synthesis of compound 10. . . . . . . . . . . . . . . . . . . . . 210

Scheme VI.1.1.21: Synthesis of compound 11. . . . . . . . . . . . . . . . 212

Scheme VI.1.2.22: Synthesis of compound 12. . . . . . . . . . . . . . . . . . . 214

Scheme VI.1.2.23: Synthesis of compound 13. . . . . . . . . . . . . . . . . . 216

Scheme VI.1.2.24: Synthesis of compound 14. . . . . . . . . . . . . . . . . . . . 218

Scheme VI.1.2.25: Synthesis of compound 15. . . . . . . . . . . . . . . . . . . 220

Scheme VI.1.2.26: Synthesis of compound 16. . . . . . . . . . . . . . . . . . . . . 222

Scheme VI.1.2.27: Synthesis of compound 17. . . . . . . . . . . . . . . . . . . . 224

Scheme VI.1.2.28: Synthesis of compound 18. . . . . . . . . . . . . . . . . . 226

Scheme VI.1.2.29: Synthesis of compound 19. . . . . . . . . . . . . . . . . . . . 228

Scheme VI.1.2.30: Synthesis of compound 20. . . . . . . . . . . . . . . . . . . 230

Scheme VI.2.1.31: Synthesis of compound 22. . . . . . . . . . . . . . . . . 233

Scheme VI.2.1.32: Synthesis of compound 23. . . . . . . . . . . . . . . . . . 235

Scheme VI.2.1.33: Synthesis of compound 24. . . . . . . . . . . . . . . . . . 237

Scheme VI.2.2.34: Synthesis of compound 26. . . . . . . . . . . . . . . . . . . . . 239 
Scheme VI.2.2.35: Synthesis of compound 27. . . . . . . . . . . . . . . . . . 241

Scheme VI.2.2.36: Synthesis of compound 28. . . . . . . . . . . . . . . . . . . . 243

Scheme VI.2.2.37: Synthesis of compound 29. . . . . . . . . . . . . . . . . . . . 245

Scheme VI.2.2.38: Synthesis of compound 30. . . . . . . . . . . . . . . . . . . . 247

Scheme VI.2.2.39: Synthesis of compound 31. . . . . . . . . . . . . . . . . . . . . 249

Scheme VI.2.2.40: Synthesis of compound 32. . . . . . . . . . . . . . . 251

Scheme VI.3.1.41: Synthesis of compound 34. . . . . . . . . . . . . . . . . . . . 254

Scheme VI.3.1.42: Synthesis of compound 35. . . . . . . . . . . . . . . . . . . . 257

Scheme VI.3.1.43: Synthesis of compound 36. . . . . . . . . . . . . . . . . . . 259

Scheme VI.3.1.44: Synthesis of compound 37. . . . . . . . . . . . . . . . . 260

Scheme VI.3.2.45: Synthesis of compound 40. . . . . . . . . . . . . . . . . . 262

Scheme VI.3.2.46: Synthesis of compound 41. . . . . . . . . . . . . . . . . . . . 265

Scheme VI.3.2.47: Synthesis of compound 42. . . . . . . . . . . . . . . . . . 268

Scheme VI.3.2.48: Synthesis of compound 43. . . . . . . . . . . . . . . . . . 271

Scheme VI.3.3.49: Synthesis of coordination cages 44, 45, 46 and 47 . . . . . . . . 274

Scheme VI.3.3.50: Synthesis of compound 44. . . . . . . . . . . . . . . . . . . . 275

Scheme VI.3.3.51: Synthesis of compound 45. . . . . . . . . . . . . . . . . . . . 2777

Scheme VI.3.3.52: Synthesis of compound 46. . . . . . . . . . . . . . . . . . . . 278

Scheme VI.3.3.53: Synthesis of compound 47. . . . . . . . . . . . . . . . . . . . 2800

Scheme VI.3.4.54: Synthesis of compound 48. . . . . . . . . . . . . . . . . . . . 281

Scheme VI.3.4.55: Synthesis of compound 49. . . . . . . . . . . . . . . . . . . . . 283

Scheme VI.3.4.56: Synthesis of compound 50. . . . . . . . . . . . . . . . . . . . 2866

Scheme VI.3.4.57: Synthesis of compound 51. . . . . . . . . . . . . . . . . . . . 288

Scheme VI.3.4.58: Synthesis of compound 52. . . . . . . . . . . . . . . . . . . . . 291

Scheme VI.1.7.59: Guests $\mathrm{G}^{1}-\mathrm{G}^{5} \ldots \ldots \ldots$. . . . . . . . . . . . 337 


\section{C.3 List of Tables}

Table III.2.2.1: $\mathrm{ONS}$ sequences for MKDH01um and MKDH01 . . . . . . . . . . 52

Table III.2.3.2: ONS $\mid$ sequence table for MKDH05 and MDKHD06 . . . . . . . . . 69

Table III.3.0.1: Comparison of $\left|\mathrm{T}_{\mathrm{M}}\right|$ for the investigated $\mid$ HJ| systems. . . . . . . . . . 82

Table V.1.2.1: Activation enthalpies . . . . . . . . . . . . . . . . . . 126

Table V.1.2.2: Values from cyclic voltammetry experiments . . . . . . . . . . . . 128

Table V.1.2.3: Comparison of HOMO-/ LUMO-energy levels . . . . . . . . . . . . 134

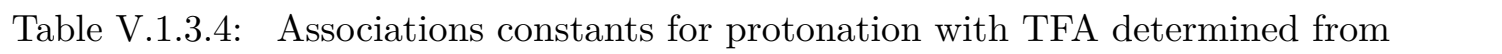
UV-vis ||TFA titrations experiments for textbfB ${ }^{t \mathrm{Bu}}, \mathbf{B}^{\mathrm{Et}}$ and $\mathbf{B}^{\mathbf{P h}}$. . 145

\begin{tabular}{|ll|l|l|l|}
\hline Table V.1.3.5: & Associations constants for protonation with & TFA & determined from \\
\hline
\end{tabular}

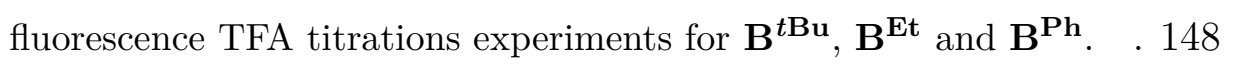

Table V.2.2.1: Comparison of relative energies of various cage stereoisomers. . . . 165

Table V.3.2.1: Activation enthalpies . . . . . . . . . . . . . . . . . . . 183

Table VI.1.8.1: Data from X-ray diffraction analysis for compounds $\mathbf{B}^{\mathrm{Et}}(\mathbf{3 5})$ and

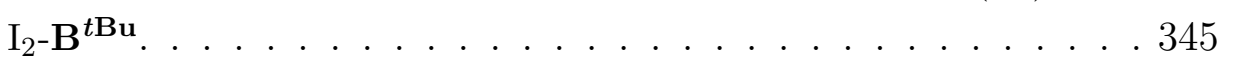

Table VI.1.8.2: Data from X-ray diffraction analysis for compounds 40, 42 and $\mathbf{4 3}$. 346

Table VI.2.1.1: DNA synthesis solutions . . . . . . . . . . . . . . . . . . . . . . . 349

Table VI.2.2.2: HPLC methods used in for analytic investigation and ONS|separation.351 


\section{C.4 Abbreviations}

\begin{tabular}{|c|c|}
\hline G & guest \\
\hline $\mathbf{H}$ & host \\
\hline$K_{\mathrm{a}}$ & association constant \\
\hline${ }^{1} \mathrm{H} \mathrm{NMR}$ & ${ }^{1} \mathrm{H}$ NMR \\
\hline bp & base pairs \\
\hline CD & circular dichroism \\
\hline CEP & $\beta$-cyanoethyl phophoramidite \\
\hline $\mathrm{CPG}$ & controlled pore glass \\
\hline $\mathrm{CV}$ & cyclic voltammertry \\
\hline $\mathrm{DCM}$ & dichloromethane \\
\hline DEA & diethanol amine \\
\hline DFT & density-function theory \\
\hline DMAP & 4-dimethylaminopyridine \\
\hline DMT & 4,4'-Dimethoxytrityl \\
\hline DNMR & dynamic NMR spectroscopy \\
\hline dsDNA & double stranded DNA \\
\hline EA & elemental analysis \\
\hline ESI-MS & electronspray ionization mass spectrometry \\
\hline FS & fluorescence spectroscopy \\
\hline FWHM & full width half maximum \\
\hline GNA & glycol nucleic acid \\
\hline GPC & gel permeation chromatography \\
\hline HJ & Holliday junction \\
\hline HPLC & high performance liquid chromatography \\
\hline IDA & inter duplex angle \\
\hline
\end{tabular}




\begin{tabular}{|c|c|}
\hline MOM & methoxymethyl acetal \\
\hline NMR & nuclear magnetic resonance spectroscopy \\
\hline ONS & oligonucelotide strand \\
\hline PAGE & polyacrylamide gel electrophoresis \\
\hline PEG & polyethylene glycol \\
\hline PNA & peptide nucleic acid \\
\hline RNA & ribonucleic acid \\
\hline SBB & supramolecular building block \\
\hline SEC & size exclusion chromatography \\
\hline SPS & solid phase synthesis \\
\hline ssDNA & single stranded DNA \\
\hline $\mathrm{T}_{\mathrm{C}}$ & coalescence temperature \\
\hline $\mathrm{T}_{\mathrm{M}}$ & denaturation temperature \\
\hline TBDMS & tert-butyldimethylsilyl \\
\hline TBM & tris-borate magnesium \\
\hline TDS & thermal differential spectrum \\
\hline TFA & trifluoroacetic acid \\
\hline THP & tetrahydropyranyl ether \\
\hline $\mathrm{TsOH}$ & p-toluenesulfonic acid \\
\hline UNA & unlocked nucleic acid \\
\hline UV-vis & ultraviolet-visible spectroscopy \\
\hline
\end{tabular}





\section{References}

[1] L.-X. Cai, S.-C. Li, D.-N. Yan, L.-P. Zhou, F. Guo, Q.-F. Sun, Journal of the American Chemical Society 2018, 140, 4869-4876.

[2] C. Bazzicalupi, A. Bianchi, E. García-España, E. Delgado-Pinar, Inorganica Chimica Acta, Special Volume: Metals In Supramolecular Chemistry 2014, $417,3-26$.

[3] G.-H. Ouyang, Y.-M. He, Y. Li, J.-F. Xiang, Q.-H. Fan, Angewandte Chemie International Edition 2015, 54, 4334-4337.

[4] J. Li, D. Yim, W.-D. Jang, J. Yoon, Chemical Society Reviews 2017, 46, $2437-2458$

[5] R. Liu, G. Zhu, G. Zhang, RSC Advances 2020, 10, 7092-7098.

[6] J. W. Steed, J. L. Atwood, Supramolecular chemistry, 2. ed., 2009.

[7] C. J. Pedersen, Journal of the American Chemical Society 1967, 89, 70177036 .

[8] J. M. Lehn, Accounts of Chemical Research 2002, 11, 49-57.

[9] B. Dietrich, J. Lehn, J. Sauvage, Tetrahedron Letters 1969, 10, 2889-2892.

[10] E. P. Kyba, M. G. Siegel, L. R. Sousa, G. D. Y. Sogah, D. J. Cram, Journal of the American Chemical Society 1973, 95, 2691-2692.

[11] E. B. Kyba, K. Koga, L. R. Sousa, M. G. Siegel, D. J. Cram, Journal of the American Chemical Society 1973, 95, 2692-2693.

[12] C. Dietrich-Buchecker, J. Sauvage, J. Kintzinger, Tetrahedron Letters 1983 , 24, 5095-5098.

[13] G. Gil-Ramírez, D. A. Leigh, A. J. Stephens, Angewandte Chemie International Edition 2015, 54, 6110-6150.

[14] J. F. Stoddart, Angewandte Chemie International Edition 2017, 56, 1109411125 . 
[15] P. R. Ashton, C. L. Brown, E. J. T. Chrystal, T. T. Goodnow, A. E. Kaifer, K. P. Parry, D. Philp, A. M. Z. Slawin, N. Spencer, J. F. Stoddart, D. J. Williams, Journal of the Chemical Society, Chemical Communications 1991, 634.

[16] J. D. Badjic, V. Balzani, A. Credi, S. Silvi, J. F. Stoddart, Science 2004, 303, $1845-1849$.

[17] C. J. Bruns, J. F. Stoddart, Accounts of chemical research 2014, 47, 21862199 .

[18] D. Ben-Amotz, Annual review of physical chemistry 2016, 67, 617-638.

[19] D. M. Huang, D. Chandler, J. Phys. Chem. B 2002, 106, 2047-2053.

[20] S. Löffler, J. Lübben, L. Krause, D. Stalke, B. Dittrich, G. H. Clever, Journal of the American Chemical Society 2015, 137, 1060-1063.

[21] G. H. Clever, P. Punt, Accounts of chemical research 2017, 50, 2233-2243.

[22] M. Cesario, C. O. Dietrich-Buchecker, J. Guilhem, C. Pascard, J. P. Sauvage, Journal of the Chemical Society, Chemical Communications 1985, 244.

[23] P. W. Atkins, J. de Paula, Physikalische Chemie, 4., vollst. überarb. Aufl., Wiley-VCH, Weinheim, 2006.

[24] S. Löffler, J. Lübben, A. Wuttke, R. A. Mata, M. John, B. Dittrich, G. H. Clever, Chemical Science 2016, 7, 4676-4684.

[25] M. Yoshizawa, M. Fujita, Pure and Applied Chemistry - PURE APPL CHEM 2005, 77, 1107-1112.

[26] S. Freye, R. Michel, D. Stalke, M. Pawliczek, H. Frauendorf, G. H. Clever, Journal of the American Chemical Society 2013, 135, 8476-8479.

[27] S. Freye, J. Hey, A. Torras-Galán, D. Stalke, R. Herbst-Irmer, M. John, G. H. Clever, Angewandte Chemie International Edition 2012, 51, 2191-2194.

[28] G. O. Bonilla-Rodriguez, Journal of Biological Chemistry 1996, 271, 48954899 .

[29] S. I. Stupp, L. C. Palmer, Chemistry of Materials 2013, 26, 507-518.

[30] C.-T. Yeung, K.-H. Yim, H.-Y. Wong, R. Pal, W.-S. Lo, S.-C. Yan, M. YeeMan Wong, D. Yufit, D. E. Smiles, L. J. McCormick, S. J. Teat, D. K. Shuh, W.-T. Wong, G.-L. Law, Nature communications 2017, 8, 1128.

[31] R. Chakrabarty, P. S. Mukherjee, P. J. Stang, Chemical Reviews 2011, 111, PMID: 21863792, 6810-6918. 
[32] T. R. Schulte, M. Krick, C. I. Asche, S. Freye, G. H. Clever, RSC Advances 2014, 4, 29724-29728.

[33] J. L. Bolliger, T. K. Ronson, M. Ogawa, J. R. Nitschke, Journal of the American Chemical Society 2014, 136, 14545-14553.

[34] K. Li, L.-Y. Zhang, C. Yan, S.-C. Wei, M. Pan, L. Zhang, C.-Y. Su, Journal of the American Chemical Society 2014, 136, 4456-4459.

[35] W. Meng, B. Breiner, K. Rissanen, J. D. Thoburn, J. K. Clegg, J. R. Nitschke, Angewandte Chemie International Edition 2011, 50, 3479-3483.

[36] M. Han, Y. Luo, B. Damaschke, L. Gómez, X. Ribas, A. Jose, P. Peretzki, M. Seibt, G. H. Clever, Angewandte Chemie International Edition 2016, 55, $445-449$.

[37] D. Fujita, Y. Ueda, S. Sato, H. Yokoyama, N. Mizuno, T. Kumasaka, M. Fujita, Chem 2016, 1, 91-101.

[38] T. R. Cook, P. J. Stang, Chemical reviews 2015, 115, 7001-7045.

[39] S. J. Dalgarno, N. P. Power, J. L. Atwood, Coordination Chemistry Reviews 2008, 252, 825-841.

[40] S. Turega, M. Whitehead, B. R. Hall, M. F. Haddow, C. A. Hunter, M. D. Ward, Chemical communications (Cambridge, England) 2012, 48, 2752-2754.

[41] S. Löffler, A. Wuttke, B. Zhang, J. J. Holstein, R. A. Mata, G. H. Clever, Chemical communications (Cambridge, England) 2017, 53, 11933-11936.

[42] M. Yamashina, Y. Sei, M. Akita, M. Yoshizawa, Nature communications 2014, 5, 4662 .

[43] P. Mal, B. Breiner, K. Rissanen, J. R. Nitschke, Science 2009, 324, 1697-1699.

[44] C. J. Hastings, M. D. Pluth, R. G. Bergman, K. N. Raymond, Journal of the American Chemical Society 2010, 132, 6938-6940.

[45] T. Murase, Y. Nishijima, M. Fujita, Journal of the American Chemical Society 2012, 134, 162-164.

[46] M. Yoshizawa, M. Tamura, M. Fujita, Science 2006, 312, 251-254.

[47] T. Murase, H. Takezawa, M. Fujita, Chemical communications (Cambridge, England) 2011, 47, 10960-10962.

[48] D. M. Vriezema, M. Comellas Aragonès, J. A. A. W. Elemans, J. J. L. M. Cornelissen, A. E. Rowan, R. J. M. Nolte, Chemical reviews 2005, 105, $1445-1489$. 
[49] M. B. Duriska, S. M. Neville, J. Lu, S. S. Iremonger, J. F. Boas, C. J. Kepert, S. R. Batten, Angewandte Chemie International Edition 2009, 48, 8919-8922.

[50] Y. R. Hristova, M. M. J. Smulders, J. K. Clegg, B. Breiner, J. R. Nitschke, Chem. Sci. 2011, 2, 638-641.

[51] M. Han, D. M. Engelhard, G. H. Clever, Chemical Society Reviews 2014, 43, 1848-1860.

[52] N. B. Debata, D. Tripathy, D. K. Chand, Coordination Chemistry Reviews 2012, 256, 1831-1945.

[53] Y.-B. Dong, P. Wang, J.-P. Ma, X.-X. Zhao, H.-Y. Wang, B. Tang, R.-Q. Huang, Journal of the American Chemical Society 2007, 129, 4872-4873.

[54] R. Custelcean, Chemical Society reviews 2014, 43, 1813-1824.

[55] G. H. Clever, S. Tashiro, M. Shionoya, Angewandte Chemie International Edition 2009, 48, 7010-7012.

[56] J. Lewis, E. Gavey, S. Cameron, J. Crowley, Chem. Sci. 2012, 3, 778-784.

[57] P. Liao, B. W. Langloss, A. M. Johnson, E. R. Knudsen, F. S. Tham, R. R. Julian, R. J. Hooley, Chemical communications (Cambridge, England) 2010, 46, 4932-4934.

[58] M. Frank, M. D. Johnstone, G. H. Clever, Chemistry - A European Journal 2016, 22, 14104-14125.

[59] R. Zhu, J. Lübben, B. Dittrich, G. H. Clever, Angewandte Chemie International Edition 2014, n/a.

[60] D. M. Engelhard, S. Freye, K. Grohe, M. John, G. H. Clever, Angewandte Chemie International Edition 2012, 51, 4747-4750.

[61] W. M. Bloch, J. J. Holstein, W. Hiller, G. H. Clever, Angewandte Chemie International Edition 2017, 56, 8285-8289.

[62] C. Tan, D. Chu, X. Tang, Y. Liu, W. Xuan, Y. Cui, Chemistry - A European Journal 2019, 25, 662-672.

[63] P. F. Kuijpers, M. Otte, M. Dürr, I. Ivanović-Burmazović, J. N. H. Reek, B. de Bruin, ACS Catalysis 2016, 6, 3106-3112.

[64] M. Kieffer, A. M. Garcia, C. J. E. Haynes, S. Kralj, D. Iglesias, J. R. Nitschke, S. Marchesan, Angewandte Chemie International Edition 2019, 58, 7982-7986.

[65] Y.-R. Zheng, K. Suntharalingam, T. C. Johnstone, S. J. Lippard, Chem. Sci. 2015, 6, 1189-1193. 
[66] M. Frank, L. Krause, R. Herbst-Irmer, D. Stalke, G. H. Clever, Dalton Transactions 2014, 43, 4587.

[67] W. M. Bloch, G. H. Clever, Chemical communications (Cambridge, England) 2017, 53, 8506-8516.

[68] A. M. Johnson, R. J. Hooley, Inorganic Chemistry 2011, 50, 4671-4673.

[69] M. Yamashina, T. Yuki, Y. Sei, M. Akita, M. Yoshizawa, Chemistry - A European Journal 2015, 21, 4200-4204.

[70] N. Kishi, Z. Li, K. Yoza, M. Akita, M. Yoshizawa, Journal of the American Chemical Society 2011, 133, 11438-11441.

[71] W. M. Bloch, Y. Abe, J. J. Holstein, C. M. Wandtke, B. Dittrich, G. H. Clever, Journal of the American Chemical Society 2016, 138, 13750-13755.

[72] L. Zhang, Y.-J. Lin, Z.-H. Li, G.-X. Jin, Journal of the American Chemical Society 2015, 137, 13670-13678.

[73] S. De, K. Mahata, M. Schmittel, Chemical Society reviews 2010, 39, 15551575 .

[74] S. Pullen, G. H. Clever, Accounts of chemical research 2018, 51, 3052-3064.

[75] R. Zhu, W. M. Bloch, J. J. Holstein, S. Mandal, L. V. Schäfer, G. H. Clever, Chemistry - A European Journal 2018, 24, 12976-12982.

[76] D. Preston, J. E. Barnsley, K. C. Gordon, J. D. Crowley, Journal of the American Chemical Society 2016, 138, 10578-10585.

[77] N. C. Seeman, Annual review of biochemistry 2010, 79, 65-87.

[78] J. Chen, N. C. Seeman, Nature 1991, 350, 631-633.

[79] F. A. Aldaye, A. L. Palmer, H. F. Sleiman, Science 2008, 321, 1795-1799.

[80] P. W. K. Rothemund, Nature 2006, 440, 297-302.

[81] S. M. Douglas, H. Dietz, T. Liedl, B. Högberg, F. Graf, W. M. Shih, Nature 2009, 459, 1154.

[82] S. D. Perrault, W. M. Shih, ACS nano 2014, 8, 5132-5140.

[83] Y.-J. Chen, B. Groves, R. A. Muscat, G. Seelig, Nature nanotechnology 2015, 10, 748-760.

[84] C. K. McLaughlin, G. D. Hamblin, H. F. Sleiman, Chemical Society Reviews 2011, 40, 5647 .

[85] Nucleic acid-metal ion interactions, (Ed.: N. V. Hud), RSC Publ, Cambridge, 2009. 
[86] D. L. Nelson, M. M. Cox, Lehninger Biochemie, Springer Berlin Heidelberg, Berlin, Heidelberg, 2009.

[87] J. S. Hardwick, D. Ptchelkine, A. H. El-Sagheer, I. Tear, D. Singleton, S. E. V. Phillips, A. N. Lane, T. Brown, Nature structural \& molecular biology 2017, 24, 544-552.

[88] T. A. Larsen, M. L. Kopka, R. E. Dickerson, Biochemistry 1991, 30, 44434449 .

[89] P. Drozdzal, M. Gilski, R. Kierzek, L. Lomozik, M. Jaskolski, Journal of biological inorganic chemistry : JBIC : a publication of the Society of Biological Inorganic Chemistry 2015, 20, 595-602.

[90] S. Ikuta, R. Chattopadhyaya, H. Ito, R. E. Dickerson, D. R. Kearns, Biochemistry 1986, 25, 4840-4849.

[91] K. Mizuuchi, M. Mizuuchi, M. Gellert, Journal of Molecular Biology 1982, 156, 229-243.

[92] S. S. Bachurin, M. E. Kletskii, O. N. Burov, S. V. Kurbatov, Biophysical Chemistry 2018, 235, 19-28.

[93] N. B. Leontis, Nucleic Acids Research 2002, 30, 3497-3531.

[94] H. A. Day, P. Pavlou, Z. A. E. Waller, Bioorganic $\&$ medicinal chemistry 2014, 22, 4407-4418.

[95] M. D. Frank-Kamenetskii, S. M. Mirkin, Annual review of biochemistry 1995, $64,65-95$.

[96] N. M. Bell, J. Micklefield, Chembiochem : a European journal of chemical biology 2009, 10, 2691-2703.

[97] M. Meng, C. Ducho, Beilstein journal of organic chemistry 2018, 14, 1293 1308.

[98] M. A. Campbell, J. Wengel, Chemical Society reviews 2011, 40, 5680-5689.

[99] P. M. Punt, G. H. Clever, Chem. Sci. 2019, 59, 620.

[100] D. M. Engelhard, L. M. Stratmann, G. H. Clever, Chemistry - A European Journal 2018, 24, 2117-2125.

[101] D. M. Engelhard, J. Nowack, G. H. Clever, Angewandte Chemie International Edition 2017, 56, 11640-11644.

[102] S. Barluenga, N. Winssinger, Accounts of chemical research 2015, 48, 1319 1331. 
[103] P. E. Nielsen, G. Haaima, Chemical Society Reviews 1997, $26,73$.

[104] W. Wang, L. M. Nocka, B. Z. Wiemann, D. M. Hinckley, I. Mukerji, F. W. Starr, Scientific reports 2016, 6, 22863.

[105] D. Monchaud, M.-P. Teulade-Fichou, Org. Biomol. Chem. 2008, 6, 627-636.

[106] G. H. Clever, C. Kaul, T. Carell, Angewandte Chemie International Edition 2007, 46, 6226-6236.

[107] P. Scharf, J. Müller, ChemPlusChem 2013, 78, 20-34.

[108] G. H. Clever, M. Shionoya, Coordination Chemistry Reviews 2010, 254, 2391-2402.

[109] S. Johannsen, N. Megger, D. Böhme, R. K. O. Sigel, J. Müller, Nature chemistry 2010, 2, 229-234.

[110] E. Meggers, P. L. Holland, W. B. Tolman, F. E. Romesberg, P. G. Schultz, Journal of the American Chemical Society 2000, 122, 10714-10715.

[111] N. Zimmermann, E. Meggers, P. G. Schultz, Bioorganic Chemistry 2004, 32, $13-25$.

[112] G. H. Clever, K. Polborn, T. Carell, Angewandte Chemie 2005, 117, 73707374 .

[113] G. H. Clever, Y. Söltl, H. Burks, W. Spahl, T. Carell, Chemistry - A European Journal 2006, 12, 8708-8718.

[114] H. Weizman, Y. Tor, Journal of the American Chemical Society 2001, 123, $3375-3376$.

[115] Y. Takezawa, S. Yoneda, J.-L. H. A. Duprey, T. Nakama, M. Shionoya, Chem. Sci. 2016, 7, 3006-3010.

[116] H. Yang, A. Z. Rys, C. K. McLaughlin, H. F. Sleiman, Angewandte Chemie International Edition 2009, 48, 9919-9923.

[117] K. Tanaka, Y. Yamada, M. Shionoya, Journal of the American Chemical Society 2002, 124, 8802-8803.

[118] J.-L. H. A. Duprey, Y. Takezawa, M. Shionoya, Angewandte Chemie International Edition 2013, 52, 1212-1216.

[119] G. Bianké, R. Häner, Chembiochem : a European journal of chemical biology 2004, 5, 1063-1068.

[120] D. M. Engelhard, R. Pievo, G. H. Clever, Angewandte Chemie International Edition 2013, 52, 12843-12847. 
[121] H. Yang, H. F. Sleiman, Angewandte Chemie International Edition 2008, 120, 2477-2480.

[122] R. Holliday, Genetical Research 1964, 5, 282.

[123] B. Alberts, A. Johnson, J. Lewis, D. Morgan, M. Raff, K. Roberts, P. Walter, J. Wilson, T. Hunt, Molecular biology of the cell, Sixth edition, Garland Science Taylor and Francis Group, New York, NY, 2015.

[124] D. M. J. Lilley, Quarterly reviews of biophysics 2000, 33, 109-159.

[125] D. M. Lilley, M. F. White, Nature reviews. Molecular cell biology 2001, 2, 433-443.

[126] F. A. Hays, J. Watson, P. S. Ho, The Journal of biological chemistry 2003, 278, 49663-49666.

[127] J. Matos, S. C. West, DNA repair 2014, 19, 176-181.

[128] K. M. Górecka, W. Komorowska, M. Nowotny, Nucleic Acids Research 2013 , 41, 9945-9955.

[129] T. M. Ashton, H. W. Mankouri, A. Heidenblut, P. J. McHugh, I. D. Hickson, Molecular and cellular biology 2011, 31, 1921-1933.

[130] P. S. Ho, Biochemical Society transactions 2017, 45, 1149-1158.

[131] I. Mukerji, C. I. Vitoc, J. Litke, E. Wheatley, O. Buzovetsky, Y. Li, D. L. Beveridge, Biophysical journal 2012, 102, 75.

[132] M. Ariyoshi, T. Nishino, H. Iwasaki, H. Shinagawa, K. Morikawa, Proceedings of the National Academy of Sciences of the United States of America 2000, 97, 8257-8262.

[133] J. Yu, T. Ha, K. Schulten, Nucleic Acids Research 2004, 32, 6683-6695.

[134] R. M. Clegg, A. I. Murchie, D. M. Lilley, Biophysical journal 1994, 66, 99-109.

[135] J. B. Rafferty, E. L. Bolt, T. A. Muranova, S. E. Sedelnikova, P. Leonard, A. Pasquo, P. J. Baker, D. W. Rice, G. J. Sharples, R. G. Lloyd, Structure 2003, 11, 1557-1567.

[136] A. Kimball, Q. Guo, M. Lu, R. P. Cunningham, N. R. Kallenbach, N. C. Seeman, T. D. Tullius, The Journal of biological chemistry 1990, 265, 65446547 .

[137] C. Joo, S. A. McKinney, D. M. J. Lilley, T. Ha, Journal of Molecular Biology 2004, 341, 739-751.

[138] M. Lu, Q. Guo, N. C. Seeman, N. R. Kallenbach, Journal of Molecular Biology 1991, 221, 1419-1432. 
[139] S. S. Martin, E. Pulido, V. C. Chu, T. S. Lechner, E. P. Baldwin, Journal of Molecular Biology 2002, 319, 107-127.

[140] J. H. Thorpe, B. C. Gale, S. C. Teixeira, C. J. Cardin, Journal of Molecular Biology 2003, 327, 97-109.

[141] O. Mendoza, M. Porrini, G. F. Salgado, V. Gabelica, J.-L. Mergny, Chemistry - A European Journal 2015, 21, 6732-6739.

[142] M. M. J. Smulders, I. A. Riddell, C. Browne, J. R. Nitschke, Chemical Society reviews 2013, 42, 1728-1754.

[143] R. Crawford, C. M. Erben, J. Periz, L. M. Hall, T. Brown, A. J. Turberfield, A. N. Kapanidis, Angewandte Chemie International Edition 2013, 125, 23402344 .

[144] L. L. K. Taylor, I. A. Riddell, M. M. J. Smulders, Angewandte Chemie International Edition 2019, 58, 1280-1307.

[145] J. S. Choi, C. W. Kang, K. Jung, J. W. Yang, Y.-G. Kim, H. Han, Journal of the American Chemical Society 2004, 126, 8606-8607.

[146] D. Mitra, N. Di Cesare, H. F. Sleiman, Angewandte Chemie International Edition 2004, 43, 5804-5808.

[147] M. Göritz, R. Krämer, Journal of the American Chemical Society 2005, 127, 18016-18017.

[148] H. Yang, F. Altvater, A. D. de Bruijn, C. K. McLaughlin, P. K. Lo, H. F. Sleiman, Angewandte Chemie International Edition 2011, 50, 4620-4623.

[149] H. Yang, C. K. McLaughlin, F. A. Aldaye, G. D. Hamblin, A. Z. Rys, I. Rouiller, H. F. Sleiman, Nature chemistry 2009, 1, 390-396.

[150] F. A. Pereira, G. H. Clever, Inorganica Chimica Acta 2016, 452, Metal-Nucleic Acid Interactions: State of the Art, 188-193.

[151] L. R. Holloway, P. M. Bogie, R. J. Hooley, Dalton transactions (Cambridge, England : 2003) 2017, 46, 14719-14723.

[152] A. M. Johnson, C. A. Wiley, M. C. Young, X. Zhang, Y. Lyon, R. R. Julian, R. J. Hooley, Angewandte Chemie International Edition 2015, 54, 5641-5645.

[153] Q.-F. Sun, S. Sato, M. Fujita, Angewandte Chemie International Edition 2014, 53, 13510-13513.

[154] S. Hiraoka, Y. Yamauchi, R. Arakane, M. Shionoya, Journal of the American Chemical Society 2009, 131, PMID: 19645469, 11646-11647. 
[155] D. A. Leigh, R. G. Pritchard, A. J. Stephens, Nature chemistry 2014, 6, 978-982.

[156] Spartan'10, version 1.1.0. Wavefunction, Inc. Irvine, CA. 2010.

[157] N. R. Kallenbach, R.-I. Ma, N. C. Seeman, Nature 1983, 305, 829-831.

[158] L. A. Howell, M. Searcey, Chembiochem : a European journal of chemical biology 2009, 10, 2139-2143.

[159] M. J. Cavaluzzi, Nucleic Acids Research 2004, 32, $13 \mathrm{e}$.

[160] J.-L. Mergny, L. Lacroix, Oligonucleotides 2003, 13, 515-537.

[161] M. D’Abramo, C. Castellazzi, M. Orozco, A. Amadei, J. Phys. Chem. B 2013, 117, 8697-8704.

[162] R. A. Cox, Biochemical Journal 1970, 120, 539-547.

[163] J.-L. Mergny, J. Li, L. Lacroix, S. Amrane, J. B. Chaires, Nucleic Acids Research 2005, 33, e138-e138.

[164] J. Kypr, I. Kejnovská, D. Renčiuk, M. Vorlíčková, Nucleic Acids Research 2009, 37, 1713-1725.

[165] M. Vorlíčková, I. Kejnovská, K. Bednářová, D. Renčiuk, J. Kypr, Chirality 2012, 24, 691-698.

[166] B. Ranjbar, P. Gill, Chemical Biology \& Drug Design 2009, 74, 101-120.

[167] T. Shida, H. Iwasaki, H. Shinagawa, Y. Kyogoku, The Journal of Biochemistry 1996, 119, 653-658.

[168] N. C. Stellwagen, Electrophoresis 2009, 30, S188-S195.

[169] A. R. Mount, C. P. Mountford, S. A. G. Evans, T. .-.J. Su, A. H. Buck, P. Dickinson, C. J. Campbell, L. M. Keane, J. G. Terry, J. S. Beattie, A. J. Walton, P. Ghazal, J. Crain, Biophysical Chemistry, Ion Hydration Special Issue 2006, 124, 214-221.

[170] P. Prasher, M. Sharma, Med. Chem. Commun. 2018, 9, 1589-1618.

[171] Z. Li, N. Kishi, K. Yoza, M. Akita, M. Yoshizawa, Chemistry - A European Journal 2012, 18, 8358-8365.

[172] Z. Li, N. Kishi, K. Hasegawa, M. Akita, M. Yoshizawa, Chemical Communications 2011, 47, 8605-8607.

[173] G. L. Eichhorn, Y. A. Shin, Journal of the American Chemical Society 1968, 90, PMID: 5725551, 7323-7328. 
[174] K. Thimmaiah, A. G. Ugarkar, E. F. Martis, M. S. Shaikh, E. C. Coutinho, M. C. Yergeri, Nucleosides Nucleotides \&6 Nucleic Acids 2015, 34, PMID: 25874941, 309-331.

[175] M. C. Gonzalez-Garcia, P. Herrero-Foncubierta, S. Castro, S. Resa, J. M. Alvarez-Pez, D. Miguel, J. M. Cuerva, E. Garcia-Fernandez, A. Orte, Frontiers in Chemistry 2019, 7, 129.

[176] In Principles of Fluorescence Spectroscopy, (Ed.: J. R. Lakowicz), Springer US, Boston, MA, 2006, pp. 277-330.

[177] F. Kaiser, A. Schmidt, W. Heydenreuter, P. J. Altmann, A. Casini, S. A. Sieber, F. E. Kühn, European Journal of Inorganic Chemistry 2016, 2016, $5189-5196$.

[178] Y. Huang, J. Xing, Q. Gong, L.-C. Chen, G. Liu, C. Yao, Z. Wang, H.-L. Zhang, Z. Chen, Q. Zhang, Nature Communications 2019, 10, 169.

[179] B. M. Laing, R. L. Juliano, Chembiochem 2015, 16, 1284-1287.

[180] C. J. Clarke, W.-C. Tu, O. Levers, A. Bröhl, J. P. Hallett, Chemical reviews 2018, 118, 747-800.

[181] C. Chen, G. Reniers in Sustainability and Life Cycle Assessment in Industrial Biotechnology, (Eds.: M. Fröhling, M. Hiete), Springer International Publishing, Cham, 2020, pp. 255-279.

[182] Y. Gu, F. Jérôme, Green Chemistry 2010, 12, 1127.

[183] T. Welton, Proceedings. Mathematical, physical, and engineering sciences 2015, 471, 20150502

[184] T. Welton, Green Chemistry 2011, 13, 225.

[185] P. Pollet, R. J. Hart, C. A. Eckert, C. L. Liotta, Accounts of chemical research 2010, 43, 1237-1245.

[186] R. Skouta, Green Chemistry Letters and Reviews 2009, 2, 121-156.

[187] P. Pollet, E. A. Davey, E. E. Ureña-Benavides, C. A. Eckert, C. L. Liotta, Green Chemistry 2014, 16, 1034-1055.

[188] H. C. Hailes, Organic Process Research \& Development 2007, 11, 114-120.

[189] M.-O. Simon, C.-J. Li, Chemical Society reviews 2012, 41, 1415-1427.

[190] D. Morrison, M. Rothenbroker, Y. Li, Small Methods 2018, 2, 1700319.

[191] A. Groß, C. Hashimoto, H. Sticht, J. Eichler, Frontiers in Bioengineering and Biotechnology 2016, 3, 211. 
[192] I. Nath, J. Chakraborty, F. Verpoort, Chemical Society Reviews 2016, 45, 4127-4170.

[193] D. L. Caulder, R. E. Powers, T. N. Parac, K. N. Raymond, Angewandte Chemie 1998, 110, 1940-1943.

[194] W. Cullen, M. C. Misuraca, C. A. Hunter, N. H. Williams, M. D. Ward, Nature chemistry 2016, 8, 231-236.

[195] K. Yazaki, Y. Sei, M. Akita, M. Yoshizawa, Chemistry - A European Journal 2016, 22, 17557-17561.

[196] M. Yamashina, S. Kusaba, M. Akita, T. Kikuchi, M. Yoshizawa, Nature Communications 2018, 9, 4227.

[197] T. B. Gasa, C. Valente, J. F. Stoddart, Chem. Soc. Rev. 2011, 40, 57-78.

[198] N. Busschaert, C. Caltagirone, W. van Rossom, P. A. Gale, Chemical reviews 2015, 115, 8038-8155.

[199] Z. D. Petrović, S. Marković, D. Simijonović, V. Petrović, Monatshefte für Chemie - Chemical Monthly 2009, 140, 371-374.

[200] Y. He, L. Wang, C. Cai, Journal of Applied Polymer Science 2011, 121, $286-291$.

[201] Z. D. Petrović, S. Marković, V. P. Petrović, D. Simijonović, Journal of molecular modeling 2012, 18, 433-440.

[202] P. Mal, D. Schultz, K. Beyeh, K. Rissanen, J. R. Nitschke, Angewandte Chemie International Edition 2008, 47, 8297-8301.

[203] L. Zanna, J.-F. Haeuw, Journal of chromatography. B, Analytical technologies in the biomedical and life sciences 2007, 846, 368-373.

[204] K. Kabiri, S. Faraji-Dana, M. J. Zohuriaan-Mehr, Polymers for Advanced Technologies 2005, 16, 659-666.

[205] J. C. Kim, M. Kim, J. Jung, H. Kim, I. J. Kim, J. R. Kim, M. Ree, Macromolecular Research 2012, 20, 746-753.

[206] Y. Chang, S. Chen, Q. Yu, Z. Zhang, M. Bernards, S. Jiang, Biomacromolecules 2007, 8, 122-127.

[207] V. Martí-Centelles, A. L. Lawrence, P. J. Lusby, Journal of the American Chemical Society 2018, 140, PMID: 29406705, 2862-2868.

[208] A. B. Grommet, J. R. Nitschke, Journal of the American Chemical Society 2017, 139, 2176-2179. 
[209] A. J. McConnell, C. J. E. Haynes, A. B. Grommet, C. M. Aitchison, J. Guilleme, S. Mikutis, J. R. Nitschke, Journal of the American Chemical Society 2018, 140, 16952-16956.

[210] T. Hayashi, Y. Ohishi, S. Hee-Soo, H. Abe, S. Matsumoto, M. Inouye, The Journal of organic chemistry 2018, 83, 8724-8730.

[211] D. Fujita, K. Suzuki, S. Sato, M. Yagi-Utsumi, E. Kurimoto, Y. Yamaguchi, K. Kato, M. Fujita, Chemistry Letters 2012, 41, 313-315.

[212] S. Zhai, Y. Ma, Y. Chen, D. Li, J. Cao, Y. Liu, M. Cai, X. Xie, Y. Chen, X. Luo, Polymer Chemistry 2014, 5, 1285-1297.

[213] M. Cai, M. Leng, A. Lu, L. He, X. Xie, L. Huang, Y. Ma, J. Cao, Y. Chen, X. Luo, Colloids and Surfaces B: Biointerfaces 2015, 126, 1-9.

[214] F. M. Bickelhaupt, E. J. Baerends, Angewandte Chemie International Edition 2003, 42, 4183-4188.

[215] F. Weinhold, Angewandte Chemie International Edition 2003, 42, 4188-4194.

[216] I. I. Schuster, A. K. Colter, R. J. Kurland, Journal of the American Chemical Society 1968, 90, 4679-4687.

[217] L. E. Harrington, J. F. Britten, M. J. McGlinchey, Canadian Journal of Chemistry 2003, 81, 1180-1186.

[218] G. Yamamoto, M. Kaneko, M. Ohkuma, M. Minoura, Chemistry Letters 2003, 32, 964-965.

[219] L. Li, A. Decken, B. G. Sayer, M. J. McGlinchey, P. Bregaint, J. Y. Thepot, L. Toupet, J. R. Hamon, C. Lapinte, Organometallics 1994, 13, 682-689.

[220] V. Blanco, D. A. Leigh, V. Marcos, J. A. Morales-Serna, A. L. Nussbaumer, Journal of the American Chemical Society 2014, 136, 4905-4908.

[221] V. Blanco, D. A. Leigh, V. Marcos, Chemical Society Reviews 2015, 44, $5341-5370$.

[222] Y. Yin, L. Yang, G. Zheng, C. Gu, C. Yi, C. He, Y. Q. Gao, X. S. Zhao, Proceedings of the National Academy of Sciences of the United States of America 2014, 111, 8043-8048.

[223] B. Lewandowski, G. de Bo, J. W. Ward, M. Papmeyer, S. Kuschel, M. J. Aldegunde, P. M. E. Gramlich, D. Heckmann, S. M. Goldup, D. M. D’Souza, A. E. Fernandes, D. A. Leigh, Science 2013, 339, 189-193. 
[224] G. de Bo, M. A. Y. Gall, M. O. Kitching, S. Kuschel, D. A. Leigh, D. J. Tetlow, J. W. Ward, Journal of the American Chemical Society 2017, 139, 10875-10879.

[225] A. Goswami, I. Paul, M. Schmittel, Chemical Communications 2017, 53, $5186-5189$.

[226] H. Ube, Y. Yasuda, H. Sato, M. Shionoya, Nature communications 2017, 8, 14296.

[227] G. S. Kottas, L. I. Clarke, D. Horinek, J. Michl, Chemical reviews 2005, 105, 1281-1376.

[228] C. A. Schalley, K. Beizai, F. Vögtle, Accounts of Chemical Research 2001, $34,465-476$.

[229] J. S. Hannam, S. M. Lacy, D. A. Leigh, C. G. Saiz, A. M. Z. Slawin, S. G. Stitchell, Angewandte Chemie International Edition 2004, 43, 3260-3264.

[230] A. Mateo-Alonso, C. Ehli, G. M. A. Rahman, D. M. Guldi, G. Fioravanti, M. Marcaccio, F. Paolucci, M. Prato, Angewandte Chemie International Edition 2007, 46, 3521-3525.

[231] J. Michl, E. C. H. Sykes, ACS Nano 2009, 3, 1042-1048.

[232] V. Balzani, A. Credi, F. M. Raymo, F. Stoddart, Angewandte Chemie International Edition 2000, 39, 3348-3391.

[233] N. Koumura, R. W. Zijlstra, R. A. van Delden, N. Harada, B. L. Feringa, Nature 1999, 401, 152-155.

[234] B. L. Feringa, Accounts of Chemical Research 2001, 34, 504-513.

[235] A. A. Kulago, E. M. Mes, M. Klok, A. Meetsma, A. M. Brouwer, B. L. Feringa, The Journal of Organic Chemistry 2010, 75, 666-679.

[236] M. D. Johnstone, E. K. Schwarze, J. Ahrens, D. Schwarzer, J. J. Holstein, B. Dittrich, F. M. Pfeffer, G. H. Clever, Chemistry - A European Journal 2016, 22, 10791-10795.

[237] C. Gropp, B. L. Quigley, F. Diederich, Journal of the American Chemical Society 2018, 140, 2705-2717.

[238] C. J. Brown, F. D. Toste, R. G. Bergman, K. N. Raymond, Chemical reviews 2015, 115, 3012-3035.

[239] O. Dimroth, R. Criegee, Chemische Berichte 1957, 90, 2207-2215.

[240] W. Chen, S. Wang, G. Yang, S. Chen, K. Ye, Z. Hu, Z. Zhang, Y. Wang, J. Phys. Chem. C 2016, 120, 587-597. 
[241] T. Takeda, H. Sugihara, Y. Suzuki, J. Kawamata, T. Akutagawa, The Journal of Organic Chemistry 2014, 79, 9669-9677.

[242] J. E. McMurry, M. P. Fleming, Journal of the American Chemical Society 1974, 96, 4708-4709.

[243] M. K. J. ter Wiel, R. A. van Delden, A. Meetsma, B. L. Feringa, Journal of the American Chemical Society 2005, 12\%, 14208-14222.

[244] E. Knoevenagel, Berichte der deutschen chemischen Gesellschaft 1898, 31, 2596-2619.

[245] W. Lehnert, Tetrahedron 1973, 29, 635-638.

[246] W. Lehnert, Tetrahedron 1974, 30, 301-305.

[247] T. Takeda, T. Akutagawa, Chemistry - A European Journal 2016, 22, 77637770 .

[248] K. D. Thériault, C. Radford, M. Parvez, B. Heyne, T. C. Sutherland, Physical chemistry chemical physics : PCCP 2015, 17, 20903-20911.

[249] M. Krick, J. J. Holstein, A. Wuttke, R. A. Mata, G. H. Clever, European Journal of Organic Chemistry 2017, 2017, 5141-5146.

[250] F. P. Gasparro, N. H. Kolodny, Journal of Chemical Education 1977, 54, $258-261$.

[251] Kirk Marat, Spinworks 4.2.4, University of Manitoba: Winnipeg, 2016.

[252] K. J. Laidler, M. C. King, J. Phys. Chem. 1983, 87, 2657-2664.

[253] O. Pytela, M. Pilný, M. Večeřa, Collection of Czechoslovak Chemical Communications 1984, 49, 1173-1181.

[254] N. Elgrishi, K. J. Rountree, B. D. McCarthy, E. S. Rountree, T. T. Eisenhart, J. L. Dempsey, Journal of Chemical Education 2017, 95, 197-206.

[255] E. Tsuchida, K. Oyaizu, E. L. Dewi, T. Imai, F. C. Anson, Inorganic Chemistry 1999, 38, 3704-3708.

[256] G. Lu, H. Yang, Y. Zhu, T. Huggins, Z. J. Ren, Z. Liu, W. Zhang, Journal of Materials Chemistry A 2015, 3, 4954-4959.

[257] Y. Patil, R. Misra, M. L. Keshtov, G. D. Sharma, J. Phys. Chem. C 2016 , $120,6324-6335$.

[258] M. V. Cooke, I. Malvacio, W. J. Peláez, A. J. Pepino, M. R. Mazzieri, G. A. Argüello, RSC Advances 2015, 5, 26255-26262. 
[259] Handbook of Computational Chemistry, 2nd ed. 2017, (Eds.: J. Leszczynski, A. Kaczmarek-Kedziera, T. Puzyn, M. G. Papadopoulos, H. Reis, M. K. Shukla), Springer International Publishing, Cham and s.l., 2017.

[260] I. Gaussian, Ed., Creating UV/Visible Plots from the Results of Excited States Calculations, 2017.

[261] M. J. Frisch, G. W. Trucks, H. B. Schlegel, G. E. Scuseria, M. A. Robb, J. R. Cheeseman, G., M. J. Frisch, G. W. Trucks, H. B. Schlegel, G. E. Scuseria, M. A. Robb, J. R. Cheeseman, G. Wallingford, CT, USA, 2009.

[262] J. Y. Lee, Y.-M. Lee, H. Kotani, W. Nam, S. Fukuzumi, Chemical Communications 2009, 704-706.

[263] J. Yuasa, S. Yamada, S. Fukuzumi, Angewandte Chemie International Edition 2008, 47, 1068-1071.

[264] P. Thordarson, Chemical Society Reviews 2011, 40, 1305-1323.

[265] D. Brynn Hibbert, P. Thordarson, Chemical Communications 2016, 52, 12792-12805.

[266] P. Wang, Y. Yao, M. Xue, Chemical Communications 2014, 50, 5064-5067.

[267] A. Liljas, L. Liljas, J. Piskur, G. Lindblom, P. Nissen, M. Kjeldgaard, Textbook of Structural Biology, WORLD SCIENTIFIC, 2009.

[268] V. R. Yella, D. Bhimsaria, D. Ghoshdastidar, J. A. Rodríguez-Martínez, A. Z. Ansari, M. Bansal, Nucleic Acids Research 2018, 46, 11883-11897.

[269] J. A. Erickson, M. Jalaie, D. H. Robertson, R. A. Lewis, M. Vieth, Journal of Medicinal Chemistry 2004, 47, 45-55.

[270] J. Janin, M. Sternberg, F1000 biology reports 2013, 5, 2-7.

[271] R. Huber, W. S. Bennett, Biopolymers 1983, 22, 261-279.

[272] C. Piguet, G. Bernardinelli, G. Hopfgartner, Chemical Reviews 1997, 97, 2005-2062.

[273] J. E. Beves, B. A. Blight, C. J. Campbell, D. A. Leigh, R. T. McBurney, Angewandte Chemie International Edition 2011, 50, 9260-9327.

[274] C. Desmarets, G. Gontard, A. L. Cooksy, M. N. Rager, H. Amouri, Inorganic chemistry 2014, 53, 4287-4294.

[275] Q.-Q. Wang, S. Gonell, S. H. A. M. Leenders, M. Dürr, I. Ivanović-Burmazović, J. N. H. Reek, Nature chemistry 2016, 8, 225-230.

[276] T. Murase, S. Sato, M. Fujita, Angewandte Chemie International Edition 2007, 46, 1083-1085. 
[277] P. Howlader, P. Das, E. Zangrando, P. S. Mukherjee, Journal of the American Chemical Society 2016, 138, 1668-1676.

[278] S. Bandi, A. K. Pal, G. S. Hanan, D. K. Chand, Chemistry - A European Journal 2014, 20, 13122-13126.

[279] R. W. Saalfrank, V. Seitz, D. L. Caulder, K. N. Raymond, M. Teichert, D. Stalke, European Journal of Inorganic Chemistry 1998, 1998, 1313-1317.

[280] Y. Chai, T. Guo, C. Jin, R. E. Haufler, L. P. F. Chibante, J. Fure, L. Wang, J. M. Alford, R. E. Smalley, J. Phys. Chem. 1991, 95, 7564-7568.

[281] E. Dietel, A. Hirsch, B. Pietzak, M. Waiblinger, K. Lips, A. Weidinger, A. Gruss, K.-P. Dinse, Journal of the American Chemical Society 1999, 121, $2432-2437$.

[282] K. Kurotobi, Y. Murata, Science 2011, 333, 613-616.

[283] H. Ueno, H. Okada, S. Aoyagi, Y. Matsuo, The Journal of organic chemistry 2017, 82, 11631-11635.

[284] S. Yang, F. Liu, C. Chen, M. Jiao, T. Wei, Chem. Commun. 2011, 47, $11822-11839$.

[285] F. Liu, F. Jin, S. Wang, A. A. Popov, S. Yang, Inorganica Chimica Acta 2017, 468, 203-208.

[286] J. Yang, B. Chatelet, V. Dufaud, D. Hérault, S. Michaud-Chevallier, V. Robert, J.-P. Dutasta, A. Martinez, Angewandte Chemie International Edition 2018, $57,14212-14215$.

[287] A. Schmidt, M. Hollering, M. Drees, A. Casini, F. E. Kühn, Dalton transactions (Cambridge, England : 2003) 2016, 45, 8556-8565.

[288] J. Han, A. Schmidt, T. Zhang, H. Permentier, G. M. M. Groothuis, R. Bischoff, F. E. Kuhn, P. Horvatovich, A. Casini, Chem. Commun. 2017, 53, 1405-1408.

[289] K. Harris, Q.-F. Sun, S. Sato, M. Fujita, Journal of the American Chemical Society 2013, 135, 12497-12499.

[290] P. M. Bogie, T. F. Miller, R. J. Hooley, Israel Journal of Chemistry 2018, 32, 975 .

[291] L. Zhao, K. Ghosh, Y.-R. Zheng, P. J. Stang, The Journal of organic chemistry 2009, 74, 8516-8521.

[292] S. Freye, D. M. Engelhard, M. John, G. H. Clever, Chemistry - A European Journal 2013, 19, 2114-2121. 
[293] M. Krick, J. Holstein, C. Würtele, G. H. Clever, Chemical Communications 2016, 52, 10411-10414.

[294] E. Persch, O. Dumele, F. Diederich, Angewandte Chemie International Edition 2015, 54, 3290-3327.

[295] L. C. Palmer, J. Rebek, Org. Biomol. Chem. 2004, 2, 3051-3059.

[296] M. D. Pluth, K. N. Raymond, Chemical Society Reviews 2007, 36, 161-171.

[297] M. Yoshizawa, J. K. Klosterman, M. Fujita, Angewandte Chemie International Edition 2009, 48, 3418-3438.

[298] V. Croué, S. Goeb, M. Sallé, Chemical communications (Cambridge, England) 2015, 51, 7275-7289.

[299] R.-J. Li, J. J. Holstein, W. G. Hiller, J. Andréasson, G. H. Clever, Journal of the American Chemical Society 2019, 141, 2097-2103.

[300] M. Han, R. Michel, B. He, Y.-S. Chen, D. Stalke, M. John, G. H. Clever, Angewandte Chemie International Edition 2013, 52, 1319-1323.

[301] M. Frank, J. Ahrens, I. Bejenke, M. Krick, D. Schwarzer, G. H. Clever, Journal of the American Chemical Society 2016, 138, 8279-8287.

[302] S. Saha, B. Holzapfel, Y.-T. Chen, K. Terlinden, P. Lill, C. Gatsogiannis, H. Rehage, G. H. Clever, Journal of the American Chemical Society 2018, 140, 17384-17388.

[303] T. W. Christian Reichardt, Solvents and Solvent Effects in Organic Chemistry (Fourth, Updated and Expanded Edition), 4th ed., Wiley-VCH, 2010.

[304] J. E. Lewis, J. D. Crowley, Supramolecular Chemistry 2014, 26, 173-181.

[305] G. H. Clever, S. Tashiro, M. Shionoya, Journal of the American Chemical Society 2010, 132, 9973-9975.

[306] A. Schmidt, A. Casini, F. E. Kühn, Coordination Chemistry Reviews 2014, 275, 19-36.

[307] M. Pan, K. Wu, J.-H. Zhang, C.-Y. Su, Coordination Chemistry Reviews 2019, 378, 333-349.

[308] Q. Zhang, L. Catti, K. Tiefenbacher, Accounts of Chemical Research 2018, 51, 2107-2114.

[309] B. Therrien, Top Curr Chem 2012, 319, 35-55.

[310] J. Kang, J. Rebek, Nature 1997, 385, 50-52.

[311] C. S. Linsley, B. M. Wu, Therapeutic delivery 2017, 8, 89-107. 
[312] P. Gupta, K. Vermani, S. Garg, Drug Discovery Today 2002, 7, 569-579.

[313] Y. Brudno, D. J. Mooney, Journal of controlled release : official journal of the Controlled Release Society 2015, 219, 8-17.

[314] T. Tagami, W. D. Foltz, M. J. Ernsting, C. M. Lee, I. F. Tannock, J. P. May, S.-D. Li, Biomaterials 2011, 32, 6570-6578.

[315] M. A. Azagarsamy, K. S. Anseth, Angewandte Chemie International Edition 2013, 52, 13803-13807.

[316] Wahajuddin, S. Arora, International journal of nanomedicine 2012, 7, 34453471 .

[317] S. R. Sirsi, M. A. Borden, Advanced drug delivery reviews 2014, 72, 3-14.

[318] C.-H. Yuan, R. West, Chem. Commun. 1997, 7, 1825.

[319] R. Velu, V. T. Ramakrishnan, P. Ramamurthy, Tetrahedron Letters 2010, 51, 4331-4335.

[320] E. N. Ushakov, M. V. Alfimov, S. P. Gromov, Macroheterocycles 2010, 3, 189-200.

[321] J. P. Dix, F. Vögtle, Chemische Berichte 1980, 113, 457-470.

[322] E. N. Ushakov, S. P. Gromov, O. A. Fedorova, M. V. Alfimov, Russian Chemical Bulletin 1997, 46, 463-471.

[323] M. P. Vladimirova, S. D. Simova, E. R. Stanoeva, M. I. Mitewa, Dyes and Pigments 2001, 50, 157-162.

[324] S.-i. Kondo, T. Takahashi, Y. Takiguchi, M. Unno, Tetrahedron Letters 2011, 52, 453-457.

[325] Y. X. Wu, J. Cao, H. Y. Deng, J. X. Feng, Spectrochimica acta. Part A, Molecular and biomolecular spectroscopy 2011, 82, 340-344.

[326] H. Zheng, D. R. Cooper, P. J. Porebski, I. G. Shabalin, K. B. Handing, W. Minor, Acta crystallographica. Section D, Structural biology 2017, 73, 223-233.

[327] T. Kuwabara, S.-n. Machida, R. Satake, Supramolecular Chemistry 2018, 30, $425-429$.

[328] S.-H. Gao, M.-S. Xie, H.-X. Wang, H.-Y. Niu, G.-R. Qu, H.-M. Guo, Tetrahedron 2014, 70, 4929-4933.

[329] H. Zhou, J. Wang, Y. Chen, W. Xi, Z. Zheng, D. Xu, Y. Cao, G. Liu, W. Zhu, J. Wu, Y. Tian, Dyes and Pigments 2013, 98, 1-10. 
[330] J. Eberhard, K. Peuntinger, R. Fröhlich, D. M. Guldi, J. Mattay, European Journal of Organic Chemistry 2018, 2018, 2682-2700.

[331] V. V. Volchkov, F. E. Gostev, I. V. Shelaev, V. A. Nadtochenko, S. N. Dmitrieva, S. P. Gromov, M. V. Alfimov, M. Y. Melnikov, Journal of fluorescence 2016, 26, 585-592.

[332] R. Welz, S. Müller, Tetrahedron Letters 2002, 43, 795-797.

[333] G. Y. Di Veroli, C. Fornari, I. Goldlust, G. Mills, S. B. Koh, J. L. Bramhall, F. M. Richards, D. I. Jodrell, Scientific reports 2015, 5, 14701.

[334] K. Amirbekyan, N. Duchemin, E. Benedetti, R. Joseph, A. Colon, S. A. Markarian, L. Bethge, S. Vonhoff, S. Klussmann, J. Cossy, J.-J. Vasseur, S. Arseniyadis, M. Smietana, ACS Catalysis 2016, 6, 3096-3105.

[335] A. Rioz-Martinez, J. Oelerich, N. Segaud, G. Roelfes, Angewandte Chemie International Edition 2016, 55, 14136-14140.

[336] B. Jash, J. Neugebauer, J. Müller, Inorg. Chim. Acta 2016, 181-187. 
